106

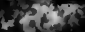

b



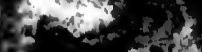

,

$=0$

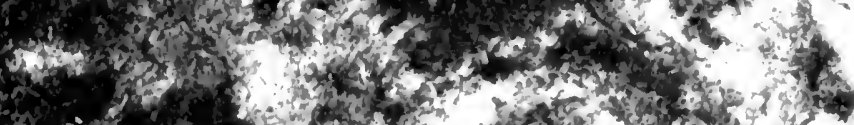

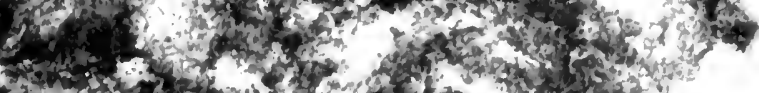

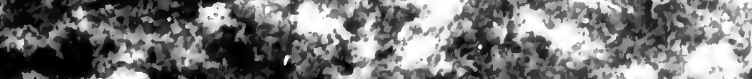

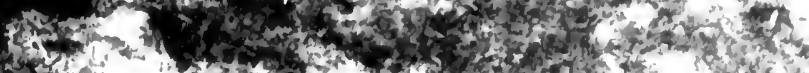

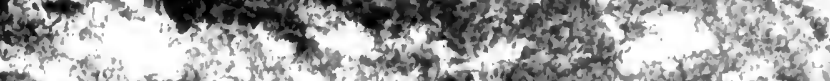

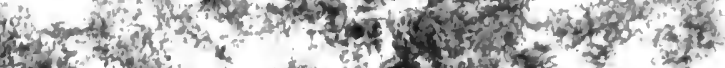

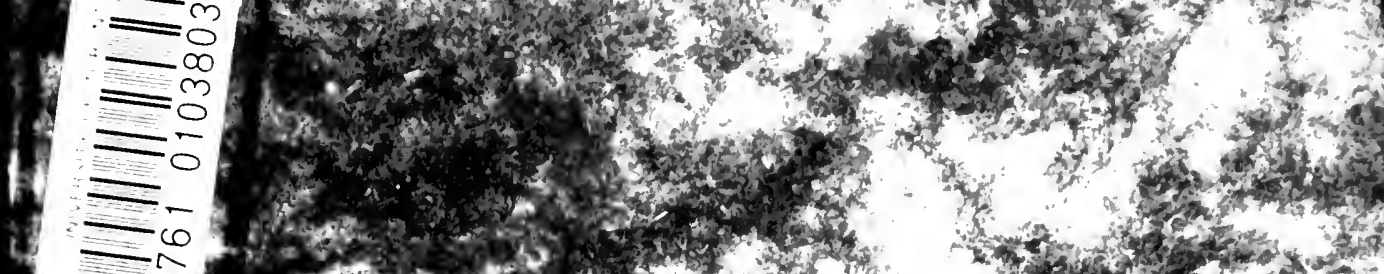

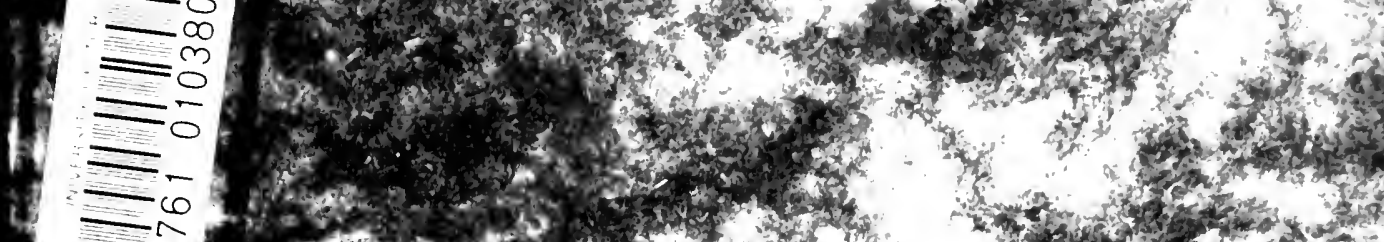

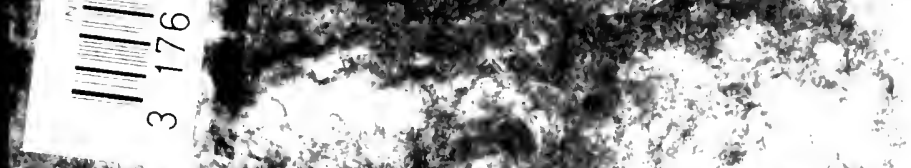

4 a

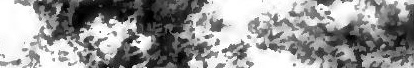

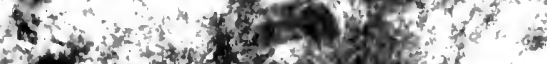

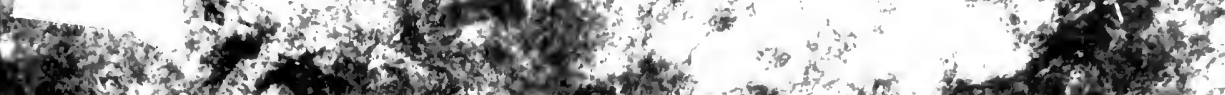

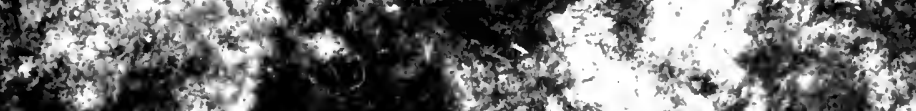

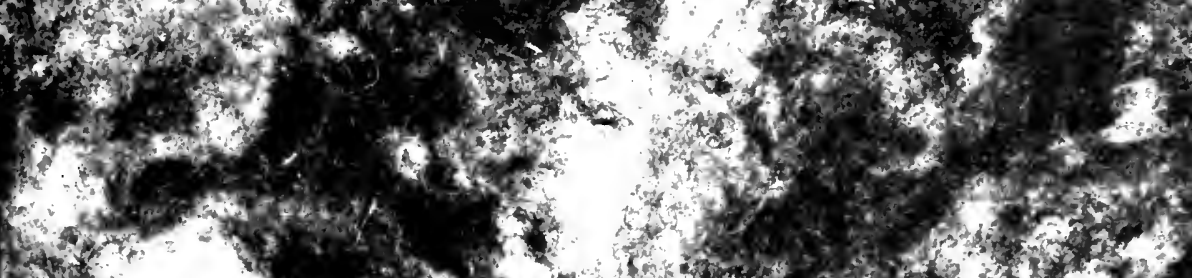

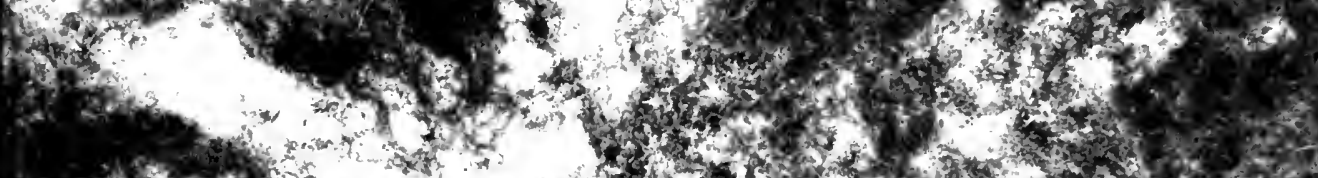

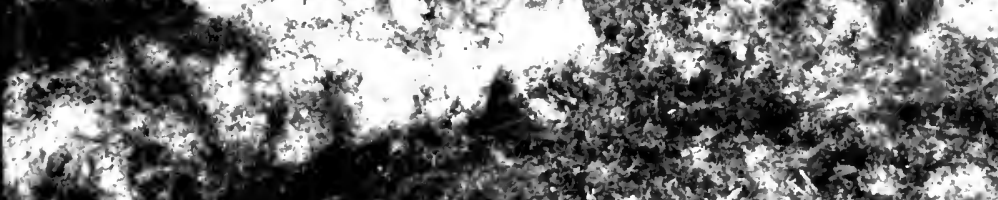
67.

(4)

1)

$x^{2}$

$4 \times$

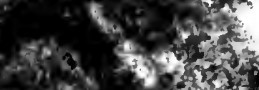

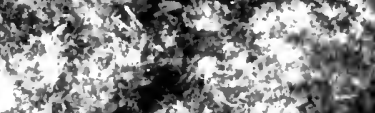

toth

B.

Wextis 



\section{EINFÜHRUNG; \\ IN DIE THEORIE DER \\ ALGEBRAISCHEN FUNKTIONEN \\ EINER VERÄNDERLICHEN}

$\operatorname{lol})$

\section{HEINRICH W. E. JUNG}

o. ö. Professor der Mathematik an der Lniversität Halle-Wittenberg

MIT 35 ABBILDUNGEN IN TEXT
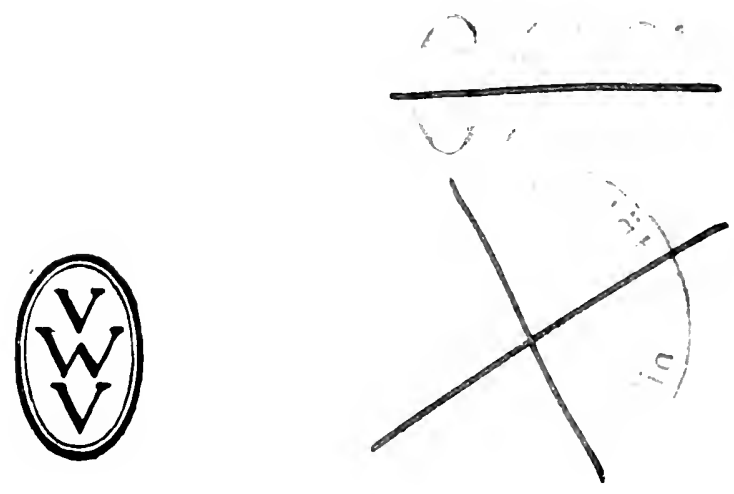

VEREINIGUNG WISSENSCHAFTLICHER

VERLEGER WALTER DE GRUYTER \& CO.

VORMALS G. J.GÖSCHEŇCHE VERLAGSHANDLCNG

J. GLITENTAG, VERLAGSBCHIANDLLNG - GEORG

REIMER - KARL J. TRÜBNER - VEIT \& COMP

BERLIN W. O UND LEIPZIC, 


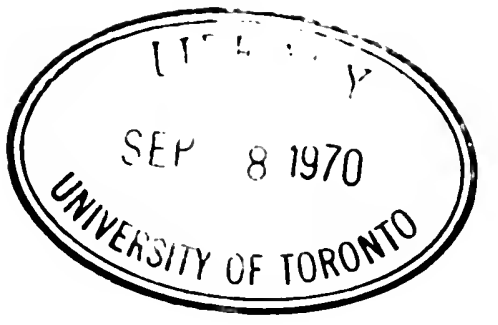

Alle Rechte, insbesondere das Úbersetzungsrecht. ron der Verlagshandlung roubehalten. 


\section{Inhaltsverzeichnis.}

Eiuleitun:

Erstes Kapitel.

Definition einer algebraischen Funktion $y$ von $x$ und ihr Verhalten in der Vingebung eines Wertes von $x$. . . . . . . . . . . . . . . . . .

$\S 1$. Definition der algebraischen Funktion. Singulare Werte der unabhängigen Veränderlichen

$\$ 2$. Verhalten von $y$ in der Lngebung eines nicht singulären Wertes von $x$

$\$ 3$. Verhalten von $y$ in der Umgebung eines singulären Wertes von $x$. .

\$4. Ein Beispiel. . . . . . . . . . . . . . . . . . . . . . . . . . 10

$\$ 5$. Ein zweites Beispiel . . . . . . . . . . . . . . . . . . . . . . 11

$\S 6$. Fortsetzung von $\S 3$. . . . . . . . . . . . . . . . . . . . . 13

$\xi$. Verhalten von $y$ in der Lmgebung eines Wertes von $x$, für den $y$ unendlich wird. . . . . . . . . . . . . . . . . . . . . . . . 17

§ 8. Verhalten von $y$ in der Umgebung von $x=\infty$. . . . . . . . . 1k

\section{Kweites Kapitel.}

Die Berechunng der Reilenentwicklungen für $y$ in der Lugebung irgendeiner Stelle

$\S 1$. Vorbetrachtungen

\$2. Das Diagramm von Pujseux . . . . . . . . . . . . . . . . . . 21

§3. Beispiele . . . . . . . . . . . . . . . . . . . . . . . 26

Drittes liapitel.

Jie Riemannsche Fläche .

$\$ 1$. Die $m$ konjugierten Funktionen . . . . . . . . . . . . . . . . . 32

.. Peispiel 1. . . . . . . . . . . . . . . . . . . . . . . 34

$\$ 3$. Beispiel 2. . . . . . . . . . . . . . . . . . 37

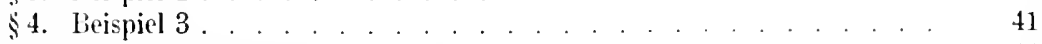

$\$$ o. Konstruktion der Riemannschen Fläche . . . . . . . . . . . . t3

$\$$ 6. Zerfallen der Riemannschen Fläche . . . . . . . . . . . . . . . 13

$\$$ 7. Die zweiblïttrige Riemannsche Flïche. . . . . . . . . . . . . . $\$ 5$

\$8. Die Punkte der Riemannschen Flïche und ihre Umgebung . . . . 45

Viertes Kapitel.

Der algebraische Körper $(x y)$ und seine Funktionen . . . . . . . . . $\quad 4 y-61$

§. Definition des Körpers. . . . . . . . . . . . . . . . . . 49

§2. Gleichung, der eine Funktion des Körpers genïgt. . . . . . . . . . 50 
\$3. Verhalten der funktionen des Kïrpers in dor Lingebung eines Punktex der Riemannschen Fläche.

4. Xull- und linendlichkeitsstellen . . . . . . . . 54

s.. Resiluen . . . . . . . . . . . . . . . 55

\$f. Ordnung einer Funktion . . . . . . . . . . . . . . . 60

: 7. Charakteristische Eigenshaton riner algebraisohen Funktion... bl

$$
\text { Fiinftrs kapitrl. }
$$

Birationale Transformationes

$\S 1$. Definition der birationalen Transformation . . . . . . . . . . . 62

$\$$ 2. Beispiel 1. . . . . . . . . . . . . . . . . . . . . . . 63

\$ 3. Beispiel : . . . . . . . . . . . . . . . . . . . 64

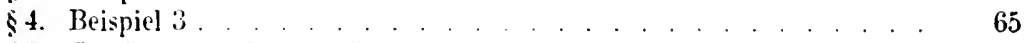

$\$ 5$. Bestimmung riner Gröbe $y$ zu einer (iröbe ș, so dab die Körper $(x y)$

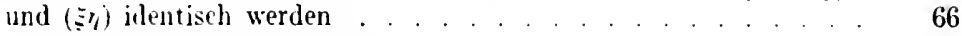

\$ 6. Anwendung auf die Beispiele "2 und 3 . . . . . . . . . 68

ז. Die bejklen Riemannsehen Flächen. . . . . . . . . . . . 70

$$
\text { Sirohstes Kapitel. }
$$

Hivisoren

1. Primteiler.

\$. Zerlegung der Funktionen des körpers in Primteiler . . . . . . 72

\$. Verzweigungsdivisoren . . . . . . . . . . . . . . . . 73

4. Divisorenklassen. . . . . . . . . . . . . . . . . . . 74

\$5. Dimension einer klasse. . . . . . . . . . . . . . . . 75

6. Die kanonische Klasse . . . . . . . . . . . . . . . . . . 78

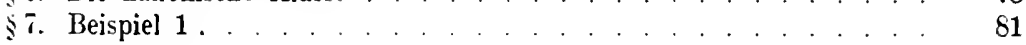

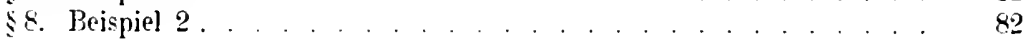

Siebentes Kapitel.

Rechnen mit systemen

\$1. Lineare Substitutionen.

\$... Inutiplikation von Systemen _ . . . . . . . . . . . . . . . 86

\$3. Quadratische Systeme . . . . . . . . . . . . . . . . . . . . 88

4. Einige besondere Systeme . . . . . . . . . . . . . . . . . 91

$\$$ 5. Quadratische Systeme, deren tlemente Funktionen einer Veränderliehen $x$ sind

\$

Achtes Kapitel.

Dir Virlarihen eines Divisor. $100-144$

\$. Jje Aufgabe . . . . . . . . . . . . . . . . . . 100

\$.. Ein Beispiel. . . . . . . . . . . . . . . . . . . . 100

\$. Basen des Kïrpers. . . . . . . . . . . . . . . . . 104

\$. Basis für die Vielfachen eines Vivisors. . . . . . . . . . . . 109

\$5. Normaltsasen. . . . . . . . . . . . . . . . . . 111

§ 6. Herstellen einer Normalbasis 1 . . . . . . . . . . . . . . . . . 113

§ 7 . Herstellen einer Normalbasis II . . . . . . . . . . . . . 116 
\$.. Herstellen einer Normalbasis 111 . . . . . . . . . . . . . . 122

\$9. Die Diskriminante einer Basis für die Vielfachen eines Divisors $a$. . 125

10. Beispiel 1. . . . . . . . . . . . . . . . . . . . . 126

11. Beispiel … . . . . . . . . . . . . . . . . . . . . . . . . . 134

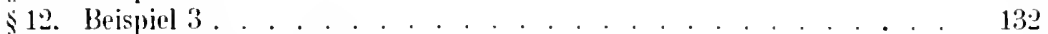

13. Komplementïre Basen . . . . . . . . . . . . . . . . . . . 133

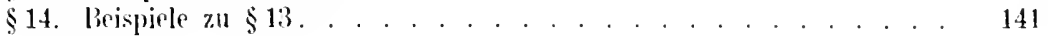

Neuntes liapitel.

I).r Riemann-Rochsche Satz.

$144-171$

1. Die ganzen Divisoren einer Klasse. . . . . . . . . . . . . . . . 144

.. Der Riemannsche Satz . . . . . . . . . . . . . . . . . . . . . 149

3. Der Riemann-Rochselıe Satz . . . . . . . . . . . . . . . . . . 150

4. Zahl der linear muabhängigen Differentiale erster Gattung . . . . . 152

5 . Funktionen mit gegebenen Unendlichkeitsstellen . . . . . . . . . 153

$\$$ 6. Körper vom Gesehlechte $p=0$. Der Lürothsehe Satz. . . . . . . $15 \overline{5}$

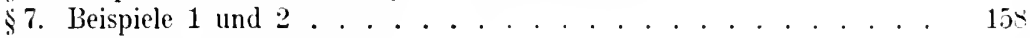

\$. Körper rom Geschlechte 1 und 2. . . . . . . . . . . . . . . . 161

\$. Beispiel 3 und 4. . . . . . . . . . . . . . . . . . . . . . 163

\$10. Weierstraßpunkte . . . . . . . . . . . . . . . . . 171

\section{Zehntes Kapitel.}

Die Kinrve $f(x, y)=0$. . . . . . . . . . . . . . . . . . . . . . . . 171-188

$\$ 1$. Die mehrfachen Punkte. . . . . . . . . . . . . . . . . . . . . 171

$\$ 2$. Die Kurrenzweige . . . . . . . . . . . . . . . . . . . . . . . 174

\$3. Gestalt reeller liurvenzweige . . . . . . . . . . . . . . . . . 175

$\$ 4$. Zahl der Schnittpunkte zweier Kurvenzweige . . . . . . . . . . 179

$\$$ ó. Der Divisor der mrhrfachen Punkte. . . . . . . . . . . . . . . 182

$\$ 6$. Beispiele . . . . . . . . . . . . . . . . . 184

$\$ 7$. ZahI der Schnittpunkte zweier linrven. . . . . . . . . . . . . . 186

s.s. Eine Formel für das Giesehlecht des Kürpers. . . . . . . . . . . 1sh

Elftes Kapitel.

bibene algebraische Kurven in homogenen Koordinaten

$1+5-213$

1. Definition der Kinre. . . . . . . . . . . . . . . . . . . . . . 188

\$.. Die Reziprokalkurve . . . . . . . . . . . . . . . . . . . . . 190

3. Die Divisoren $r$ und $\mathbf{r}^{\prime}$. . . . . . . . . . . . . . . . . . . . . 198

4. Der Divisor der nehrfachen l'unkte und der der mehrfachen Tangenten 195

$\$$. Die Hessesche Kurve. . . . . . . . . . . . . . . . . . . . . . 196

$\$$ 6. Plïckersche Formelı . . . . . . . . . . . . . . . . . . . . . . 197

†. Zahl der Sehnittpunkte zweier Kurvm. . . . . . . . . . . . . . 198

$\$$ s. Unendlich ferne Punkte . . . . . . . . . . . . . . . 198

\$9. Der Noethersche Satz. Adjungierte Funktionen. . . . . . . . . . $\quad 200$

10. Beispirle . . . . . . . . . . . . . . . . . . . . . . 204

\section{Zwöltes Kapitel.}

Ije Art des Zusammenhanges einer Riemannschen Fläche . . . . . . . . . 214-20.:

$\S 1$. Definitionen. . . . . . . . . . . . . . . . . . . . . . . 214

\$. Einfach zusammenhängende Flächen. . . . . . . . . . . . . . . 215 
Seite

si. lie Crduung des Zusammenhanges einer Fläche . . . . . . . . 216

4. Brispiete. . . . . . . . . . . . . . . . 218

5. Zerlegung einer Fläche in eine einfach zusammenhängende. . . . . 219

6. Ordnung des Zusammenhanges einer Riemannschen Fliache. . . . . 229

:. Kanonische Zerschneidung der Riemannschen Fliche . . . . . . 203

\section{Wreizehntes kapitel.}

lie lntegrale. . . . . . . . . . . . . . . . . . . . . . 226-244

\$ 1. Definition der Integrale. . . . . . . . . . . . . . . . . . . . . 226

ร... Verhalten der Integrale in der Lngebung einer stelle. . . . . . . 228

3. Verhalten ter Integrale anf der Riemannschen Fläche. . . . . . 229

4. Nie Periodizititsmoduln . . . . . . . . . . . . . . . . 231

5. Integrale erster Gattung. . . . . . . . . . . . . . . . . . 234

6. Integrale zweiter Gattung. . . . . . . . . . . . . . . . . 235

§. Elementarintegrale zweiter Gatming . . . . . . . . . . . . . 236

§. Der Weierstrabsche Lïckensatz . . . . . . . . . . . . . . 239

\$9. Integrale dritter Gattung. . . . . . . . . . . . . . . . . 242

Namen- und sachregister . . . . . . . . . . . . . . 245-246 


\section{Einleitung.}

Die Entduekung der analytisch-geometrisehen Methode dureh Deseartes gab den Anlaß, Kurven zu untersuchen, die durch eine algebraisebe Gleichung zwischen den Koordinaten gegeben waren. Dabei botrachtete man dann auch die eine Koordinate als Funktion der andern und hatte so dom Wesen nach den Begriff der algebraischen Funktion. Newtrn war der erste, der Reihenentwicklungen benutzte, um die Ordinate als Funktion der Abszisse darzustellen. Zur Bestimmung der Exponenten und koeffizienten benutzte er eine graphische Methode, das nach ihm oder auch nach Puiseux benannte Diagramm. Aber Newton lag nicht so sehr daran, die algebraische Funktion zu untersuchen als die Gestalt der algebraischen Kurve zu ermittehn. Auch beschränkte er sich auf reell. Werte. Ein weiterer Fortsehritt konnte erst kommen, als die Funktionentheorie durch Cauchy eine Entwicklung erlangt hatte, die eine Anwendung auf die algebraisehen Funktionen gestattete und als dureh Gaufs und Cauchỵ die imaginären Zahlen Bürgerreeht in der Mathematik erlangt hatten. Cauchy zeigte, in welcher Art sich algehraische Funktionen in der Umgebung einer regulären oder singulären Stelle in Reihen entwickeln ließen. Puiseux (1850) zcigte im Anschluß an das Nowtonsche Verfahr'n, wie diese Reihen praktisch berechnet werden konnte'n, und ving über Cauchy hinaus, indem er die algebraische Funktion nicht nur in der Ungehung einer Stelle betrachtete, sondern den Verlauf der ganzen mehrwertigen Funktion untersuchte. Auch dir Integrale algebraiseher Funktionen und ihre Perioden zog er in den Kreis seiner Betrachtungen, ohn" aber zu voller Klarheit über die vorliegenden Verhältnisse gelangen zu können.

Erst Riemann brachte das Verhalten der mehrdeutigen algebraisehen Funktion zu voller Anschaulichkeit, indem er sie als eindeutige Funktion auf einer mehrblättrigen Fläche, die nach ihm benannt wurde, auffaßte (1857). Rixmann ging bei der Untersuchung der algehraisehen Funktionen von ihren Integralen aus, benutzte also transzendente Hilfsmittel. Integrale von algebraischen Funktionen waren schon früh betrachtet, zunächst die elliptisehen Integrale, dann hyperelliptische. Erst als Abcl (1826-1829) in seinem berühmten Theorem zeigte, daß nicht nur für diese Integrale, sondern allgemein für Integrale beliebiger algehraischer 
Funtionen oin .riditionstheorem bestand, begann man sieh auch mit

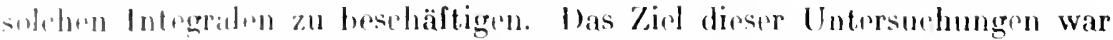
die Lïsung des von Jacoli (1832) formulierten Umkehrproblems von Integralen algobraiseher Funktionen, das zor ciner Verallgemeinerung der olliptischen Funktionen auf periodische Funktionen mehrerer Veränderlinher finhte. liemann st ïtztr sich auf dir lirgehnisse dieser Untersurhumen und der von Cauchy und P'uiseux, und es gelang ihm, die llaupteigensehaften der algohraisehen Funktionen und ihrer Integrale zu cuthitten. So pinfach die beweise von Riemann auch sind, so hat seine Methode dow wesentiehe Mängel. Kiemann dofiniorte die Integrale und Funktion'n dureh ihro charaktristischen Eignschaften und bewies ihre Existonz mit llilfe des l)irichletschen Prinzips. Aber der Beweis dieses l'rinzijs prfordort Hilfsmittel, die gar nichts mit den algebraischen Funktionen zu tun haben. Ferner ist zwar die Existenz der Integrale gesiehert, aber man hat keine Methode, sie wirklich aufustellen. Und zu den Sätzen übur die algobraischen Funktionen kommt Riemann orst auf dem Unwege üler die Integrale. So ist auch der Beweis des RiemannRochschen Satzes zuerst auf diesem Wege geführt.

E; lag nahe zu versuchen, die schönen Ergebnisse Riemanns unmittelbar durch Ausgang von der die algebraische Funktion definierenden Gleichung zu rrhalten. Im wesentlichen sind es drei Wege, die zu diesem Ziel greührt haben.

1. Die algebraisch-geometrische Methode. Begonnen von Clebsch und Jordan, wurde sio von Brill und Noether ganz ohne Benutzung transzendenter llilfsmittel ausgebildet. Die Grundlage dieser Theorie hildet der Noethersche Satz. Es wird eine algebraische Kurve $f$ zugrunde gilegt. Irgendeine andere Kurve $g$ schneidet auf $f$ eine Punktgruppe aus. Wenn aber umgekchrt irgendeine Punktgruppe auf $f$ gegeben ist, sr) gibt es im allgemeinen keine Kurve $g$, die diese ausschneidet. Es werden dann linear Kurvenscharen betrachtet. Eine solche Sehar kann mit $f$ feste und bewegliche Schnittpunkte haben. Zwei Punktgruppen, $C_{1}$ und $G_{i}$, die aus beweglichen Schnittpunkten bestehen und von zwei Kurven d.r. Schar ausgeschnitten werden, heißen äquivalent. Sind $g_{1}=0$ und $g_{2}=0$ dir Gleichungen der Kurven, die $f$ außer in den festen Punkten in den Gruppen $G_{1}$ und $G_{2}$ schneiden, so ist der Quotient von $g_{1}$ und $g_{2}$ eine rationale Funktion der Koordinaten, die an den Stellen $G_{1}$ Null und an den Stellen $G_{2}$ umendlich wird. Sind umgekehrt $G_{1}$ und $G_{2}$ irgend zwei Punktgrupera auf $f$, so gibt es im allgemeinen keine rationale Funktion der Korordinaten, die nur an den Stellen $G_{1}$ Null und nur an den Stellen $G_{2}$ unendlich wird. Zunächst mul die Anzahl der Null- und Unendlichkeitsstellar discolle sein. Aber diese Bedingung ist im allgemeinen keineswegs hinrrithend, sonderx man kann nur einen Teil der Null und Un- 
endlichkeitsstellen vorschreiben. Eine bestimnte: Anzahl $p$ ist dureh die andern mitbestimmt. Diese Zahl $p$ ist das Geshlonlat der Kurve. Öber diese Verhältnisse gibt der Rirmann-Roclssche Sat\% Aufsehlub, und or wird bei der geometrisehen Methode dureh dir Untorsuchung äquivalenter Punktgruppen bewiesen. Iliese Untersuchung wird durch den Noetherschen Sat\% wesentlich vereinfaelst, indem mit ihm gezeigt wird, daß man sich auf solehe Punktgruppen besehränken kann, die durch dir sogenannten adjungicrten Kurven ausgesehnitten werden. Die Hauptsehwierigkeit bestand in der Bestimmung der Zahl der Sehnittpunkte, die awei Kurven $f$ und $g$ in einem Punkte haben, wo sie beide singulär sind. Es gelang aber Noether, diese Schwierighrit vollkommen zu überwinden, indem er zeigte, wie man eine noch so komplizierte Singularität sich aus einfachen Singularitäten zusammengesetzt denken kann.

2. Die funktionentheoretisehe Methode. Es ist die Methode von Weierstraß. Er zeigt zunächst, wie sich eine algebraische Funktion $y$ einer unabhängigen Veränderlichen $x$ in der Umgebung einer Stelle der unabhängigen Veränderlichen verhält. Dann betrachtet er die Gesamtheit aller rationalen Funktionen von $x, y$, also den Körper $(x y)$ und geht zu der Aufgabe über, Funktionen mit $\varrho$ gegebenen Unendlichkeitsstellen herzustellen. Es zeigt sich, daß bei beliebiger Wahl der $\varrho$ Stellen die Aufgabe nur möglich ist, wenn $\varrho$ mindestens gleich $p+1$ ist, wo $p$ das Geschlecht der algebraischen Funktion ist. Weierstraß definiert das Geschlecht geradezu durch diese Eigenschaft. Sind dann $p$ Stellen beliebig aber fest gewählt und ist $p$ irgendeine andere Stelle, so zeigt Weierstraß, daß es immer eine Funktion $g_{i}(p)$ gibt, die an den $p$ festen Stellen von der ersten Ordnung und an der Stelle \& genau von der $\lambda$-ten Ordnung unendlich wird. Damit kann Weierstraß irgendeine algebraische Funktion $R$ des Körpers gewissermaßen in Partialbrüche zerlegen. Wird $R$ an den Stellen $p_{1}, p_{2}, \ldots p_{1}$ unendlich und in $p_{i}$ von der Ordnung $\lambda_{i}$, so läßt sich $R$ in der Form darstellen

$$
\frac{\sum}{i}\left(a_{11} g_{1}\left(k_{i}\right)+a_{z i} g_{2}\left(k_{i}\right)+\cdots+a_{\lambda_{i} i} g_{\lambda_{i}}\left(f_{i}\right)\right) \text {, }
$$

wo die $a_{k l}$ konstant sind. Diese müssen dann noch $p$ linearen Gleichungen genügen, die ausdrücken, daß $R$ in den $p$ festen Stellen nicht unendlich wird. So ergibt sich der Riemann-Rochsche Satz.

Weierstraß leitet dann die Eigensehaften der Integralo her, und zwar ohne Benutzung der Riemannschen Fläche.

3. Die arithmetische Methode. Während Weierstraß die Funktionen des Körpers $(x y)$ charakterisiert durch Angabe ilhrer Unendlichkeitsstellen und der Art ihres Unendlichwerdens und so zu einer Verallgemeinerung der Partialbruchzerlegung der rationalen Funktionen kommt, eharakterisieren Dedekind und Weber die Funktionen dureh 
Einleitung.

Angahe ihrer Xill- und Unendlichkeitsstellen. Ihre Methode ist whr vereinfacht durch Hensel und Landsberg. Da man, wie schon oben gesagh, die Null- und Unendlichkeitsstellen einer Funktion nicht sämtlich beliehig vorschreiben kann, sı worden zunächst mur dicjenigen Funktionen bestimmt, die an gegebenen endlichen Stellen mindestens von gegebener Grdnung Null und höehatens von gegebener (Ordnung unendlich werden. Aus diesen werden dann diejenigen ausgesurht, die sich auch im Un"ndlichen in gewünsehter Wrise verhalten. Die gefundenen Funktionen haben dann auber den angegubenen keine Unendlichkeitsstellen, wohl aber im allgemeinen noch $p$. vullstellen, auf dir man keinen Einflub hat.

Lås folgende Buch soll eine Einführung in die Theorie der algebraischen Funktionen sein. Ich habe mich dalıer irn Stoffumfang beschränkt und mich bemüht, die benutzten llothoden durch einfache Zahlenbeispiele zu erläutern, und hoffe, daß der Leser mit den grundlegenden Methoden lurch das studium dieses Buches hinreichend vertraut wird, um dann umfangruichere Larstellungen lejcht verstehen zu können. Im wesentlichen sind die Methoden von Hensel und Landsberg benutzt.

Von ausführlichen Darstellungen der Theorie der algebraischen Funktionen seien empfohlen:

Franceseo Severi, Vorlesungen über algebraisehe Geometrie, Geometrir auf einer Kurva, Riemannsehe Flächen, Abelsche Integrale. Deutsch von E. Löffler. (B. G. Teubner, Leipzigu. Berlin 1921.) Hier werden die BrillVoethersehen Methoden benutzt.

Karl WeirstraB, Vorlesungen über die Theorie der Abelschen Transzendenteri. Grommelte mathematische Werke, Bd. IV.

Kurt [Hense] und Georg Landsberg, Theorie der algebraischen Funktionen ciner Variabeln und ihre Anwendung auf algebraische Kurven und Abrasche Integrale. (B. G. Teubner, Leipzig 1902.) Im folgenden zitiert mit $\mathrm{H}-\mathrm{L}$.

Zusammenfassende Darstellungen, die auch vollständige Literaturangahen enthalten, sind:

A. Brill und M. Noether. Die Entwicklung der Theorie der algebraischen Furktionen in älterer und neuerer Zeit. Jahresb. d. deutsch. Nath. Ver. 3 (1894).

EnzykJopädie der mathematischen Wissenschaften, II B 2, Algebraische Funktionen und ihre Integrale von W. Wirtinger (1901), und IIC5, Arithmetische Theorie der algebraischen Funktionen von K. Hensel (1921). 
Erstes $\mathrm{K}$ apitel.

\section{Definition einer algebraischen Funktion $y$ von $x$ und ihr Verhalten in der Umgebung irgendeines Wertes von $x$.}

\section{$\$ 1$. Definition der algebraischen Funktion. Singuläre Werte der unabhängigen Veränderlichen.}

Es sei

$$
f(x, y)=a_{0}(x) y^{m}+a_{1}(x) y^{m-1}+\cdots-a_{m}(x)
$$

eine ganze unzerlegbare rationale Funktion ron $x, y$. Sie sei in $x$ vorn Grade $l$ und in $y$ vom Grade $m$. Sie sei also, wie wir kurz sagen wollen, in $x, y$ rom Grade $(l, m)$. Durch die Gleichung

$$
f(r, y)=0
$$

wird $y$ als Funktion von $x$ definiert. Man nenut die so definierte Funktion eine algebraische Funktion.

Zu einem endlichen Werte von $x$ ergeben sich aus (l) im allgemeinen $m$ verschiedene und endliche Werte von $y$. Unendlich grobe Werts von $y$ können sich nur für solche Werte von $x$, die Nullstellen von $a_{0}(x)$ sind, oder für unendlich große Werte von $x$ ergeben. Gleiche endliche Wertw von $y$ rrhalten wir nur für die Werte von $x$, die Nullstellen der Ijiskriminante $D(x)$ von $f(x, y)=0$ sind. Diese Dishriminante $D(x)$ ist eine ganze rationale Funktion von $x$. Sie verschwindet nicht identisch, da wir angenommen haben, daß $f(x, y)$ unzerleghar ist, also heinen quadratischen Faktor hat und daher die Gleichung $f(x, y)=0$ nicht für alle Verte von $x$ gleiche Wurzeln haben kann. Bezeichnen wir die $m$ Werte, die $y$ für einen Wert von $x$ annimmt, mit $y_{1}, y_{2}, \ldots y_{m}$, so können wir schreiben:

$$
f(x, y)=a_{0}\left(y-y_{1}\right)\left(y-y_{2}\right) \cdots\left(y-y_{m}\right)
$$

und

$$
D(x)=a_{0}^{m} \prod_{i=1}^{m}\left(y_{i}-y_{u}\right)=\prod_{i=1}^{m} \frac{\partial f}{\partial y}\left(x, y_{i}\right) \text {, }
$$

wo in dem ersten Produkt $\lambda$. und "u unabhängig voneinander die Wert" von 1 his $m$ durchlaufen sollen. 
Die Werte von $x$, für die $y$ unentlich wird oder für die zwei oder mehrere der Werte von $y$ einander gleich werden, sind also nur in endlieher Zahl vorhanden. Wir nennen diese Werte von $x$ singulär. Zu ihnen wollen wir jedenfalls den Punkt $\infty$ rechnen.

Die Werte der unabhängigen Veränderlichen $x$ denken wir uns auf der Giulschen Zahlenebene ausgebreitet oder auf der Einheitskugel, indem wir uns die Ebene stereographisch auf eine Kugel in folgender Weise projizicrt denken (Abb. 1).

Wir machen die Ebene zu einer Berührungsebene der Kugel, und zwar -oll der Nullpunkt der Berührungspunkt sein. Er heiße $O$. Der $O$ diametral

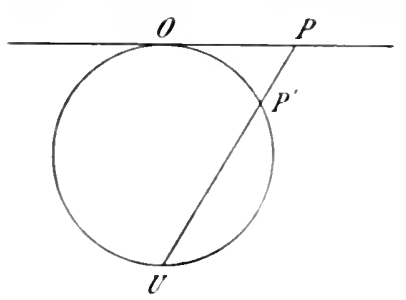

Abb, 1 . gegenïberliegende Punkt der Kugel sei $U$. Ist $P$ irgendein Punkt der Ebene, so soll ihm auf der Kugel der Punkt $P^{\prime}$ entsprechen, in dem die Gerade $U P$ die Kugel trifft; diesem Punkt $P^{\prime}$ ordnen wir den Wert von $x$ zu, der dem Punkt $P$ in der Gaußschen Zahlenebene zugeordnet ist. Dem unendlich fernen Punkt der Zahlenebene entspricht also der Punkt $U$. Der Vorteil dieser Übertragung auf die Kugel ist vor allem der, dab auch der unendlich ferne Punkt der Zahlenebene mit seiner Umgebung der Ansehauung bequem zugänglich wird.

Wir bezeichnen die Gaubsche Zahlenebene oder Kugel mit $E$. Wir schließen aus $E$ zunächst den unendlich fernen Punkt durch einen Kreis um den Nullpunkt mit einem so großen Radius aus, daß außerhalb dieses Krreises außer $\infty$ kein singulärer Punkt liegt. Dann schließen wir die anderen singulären Punkte durch kleine Kreise aus, deren Radien wir beliebig klein wählen können und jedenfalls so klein wählen, daß sie sich gegenseitig nicht ïßerdecken. Die übrigbleibende Fläche bezeichnen wir mit $E^{\prime}$.

\section{$\$$ ‥ Verhalten von $y$ in der Umgehung eines nicht singulären Wertes von $x$.}

Es sei $a$ ein Punkt in $E^{\prime}$. Für $x=a$ liefert uns die Gleichung $f(x, y)$ $=0 m$ endliche und voneinander verschiedene Werte von $y$, die wir mit $b_{1}, b_{-}, \ldots b_{m}$ bezeichnen. Wir greifen einen dieser Werte heraus, etwa $b_{1}$ und entwickeln $f(x, y)$ nach Potenzen von $x-a, y-b_{1}$ und erhalten:

(4) $f(x, y)=A(x-a)+B\left(y-b_{1}\right)+C(x-a)^{2}+D(x-a)\left(y-b_{1}\right)+\cdots=0$.

Der Koeffizient von $y-b_{1}$ ist nach dem Taylorschen Satze

$$
B=\frac{\partial f}{\partial y}\left(a, b_{1}\right)
$$

und ist von Null verschieden, weil sonst nach (3) die Diskriminante $D(x)$ 
für $x=a$ verschwimlen wïrde. Daher folgt aus rinem Sater der Funk-

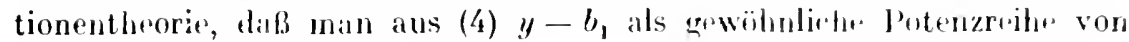
$x-a$ berechnen kann

$$
y-b_{1}=E_{1}(x-a) \text { wder } y=b_{1}+E_{1}(x-a) \text { 餿 }(x-a) \text {, }
$$

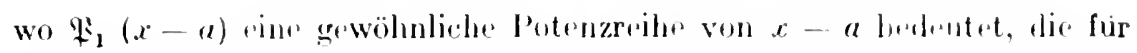
$x=a$ gleich $b_{1}$ wird. Der konvergenzkreis der keihe reidh mindestens bis zum nälhsten singulären Punkt. Eine solehe Eintwirklung nemen wir mit Weierstrab ein Element oder einen Zwoig der Funlion y. Der Punkt $a$ heißt Mittelpunkt des Elements. Da wir statt $b_{1}$ wenso inen der anderen $m-1$ Werte $b$, hätten nehmen können, so folgt, daß wir für die Umgebung dines nicht singulären Punktes a für unsere Funktion y in ganzen $m$ verschiedene Rohenentwicklungen erhalten

$$
y=\mathfrak{P}_{k}(x-a), \quad(k=1,2, \ldots m) .
$$

die nach steigenden positiven ganzen Potenzen von $x$-a fortschreiten. Diese Entwicklungen liefern uns alle Werte von $y$ in der Umgelung von $a$; denn sie liefern uns $m$ Werte von $y$ zu jedem $x$. Es ist also $y$ in der Fläche $E^{\prime}$ eine $m$-dentige analytisehe Funktion, die sich überall in $E^{\prime}$ wie eine rationale Funktion verhält.

Nun können wir die ganze Flächr $E^{\prime}$ mit einer endlichen Zahl von Kreisen überdecken, von denen keiner einen der singulären Punkte im Innern enthält, und deren Mittelpunkte in $E^{\prime}$ liegen. Die Flächen der Kreise können zum Teil außerhalb $E^{\prime}$ liegen und werden sich zum Teil überdecken. Wir können diese Kreise auf unendlich viele verschiedene Arten wählen. Es sei auf irgendein' Art $E^{\prime}$ in dor angegebenen Wrise mit Kreisen übrdeckt. Es seien im ginzen $N$ kireise. Ihre Nittelpunkte seien $a_{1}, a_{2}, \ldots a_{\backslash} . Z_{u}$ jeden dieser Punkte $a_{k}$ gehören, wie wir eben gesehen haben, m Elemente der Funktion y, die den betreffenden Punkt zum Mittelpunkt haben, und jede der $m$ Potenzreihen, die diese $m$ Elemente darstellen. konvergiert in dem keise mit dem Mittelpunkt $a_{k}$. Wir sehen daraus. dab wir den ganzen Wertvorrat der Funktion $y$ innerhalb von $E^{\prime}$ durch $m X$, also durch eine endliche Zahl von Elementen darstellen können.

\section{$\$ 3$. Verhalten ron !/ in der Umgebung eines singulären Wertes voll $x$.}

Es wi jotzt a einer der singulären Werte von $x$. Wir unterscheiden dre Fälle, die wir der Reihe nach betrachten. 1. $a$ ist nicht der unendlich ferne Punkt und os wird für $x=a$ keiner der Werte von $y$ unendlich; 2. $a$ ist nicht der mendlich ferne Punkt und es wird für $x=a$ wenigstens ainer der Worte von y unendlich; 3. $a$ ist der unendlich ferne Punkt.

Wir hesihreibun um a zwei Kreise mit den Radien $r_{1}$ und $r_{2}$. Es sei $r_{1} \cdot r_{2}$. $r_{1}$ können wir beliebig klein wählen und $r_{2}$ so klein, daß innerhalb 
des Kreises mit dem Radius $r$ a keiner der anderen singulären Punkte liegt. Den so entstehenden lireisring nennen wir $T$. Wir wählen in $T$ einen Punkt c. Da er nach unserer Annahme sicher nicht zu den singulären Punkten gehört, so gehören zu ilum als Mittalpunkt $m$ Elemente von $y$, die wir mit $y_{1}, y_{2}, \ldots y_{m}$ bezeichnen wollen. Jedes dieser Elemente ist gevaben durch cine gewöhnliche Potenzreile von $x-c$ :

$$
y_{k}=H_{k}(x-c) \text {. }
$$

Wir betrachten zunächst das Element $y_{\text {, }}$ und setzen es analytisch fort längs eines Weges, der ganz in dem Kreisring $T$ bleibt und den Punkt $a$ einmal in positivem Sinne umkreist, his wir wieder zu der Stelle $c$ zurückkommen. Die Potenzreihe von $x-c$, zu der wir so gelangen, sei

$$
\bar{y}=\overline{\mathbb{B}}(x-c) \text {. }
$$

Dirs Funktionselement $\bar{y}$ muB mit einem der Elemente $y_{1}, y_{2}, \ldots y_{m}$ identisch sein, weil $f\left(x, y_{1}\right)$ identisch $N_{11} l l$ ist und bei der analytischen Fortsetzung diese Eigenschaft erhalten bleibt, also auch $f(x, \bar{y})$ identisch verschwinden muß. Wenn wir das Element $\bar{y}$ wieder rückwärts analytisch fortsetzen, also auf einem Wege, der den Punkt $a$ einmal in negativem Sinne umkreist, so kommen wir wieder zu $y_{1}$ zurück. Da in dem Kreisring kein singulärer Punkt liegt, so ist die Wahl der Wege bei der analytisehen Fortsetzung gleichgültig, wenn wir nur ganz innerhalb des Kreisringes bleiben und den Punkt a nur einmal umlaufen. Der einfachste Fall ist der, da $\bar{y}$ mit $y_{1}$ übereinstimmt. In diesem Falle ist die durch das Element $y_{1}$ und seine analytischen Fortsetzungen in dem Kreisring $T$ definierte Funktion $y_{1}$ in der Umgebung von $a$ eine eindeutige analytische Funktion. Da sie in $a$, wie wir vorausgesetzt haben, nicht unendlich wird, so läßt sie sich nach dem Cauchysehen Satze darstellen durch eine gewöhnliche Potenzreihe von $x-a$ :

$$
\bar{y}_{1}=\overline{\mathfrak{P}}_{1}(x-a) \text {. }
$$

Diese Reihenentwicklung muß konvergieren mindestens in dem Kreise mit dem Radius $r_{2}$. Sie liefert uns auch wieder ein Element unserer algebraischen Funktion $y$, diesmal eines, dessen Mittelpunkt ein singulärer Punkt von $x$ ist.

Nicht so einfach ist es, wenn $\bar{y}$ nicht mit $y_{1}$ identisch ist. Es sei $\bar{y}$ etwa mit $y_{2}$ identisch. Dann setzen wir $y_{2}$ analytisch fort auf einem Wege, der in dem Kreisring liegt und den Punkt a einmal in positivem Sinne umkreist, bis wir wieder nach $c$ gelangen. Wir kommen dann wieder $\mathrm{zu}$ einer gewöhnlichen Potenzreihe $\overline{\bar{y}}$ von $x-c$, die wieder mit einem der Elemente $y_{1}, y_{2}, \ldots y_{m}$ identiseh sein muß. Wenn wir $\overline{\bar{y}}$ auf dem Wege, auf dem wir gekommen sind, analytisch fortsetzen, so kommen wir, wenn wir den Punkt $a$ einmal in negativem Sinne umlaufen haben, wieder zu $y_{2}$, 
und wenn wir ihn zweimal mmlaufen haben, weder zu $y_{1}$. Wenn wir also y analytisch fortsetzen auf einem Wege in dem Kreisring, der den Punkt a einmal in negativem Sinne umkreist, so kommen wir zu $y_{2}$, machen wir dassebe mit $y_{2}$, so konmen wir zu $y_{1}$. Es kann also keinesfalls $\bar{y}$ mit $y_{2}$ identiseh sein. Wohl aber kann y mit $y_{1}$ identiseh sein. Wir wollen gleich den algemeinsten Fall betrachten. Es sei $\ddot{y}$ etwa identisch mit $y_{3}$. Dann setzen wir wieder $y_{3}$ auf rinem Wege fort, der den l'unkt a einmal in positivem Sinne umkreist, und gelangen so zu einer l'otenzreihe von $x-c$, die wieder mit einer der keihen $y_{1}, y_{2} \ldots y_{m}$ übereinstimmen muB, etwa mit $y_{4}$. Fahren wir so fort, so müsen wir schlieblich zu ciner lotenzreihe kommen, die schon einmal da war, da ja im ganzen nur $m$, also eine entlich" Zahl vorhanden ist. Es sei

$$
y_{1}, y_{2}, \ldots y_{1}
$$

die Reihe der versehiedenen Elemente, die auf die angegebene Art der Reihe nach entstehen. Aus $y_{\text {u }}$ möge bei wejterer Fortsetzung des Verfahrens $y_{3}$ hervorgehen, und es sei $y_{3}$ das erste Element, das mit einem der vorhergehenden identiseh ist. Es ist $\beta$ also gleich einer der Zahlen 1, $2, \ldots \ldots$. Wenn wir $y_{3}$ auf rinem Vege analytisch fortsetzen, der a einmal in negativem Sinne umkreist, so muß es übergehen in $y_{\kappa}$, aus dem es entstanden ist; andererseits aber, wenn $\beta \neq 1 \mathrm{in} y_{\beta-1}$, aus dem es auch entstanden ist. Es muß also, wenn $\beta \neq 1, y_{k}$ mit $y_{3-1}$ übereinstimmen, was nicht sein kann, da wir angenommen haben, daß die Elemente $y_{1}, y_{2}, \ldots y_{\alpha}$ voneinander versehieden sind. Es ist also $\beta=1$. Aus $y_{u}$ geht also, wenn $x$ den Punkt $a$ einmal in positivem Sime umläuft, wieder $y_{1}$ hervor, aus $y_{1}$ bei nochmaligem Unlauf $y_{2}$ usw.; die Elemente $y_{1}, y_{2}, \ldots y_{a}$ vertausehen sich also zyklisch, wenn $x$ den Punkt a einmal in positivem sinne umkreist.

Der Punkt $c$, der ja in dem Kreisringe $T$ beliebig gewählt werden kann, liege nicht auf der zur Achse der reellen Zahlen dureh a gezogenen parallelen. Wir denken uns den Kreisring längs des in ibm liegenden Stückes dieser Parallelen zerschnitten und verbieten $x$ diesen Schnitt, den wir $s$ nennen wollen, zu übersehreiten. Wir erhalten so ein einfach zusammenhängendes Gehiet, das begrenzt ist von den bejelen Kreisen mit den Radien $r_{1}$ und $r_{2}$ und von dem zweimal zu nehmenden Sehnitt $s$. Wir bezeichnen dies Gebiet mit $T^{\prime \prime}$. In $T^{\prime}$ liegt kein singulärer Punkt von $y$. Es läßt sich daher jedes der Elemente $y_{1}, y_{2}, \ldots y_{\star x}$ in $T^{\prime}$ analytisch fortsetzen und jedes bildet mit allen seinen analytischen Fortsetzungen in $T^{\prime}$ eine innerhalb $T^{\prime}$ eindeutige analytische Funktion, die in der Umgebung eines jeden Punktes von $T^{\prime}$ in eine gewöhnliche Potenzreihe entwiekelt werden kann. Wir bezeichnen die so aus dem Element $y_{i}$ entstehende Funktion auch mit $y_{k}$. Überschreitet $x$ den Schnitt $s$ cinmal in 
prositivem simme, so geht die Funktion $y$ über in $y_{p+1}$; üherschreitet $x$ den shnitt rinmal in ungativem Sinne, so geht $y$ ïher in $y$ 's-1. Der Index ist nach dem Votul a zu nehmen. Die aus einem der Elemente $y_{\text {: }}$ und allen seinen analytischen Fortsetzmngen innerhalb des unzerschnittenen Kreisringes butebende Funktion $y^{(1)}$ ist also eine a-deutige Funktion von $x$. Dir Werte, die sie für einen Wert von $x$ haben kann, sind $y_{1}(x), y,(x), \ldots$ $y_{\text {، }}(x)$. Welchen Wert sie hat, hängt davon ab, von welchem der Elemente y: man ausgeht und welehen Weg $x$ beschrieben hat. Geht man z. B. von dem Elemente $y_{1}$ aus uni hat $x$ einen Weg beschrieben, der den Schnitt $p$-mal in positivem und $n$-mal in negativem Sinne überschreitet, so ist $y^{(1)}(x) \quad y_{1+p-n}$, wo der lndex nach dem Modul a zu nehmen ist.

\section{\$. Ein Beispiel.}

Wir betrachten zunächst folgendes Beispiel. Der späteren Verwendung wegen hezeichnen wir die zu betrachtende Funktion mit $\xi$ statt $y$. Es sei

$$
\xi=\mid a-a=(x-a)^{\frac{1}{\alpha}} \text {. }
$$

Wir führen Polarkoordinaten $r, q$ in der $x$-Ebene mit dem Pole $a$ und $\varrho, \psi$ in der 5 -Ebene nit dem Nullpunkte als Pol ein, indem wir setzen

$$
x-a=r e^{\prime i}, \quad \xi=\varrho e^{\psi i} .
$$

Es ist dann zufolge (5)

$$
\rho e^{\psi i}=\sqrt[1]{r} e^{\frac{p i}{\alpha}},
$$

woraus wir schließen, daß wir setzen können

$$
\varphi=i r, \quad \psi=\frac{\varphi}{x} \text {. }
$$

Unter $\mid r$ ist der reelle positive Wert der Wurzel zu verstehen. Es sei zunächst $0 \leq q<2 \pi$. Beschränken wir $x$ auf die zerschnittene Ringfläche $T^{\prime}$, so bleibt $r$ inmer zwischen 0 und $2 \pi$ und es liegt $\psi$ zwisehen 0 und $2 \pi / \alpha$. Da zu einem gegebenen Werte von $x$ ein und nur ein Wert von $\varphi$ gehört, für den $0-g<2 \pi$, und sich nach $(6)$ aus $q$ und $r$ eindeutig $\phi$ und $\varrho$ ergeben, so gehört unter den gemachten Annahmen zu einem Wert von $x$ in und nur ein Wert von $\xi$. Wir bezcichnen die so definierte innerhalb $T^{\prime}$ rindeutige Funktion von $x$ mit $\xi_{1}$. Für sie ist $0 \leq \psi<2 \pi / \alpha$. Gehen wir von dins.r Funktion aus und lassen $x$ den Schnitt $k$-mal in positivem Sinne üherschreiten, so nimmt \& um $2 \pi k$ zu, also $\psi$ um $2 \pi k / \alpha$. Es geht daher $\xi_{1}=\rho e^{y i}$ über in $\varrho e^{\left(\psi+\frac{2 \pi}{c} k\right) i}=e^{2 \frac{2 \pi i}{a} k} \xi_{1}$. Wir bezeichnen die a-ten Einheitswurzeln, die hier auftreten, mit 


$$
(1)_{k}=e^{2 \pi i}=(1)_{1}^{k} \text {. }
$$

Es ist für positives oder negatives ganzzahliges $k$ und $l$

Wir setzen

$$
\omega_{k} \cdot \omega_{l}=\omega_{1}{ }^{k}\left(\omega_{\mathbf{1}}^{l}=\omega_{\mathbf{1}}^{k+l}=\omega_{k} \quad\right. \text { । }
$$

$$
\omega_{k} \Xi_{1}=\Xi_{k} \quad 1 \text {. }
$$

Da $e^{2} x^{i n}=1$, wenn $n$ eine ganze Zahl ist, so ist ()$_{k}=1$, wenn $k$ durch a teilbar ist und $\left(\omega_{k}=\omega_{l}\right.$, wenn $k \quad l(\bmod \Omega)$. Von den Funktionen $\xi_{1}$, $\xi_{2}, \ldots \xi_{k}, \xi_{k+1}, \ldots$ sind daher nur die ersten $\alpha$, nämlich $\xi_{1}, \xi_{2}, \ldots \xi_{\text {u }}$ voneinander verschicden, während $\xi_{a+1}=\xi_{1}$, $\xi_{a+2}=\xi_{2}$ usw. Da $\xi_{1}$ in $T^{\prime}$ eindeutig ist, so ist aucl jede der Funktionen $\Xi_{l}=(1)-1 \Xi_{1}$ in $T^{\prime}$ eindeutig. Überschreitet $x$ den Srhnitt einmal in positivem Sinne, so geht $\xi_{1}$ über in $\omega_{1} \xi_{1}$, also $\Xi=\left(\omega_{l}-1 \xi\right.$ in $\omega_{l}-1 \omega_{1} \xi_{l}=\omega_{l} \xi_{1}=\xi_{l+1}$. Wir Funktionen $\xi_{l}$ vertausehen sich also zyklisch. Alle $\xi$ zusammen liefern uns die Funktion $\xi$ innerhalb des unzerschinittenen kreisringes. Es ist also $\Xi$ eine $\alpha$-deutige Funktion von $x$. Sie hat bei gegebenem $x$ einen der Werte $\xi_{1}(x), \xi_{2}(x), \ldots$ $\xi_{a}(x)$. Welchen sie hat, hängt davon ab, von welcher der Funktionen $\xi_{k}$ wir ausgehen und welchen Weg $x$ beschrieben hat. Gehen wir von $\xi_{1}$ aus und beschreibt $x$ einen Weg, der den Schnitt $p$-mal in positivem und $n$-mal in negativem Sinne überschreitet, so ist $\xi(x)=\xi+p-n$, wo der Index nach dem Modul $\alpha$ zu nehmen ist. Wir sehen also, daß sich $\xi$ in der Umgebung von $a$ genau so verhält wie die Funktion $y^{(1)}$ in $\S 3$. Wir werden sehen, daß das einen tieferen Grund hat.

\section{\$5. Ein zweites Beispiel.}

Wir betrachten noch einen zweiten besonderen Fall, nämlich die allgemeinste reine Gleichung in $y$. Sie hat die Form

$$
f(x, y) \quad y^{m}-r(x)=0,
$$

wo $r(x)$ eine rationale Funktion von $x$ ist. Die Funktion $r$ läßt sich wie jede rationale Funktion in der Umgebung jeder Stelle $x=a$ in eine nach steigenden Potenzen von $x-a$ fortschreitende Reihe entwickeln, wobei unter $x-a$ zu verstehen ist $x^{-1}$, wenn $a=\infty$. Nur für eine endliche Zahl von Stellen können in der Entwicklung negative Potenzen auftreten und auch nur in endlicher Zahl. Nehmen wir die niedrigste Potenz von $x$ - a vor die Klammer, so können wir schreiben

$$
r(x)=(x-a)^{\prime} E(x-a),
$$

wo $\lambda$ eine ganze positive oder negative Zahl oder Null ist und wo $E(x-a)$ eine gewöhnliche P'otenzreihe von $x-a$ ist, die für $x=a$ nieht Null wird. Eine solche Potenzreihe nennt man eine Einheit für $x=a$. Wir werden von solchen Einheiten und ihren Eigonsehaften häufig Gebrauch machen. Es seien daher ihre Eigenschaften angegeben, soweit wir sie gebrauchen. 
1. Der reziproke Wert einer Einheit für $x=a$, also ihre (-1)-te Potenz ist wieder eine Einheit für $x=a$.

Allgemeiner ist jede ganzzahlige oder rational gebrochene Potenz oiner länheit für $x=a$ wieder ein" solche linheit.

2. Der Logarithmus aner Einheit för $x=a$ ist eine gewöhnliche potemzreihe ron $x-a$.

Der Bewris dieser Sütr sei kurz angedeutet. Es sei $E$ eine Einheit für $x=a$ und os sei

$$
E(x-a)=e_{0}+e_{1}(x-a)+e_{2}(x-a)^{2}+\cdots, \quad\left(e_{0} \neq 0\right) .
$$

Wir schreiben

$$
E(x-a)=e_{0}\left\{1+{ }_{e_{0}}^{e_{1}}(x-a)+\frac{e_{2}}{e_{0}}(x-a)^{2}+\cdots\right\}=e_{0}(1+z) .
$$

Da $z$ fïr $x=a$ zu Null wird, so können wir $x-a$ so klein annehmen, daß $z<1$. Ist $n$ irgendeine rationale Zahl, so ergibt der binomische Satz

$$
\{E(x-a)\}^{n}=e_{0}^{n}(1+z)^{n}=e_{0}^{n}\left\{1+n z \frac{n(n-1)}{2} z^{2}+\cdots\right\} \text {. }
$$

Setzt man hierin für $z$ seinen Wert ein und ordnet nach Potenzen von $x-a$, so erhält man für $E(x-a)$ eine gewöhnliche Potenzreihe, die für $x=a$ den Wert $e_{0}^{v}$ annimmt, also nicht Null wird. Damit ist der erste Satz bewiesen.

Aus (9) folgt ferner

$$
\ln E(x-a)=\ln e_{0}+\ln (1+z)=\ln e_{0}+\left(z-\frac{1}{2} z^{2}+\frac{1}{3} z^{3}-\cdots\right) \text {. }
$$

Setzt man hierin für $z$ seinen Wert und ordnet nach Potenzen von $x-a$, si) erhält man für $\ln E$ eine gewöhnliche Potenzreihe von $x-a$.

Wir werden eine Reihe auch dann eine Einheit nennen, wenn sie nach ganzen positiven Potenzen einer ganzzahligen Wurzel von $x-a$ fortschreitet und für $x=a$ nieht Null wird. Schreitet solch eine Reihe etwa

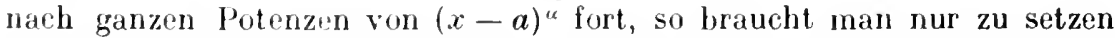
$(x-a)=\xi^{\prime \prime}$, um den vorigen Fall zu haben. Daraus sieht man, daß auch für diese Einheiten die beiden angegebenen Sätze gelten.

Wir krhren zur Betrachtung der reiner. Gleichung (7) zurück. Aus ihr und aus (8) folgt

$$
y=\mid \overline{r(x)}=(x-a)^{\frac{\dot{a}}{m}}\left\{E(x-a)^{\frac{1}{\frac{1}{m}}}=\omega(x-a)^{\frac{\partial}{m}} E_{1}(x-a),\right.
$$

wo $E_{1}$ eine Einheit für $x=a$ ist, und wo $\omega$ irgendeine $m$-te Einheitswurzel ist. Wir erhalten so, den $m \boldsymbol{m}$-ten Einheitswurzeln entsprechend, $m$ Entwicklungen, die mit $y_{1}, y_{2}, \ldots y_{m}$ bezeichnet seien. 
Ist $a$ weder time Null- noch aine Unendlichkeitsstelle von $r(x)$, ist also $\lambda=0$, so erhalten wir $m$ gewöhnlicht l'otenzroihen von $x-a$, und es geht jode in sich sellist ïber, wenn $x$ einen gesehlossenen Weg um $a$ besehroibt. Dasselbo gilt, wonn $\lambda$ durch $m$ teilhar ist. Singuläre Stellon für $y$ sind also hörhstens die Null und Unendlichkeitsstellen von $r(x)$.

Ist 7 nicht Null und nicht dureh $m$ trilbar, so sei $\delta$ der größte gemeinsame Triler von $i$ unl $m$ und es sei $i=\| d, m=\gamma d$. E- wirl dann

$$
y=(i)(x-a)^{a} E_{1}(x-a) \text {, }
$$

wo " und $\alpha$ teilerfremd sind. Es sei $y_{1}$ rino dieser $m$ Entwieklungen. I Imkreist $x$ den Punkt $a$ einmal, so geht $E_{1}$ wieler in sich über, aher $(x-a)^{*}$ $=\left\{(x-a)^{\frac{1}{\prime \prime}}\right\}^{\prime \prime}$ nimmt den Faktor $\omega_{1}^{\prime \prime}=\omega_{n}$ an, wo, wie im vorigen Beispiel, $\omega_{1}=e^{-\bar{t}}$. Es geht also $y_{1}$ in eine andere der Entwicklungen $y_{k}$ ïber, nämlich in $\omega_{u} y_{1}$. Diese sei mit $y_{2}$ bezeichnet. Setzen wir allgemeiner

$$
y_{l}=\omega_{a}^{l-1} y_{1}, \quad(l=1,2, \ldots \alpha)
$$

so sind diese $\alpha$ Entwicklungen unter den $m$ Entwicklungen $y_{1}, y_{2}, \ldots y_{\prime \prime}$ enthalten und sie vertauschen sich zykliseh, wenn $x$ den Punkt a einmal umkreist. Ist $\alpha<m$, so sei $y_{u+1}$ eine von den a Entwicklungen (10) verschiedene Entwicklung. Setzen wir

$$
y_{c+l}=()_{u}^{l-1} y_{a+1}, \quad(l=1,2, \ldots x)
$$

so sind auch diese $\alpha$ Entwicklungen unter den $m$ Entwicklungen $y_{1}, y_{2}, \ldots y_{m}$ enthalten und sic sind von den a Entwicklungen (10) versehieden. Auch sie vertaushen sich zyklisch, wenn $x$ einen geschlossenen Weg um a beschreibt. Ist $m>2 a$, so kömmen wir in dieser Weise fortfahren und erhalten das Ergebnis, daß die $m$ Entwicklungen $y_{1}, y_{2}, \ldots y_{m}$ sich in ¿Gruppen von je a Entwicklungen so anordnen lassen, daB die Entwicklungen jeder Gruppe sich zyklisch vertausehen, wenn $x$ den Punkt $a$ cinmal umkreist. Bleibt $x$ in dem um den Punkt $a$ abgegrenzten Ringgebiet. so kann jede Entwicklung einer Gruppe in jede andere derselben Gruppe übergeführt werden, dadurch, daß $x$ den Punkt a ein oder mehrere Male umkreist. Es ist aber nicht möglich, auf diese Art eine Entwicklung einer Gruppe in eine einer anderen ïberzuführen.

\section{\$6. Fortsetzung von $\$ 3$.}

Wir kehren zur Betrachtung der Funktion $y^{(1)}$ zurïck und wollen sie auffassen als Funktion der in $\$$ \& betrachteten Größe $\Xi$. Wir führen also durch die Gleichung (5) oder

$$
x=a+\xi^{\prime \prime}
$$


statt $x$ rime nete mobhängige Verändrline $\Xi$ ein. Wir haben $x$ beschrinkt anf den Kreisring $T$. fïr den $r_{1}, \quad x-a \mid \cdot r_{2}$, so daB zufolge (ti) anth

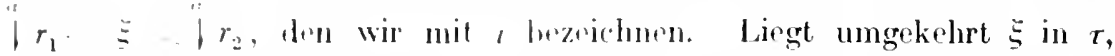
so liegt it in $T$. Da nach $(i): f=\arg x$,-mal so groß ist wie $\psi=\arg \xi$, so gilt ferner folgendus. Wron winkel 2- I durchläuft, so durchlïuft $x$ ten ganzen ling $T$. Wir teilen in I kungruente Soktoren mit lem Mittelpunktswinkel $2 \pi / a$, die wir der

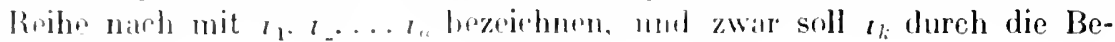
dingung

$$
(k-1)^{2 \pi} \quad \xi^{\prime}<k^{2 \pi}
$$

bestimmt win. buthreibt $\fallingdotseq$ einen der Sektoren $r_{k}$, so beschreibt $x$ den zershnittenen Kreisring $T^{\prime}$. ohne den Schnitt zu übersehreiten.

Es sei $\Xi_{0}$ irgendein Wert von $\Xi$ in $t$. Der eindeutig bestimmte zugehörig Wert von $x$, nämlich $a+\xi_{0}^{\prime}$, sei mit $x_{0}$ bezeichnet. Aus (11) folgt

$$
x=a+\left[\Xi_{0}+\left(\xi-\Xi_{0}\right)\right]^{e}
$$

oder nach flem binomischen Satze

(12) $x-x_{0}=\alpha \xi_{0}-1\left(\xi-\xi_{0}\right)+\frac{a(u-1)}{2} \xi_{0}-2\left(\xi-\xi_{0}\right)^{2}+\cdots+\left(\xi-\xi_{0}\right)^{\alpha}$.

Da $x_{0}$ in $T$ liegt, so läBt sich jede der Funktionen $y_{i}$ in eine gewöhnliche nach Potenzen von $x-x_{0}$ fortschreitende Potenzreihe entwickeln und diese geht unter Benutzung von (12) in eine gewöhnliche Potenzreihe von $\xi-\xi_{0}$ über. Daher lassen sich die Funktionen $y_{k}$ in der Umgebung jeder Stelle $\Xi_{0}$ von $t$ in rine gewöhnliche Potenzreihe von $\xi-\xi_{0}$ entwickeln. Wir wählen im lesonfleren $\xi_{0}$ innerhalb $\tau_{1}$ und denken uns $y_{1}$ nach Potenzen von $x-x_{0}$ entwickelt und dann mit Hilfe von (12) diese Entwicklung verwandelt in eine Potenzreihe von $\xi-\xi_{0}$. Wenn wir die so erhaltene Potenzroihe innerhalh, $t$ analytisch fortsetzen, so erhalten wir cine analytisehe Funktion, die mit, $(\xi)$ bezeichnet sei. In der Umgebung von $\xi_{0}$ ist nach der befinition, $(\xi) \quad y_{1}(x)$, wenn $x$ aus der Gleichung (11) bestimmt wird. Durchläuft $\xi$ das Gebiet $\tau_{1}$, so bleibt $x$ in $T^{\prime}$ und es bleibt die Gleichung,$(\xi)=y_{1}(x)$ bestehen. Geht aber $\xi$ von $\tau_{1}$ nach $\tau_{2}$, so geht $x$ unmal in positivem Sinne über den Schnitt $s$ und $y_{1}(x)$ geht in $y_{2}(x)$ iher. Befindet sich also 5 in $\tau_{\dot{2}}$, so besteht die Gleichung $\eta(\xi)=y_{2}(x)$, wnn $x$ wirder aus der Gleichung (11) bestimmt wird. Geht $\xi$ von $\tau_{2}$ nach $r_{3}$, so ïbcrschreitet $x$ zum zweiten Male den Schnitt $s$ in positivem Sinne und $y_{2}(x)$ geht in $y_{3}(x)$ über. Wenn also $\xi$ in $\tau_{3}$ liegt, so ist $\eta(\xi)$ $-y_{3}(x)$. So können wir weiter schließen. Wenn schließlich $\xi$ aus $\tau_{\alpha}$ in 
$r_{1}$ übergeht, so ïberschreitet $x$ zum a-tun Malu dru Schnitt $s$ in positivim Sinne und wir kommen wieder zu $y_{1}(x)$. Es ist also

$$
y(5) \quad y_{k}(x)
$$

wenn 5 in $r_{k}$ liegt und $x$ aus (11) bestimmt wird. l)ablist es gloichgültig, welchen Woug in $r$ brschrimben hat. Es kann 5 anch den Nullpunkt beliebig oft mmkreist haben. Es ist daher y (5) in t eine eindentign analytione Funktion mod es ist hei passender Zuordnung von 5 und $x$

$$
\text { , (5) } \quad y^{(1)}(x) \text {. }
$$

Da nach Voraussetzung $y^{(1)}(x)$ endlich bleibt, wenn $x$ sirh dem l'unkto $a$ nähert, so bleibt y) (ङ) endlich, wenn $\Xi$ sich dem Punkte O näh+ot. Nach dem Cauchysehen Satze ist daher y (૬) in eine gewöhnlinh Putenzreihe von 5 entwickelbar $y=\$(5)$. Aus (11) und (13) folgt dann, daßs sich $y^{(1)}$ in der Umgehung von $a$ in eine gewöhnliche Potenzreihe von $(x-a)^{\text {" }}$ entwickeln läßt:

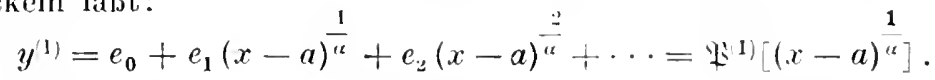

Wenn wir $x$ erlauben, sich in $T$ beliebig zu bewegen, also auch den Punkt $a$ beliebig oft $\mathrm{zu}$ umkreisen, so liefert uns die Reihe (14) alle Werte von $y^{\prime \prime}$. Sie liefert uns zu jedem Werte von $x$ in $T$, den $\alpha$ Werten entsprechend, die $(x-a)^{\frac{1}{a}}$ für einen Wert von $x$ liat, " Werte der algebraischen Funktion $y$. Wenn wir aber $x$ auf $T^{\prime}$ beschränken, so liefert uns die Reihe (14) nur eine der Funktionen $y_{k}$. Welche dieser Funktionen das ist, hängt davon ab, welchen Wert wir der a-ten Wurzel aus $x-a$ beilegen. Wählen wir $(x-a)^{a}=\Xi$ so, daß $\zeta$ in $\tau_{k}$ liegt, so ist bei unseren Festsetzungen der Wert der Reihe (14) gleich $y_{k}$. Verstehen wir im besonderen unter $\mid(x-a)$ den Wert von $(x-a)^{*}$, der in $t_{1}$ liegt, nehmen wir also nach früherev Bezeichnung $(x-a)^{\frac{1}{\alpha}}=\xi_{1}$ an, so ist der Wert von $(x-a)^{\frac{1}{\alpha}}$ in $r_{k}$ gleich $\left(\omega_{k-1} \mid(x-a)=\omega_{k}-1 \xi_{1}=\xi_{k}\right.$. Es bestehen daher die Entwicklungen

$$
\begin{aligned}
& \left.y_{k}=e_{0}+e_{1}()_{k}-1 \sqrt[\alpha]{x-a}+e_{2}\left(\omega_{k-1}\right)^{\alpha} x-a\right)^{2}+\cdots \\
& (k=1,2, \ldots(t)
\end{aligned}
$$

wo $x$ auf das Gobiet $T^{\prime}$ zu beschränken ist. Wir nennen diese a Entwicklungen zueinander adjungiert. Die Entwicklungen gehrn aus einer von ihnen dadurch hervor, daß man $x$ den Punkt $a$ umlaufen läBt, da allgemein aus der Entwicklung für $y_{i}$ die für $y_{i}+1$ entsteht, wenn man $x$ den Punkt $a$ einmal in positivem Sinne umlaufen läßt. Daher liefert uns jede der Ent- 
wicklungen alle Werte von $y^{11}$, wenn wir $x$ die Uberschreitung des Schnittes $s$ gestatten. Wir sagen daher von adjungierten Entwicklungen, sie hängen in der Ungebung von $a$ miteinander zusammen.

Wir bezeichnen den Teil der algebraischen Funktion $y$, der uns durch (14) gegeben wird, wieder als Element oder Zweig der Funktion $y$ und die durch die Entwicklungen (15) gegebenen Teile von $y$ als Teilplemente oder Teilzweige.

Fassen wir in der Entwicklung (11) für $y^{(1)}$ immer die Glieder zusammen, wo die Exponenten von $x-a$ sich nur um ganze Zahlen unterscheiden, so erhalten wir sie in der Gestalt

$$
y^{1)}=p_{1}+p_{2}(x-a)^{\frac{1}{\alpha}}+p_{3}(x-a)^{\frac{2}{\alpha}}+\cdots+p_{c(}(x-a)^{\frac{\alpha-1}{\alpha}},
$$

wo die $p_{k}$ gewöhnliche Potenzreihen von $x-a$ sind. Ein Glied, das in einem der Summanden $p_{l}(x-a)^{\frac{l-1}{\text { " }}}$ vorkommt, kann sich nicht gegen Glieder in anderen Summanden fortheben, da Glieder aus verschiedenen Summanden nie dieselbe Potenz von $x-a$ zum Faktor haben. Soll z. B. $y^{(1)}$ für $x=a$ endlich bleiben, so darf in keinem Summanden eine negative Potenz von $x-a$ enthalten sein. Das aber ist dann und nur dann der Fall, wenn die Potenzreihen $p_{k}$ keine negative Potenz von $x-a$ enthalten, wic man sofort sieht, wenn man bedenkt, da $B$ die $p_{k}$ nur ganzzahlige Potenzen von $x-a$ enthalten.

Ist $m>a$, so gehen wir von dem Element $y_{u+1}=\mathfrak{B}_{\alpha+1}(x-c)$ aus und setzen es analytisch fort auf einem Wege, der in $T$ liegt und den Punkt $a$ einmal in positivem Sinne umkreist. Dadurch gehe $y_{a+1}$ über in $\bar{y}$. Es muß dann $\bar{y}$ wieder eine Wurzel der Gleichung $f(x, y)=0$ sein und muß also eines der Elemente $y_{1}, y_{2}, \ldots y_{m}$ sein. Es kann aber nicht gleich einem der Elemente $y_{1}, y_{2}, \ldots y_{1}$ sein. Denn, wenn $x$ in negativem Sinne den Punkt $a$ umkreist, so gelit $\bar{y}$ wieder in $y_{u+1}$ über, aber jedes der Elemente $y_{1}$, $y_{2}, \ldots y_{u}$ geht dabei wieder in eins dieser Elemente über und nicht in $y_{\alpha+1}$. Es sei $\bar{y}=y_{a+2}$. Lassen wir $x$ noch mal den Punkt $a$ in positivem Sinne umkreisen, so gehe $y_{\alpha+2}$ über in $y_{a+3}, y_{u+3}$ in $y_{u+4}$ usw., schließlich $y_{a+\beta}$ in $y_{a+\beta+1}$ und es sei $y_{a+\beta+1}$ das erste Element, das gleich einem der vorhergehenden ist. Dann läßt sich wieder wie oben nachweisen, $\mathrm{da} B y_{\alpha+\beta+1}$ $=y_{a+1}$ sein muß. Es vertauschen sich also die $\beta$ Elemente $y_{a+1}, y_{a+2}$, $\ldots y_{a+q}$ auch zyklisch, wenn $x$ den Punkt $a$ einmal umkreist. Wir schließen, wie oben, daß wir für die Funktion $y$ in der Umgebung von $a$ noch ein zweites Element $y^{2 /}$ haben, das gegeben ist durch eine Entwicklung von der Form

$$
y^{(2)}=\mathfrak{B}^{(2)}\left[(x-a)^{\frac{1}{3}}\right]=c_{0}+c_{1}(x-a)^{\frac{1}{3}}+c_{2}(x-a)^{\frac{2}{3}}+\cdots,
$$

wo $\mathfrak{B}(2)$ eine gewöhnliche Potenzreihe von $(x-a)^{\frac{1}{\beta}}$ ist. Diese Reihe gibt 
uns, den $\beta$ Werten von $(x-a)^{\beta}$ entsprechend, $\beta$ Werte von $y$ zu einem Werte von $x$. Beschräuken wir $x$ auf $T^{\prime \prime}$, so haben wir statt (16) $\beta$ arljungierte Entwicklnugen.

Ist $\alpha+\beta=m$, so gehen wir aus von dem Elrment $y_{a+\beta+1}$ $=\mathfrak{B}_{a+\beta+1}(x-c)$ mud verfahren wie oben. Wir wollen aber annehmen, $\mathrm{da} \alpha+\beta=m$ soi. Das genügt, um auch den allgemrinsten Fall zn übersehen. Das Ergebnis ist dann folgendes:

Ist $a$ ein im Endlichen liegender singulärer Punkt und wird für $x=a$ keiner der Werte von y unendlich, so lassen sich die Wurzeln von $f(x, y)$ $=0$ in der Umgebung von $a$ in eine endliche Zahl von Gruppen teilen, etwa in zwei, so daß die Wurzeln in jeder Gruppe sich zyklisch vertausehen, wenn $x$ den Punkt $a$ umkreist (bei einem Wege, der auBer $a$ keinen singulären Punkt einschließt). Enthalten die Gruppen $\alpha$ und $\beta$ Wurzeln, so haben wir in der Umgebung von $a$ zwei Reihenentwicklungen für $y$ von der Form:

$$
y=\mathfrak{F}^{(1)}\left[(x-a)^{\frac{1}{1 t}}\right], \quad y=\mathfrak{F}^{(2)}\left[(x-a)^{\frac{1}{\gamma}}\right],
$$

wo die $\not 3$ gewöhnliche Potenzreihen ihrer Argumente sind. Aus der ersten Entwicklung gehen noch $\alpha-1$, aus der zweiten $\beta-1$ adjungierte hervor. dadurch, daß die Wurzeln von $x-a$ durch ihre verschiedenen Werte ersetzt werden. Wir bekommen also im ganzen $\alpha+\beta=m$ Entwicklungen und bekommen, wie es sein $\mathrm{mu} \beta, \alpha+\beta=m$ Werte von $y$ zu jedem Wert von $x$ in der Umgebung von $a$. Wir nennen diese $m$ Entwicklungen zueinander konjugiert.

Es sei noch darauf hingewiesen, daB sehr wohl der Fall eintreten kann. daß die Zahl der Gruppen gleich $m$ ist, daß also jede Gruppe nur eine einzige Wurzel enthält. Wir haben dann, genau wie bei einem nicht singulären Punkt $m$ Reihenentwicklungen, die nach ganzen Potenzen von $x-a$ fortschreiten. Der (unwesentliche) Unterschied ist nur der, daß zwei oder mehrere dieser Entwicklungen für $x=a$ denselben Wert annehmen.

\section{s. T. Verhalten von $y$ in der Umgebung eines Wertes von $x$, für den $y$ unendlich wird.}

Wir nehmen jetzt den Fall, wo für $x=a$ eine oder mehrere Wurzeln vou $f(x, y)=0$ unendlich werden, wo aber $a$ endlich ist. Es muß dann a eine Nullstelle von $a_{0}(x)$ sein, dem Koeffizienten von $y^{m}$ in $f$. Es sei $a_{0}(x)$ durch $(x-a)^{\prime \prime}$ teilhar. Wir setzen $a_{0}(x)=(x-a)^{\delta} a_{0}^{\prime}(x)$. Statty führen wir cine neur abhängige Veränderliche ein, durch die Gleichung

$$
\eta=(x-a)^{d} y \text {. }
$$

Die Gleichung $f=0$ goht nach Multiplikation mit $(x-a)^{(m-1) d}$ über in

$$
a_{0}^{\prime} \eta^{m}+a_{1} \eta^{m-1}+(x-a)^{d} a_{2} \eta^{m-2}+\cdots+(x-a)^{m-1) d} a_{m}=0 .
$$


Da für $x=a$ der koeffizient von $\iota^{m}$ nicht Null wird, so können wir auf diese Calcichung unsere bishrrigen Ergebnisse anwenden. Wir erhalten also. wonn wir gleich den allgemeinen Fall annohmen, in der Umgebung von $x=a$ eine oder mehrere Entwicklungen, die nach positiven ganzen oder gubrochenen Potenzen von $x$-a fortschreiten. Aus der Gleichung (17) folgt dann, daß wir awh fiir $y$ Entwicklungen nach steigenden Potenzen von $x-a$ oder ciner gebrochenen Potenz von $x-a$ erhalten, da aber diese Entwicklungen ane endliche Zabl von negativen Potenzen enthalten können, da $y$ aus y, entsteht dureh Oivision mit $(x-a)^{\delta}$. Es brauchen aber nicht alle Entwicklungen von $y$ in der Umgebung von $a$ negative Potenzen enthalten. Es kann ja sein, daß eine oder mehrere der Entwicklungen für $y x-a$ in der Potenz $\delta$ oder in höherer Potenz als Faktor enthalten. Aber mindestens eine Entwicklung von $y$ muß wirklich negative Potenzen enthalten, wenn $a_{0}(x)$ durch $(x-a)$ teilbar ist, weil in diesem Fallo mindestens eine Wurzel von $f(a, y)=0$ unendlich grob sein muB.

\section{s S. Verhalten von $y$ in der Umgebung von $x=\infty$.}

Wir betrachten schließlich noch das Verhalten von $y$ für unendlich große Werte ven $x$. Zu dem Ende setzen wir $x=1^{\prime} z$. Der Umgebung des unendlich fernen Punktes der $x$-Ebene entspricht dic Umgebung des Nullpunktes der $z$-Ebene. Wir haben also $y$ als Funktion von $z$ in der Umgebung des Punktes $z=0$ zu betrachten. Die Gleichung $f(x, y)=0$, die in $x$ vom Grade $l$ sein sollte, geht nach Multiplikation mit $z^{l}$ über in eine Gleichung $G(z, y)=0$, wo $G(z, y)$ eine ganze rationale Funktion von $z, y$ ist. Aus den bisher gefundenen Ergebnissen folgt, daß wir für $y$ aus dieser Gleichung eine oder mehrere Entwicklungen erhalten. Jede dieser Entwicklungen schreitet nach ganzen positiven Potenzen von $z=$ $1 / x$ oder ciner gebroclienen Potenz von $z$ fort und sie kann höchstens eine endliche Anzahl von negativen Potenzen von $z$ unthalten. Diese Entwicklungen geben uns die Funktion y für die Umgebung des Punktes $z=0$ oder $x=\infty$. Es gilt also für den Fall, da $\beta$ der Punkt $a$ der unendlich ferne Punkt der $x$-Ebene ist, dasselbe wie für endliches $a$, wenn man nur für $a=\infty$ unter $x-a$ versteht $1 / x$.

I) amit wissen wir, wie sich die algebraische Funktion $y$ in der Umgebung irgendeines Punktes der $x$-Elone verhält.

Wir haben schon früher gesehen (am Ende von $\S 2$ ), daß der gesamte Wertrorrat von $y$, soweit er für solche Werte von $x$ angenommen wird, die in $E$ ' liegen, durch eine endliche Zahl von Elementen dargestellt werden kann. Da wir jetzt gesehen haben, daß auch zu jedem der in endlicher Záhl vorhandenen singulären Punkte von $x$ nur eine endliche Zahl von Elementen gehört, so folgt: Wir können den gesamten Werte- 
Zweites Kapitw. Berehunug der Reihenentwioklungen. \$1. Vorbetrachtumgen.

vorrat der algobaisthen funktion $y$ dureh eine endliche Zahl von Elomenten darstellen. Jedes dirser Elemente ist, wenn a sein llittelpunkt ist, eine Potenzreibe, die nach steigenden ganz'n Potenzen von $x-a$ oder piner ganzzahligen positiven Wurzel aus $x$ - a fortschreitet und dir höchstens rine endlichr. Zahl von negativen Exponenten enthält. Dahei ist unter $x-a$ zu verstehen $1 / x$, wenn $a=\infty$. Negative und gebrochene Exponenten können nur an einer endlichen Zahl von Punkten vorkommen.

Zweites Kapitel.

\section{Die Berechnung der Reihenentwicklungen für $y$ in der Ungebung irgendeiner Stelle.}

\section{s 1. Vorbetrachtungen.}

Nichdem wir festgestellt haben, daß wir $y$ in der Umgebung jeder Stelle $a$ in nach steigenden Potenzen von $x-a$ fortschreitende Reihen entwickeln können und wie diese beschaffen sind, gehen wir dazu über zu zeigen, wie man diese Entwicklungen praktisch berechnen kann. Wir setzen:

$$
x-a=z
$$

und, wenn a dir unendlich ferne Stelle ist,

$$
\frac{1}{x}=z \text {. }
$$

Die Gleichung $f(x, y)=0$ schreiben wir (wenn $a=\infty$, nach Multiplikation mit $z$ ) in der Form

$$
g(z, y)=c_{0}+c_{1} y+c_{2} y^{2}+\cdots+c_{m} y^{m}=0 .
$$

wo die $c$ ganze rationale Funktionen von $z$ sein sollen. Es sei das Anfangsglied von $c_{k}$, wenn wir $c_{k}$ nach steigenden Potenzen von $z$ ordnen, gleich $g_{k} z^{\text {"k }}$, so daß wir unsere Gleichung auch schreiben können:

(1) $g(y, z) \quad\left(g_{0} z^{u_{0}}+\cdots\right)+\left(g_{1} z^{\alpha_{1}}+\cdots\right) y+\cdots+\left(g_{m} z^{\prime \prime m}+\cdots\right) y^{m}=0$.

In diese Gloirhung setzen wir mit unbestimmten Koeffizienten und Exponenten ein

$$
y=e_{1} z^{k_{1}}+e_{2} z^{k_{2}}+\cdots,
$$

wobei wir voraussetzen $\varepsilon_{1}<\varepsilon_{2}<\varepsilon_{3} \ldots \ldots$ und die Koeffizienten $e_{k}$ als von Null verschieden. Wir erhalten

$$
\begin{gathered}
\left(g_{0} z^{\alpha_{0}}+\cdots\right)+\left(g_{1} z^{k_{2}}+\cdots\right)\left(e_{1} z^{k_{1}}+\cdots\right)+\left(g_{2} z^{\alpha_{z}}+\cdots\right)\left(e_{1}^{2} z^{2 \varepsilon_{1}}+\cdots\right) \\
+\cdots+\left(g_{m} z^{\alpha_{m}}+\cdots\right)\left(e_{1}^{m} z^{m \varepsilon_{1}}+\cdots\right)=0 .
\end{gathered}
$$


wo in jeder Klammer nur das Glied niedrigster Ordnung hingeschrieben ist. Soll dir Reihe (2) rine Lösung sein, so muB der in (3) auf der linken Seite stehende Ausdruck identisch Null sein. Multiplizieren wir also in (3) die Klammern aus und ordnen nach Potenzen von $z$, so müssen sich die Potenzen mit gleichen Exponenten fortlueben. Es müssen sich also auch die Glieder fortheben, in denen z den kleinsten Exponenten hat. Damit aber diese Glieder sich gregenseitig aufheben können, müssen mindestens zwei von ihnen da sein. Wir werden sehen, da $B$ uns diese Bedingung den Exponenten $\varepsilon_{1}$, endlich vieldeutig, liefert. Die Bedingung, da $B$ die dann mindestens in zwei Exemplaren vorhandenen Glieder niedrigster Ordnung sich fortheben. liefert uns den ersten Koeffizienten $e_{1}$.

Die Glieder niedrigster Ordnung in (3) sind sicher unter denen enthalten, die sich ergeben. wem wir in (3) in den Klammern nur die Anfangsglieder berücksichtigen, also unter den Gliedern

$$
g_{0} z^{\kappa_{0}} \cdot g_{1} e_{1} z^{\alpha_{1}+\varepsilon_{1}}, \quad g_{2} e_{1}^{2} z^{\alpha_{2}+2 \varepsilon_{1}}, \quad g_{3} e_{1}^{3} z^{\alpha_{3}+3 \varepsilon_{1}}, \ldots g_{m} z^{u m+m \varepsilon_{1}} .
$$

Wir lraben also $\varepsilon_{1}$ so zu bestimmen, daß unter den Exponenten

$$
\iota_{0}+0 \cdot \varepsilon_{1}, \quad \iota_{1}+1 \cdot \varepsilon_{1}, \quad \alpha_{2}+2 \cdot \varepsilon_{1}, \ldots \alpha_{m}+m \varepsilon_{1}
$$

der kleinste mindestens zweimal vorkommt.

Wir betrachten erst ein Beispiel. Die Gleichung zwischen $x$ und $y$ sei

$$
x^{2}+2 x^{3}-\left(x+x^{2}\right) y+x y^{2}+y^{3}=0 .
$$

Wir wollen die Entwicklungen von $y$ in der Umgebung des Punktes $x=0$ betrachten. Wir hätten $x=z$ zu setzen, wollen aber der Einfachheit halber den Buchstaben $x$ beibehalten. Es ist hier

$$
\alpha_{0}=2, \alpha_{1}=1, \alpha_{2}=1, \alpha_{3}=0,
$$

also die Reihe (5) der Exponenten

$$
2,1+\varepsilon_{1}, 1+2 \varepsilon_{1}, 3 \varepsilon_{1} .
$$

Da dic Gleichung (6) für $x=0$ übergeht in $y^{3}=0$, so folgt, daß für $x=0$ alle Wurzeln von (6) den Wert Null annehmen. Es ist also für jede der gesuchten Entwicklungen $\varepsilon_{1}>0$. Dann aber folgt, daß unter den Zahlen (7) die Zahl $1+2 \varepsilon_{1}$ immer größer ist als $1+\varepsilon_{1}$, also bei keiner erlaubten Wahl von $\varepsilon_{1}$ eine der kleinsten Zahlen sein kann. Wir können uns also beschränken auf die Betrachtung der Zahlen

$$
2, \quad 1+\varepsilon_{1}, \quad 3 \varepsilon_{1} .
$$

Es sind zwei Bedingungen, die zur Bestimmung von $\varepsilon_{1}$ führen. 1. Es müssen zwei der Zahlen (8) einander gleich sein und 2. diese Zahlen müssen kleiner als die dritte sein (oder ihr gleich). Die erste Bedingung können wir auf drei Arten erfüllen: 

I. $2=1+\varepsilon_{1}, \varepsilon_{1}=1$;
II. $2=3 \varepsilon_{1}, \quad \varepsilon_{1}=\begin{aligned} & 2 \\ & 3\end{aligned}$;
1II. $1+\varepsilon_{1}=3 \varepsilon_{1}, \varepsilon_{1}=\begin{aligned} & 1 \\ & 2\end{aligned}$.

Die drei Zahlen (8) sind in den drei Fällen:
I. $2,2,3$;
II. $2, \frac{5}{3}, 2$;
III. $2, \frac{3}{2}, \frac{3}{2}$

Wir sehen, daß im Falle I und III die beiden gleichen Exponenten auch die kleinsten sind, im Falle II aber nicht. Wir haben also zwei mögliche Werte für $\varepsilon_{1}$ :

$$
\text { 1. } \varepsilon_{1}=1, \quad \text { 2. } \varepsilon_{1}=\frac{1}{2} \text {. }
$$

Die Glieder (4), auf die es zunäehst ankommt, sind in unserem Beispiel

$$
x^{2}, \quad-e_{1} x^{1+\varepsilon_{1}}, \quad e_{1}^{2} x^{1+2 \varepsilon_{1}}, \quad e_{1}^{3} x^{3 \varepsilon_{1}} .
$$

Im Falle 1, wo $\Sigma_{1}=1$, werden das erste und zweite Glied von derselben und niedrigsten Ordnung. Sollen sie sich fortheben, so muß $e_{1}=1$ sein. Im Falle 2, wo $\varepsilon_{1}=\frac{1}{2}$, werden das zweite und vierte Glied von derselben und niedrigsten Ordnung. Sie heben sich fort, wenn $-e_{1}+e_{1}^{3}=0$, also, da $e_{1}$ von Null versehieden angenommen ist, wenn $e_{1}= \pm 1$. Wir erhalten also für $y$ in der Umgehung von $x=0$ drei Entwieklungen, die so anfangen

$$
y=x+\cdots, \quad y=x^{\frac{1}{2}}+\cdots, \quad y=-x^{\frac{1}{2}}+\cdots
$$

\section{\$̊. I) Das Diagramm vou Puiseux.}

Wir kehren zu dem allgemeinen Fall zurück. Man kann in jedem Fall zur Bestimmung von $\varepsilon_{1}$ so verfahren, wie wir es bei dem Beispiel getan haben, indem man nämlich auf alle möglichen Arten zwei der Exponenten (5) einander gleichsetzt und zusieht, ob diese dann die kleinsten werden oder nicht. Dies Verfahren ist aber in den meisten Fällen reeht mühsam. Viel ühersichtlicher und einfacher ist das Verfahren von Puiseux, das wir jetzt auseinandersetzen. Wir zeichnen in einem reehtwinkligen Koordinatensystem mit dem Anfangspunkt $O(\Lambda \mathrm{bb} .2)$ die $m+1$ Punkte mit den Koordinaten

$$
0, \alpha_{0} ; 1, \alpha_{1} ; 2, \alpha_{2} ; \ldots m, \alpha_{m} .
$$




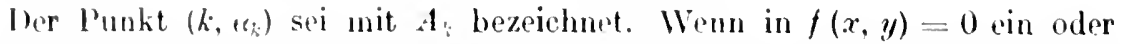
mehrere Potenzen vou $y$, z. B. $y^{2}$ und $y^{j}$ fehlen, so sind die entsprechenden l'unkte $A$, also in dem Beispinl $A_{2}$ und $A_{5}$, fortzulassen. Wenn man will, hann man sich dir entsprechenden $\alpha$, also $\epsilon_{2}$, und $\alpha_{5}$, unendlich groß denken.

Bestimmen wir einen Winkel $x$ dureh die Gleichung

$$
\operatorname{tg} t=\varepsilon_{1},
$$

so könun wir die Exponnten (5) selar pinfarh geometrisch darstellen, indem wir durelı die Punkte A Gerade zichen, die mit der negativen Alszissenachse den Winkel $r$ cinschließen. Die Gerade durch $A_{k}$ möge die Ordinatenachse in $B$ sehneiden. Dann ist das von der Ordinatenachse abgeschnittene Stïck $O B_{k}=a_{k}+k \operatorname{tg} \tau=\alpha_{k}+k \varepsilon_{1}$. Wir müssen $\tau$, also die Noigung der Geraden so wählen, daß mindestens zwei der

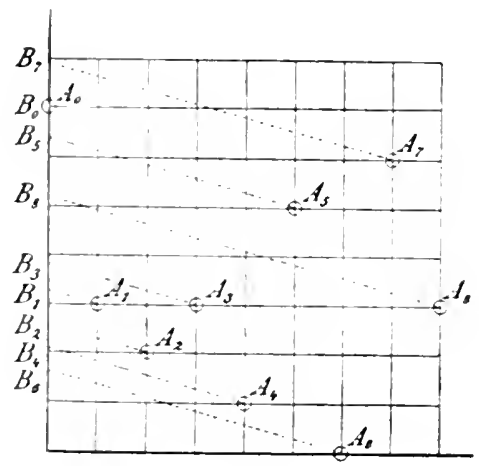

Abb. 2.

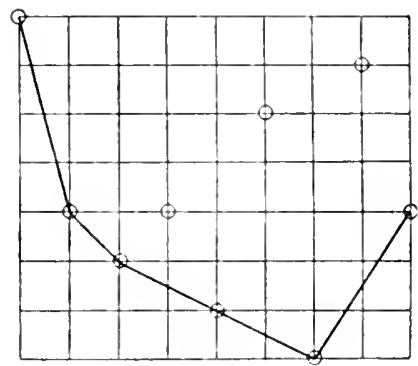

Abb. 3.

Strecken $O B_{k}$ einander gleich werden und daß keine der anderen Strecken $O B_{k}$ diese an Größe übertreffen, daß also, anders ausgedrückt, mindestens zwei Punkte $B_{k}$ zusammenfallen und keiner der anderen Punkte $B_{i:}$ tief + r liegt. Sollen aber z. B. $B_{1}$ und $B_{2}$ zusammenfallen, so müssen $A_{1}$ und $A_{2}$ auf derselben Geraden liegen, so da $B$ das zugehörige $\tau$ gleich dem Winhol ist, den die Gerade $A_{1} A_{2}$ mit der negativen Abszissenachse bildet. Soll fermer bei dirser Wahl von $\tau$ keiner der anderen Punkte tiefer liegen als $B_{1}=B_{2}$, so darf keiner der Punkte $A_{k}$ unterhalb der Geraden $A_{1} A_{2}$ liegen. Um also alle zulässigen Verte von $\tau$ zu finden, haben wir alle Graden aufzusuchen, die durch mindestens zwei der Punkte $A_{k}$ gehen und die so liegen, daß keiner der Punkte $\boldsymbol{A}_{k}$ unterhalb dieser Geraden lirgt. Alle Guraden dieser Art finden wir auf folgende Art. Wir leg'n rine Gerade durch den Punkt $A_{0}$ und lassen sie zunächst mit der Ordinatenarhse zusammenfallen und drehen sie dann solange um $A_{0}$ entgrgen dem Sinne des Uhrzeigers, bis ein zweiter der Punkte $A_{k}$ auf der Geraden liegt, otwa der Punkt $A_{\lambda}$. Liegen auf der Geraden $A_{0} A_{\lambda}$ mehr 
als zwei der Punkte $A_{k}$, so soll unter $A_{z}$ der am weitesten rechts liegende unter diesen Punkten verstanden sein. Bie Gurade $A_{0} A_{\lambda}$ ist damn eine der gesuchten Geraden. Wir lassen die Grerate sieh weiter drehen in demselben Sinne, aber $n$ den Punkt $A_{2}$, bis wieder riner der Punkte $A_{6}$ auf der Geraden liegt. Es sei etwa der l'unkt $A_{1}$. Liegen aubur $A_{\lambda}, A_{u}$ auf der Geraden $A_{2} A_{n}$ noch andere der l'unkte $A_{k}$, so sell wieder $A_{n}$ der am weitesten rechts grome Punkt sein. $A_{\lambda} A_{u}$ ist aluch rine der gesuchten Geraden. Wir lassen die Gerade sich woiter in demselloen sinne drehen, abre um den Punkt $A_{u}$, bis wieder ein weiterer Punkt anßer $A_{\text {u }}$ auf ihr liegt. Es sei der Punkt $A_{v}$. Liegen außer $A_{n}$ und $A_{v}$ dann noch andere Punkte $A_{k}$ anf der Geraden, so sollen wieder alle zwischen $A_{u}$ und $A_{v}$ liegen. $A_{n} A_{1}$ ist dann auch eine der gesuchten Geraden. In dieser Weise fahren wir fort, bis wir zu einer Geraden kommen, die den am weitesten rechts liegenden Punkt $A_{m}$ enthält. Um einen bestimmten Fall vor Augen zu haben, nehmen wir an, das sei die Gerade $A_{v} A_{m}$. Wir erhalten dann vier Gerade der verlangten $A$ rt, nämlich $A_{0} A_{z}, A_{z} A_{u}, A_{u} A_{v}, A_{v} A_{m}$. In dem in der Abb. 3 dargestellten Fall rrgeben sich nach dem angegohenen Verfahren die vier Geraden $A_{0} A_{1}, A_{1} A_{2}, A_{2} A_{6}, A_{6} A_{8}$ (Ab). 3). Die Strecken $\overline{A_{0} A_{\lambda}}, \overline{A_{\lambda} A_{u}}, \overline{A_{u} A_{r}}, A_{r} A_{m}$ bilden einen nach unten überall konvexen und nach oben offenen Streckenzug von der Art, daß keiner der Punkte $A_{k}$ unterhalb von ihm liegt; der Tangens des Neigungswinkels dieser Streeken gegen die negative Abszissenachse gibt uns die erlaubten Werte von $\varepsilon_{1}$. Für den Fall der Abb. 2 u. 3 bekommen wir also vier Werte von $\varepsilon_{1}$, und zwar die Werte $4,1, \frac{1}{2},-\frac{3}{2}$.

Für jodes $\varepsilon_{1}$, das wir gefunden haben, finden wir den zugehörigen Wert des Koeffizienten $e_{1}$, indem wir aus den Gliedern (4) diejenigen aussuchen, die für das betreffende $\varepsilon_{1}$ dieselbe Potenz von $z$ als Faktor haben und die Bedingung dafür aufstellen, daß sie sich gegenseitig aufheben. Nehmen wir z. B. das $\varepsilon_{1}$, das zu der Streeke $A_{\lambda} A_{n}$ gehört. Es werden für diesen Wert von $\varepsilon_{1}$ alle diejenigen Exponenten $\boldsymbol{\alpha}_{k}+k \varepsilon_{1}$ einander gleich, deren zugehörige Punkte $A_{k}$ auf der Strecke $A_{2} A_{u}$ liegen, also vor allem $\varkappa_{1}+\lambda_{\varepsilon_{1}}$ und $\iota_{u}+\mu \varepsilon_{1}$. Liegt auf $A_{\lambda} A_{u}$ keiner der Punkte $A_{k}$ außer $A_{\lambda}$ und $A_{u}$, so werden auch nur diese beiden Exponenten gleich und gleiehzeitig die kleinsten. Sollen die entsprechenden der Glieder (4) sich fortheben, so $\mathrm{muB}$ sein

$$
g_{\lambda} e_{\hat{1}}^{\dot{\lambda}}+g_{u} e_{1}^{u}=0
$$

oder, da $e_{1}$ als von Null versehieden vorausgesetzt werden konnte und $\boldsymbol{\mu}>\lambda$ ist,

$$
g_{\lambda}+g_{u} e_{1}^{u-\lambda}=0 .
$$

Wir erhalten also eine Gleichung vom Grade $\mu-\lambda$ für $e_{1}$, und zwar eine reine Gleichung. Liegen auf $A_{\lambda} A_{u}$ noch andere Punkte $A_{k}$, die dann 
nach unseren Festsetzungen zwischen $A_{\lambda}$ und $A_{\mu}$ liegen, etwa $A_{0}$ und $A_{0}$, so werden für das gewählte $\varepsilon_{1}$ die vier Exponenten

$$
\alpha_{\lambda}+\lambda \varepsilon_{1}, \quad \alpha_{0}+\varrho \varepsilon_{1}, \quad \alpha_{o}+\sigma \varepsilon_{1}, \quad \alpha_{\mu}+\mu \varepsilon_{1}
$$

anander gleich, und es erhalten die vier Glieder (4) mit diesen Exponenten fiir das gewählte $\varepsilon_{1}$ dieselbe und zugleich die kleinste Ordnung. Sollen sie sieh fortheben, so muß sein

$$
g_{\lambda} e_{1}^{\lambda}+g_{0} e_{i}^{u}+g_{o} e_{1}^{o}+g_{u} e_{1}^{u}=0
$$

oder, da $e_{1} \neq 0$ und $\lambda<\varrho<\sigma<\mu$,

$$
g_{\lambda}+g_{v} e_{i}^{o-\lambda}+g_{v} e_{1}^{\sigma-\lambda}+g_{\mu} e_{1}^{u-\lambda}=0 .
$$

Wir erhalten also wieder eine Gleichung vom Grade $\mu-\lambda$, freilich diesmal keine reine Gleichung. Die Werte von $e_{1}$, die uns diese Gleichung liefert, können also sehr wohl teilweise oder auch alle einander gleich sein.

Wir erhalten also eine endliche Zahl von möglichen Exponenten $\varepsilon_{1}$; wenn wir bei unserem Beispiel bleiben, vier. Zu jedem $\varepsilon_{1}$ ergibt sich eine endliche Zahl von Werten für den Koeffizienten $e_{1}$, und zwar ergeben sich zu den durch die Strecken $\bar{A}_{0} A_{\lambda},{\overline{A_{\lambda} A_{u}}}_{A_{u} A_{v}},{\overline{A_{v}} A_{m}}_{m}$ bestimmten Werten von $\varepsilon_{1}$ der Reihe nach $\lambda, u-\lambda, v-\mu, m-v$ Werte des Koeffizienten $e_{1}$, so daß wir für die gesuchte Entwicklung $y=e_{1} z^{\varepsilon_{1}}+e_{2} z^{\varepsilon_{2}}+\cdots$ im ganzen $\lambda+(\mu-\lambda)+(\nu-\mu)+(m-\nu)=m$ Anfangsglieder $e_{1} z^{\varepsilon_{1}}$ erhalten, die aber nicht alle voneinander verschieden zu sein brauchen.

$\mathrm{Zu} \mathrm{Abb.} 3$ würde z. B. folgende Gleichung führen

$$
\begin{gathered}
2 z^{7}-z^{9}-z^{3} y+\left(4 z^{2}+z^{3}\right) y^{2}+\left(z^{3}-z^{4}\right) y^{3}-4 z y^{4}+7 z^{5} y^{5} \\
+\left(1-z^{2}\right) y^{6}+5 z^{6} y^{7}+z^{3} y^{5}=0 .
\end{gathered}
$$

Hier ist

$$
g_{0}=2, g_{1}=-1, g_{2}=4, g_{3}=1, g_{4}=-4, g_{5}=7, g_{6}=1, g_{7}=5, g_{8}=1 \text {. }
$$

Wir haben, wie wir schon festgestellt haben, 4 Werte für $\varepsilon_{1}$, nämlich 4, $1, \frac{1}{2},-\frac{3}{2}$. Für $e_{1}$ ergeben sich folgende Gleichungen:

1. $\varepsilon_{1}=4$. Auf der ersten Strecke des Streckenzuges in Abb. 3 liegen $A_{0}, A_{1}$, also haben wir dis Gleichung

$$
g_{0}+e_{1} g_{1}=0 \quad \text { oder } \quad 2-e_{1}=0, \quad e_{1}=2 .
$$

2. $\varepsilon_{1}=1$. Auf der entsprechenden Strecke $\overline{A_{1} A_{2}}$ liegen $A_{1}, A_{2}$, also ist hier

$$
g_{1}+e_{1} g_{2}=0 \quad \text { oder } \quad-1+4 e_{1}=0, \quad e_{1}=\frac{1}{4} \text {. }
$$

3. $\varepsilon_{1}=\frac{1}{2}$. Es liegen $A_{2}, A_{4}, A_{6}$ auf der folgenden Strecke, also ist $g_{2}+e_{1}^{2} g_{4}+e_{1}^{4} g_{6}=0$ oder $4-4 e_{1}^{2}+e_{1}^{4}=0, e_{1}= \pm \sqrt{2}, \pm \sqrt{2}$. 
4. $\varepsilon_{1}=-\frac{3}{2}$. Auf der entsprechenden Strecke liegen $A_{6}, A_{8}$, also ergibt sich

$$
g_{8}+e_{1}^{2} g_{8}=0 \text { oder } 1+e_{1}^{2}=0, e_{1}= \pm V-1
$$

Wir erhalten daher für die Entwicklung der dureh die Gleichung (9) definierten Funktion $y$ in der Umgebung von $z=0$ im ganzen 8 Entwicklungen mit den Anfangsgliedern

$2 z^{4}, \frac{1}{4} z, \sqrt{2} z^{\frac{1}{2}}, \sqrt{2} z^{\frac{1}{2}},-\sqrt{2} z^{\frac{1}{2}},-\sqrt{2} z^{\frac{1}{2}}, \sqrt{-1} z^{-\frac{3}{2}},-1-1 z^{-\frac{3}{2}}$,

von denen zweimal zwei einander gleich sind.

Die Bestimmung der weiteren Glieder der Reihenentwicklungen läßt sich in derselben Weise ausfülıren, wie die Bestimmung des Anfangsgliedes. Ist $e_{1} z_{-}^{k_{1}}$ eins der gefundenen Anfangsglieder, so setzen wir

$$
y=e_{1} z^{\varepsilon_{1}}+y_{1}
$$

in die Gleichung (1) zwischen $y$ und $z$ ein. Wir erhalten dann eine Gleichung von der Form

$$
\left(h_{0} z^{\beta_{0}}+\cdots\right)+\left(h_{1} z^{\beta_{1}}+\cdots\right) y_{1}+\cdots+\left(h_{m} z^{\zeta_{m}}+\cdots\right) y_{1}^{m}=0,
$$

in der freilich gebrochene Potenzen von $z$ vorkommen können, die aber im übrigen ganz so aussieht wie die Gleichung (1). In dieser Gleichung setzen wir

$$
y_{1}=e_{\dot{z}_{\mathrm{z}}} z^{\varepsilon_{2}}+e_{3} z^{\varepsilon_{3}}+\cdots
$$

und bestimmen $\varepsilon_{2}$ und $e_{2}$ geradeso, wie wir $\varepsilon_{1}$ und $e_{1}$ bestimmt haben. Dabei ist zu berücksichtigen, daß nur solche Werte von $\varepsilon_{2}$ in Betracht kommen, die größer sind als $\varepsilon_{1}$. Es ergeben sich auch $\varepsilon_{2}$ und $e_{2}$ endlich vieldeutig. Sind $\varepsilon_{2}$ und $e_{2}$ zwei der gefundenen Werte, die zusammen gehören, so setzt man in (10) ein

$$
y_{1}=e_{2} z^{\varepsilon_{2}}+y_{2}
$$

und erhält eine Gleichung in $z$ und $y_{2}$, die in derselben Weise zu behandeln ist wie die Gleichungen (1) und (10).

Auf diese $\Lambda$ rt erhalten wir für $y=e_{1} z^{\varepsilon_{1}}+y_{1}=e_{1} z^{\varepsilon_{1}}+e_{2} z^{\xi_{2}}+y_{2}$ $=e_{1} z^{k_{1}}+e_{2} z^{k_{2}}+e_{3} z^{\xi_{3}}+\ldots$ eine endliche $Z$ ahl von Reihenentwieklungen, die der Gleichung (1) jedenfalls formal genügen. Wir erhalten aber dureh unser Verfahren alle Reihenentwieklungen dieser Art. Unter diesen sind also sicher auch die $m$ konvergenten Reihenentwicklungen enthalten, deren Vorhandensein in $\S 2 \mathrm{u}$. $\S 3, \mathrm{Kap}$. I bewiesen ist. Es könnte aher der Fall eintreten, daß uns unser Verfahren noch weitere, der Gleichung (1) nur formal genügende Entwicklungen liefert. Wir können aber leicht zeigen, daß das nicht der Fall sein kann. 
Lis sein $y_{1}, y_{2} \ldots y_{m}$ die $m$ Wurzoln moserer Gleichung (1) $g(y, z)$

0 in der l'mgebung von $z=0$. Es sind also die $y_{k}$ Potenzreihen nach ganten uder gohrochenen Potenzen vin $\approx$ Dann ist identisch

$$
g(y, z) \quad c_{m}\left(y-y_{1}\right)\left(y-y_{2}\right) \ldots\left(y-y_{m}\right) .
$$

Ergah sich dureh unser Virfahren now wine von den $y_{k}$ verschiedene Pontenzreihe $\bar{y}$, dir der (ilridung $g=0$ formal geniugt. so müßte $g(\dot{y}, z)$ und also aum

$$
c_{m}\left(\bar{y}-y_{1}\right)\left(\bar{y}-y_{2}\right) \ldots\left(\bar{y}-y_{m^{\prime \prime}}\right)
$$

identiseh Null werden, jedenfalls dann. Wron man die klammern ausmultipliziert mul nach Potenzen von $z$ ordnet. l)as aber ist auch rein formal mur möglich. won dir Reihe $\bar{y}$ mit riner der Reihen $y_{k}$ übereinstimmt.

\section{\$3. Beispiele.}

1. Wir betrachton als erstes Beispiel die schon früher behandelte Cileichung (6) $\$ 1$

$$
x^{2}+2 x^{3}-\left(x+x^{2}\right) y+x y^{2}+y^{3}=0 .
$$

E: soll wieder $y$ als Funktion von $x$ in der Umgebung von $x=0$ betrachtet werden. Die Glieder, auf die es bei der Bestimmung des Anfangskeffizienten $e_{1}$ und des Anfangsexponenten $\varepsilon_{1}$ inkommt, sind hier (vgl. (') $\$ 1$ )

$$
x^{2}, \quad-e_{1} x^{1+\varepsilon_{1}}, \quad e_{1}^{2} x^{1} \quad 2^{2} \varepsilon_{1}, \quad e_{1}^{3} x^{3 \xi_{1}} .
$$

Aus dem Puiseuxschen Diagramm (Abb) 4) rrgeben sieh für $\varepsilon_{1}$ die Werte 1 und $\frac{1}{2}$, die wir auch früher auf anderem, weniger rinfachem Wege gefunden haben.

$$
\dot{r}_{1}=1 . \quad y=e_{1} x+\cdots .
$$

La $A_{0}, A_{1}$ auf der Strecke liegen, die zu $\varepsilon_{1}=1$ führt, so werden für $y=e_{1} r+\cdots$ die briden rerten der Glieder (12) von gleicher und niedrigster Ordnung. Sir helen sich fort, wenn

wir setzen atio in (11)

$$
1-e_{1}=0, e_{1}=1 \text {; }
$$

$$
y=x+y_{1}
$$

ind rirhalt, n

$$
3 x^{3}-\left(x-4 x^{2}\right) y_{1}+4 x y_{1}^{2}+y_{1}^{3}=0 \text {. }
$$

Zur Bwstimmung von $\varepsilon_{2}$, e. kommt es auf die Glieder an:

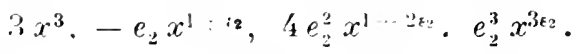

bas Puiseuxshe biagramm (Abb, 5) crgibt, wenn man beachtet, daß $r_{2}, r_{1}$ sein mub. $\therefore_{2}=2$. Auf der rntsprechenden Strecke liegen $A_{0}, A_{1}$. 
Also werden für $\varepsilon_{2}=2$ das orste und zwrite Glied (15) von derselben und niedrigsten Ordunng. Sie heben sich fort, wonn

$$
3-e_{2}=0, \quad e_{2}=3 \text {. }
$$

Wir setzen also in (1'́) pin

$$
y_{1}=3 x^{2}+y_{2}
$$

und erhalten

$$
\begin{gathered}
12 x^{4}+36 x^{5}+27 x^{6}-\left(x-4 x^{2}-24 x^{3}-27 x^{4}\right) y_{2} \\
+\left(4 x+9 x^{2}\right) y^{2}+y_{2}^{3}=0 .
\end{gathered}
$$

Die Glieder, auf die es zur Bestimmung von $\varepsilon_{3}, e_{3}$ ankommt, sind hier

$$
12 x^{4},-e_{3} x^{1+z_{3}}, 4 e_{3}^{2} x^{1} z_{3}^{2}, e_{3}^{3} x^{3 z_{3}} .
$$

Aus dem Puiseuxschen Diagramm (Abb. 6) ergibt sich, wenn man be-

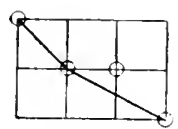

Abb. 4

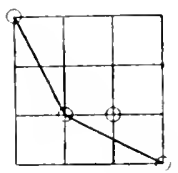

Abr.

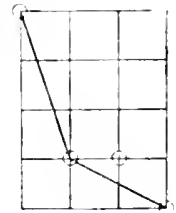

Ahb. 6.

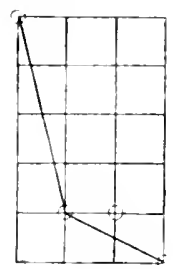

Abb. 7 .

denkt, daß $\varepsilon_{3}>\varepsilon_{2} \operatorname{sein} \mathrm{muß}, \varepsilon_{3}=3$. Für $e_{3}$ erhalten wir die Gleichung, da wieder die beiden ersten Glieder in (18) sich fortheben müssen,

$$
12-e_{3}=0, \quad e_{3}=12 \text {. }
$$

Wir haben also zu setzen in (17)

$$
y_{2}=12 x^{3}+y_{3} \text {. }
$$

Wir wollen nur noch $\varepsilon_{4}, e_{4}$ bestimmen und brauchen daher nur die Anfangsglieder zu berechnen. Das gibt

$$
84 x^{5}+\cdots-(x+\cdots) y_{3}+(4 x+\cdots) y_{3}^{2}+y_{3}^{3}=0 \text {. }
$$

Aus dem Puiseuxschen Diagramm (Abb. 7) ergibt $\operatorname{sich} \varepsilon_{4}=4$ und dann $e_{4}=84$, also

$$
y_{3}=84 x^{4}+\cdots
$$

Aus den Gleichungen (13), (16) und (20) ergibt sich für $y$ folgende Entwicklung

$$
y=x+3 x^{2}+12 x^{3}+8^{\prime} x^{4}+\cdots .
$$

2.

$$
\varepsilon_{1}=\frac{1}{2} \cdot y=e_{1} x^{\frac{1}{2}}+\cdots \text {. }
$$

Da die Punkte $A_{1}, A_{3}$ auf der Strecke liegen, die zu $\varepsilon_{1}={ }_{2}^{1}$ führt, werden 
für $\varepsilon_{1}=\frac{1}{2}$ das zwoite und viorte Giliod (12) von derselben und nicdrigsten

ordunng. Site hohen sich fort, wemn

$$
-e_{1}+e_{1}^{3}=0, e_{1}= \pm 1 \text {. }
$$

Wir haben also eine Entwicklung $y=+x^{2} \cdots$ und eine $y=-x^{2}+\cdots \cdots$ $\mathrm{Es}$ ist aber nicht etwa nötig, beide Entwicklungen weiter zu berechnen. Es enügt, eine von ihnen zu bestimmen, da wir die andere aus dieser einfach dadurch bekommen, daß wir $x$ einen geschlossenen Umlauf um den punkt 0 machen lassen, also $x^{2}$ durch $-x^{2}$ ersetzen.

Wir berechnen div Reihe $y=+x^{2}+\cdots$ und setzen

$$
y=x^{\frac{1}{2}}+y_{1}
$$

in (11) ein. Wir erhalten

$$
2 x^{2}-x^{2}+2 x^{3}+\left(2 x+2 x^{2}-x^{2}\right) y_{1}+\left(3 x^{2}+x\right) y_{1}^{2}+y_{1}^{3}=0 \text {. }
$$

Die Glieder, auf die es bei der Bestimmung von $\varepsilon_{2}, e_{2}$ ankommt, sind hier

$$
2 x^{2}, 2 e_{2} x^{1 \cdots \varepsilon_{2}}, 3 e_{2}^{2} x^{1}+2 \varepsilon_{2}, \quad e_{2}^{3} x^{3 \varepsilon_{2}} .
$$

Aus dem Puiseuxsehen biagramm (Abb. 8) ergibt sich, da $\varepsilon_{2}>\varepsilon_{1}$ sein muß. $\varepsilon_{2}=1$ und ferner, daß für $\varepsilon_{2}=1$ das erste und zweite Glied (24) von derselben und niedrigsten Ordnung werden. Sie heben sich fort, wenn $2+2 e_{2}=0$. $e_{2}=-1$. Wir setzen also

in (13) ein und prhalten

$$
y_{1}=-x+y_{2}
$$

$$
3 x^{3}+\left(2 x-4 x^{\frac{3}{2}}\right) y_{2}+\left(3 x^{\frac{1}{2}}-2 x\right) y_{2}^{2}+y_{2}^{3}=0 \text {. }
$$

Zur Bestimmung von $\varepsilon_{3}, e_{3}$ kommt es an auf die Glieder

$$
3 x^{3}, 2 e_{3} x^{1+\varepsilon_{3}}, 3 e_{3}^{2} x^{\frac{1}{2}+2 \varepsilon_{3}}, e_{3}^{3} x^{3 \varepsilon_{3}} .
$$

Wir finden (Abb. 9) $\varepsilon_{3}=2,3+2 e_{3}=0, e_{3}=-\frac{3}{2}$.

Wir setzen

$$
y_{2}=-\frac{3}{2} x^{2}+y_{3}
$$

in (26) ein, beschränken uns aber auf die Bercehunng der Anfangsglieder, dis wir nur noch $\varepsilon_{\mathbf{4}}, e_{\mathbf{4}}$ bestimmen wollen. Wir erhalten

$$
6 x^{2}+\cdots+(2 x+\cdots) y_{3}+\left(3 x^{2}+\cdots\right) y_{3}^{2}+y_{3}^{3}=0 \text {. }
$$


Die Glieder, auf die es ankommt, sind also

$$
6 x^{7}, \quad 2 e_{4} x^{1+4}, \quad 3 e_{4}^{2} x^{1}+2 *, e_{4}^{3} x^{3+} \cdot
$$

Aus (Abb. 10) ergiht sich $\imath_{4}=\frac{5}{2} ; e_{4}=-3$, alst)

$$
y_{3}=-3 x^{j}+\cdots \text {. }
$$

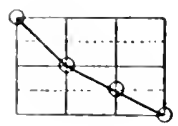

Abb. 8 .

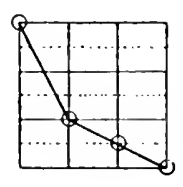

Abb. 9.

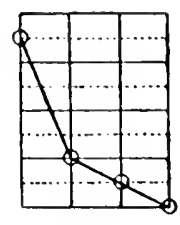

Ab). 10 .

Aus den Gleichungen (22), (25), (27) und (28) bekommen wir die Entwicklung

$$
y=x^{2}-x-\frac{3}{2} x^{2}-3 x^{\frac{5}{2}}+\cdots
$$

aus der sich durch Vertauschen von $x^{\frac{1}{2}}$ mit $-x^{\frac{1}{2}}$ die dritte Entwicklung ergibt

$$
y=-x^{\frac{1}{2}}-x-\frac{3}{2} x^{2}+3 x^{\frac{5}{2}}+\cdots
$$

Der Gang der Rechnung wird durch dieses ausführlich behandelte Beispiel klar geworden sein. Es seien noch drei Beispiele angegeben, aber der Gang der Prechnung nur kurz angedeutet.

$$
\text { II. } f(x, y) \cdots 1+x+\frac{1}{4} x^{2}+x^{4}-\left(2+x+x^{3}\right) y+y^{2}=0 \text {. }
$$

Auch hier wollen wir die Entwicklungen von $y$ für die Stelle $x=0$ bestimmen.

1. $-2 e_{1} x^{\varepsilon_{1}}, e_{1}^{2} x^{2 \varepsilon_{1}}$; Abb. 11. $\varepsilon_{1}=0 ; 1-2 e_{1}+e_{1}^{2} \equiv\left(1-e_{1}\right)^{2}=0, e_{1}=1$.

$$
\begin{aligned}
& y=1+y_{1}, \frac{1}{4} x^{2}-x^{3}+x^{4}-\left(x+x^{3}\right) y_{1}+y_{1}^{2}=0 . \\
& { }_{4}^{1} x^{2},-e_{2} x^{1+\varepsilon_{2}}, e_{2}^{2} x^{2 \varepsilon_{2}} ; \\
& \text { Ab. 12. } \varepsilon_{2}=1 ; \frac{1}{4}-e_{2}+e_{2}^{2}=\left(\frac{1}{2}-e_{2}\right)^{2}=0, e_{2}=\frac{1}{2} \text {. } \\
& y_{1}=\frac{1}{2} x+y_{2:}, x^{3}-\frac{1}{2} x^{4}+x^{3} y_{2}-y_{2}^{2}=0 \text {. } \\
& x^{3}, e_{3} x^{3+\varepsilon_{3}},-e_{3}^{2} x^{2 \varepsilon_{3}} ; \text { Abb. 13. } \varepsilon_{3}=\frac{3}{2}, 1-e_{3}^{2}=0 e_{3}= \pm 1 \text {. }
\end{aligned}
$$


Es genügt auch hier, mit dem Werte $e_{3}=+1$ weiter zu rechnen. Die Entwicklung, für die $e_{3}=-1$, erhalten wir aus der anderen, indem wir $x^{\frac{1}{2}}$ durch $-x^{2}$ ersetzen.

$$
\begin{gathered}
y_{2}=x^{2}+y_{3}, \frac{1}{2} x^{4}-x^{\frac{9}{2}}+\left(2 x^{2}-x^{3}\right) y_{3}+y_{3}^{2}=0 . \\
\frac{1}{2} x^{4}, 2 e_{4} x^{\frac{3}{2}} \text {. } e_{4}^{2} x^{2 \varepsilon_{4}} ; \text { Ab b. 14. } \varepsilon_{4}=\frac{5}{2}, e_{4}=-\frac{1}{4} \\
y_{3}=-\frac{1}{4} x^{\frac{5}{2}}+\cdots
\end{gathered}
$$
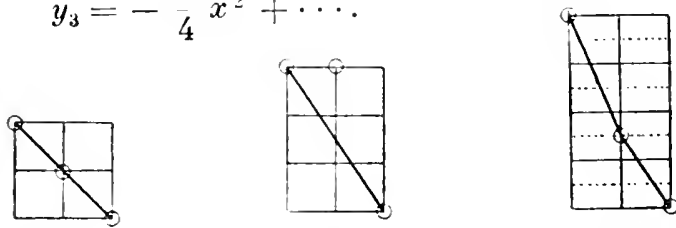

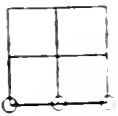

Abb. 11

Wir erhalten also die Entwicklung

$$
y=y_{1}=1+\frac{1}{2} x+x^{\frac{3}{2}}-\frac{1}{4} x^{\frac{5}{2}}+\cdots
$$

und die hieraus durch Vertauschen von $x^{\frac{1}{2}}$ mit $-x^{\frac{1}{2}}$ hervorgehende

$$
y=y_{2}=1+\frac{1}{2} x-x^{2}+\frac{1}{4} x^{\frac{5}{2}}+\cdots .
$$

III. $f(x, y) \equiv x^{2}+4 x^{3}+4 x^{4}-\left(2 x+4 x^{2}+2 x^{5}\right) y+y^{2}=0$.

Auch hier wollen wir $y$ in der Umgebung von $x=0$ betrachten.

$$
\begin{gathered}
x^{2},-2 e_{1} x^{1+\varepsilon_{2}}, e_{1}^{-3} x^{2 \varepsilon_{1}} ; \text { Abb. 15. } \varepsilon_{1}=1,1-2 e_{1}+e_{1}^{2}=0, e_{1}=1 . \\
y=x+y_{1}, 4 x^{4}-2 x^{6}-\left(4 x^{2}+2 x^{5}\right) y_{1}+y_{1}^{2}=0 .
\end{gathered}
$$

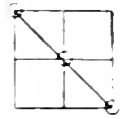

Abb. 15 .

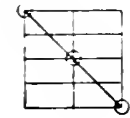

Abb. 16.

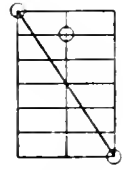

Abb. 17.

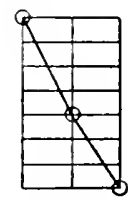

Abb. 18.

$$
\begin{gathered}
4 x^{4},-4 e_{2} x^{2+\iota_{2}}, e_{2}^{2} x^{2 t_{2}} ; \text { Abb. 16. } \varepsilon_{2}=2,4-4 e_{2}+e_{2}^{2}=0, e_{2}=2 . \\
y_{1}=2 x^{2}+y_{2}, 2 x^{6}+4 x^{7}+2 x^{5} y_{2}-y_{2}^{2}=0 . \\
2 x^{6}, 2 e_{2} x^{5+\varepsilon_{3}},-e_{3}^{2} x^{2 t_{3}} ; \text { Abb. 17. } \varepsilon_{3}=3,2-e_{3}^{2}=0, e_{3}= \pm \sqrt{2} .
\end{gathered}
$$

Auch hier hrauchen wir nur mit einem Wert von $e_{3}$ weiter zu rechnen. Die zweite Entwicklung geht zwar dieses Mal nicht dadurch aus der anderen 
hervor, daß $x$ den l'unkt 0 umläuft, wohl aber didurch, daß man $/ 2$ durch - $\sqrt{2}$ ersetzt.

$$
\begin{gathered}
y_{2}=12 x^{3}+y_{3}, 4 x^{7}+2 \mid 2 x^{8}-\left(2 \mid 2 x^{3}-2 x^{5}\right) y_{3}-y_{3}^{2}=0 . \\
4 x^{7},-2 e_{1} / 2 x^{3},-e_{4}^{2} x^{2} ; \text { Abb. 18. } x_{4}=4_{4}, e_{4}=+\sqrt{2} . \\
y_{3}=1 / 2 x^{4}+\cdots .
\end{gathered}
$$

Wir haben also folgende Entwicklungen

$$
\begin{aligned}
& y=y_{1}-x+2 x^{2}+12 x^{3}+12 x^{4}+\cdots, \\
& y=y_{2} \quad x+2 x^{2}-12 x^{3}-\sqrt{2} x^{4}+\cdots
\end{aligned}
$$

IV.

$$
f(x, y) \ldots x^{2}+x y-y^{3}=0 .
$$

Wir wollen die Entwicklungen für $y$ in der Umgebung von $x=\infty$ bestimmen und setzen daher $x=1 / z$. Wir erhalten

$$
g(z, y) \quad 1+z y-z^{2} y^{3}=0 .
$$

Wir haben hier einen Fall, wo eine der Potenzen von $y$, nämlich $y^{2}$, fehlt.

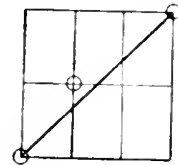

Abb. 19.

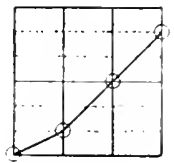

Abb. 20.

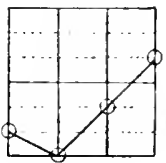

Abb. 21 .

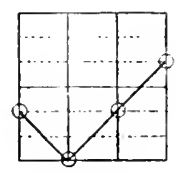

Abb. 22 .

In dem Puiseuxschen Diagramm (Abb. 19) fehlt daher der Punkt $A_{2}$. Die Rechnung gestaltet sich folgendermaßen

$$
\text { 1. } e_{1} z^{1+\varepsilon_{1}},-e_{1}^{3} z^{2-3 k_{1}} ; \text { Abb. 19. } \varepsilon_{1}=-\frac{2}{3}, e_{1}=\ddot{\|} \text {. }
$$

Es genügt, mit dem Werte $e_{1}=1$ woiter zu rechnen. Die andern Entwicklungen erhalten wir, indem wir in der errechneten $\sqrt[3]{x}$ durch ihre anderen Werte $\left.\varepsilon\right|^{3} x$ und $\varepsilon^{2} \mid x$ ersetzen, wo $\varepsilon=\frac{1}{2}(-1+1-3)$ die eine primitive dritte Einheitswurzel ist.

$$
\begin{aligned}
& y=z^{-\frac{2}{3}}+y_{1}, 1-\left(3 z^{\frac{1}{3}}-z^{\frac{2}{3}}\right) y_{1}-3 z y_{1}^{2}-z^{3} y_{1}^{3}=0 . \\
& 1,-3 e_{2} z^{3^{++t_{2}}},-3 e_{2}^{2} z^{1+2 \varepsilon_{2}},-e_{2}^{3} z^{\frac{5}{3}+3 \varepsilon_{2}} ; \text { Abb. } 20 . \varepsilon_{2}=-\frac{1}{3}, e_{2}=\frac{1}{3} \text {. } \\
& y_{1}=\frac{1}{3} z^{-\frac{1}{3}}+y_{2}, \frac{1}{27} z^{\frac{1}{3}}+\left(3+z^{\frac{1}{3}}+\frac{1}{3} z^{3}\right) y_{2}+\left(3 z^{\frac{2}{3}}+z\right) y_{2}^{2}+z^{3} y_{2}^{3}=0 \text {. } \\
& \frac{1}{27} z^{\frac{1}{3}}, 3 e_{3} z^{t_{3}}, 3 e_{3}^{-\frac{2}{3} z^{3}+2^{2},}, e_{3}^{3} z^{\frac{4}{3}+3 \varepsilon_{3}} ; \text { Abb. 2l. } \varepsilon_{3}=\frac{1}{3}, e_{3}=-\frac{1}{81} .
\end{aligned}
$$




$$
\begin{aligned}
& y_{2}=-\frac{1}{s 1^{\frac{1}{3}}} z^{\frac{1}{3}}+y_{3}, \frac{1}{s 1^{2}} z^{3}+\cdots-(3+\cdots) y_{3}-\left(3 z^{\frac{2}{3}}+\cdots\right) y_{0}^{2}-z^{\frac{4}{3}} y_{3}^{8}=0 . \\
& \frac{1}{S 1} z^{3} \cdot-3 e_{4} z^{k_{4}},-3 e_{4}^{2} z^{\frac{2}{3}+2 z_{4}},-e_{4}^{3} z^{4+3 z_{4}} ; \text { Abb. } 22 . \varepsilon_{1}=\frac{2}{3}, e_{4}=\frac{1}{243} \text {. } \\
& y_{3}=\frac{1}{243} z^{3}+\cdots
\end{aligned}
$$

Wir erhalten so die Entwicklung

$$
y=y_{1}-z^{-\frac{2}{3}}+\frac{1}{3} z^{-\frac{1}{3}}-\frac{1}{81^{-\frac{1}{3}}}+\frac{1}{243} z^{\frac{2}{3}}+\cdots
$$

oder

$$
y=y_{1} x^{\frac{2}{3}}+\frac{1}{3} x^{\frac{1}{3}}-\frac{1}{81} x^{-\frac{1}{3}}+\frac{1}{243} x^{-\frac{2}{3}}+\cdots
$$

und die beiden adjungierten

$$
\begin{gathered}
y=y_{2} \quad \varepsilon^{2} x^{\frac{2}{3}}+\frac{\varepsilon}{3} x^{\frac{1}{3}}-\frac{\varepsilon^{2}}{81} x^{-\frac{1}{3}}+\frac{\varepsilon}{243} x^{-\frac{2}{3}}+\cdots, \\
y=y_{3}-\varepsilon x^{\frac{2}{3}}+\frac{\varepsilon^{2}}{3} x^{\frac{1}{3}}-\frac{\varepsilon}{81} x^{-\frac{1}{3}}+\frac{\varepsilon^{2}}{243} x^{-\frac{2}{3}}+\cdots
\end{gathered}
$$

Da in der Gleichung $f(x, y)=x^{2}+x y-y^{3}$ das Glied mit $y^{2}$ fehlt, so muß die Summe $y_{1}+y_{2}+y_{3}$ der Wurzeln identisch Null sein. Da $1+\varepsilon+\varepsilon^{2}=0$, so bestätigt man das leicht. Aus demselben Grunde fehlen in den gefundenen Entwicklungen die ganzzahligen Potenzen von $x$.

$$
\text { Drittes Kapitel. }
$$

\section{Die Riemannsche Fläche.}

\section{$\$ 1$. Die $m$ konjugierten Funktionen.}

Wir haben im vorhergehenden gesehen, wie sich die durch die Gleichung $f(x, y)=0$ definierte Funktion $y$ in der Umgebung eines Punktes der $x$-Ebene verhält. Wir wollen uns jetzt über den Verlauf von $y$ in der ganzen $x$-Ebene Klarheit zu verschaffen suchen. Wir bezeichnen wieder die Gaußsche $x$-Ebene oder Kugel mit $E$. Diejenigen Punkte $a$, wo nicht alle Entwicklungen von $y$ nach ganzen Potenzen von $x-a$ fortschreiten, seien bezeichnet mit $\mathfrak{B}_{1}, \mathfrak{B}_{2}, \ldots \mathfrak{B}_{h}$. Wir schneiden diese Punkte durch kleine Kreise, deren Mittelpunkte diese Punkte sind, aus $E$ aus. Die Kreise müssen jedenfalls so klein sein, daß ihre Flächen sich gegenseitig nicht überdecken. Es sei ferner $\mathfrak{B}_{0}$ irgendein Punkt der $x$-Ebene, der außerhalb der $h$ um die Punkte $\mathfrak{Z}_{1}, \mathfrak{B}_{2}, \ldots \mathfrak{B}_{h}$ gezeichneten Kreise liegt. Wir ziehen von $\mathfrak{S}_{0}$ nach den Punkten $\mathfrak{B}_{1}, \mathfrak{B}_{2}, \ldots \mathfrak{B}_{h}$ Linien, $l_{1}, l_{2}, \ldots l_{h}$, die sich nicht selbst und nicht untereinander treffen. Längs dieser Linien $l_{k}$ denken 


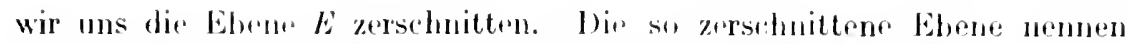
wir $E_{1}$. Dorch joten Sehnitt $l_{k}$ entstehen zwei l3ogrenzungslinien, die wir als rechtes und linkes Ufer untershoiden wollen, und zwar soll das rechte Ufer dasjenige soin, das rechts liegt, wenn man ven $\mathscr{L}_{0}$ marh $\mathscr{W}_{k}$ geht.

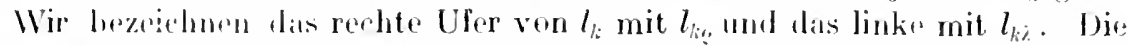
Elene obler lingel $E_{1}$, je nachdom man sich's vorstollen will, hat oine

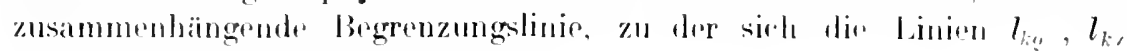
zusammensehlieBon, mod zwar hei passendor Wahl dor bezeichnong in der Ripihenfolge $l_{1 ;} l_{1:} l_{2 ;} l_{2,} l_{3 ;} l_{3, \ldots} \ldots$ $l_{\text {h, }} l_{\text {he. }}$ (Abh. 2?.)

Es sid c rin Junkt in Innorn Jur Ebene $E_{1}$. Wir haben $m$ Entwieklungen für $y$ in der Umgebung von $c$, die nach ganzen Potenzen ven $x-c$ fortschreiten. Wir bezrichnen sie mit $\mathbb{R}_{1}(x-c), \mathfrak{H}_{2}(x-c), \ldots$ $\mathscr{H}_{1,}(x-c)$. Wir setzen das Funktionselement $t_{1}(x-c)$ in $E_{1}$ analy-

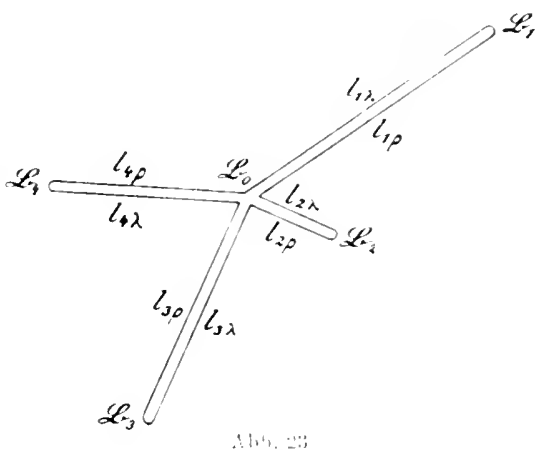
tisch fort. Dio Funktion, die wir auf diese Art aus $\$_{1}(x-c)$ erhalten, nennen wir $y_{1}$.

Da $E_{1}$ eine einfach zusammenhängende Fläche ist, und da $y_{1}$ in $E_{1}$ sich überall wie eine rationale Funktion von $x$ verhält, das heißt sich in ler Umgebung jeder Stelle a im Innern von $E_{1}$ in eine Potenzreihe entwickelu läBt, die nach stejgenden ganzen Potenzen von $x$ - a fortschreitet, wobri nur für rine ondliche Zahl von Stellen negative Potenzen und immer nur in endlicher Zahl auftreten, so ist $y_{1}$ in $E_{1}$ cine oindentige analytische Funktion von $x$.

Wir erhalten ebenso eine eindeutige analytisch Funktion von $x$, die wir mit $y_{i}$ bezeichnen wollen, wenn wir das Element $\mathbb{W}_{k}(x-c)$ in $E_{1}$ analytisch fortsetzen. So orhalten wir die $m$ in $E_{1}$ eindeutigen analytischen Funktionen $y_{1}, \ldots y_{n}$ vor $x$. Wir nennen sie zueinander $k$ onjugiert. Wir geben jeder dieser Funktionen ein eigenes Blatt oder eine eigene Kugelfläche. Diese bezeichnen wir mit $E_{1}, E_{2} \ldots E_{m}$. Sie sollen alle zu $E_{1}$ kongruent sein. Wir denken uns die Werte von $y_{i}$ auf der Kugelfläche $E_{k}$ ausgebreitet. Diese $m$ Flächen legen wir so übereinander, daß die Punkte, die demselben Werte von $x$ entsprechen, aufeinander liegen.

Wir wollen diese $m$ getrennten Blätter nach dem Vorgange Riemanns zusammenheften längs der Schnitte $l_{k}$ in einer noch wäher zu bosprechenden Weise, so daß wir statt der $m$ einzelnen losen Blätter eine einzige $m$-blättrige Fläche erhalten, auf der dann, wie wir sehen werden, $y$ eine eindeutige Funktion des Ortes ist. 


\section{\$ุ. Boispiel 1.}

Es sei $y$ als algebraische Funktion von $x$ definiert dureh die Gleichung

$$
y^{2}-x^{2}-1=0 \text {. }
$$

Es ist $y=1 x^{2}+1$. Es sei a rin endlicher Punkt, der $x$-Ebene. Wir setzen $x-a=z$ und erhalten

$$
y=11+a^{2}+2 a z+z^{2} .
$$

Ist $1+a^{2} \neq 0$, also $a \neq \pm i$, so folgt weiter:

$$
y= \pm 11+a^{2}\left(1+\frac{2 a}{1+a^{2}} z+\frac{z^{2}}{1+a^{2}}\right)^{2} \text {. }
$$

Wir erhalten also für $y$ nach dem binomischen Satze zwei gewöhnliche Potenzreihen, die nach Potenzen von $z=x-a$ fortschreiten und die sich nur durch das Vorzeichen unterscheiden.

Für $a=i$ haben wir

$$
y=\left|2 i z^{\frac{1}{2}}\left(1+\frac{z}{2 i}\right)^{\frac{1}{2}}=\right| 2 i(x-i)^{\frac{1}{2}}|1-i 2(x-i)|^{i} .
$$

Wir erhalten also eine Entwicklung, die nach gebrochenen Potenzen von $x-i$ oder nach ganzen Potenzen von $(x-i)^{\frac{1}{2}}$ fortschreitet. Ebenso erhalten wir für $a=-i$ eine Entwicklung, die nach ganzen Potenzen von $(x+i)^{\frac{1}{2}}$ fortschreitet. Wir haben also für endliche Werte von $x$ zwei singuläre Punkte, $i$ und $-i$.

Um noch $y$ für unendlich große Werte von $x$ zu betrachten, setzen wir $x=1 / z$ und haben

$$
y= \pm \frac{1}{z} \sqrt{1+z^{2}}
$$

Wir erhalten also für $y$ zwei nur durch das Vorzeichen verschiedene Potenzreihen, die nach ganzen Potenzen von $z=x^{-1}$ fortschreiten.

Wir bezeichnen $i$ mit $\mathfrak{B}_{1}$ und $-i$ mit $\mathfrak{R}_{2}$, um die Bezeichnung des allgemeinen Falles zu haben, und nehmen als Punkt $\mathfrak{B}_{0}$ den Nullpunkt. Als Linien $l_{1}, l_{2}$, nehmen wir den Teil der Achse der rein imaginären Zahlen, der zwischen $-i$ und $+i$ liegt. Wir zerschneiden also die $x$-Ebene längs der Strecke von $-i$ bis $+i$. Wir bezeichnen diesen aus $l_{1}$ und $l_{2}$ bestehenden Schnitt mit $l$. Unter $l_{0}$ verstehen wir das Ufer von $l$, das rechts liegt, wenn man von $-i$ nach $+i$ hinsieht; unter $l_{\dot{\lambda}}$ das andere. Da in unserem Beispiel $m=2$, so haben wir zwei solche $x$-Ebenen zu nehmen, die wir mit $E_{1}$ und $E_{2}$ bezeichnen (Abb. 24). 
Als Punkt $c$ nehmen wir den Punkt 1. Hier haben wir die Entwicklungen

$$
\begin{gathered}
\mathfrak{S}_{1}(x-1) \quad 12+\frac{1}{2} / 2(x-1)+\cdots, \$_{2}(x-1)-\sqrt{2}-\frac{1}{2} \sqrt{2}(x-1) \\
+\cdots
\end{gathered}
$$

Wir können zwar die analytische Fortsetzung praktisch nicht ausführen, aber wir können in diesem einfachen Falle doeh angeben, welche von den beiden an einer Stelle $a$ geltenden Entwieklungen ein Element von $y_{1}$ und welehe eines von $y_{2}$ ist. $y_{1}$ hat an der Stelle 1 den Wert $+1 / 2$. Der reelle Teil von $y_{1}$ ist also für $x=1$ positiv, und da er sich mit $x$ stetig ändert, so kann er nur negativ werden, wenn er vorher Null wird. Das aber kann nur eintreten, wenn $y_{1}$ rein imaginär wird. Dazu ist notwendig und hinreichend, daß $1+x^{2}$ reell und negativ ist, also $x$ rein imaginär und absolut größer als 1 ist.

Es ist daher der reelle Teil von $y_{1}$ nur Null für diejenigen Punkte der Achse der rein imaginären Zahlen, die außerhalb der Strecke von $-i$ bis $+i$ liegen. Daraus folgt zunächst, daß $y_{1}$ für alle Punkte rechts von der imaginären Achse, die Punkte der imaginären Achse selbst nicht mit gerechnet, dureh dasjenige

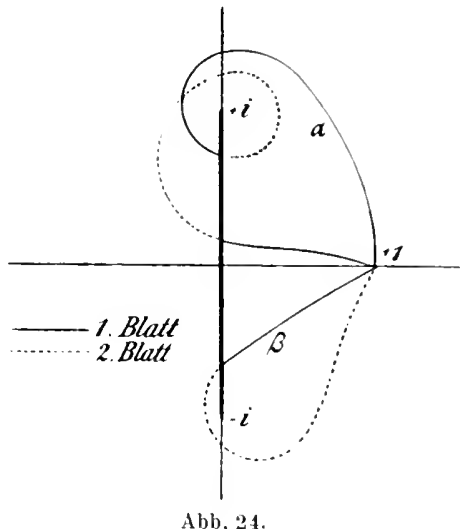

Abb. 24. Element gegeben ist, dessen reeller Teil positiv ist. Lassen wir $x$ auf

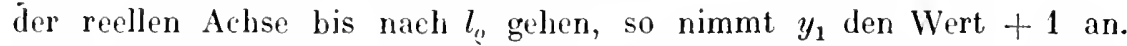
Lassen wir $x$ längs $l_{0}$ weitergehen bis nahe an $+i$, so bleibt $y_{\mathbf{1}}$ reell und positiv und nähert sich dem Werte Null. Wir lassen $x$ nicht bis ganz nach dem Punkte $i$ gehen, sondern nur bis nahe heran und lassen es dann auf einem kleinen Kreise in positivem Sinne um den Punkt $i$ herumgehen. Haben wir den Kreis halb durehlaufen, befinden wir uns also wieder auf der imaginären Achse, aber außerhalb der Strecke von $-i$ bis $+i$, so hat $\sqrt{x-i}$ den Faktor $i$ bekommen und also zufolge der in der Umgebung von $i$ geltenden Reihenentwieklung auch $y$. Es ist also $y$ von positiven reellen Werten zu positiven rein imaginären Werten übergegangen. Daraus folgern wir, daß $y_{1}$ längs der positiven imaginären Achse oberhalb $+i$ rein imaginäre positive Werte hat. Lassen wir $x$ den kleinen Kreis um $+i$ ganz durchlaufen, so daß wir nach einem nahe bei $i$ golegenen Punkte von $l_{2}$ kommen, so nimmt $\sqrt{x-i}$ und also auch $y$ den Faktor -1 auf, es ist also $y_{1}$ längs $l_{\lambda}$ reell und negativ. Daraus schließen wir weiter, daß links von der imaginären Achse $y_{1}$ überall 
einen negativen redten Tril hat, da der reelle Teil ja nur auf der imaginären Achse Null werden kann. Wir lassen jetzt $x$ längs 7 , weitergehen bis nahe an - i. Es bleibt $y_{1}$ negativ ronll. Dann lassen wir $x$ auf einem kleinen Kreise in prositiom Sinne un - $i$ herumghen. Hat $x$ den Kreis halb durehlanfon, se nimmt $\mid x+i$ und atso zufolge der Reihenentwicklung fur $y_{1}$ in der Imgebung von $i$ auch $y_{1}$ den Faktor $i$ auf, wird also negativ rein imaginär. Daraus s.hlinßen wir, dab $y_{1}$ auf dem Teil der imaginären Achse, der unterhalh von - $i$ liegt, negativ rein imaginär ist. Lassen wir $x$ den klein'n kreis um - $i$ ganz durehlaufen, so kommt $x$ wieder zu rinem Punkt auf $l_{\text {.. }}$. Es nimmt $\mid x+i$ und also auch $y_{1}$ den Faktor -1 anf, wird also wieder positiv reell, wie es sein muß. Es kann uns dies auch eine Bestätigung dafür sein, daß $y_{1}$ wirklich in $E_{1}$ eindeutig ist.

J lis Ergebnis fass wir zusammen. Es ist rechts von der imaginären A.his' der reelle Teil von $y_{1}$ positiv, links von der imaginären Achse negativ. Auf dor imaginären Achse selbst ist $y_{1}$ unterhalb $-i$ negativ rein imaginär. zwischen $-i$ und $+i$ längs $l_{n}$ positiv. längs $l_{i}$ negativ reell, (berhalb $+i$ ist $y_{1}$ positiv rein imaginär. Dadureh ist für jeden Punkt pindeutig bestimmt, welche von den beiden vorhandenen Entwicklungen $y_{1}$ darstellt. Die andere stellt dann $y_{.:}$dar. Wir wollen z. B. bestimmen, wekhe Entwicklung $y_{1}$ in der Umgebung dos unendlich fernen Punktes darstellt. Wir haben dis Entwirklungen

$$
y=x+\frac{1}{2 x}-\begin{array}{cc}
1 & 1 \\
2 & x^{3}
\end{array}+\cdots, \quad y=-x-\begin{aligned}
& 1 \\
& 2 x
\end{aligned}+\frac{1}{8} \frac{1}{x^{3}}-\cdots .
$$

1) Re Reihen geven für rein imaginäre Werte von $x$ rein imaginäre Werte von $y$, da sie nur ungrade Potenzen von $x$ enthalten. Sie konvergieren aber nur für $x \mid$ 1. Die erste ist für großes $x$ annähernd $x$. Sie gibt also, wenn $x$ auf der imaginären Achse oberhalb $+i$ liegt, positive, und wern $x$ unterhall, $-i$ liegt. negative rein imaginäre Verte von $y$. Sie stellt also $y_{1}$ dar. Die zweite Reihe demnach $y_{2}$.

Wir denken uns die Werte von $y_{1}$ in der Ebere $E_{1}$ und die von $y_{2}$ in der $E_{1}$ kongruenten Ebene $E_{2}$ ausgebreitet. $y_{1}$ ist in $E_{1}$ und $y_{2}$ in $E_{2}$ -ine eindeutige analytische Funktion. An $l$ sind die Funktionen unstetig. Wir können hier auch genau angeben in wolcher Art. Es sei $\xi$ ein Punkt von $l$. Wir müssen unterscheiden, ob wir ihn als Punkt von $l_{g}$ oder $l_{\lambda}$ auffassen wollen. Demnach unterseheiden wir zwisehen $\xi_{\text {. }}$ und $\xi_{\text {;. }}$ Es ist, wi wir gesehen haben, $y_{1}\left(\xi_{0}\right)$ positiv rerll und $y_{1}\left(\xi_{j}\right)$ negativ reell. Es können sich aber zwei Werte von $y=11+x^{2}$ für denselben Wert von $x$ nur durch das Vorzeichen unterseheiden und es ist daher $y_{1}\left(\xi_{0}\right)=-y_{1}\left(\xi_{i}\right)$. La $y_{2}$ sich von $y_{1}$ immer nur durch das Vorzeichen unterscheidet, so ist woitur.

$$
y_{2}\left(\xi_{1}\right)=-y_{1}\left(\xi_{1}\right)=y_{1}\left(\xi_{i}\right) . \quad y_{2}\left(\xi_{i}\right)=-y_{1}\left(\xi_{i}\right)=y_{1}\left(\xi_{3)}\right) .
$$


Das gilt für jeden l'unkt $\Xi$ von $l$. Wenn wir alse don hand $l_{\theta}$ von $E_{1}$ mit dem Rand $l_{\text {; }}$ von $E_{2}$ vrobinden und den Rand $l_{n}$ von $E_{2}$, mit tem Rand $l_{\text {; }}$ von $E_{1}$, also festsetzen, daß $x$, wenn es $l$ ïherschroitet und sich zunächst in $E_{1}$ befindet, von $E_{1}$ in $E_{2}$, mud, womes sinh in $E_{2}$ befindet, in $E_{1}$ eintreten soll, so sehlirßin sich die Werte von $y_{1}$ und $y_{2:}$ an $l$ stetig aneinander. Auf der Fläche $R$, die auf die angegebene $A_{1}$ t aus $E_{1}$ und $E_{2}$ entsteht, ist also $y$ eimr pindeutige Funktion des Ortes.

Die Flächr $R$ hribt eine Riemannsche Fläche. Sie besteht in unserem Fallr aus zwoi Blättern. Diese Blättor hängen längs $l$ krenzweis' zusammen. $l_{n}$ von $E_{1}$ ist mit $l_{2}$ von $E_{2}$ und $l_{;}$von $E_{1}$ mit $l_{n}$ von $E_{2}$ vereinigt. Die Fläche $\grave{R}$ durchsistzt sich also längs der Linie l. Ein Durchschnitt längs der reellen Achso sieht so aus (Abb. 25). $E_{1}$ und $E_{2}$ erscheinen als nah (eigentlich unendlicl nah) nebeneinander verlaufende Linien, $l$ als Punkt. Denken wir uns die Fläche $R$ materiell, etwa aus Papier hergestellt, und lassen wir $x$ vom Punkte $x=1$ in $E_{1}$ aus längs der reellen Achse nach $l$ wandern und über $l$ hinaus, so kann $x$, ohne die Fläche $R$ zu verlassen, stetig sich so bewegen, daB es in $E_{1}$ bleibt, also nicht nach $E_{2,}$ kommt (vgl. den gefiedorten Pfeilin Abb. 25), ja, es muB $x$ das Papier sogar durchsetzen, wenn es, wie es soll, nach $E_{2}$ kommen will (vgl.

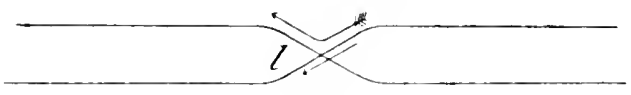

Ibb. 25 den ungefiederten Pfeil, Abb. 25). Aber das materielle Modell gibt eben kein genaues Bild der Kiemannselıen Fläche. Will man diese Schwierigkeit gern vermeid's, so kann man die Verbindung der Ebene $E_{1}$ und $E_{2}$ in folgender Weise vorwehmen. Der Kürze wegen bezeichnen wir die Ränder $l_{0}, l_{\text {; }}$ von $E_{1}$ mit $l_{0,1}, l_{j, 1}$ und die von $E_{2}$ mit $l_{0,2}, l_{j_{2}}$. Wir verbinden diejenigen Punktr von $l_{i, 1}$ mit dren entsprechenden von $l_{a s}$, in denen $x$ rin rationales Vielfarhes von $i$ ist, durch unendlich dünne Fäden oder Stäbehen. Wir erhaltrn so ein Gitter. Wir verbinden ferner diejenigen l'unkte von $l_{\text {i. }}$ und dir entsprechenden von $l_{n g}$, in denen $x$ ein irrationales Vielfaches von $i$ ist, durch unendlich dünne Stäbchen. Sie bilden ein zweites Gitter. Die Stäbe des ainen Gitters gehon durch die Lücken des anderen. Dann kann $x$ auch ohne weitero Festsetzung in der Fläche $R$ nur so wandern, wie es soll. Jedesmal, wenn es $l$ üborschreitet, kommt es in das andere Blatt. Wie man sich das auch vorstellen mag, wesentlicli ist, daB $x$ jedesmal, wenn es $l$ ülerschreitet von drm einen Blatt in das andere übertritt.

\section{\$3. Beispiel ‥}

Es sei $y$ definiert durch die Gleichung

$$
y=1 x+1 x-1
$$


Da $\left.\right|^{3} x$ dreiwertig und $\sqrt{x}-1$ zweiwertig ist und wir diese Werte beliehig miteinander kombinieren können, so hat $y$ für einen Wert von $x$ sochs im allgemeinen verschiedene Werte. Die rationale Gleichung. der $y$ gentigt, ist daher vom 6 . Grade. Diese Gleichung lautet

$$
\begin{gathered}
y^{6}-3(x-1) y^{4}-2 x y^{3}+3(x-1)^{2} y^{2}-6 x(x-1) y \\
-\left(x^{3}-4 x^{2}+3 x-1\right)=0 .
\end{gathered}
$$

Aus der Gleichung (38) folgt, dab nur die Punkte $x=0, x=1$ und $x=x$ singulär sind. Für die Ungebung von $x=0$ haben wir erstens die Entwicklung:

$$
y=i+x^{3}-\frac{i}{2} x-\frac{i}{8} x^{2}+\cdots,
$$

die wir einfach dadurch erhalten, daß wir $\sqrt{x-1}$ nach dem binomischen Satze nach Potenzen von $x$ entwickeln. Aus der Reihe (3) gehen noch zwei andere hervor, dadurch, daß wir $x^{\frac{1}{3}}$ ersetzen dureh $\varepsilon x^{\frac{1}{3}}$ und $\varepsilon^{2} x^{\frac{1}{3}}$, wo $\varepsilon=\frac{1}{2}(-1+i \mid \overline{3})$ eine dritte Einheitswurzel. Diese Reihen bezeichnen wir mit $\left(3_{1}\right)$ und $\left(3_{2}\right)$. Außerdem haben wir noch die Entwicklung:

$$
y=-i+x^{\frac{1}{3}}+\frac{i}{2} x+\frac{i}{8} x^{2}+\cdots
$$

die wir erhalten, wenn wir den anderen Wert von $\mid x-1$ benutzen. Auch aus (4) gehen noch zwei Entwicklungen hervor, indem wir $x^{\frac{1}{3}}$ durch $\varepsilon x^{\frac{1}{3}}$ und $\varepsilon^{2} x^{3}$ ersetzen. Wir bezeichnen sie mit $\left(4_{1}\right)$ und $\left(4_{2}\right)$. Im ganzen haben wir also, wie es sein muß, 6 Entwicklungen. Diese hängen zu je dreien in der Umgebung von $x=0$ zusammen. Wenn wir $x$ den Nullpunkt umkreisen lassen, so können wir die drei Reihen (3) ineinander überführen und ebenso die drei Reihen (4). nicht aber eine der Reihen (3) in eine der Reihen (4).

Für die Ungebung des Punktes 1 haben wir, indem wir $\sqrt[3]{x}$ nach Potenzen von $x-1$ entwickeln, je nachdem, welchen Wert von $\sqrt[3]{x}$ wir benutzen, eine der folgenden Entwicklungen:

$$
\begin{aligned}
& y=1-(x-1)^{\frac{1}{2}}+\frac{1}{3}(x-1)-\frac{1}{9}(x-1)^{2}+\cdots, \\
& y=\varepsilon+(x-1)^{\frac{1}{2}}+\frac{\varepsilon}{3}(x-1)-\frac{\varepsilon}{9}(x-1)^{2}+\cdots, \\
& y=\varepsilon^{2}+(x-1)^{\frac{1}{2}}+\frac{\varepsilon^{2}}{3}(x-1)-\frac{\varepsilon^{2}}{9}(x-1)^{2}+\cdots
\end{aligned}
$$


Aus jeder geht noch eine zweite hervor dadurch, daB wir $(x-1)^{\frac{1}{2}} \mathrm{ar}$ setzen durch - $-(x-1)^{2}$. Wir haben im gauzen 6 konjugierte Entwicklungen. Diese hängen in der Umgebung von $x=1$ paarweise zusammen.

Für die Ungebung des nnendlich fernen Punktes finden wir

$$
\begin{aligned}
y=x^{\frac{1}{3}}+x^{\frac{1}{3}} \sqrt{1-{ }^{1}} & =x^{\frac{1}{3}}+x^{2}-\frac{1}{2}\left(\begin{array}{c}
1 \\
x
\end{array}\right)^{1}-\frac{1}{8}\left(\begin{array}{l}
1 \\
x
\end{array}\right)^{3}+\cdots \\
& =x^{6}+x^{6}-\frac{1}{2}\left(\frac{1}{x}\right)^{3}-\frac{1}{8}\left(\begin{array}{l}
1 \\
x
\end{array}\right)^{6}+\cdots,
\end{aligned}
$$

also eine Reihenentwicklung, die nach steigenden ganzen Potenzen von $\left(\begin{array}{l}1 \\ x\end{array}\right)^{\prime}$ fortschreitet. Lassen wir $x$ den unendlich fernen Punkt in positivem Sinne umkreisen, so erhalten wir nach jedem Umlauf eine neue Entwicklung, und zwar im ganzen sechs. Diese sind der Reihe nach, wie sie sich auf die angegebene Art ergeben:

$$
\begin{aligned}
& x^{\frac{1}{3}}+x^{\frac{1}{2}} \sqrt{1-x^{-1}}, \varepsilon^{2} x^{\frac{1}{3}}-x^{\frac{1}{2}} \sqrt{1-x^{-1}}, \quad \varepsilon x^{\frac{1}{3}}+x^{\frac{1}{2}} \sqrt{1-x^{-1}}, \\
& x^{3}-x^{\frac{1}{2}} \sqrt{1-x^{-1}}, \varepsilon^{2} x^{\frac{1}{3}}+x^{2} \sqrt{1-x^{-1}}, \quad \varepsilon x^{\frac{1}{3}}-x^{\frac{1}{2}} \sqrt{1-x^{-1}} .
\end{aligned}
$$

Dabei ist unter $\sqrt{1-x^{-1}}$ diejenige Entwicklung von $\sqrt{1-x^{-1}}$ zu verstehen, die für $x=\infty$ den Wert 1 annimmt. Es ist zu beachten, daß, wenn $x$ sich in positivem Sinne um den unendlich fernen Punkt bewegt, es sich auf einem sehr großen Kreise um den Nullpunkt in negativem Sinne bewegt. Die sechs Entwicklungen in der Umgebung des unendlich fernen Punktes hängen also alle in der Umgebung dieses Punktes zusammen.

Wir nennen die Punkte $0,1, \infty$ in dieser Reihenfolge $\mathfrak{B}_{1}, \mathfrak{R}_{2}, \mathfrak{B}_{3}$ und nehmen als $\mathfrak{Z}_{0}$ den Punkt $i$ und führen längs der Strecken $\overline{\mathcal{S}_{0}} \mathfrak{B}_{1}, \overline{\mathcal{B}_{0}} \mathbb{S}_{2}$. die Schnitte $l_{1}, l_{2}$ aus. Als Schnitt $l_{3}$ nehmen wir den Strahl, der von $\mathcal{B}_{0}$ aus parallel zur Achse der positiven reellen Zahlen nach oo geht. Für Punkt $c$ nehmen wir den Punkt ${ }_{2}^{1}$. Für $x=\frac{1}{2}$ sollen $y_{1}, y_{2}, \cdots y_{6}$ die Werte

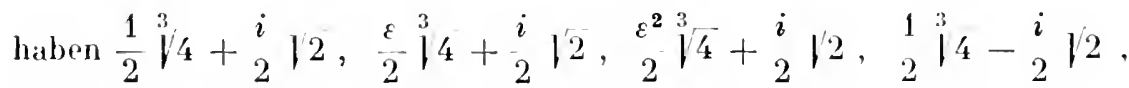
${ }_{2}{ }^{3} / 4-{ }_{2}^{i} / 2, \varepsilon_{2}^{2} / 4-{ }_{2}^{i} / 2$, wo $/ 4$ und $/ 2$ reell und positiv genommen werden sollen. Wenn $x$ einmal in positivem Sinne um den Punkt 0 herumgeht, sobleibt $/ x-1$ ungeändert, $\sqrt[3]{x}$ nimmt den Faktor $\varepsilon$ an. Es geht also $y_{1}$ über in $y_{2}, y_{2}$ in $y_{3}, y_{3}$ in $y_{1}$ ferner $y_{4}$ in $y_{5}, y_{5}$ in $y_{6}, y_{6}$ in $y_{4}$. Wir nehmen $6 x$-Ebenen $E_{1}, E_{2}, \ldots E_{6}$, die alle längs der Linien $l_{1}, l_{2}, l_{3}$ zerschnitten sind. 
Diese Ebenen haben wir längs $l_{1}$ so miteinander zu vereinigen, wie in der Abb. 26 angegehen. Da $z$. B. $y_{1}$ in $y_{2}$ übergeht, wenn $x$ den Schnitt $l_{1}$ vom

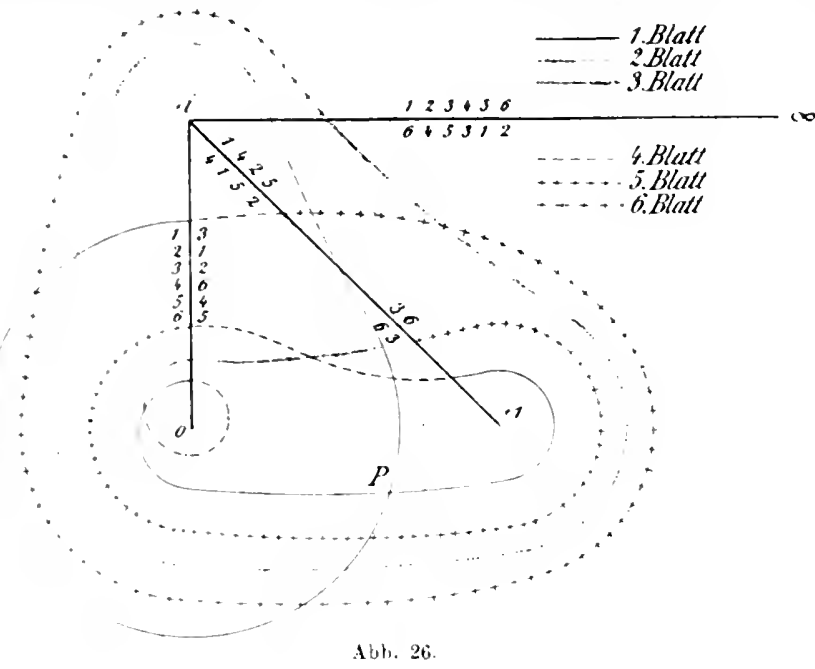

linken zum rechten Ufer überschreitet, so muß $r$ hierbei aus $E_{1}$ nach $E_{2}$ kommen. Wir haben also das linke Ufer von $E_{1}$ mit dem rechten von $E_{2}$ zu vereinigen, was durch die beiden nebeneinander stehenden Zahlen 2, 1 ausgedrückt ist. Wir wollen überhaupt in folgender Weise angeben, wie die Blätter längs eines Schnittes verbunden sind. Gelangt man beim Überschreiten eines Schnittes von links nach rechts aus dem $\iota-t e n$ ins $\beta$-te Blatt, so wollen wir auf die linke Seite des Schnittes irgendwo a hinschreiben und rechts dicht daneben $\beta$.

Lassen wir $x$ inmal in positivem Sinne um den Punkt 1 gehen, so ändert $\operatorname{sich} \mid x$ nicht, $\mid x-1$ geht in $-\mid x-1$ über. Es geht also $y_{1}$ über in $y_{4}, y_{2}$ in $y_{5} y_{3}$ in $y_{6}$ und umgekehrt $y_{4}$ in $y_{1}, y_{5}$ in $y_{2}, y_{6}$ in $y_{3}$. Wir haben also die Blätter $E_{k}$ längs $l_{2}$ so zu vereinigen, wie in Abb. 26 angegeben. Lassen wir schließlich $x$ einmal in positivem Sinne um den Punkt $\infty$ herumgehen, so geht $\mid x$ über in $\varepsilon^{2} \mid x$ und $\mid x-1$ in $-\mid x-1$, also geht über $y_{1}$ in $y_{6}, y_{6}$ in $y_{2}, y_{2}$ in $y_{4}, y_{4}$ in $y_{3}, y_{3}$ in $y_{5}, y_{5}$ wieder in

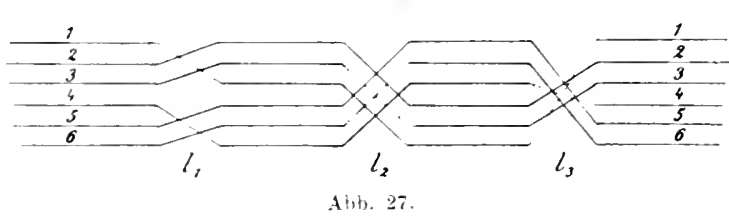

$y_{1}$, so daß wir die Blättคr längs $l_{3}$ so zusammenfügen müssen, wie in der Abb. 26 angegeben.

In Abb. 27 ist ein Durehsehnitt durch die entstandene sechsblättrige Riemannsche Fläche gezeichnet, und zwar längs einer Geraden, die $l_{1}, l_{2}, l_{3}$ schneidet, der erkennen läßt, wie die Blätter längs $l_{1}, l_{2}, l_{3}$ zusammenhängen. Nur zu Erhöhung der Übersicht sind die Verbindungslinien zum Teil punktiert. Aus dieser Abbildung geht auch deutlich hervor, daß man allein durch Überschreiten von $l_{1}$ aus dem 
ersten Blatte ins zweite und dritte golang'n kann, aber nicht ins virert, fünfte oder sechstr, daB man allein dureh C̈bersehroiten von $l_{2}$ aus dem ersten Blatt wohl ins vierte gelangen kam, aber in krines der andren. Eine Probn für die Richtigkeit der atugeführtm Verbindungen ist folgendes. Wenn man von irgendeinem Punkte ans in irgendeinem Blatte einen Weg beschreibt, der nur $\mathfrak{L}_{0}$ umkreist, nicht aber rinen der anderen Punkte $\aleph_{k}$, so muB man, da $\aleph_{0}$ kein singulärer l'unkt ist. schon nach einem Umlauf wieder zum Ausgangspunkte zuruikkonmen (also, auch ins selbe Blatt). Ich überlasse es dem Leser, dies zu bestätigen. In der Abb. 26 sind zwei geschlossene Kurven grzeichnet, dir sich nur in einem Punkte treffen.

\section{\$4. Beispiel 3 .}

Es sei $y$ definiert durch die Gleichung

$$
f(x, y) \quad x^{2}+x y-y^{3}=0 \text {. }
$$

Wie wir in Kap. II 2 sahen, wo wir diese Gleichung als Beispiel IV behandelten, hahen wir im Unendlichen eine nach Potenzen von $\left(\begin{array}{l}1 \\ x\end{array}\right)^{\frac{1}{3}}$ fortschreitende Reihe, so dab wir dort einen singulären Punkt haben. Um zu sehen, wo etwa im Endlichen noch singuläre Punkte vorhanden sind. sehen wir zu, wo $\frac{d j}{d y}$ Null wird. Es ist

$$
\frac{d f}{d y}=x-3 y^{2}
$$

und das ist gleichzeitig mit $f$ Null, für $x=y=0$ und für $x=\begin{gathered}\text { 't } \\ 27\end{gathered}, y=-\frac{2}{9}$. Es sind also $x=0$ und $x={ }_{27}^{4}$ dir.rinzigenim Endlichen lirgenden Punkt.. die singulär sein können.

Wir erhalten in dre Lmgelsung von $x=0$ pine nach ganzen Potenz+n von $x$ und wine nach ganzen Potenzm von $x$ - fortschreitende Reihe und in der Umgebung von $x=\frac{4}{27}$ rin nach ganzen Potenzen von $\left(x-\frac{1}{27}\right)$ und pine nath ganzen Potenzen von $\left(x-\frac{1}{27}\right)^{2}$ fortschreitend Reihe.

Als Punkt $\mathfrak{B}_{0}$ nehmen wir den Punkt $i$ und ziehen die Schnitte $l_{1}, l_{2}, l_{3}$ vun $W_{0}$ nach $0, \frac{4}{27}, \infty$, in der in Abb. 28 angegebenen Weise. Wa unser. Filrichung in $y$ vom dritton Gradı ist, haben wir drei längs $l_{1}, l_{2}, l_{3}$ zer- 
schnittene Ehenen $E_{1}, E_{2}, E_{3}$ nötig, in denen wir uns die Funktionen $y_{1}$, $y_{2}, y_{3}$ ausgehreitet denken. Im Unendlichen hängen alle drei Funktionen $y_{1}, y_{2}, y_{3}$ zusammen. Da wir die Bezeichnung willkürlich wählen dürfen, kïnnen wir annohmen, daß $y_{1}$ in $y_{2}, y_{2}$ in $y_{3}, y_{3}$ in $y_{1}$ übergeht, wenn $x$ den Punkt oo cinmal in positivem Sinne umkreist. Wir müssen dann die Blïtter lïngs $l_{3}$ so zusammenheften, wie in der Abbildung angegeben.

Im Punkte ${ }_{27}^{4}$ hängen zwei der Wurzeln zusammen und gehen ineinander über, wenn $x$ den Punkt ${ }_{27}^{4}$ einmal umläuft. Da die Wurzeln $y_{1}, y_{2}, y_{3}$ noch gleichberechtigt sind, so können wir die Bezeichnung so wählen,

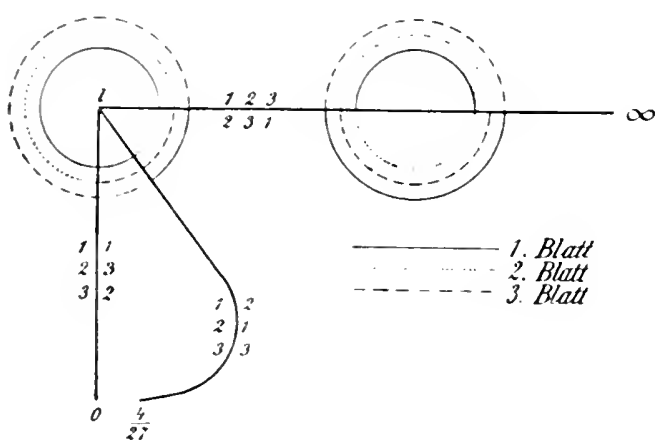

Abb. 28 .

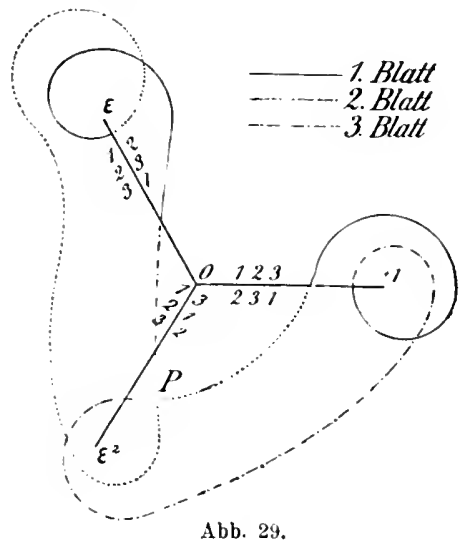

Abb. 29 .

daßin $\frac{4}{27}$ die Wurzeln $y_{1}$ und $y_{2}$ zusammenhängen. Dann müssen wir längs $l_{2}$ die Blätter so zusammenheften, wie in der Abb. 28 angegeben. Wie wir die Blätter längs $l_{1}$ zusammenheften müssen, ergibt sich jetzt daraus, daß wir nach einmaligem Umkreisen des Punktes ${ }^{2}{ }_{0}$ wieder in dasselbe Blatt gelangen müssen, gleichgültig in welehem Blatt wir uns beim Beginn des Umkreisens befinden. Es ergibt sich, daß längs $l_{1}$ die Blätter $E_{2}$ und $E_{3}$ zusammenhängen.

Wir haben also für die Funktion $y$ eine Riemannsche Fläche hergestellt, können aber nicht entscheiden, welche von den drei in der Umgebung irgendeines Punktes $x$ geltenden Reihenentwicklungen für das erste, zweite oder dritte Blatt gilt. Las zu entscheiden, würde noch allerhand Überlegungen notwendig machen. Am schnellsten kommt man wobl zum Ziel, indem man die Gleichung nach $y$ mit der Cardanischen Formel auflöst.

In Abb. 29 ist noch die Riemannsche Fläche dargestellt, die zu der Gleichung $x^{3}+y^{3}-1=0$ gehört. Es sind auf ihr zwei geschlossene Knrven gezeirhnrt, die sich nur in einem Punkte $P$ schneiden. 


\section{\$ 5. Konstruktion der Riemanmschen Flïche.}

Wir haben (vgl. §1) $m$ Blätter $E_{\mathrm{I}}, E_{2}, \ldots E_{m}$, dir längs der $h$ Linien

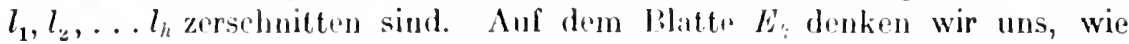
das oben auspinandergesetzt ist, die Funktion ". ansbreitet. Es sei $\xi$ ein beweglicher Punkt von $l_{1}$. Wir unterseheiden wieder, ob der Punkt anf dem rechten oder linken Ufer von $l_{1}$ liegend gedacht wreden soll, und nennen ihn je nachdem $\xi_{0}$ oder $\xi_{2}$. Die Funktionen

$$
y_{1}\left(\xi_{1,}\right), y_{2}\left(\xi_{10}\right), \ldots y_{m}\left(\xi_{0}\right)
$$

sind voneinander verschieden und sie stellen alle $m$ Wurzeln von $f(x, y)$ $=0$ dar, wenn $x$ ein Punkt von $l_{1}$ ist. Dasselbe gilt für die Funktionen

$$
y_{1}\left(\xi_{2}\right), y_{2}\left(\xi_{\lambda}\right), \ldots y_{m}\left(\xi_{\lambda}\right) \text {. }
$$

Es können sich daher die Funktionen (5) nur durch die Reihenfolge von den Funktionen (6) unterseheiden. Bezeichnen also $\alpha_{1}, \alpha_{2}, \ldots \alpha_{m}$ die Zahlen $1,2, \ldots m$ in passend gewählter Reihenfolge, so ist

$$
y_{1}\left(\xi_{\lambda}\right)=y_{\alpha_{1}}\left(\xi_{0}\right), y_{2}\left(\xi_{\lambda}\right)=y_{\omega_{2}}\left(\xi_{0}\right), \ldots y_{m}\left(\xi_{2}\right)=y_{\alpha_{m}}\left(\xi_{0}\right) \text {. }
$$

Wenn wir also $l_{1 \lambda}$ von $E_{k}$ vereinigen mit $l_{10}$ von $E_{\alpha_{k}}$, so schließen sich die Funktionen $y_{1}, y_{2}, \ldots y_{m}$ längs der $m$-Schnitte $l_{1}$ stetig ancinander. Jedes der $2 m$ längs $l_{1}$ vorhandenen Ufer wird mit einem anderen und nur mit einem verbunden, so daß kein freier Rand bleibt. Geht $x$ über $l_{1}$ einmal vom linken zum rechten Ufer hinüber, so kommt es, wenn es vorher in $E_{k}$ war, nach $E_{c_{k}}$ und es geht $y_{i}$ über in $y_{c_{k}}$. Das tritt im besonderen ein, wenn wir $x$ einen Umlauf in positivem Sinne um den Punkt $B_{1}$ machen lassen. Es tritt also auch dann unter $y_{1}, y_{2}, \ldots y_{m}$ die Vertauschung ein, daß $y_{k}$ in $y_{c_{k}}$ übergeht. Diese Vertauschung muß init der übereinstimmen, die sich aus den für die Umgebung von ${ }_{2}{ }_{1}$ geltenden Reilenentwicklungen ergibt.

Ebenso wie längs $l_{1}$ kann man die Blätter $E_{1}, E_{2}, \ldots E_{m}$ längs der anderen Schnitte $l_{k}$ so zusammenfügen, daß sich auch dort die $m$ Zweige $y_{1}, y_{2}, \ldots y_{m}$ stetig zusammenschließen. Damit haben wir bewiesen, daB es zu jeder algebraischen Funktion $y$ von $x$ eine Riemannsche Fläche gibt, auf der $y$ eine eindeutige Funktion des Ortes ist. Die $m$ Funktionen $y_{1}, y_{2}, \ldots y_{m}$, die zusammen die Funktion $y$ ausmachen, nennen wir die konjugierten Funktionen von $y$. Wir nehmen auch wohl im besonderen $y=y_{1}$, an und nennen $y_{2}, y_{3}, \ldots y_{m}$ konjugiert zu $y=y_{1}$.

\section{S 6. Zerfallen der Riemannschen Fläche.}

Wir haben die Riemannsehe Fläche aus den Ebenen $E_{1}, E_{2}, \ldots E_{n}$. erhalten dadurch, daß wir sie längs der Schnitte $l$ miteinander verbunden haben. Es entsteht die Frage, ob die so erhaltene Flärhe aus einem einzigen 
Stïck besteht uder aus mehreren untereinander nicht zusammenhängenden Toilen. In resten Falle kann der Punkt $x$ ans jeelem Blatt in jodes andere gelangen. ohne die Fläche zu verlassen, im zweiten nicht. Es zerfalle etwa die Riomannsche Fläche $R$ in zwei Teile $R_{1}, R_{2}$, die miteinander keinen Zusanmenhang haben. $R_{1}$ möge bestehen aus den Blättern $E_{1}, E_{2}, \ldots E_{\alpha}$ und $R_{2}$ aus den Blätteru $E_{x+1}, E_{t+2}, \ldots E_{m}$. Es kann dann $x$ aus keinem der Blätter $E_{1}, E_{2} \ldots E_{a s}$ in eins der Blätter $E_{a}+1, E_{w}+\ldots, \ldots E_{m}$ gelangen. Das hedentet aber, da $B$ keine der Funktionen $y_{1}, y_{2}, \ldots y_{t}$ durch analytisehe Fortsetzung ühergehen kamn in eine der Funktionen $y_{a+1}, y_{a}+2, \ldots y_{m}$. Besehreibt also $x$ in der cinfachen $x$-Ebene pine geschlossene Kurve, so vertausehen sich die a Funktionen $y_{1}, y_{,} \ldots y_{\text {a }}$ nur mntereinander, so daß jede symmetrische Funktion von ihnen ungeändert bleibt, also eins eindentige analytische Funktion von $x$ ist. In der Funktion

$$
\left(y-y_{1}\right)\left(y-y_{2}\right) \ldots\left(y-y_{12}\right)=y(x, y)
$$

sind daher die Koeffizienten der Potenzen von y mondeutige analytische Funktionen von $x$. Da es auBerdem ganze rationale Funktionen von $y_{1}, y_{2}, \ldots y_{\alpha}$ sind, so können sie nur unendlich werden, wenn $y_{1}, y_{2}, \ldots y_{r}$ "s werden. Sie können daher nur an einer endlichen Zahl von Stellen unendlich werden und jedesmal nur von endlicher Ordnung, so daß sie nur eine endlich. Zahl von Polen haben und sonst kein singulären Stellen. Sir sind daher rationale Funktionen von $x$. Demnach ist $g(x, y)$ eine ganze rational. Funktion von $y$ vom Grade $\iota$, deren Koeffizienten rationale Funktionen von $x$ sind. Zufolge der Definition von $g$ genügen die a Funktionen $y_{1}, y_{2}, \ldots y_{a}$ der Gleichung $g(x, y)=0$. Ebenso läBt sich zeigen, daß die $m-$ « ïbrigen $y_{k}$ einer Gleichung

$$
h(x, y) \quad\left(y-y_{t+1}\right)\left(y-y_{a}+2\right) \ldots\left(y-y_{m}\right)=0
$$

rom Grade $m$ - a in $y$ genügen, deren Koeffizienten rationale Funktionen von $x$ sind. Aus den Gleichungen (7), (8) und (2), § 1 folgt dif in $x$ und $y$ identische Gleichung

$$
f(x, y) \quad a_{0}(x) g(x, y) h(x, y),
$$

-1 r lab $f(x . y)$ rational zerfällt. Wenn umgekehrt $f(x, y)$ rational zerfällt, $\therefore$ kann man jeden Fatitor für sich Null setzen und für sich behandeln. Man erhält dann für jeden eine besondere Riemannsche Fläche, so daß die Rirmanusche Fläche von $f=0$ in so viele getrennte Teile zerfällt, wir $f(x, y)$ rationale Faktoren hat. Wir sehen also:

lir zu $f(x, y)=0$ gehörige Riemannsche Fläche zerfällt laun und nur dann, wenn $f(x, y)$ in rationale Faktoren zerfällt, und sie zerfällt in so viele gotrennte Teile, wie $f(x, y)$ rationale Faktoren hat. 


\section{s. Die zweiblittrige Riemannsche Fliche.}

Die bei unseren allgemeinen Brtrachtungen und auch bej den Beispielen benutzte Art der llerstellung der Rirmanusehen Flärhe ist nicht immer die rinfachste. Hazu wollen wir noch ein besonders wichtiges Bei-

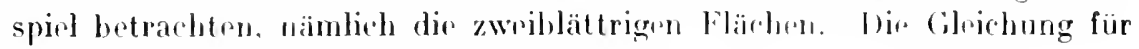
y mub in y vom zwriten Grade seint, nwa

$$
A y^{2}+B y+C=0
$$

wo $A, B, C$ ganze rationale Funktionen von $x$ sein sollen. Die Diskrimininte $B^{2}-4 A C$ kann einen quadratischen Faktor hahen. Wir setzen

$$
B^{2}-4 A C=Q^{2} G
$$

wn G' krinn'n mehrfachen Faktor mehr onthält. Da

$$
y=-\frac{1}{2} A(B+Q \mid G) \text {. }
$$

so folgt, daß $y$ an den Nullstellen von $G$ singulär ist und in Endlichen nur an diesen. Der unendlich ferne Punkt ist singulär. Wrm der Grad von $G$ ungrade ist, sonst nieht. Wir beschränken uns aul den Fall, wo der Grad von $G$ grade ist und bezeichnem ihn mit $2 g$. Dic Nullstellen von $G$ seien $a_{1}, a_{2}, \ldots a_{2 y}$. Wir nehmen zunächst eime einfiche $x$-Ebene, ziehen in ihr $g$ Linien $l_{1}, l_{2}, \ldots l_{l}$, die sich selbst und untereinander nicht schneiden, und zwar eine von $a_{1}$ nach $a_{2}$, eine von $a_{3}$ nach $a_{4}$ usw. und schließlich eine von $a_{2 g} \ldots$, nach $a_{27}$, und zerschueiden die Ebene längs der Linien $l_{k}$. Die zerschnittene Ebene nennen wir $E_{1}$. Im Innern von $E_{1}$ wählen wir irgendeinen Punkt $c$. Für die Umgetung von $c$ lwstehen zwei Entwicklungen für $y$, die sich nur durch das Vurzeichen von $f G$ unterseheiften. Setzen wir diese Entwieklungen in $E_{1}$ analytisch fort, so erhalten wir in $E_{1}$ zwei Funktionen $y_{1}$ und $y_{\text {- }}$, die in $E_{1}$ cindeutig sind. Denn wenn $x$ rinc'n der Punkt $a_{k}$ einmail umläuft, su ändert $\mid G$ sein Zeichen, es würde $y_{1}$ in $y_{2}$ und $y_{2}$ in $y_{1}$ übergehen. Aber wegen der Schnitte $l_{n}$ kann in $E_{1}$ $x$ immer nur eine grade Zahl von Punkten $a_{k}$ umlaufon und daher sind $y_{1}$ und $y_{2}$ eindeutig in $E_{1}$ und können nicht ineinander übergoführt werden, solange $x$ die Linien $l_{k}$ nicht ïberschreitet. Wir denken uns $y_{1}$ in $E_{1}$ ausgubreitet und $y_{2}$ in einer zu $E_{1}$ kongruenten Ebene $E_{2}$. Virbinden wir die Ränder dieser Ebenen an den Sehnitten $l_{k}$ krenzweise miteinandur, so erhalten wir de Kiemannsehe Fläche, anf der $y$ eindeutig ist. Hiese Konstruktion der Kiemannschen Fläche ist hier einfacher und ühersichtlicher als die für d’n allgimeinen Beweis benutzte.

\section{s. S. Die Punkte der Riemannschen Flaiche und ihre Umgebung.}

Unter der Umgebung eines Punktes $P$ der GauBschen Zahlen-Ebene oder-Kugel verstehen wir die Fläche eines Kreises, der den gegebenen 
Punkt $P$ als Vittelpmot hat. Dureh den Kreis wird die Gaußsche Zahlenkugel in zwei Gehiete geteilt derart, daß man die Umgebung des Punktes $P$ nicht verlassen kann, ohne den kreisumfang zu überschreiten. Zerschneiden wir die Fläche längs des Lroisumfanges, so wird die Umgebung des Punktes $P$ ganz von der Zalilenkugel losgelöst. Diese Eigenselaft muß dic Umgebung eines Punktes $P$ der Riemannschen Fläche auch haben, weil wir sonst nicht entseheiden können, ob ein Punkt der Umgebung vin $P$ angehört oder nicht.

Wir betrachten diejenigen Punkte der Rirmannschen Fläche $R$, für die $x$ einen bestimmten Wort, etwa den Wert a hat. Wir unterscheiden zwei llauptfälle. 1. a ist kniner der Punlite $\mathfrak{B}_{1}, \mathfrak{B}_{2}, \ldots \mathfrak{B}_{h}$, II. $a$ ist einer der Punkte $\mathfrak{S}_{1}, \mathbb{B}_{2}, \ldots \mathfrak{W}_{h}$.

I. Hier unterscheiden wir drei Unterfälle.

1. a liegt auf keiner der Linien l, längs deren wir die $m$ Blätter miteinander verbunden haben. Dir $m$ Blätter verlaufen in der Umgebung von a getrennt. Sie liegen übereinander, wie die Blätter eines Buches. Wir haben $m$ Punkte, $\mathfrak{p}_{1}, \mathfrak{p}_{2}, \ldots \mathfrak{p}_{m}$ in $R$, für die $x$ den Wert $a$ hat. Es liege $\mathfrak{p}_{*}$ in $E_{k}$. Unter der Umgebung eines dieser Punkte, z. B. des Punktes $\mathfrak{p}_{1}$, verstehen wir die Fläche eines Kreises, der in $E_{1}$ liegt, dessen Mittelpunkt $p_{1}$ ist und dessen Halbmesser so gewählt ist, daß keiner der Punkte $\mathcal{B}_{1}, \mathfrak{B}_{2}, \ldots \mathfrak{B}_{h}$ im Innern des Kreises liegt. Der Einfachheit halber wollen wir ferner noch annehmen, obwohl das nicht notwendig ist, dab auch keiner der Punkte der Linien $l_{1}, l_{z}, \ldots l_{h}$ im Innern des Kreises liegt. Machen wir dieselbe konstruktion bei den Punkten $\mathfrak{p}_{2}, \ldots \mathfrak{p}_{m}$, so erhalten wir $m$ Kreisflächen, die übereinander liegen und voneinander ganz getrennt verlaufen.

2. Es sei $a$ einer der Punkte von $l_{1}$ aber nicht $R_{0}$ oder $B_{1}$. Wir zeichnen um $a$ in jedem der $m$ Blätter von $R$ einen Kreis, dessen Radius so klein zu wählen ist, daß keiner der Punkte $\mathfrak{B}_{k}$ in ihm liegt, daß außer $l_{1}$ keine der Linien $l_{l}$ ihn durchsetzt und daß $l_{\mathbf{1}}$ ihn nur in zwei Teile zerlegt, und denken uns die Fläche $R$ längs dieser Kreise zerschnitten. Wir haben in der Umgebung von $a$ wieder $m$ Flächenstücke, die sich zwar längs $l_{1}$ durchsetzen, die aber nach unseren Festsetzungen nicht untereinander zusammenhängen. Wir bezeichnon diese Flächenstücke (Kreisflächen) mit I, II, III, .. Nehmen wir als Beispiel die Linie $l_{3}$ in Abh. 28. Längs $l_{3}$ haben wir den oberen Teil des ersten Blattes mit dem unteren des zweiten verbunden. Es bestoht also die Kreisfläche I aus einem Halbkreise, der oberhalb $l_{3}$ im ersten Blatt liegt, und aus einem Halbkreis, der unterhalb $l_{3}$ im zweiten Blatt lifgt. Das Flächenstück II besteht aus einem im zweiten Blatt oberhalb $l_{3}$ liegenden Halbkreis und aus einem im dritten Blatt unterhalb $l_{3}$ liegenden Halbkreis. Schließlich besteht das Flächenstück III aus einem Halbkrris oberhalb $l_{3}$ im dritten Blatt und aus einem Halbkreis unterhalb $l_{3}$ im erstrn Blatt. Diese drei Kreisflächen I, II, III durchsetzen sich zwar 
längs $l_{3}$, hängen aber nicht miteinander zusammen. Befindet sich $x$ in einer der Kreisflärhen I, II, III, so kann es nicht heraus, ohne den Umfang des Kreises zn ülersehreiten.

Wir haben also geradeso, wie im Falle $\mathrm{I}_{1}$, auch im Falle $\mathrm{I}_{2}$ für $x=a$ im ganzen $m$ Punkte der Riemannschen Fläche und jeder dieser hat als Ungebung eine schlichte Kreisfläche.

3. Es sei a der Punkt $\mathfrak{B}_{0}$. Auch hier zerschneiden wir jedes rer $m$ Blätter der Fläche $R$ längs eines Kreises um den Punkt $\$_{0}$ als Mittelpunkt, dessen Halbmesser so klein zu wählen ist, daß keiner der Punkte $\mathfrak{S}_{1}, \mathfrak{B}_{2}, \ldots \mathfrak{B}_{h}$ in seinem Innern liegt und da jede der $h$ Linien $l_{k}$ den Umfang des Kreises nur einmal trifft. Wir erhalten dann $m$ Flächenstürke, die sich zwar längs der Linien $l_{k}$ durchsetzen, die aber wieder nicht zusammenhängen und die wir mit I, II, III, ... bezeichnen. Jede dieser Kreisflächen enthält im allgemeinen Stücke aus mehreren der $m$ Blätter von $R$. So besteht z. B. in Abb. 29 jede der drei Kreisflächen I, II, III zu je einem Drittel aus einem Stück des ersten, einem des zweiten und einem des dritten Blattes. Man vgl. auch Abb. 26 u. 28.

Wir haben also auch bei $\mathfrak{B}_{0} m$ Punkte der Riemannschen Fläche, deren Umgebungen schlichte Kreisflächen sind.

II. Es sei $a$ einer der Punkte $\mathfrak{B}_{1}, \mathfrak{B}_{2}, \ldots \mathfrak{B}_{h}$, etwa der Punkt $\mathfrak{B}_{\mathbf{1}}$. Es möge in der Umgebung von $x=a$ für $y$ (unter anderem) eine Entwicklung gelten, die nach ganzen Potenzen von $(x-a)^{\frac{1}{\alpha}}$ fortschreitet. Sie liefert a adjungierte Entwicklungen, die mit $y_{1}, y_{2}, \ldots y_{\alpha}$ bezeichnet seien. Die Bezeichnung sei so gewählt, daß $y_{1}, y_{2} \ldots y_{\alpha}$ sich zyklisch vertauschen, wenn $x$ den Punkt $\mathfrak{B}_{1}$ einmal in positivem Sinne umkreist, also $l_{1}$ einmal von links nach rechts überschreitet. Ferner seien die Blätter $E_{k}$ so geordnet, da $B$ die Werte von $y_{k}$ in $E_{k}$ ausgebreitet sind. Da, wenn $x$ den Schnitt $l_{1}$ von links nach rechts überschreitet, $y_{1}$ übergeht in $y_{2}, y_{2}$ in $y_{3}, \ldots y_{\alpha}$ in $y_{1}$, so sind die Blätter $E_{1}, E_{2}, \ldots E_{\alpha}$ längs $l_{1}$ so miteinander verbunden, daß $x$, wenn es $l_{1}$ von links nach rechts überschreitet, aus $E_{1}$ in $E_{\text {. }}$, aus $E_{2}$ in $E_{3}, \ldots$ aus $E_{\alpha}$ in $E_{1}$ gelangt. Wir zeichnen in jedem der « Blätter $E_{1}, E_{2}, \ldots E_{\text {tc }}$ einen Kreis um $\mathfrak{S}_{1}$ mit so kleinem Halbmesser, daß keiner der Punkte $\mathfrak{B}_{2}, \mathfrak{B}_{3}, \ldots \mathfrak{B}_{\alpha}$ im Innern der Kreise liegt. Die Kreisflächen seien bezeichnet mit $K_{1}, K_{2}, \ldots K_{a}$. Sie hängen miteinander zusammen längs der Linie $l_{1}$ und bilden, wie wir gleich sehen werden, ein einziges Flächenstück mit nur einer Begrenzungslinie. Gehen wir nämlich von einem Punkte $A$ des Kreisumfanges im ersten Blatte aus und gehen auf dem Kreisumfang in positivem Sinne in $R$ weiter, so kommen wir nach einem Umlauf nicbt nach $A$ zurück, sondern in den unter $A$ liegenden Punkt des zweiten Blattes. Wandern wir aul dem Umfange in demselben Sinne weiter, so kommen wir nach einem weiteren Umlaufe 
7.11 d'm moter A liegunden l'unkt des dritten Biattes. Erst nach a-maligem linlanfe kommen wir nath A zurïck. Lie a Umfängo der lireisflächen $K_{1} . K_{2} \ldots K_{12}$ s.hlinkens sich also zu riner pinzigen geschlossenon Kurve

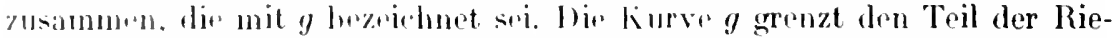

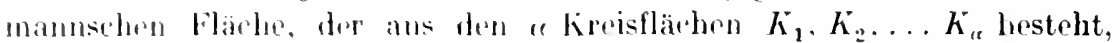

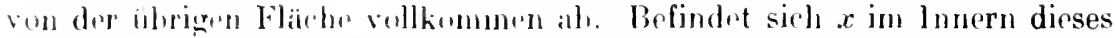

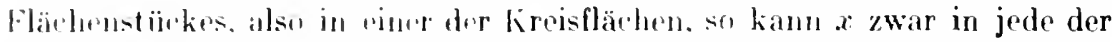

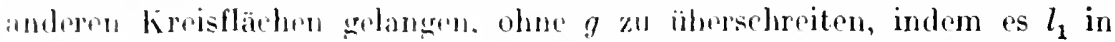
passonder Weise rin uder mehrere Maln iiberschreitet; os kann aber $x$ aus len Kreisflächen nicht herans, ohn"g zu ïbersehreiten. Das von den kreisfächen gehildot" Hächenstïck ist pinfach zusammenhängend. Es

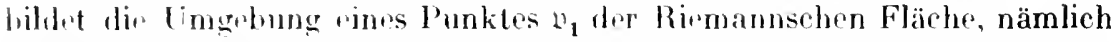

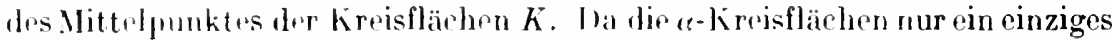
Fähenstürk hilden, so können wir anch ihre Mittelpunkte nur als cinen Tunkt der Riumannschen Fläche rochmen. Man nennt einen solchen Punkt pinen Verzwrigungspunkt oder IVindungspunkt ler Verzweigungsorrlmung oder auch kurz der Ordnung " - 1. Man bezeichnet ihn auch als («-b]ättrigen Verzweigungs- oder Windungspunkt. Ein gewöhnlicher lunkt der Biomannschen Fläche kann aufgefaßt werden als einblättriger Verzwoigungspunkt oder als riner von der nullter Ordnung.

llat $y$ in der Umgrhung von $x-a$ amBer der nach ganzen Potenzen vou (.r - a)" fortsurutenden noch andere Entwicklungen, etwa eine nath ganzen Poteruzen ven $(x-a)^{1}$ r und pine nach ganzen Potenzen von 1

$(x-a)$; fortschroitende. so liegen bei $x=a$ im ganz'n drei Punkte der Riemannsehen Fläclu. nämlieh drei Verzwestrungspunkte der Ordnungen $" 1 . j-1 . ;-1$.

Man kann sich Jeicht ein Modell eimes Windungspunktes herstellen, z. B. "ines dritter (Ordnung. Man schneide sich aus Papier vier Kreise aus und zrrsrhneide siø läng eines Halbmessors. J)is linken und rechten Ufor diesur Schnitte soien hezeichnet mit $l_{k \prime}, l_{l, k}(k=1, \ldots 4)$. Dann kJolw man $l_{1, \text { nit }} l_{2}$, zusammen, $l_{2,}$ mit $l_{3}$. $l_{2,}$ mit $l_{4}$. Hierdurch erhält man ein Flärhe, dir Thnlirhkeit mit einer Schraubenfläche hat. Um den Windungspunkt mit seiner Umgebung vollkommen zu haben, muß man sirh norh $l_{\text {: }}$ mit $l_{1}$, verbunden denken, was freilich praktisch nicht ausführlarr ist. Jor Umgrbungen der Verzweigungspunkte können sich Indunigfareh durchsetzen (ohne zusammenzuhängen). Man betrachte $z$. B. dir. Punkt. $i_{2}$. $\mathcal{B}_{3}$ der Abb. 26 genaurr.

l)as Ergronis fassen wir zusammen:

Jadrin Punkt der Riemannschen Fläche kann eine vollkommin aligrgronzt. Umgebung zugewiesen werden, die 
auch belirbig kloin gewählt worden kant. Sie bostrht aus riner sehlichtell kroisfläche oder alus "s shlatubenartig mit-

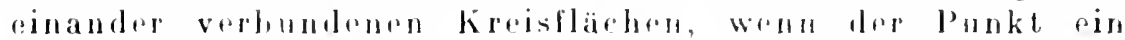
Vorzweigungspunkt der Ordnung $4-1$ ist. I)urchläuft $x$ dir ganze bregrougng ler Umgrbung, so niment arg $x$ um

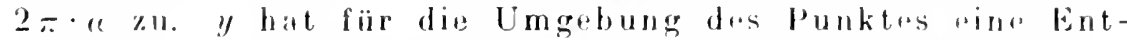
wicklung, dir nach ganzen P'otenzun von (x-a)a fortsehreitet, wonn $r$ in dem lount den Wurt a annimmt. Im allgemeinen ist $a=1$, dir Umgebung also eine schlichtr Kroislärhe. Es gibt nur eine ondliche Zahl von Pankten aul der liemannschen liache, für die $u>1$. Dirs sind dir rigentlirben Verzwoigungs- oder IVindungspunkte.

Dic Summe der Verzweigungsordnungen aller Punkte ist alst "ine "ndliche Zahl, die mit $w$ oder genauer mit $w_{x}$ bezeichnet werden soll und die die Verzweigungszahl dor Fläche T genannt wird. Für die in dem Beispiel behandelten Flärhen können wir die Verzwrigungszahlen leicht angeben. Es ist in Beispiel I (Abb. 24) $w=1+1=2$, in Buspiel II (Abb. 26) $w=2 \cdot 2+3 \cdot 1+5=12$, in Beispicl III (Abb. 28) $u=1+1+2=4$, in Beispiel IV (Abb. 29) $w=2+2+2=6$. Es ist also in den Beispielen $w$ immer eine grade Zahl. Wir werden spätor srhen, daß das kein Zufall ist, sondern da $w w \mathrm{immer}$ grade ist.

Viertes kapitel.

\section{Der algebraische Körper ( $x y)$ und seine Funk- tionen.}

\section{$\$$ 1. Definition des Körpers.}

Gleichzeitig mit $y$ wird auch jorle rationale funktion von $x, y$ em. algebraische Funktion von $x$. Ist nämlich $R(x, y)=\xi$ eine solche Funktion, so erhalten wir durch Elimination von $y$ aus den beiden Gleichungen $f(x, y)=0, R(x, y)-\xi=0$ eine algebraisch. Gleichung zwischen $\xi$ und $x, \mathrm{~s} \theta$ daß $\Xi=R(x, y)$ cine algebraische Funktion von $x$ ist. linispicle für diese Elimination finden sich im Kap. V. Wir werden auf die zwistlen bestehende Gleirhung weiter unten genauer eingehen. Dit mur rationale Funktion von $x, y$ rine eindeutige Funktion ihrer Argumente ist, und $y$ rine eindeutig" Funktion auf der Riemannschen Flach", so ist auch jede rationale Funktion von $x, y$ cine eindeutige funktion auf der Rienannschen Fläche. 
Wenn wir zwei rationale Funktionen von $x, y$ addieren, subtrahicren, multiplizieren, dividieren, erhalten wir immer wieder eine rationale Funktion von $x, y$. Dabei sind die konstanten mit zu den rationalen Funktionen gerechnet. Im Bereiche der rationalen Funktionen von $x, y$ sind also die vier Grundrechenarten unbesehränkt (mit Ausschluß der Division dureh Null) ausführbar. Jie Gesamtheit der rationalen Funktionen von $x, y$ hildet also, wie man sagt, cinen körper, und zwar einen algebraisehen körper einer unabhängigen Veränderlichen. Wir bezeichnen ihn nit $(x y)$.

Sind $\Xi=R_{1}(x, y)$, und $y_{i}=R_{2}(x, y)$ zwei Funktionen des Körpers $(x y)$, so erhalten wir aus den drei Gleichungen $R_{1}(x, y)-\xi=0$, $R_{2}(x, y)-y=0, f(x, y)=0$ durch Elimination von $x, y$ eine algebraische Gleichung zwischen $\vdots$ und $\%$. Es kann eintreten, daß diese Gleichung 5 oder ? nicht enthält. Die andere Funktion ist dann, wie aus der Gleichung folgt, eine konstante.

Wir fassen tas bisherige Ergebnis zusammen in den

Satz I. Die rationalen Funtionen ron $x, y$ bilden einen algebraischen Körper einer Veränderlichen. Jede Funktion des Körpers ist eine algebraisehe Funtion von $x$, die auf der über der $x$-Ebene ausgebreiteten Riemannschen Fläche, auf der $y$ eindeutig ist, gleichfalls eindeutig ist. Zwischen je zwei Funktionen des Körpers besteht eine algebraisehe Gleichung.

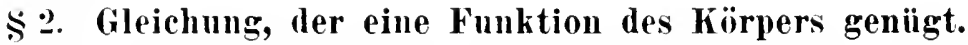

Es seien $y_{1}, y_{2}, \ldots y_{m}$, wie früher, die $m$ konjugierten Funktionen von $y$. Sie sind die $m$ Wurzehn der Gleichung $f(x, y)=0$. Wir nennen, wemn $R(x, y)$ eine Funktion des Körpers $(x y)$ ist, die $m$ Funktionen $R\left(x, y_{1}\right), R\left(x, y_{2}\right), \ldots R\left(x, y_{m}\right)$ die konjugierten Funktionen von $R$. Indem wir speziell $y=y_{1}$ annehmen, nennen wir auch die $m-1$ Funktionen $R\left(x, y_{2}\right), \ldots R\left(x, y_{m}\right)$ die konjugierten zu $R(x, y)$ $R\left(x, y_{1}\right)$. Zur Abkürzung bezeichnen wir $R\left(x, y_{k}\right)$ mit $R_{k}$. Da die $y_{k}$ die Wurzeln der Gleichung $f(x, y)=0$ sind, deren Koeffizienten rationale Funktionen vou $x$ sind, so ist jede rationale symmetrische Funktion der $y_{k}$ als rationale Funktion von $x$ darstellbar. Es ist also auch jede symmetrische rationale Funktion der $R_{k}$ als rationale Funktion von $x$ darstellbar. Unter diesen Funktionen seien hervorgehohen, die Summe, die Spur von $R$, und das Produkt, das Norm von $R$ genannt wird. Also

$$
\begin{aligned}
& \text { Spur }(R)=R_{1}+R_{2}+\cdots+R_{m}, \\
& \text { Norm }(R)=R_{1} R_{2} \cdots R_{m} .
\end{aligned}
$$

Die Funktion

$$
G(\xi, x)=\left(\xi-R_{1}\right)\left(\xi-R_{2}\right) \ldots\left(\xi-R_{m}\right)
$$


gehört auch bei beliebigen $\stackrel{5}{5}$ zu den rationalen symmetrisehen Funktionen der $R_{k}$ und ist also rine rationale funtion von $x$. Sie ist anBerdem, wie aus ihrer Definition folgt, rine ganzr rationale Funktion von $\xi$ vom Grade. m. Die Geichung

$$
G(\xi, x)=0
$$

lat offenbar die Wurzelı $\xi=R_{1}, R_{2}, \ldots R_{m}$. Es ist die Gleichung, der dir Funktion $R(x, y)$ genügt. Es sei z. B.

Es ist dann

$$
f(x, y) \quad y^{2}-x y+1=0, \quad R=\frac{x+y}{x-y} \text {. }
$$

und es wird

$$
y_{1}+y_{2}=x, y_{1} y_{2}=1
$$

$$
\begin{aligned}
G \xi, x) & \left(\xi-\frac{x+y_{1}}{x-y_{1}}\right) \xi-\left(\xi-\frac{x+y_{2}}{x-y_{2}}\right) \\
& \xi^{2}-\frac{\left(x+y_{1}\right)\left(x-y_{2}\right)+\left(x-y_{1}\right)\left(x+y_{2}\right)}{\left(x-y_{1}\right)\left(x-y_{2}\right)} \xi+\begin{array}{l}
\left(x+y_{1}\right)\left(x+y_{2}\right) \\
\left(x-y_{1}\right)\left(x-y_{2}\right)
\end{array} \\
& \xi^{2}-x^{2}-\left(y_{1}+y_{2}\right) x+y_{1} y_{2} \\
= & \xi^{2}-2\left(x^{2}-1\right) \xi+2 x^{2}+1 .
\end{aligned}
$$

Dem Leser sei empfohlen, die Beispiele des nächsten Kapitels in derselben Weise zu behandeln.

Die Koeffizienten in $G(\xi)$ sind ganze rationale Funktionen der $\boldsymbol{R}_{k}$, können also nur unendlich werden, wenn eine oder mehrere der Funktionen $R_{k}$ unendlich wird. Weiß man also von einer Funktion $R$ des Körpers, daß sie nirgends unendlich wird, so können in der Gleichung $G(\xi)=0$ die Koeffizienten nicht unendlich werden, müssen also, da es rationale Funktionen von $x$ sind, konstant sein. Dann aber wird $R$ als Wurzel der Gleichung $G(\xi)=0$ auch konstant.

Satz II. Eine Funktion des Körpers, die nirgends unendlich wird, ist eine konstante.

Die Gleichung $(r(\xi, x)=0$ kann rational zerfallen. Es sei etwa:

$$
G(\xi, x)-G_{1}(\xi, x) G_{2}(\xi, x) \ldots G_{p}(\xi, x)
$$

wo die $G_{k}(\xi, x)$ rationale Funktionen von $\xi, x$ sein sollen, und zwar ganz in $\xi$. Wir setzen die Faktoren $G_{k}(\xi, x)$ als rational unzerlegbar voraus. Den Kurfizienten der höchsten Potenz von $\xi$ nehmen wir in allen Faktoren gleich 1 an. Die Gleichung $G_{1}(\xi, x)=0 \mathrm{muB}$ mindestens eine der Funktionen $R_{k}$ als Wurzel haben, etwa $R_{1}$. Es ist dann identisch

$$
G_{1}\left(R_{1}, x\right)=G_{1}\left[R\left(x, y_{1}\right), x\right]=0 .
$$

Diese Gleichung bleibt richtig bei analytischer Fortsetzung. Dadurch können wir aber $y_{1}$ in jerle der konjugierten Funktionen $y_{2}, y_{3}, \ldots y_{m}$ überführen. Es ist also ganz allgemein für $k=1,2, \ldots m$

$$
G_{1}\left[R\left(x_{1} y_{k}\right), x\right] \cdots G_{1}\left(R_{k}, x\right)=0 \text {. }
$$


Es sind also alle $m$ Funktionen $R_{k}$ Wurzeh ron $G_{1}(\xi, x)=0$. Der Grad von $G_{1}(\Xi, x)$ in $\Xi$ sei $q$. Wenn dann $q<m$, so können die $R_{k}$ nicht alle voneininder verschieden sein, sondern unr $q$. So viele müssen aber auch verschieden sin, da $G_{1}(\xi, x)=0$ rational muterlegbar spin soll, also keine mehrfachen Wurzeln hahen kann. Es sien $R_{1}, R_{2} \ldots R_{\eta}$ voneinander versehioden. Setzen wir

sol ist

$$
g(\xi, x) \quad\left(\xi-R_{1}\right)\left(\xi-R_{2}\right) \ldots\left(\xi-R_{\eta 1}\right) .
$$

$$
\left(i_{1}(\xi, x) \quad g(\xi, x)\right. \text {, }
$$

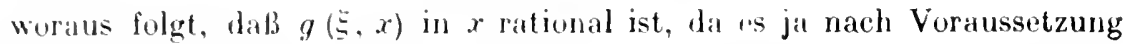
$G_{1}($ E. $x)$ ist.

Genau dieselben Schlüsse gelten für dir anderen Faktoren $G_{k}(\xi, x)$. Es sind daher alle $p$ Faktoren $G_{k}$ gleich $g(\xi, x)$ and es wird

$$
G(x, \leftrightarrows)-[g(x, \Xi)]^{\prime \prime} .
$$

Hieran folgt

$$
m=\mu \cdot q
$$

und, daß dir $m$ Funktionen $R_{k}$, wenn sie nicht alle voneinander verschieden sind, in $q$ Gruppen von je $p$ einander gleichen zerfallen.

Wir haben also

Satz III. Jedr Funktion $R(x, y)=R\left(x, y_{1}\right)$ des Körpers $(x y)$ genügt mit ihron konjugierten winer Gleichung $G(\xi, x)$ $=0 m$-ten Grades, deren Koeffizienten rationale Funktionen von $x$ sind. Die Funtion $G(\xi, x)$ ist entweder rational unzerleglar oder sie ist die Potenz, wtwa dio $p$-te, einer unzerlegbarin Funktion $g(\xi, x)$, dir in $\xi$ ganz ist und die vom Grade $q$ in 5 sei. Es ist dann $m=p \cdot q$ und die $m$ konjugierten Funktionen $R\left(x, y_{1}\right), R\left(x, y_{2}\right), \ldots R\left(x, y_{m}\right)$ zerfallen in $q$ Ciruppen von je $p$ einander gleichen.

\section{\$ 3. Verhalten der Funktionen des Körpers in der Umgebung eines Punktes der Riemannschen Fläche.}

Es sui ${ }^{2}$ rin Punkt dor Rirmannschen Fäehe, und zwar rin Verzweigungspunkt der Ordnung $"-1$. Ist $u=1$, so ist $p$ ein gewöhnlicher Punkt der Fläche. $x$ habe in ihn den Wrert a. Es läbt sieh dann $y$ in der Umgrbung von bals Protrugreihe darstellen. dir nards ganzen steigenden

Potrnzen von $(r-a)^{\prime 2}$ fortschreitet. Wrinn $a=\infty$, so ist muter $x-a$ zu verstehen $\frac{1}{x}$. Setzen wir die Reihenentwicklung von $y$ in eine Funktion $R(x, y)$ des körpers ein, so rohalten wir anth für $R$ eine nach ganzen

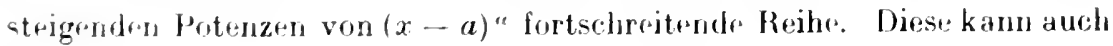


nur rinn "mdliche Zahl von negativen Potenzen enthalten. Ist die nindrigste l'otenz von $(x-a)^{\prime \prime}$, dio in ihr vorkommt, die $r$-te, so ist fur die Ungehung von p'

$$
R(x, y)=(x-a)^{\prime \prime} E\left[(x-a)^{\prime \prime}\right] .
$$

wo $E$ rim gewöhnliche Potenzreihe von $(x-a)$ " ist, die für $x=a$ nicht versehwindet, wo also $E$ eine Einheit für dir Strll $x=a$ ist.

Die Funktionen des Körpers $(x y)$ verhalten sich also in der Um gehung riurs Stelle p der Riemannsehen Fläche wio $y$. Es kann rintrutיn, daß sich oine Funktion in gewissem Sinne einfacher verhält als $y$. Jie Reihenentwicklung für eine Funktion $R$ kann nämlich unter Umständen nur solebe Jotenzen von $(x-a)$ " onthalten, deren Exponenten alle mit " denselben gemeinsamen Teiler $\delta$ haben. Ist $\epsilon^{\prime}=\delta, \beta$, so läßt sich die Entwicklung vou $R$ auch als Potenzreihe schreiben, die nach ganzen Potenzen

von $(x-a)^{x}$ fortsehreitet. Nehmen wir z. B. den durch die Gleichung (2), Kap. III, §3. S. 37 definierten Körper. Die zugehörige Riemannsche Fläche hat nur eine unendlich ferne Stelle und dort ist $y$ in die nach ganzen Potenzen von $x^{-\frac{1}{6} \text { fortsehreitende Reihe entwickolbar. dir auf Seite } 39}$ angegelon ist. Jie Funktion

$$
\begin{gathered}
y^{3}+3(x-1) y-x \\
3 y^{2}+x-1
\end{gathered}
$$

"rgibt aber, wenn man die Keihe für $y$ einsetzt, rinr Eutwicklung, die nur

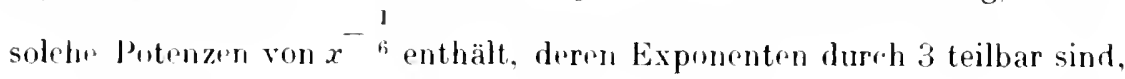
so dab $R$ sich in rine nach ganzen Potenzen von $x^{-\frac{1}{2}}$ fortschreitende Reihe "ntwickeln läßt für die Umgebung des unendlich fornen Punktes. Am einfachsten sieht man das daran, daB unter Benutzung der zwisehen $x$ and $y$ bestehenden Gleichung $R$ übergeführt werden kann in $\mid x-1$. Iirraus resioht man ferner, daß sich $R$ in der Umgebung der beiden Verzweigungspunkte zweiter Ordnung, die bei $x=0$ liegen, in eine Roihe entwickelı läßt, die nur ganze Potenzen von $x$ enthäilt.

Das rinfachste Beispiel sind die rationalen lunktionen von $x$, die ja dem Kïrper angrhöron, und sich äberall nach ganzen Potenzen entwickeln lassen.

Wir hahen also den

Satz IV. Jede Funktion $R$ des Körpers läBt sich für dis Umgebung eines a-fachen Verzwrigungspunktes der über dor 
$x$-Ebene ausgebreiteten Riemannschen Fläche, in dem $x$ den Wert a hat, in der Form darstellen

$$
R=(x-a)^{\alpha} E\left[(x-a)^{a}\right],
$$

wo $E$ eine gewöhnliche Potenzreihe von $(x-a)^{\alpha}$ ist, die für $x=a$ nicht verschwindet, und wo $r$ eine positive oder negative ganze Zahl oder Null ist $\left(x-a=x^{-1}\right.$, wenn $\left.a=\infty\right)$.

\section{\$t. Null- und Unendlichkeitsstellen.}

Hat eine Funktion $R$ in der Umgelung des a-fachen Verzweigungs-

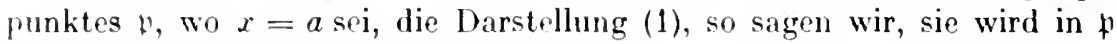
von der Ordnung $r$ Null, wenn $r>0$, und sie wird von der Ordnung $-r$ unendlich, wenn $r<0$ oder sie ist in $b$ von der Ordnung $r$ oder hat die Urdnungszahl $r$. Wir werden später Kap. VIII, $\$ 7$ sehen, daß es für jede Stelle Funktionen des Körpers gibt, die dort von der ersten Ordnung -ind. Eine solche Funktion, die in $p$ von der ersten Ordnung Null wird, wi t. Es ist dann in der Umgebung von

$$
t=e_{1}(x-a)^{\frac{1}{a}}+e_{2}(x-a)^{\frac{2}{a}}+\cdots,
$$

wo $e_{1} \neq 0$. Dann aber kann man nach einem Satze der Funktionentheorie aus dieser Gleichung umgekehrt $(x-a)^{i t}$ als Potenzreihe von $t$ ausrechnen und erhält

$$
(x-a)^{\frac{1}{u}}=c_{1} t+c_{2} t^{2}+c_{3} t^{3}+\cdots,
$$

wo $c_{1}=\frac{1}{e_{1}} \neq 0$. Haben wir eine Funktion $R$ des Körpers in eine nach Potenzen von $(x-a)^{\frac{1}{u}}$ fortschreitende Reihe entwickelt, so können wir diese Entwicklung mit Hilfe von (2) verwandeln in eine nach ganzen Potenzen von $t$ fortschreitende Entwicklung. Hat die Entwicklung von $\boldsymbol{R}$

nach Potenzen von $(x-a)^{\star}$ die Gestalt (1), so wird die Entwicklung nach Potenzen von $t$ so aussehen

$$
R(x, y)=t^{r} e(t),
$$

wo $e(t)$ eine gewöhnliche Potenzreihe von $t$ ist, die für $t=0$ nicht verschwindet, also eine Einheit ist für $t=0$. Der Exponent $r$ ist in beiden Gleichungen (1) und (3) derselbe. Ist also $t$ irgendeine Funktion des Körpers, die an einer Stelle $\mathfrak{p}$ der Riemannschen Fläche von der ersten Ordnung Null wird, so läßt sich jede Funktion des Körpers in der Umgebung von f darstell $n$ als eine nach ganzen steigenden Potenzen von $t$ fortschreitende Roihe. Hat diese Darstellung für eine Funktion $R$ die Form $R=\boldsymbol{t}^{r} e(t)$, 
wo $e(t)$ eine Einhrit fiur $t=0$ ist, so sigen wir in libureinstimmung mit der oben gegebenen Definition: $R$ wird an der Stulle pon der Ordnung $r$ Null oder von dep tordnung $-r$ mendlich. Ist $r<0$, wird also $R$ an der Stelle " morndlich, und setzen wir $r \quad s e(t) e_{0}+e_{1} t+e_{2} t^{2}$ $+\cdots$ so wiril

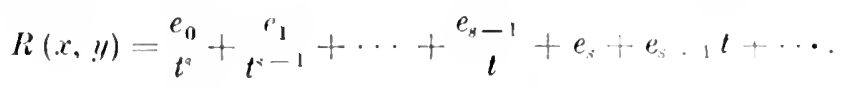

Di* Reihe enthält also nur eine ondliclur Zahl von negativen Potenzen und $R$ wird unendlich wie eine rationale Funktion von $t$. Solohe Unendliehkeitsstellen nent man aber Pole. Die Funktion $R$ ist in der Umgotung jeder Stelle $p$, wo $r>0$, in cine gewöhnliche Potenzrrihe entwickelbar nach Potenzen einer Funktion des körpers, die in $\mathfrak{p}$ von der arsten Ordnung ist. Die Funktionen des Körpers haben also als einzige singuläre Stellen Pole und haben wesentlich singuläre Stcllen überhaupt nicht. Hieraus sehon kann mau ersehen, daß eine Funtion des Körpers nur eine endlich. Zahl von Unendlichkeitsstellen haben kann. Hätte sie nänlich unendlich viele, so wärden sie eine Häufungsstelle haben, und diese mübte eine wesentlich singuläre Stelle der Funktion sein. Wir können aber auch so schließen. Jede Funktion $P$ des Körpers genügt einer Gleichung, deren Koeffizienten ganze rationale Funktionen von $x$ sind. Ist der Koeffizient der höchsten Potenz von $R$ in dieser Gleichung gleich $a(x)$, so kann $R$ nur unendlich werden für die Nullstellen von $a(x)$ und für unendlich grobes $x$, also nur für eine endliehe Zahl von Stellen.

\section{s. b. Residuen.}

Sind $\Xi$ und $y$ zwei Funktionen des Körpers $(x y)$, so läbt sich das Differential $; d \xi$ in der Umgebung einer Stelle $p$ in der Form darstellen

$$
\text { y } d \xi=P(t) d t
$$

wo $t$ in p von der ersten Ordnung Null wird und wo $P(t)$ eine nach steigenden von $t$ fortschreitende Potenzreihe ist, die höchstens eine endliche Zahl von negativen Potenzen enthält. Kommt in $P(t)$ die $(-1)$ te Potenz von $t$ vor, so heißt der Faktor dieser Potenz das Residuum des Differentials $\eta d \xi$ für die Stelle p.

Wir beweison zunächst, daß das Residuum unabhăngig von der Wahl von $t$ ist. Es sei

$$
P(t)=p_{-i} t^{-i}+\cdots+p_{-1} t^{-1}+p_{0}+\cdots=\sum_{k=-\lambda}^{\infty} p_{k} t^{k} .
$$

Es sei ferner $\iota$ eine zweite Funktion des Körpers, die an der Stelle p von der ersten Ordnung Null wird. Dann muB zwischen $t$ und $t$ eine Gleichung bestehen von der Form

$$
t=\tau e(\tau)
$$


wo $\varepsilon(t)$ eine Einheit für die Stelle $t=0$ ist. Da $e(t)$ für $t=0$ nicht Null wird, so läßt sich $\log e(t)$ als gewöhnliche Potenzreihe von $t$ darstellen, dir mit $h,(r)$ bezeichnet sei. Aus (6) folgt

$$
\log t=\log t+\log e(t)=\log t+h_{-1}(t)
$$

und hipraus durch Differentiation

$$
d t=\frac{d t}{t}+h_{-1}^{\prime}(t) d t .
$$

Da $e(\tau)$ eine Einheit ist. so sind alle positiven und negativen ganzzahligen Potenzen von $e(t)$ auch Einheiten. Es sei $e^{k}(r)$ mit $e_{k}(\tau)$ bezeichnet. Aus (6) folgt für $k \neq-1$

$$
k_{k+1} t^{k+1}=\frac{1}{k+1} t^{k+1} e_{k+1}(r)=h_{k}(r) .
$$

wo $h_{k}(\tau)$ eine Potenzreihe von $r$ ist. die höchstens eine endliche Zahl von negativen Potenzen enthălt. Durch Differentiation folgt

$$
t^{k} d t=h_{k}^{\prime}(\tau) d t \text {. }
$$

Aus den Gleichungen (4), (5), (7). (8) folgt

$$
r_{i} d \Xi=P(t) d t=\left|p \quad r^{-1}+\underset{k=-i}{+\infty} p_{k} h_{k}^{\prime}(\tau)\right| d \tau .
$$

Bedenkt man, dab die. Ableitung einer Potenzreihe von $\tau$ nach $\tau$ nie die $(-1)$-te Potenz von $t$ enthält, so folgt hieraus, daß der Koeffizient von $\tau^{-1}$ gleich dem von $t^{-1}$ gleich $p_{-1}$ ist, daß also das Residuum unabhängig ist von der Wahl von $t$. Wesentlich für den Beweis ist das Bestehen der Gleichung (6). Es ist also nicht notwendig, daß $\tau$ eine Funktion des Kör1

pers ist. Man kann im besonderen auch $t$ oder $t$ gleich $(x-a)^{\alpha}$ wählen.

Da jede Funktion des Körpers $(x y)$ nur eine endliche Zahl von Unendlichkeitsstellen hat, so hat auch jedes Differential nur eine endliche Zahl ron Residuen. Es gilt der wichtige

Satz V. Dir Summe der Residuen eines Differentials des Körpers $(x y)$ ist Null.

Wir beweisen den Satz erst für den Körper der rationalen Funktionen von $x$. Sind $气$ und, rationale Funktionen von $x$, so läßt sich das Differential,$d \xi$ darstellen in der Form

$$
r d \xi=r_{d x} \frac{d \xi}{d x} d x=r(x) d x \text {, }
$$

wo $r(x)$ eine rationale Funktion von $x$ ist. Wir betrachten die Funktion $r$ in der Umgebung der Stelle $x=a$. Ist $a$ endlich, so ist $x-a$, ist $x$ unendlich. so ist $x^{-1}$ aine Funktion erster Ordnung. Es sei $a$ zunächst endlich. 
Dann können wir setzen $x-a=t$. Lis wird $d x=d t$, mnd wenn in der Umgebung von a

$$
\begin{gathered}
r(x)=r_{u}(x-a) "+r_{11-1}(x-a)-" 1+\cdots+r_{1}(x-a) 1+r_{10} \\
\quad+r .1(x-a)+\cdots .
\end{gathered}
$$

so wird

$$
\text { 1 } d \xi=r(x) d x=\left(r_{1} t^{-1}+\cdots+r_{1} !+1+\cdots\right) d t .
$$

baher ist das Residuum von $y d \xi$ für die Stelle $a$, wenn $a$ endlich, gleich dem Faktor von $(x-a)^{-1}$ in der Entwicklung von $r(x)$ nach strigenden Potruzen von $x$ - $a$. Es sei zweitens a unendlich. Dann können wir setzen $x=t$ ',$d x=-t^{-2} d t$. Wenn in der Umgebung von $x=\infty$

$$
r(x)=r_{1} x^{1}+r_{1}, x^{1} \quad 1+\cdots+r_{0}+r_{1} x^{-1}+r_{-2} x^{-2}+\cdots,
$$

$\mathrm{so}$ ist dort

$$
\begin{gathered}
y d \Xi=r(x) d x=-\left(r_{1} t^{-1}-{ }^{2}+r_{1} t^{-1-1}+\cdots+r_{0} t 2+r, t^{-1}\right. \\
\left.+r_{2}+\cdots\right) d t .
\end{gathered}
$$

Das Residuum von $y d \xi$ ist also für die Stelle $\infty$ gleich $-r_{-1}$, also gleich dem negativen Faktor von $x^{-1}$ in der Entwicklung $r(x)$ nach fallenden Potenzen von $x$.

Die Funktion $r(x)$ läßt sich wie jede rationale Funktion in Partialbrüche zerlegen, also in der Form darstellen

$$
\begin{aligned}
& r(x)=G(x)+\underset{(x-a)^{i}}{A}+\frac{A_{j-1}}{(x-a)^{i-1}}+\cdots+\frac{A_{1}}{x-a} \\
& +\underset{(x-b) "+B_{u-1}}{B_{u}}+\cdots+\begin{array}{c}
B_{1} \\
x-b
\end{array} \\
& +\underset{(x-c)^{\prime}+}{C^{\prime}}+\frac{C_{v-1}^{\prime}}{(x-c)^{\prime-1}}+\cdots+\frac{C_{1}}{x-c},
\end{aligned}
$$

wo $G(x)$ eine ganze rationale Funktion von $x$ ist, wenn wir annehmen, daB $r(x)$ im Endlichen die drei Unendlichkeitsstellen $a, b, c$ von den Ordmungen $i, \mu, v$ hat. Die Residuen an diesen Stellen sind $A_{1}, B_{1}, C_{1}$, ihr. Summr $A_{1}+B_{1}+C_{1}$. Um das Residuum für $x=\infty$ zu bestimmen, haben wir den Faktor von $x^{-1}$ in der Entwicklung von $r(x)$ nach steigenden Potruzen von $x^{-1}$ aufzusuchen. Dir Glieder $(x-a)^{-k},(x-b)^{-k}$. $(x-c)^{-h}$ liefern, da sie für unendlich großes $x$ von der Ordnung $k$ Null werden, tuur solche Potenzen von $x^{-1}$, deren Exponent gleich oder größer ist als $k$. Jaher liefern nur die Summanden $A_{1}(x-a)^{-1}, B_{1}(x-b)^{-1}$, $C_{1}(x-c)^{-1}$ Gilieder mit dem Faktor $x^{1}$, und zwar die Glieder $A_{1} x^{-1}$, $B_{1} x^{-1}, C_{1} x^{-1}$, so dab der Faktor von $x$ 'gleieh $A_{1}+B_{1}+C_{1}$ ist und das Residuum gleich - $\left(A_{1}+B_{1}+C_{1}\right)$. Dip Summe aller Residuen ist also $A_{1}+B_{1}+C_{1}-\left(A_{1}+B_{1}+C_{1}\right)=0$.

Wir beweisen den Satz über die Summe der Residuen eines I)iffrrentials des Körpors $(x y)$, indem wir ihn zurüekführen auf den eben be- 
wiesenen Satz. Mit Hilfe der Gleichung $f(x, y)=0$ können wir schreiben

$$
\begin{aligned}
& \text { af } \\
& \gamma_{1} d \xi=1\left(\hat{c}_{\hat{c} x} d x+\frac{\partial \xi}{\partial y} d y\right)=1\left(\left(\begin{array}{cc}
\partial \xi & \hat{\partial} \xi \partial x \\
\partial x & \partial y \partial f
\end{array}\right) d x=R(x, y) d x,\right. \\
& \text { तy }
\end{aligned}
$$

wo $R(x, y)$ eine rationale funktion von $x, y$ ist. Wir bezeichnen die konjugierten Funktionen von $R$ mit $R_{1}, R_{2}, \ldots R_{m}$ und die Spur $R_{1}+R_{2}$ $+\cdots+R_{m}$ von $R$ mit $r(x)$. Es ist $r$ eime rationale Funktion von $x$. Wir werden zeigen, daß die Summe der Residuen, die $y, d \xi=R(x, y) d x$ an denjenigen Stellen der Riemannsehen Fläehe hat, wo $x=a$, gleich ist dem Resiluum von $r(x) d x$ fïr $x=a$, und zwar gleichgültig, ob $a$ endlich ist oder nicht. Haben wir das bewiesen, so folgt, daß die Summe der Residuen von, $d \xi$ gleich ist der Summe der Residuen von $r(x) d x$, also nach dem eben bewiesenen Satze gleich Null.

Es sei zunächst $a$ endlich. Es mögen etwa bei $x=a$ zwei Punkte der Riemannschen Fläche liegen, $\psi_{1}$ und $p_{2}$. Sie seien ein $a$-facher und ein $\beta$-facher Windungspunlit. Der Fall a oder $\beta$ gleich 1 soll nicht ausgeschlossen sein. In der Umgebung von $p_{1}$ sei

$$
\begin{aligned}
R(x, y) & =A_{j}(x-a)^{-\frac{\lambda}{a}}+A_{j-1}(x-a)^{-\frac{\lambda-1}{\alpha}}+\cdots+A_{k}(x-a)^{-1} \\
& +A_{a-1}(x-a)^{-\frac{\alpha-1}{\alpha}}+\cdots=\sum_{k=-\lambda}^{+\infty} A_{-k}(x-a)^{k} .
\end{aligned}
$$

Setzen wir in $R d x$

$$
x-a=t^{\alpha}, d x=\alpha t^{t r-1} d t,
$$

so wird nach dem oben (S. 56) Bewiesenen das Residuum von $R d x$ gleich dem Faktor von $t^{-1}$, also gleich $a A_{\omega}$, d. h. gleich dem mit $\alpha$ multiplizierten Koeffizienten von $(x-a)^{-1}$ in der Entwicklung von $R$ in der Umgrobung von $\mathfrak{p}_{1}$.

Ebenso ist das Residuum von $\eta_{1} d \xi$ für die Stelle $p_{2}$ gleich dem mit $\beta$ multiplizierten Koeffizienten - er sei mit $B_{1}$ bezeichnet - von $(x-a)^{-1}$ in der Entwicklung von $R(x, y)$ in der Umgebung von $p_{2}$. Die beiden bei $x=a$ liegenden Stellen liefern daher zur Summe der Residuen von $\eta d \xi$ den Beitrag

$$
\alpha A_{u}+\beta B_{\beta} .
$$

Wir bestimmen jetzt das Residuum von $r(x) d x$ an der Stelle $a$. Bei passender Wahl der Bezeichnung sind in der Umgebung von $x=a$ die Funktionen $R_{1}, R_{2}, \ldots R_{u}$ gegeben dureh die Reihe (10) und die $\alpha-1$ zu ihr adjungierten, die aus ihr dadurch hervorgehen, daß man $x$ den Punkt $a$ ein oder mehrere Nale umkreisen läßt. Die « Entwicklungen stimmen also in den Gliedern überein, die ganzzahlige Potenzen von $x-a$ enthalten, so da $\beta$ in allen der Faktor von $(x-a)^{-1}$ gleich $A_{a x}$ ist. 


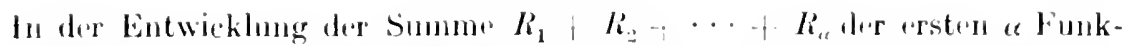
tionen $R_{i}$ hat daher $(x-a)^{1}$ den faktore $A_{a b}$. Ebenso folgt, da $B$ in der

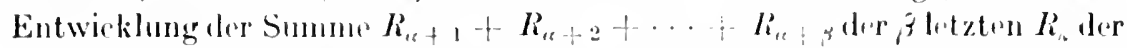
Faktor von $(x-a)$ ghteinh $\beta B_{3}$ ist. Also ist das linsiduum von $r d x$ $=\left(R_{1}+R_{2}+\cdots+R_{n}\right) d x$ an der Stoll a ghoind $A_{\alpha}+\beta B_{s}$, was init (11) ïhroinstimmt. Dies alles gilt, solange $a$ endlich ist.

Wir betrachten jetzt das lofferential $y d \xi$ in der l Imgelung von $x=s$. Dort mögen aueh wierler zwei Punkte $f_{1}, f_{2}$ der liemannschen Fläche lingen, die Verzweigungspunkte der Ordnung $\alpha-1$ und $\beta-1$ soien. In der Umgelung von tr möge sein

$$
R(x, y)=A_{i} x^{\lambda}+A_{i-1} x^{i-1}+\cdots+A_{-u} x^{-1}+A_{-u-1}^{-a+1} x^{u}+\cdots
$$

$$
=\sum_{k=i}^{\infty} A_{k} x^{k} \text {. }
$$

Setzen wir in $R d x$

$$
r=t^{-*}, d x=-\mathrm{a} t^{-u-1} d t,
$$

so wird wieder das Residuum vou $R d x$ gleich dem Faktor von $t^{-1}$, also gleich-a $-A_{-\alpha}$, d. h. gleich dem mit - " multiplizierten Koeffizienten von $x^{-1}$ in der Entwicklung ron $R(x, y)$ in der Umgebung von $f_{1}$.

Ebenso ist das Residuum von $\gamma_{j}{ }_{S}$ für die Stelle $p_{: \text {gleich }}$ gem mit - $\beta$ multiplizierten Koeffizienten von $x^{-1}-$ er heiße $B_{-\beta}-$ in der Entwicklung von $R(x, y)$ in der Umgebung von $\mathfrak{p}_{2}$. Dje beiden bei $x=\infty$ liegenden Stellen liefern also zu der Summe der Residuen von $\eta d \Xi$ den Beitrag

$$
-\left(u A_{-\lll}+\beta B_{-x}\right) \text {. }
$$

Wir bestimmen weites das Residuum von $r(x) d x$ für $x=\infty$. Unter don $m$ konjugierten Funktionen $R_{k}$ sind a, deren Entwicklungen nach Potrnzen von $x^{-1}$ in der Umgobung von $x=\infty$ gegeben sind durch (12) und die dazu adjungierten Entwicklungen; in diesen haben die ganzzahligen Potenzen von $x$ denselben Faktor, so da $B$ im besonderen $x^{-1}$ in diesen a Entwicklungen den Koeffizienten $A_{-}$"hat. Die " in Rede stehenden Funktionen $R_{k}$ liefern daher zu dem Koeffizienten von $x^{-1}$ in der Entwicklung von $r(x)=R_{1}+R_{2}+\cdots+R_{m}$ den Beitraged $A_{-}$. Ebenso zeigt man, daß die $\beta$ anderen Funktionen $R_{h}$ zu dem Faktor von $x^{-1}$ in der Entwicklung von $r(x)$ den Beitrag, $\beta B_{-3}$ ljefern. Das Risiduum von $r(x) d x$ für $x=\infty$ ist daher $-\left(\alpha A_{-a}+\beta B_{-\beta}\right)$, was mit (13) ibereinstimmt. Damit ist der Salz $\mathrm{V}$ bewiesen.

Nebenbei haben wir folgendes Ergebnis erhalten, das wir ausspreehen in Satz VI. Schroiben wir das Differential ,d de des Körpers $(x y)$ in der Form $R(x, y) d x$, so ist sein Rosidum an einer Strlle p, wo $x$ den Wert a hat und die eine c-fache Verzwei- 
gungsstolle in bezug anf $x$ ist, gloich dem mit + a multiplizierten koeffizienten von $(x-a)^{-1}$ in der für die Ungebung von goltendon Roihenentwicklung, wenn a endlich ist, und gleich dom mit - r multiplizierten korfizienten von $x^{-1}$, wenn a unendlieh ist.

\section{\$ 6. Ordnung einer Funktion.}

Es sei $R$ irgendeine Funktion des lïrpers $(x y)$. Wir wenden den Satz $V$ an auf das Differential $d R / R$. Es sei $t$ wieder eine Funktion aus $(x y)$, die an einer Stelte " von der orsten Ordnung Null wird. Es sei ferner für dir I'mgehung vol]

$$
R=t^{\prime} E(t)
$$

wo $E(t)$,ine Einheit für $t=0$ sei, Es sei also $\lambda$ die Ordnungszahl von $R$ an tir Stelle $p$, so flaß $R$ dort von der Ordnung $\lambda$. Null wird $(\lambda>0)$ oder von der Ordnung $-\lambda$. unendlieh $(\lambda<0)$ orler weder Null noch unendlieh wird $(i=0)$. Aus (14) folgt

$$
\log R=\lambda \log t+\log E(t)=i \log t+P(t),
$$

wo $P(t)$ eine gewöhnlirhe Potrnzreihe von $t$ ist. Dureh Differentiation ergibt sich

$$
\frac{d R}{R}=\left|\begin{array}{l}
i \\
t
\end{array}+P^{\prime}(t)\right| d t
$$

1)a $P^{\prime}(t)$ als Ableitung einer Potenzreihe nicht die (-1)-te Potenz von $t$ -nthält, so ist das Residuum von $d R / R$ für die Stelle $\mathfrak{p}$ gleich $\lambda$, also gleich der Ordnung von $R$ in p. Wenden wir den Satz $\mathrm{V}$ an, so folgt, daß die Summe aller Ordnungszahlen einer Funktion des Körpers $(x y)$ gleich Null ist, oder daß die Summe der positiven Ordnungszahlen gleich der der negativen ist. Kerhnen wir jede Null- und Unendlichkeitstelle so oft, wie ihre Ordnung angibt, so können wir auch sagen, jede Funktion $R$ des Körpers wird geradese oft Null wie unendlich. Ist $C$ irgendeine Konstante, so hat flie Funktion $R-C$ dieselben Unendlichkeitsstellen wie $R$; sie wird also geradeso oft Null wie $R$, d. h. die Funktion $R$ nimmt den Wert $C$ geradrso oft an wie den Wert Unendlirh. J) $C$ rine beliebige Konstante ist, so haben wir den

Satz VIl. Eine Funktion des Körpers $(x y)$ nimmt jeden Wert glrieh of an. Jie Zahl, die angibt, wie oft eine Funktion jeden Wert annimmt, heißt ihre Ordnung.

Im brsonderen folgt hieraus, daß eine Funktion des Körpers, die irgend einen Wert, z. B. Null oder Unendlich, nicht annimmt, nur eine Konstante srin kann. 


\section{\$ิ 7 . Charakteristische Eigenschaften einer algebraischen Funktion.}

Wir hiseisen norh den

Satz VIII. Eine m-dentige analytiselu Funktion $R$ von $x$, die nur Pole hat, ist rine algebraische finnktion von $x$. Ist foruer $R$ eindeutig auf der Ricmannschon Fläehr, auf der $y$ eindentig ist, und hat sie nur Pole, so ist $R$ rimr rationale Funktion von $x$ und $y$.

Zunächst folgt aus der Voraussetzung, dab $R$ nur rine mulliche Zahl von Unendlichkeitsstellen haben hann. Hätte sir unendlich viele, so hätten diese rine lläufungsstelle und diese müßte ein wesentlich singulärer Punkt sein. Sind $R_{1}, R_{2}, \ldots R_{m}$ die $m$ Zweige der Funktion $R$, su vertauschen sich diese nur untereinander, wenı $x$ einen geschlossen+n Weg durehläuft. Es ist also jede rationale symmetrisetor Funktion von $R_{1}, R_{2}, \ldots R_{m}$ cine eindeutige Funktion von $x$, die auch nur Pole babmi kann und die daher eine rational Funktion von $x$ ist. Daher ist

$$
\left(\xi-R_{1}\right)\left(\xi-R_{2}\right) \ldots\left(\xi-R_{m}\right)=G(\xi, x)
$$

eine rationale Funktion von $x$. Es genügt also $R$ einer algebraischrn Gleichung $G(\xi, x)=0$ und ist eine algebraische Funktion.

Ist ferner $R$ eindeutig auf der Riemannschen Fläche, auf der $y$ eindeutig ist, so können wir die $m$ Zweige von $R$ den $m$ konjugierten Funktionen $y_{1}, y_{2}, \ldots y_{m}$ zuordnen, so daß für $y=y_{\text {" }}$ auch $R=R_{u}$ ist. Ist $f(x, y)=0$ die Gleichung, der $y$ genügt, und bezeichnen wir mit $f_{2}^{\prime}(y)$ die Ableitung von $f$ nach $y$, so haben wir nach der Lagrangeschen Interpolationsformel

$$
\begin{gathered}
R(x, y)=\left.f(x, y)\right|_{\left(y-y_{1}\right) f_{2}^{\prime}\left(y_{1}\right)} ^{R_{1}}+\frac{R_{2}}{\left(y-y_{2}\right) f_{2}^{\prime}\left(y_{0}\right)} \\
\left.+\cdots+\underset{\left(y-y_{m}\right) f_{2}^{\prime}\left(y_{m}\right)}{R_{2}}\right] .
\end{gathered}
$$

Bedenkt man, daß $f(x, y)=\mathbb{e}_{0}(x)\left(y-y_{1}\right)\left(y-y_{2}\right) \ldots\left(y-y_{m}\right)$, so sieht man, $d_{a} B$ in dieser Gleichung rechts eine ganze rationale Funktion von $y$ höchstens vom Grade $m-1$ steht. Ferner ist die rechtsstehende Funktion symmetrisch von $y_{1}, y_{2}, \ldots y_{m}$ abhängig und also eine rationale Funktion von $x$. Es läßt sich also unsere Funktion $R$ als ratiunale Funktion von $x$. $y$ darstellen. Wir finden nebenbei noch den

Satz IX. Jede Funktion des Körpers $(x y)$ läßt sich darstellen als ganze rationale Funktion von $y$, deren Grad höchstens gleich $m-1$ ist und deren Koeffizienten rationale Funktionen von $x$ sind. 
Fiinftes liapitel.

\section{Birationale 'Transformationen.}

\section{\$1. Definition der birationalen Transformation.}

Wir haben bisher bei unseren Betrachtungen eine Größe des Körpers bevorzugt, nämlich die Größe $x$, die wir als unabhängige Veränderliche genommen haben. Wir wollen jetzt zeigen, daß man jede nicht konstante Größe des lï̈rpers $(x y)$ als unabhängige Veränderliche wählen kann. Es sei $\xi=R(x, y)$ eine nicht konstante Größe des liörpers $(x y)$. Ist $\eta$ irgendeine andere Größe des Körpers, so besteht zwischen $\xi$ und $\eta$ eine algebraische Gleichung; die Gesamtheit der rationalen Funktionen von $\xi$, ${ }_{1}$ ist also ein algebraischer Körper ( $\left.\xi_{y}\right)$. Da wir $\xi$ und $\eta$ als Funktionen des Körpers $(x y)$ anmehmen, so sind $\xi$ und $\gamma$ rational dureh $x, y$ darstellbar, und dasselbe gilt also von jeder rationalen Funktion von $\xi, \eta$. Es ist also jede Funktion des Körpers (E, gleichzeitig eine Funktion des Körpers $(x y)$ : Der Körper $\left(\xi y_{1}\right)$ ist in dem Körper $(x y)$ enthalten. Aber es kann bei beliebiger Wahl von $\eta$ sehr wohl eintreten, daß nicht umgekehrt auch jede Funktion aus $(x y)$ in $\left(\xi r_{)}\right)$enthalten ist. Wir werden aber zeigen, daß man zu jeder Funktion $\xi$, die nicht konstant ist, eine Funktion , so finden kann, daß auch jede rationale Funktion von $x, y$ eine rationale Funktion von $\xi, \eta$ wird. Es werden dann die Körper $(x y)$ und $(\xi)$ identisch. Man nennt in diesem Falle den Übergang von den veränderlichen $x, y$ zu den neuen $\xi, \eta$ eine birationale Transformation.

Wie wir in $\S 2$ des vorigen Kapitels gesehen haben, genügt $\xi$ einer unzerlegbaren Gleichung $g(x, \xi)=0$, deren Koeffizienten rationale Funktionen von $x$ sind und deren Grad $q$ ein Teiler von $m$ oder $m$ ist. Sie sei in $x$ vom Grade $e$. Wir erhalten diese Gleichung dadurch, daß wir aus den beiden Gleichungen

$$
R(x, y)-\xi=0, \quad f(x, y)=0
$$

die Größe y eliminieren. Jies kann dadurch geschehen, daß wir die beiden Funktionen $R(x, y)-\xi$ und $f(x, y)$ als ganze rationale Funktionen von y allein betrachten und ihren größten gemeinsamen Teiler aufsuchen und den letzten (von $y$ unabhängigen) Rest gleich Null setzen. 
Es sei

\section{\$ับ. Beispiel 1.}

(2)

$$
\begin{aligned}
& f(x, y) \quad y^{3}-x=0 ; \quad \Xi=x^{2}+y^{2} \quad R(x, y) \text {. } \\
& y^{3}-x: y^{2}+x^{2}-5=! \\
& y^{3}+\left(x^{2}-5\right) y \\
& -\left(x^{2}-\xi\right) y-x \\
& y^{2}+x^{2}-\xi:\left(x^{2}-\xi\right) y+x=\frac{y}{x^{2}-\xi-\left(x^{2}-\xi\right)^{2}} \\
& y^{2}+x^{x}-\xi \\
& -\frac{x y}{x^{2}-\xi}+x^{2}-\xi
\end{aligned}
$$

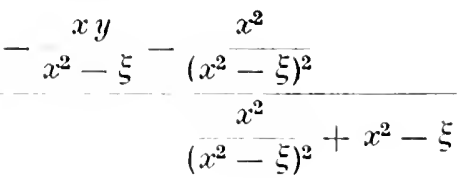

Sollen also die beiden Gleichungen $f=0$ und $R=\xi$ in diesem Falle gleichzeitig bestchen, so muß

$$
\frac{x^{2}}{\left(x^{2}-5\right)^{2}}+x^{2}-\xi=0
$$

sein. Die Gleichung $g(x, 5)=0$ lintet also

$$
g(x, \Xi)=\left(x^{2}-5\right)^{3}+x^{2}=0 .
$$

Die Gleichung ist in 5 vom dritten Grade und sie ist unzerlegbar. Es sind also die drei konjugierten Werte von 5 voneinander versehieden. Das können wir hier leicht bestätigen. Es ist nämlich $\xi=x^{2}+y^{2}=x^{2}+\sqrt[3]{x^{2}}$

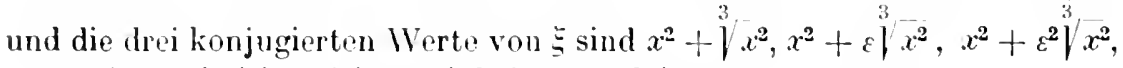
wo $\&$ eine primitive dritte Einheitswurzel ist.

Aus der Rechnung ergiht sich, da $B$ für $g(x, \xi)=0$ folgende Gleichungen hostrhon:

$$
\begin{gathered}
y^{2}+x^{2}-\xi=\left[\left(x^{2}-\xi\right) y+x\right]\left[\begin{array}{c}
y \\
x^{2}-\xi
\end{array}-\frac{x}{\left(x^{2}-\xi\right)^{2}}\right], \\
y^{3}-x=\left[y^{2}+x^{2}-\xi\right] y-\left[\left(x^{2}-\xi\right) y+x\right] \\
\left.=\mid\left(x^{2}-\xi\right) y+x\right]\left[\begin{array}{c}
y^{2} \\
x^{2}-\xi
\end{array}-\left(x^{2}-\xi\right)^{2}-1\right] .
\end{gathered}
$$

Es haben also $y^{3}-x$ und $y^{2}+x^{2}-\xi$ für $g(x, \xi)=0$ den gemeinsamen Teiler $\left(x^{2}-\xi\right) y+x$. Die Gleichungen (2) können also gleichzeitig nur bestehen, wenn $g(x, 5)=0$ und wenn $\left(x^{2}-5\right) y+x=0$, und sie sind andererseits erfült, wenn diese beiden Gleichungen bestehen. Es ergibt 
sich aber itls $\left(x^{2}-\Xi\right) y+x=0$ die firöße $y$ als rationale Funktion vin $x$ und $三$.

$$
y=\Xi-x^{2}
$$

Wählen wir also als Größe, die Größr $x$, so hahen wir die Transformationsformeln

$$
\Xi=x^{2}+y^{2}, \quad, 1=x,
$$

alls denen sich mongekehrt. wie eben hewiesen, ergibt

$$
x=1, \quad y=\frac{1}{\xi-1^{2}},
$$

$s 0$ dab der hörper $(x y)$ mit dem Körper $\left(\Sigma_{y}\right)$ identisch ist. Zwischen $x, y$ hesteht $\left\{\mathrm{i}\right.$. Gleichung $f(x, y)=y^{3}-x=0$ und zwischen $\Xi,{ }^{\prime}$ die Glei.hung $g(1, \Xi) \quad\left(1^{2}-\Xi\right)^{3}+y^{2}=0$. Es ergibt sich aus dem Vorhergehenden. daB aus den Gleichungen (3) die Gleichungen (4) nur folgen, wnn $f(x, y)=0$ angenommen wird, und daß auch (3) aus (4) nur folgt für $g(1, \xi)=0$, aber es sei doch ausdrücklich darauf hingewiesen.

DaB wir hier so einfach zum Ziele gelangen, daB wir nämlich als y vinfich $x$ wählen können. liegt daran, daß die Gleichungen (1), nämlich $f(x, y)=0$ und $R(x, y)-\xi=0$ unter der Voraussetzung $g(x, \xi)=0$ und aufgefaßt als Gleichungen für $y$, nur eine gemeinsame Wurzel haben. Jedesmal, wenn dirs eintritt, und nur dann ist der gröbte gemeinsame Teiler von $f(x, y)$ und $R(x, y)-\xi$, der sich auf rationalem Wege bestimmen läBt, in $y$ vom ersten Grade. Er sei $a(x, \xi) y+b(x, \xi)$. So bestehen die Gleichungen $f(x, y)=0$ und $R(x, y)-\xi=0$ dann und nur dann gleichzeitig, wenn gleichzeitig $g(x, \xi)=0$ und $a y+b=0$, und umgekehrt. Aus der letzten Gleichung ergibt sich aber $y$ als rationale Funktion von $x$ und $\Xi$. Und man kann weiter schließen wie in dem Beispip].

Es sei

\section{\$ุ. Beispiel ?.}

$$
f(x, y) \quad y^{6}-\left(1-x^{2}\right) x^{2}=0, \quad \xi=\begin{aligned}
& y^{3} \\
& x
\end{aligned} .
$$

Wir eliminieren $y$ aus diesen beiden Gleichungen und bestimmen zugleich den größten gemeinsamen Teiler der beiden Funktionen

$$
\begin{aligned}
& y^{6}-\left(1-x^{2}\right) x^{2}, \quad y^{3}-x \xi . \\
& y^{6}-\left(1-x^{2}\right) x^{2}: y^{3}-x \xi=y^{3}+x \xi \\
& y^{6}-x \xi y^{3} \\
& x \xi y^{3}-\left(1-x^{2}\right) x^{2} \\
& x \xi y^{3}-x^{2} \xi^{2} \\
& \text { Rest } x^{2}\left(x^{2}+\xi^{2}-1\right) .
\end{aligned}
$$


Sollen die Gleichungen (5) zusammen bestepren, so muB also sein

$$
g(x, \xi) \quad x^{2}+52-1=0 .
$$

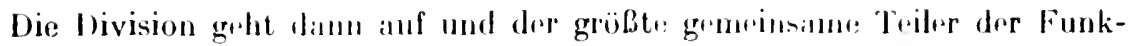

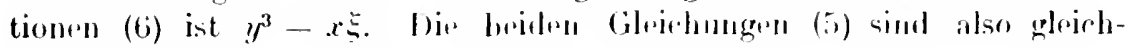
bedrutend mit

$$
x^{2}+5^{2}-1=0, \quad y^{3}-x \xi=0 \text {. }
$$

Hire habren demuach div Gloichumgen (5) fïr $g(x, \xi)=0$ drei Wirzoln gemoinsam, nämlich die droi Wurzeln von $y^{3}-x_{5}^{5}=0$. H)r Körper $\left(x_{5}^{5}\right)$, drfiniert durch die Gleichung $x^{2}+\Xi^{2}-1=0$, ist uicht gllidh drm Körper $(x y)$, sondern nur cin Trilkörper von $(x y)$. So gehört z. l'. $y=\mid \begin{aligned} & \mid x \xi \\ & \mid x \xi\end{aligned}$ dem Körper $(x \xi)$ nicht an. Die Gloifhung $g(x, \xi)=0$ ist hime nicht vom Grade $m=6$, sondern nur vom Grado $q=2$ in 5 . Die 6 konjugiorten Werte von $\Xi$ sind also zu je drejen rinander gledele, was man leicht bestätigt. wenn man bedenkt. $\mathrm{da} B \xi=y^{3}=11-x^{2}$ eine zweiwertige Funktion von $x$ ist.

Wie wir in diesem Falle pille passendo Größr y linden, werten wir weiter unten sehen.

Es sei

\section{\$4. Beispiel 3.}

$$
\begin{gathered}
f(x, y) \quad y^{4}+(8-10 x) y^{2}+16-24 x+9 x^{2} \\
\Xi=-\frac{y^{3}+(4-7 x) y}{2(4-3 x)}=R(x, y) .
\end{gathered}
$$

Zur Elimination von $y$ rechuen wir

$y^{4}+(8-10 x) y^{2}+\left(16-24 x+9 x^{2}\right): y^{3}+(4-7 x) y+2(4-3 x) \xi=y$ $y^{4}+(4-7 x) y^{2}+2(4-3 x) \xi y$

Rest $(4-3 x)\left(y^{2}-2 \xi y+4-3 x\right)$

$y^{3}+(4-7 x) y+2(4-3 x) \xi: y^{2}-2 \xi y+4-3 x=y+2 \xi$

$y^{3}-2 \xi y^{2}+(4-3 x) y$

$2 \xi y^{2}-4 x y+2(4-3 x) \xi$

$2 \Xi y^{2}-4 \xi^{2} y+2(4-3 x) \xi$

Rest $4\left(\Sigma^{2}-x\right) y$

Es ist also hior $g(x, \xi)=\xi^{2}-x$ und der größte gemeinsame Teiler von $f(x, y)$ und $R(x, y)-\xi$ ist $y^{2}-2 \xi y+4-3 x$. Die Gleichungen (7) sind daher gleichwertig mit den Gleichungen

$$
g(x, \xi) \quad \xi^{2}-x=0, \quad y^{2}-2 \xi y+4-3 x=0 .
$$

Auch hier ist der Körper $(x \xi)$ nur ein Teilkörper vom Körper $(x y)$. Dir. Gleichung $g(x, \xi)=0$ ist in $\xi$ nicht vom Grade $m=4$, sondern nur vom Grade $q=2$. 


\section{\$. 5. Bestimumng einer Größe $y$ zu ciner Gröbe $\Xi$, so dab die liörper $\left(x^{\prime} y\right)$ und $\left(\Xi_{3}\right)$ identisch werden.}

Wir knhren zum allgemeinen Fall zurärk. Die Gleichung $g(x, \xi)=0$ ist. wi, wir angonommen, in $x$ rom Grade $e$ und in $\Xi$ vom Grade $q$. Es s.i wir frïher $q \cdot p=m$. Dì $m$ kinnjugierten Werte von $\Xi$ nämlich $\xi_{a}$ $=R\left(x, y_{u}\right)$ sind, womn $p>1, z u j$ j pinander gleich. Es sei etwa $\xi_{a}=\xi_{3}$,

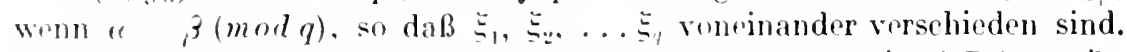
W.r gröbte gemeinsam Teiler von $f(x, y)$ und $R(x, y)-\xi$ sei $D(y ; x, \xi)$; or soi in $y$ vom Grarle $n$. Wir können $n$ sofort bestimmen. Ist $(x, \xi)$ irgendrin Wertepaar. das der Gleichung $g(x, \xi)=0$ genügt, so haben die Gleichungen

$$
f(x, y)=0, \quad R(x, y)-\xi=0
$$

mindestns.inn Wurzel $y$ gemeinsam, etwa $y_{1}$. Da aber $R\left(x, y_{c}\right)=R\left(x, y_{1}\right)$, wenn " $1(\bmod q)$ und also gleichzeitig mit $R\left(x, y_{1}\right)-\xi=0$ auch $R\left(x, y_{\star}\right)-\xi=0$ für jodes a $1(\bmod q)$, so lıaben die Gleichungen $(8)$ die $p$ Wurzeln $y_{1}, y_{1+1}, y_{2 y-1}, \ldots y_{(1-1, q+1}$ gemeinsam und für be-

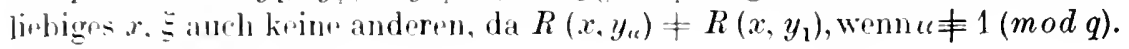
Daher ist $n=p$.

Zu rinem Werte von $x$, etwa $x_{0}$, gehören zufolge der Gleichung $g(x, \xi)=0 q$ Wrte von $\Xi$ dir mit $\xi_{0}^{1}, \xi_{0}^{2}, \ldots \xi_{0}$ bezeichnet seien. Zu jedem

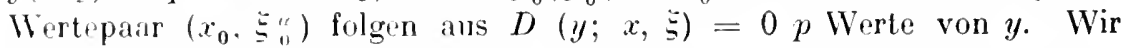
rhalten also zu einem Werte von $x$ im ganzen $q \cdot p=m$ Werte von $y$, wie es sein muß. Der Körper $(x y)$ kann also so erhalten werden, daß man erst den Körper $(x \xi)$ durch die Gleichung $g(x, \xi)=0$ definiert und dann $y$ durch die Gleirhung $I)(y ; x, \Xi)=0$ vom Grade $p$ definiert. Der Körper $(x y)$ ist, wie man sagt, ein Überkörperdes Körpers $(x \xi)$ vom Relativgrade $p$.

lst also $p=1$, so können wir $1=x$ setzen. Dann ist ja die Gleichung $L(y ; x \xi)=0 \mathrm{in} y \mathrm{rom}$ ersten Grade, und es folgt aus ihr $y$ als rationale Funktion won $x$ und (Beispiel 1). Wir haben bisher das Ergebnis:

Satz I. Genügt eine Funktion $\xi$ des Körpers $(x y)$ einer unzerlegbarm Girichung vom Grade $m$, wo $m$ die Ordnung von $x$ int, so ist der Körper $(x \xi)$ gleich dem Körper $(x y)$. Gröigt aber 5 nur piner unzerlegbaren Gleichung vom Grade $q<m$. so ist zunärh-t $q$ ein Teiler von $m$. y genügt einer unzerlegharm Gleichnng $D(y ; x, \xi)=0$ vom Grade $p=m / q$, deren Keffizirnton rationale Funktionen von $x$, $\xi$ sind. Der Körper (ry) ist ain Körpor vom lelativgrade $p$ über dem Körper ( $x$

Im Fall, $p>1$ findm wir ein passendes $y$ dureh folgende Betrachtungen. Es swi , gofunden. Die Gleichung zwischen $\xi$ und $\eta$ sei $r(\xi, \eta)=0$. 
Sie sei in y vom Grade $\mu$. Dann gehör"n im allg"meinen "verschiedene Werte von y zu einem Werte von 5 . Es ist also Annahme gleich $(x y)$ ist, von der Ordnung $!$. Wir werden daher nur dann erwarten können, daß y, eine passende Größe ist, wenn zu einem Werte von $\Xi$ so viele Werte von y gehören, wie die Orinung von $\xi$ angibt.

Zu einem Werte von $\Xi$ gehören zufolge der Glejchung $g(x, \xi)=0$. $e$ Werte von $x$. Zu einem Wertepaar $x, \xi$ gehören zufolge der Gleichung $D(y ; x \xi)=0 p$ Werte von $y$. Zu einem Werte $\xi$ gellören also ep Wertepaar $x, y$. Es ist daher $\|=e p$. Man kann leicht Funktionen angeben, die ep verschiedene Werte für einen gegebenen Wert von $\xi$ annehmen. Die einfachste ist

$$
w=u x+v y
$$

wo unter $u$ und $v$ zunächst noch unbestimmte Konstanto verstanden sein sollen. Sind $x_{1}, x_{i}, \ldots x_{e}$ die Werte von $x$, die einem Wert von $\Xi$ entsprechen, und $y_{a 1}, y_{\star 2}, \ldots y_{a »}$, die Werte von $y$, die dem Wertepaar $\xi, x_{a}$ entsprechen, so nimmt für einen Wert von $\xi$ die Größe $w$ die $e p=\|$ Werte

$$
u x_{\iota}+v y_{u,}(\alpha=1,2, \ldots e ; \beta=1,2, \ldots p)
$$

an, die wir in irgendeiner Reihenfolge mit $w_{1}, w_{z} \ldots w_{u}$ bezeichnen. Wenn $\xi$ irgendeinen geschlossenen Weg durchläuft, so vertauschen sich die $x_{*}$ untereinander. Geht dabei z. B. $x_{1}$ in $x_{2}$ über, so geht $D\left(y ; x_{1}, \xi\right)$ übcr in $D\left(y ; x_{2}, \xi\right)$, und es müssen daher die Größen $y_{1 ;}$, abgesehen von der Reihenfolge, übergehen in die Größen $y_{23}$. Bei rinem geschlossenen Umlauf von $\xi$ vertauschen sich daher dif $w_{k}$ höchstens untereinander, so daß die symmetrischen rationalen Funktionen der $w_{k}$ rationale Funktionen von $\xi$ sind. Diese sind bei unbestimmtem $u$ und $v$ bei nieht speziellem $\xi$ voneinander versehieden. Denn für nicht speziell gewähltes $\Xi$ hat weder die Gleichung $g(x, \Xi)=0$, noch haben die Gleichungen $D\left(y ; x_{\star}, \Xi\right)=0$ gleiche Wurzeln.

Die Größe $w$ genügt einer Gleichung , ‘-ten Grades, deren Koeffizienten rationale Funktionen von $\Xi$ sind, nämlich der Gleichung

$$
\zeta(w, \zeta) \quad\left(w-w_{1}\right)\left(w-w_{2}\right) \ldots\left(w-w_{u}\right)=0 \text {. }
$$

$\zeta(w, \zeta)$ ist eine ganze rationale Funktion von $u, v$. Setzen wir $w=u x$ $+v y$, so ist die Gleichung $\zeta(w, \zeta)=0$ identisch befriedigt, auch in bezug auf $u, v$. Wir dürfen sie daher nach $u$ und $v$ differentiieren und erhalten, wegen $\partial w, \partial u=x, \partial w / \partial v=y$,

$$
x \frac{\partial \psi}{\partial w}+\frac{\partial \psi}{\partial u}=0, \quad y \frac{\partial \psi}{\partial w}+\hat{\partial} \hat{v}=0
$$

oder

$$
x=-\begin{gathered}
\partial \psi \\
\frac{\partial u}{\partial \psi} \\
\frac{\partial w}{\partial w}
\end{gathered}, \quad y=-\frac{\partial v}{\partial \psi} .
$$


Da dir Glcichung $\varsigma^{\prime}=0$ bei boliehigem $\stackrel{\Xi}{\Xi}, u$, e keine mehrfachen Wurzeln

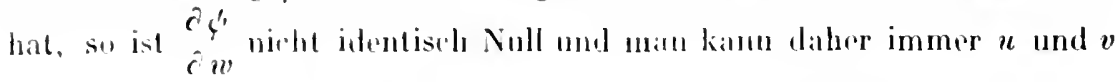

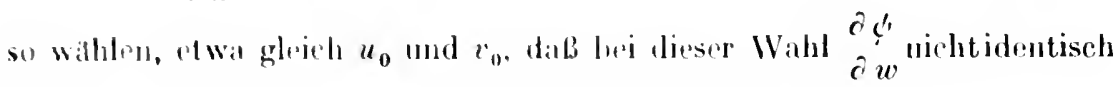
Null ist. Wir setzen dies spezinlle w gleich 1, also

$$
i_{1}=v_{0} r+v_{0} y \text {. }
$$

Es werdin dimn, wir ans din Gluidungen (9) hervergeht, $x$ und $y$ rationale Funktionen von $\xi_{1}$, D D anderersits $气$ und , rationale Funktionen von $x, y$ sind, so ist damit hwweson, dab man zu eimer Funktion $\Xi$ des körpers $(x y)$. die nicht konstant ist, immer rim zweite funktion y finden kann, so daß der liörper $(x y)$ mit dem Körper $\left(\xi_{1,}\right)$ idrutisch ist. Wir fassen das Ergethis in folgrnd Sätze zusammen, wobei wir gleichzeitig den SatzIII, Kap. IV $\$ 2$ der sich auf $x$ als unablängige Vor̈̈nderliche bezicht, anwinden auf $\xi$ als mahhängige Veränderliche.

Satz II. Es sei gnine nicht konstante Größe des Körpers $(x y)$. thre Ordnung sei $\mu$. Ist dann, rine zwoite Größe des Körpers, so hesteht zwischen $\Xi$ und, cine unzerlegbare Gleichung $f(\xi, 1)=0$, deren Grad in bezug aul gleich "l oder gleich einem Teiler von $\mu$ ist. Jann und nur dann, wenn der Grad der Gleichung " ist, ist der Körper ( $\left.\xi_{1}\right)$ identiseh mit lem hörper $(x y)$. Der Grad der Gleichung zwischen 5 und, ist in dirsem Falle in bezug auf gleich der Ordnung $\lambda$ von Ist abor der Grad der Gleichung $q=0$ in gloirh $\mu / p$, so ist er in 5 rom Grade $\lambda / p$.

SulzIII. Zu jeder nicht konstanten Größe gdes Körpers (xy) Jäbt sich (auf unendlich viele Arten) eine Größe $\eta$ aus

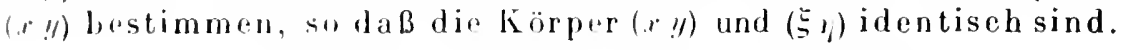

\section{\$. Anwendung auf die Beispiele 2 und 3.}

Boispiel 2. Wir bestimmen zunächst die Ordnung von $\xi$. Es ist $\xi=11-x^{2}$, also in der Umgehung des unendich fernen Punktes der $x$-Ebene $\xi=x \mid-1+\cdots$. Die über der $x$-Ebene ausgebreitete Riemannsthe Fläche ist 6hlättrig. Sio hat für mendlich großes $x$ zwei zweifarhe Vorzwigungspunkte, wie daraus folgt, dab dort $y=+1-1 x^{\frac{2}{3}}+\cdots$ oder $y=-\breve{l}-1 x^{3}+\cdots$. Da $\xi=11-x^{2}$ für unendlich großes $x$ unendlich wird wie $x$, so wird $\xi$ in jedem der beiden bei $x=\infty$ übereinanderliegenden Verzweigungspunkten von der Grdnung 3 unendlich, hat also, da es sonst nirgends unendlich wird, im ganzen 6 Unendlichkeitsstellen. Die Ordnung von $\xi$ ist daher $\mu=6$. Wir konnten das auch schon aus der 
Gleichung $g(x, \zeta)=0$ ersehen. I)inse ist in 5 vom Grode $q=2$, während $m=6$ ist. Lis ist also $p=m 2-3$. Hir (ideichung $g(x, \xi)=0$ ist fermer in $x$ vom Cirade $c=2$. Also ist $u=e p=6$

Wir setmon

$$
w=n x+b y \text {. }
$$

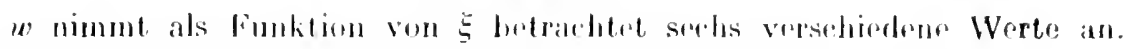
Das könmon wir hier sofort äbersihen, deno es ist

$$
w=u\left|1-\xi^{2}+v\right| \xi \mid 1-\xi^{2} \text {. }
$$

Hieraus ergibt sich anch am einfachsten die Gleichung $\ell^{\prime}(u, \xi)=0$, nämlich

$$
\begin{array}{r}
\xi^{\prime}(w, \xi)=u^{6}-3\left(1-\xi^{2}\right) u^{2} u^{4}-3\left(1-\xi^{2}\right)\left|2 v^{3} \xi-u^{3}\left(1-\xi^{2}\right)\right| u w^{2} \\
-\left(1-\xi^{2}\right)\left\{v^{3} \xi+\left.u^{3}\left(1-\xi^{2}\right)\right|^{2}=0 .\right.
\end{array}
$$

Es wird ilso

$$
\begin{aligned}
& \partial^{4} \\
& \partial u=\left(1-\xi^{2}\right)\left\{u u^{4}+\left|v^{3} \xi-2 u^{3}\left(1-\xi^{2}\right)\right| w^{2}+u^{2}\left(1-\xi^{2}\right) \mid v^{3} \xi+u^{3}\left(1-\xi^{2}\right)\right. \\
& \partial \varphi^{\prime}=\quad w\left\{w^{4}-2\left(1-\xi^{2}\right) u^{2} u^{2}-\left(1-\xi^{2}\right)\left|2 v^{3} \xi-u^{3}\left(1-\xi^{2}\right)\right| u\right\} \\
& \partial u \\
& \partial \psi \\
& l=\begin{array}{l}
\partial v \\
\partial \xi^{\prime}
\end{array}=w_{\left\{u^{4}-2\left(1-\xi^{2}\right) u^{2} u^{2}-\left(1-\xi^{2}\right)\left|2 v^{3} \xi-u^{3}\left(1-\xi^{2}\right)\right| u\right\}}^{\left(1-\xi^{2}\right)\left\{3 u u^{2}+\left|v^{3} \xi+u^{3}\left(1-\xi^{2}\right)\right| v^{2} \xi\right.} . \\
& \partial w
\end{aligned}
$$

Würden wir hier $u=1, v=0$, alsn $w=x$ wählen, so wird $\psi$ zerlegbar und erhält mehrfache Faktoren. Es wird nämlich dann $\varphi^{4} \quad\left[w^{2}-\left(1-\xi^{2}\right)\right]^{3}$. Fis wird daher $\frac{\partial c^{\prime}}{\partial w^{\prime}}$ zugleich mit $c^{\prime} z u$ Null und die Werto für $x$ und $y$ vorlieren ihren Sinn. Dagegun können wir wihlen $u=0, v=1$, also $u=y=\eta$. Es wird

$$
\zeta^{\prime \prime} \quad w^{6}-\xi^{2}\left(1-\xi^{2}\right) \quad y^{6}-\xi^{2}\left(1-\xi^{2}\right) .
$$

bleibt also unzerlegbar. Ferner wird

$$
\begin{aligned}
& x=\begin{array}{c}
\left(1-\xi^{2}\right) \xi^{2}{ }^{2} \\
1^{5}{ }^{5}
\end{array}=\begin{array}{c}
\left(1-\xi^{2}\right) \xi_{1}{ }^{3} \\
1^{6}{ }^{6}
\end{array}=\begin{array}{c}
\left(1-\xi^{2}\right) \xi \eta^{3} \\
\xi 2\left(1-\xi^{2}\right)
\end{array}=\eta^{3}, \\
& y=\frac{\left(1-\xi^{2}\right) \xi^{2}}{y^{5}}=\frac{\left(1-\xi^{2}\right) \xi^{2} 1}{1^{6}}=1 .
\end{aligned}
$$

"in Ergehnis, desen Richtigkejt man ohne woiteres rinsieht.

Brispiel 3. Wir hestimmen and hier zun̈irhst dir Ordnung von Lis ist, wie aus $g(x, \xi)=0$ folgt $\Xi=\mid x$. Es wird daher $\Xi$ Null nur für $x=0$. bie Rirmannsehe liärhe, die über der $x$-Ebene ausgebreitet ist, ist hier Ablättrig. Sir hat im l'unkte $x=0$ zwei Verzweigungsponkte erster Ordmung, wir sich ergibt, wemn man dis. Gleichung $f(x, y)=0$ nach $y$ auflöst. In jedem dieser Punkte wird $\xi$ von der arsten Ordmung Null und sonst 
nirgends. Lis ist also $ミ$ von der Ordnung $\|=2$. Auch hier ist, wie es sein muB. $\iota=e p$, da $e=1, p=2$.

setzen wir

$$
u=u x+v y .
$$

so rrgibt sich zunächst aus $g(x, \xi)=0$ und $D(y ; x, \xi)=0 w=u \xi^{2}$ $\left.+v(\xi+2) \xi^{2}-1\right)$ und daraus

$$
\zeta^{\prime}(u, \Xi) \quad u^{2}-2\left(v \Xi+u \Xi^{2}\right) u+4 v^{2}-3 v^{2} \Xi^{3}+2 u v \xi^{3}+u^{2} \xi^{4}=0 .
$$

Li ward also

$$
\begin{aligned}
& \text { ô } \\
& x=-\frac{\partial u}{\partial \xi^{\prime}} \begin{array}{c}
\partial w \\
\partial w-\left(v \xi+u \xi^{2}\right)
\end{array}, \begin{array}{c}
\xi^{2}\left(w-v \xi-u \xi^{2}\right) \\
w-(v \xi
\end{array}, \\
& \text { c) } \\
& y=-\frac{\partial v}{\frac{\partial \psi}{\partial w}}=\frac{w \xi-4 v+3 v \xi^{2}-u \xi^{3}}{w-\left(v \xi+u \xi^{2}\right)} .
\end{aligned}
$$

Wir dürfen z. B. wählen

Dann wird

$$
u=0, \quad v=1, \quad w=y=1 .
$$

$$
\begin{aligned}
& \text { ! } \quad w^{2}-2 \xi w+4-3 \xi^{2} \quad \imath^{2}-2 \xi^{2}+4-3 \xi^{2}=0 \text {, } \\
& x=\xi^{2}, \quad y=\begin{array}{c}
\Xi_{1}-4+3 \xi^{2} \\
1, \xi
\end{array} \text {. }
\end{aligned}
$$

Aus der Gleichung $\zeta(,, \xi)=0$ folgt aber $\eta(\eta-\xi)=\xi \eta-4+3 \xi^{2}$, so dal $y$. wie es sein mub, gleich, wird.

Man könnte auch wählen $u=v=1$. Man kann hier überhaupt $u$ und $v$ beliebig wählen, wenn man nur $v$ von Null versehieden annimmt.

\section{\$ 7. Die beiden Riemannschen Flächen.}

Geradeso wie wir über der $x$-Elene eine Rie m an n sche Fläche hergestellt habmn, obenso können wir übor der $\Xi$-Ebene eine Riemannsche Fläche herstellen, auf der auch die Funktionen des körpers eindeutige Funktionen des (urtes sind. Sie wird aus "Blättern bestehen, wenn "e wieder die Ordnung von $\Xi$ ist. Sie wird Vorzweigungspunkte enthalten, deren Zahl und Amrrdnung ganz andres suin kann, als bri ler über der $x$-Ebene konstrui rtrn. Jst der Körper $(x y)$ z. B. definiert durch die Gleichung $y^{2}=1$ $-r^{4}$, sn ist die über der $x$-Ebene ausgebreitete Riemannsche Fläche zw.iblättrig und hat als Verzweigungspunkte erster Ordnung die Punkte 1, $-1, i,-i$. Nehmen wir $\xi=y$ als muabhängige Veränderliche und twa $x=1$, so daß der Körper $(x y)$ gewiß mit $\left(\xi_{1}\right)$ identisch ist, so ist $1=11-\xi^{2}$ and die Riemannsche Fläche über der $\xi$-Ebene ist vier- 
biattrig. Sir lat in den Punkten +1 und -1 je rinen dreifarchen und im Unendlirhen awei einfache Verzweigungspmete. Da wir 5 auf un-

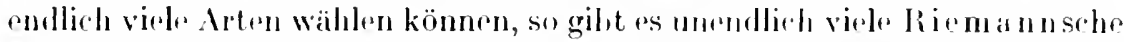
Flärhen, auf denen wir die Funktionen desselben aldowalashen körpers $(x y)$ als rindeutige Funktionen des Ortes hetrathten können. So verschieden diese anch anssehen können, so haben sie dor.h gawissa Eigensehaften gemuinsim, worauf wir aber erst später ringehen.

Jedem P'unkte und seiner Umgelung riner dirser Rirmannschen Fläehen, die zu dem Körper $(x y)$ gehören, entspricht rin Punkt und seine Umgrhung auf jeder anderen. Wir können daher die Stellen eines algebraischen Körpers und ihre Umgebung mit IIilfe irgendeiner Größe $\Xi$ des Körpers definieren, die nicht konstant ist. Ja, wir können aurch jede Stelle mit Hilfe einer anderen Größe $\xi$ definieren, wie es uns geradr am einfachsten erscheint.

Eine Stelle, die in der einen Fläche eine Verzweigungsstelle ist, brauclut es keineswegs in der anderen zu sein oder kann eine von anderer Verzweigungsordnung sein, wie das schon das Beispiel lehrt, was wir eben betrachteten. Sprechen wir also von einer Verzwoigungsstelle, so muß immer hinzugefügt werden, welche Größe als unabhängige Veränderliche betrachtet werden soll.

Zum Schlusse sei noch bemerkt, daß man die birationale Transformation vor allem dazu benutzen kann, statt der gegebenen Gleichung $f(x, y)=0$ eine andere $g\left(\xi, y_{1}\right)=0$ zu erhalten, die den algebraischen Körper geradeso gut definiert und die einfacher ist oder für die besondere Aufgabe, die man gerade hehandeln will, brauchbarer. Wir werden darauf noeh zuriickiommen.

\section{Srehstes Kapitel.}

\section{Divisoren.}

\section{$\$ 1$. Primteiler.}

Einæ Stelle des algebraischen körpers können wir jetzt mit seiner Ungebung so definieren. Ist $t$ irgenteine Ciröße, die an der Stelle von möglichst niedriger Ordnung Null wird, so lassen sich alle Funktionen des Körpers als Potenzreihen darstrillen, dir nach ganzen steigenden Potenzen voin $t$ fortsehreiten und die höchstens aine endliche Zahl von negativen Potenzen enthalten. Sotzen wir in die Reihenentwicklung, dir sich für eine Funktion $R$ des Körpers ergiht, $t=0$, so rrlalt'n wir den Wert, den die Funktion an der betreffenden Stelle annimmt.

Wir bezeichnen die Stellen des körpers wir hisher mit kleinen deutschen Buchstaben. Es sei etwa w eine Stelle. Wir ordnen jeder Stelle einen 


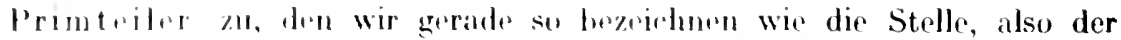
Stolle den Printriler p. Ilat eine Funktion $b$ in der Umgebung von $p$ die larstollung

$$
R=t^{\prime} E(t) .
$$

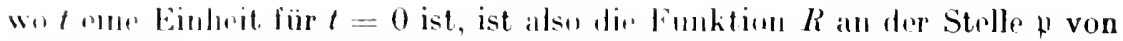

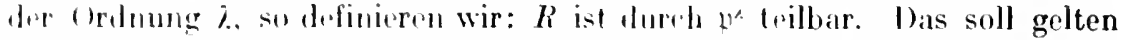
fiir pusitives und negatives $i$ und anch, wenn $\lambda=0$. Ein Produkt aus mehreren Primteileru, jeden in einer positiven oder negativen ganzzahligen Petenz genommen, nenten wir einen Divisor. Ein l)ivisor heißt ganz, wenn keiner der Exponenten negativ ist. Die Summe alher Exponenten heißt die Ordnung des Invisors. So hat z. B. dre Divisor $\psi_{1}^{3} p^{-2} p^{-2}$ die Orduning - 1 .

\section{\$. Z. Zcrlegung der Funktionen des Körpers in Primteiler.}

Da wir gesehen haben, daß jedr. Funktion des körpers nur eine endliche Zahl von Null- und Unendlirhkritsstellen hat, so enthält jede Funktion nur eine endliche Zahl von Primtrilern. Wir können sie daher in eindeutiger Wrise in Primteiler zerlengu. Auf diese Art wird jeder Funktion des Körpers ein ganz Jestimmter Jivisor zugeordnet. Es sei $R$ rine Funktion des Körpers und as sei, in Primtriler zerlegt,

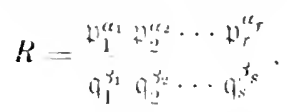

wo wir die a und $\beta$ als positiv annehmen wollen. Solch eine Zerlegung ist nur cin äbersirhtlicher Ausdrurk dafür. wo und wie $R$ Null und unendlich wird. Zwei Funktionen. deren Zerlegung denselben Divisor ergibt, hahen also dieselben Null- und Unendlichkeitsstellen und unterschriden sich daher nur um einon krunstanten Faktor. Wir nennen die ganzen Divisoren

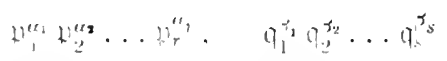

den Zähler und den Nenner von $R$. Dir Ordnung des Zählers von $R$ ist $u_{1}-a_{2}+\cdots+a_{r}$ und sie ist nichts anderes als die Zahl der Nullstellen von $R$, jodre so oft grechnet, als ihro Violfachheit angibt. Ebenso ist die Ordnung dos Nonners, nämlich $\beta_{1}+\beta_{2}+\cdots+\beta_{3}$ gleich der Zahl der Unmollirhkeitsstmen von $R$. Alber wir halen bewiesen, daß die Zahl der Nillotellon einer Funktion des Körpers immer gleich der Zahl ihrer Cnemllirhkoitsstallen ist. Daher haben der Zähler und der Nenner von $R$ distl,. Crdnung, und diese Ordnung stimmt überein mit der Ordnung der Funktion $R$, und die Ordnung $\Sigma u-\Sigma \beta$ des der Funktion $R$ entsprorhendon Divisors ist Null. Wir haben den Satz: 
Jede Funktion des Körpers läbt sich rindentig in eine endliche Zahl von Primteilern zrrlegrn. Jir Ordnung des so entstehendon Divisors ist Null.

Es sei gleich bemerkt, daß im allgenurinen nicht das Umgekehrte gilt, daß also nirht jedem Divisor der Ordnung Null rine Funktion des Körpers entspricht.

Wir bezeiehnen den Zähler einer lunktion $R$ des Körpers auch mit $l_{k}$ und den Nenuer mit $n_{k}$, so daß

$$
R=\frac{l_{k}}{n_{H^{\prime}}} \text {. }
$$

Die Ordnung von $R$ bezeichnen wir mit $n_{k}$. Sic ist gleichzeitig die ()rdnung der Divisoren $l_{k}$ und $n_{k}$.

\section{$\$ 3 . \quad$ Verzweigungsdivisoren.}

Als Beispiel betrachten wir die Zerlegung von $x-a$. Die Ordnung von $x-a$ ist $m$, wo $m$ der Grad der Gleichung $f(x, y)=0$ in $y$ ist. Es möge zunächst von den Stellen der über der $x$-Ebene ausgebreiteten Ri $\rho$ mannschen Fläche, für dic $x$ den Wert $a$ hat, keine eine Verzweigungsstelle sein. Dann liegen dort $m$ Stellen übereinander. $Z u$ jeder dieser Stellen gehört ein Primteiler. Diese $m$ Primteiler bezeichnen wir mit $\mathfrak{p}_{1}, \mathfrak{p}_{2}, \ldots \mathfrak{w}_{m}$. Für die Umgebung cines jeden dieser Punkte lassen sich die Funktionen des Körpers nach ganzen Potenzen von $x$ - $a$ entwickeln. In jedem dieser Punkte hat daher $x-a$ die Ordnung 1 , so da $B-a$ genau durch die reste Potenz eines jeden der Primteiler $w_{k}$ teilhar ist. Es wird

$$
1_{x-a}=p_{1} p_{2} \ldots n_{m} \text {. }
$$

Es möge ferner ein «-facher, win $\beta$-facher und rin $\gamma$-facher Verzweigungspunkt dort vorhanden sein, wo $x$ den Wert $a$ annimmt. Wir haben damn nur drei Punkte $p_{1}, p_{2}, p_{3}$ lei $x=a$ und auch nur drei Primteiler $p_{1}, k_{2}, f_{3}$. Für die Ungebung von $f_{1} l_{1}$ assen sich alle Funktionen des körpers

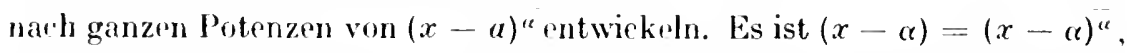
also von der Ordnung a an der Stelle $f_{1}$, so daß $x-a$ genau durch $p_{1}^{\prime \prime}$ teilhar ist. Ehenso ist os dureh +3 und $\mu_{3}$ teilbar. Wir haben also in dissem Falle

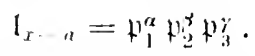

Es enthält alsu $\left.\right|_{x-n}$ die Primteiler, die zu Stellen gohören, an denen $x$ den Wert $a$ hat, und es enthält einen dieser Primteiler nur dann mehrfach, wenn die Stelle, zu der er gehört, ein Verzweigungspunkt in bezug auf $x$ ist. Ist die Verzweigungsordnung gleich $a-1$, so enthält $l_{x-a}$ den betreffenden Primteiler genau in der a-ten Potenz. Was für $x$ gilt, gilt natürlich auch für jede andere Größe des körpers. Wie wir gesehen haben, gibt es in bezug auf jede Größe $\xi$ des Körpers eine endliche Zahl von Ver- 
zweigungsstelle'n. Dir z.1 diesen Stelten gohörenden Primteiler heißen Verzweigungs-Primteilerin bezug auf Primteiler-jeder in der Potenz genommen, dir seine Verzweigungsordnung angiht - heißt Verzwigungsdivisurvon Die ordnung dieses lovisors leißt die Verzweigungszahl von oder in bezug auf $\Xi$ und soll mit $w$ berichnet werden. Vgl. Kap. III, §8.

\section{\$4. Divisorenklassen.}

Wir teilen die Divisoren in Klassen ein, indem wir zwei Divisoren dann und uur dann in diesclbe Klasse rechnen, wenn der Quotient piner Funktion des Körpersentspricht. So bilden die Divisoren, die den Funktionen des Körpers entsprechen, eine Klasse. Sind nämlich $r_{1}, r_{2}$ zwei solehe Divisoren und $R_{1}$ und $R_{2}$ die Funktionen, denen sie entsprechen, so entspricht auch ${ }_{r_{2}}^{r_{1}}$ einer Funktion des Körpers, nämlich der Funktion $\begin{aligned} & R_{1} \\ & R_{2}\end{aligned}$. Es gehören daher $r_{1}$ und $r_{2}$ zı derselben Klasse. Ist andererseits $r$ irgendein Divisor der Klasse zu der $r_{1}=R_{1}$ gehört, so ist nach Definition ${ }_{{ }^{c}}$ eine Funktion $R$ des Körpers und daher entspricht auch r einer Funktion des Körpers, nämlich der Funktion $R R_{1}$. Die Funktionen des Körpers bilden also wirklich eine Klasse für sich, die alle und nur Funktionen des Körpers enthält. Sie heißt die Hauptklasse.

Die Divisoren der Hauptklasse haben die Eigenschaft, daß das Produkt von irgend zweien oder ihr Quotient wieder der Klasse angehört. Allgemeiner ist ein irgendwie aus Divisoren der Hauptklasse multiplikativ zusammengesetzter Divisor wieder ein Divisor der Hauptklasse.

Wir können jetzt auch definieren: Zwei Divisoren gehören dann und nur dann zu derselben KJasse, wenn ihr Quotient ein Divisur der Hauptklasse ist. Es sind daher alle Divisoren, die mit einem Divisor a in dieselbe Klasse gehören, in der Form

darstellbar, wa $r$ ein Jivisor der Hauptklasse ist, und umgekehrt gehört jedrer Divicor der Form ar mit q in dieselbe Klasse. Da die Divisoren der llauptklasse die Ordnung Null haben, so haben die Divisoren q $r$ alle dieselbe (Jrdnung wie a, so daß dir. Divisoren riner Klasse alle dieselbe Ordnung haben, die die Ordnung der Klasse heißt. Durchläuft $r$ die Divisoren der Hauptklasse, so durchläuft $q \mathfrak{r}$ alle und nur die Divisoren der Klasse, der q éngrhört. Wir bezeichnen sie mit (q). Ist 5 irgendein anderer bivisor, so durchläuft auch $\tilde{a}$ alle und nur die Divisoren einer Klasse, die wir mit ( $)$ bezeichnen. Wir betrachten zunächst den Fall, wo 5 lar Klasse $(q)$ angehört. Es ist dann $s=q r_{0}$, wo $r_{0}$ der Hauptklasse 


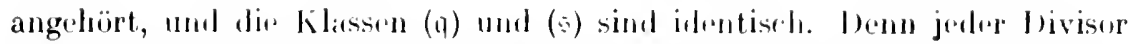

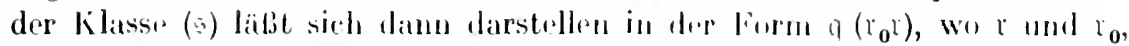
also auch $\mathrm{rl}_{0}$ dher Hauptklasse angehören, und gohö̈t dahor der Klasse (a)

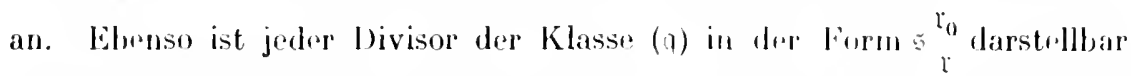
und grehört demuach der lilasse (s) an. Es ist dahre ganz gloirlogïltig, welehen Divisor der Klasse man benutzt, um dir Klasse zu definirern. Ist q' ingendoin Divisor der Klasse, so durehlänft $q^{\prime} r$ die Wivisoren dor Klasse und nur diese, wonn a dir Divisoren dor llauptklasse durchläuft. Jede Klasse ist durch irgendeinen ihror Divisoren vollständig bestimmt. Es sei ferner s nicht in der Klasse (q) enthalten. Jann haben die Klassen (1) und (s) keinen Divisor gemoinschaftlich. Iätten sie nämbich rines Divisor a' gerneinsam, so könnten wir dir Jivisoron von (q) sowohl wir die von (s), und zwar jeden einmal und nur pinmal erhalten, wenn wir in $q^{\prime}$ ? den Divisor $r$ alle Divisoren der IIauptklasso durchlaufon lassen. Die Klassen wärem also identisch und s würde gegen die Voraussetzung der Klasse (१) angehören.

Wir haben also: Jeder Divisorgehörteinerund nuroiner Klass" an oder: Zwei Klassen sind entweder identiseh orler haben gar keinen Divisor gemeinsam. Wir können daher auch zwei Divisoren $\mathfrak{q}_{1}, \mathfrak{q}_{2}$, die derselben Klasse angehören, äquivalent nennen, in Zeichen $\mathfrak{q}_{1}-\mathfrak{q}_{2}$. Denn es gilt nach dem Bewiesenen folgendes:

1. Jeder Divisor ist sich selbst äquivalent.

2. Wenn zwei Đivisoren zu einem rlriten äquivalont sind, so sind sie untereinander äquivalent.

Sind $\left(q_{1}\right)$ und $\left(q_{2}\right)$ irgend zwei Klassen, also $q_{1}$ und $q_{2}$ irgembreleh" Divisoren aus ihnen, so lerißt die Klasse $\left(a_{1} \theta_{2}\right)$ iln Produkt und dir Klasse $\left(\begin{array}{l}\pi_{1} \\ \eta_{2}\end{array}\right)$ ilır Quotient. Diese Klassen sind nach dem Bewiesemen vollständig bestimmt, und es ist gleichgültig, wrlehe Divisoren $\eta_{1}$, $\eta_{2}$ man aus den Klassen $\left(a_{1}\right)$ und $\left(q_{2}\right)$ zur Definition der Klassen $\left(q_{1} q_{2}\right),\left(\begin{array}{l}a_{1} \\ q_{2}\end{array}\right)$ benutzt. Wir kömmen also dir Klassen belingig multiplizieren und dividieren. Habei spint dir Ilauptliasse die Rolle der Einleit, la in ihr der Divisor I vorhanden ist und man diesen beim Multiplizieren oder Dividieren mit dre Jauptklasse als Repräsentanten dieser Klasse nelumen kann.

\section{\$ุ.. Dimension einer Klasse.}

In einer Klasse können ganze bivisoren vorhanden sein. Es sei in einer Klasse (१) mehr als ein ganzer Ijivisor vorhanden, also mindestens zwei voneinander verschiodene $!_{1}, ?_{2}$. Es ist dann $?_{!_{1}}$ einr finktion des 
Korpers. die mit $G_{2}$ bezeichnet sei. Sind $\lambda_{1}$ und $\lambda_{2}$ Konstante, so kann die Funtion $\lambda_{1}+\lambda_{2} \theta_{2}$ nur unendlich werden, wo $g_{2}$ unendlich wird, und nicht ron höherer Ordnung als $G_{2}$. Schreiben wir daher, in Faktoren zerlogt.

$$
i_{1}+i_{2} i_{2}=\frac{a_{0}}{a_{1}} \text {. }
$$

$\therefore$ ist 9 sicher ein ganzer Divisor. Dieser Divisor ist vollständig bestimmt hureh $\lambda_{1}, \lambda_{2}$, und da rein formal

so schreibt man winfach

$$
\lambda_{1}+\lambda_{2} a_{22}=\frac{\lambda_{1} n_{1}+\lambda_{2} n_{2}}{n_{1}}
$$

$$
\eta=\lambda_{1} !_{1}+\lambda_{2} !_{2} \text {. }
$$

Wir erhalten auf diese Art unendlich viele ganze Divisoren g, die alle der Klass (a) angehören. da ja $\frac{n}{a_{1}}$ gleich einer Funktion des Körpers ist und daher $n-?_{1}$. Es kann sein, daB in der Klasse (q) andere als diese ganzen Divisoren nicht enthalten sind. Es kann aher auch sein, daß außer diesen norh andere ganze Divisoren vorhandın sind. Es sei $\bigcap_{3}$ einer dieser Divisoren. Es ist auch $\frac{C_{3}}{?_{1}}$ pino Funktion des Körpers, die mit $G_{3}$ bezeichnet sei. Sind $\lambda_{1}, \lambda_{2}, \lambda_{3}$ irgendwelche liunstante, so ist $\lambda_{1}+\lambda_{2} G_{2}+\lambda_{3} g_{3}$ eine Funktion des körpers, die wieder hörhstens den Nenner $a_{1}$ haben kann, so dab, in Primteiler zerlegt.

$$
r_{3}=\stackrel{?}{!} !_{1} .
$$

wo !? sicher ein ganzer Divisor ist, der äquivalent $z u a_{1}$ ist und daher der Klass: (q) angehört. Wir schreiben wiedor symbolisch

$$
q=i_{1} \Omega_{1}+i_{2} \varrho_{2}+i_{3} \Omega_{3} \text {. }
$$

Unter diesen Divisoren sind die in der Form (1) darstellbaren natürlich mit enthalten. Keiner dieser Divisoren kann identisch Null sein, weil sonst für die Konstanten $c_{1}, c_{2}, c_{3}$ eine Gleichung der Form

$$
c_{1} \Omega_{1}+c_{2} ?_{2}+c_{3} \Omega_{3}=0
$$

bestehen müßtr. Wäre hier $c_{3}=0$. so würde folgøn, da $\beta \frac{Q_{2}}{a_{1}}=G_{2}=-\frac{c_{1}}{c_{2}}$, also konstant wäre, so daß $\bigcap_{2}=\bigcap_{1}$ scin würde gegen unsere Annahme. Ist aber $c_{3}$ von Null versehieden, so folgt

$$
\mathrm{q}_{3}=-\frac{c_{1}}{c_{3}} \mathrm{q}_{1}-{ }_{c_{3}}^{c_{2}} \mathrm{q}_{2} \text {. }
$$

Es wäre also gegen unsere Annahme $\varrho_{3}$ in der Form $\lambda_{1} \mathfrak{g}_{1}+\lambda_{2} \mathfrak{g}_{2}$ darstellbar. 
lis sind wirder awoi Fälle möglich. Entwollor es gibt aubur den in der form (2) entlaltenen ganzen l)ivisoren krime amblemen in der Klasse (q)

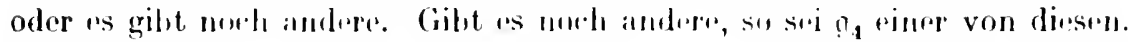
Es sind dann all. dir dureh $i_{1} ?_{1}+\cdots+i_{1} !_{4}$ mit konstanten $\lambda_{\text {. de }}$

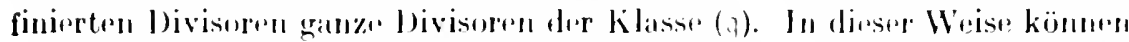

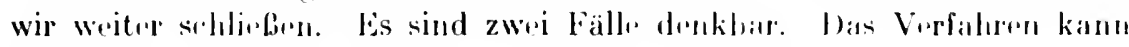

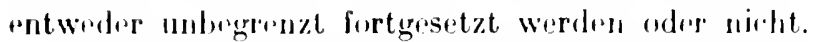

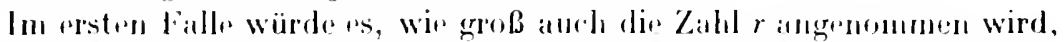

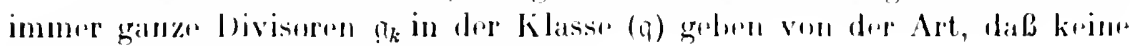
identische Gloichung von der from

$$
c_{1} !_{1}+c_{2} !_{2}+\cdots+c_{r} !_{r}=0
$$

mit konstanten $c$ bestaht. bezeirlunen wir dir Fusktion, die dern lovisor

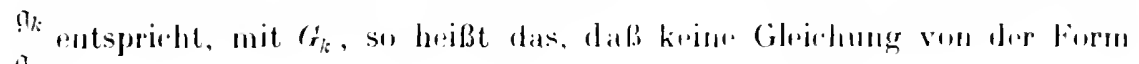
?1

$$
c_{1}+c_{2} i_{2}+c_{3} !_{3}+\cdots+c_{r} c_{i_{r}}=0
$$

identisch lustehon kann. Die Funktionen $G_{2}, G_{3}, \ldots G_{r}$ haben allo als Unondlichkeitsstollen höclıstens die Stellen, zu demen die Primtriler von $l_{1}$ gelı̈ren, und werden an ciner dieser Stellen höchstens von der ()rdnung a unendlich, wenn der zu dieser Stelle gehörende Primtuiler in ? in der reten Putenz enthalten ist. Ist die Ordunng von ? gleirh g, so hat also jede Funktion der Form

$$
\text { G } \quad c_{1}+c_{2} G_{2}+c_{3} G_{3}+\cdots+c_{r} G_{r}
$$

wo die $c$ koustant sind, höchstens $g$ Umendlichkeitsstellen und also auch höchstens $g$ Nullstellen. Wenn aber $r>g+1$, und das können wir annehmen, da wir ja $r$ belinbig grob voranssietz'n dürlen, so können wir die konstanten

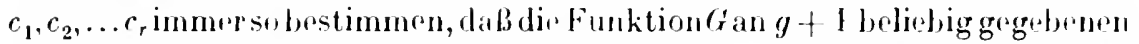
Stellen versehwindet. Sind nämlirh $G_{2}^{2}, G_{i}^{1}, \ldots G_{r}(k=1,2, \ldots g+1)$ die Werte, die die Funktionen $G_{2}, G_{3} \ldots G_{r}$ an $g+1$ irgendwie (nur verschieden von den Unendlichkoitsstellen von $G$ ) gewählten Strllen annehmen. s) brauchen wir nur die $g+1$ linearen homogenen Gleichungen zu lösen

$$
\lambda_{1}+\lambda_{2} G_{2}^{k)}+\lambda_{3} G_{3}^{(i)}+\cdots+\lambda_{r} G_{r}^{k}=0(k=1,2, \ldots g+1) \text {. }
$$

und dies" haben immer mindestens eine von Null verschiodene lïsung $c_{1}, c_{2} \ldots c_{r}$, wenn nur $r>g+1$. Dir Funktion $c_{1}+c_{2} G_{2}+\cdots-c_{r} G_{r}$ würde dann bui höchstrns $g$ Unondlichkritsstollen $g+1$ Nullstellen haben. Sie muB dah+r identisch Null sein. Es besteht also doch cine Gleichung der Form (4) und damit eine der Form (3). Der erste denkbare Fall ist also nicht mögli.lı und es bleibt nur der zwrite iilrigr. Daraus ergibt sich der Satz:

Sind in ciner klasse $(q)$ überhaupt ganz" loivisoren rnthalten, so gilit is immer eine endliche Zahl s von ganzen 
Mivisoren $\eta_{1}, \eta_{2}, \ldots !_{s}$ in der Klasse, so daB erstens keine (iloichung von der Form

$$
c_{1} !_{1}+c_{2} !_{2}+\cdots+c_{s} !_{s}=0
$$

mit kunstanten $c$ identisch besteht und da $B$ zweitens alle und mur dir eranzen bivisoren der klasse (n) in der Form darstellhar sind

wo die $i$ konstant sind.

$$
i_{1} !_{1}+\lambda_{2} \eta_{2}+\cdots+\lambda_{s} !_{s}
$$

Wir nennen ganze loivisoren $!_{1}, !_{2}, \ldots n_{2}$ zwischen denen keine Gleichung der Form (3) besteht. linear unablängig. Wir können daher auch kïrzer sagen:

Es gibt in jeder Klasse eine endliche Zahl linear unabhängiger ganzer loivisoren.

Ilirrin ist auch der Fall eingeschlossen, wo es in der Klasse gar keinen ganzen I)ivisor gibt.

Die Zahl der in einer Klasse (१) enthaltenen linear unabhängigen ganzen l)ivisoren heißt die Dimension der Klasse und wird mit $\{q\}$ bezeichnet. Die Hauptklasse hat die Dimension 1. Denn in ihr ist der ganze l)ivisor 1 enthalten und kein anderer; wäre noch ein anderer vorhanden, etwa $!$, so müßte $n=\frac{?}{1}$ einer Funktion des Körpers entsprechen und diese Funktion würde Nullstellen, aber keine Unendlichkeitsstellen haben. Es ist also

$$
\{1\}=1 \text {. }
$$

Aus unseren Betrachtungen ergibt sich, daß, wenn die Ordnung einer Klasse (1) gleich $g$ ist, dann

$$
\{q\} \leq g+1 \text {. }
$$

Da ferner alle Divisoren einer Klasse dieselbe Ordnung haben und ein ganzer Divisor immer eine positive Ordnung hat - abgesehen von dem Divisor 1 mit der Ordnung Null -, so ist die Dimension jeder Klasse mit negativer Ordnung oder der Ordnung Null gleich Null mit Ausnabme allein der Hauptklasse, deren Ordnung Null ist und deren Dimension gleich 1 ist.

Die Bestimmung drr in einer Klasse enthaltenen ganzen Divisoren und der Dimension einer Klasse wird uns in den beiden nächsten Kapiteln beschäftigen.

\section{\$ (6. Die kanonische Klasse.}

AuBer der Hauptklasse ist noch eine andere Klasse von besonderer Wichtigkeit, die wir jetzt definieren wollen. Es sei $\zeta$ irgendeine Funktion des Körpers. Es sei für die Umgebung irgendeiner Stelle $\mathfrak{w}$

$$
\xi=P(t), d \xi=P^{\prime}(t) d t,
$$


wo $t$ in $p$ von der arstrn Ordnung ist mul wo $P(t)$ eine nach steigenden Potenzen von $t$ fortschreitende lieihe ist. Wenn $P^{\prime}(t)=t^{\prime} E(t)$, wo $E(t)$ eine Einhoit für dir. Stolle $t=0$ ist, so sagen wir, das lofferential $d \xi$ ist durch $p^{2}$ teillar, ghlelegülig, ob i. positiv, negativ oder Null ist. Jer Exponent $\lambda$ kann nur für bine molliche Zahl vou Stellen von Null verschieden sein, wie wir jotat zoigen wollen. Wir nuterscheiden die beiden Fäle, wo $\xi$ an der betrarhtoten Stelle endlieh bleilot und es mnendlich wird. Im ersten Falle sei $\Xi_{0}$ der Wert von $\Xi$ an der Stelle. Es sei

$$
\Xi=\Xi_{0}+e_{0} t^{a}+e_{1} t^{t} \cdot 1+\cdots,\left(e_{0} \neq 0, \|>0\right) \text {. }
$$

Es wird

$$
d \xi=\left(u e_{0} t^{\prime \prime-1}+\cdots\right) d t,
$$

also $\lambda_{0}=\pi-1$. Aus der Gleichung (5) folgt aber, daß $\xi-\xi_{0}$ durch pet teilbar ist, daß also $p$ ein Verzweigungsprimteiler in bezug auf $\Xi$ von der Ordnung $a-1$ ist. Es ist also im ersten Falle $\lambda \neq 0$ nur für diejenigen Primteiler, die Verzweigungsprimteiler in bezug auf 5 sind. Soleher gibt es nur eine endliche Zahl. Da es ferner nur eine endliche Zahl von Stellen gibt, für die der zweite Fall eintritt, an denen nämlich 5 unendlich wird, so kann 2. nur für eine endliche Zahl von Stellen von Null verschieden sein. Wir betrachten den zweiten Fall genauer. Es sei an einer Stelle $p$

$$
\begin{gathered}
\Xi=e_{0} t^{-\beta}+e_{1} t^{-\beta+1}+\cdots,\left(\beta>0, e_{0} \neq 0\right) \\
d \xi=\left(-\beta e_{0} t^{-\beta-1}+\cdots\right) d t,
\end{gathered}
$$

so daß in diesem Falle $\lambda=-(\beta+1)=+(\beta-1)-2, \beta$ wird. Ferner folgt, daß $\xi^{-1}$ durch $w^{\beta}$ teilbar ist, daß also der Ordnung $\beta-1$ ist und daß ferner der Nenner $\eta_{\xi}$ von $\xi$ genau dureh

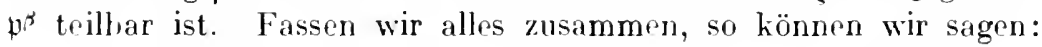

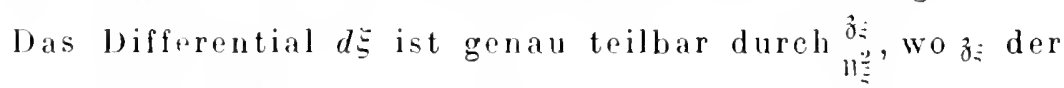
Vorzweigungsdivisor und li der Nennerdivisor von $\Xi$ ist.

Der Divisor, durch den ein Differential teilbar ist, hängt wesentlich al, von dem Differential, aber wir zeigen jetzt, daß alle diese Divisoren derselben Klasse angehören. Es sei ־ irgendeine (nicht konstante) zweite Funktion des Körpers. Wir bezeichnen die Divisoren, durch die $d \xi$ und $d_{s}^{c}$ teilbar sind, mit $v$ und $w$. Wählen wir y passend, so können wir alle Funktionen des Körpers als rationale Funktionen von $\xi$ und $y$ darstellen, also auch $\sqsubseteq$. Es sei $\zeta=R(\xi, 1)$. Die zwischen $\xi$ und , bestehende unzerlegbare Gleichung sei $g(\xi, y)=0$. Es ist

und

$$
d \zeta=\frac{\partial R}{\partial \xi} d \xi+\frac{\partial R}{\partial y_{y}} d_{1}, \quad \frac{\partial \mathscr{y}}{\partial \xi} d \xi+\frac{\partial y}{\partial y} d y=0
$$

$$
d \xi=\frac{w}{v}=\frac{\partial R}{\partial \xi}-\frac{\partial \xi}{\partial \eta} \partial \eta,
$$


also gleich riner rationalen Funktion ron $\xi, y$ und damit gleich einer Funtion des Körpers. Damit ist bewiesen, daß $\mathfrak{v}-\mathfrak{w}$.

l)ie Klasse, zu der hiernach alle Divisoren gehören, die den Differentialen der Funktionen des Körpers $(x y)$ entsprechen, heißt die Differentialklasse oder die kanonische Klasse. Die Ordnung der Differentialklasse ist, wie wir später sehen werden, immer grade und wird mit $2 p-2$ bezeichnet. Die Zahl $p$ heißt das Gesclilecht des Körpers. Ihre eigentlirh" Brdentung lernen wir später (Kap. VIII, \$4) kennen. Da alle Divisoren einer Klasse diesulle Ordnung haben und diese nichts anderes ist als die (Irdnung der Klasse, so ist für jede Funktion $\Xi$

$$
w_{\vdots}-2 n_{\vdots}=2 p-2 \text {. }
$$

da $w_{i}-2 n_{-}$dir. Ordnung des Divisors ${ }_{3:}^{3:}$ ist. Ls ist danach der Wert von $u_{\vdots}-2 n$; unabhängig von $\Xi$. In Worten heißt das:

1)i" Difforenz der Verzweigungszahl und der doppelten Zahl der Blätter ist dieselbe für alle Riemannschen Flächen, auf denen die Funktionen des Körpers eindeutig sind. Man setzt diese Zahl gleich $2 p-2$ und nent $p$ das Geschlecht des Körpers. Es ergibt sich noch

Sind $\Xi$ und $\sqsubseteq$ irgend zwei nicht konstante Größen des Körpers, so ist $d \xi$

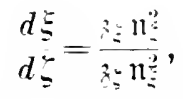

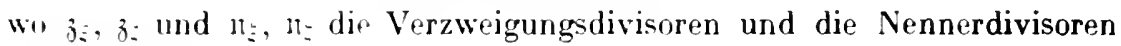
von $\Xi=\operatorname{sind}$.

IIir gehen noch auf die Bedeutung der Differentialklasse ein. Ist $\xi$ irgendrine nicht konstante Größe des Körpers, so gehört zu $d \xi$ der Divisor $3_{5} "_{\xi}{ }^{2}$ der Differentiallilasse. Ist $R$ eine Funktion des Körpers, so ist $\boldsymbol{R} \boldsymbol{d} \boldsymbol{\xi}$ eirs sriner Differentiale. Diesem entspricht der Divisor

$$
\mathrm{q}=\mathrm{r}_{\xi} \mathrm{n}_{\xi}{ }^{2},
$$

wenn $r$ der Divisor ist, den wir durch Zerlegung von $R$ in Primteiler erhalten. Da $r \sim 1$, so ist $q \sim n_{z}{ }^{2}$, grhört also der kanonischen Klasse an. Ist umgekehrt $q$ irgendein Divisor dirser Klasse und $\xi$ irgendeine nicht konstante Größe des Körpers, so ist der Divisor

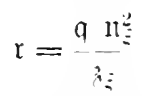

ein Divisor der Hauptklasse, entspricht also einer Funktion $R$ des Körpers, und es ist $R d \xi$ ein Differential des Körpers, dem der Divisor q entspricht. Wir haten also: 
Jedem lifforential des Körpers entspricht ein Divisor der Differentialklasse, der angibt, wie das Differential Null und unendlich wirl, und umgekehrt ontspricht jedem Divisor der Differntialklasse oin Differential des Körpers.

\section{\$. Beispiel 1.}

Die Gleichung zwischen $x$ und $y$ sei

$$
f(x, y) \quad y^{2}+x^{5}-1=0 .
$$

Nehmen wir $x$ als unabhängige Veränderliche, so ist $y=11-x^{5}$. Die Riemannsche Fläche über der $x$-Ebene ist zweiblättrig, $n_{x}=2$. Sie hat 6 Verzweigungspunkte erster Ordnung, nämlich, wenn $\omega$ eine primitive fiinfte Einheitswurzel ist, die Punkte $1,(1),()^{2}, \omega^{3}, \omega^{4}$ und den Punkt $\propto$. Die zu diesen Punkten gehörenden Primteiler seien bezeichnet mit $\mathfrak{b}_{1}$, $m_{2}, \ldots w_{6}$. Es ist $w_{s}=6$ und

$$
w_{x}-2 n_{x}=2 p-2=2 .
$$

Nehmen wir $y$ als unabhängige Veränderliche, so ist $x=\sqrt[5]{1-y^{2}}$. Die Riemannsche Fläehe über der $y$-Ebene hat fünf I3lätter; $n_{y}=5$. Sie hat drei Verzweigungspunkte vierter Ordnung, nämlich $+1,-1, \infty$. Die zu den ersten beiden Stellen gehörenden Primteiler bezeichnen wir mit $\mathfrak{v}_{1}, \mathfrak{v}_{2}$. Zu der dritten gehört der Primteiler $\mathfrak{w}_{6}$. Es ist $w_{y}=3 \cdot 4=12$ und

$$
w_{y}-2 n_{y}=2 \text {. }
$$

Da $2 p-2=2$, so ist das Geschlecht des hier betrachteten Körpers $p=2$.

Genauer haben wir folgendes. Die Riemannsehe Fläche über der $x$-Ebene hat nur einen unendlich fernen Punkt, der ein Verzweigungspunkt erster Ordnung ist und zu dem der Primteiler $w_{6}$ gehört. Es ist also

Ähnlich findet man, daß

$$
\mathrm{u}_{. c}=\mathrm{w}_{6}^{2} \text {. }
$$

Ferner ist

$$
\mathfrak{n}_{y}=\mathfrak{w}_{4 ;}^{5} \text {. }
$$

also

$$
z_{x}=\mathfrak{w}_{1} \mathfrak{w}_{2} \ldots \mathfrak{w}_{6}, z_{y}=\mathfrak{v}_{1}^{4} \mathfrak{v}_{2}^{4} \mathfrak{w}_{6}^{4},
$$

$$
d y=\frac{z_{y} \mathfrak{w}_{x}^{2}}{z_{x} w_{y}^{2}}=\frac{\mathfrak{v}_{1}^{4} \mathfrak{v}_{2}^{4} w_{6}^{4} \mathfrak{w}_{6}^{4}}{w_{1} w_{2} \ldots w_{6} w_{6}^{10}}=\frac{\mathfrak{v}_{1}^{4} \mathfrak{v}_{2}^{4}}{w_{1} w_{2} w_{3} w_{4} w_{5} w_{6}^{3}} .
$$

Andererseits ist

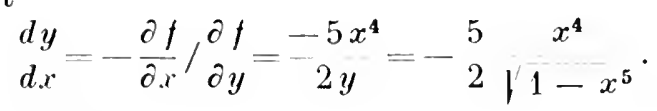

Die Funktion $-\frac{5 x^{4}}{2 y}$ wird tatsächlich, wie am einfachsten aus der Form

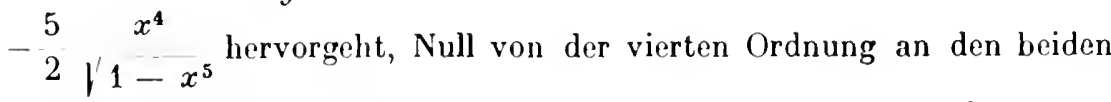


Stellen. wo $x=0, y= \pm 1$, zu denen dir Primteiler $v_{1}$, $u_{2}$ gehören, mul unmollich von der ersten Ordnung an den Stellen, zu denen die b'rimteilı $w_{1}, w_{2}, \ldots w_{5}$ gehören, aher unemllich dritter Ordnung für $x=x$.

\section{$\$$ S. Beispiel :.}

Als zwrites Beispiel nehmen wir das boispiel 3 in Kap. V, \$ 4 . Der körper besteht in diesem Fall aus allen rationalen Funktionen von $x$ und $y$, wenn zwischen $x$ und $y$ die Gleichung besteht

$$
f(x, y)=y^{4}+(8-10 x) y^{2}+(1-3 x)^{2}=0,
$$

oder. wenn wir setzen

$$
\Xi=-\frac{y^{3}+(4-7 x) y}{2(4-3 x)}, \quad 1=y,
$$

aus allen rationalen Funktionen von $\xi$, , went zwischen $\Xi$ und $\eta$ die Gleirhung besteht

$$
1^{2}-2 \xi_{1}+4-3 \xi^{2}=0
$$

wie wir in Kap. V, $\$ 4$ gesehen haben. Nehmen wir $x$ als unabhängige Veränderliche, so haben wir eine vierblättrige Riemannsrhe Fläche. Die Verzweigungspunkte können nur dort liegen, wo $\frac{\partial y}{\partial y}=0$ wird, oder im Punkte $x=s . \quad \begin{aligned} & \partial f \\ & \partial y\end{aligned}=4 y\left(y^{2}+4-5 x\right)$ wird Null für folgend Punkte:

$$
\text { 1. } x=\frac{4}{3}, y=0 ; 2 . x=0, y=+2 i ; 3 . x=0, y=-2 i ; 4 . x=1 \text {, }
$$
$y=1 ; 5 . x=1, y=-1$. Dazu kommt noch als sechster Punkt, der untersucht werden muß, der Punkt $x=x$. Da wir dies Beispiel auch später noch benutzen wollen, seien die Entwicklungen von $y$ für die sechs Fälle angegeben. Sie sind nach den Methoden des Kap. II zu bestimmen.

$$
\text { 1. } y= \pm \frac{3}{4} \mid 3\left(x-\frac{4}{3}\right)\left[1-\frac{15}{16}\left(x-\frac{4}{3}\right)+\frac{189}{128}\left(x-\frac{4}{3}\right)^{2}+\cdots\right] \text {. }
$$

Wir haben also zwei verschiedene Entwicklungen in der Umgebung von $x=\frac{4}{3}$, die die Eigenschaft haben, daß für $x=\frac{4}{3}$ die Größe $y$ den Wert Null annimmt. Die zu diesen Stellen gehörenden Primteiler bezeichnen wir mit $b_{1} . y_{2}$. Sie sind nicht Verzweigungsprimteiler in bezug auf $x$.

$$
\begin{aligned}
& \text { 2. } y=2 i+x^{2}-i x-\frac{i}{4} x^{2}+\cdots, \\
& \text { 3. } y=-\left(2 i+x^{2}-i x-{ }_{4}^{i} x^{2}+\cdots\right) \text {. }
\end{aligned}
$$

Die zu diesen beiden Entwicklungen gehörenden Primteiler seien mit 


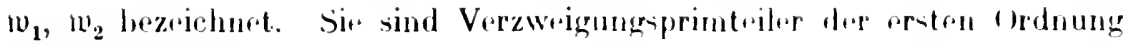
in brzugr auf $x$.

$$
\begin{aligned}
& \text { 4. } y=1+2(x-1)^{2}+\frac{1}{2}(x-1)-\frac{1}{8}(x-1)^{2}+\cdots \\
& \text { 5. } y=-\left|1-2(x-1)^{2}+\frac{1}{2}(x-1)-\frac{1}{8}(x-1)^{2}+\ldots\right| .
\end{aligned}
$$

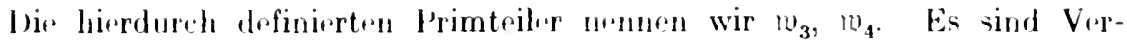
zwrigungsprimteiler arster Ordnung in bezug auf $x$.

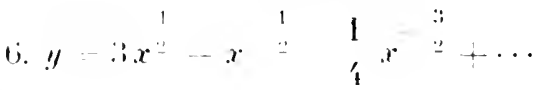

$$
y=x^{2}-x^{-1}+1, x^{-2}+\cdots
$$

Dir Riemannsche Flächr hat daher hei $x=$ swei Punkte, die beide Verzweigungspunkter erster Ordnung sind. Tie zugohörigen Primteiler seien mit $\dddot{w}_{5}, w_{6}$ bezeichnet.

Die über der $x$-Ebene ausgebreitetr Riemannsch Fläche hat vier Blätter und sechs Verzwrignngspunkte erster Ordnung. Es ist also

$$
n_{s}=4, w_{s}=6, w_{s}-2 n_{s}=2 p-2=-2, p=0 .
$$

Nehmen wir $\vdots$ als unabhängige Veränderliche und konstruieren di Riemannsche Flärh" übrr der E-Ebrne, so erhalten wir eine zweiblättrige Flärhe, wie aus dor Gleichung (8) folgt. Aus dieser Gleichung ergibt sich $1=\Xi \pm 2 \mid \xi^{2}-1$, woraus man ersieht, daß die Fläche bei $\Xi=+1$ und $\Xi=-1$ Verzweigungspunkte rester Ordnung hat und sonst keine Verzwrigungspunktı. Die zu diesen Stellen gehörondon Primteiler sinol die Primtriler $\omega_{3}$. $w_{4}$. Es ist

$$
n_{-}=2, w_{z}=2, w_{-}-2 n_{z}=-2=u_{r}-2 n_{s} .
$$

Wir wollen aurh norh $\begin{aligned} & d x \\ & d \xi\end{aligned}$ bestimmen. Wir zerlegen erst $x$ und $\Xi$ in Primteiler. Die Riemannschr Fläche hat für $x=0$ zwei Vrrzwrigungspunkte mit den Primteilern $w_{1}, w_{2}$. Der Zähler $l_{s}$ von $x$ ist daher $i_{r}=10_{1}^{2} 1 w_{2}^{2}$. Für unendlich großes $x$ hat die Fläche zwei Verzweigungspunkte erster Ordnung, denen die Primteiler $\mathfrak{w}_{5}, \mathfrak{w}_{6}$, entsprechen. Der Nanner von $x$

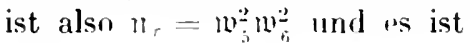

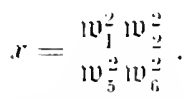

Für $\xi=0$ ist,$(\quad y)= \pm 2 i$ und für $x$ ergibt sich aus der Definitionsgleichung von $\Xi$ als zugehöriger Wert 0 . Es wird also der Zähler !: von $\Xi$ nur die Primteiler $w_{1}, w_{2}$ "nthalten. Da die über der E-Ebene ausgebreitete 
Riemannsche Fläche hei $\xi=0$ zwei gewöhnliche Punkte hat, so müssen zu diesen beiden die Primteiler $w_{1}, w_{2}$ gehören und es wird $\xi$ an diesen Stellen mur von der ersten Ordnung Null, so daß $\mathbb{1}_{\xi}=\mathfrak{w}_{1} \mathfrak{w}_{2}$. Man findet dasselbe Ergebnis, wenn man die Entwicklungen 3. und 4., dureh die $m_{1}$ und $w_{2}$ definiert sind, in die Definitionsgleichung von $\xi$ einsetzt und nach Potenzen von $x$ entwickelt. Die Entwicklungen beginnen mit der $\frac{1}{2}$-Potenz von $x$. Für $\xi=x$ hat die Riemannsche Fläche über der $\xi$-Ebene zwei gewöhnliche Punkte. Da für $\xi=\infty$ auch $\eta$ und also auch $y$ unendlich ist, so ist auch $x$ unendlich; denn $y$ wird nur für unendliches $x$ unendlich. Die beiden Primteiler, die den beiden Punkten bei $\xi=\infty$ entsprechen, sind also $w_{5}$ und $w_{6}$. Es wird $w_{5}=w_{5} w_{6}$ und

$$
\xi=!_{\vdots}=\frac{w_{1} w_{2}}{w_{5} w_{6}} \text {. }
$$

Vergleichen wir (9) mit (10), so sehen wir, daß $\xi^{2}$ und $x$ sich nur dureh cinen konstanten Faktor unterscheiden können, da ihre Zerlegung in Primteiler dieselbe ist. Es ist ja auch in der Tat $\xi^{2}=x$, wie wir in Kap. V, $\S 4$ gesehen haben.

Es ist ferner also

$$
j_{j}=w_{1} w_{2} \ldots w_{6}, \quad i_{i}=w_{3} w_{4},
$$

$$
d x=\frac{z_{x} w_{\xi}^{2}}{z_{\xi} w_{5}^{2}}=\frac{w_{1} w_{2} w_{3} w_{4} w_{5}^{3} w_{6}^{3}}{w_{3} w_{4} w^{4} w_{6}^{4}}=\frac{w_{1} w_{8}}{w_{5} w_{6}} .
$$

Es kann sich also $\frac{d x}{d \xi}$ nur durch einen konstanten Faktor von $\xi$ unterseheiden. Es ist ja auch $x=\xi^{2}$ und also $\frac{d x}{d \xi}=2 \xi$.

Sicbentes Kapitel.

\section{Rechnen mit Systemen.*)}

\section{$\$ 1$. Lineare Substitutionen.}

Zwischen den $m$ Größen $\xi_{1}, \xi_{2}, \ldots \xi_{m}$ und den $m$ Größen $\eta_{1}, \eta_{2}, \ldots \eta_{m}$ mögen die $m$ linearen Gleichungen bestehen

$$
\begin{aligned}
& \eta_{1}=p_{11} \xi_{1}+p_{12} \xi_{2}+\cdots+p_{1 m} \xi_{m}, \\
& \eta_{2}=p_{21} \xi_{1}+p_{22} \xi_{2}+\cdots+p_{2 m} \xi_{m}, \\
& -----------1-p_{m m} \xi_{m} .
\end{aligned}
$$

-) Eine aufführliche Darstellung findet man in L. Kronecker, Vorlesun'en über die Theorip der Determinanten, bearbeitet und fortgeführt von K. Hensel, Bd. I (Teubner. Leipzig 1903). 
Man sagt in dirsem Falle: die y geh'n durch eine lineare homogene Substitution aus den $\xi$ hervor. Die Substitution ist bestimmt durch das System ihrer $m^{2}$ Koeffizienten $p_{k l}$, das wir kur\% mit $p$ bezeichnen, und zwar sei

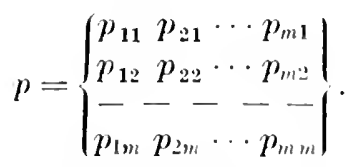

Die Anordnung der Elemente im System $p$ ist, worauf ausdrücklich hingewiesen sei, nicht dieselbe wie in den Gleichungen (1), sondern es sind gegenüher (1) Ilorizontal- und Vertikalreihen vertauscht. Das System, das aus $p$ durch Vertausehen der Horizontal- mit den Vertikalreihen hervorgeht, also die $p_{k l}$ in derselben Anordnung wie in (1) zeigt, heißt das zu $p$ adjungierte System und wird mit $\vec{p}$ bezeichnet.

Wir nennen die durch (1) vermittelte Substitution auch kurz die Substitution $p$ und schreiben sie abgekürt in der Form

$$
\eta=\xi p \text {. }
$$

Wie aus den $\Xi$ durch die Substitution $p$ die Größen $y$ hervorgehen, so können wir aus den $\eta$ durch eine zweite lineare Substitution $q$ neue Größen $\xi$ hervorgehen lassen, also durch die Gleichungen

$$
\begin{aligned}
& \zeta_{1}=q_{11} \eta_{1}+q_{12} \eta_{2}+\cdots+q_{1 m} \eta_{m}, \\
& \zeta_{2}=q_{21} \eta_{1}+q_{22} \eta_{2}+\cdots+q_{2 m} \eta_{m}, \\
& \overline{-}----------- \\
& \zeta_{m}=q_{m 1} \eta_{1}+q_{m 2} \eta_{2}+\cdots+q_{m m} \eta_{m} .
\end{aligned}
$$

Diese Gleichungen schreihen wir auch in der Form

$$
=={ }_{1} q \text {. }
$$

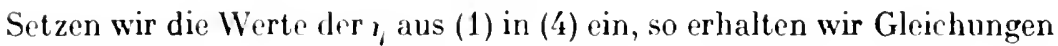
der Form

$$
\begin{aligned}
& \xi_{1}=r_{11} \xi_{1}+r_{12} \xi_{2}+\cdots+r_{1 m} \xi_{m}, \\
& \zeta_{2}=r_{21} \xi_{1}+r_{22} \xi_{2}+\cdots+r_{2 m} \xi_{m}, \\
& ---\overline{-}--\overline{-}-----1 \\
& \zeta_{m}=r_{m 1} \xi_{1}+r_{m 2} \xi_{2}+\cdots+r_{m m} \xi_{m},
\end{aligned}
$$

also eine lineare Substitution $r$, die die $\xi$ in die $\zeta$ überführt. Wir schreiben wieder kurz

$$
\zeta=\xi r .
$$

Man findet

$$
\begin{aligned}
& r_{1 \|}=p_{1 \|} q_{11}+p_{2 a} q_{12}+\cdots+p_{m 《} q_{1 m}, \\
& r_{2 a}=p_{1 \alpha} q_{: 1}+p_{2 \alpha} q_{22}+\cdots+p_{m \alpha} q_{2 m} \text {, } \\
& r_{m a}=p_{1 \alpha} q_{m 1}+p_{z \alpha} q_{m 2}+\cdots+p_{m \alpha} q_{m m} . \\
& (\alpha=1,2, \ldots m) \text {. }
\end{aligned}
$$




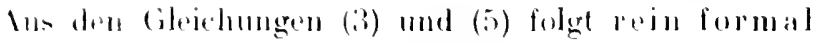

$$
\because \quad \Xi p q \text {. }
$$

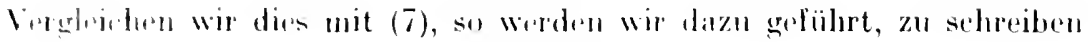

$$
r=p q \text {. }
$$

W, finition 1. Nan mennt laher die durch das Musführen

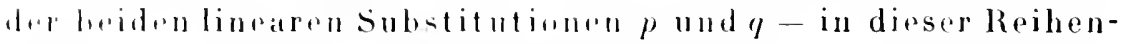
folgr - rntstehende linrare Substitution $r$ aus $p$ und $q \mathrm{zu}$ sammongusetzt odor auch geradozu das lerudukt vou $p$ und $q$. Elonso nenut man das Systrm ras leodukt der Systeme l' $11 \mathrm{nd} \varphi$.

\section{\$... Multiplikation von Systemen.}

Wï nussin uns zunächst die dureh die Gloichungen (8) gegebene Mnltiplikationsregel für Systeme möglichst klarmachen, da wir davon ausgiobigen Gebrauch machen werden.

Es sejull

$$
\begin{aligned}
& a_{1}, a_{2}, \ldots a_{m}, \\
& b_{1}, b_{2}, \ldots b
\end{aligned}
$$

жwi keihen von jum Größen. Dann verstuht man unter dem Produkte der liejden Reihen die Summo der Jrodukte je zwejer antsprechender Cimbilu. also don Ausdrurk

$$
a_{1} b_{1}+a_{2} b_{2}+\cdots+a_{n} b_{m} \text {. }
$$

Dofinition Il. Essojen p und q zwei Systeme. Dann ver-tuht man unter dim Produkte pq von $p$ und q das System, das

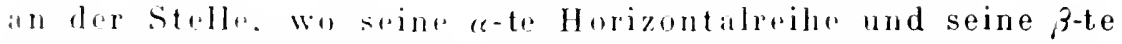
Vertikalreihrsich troffon, das lerodukt ausdera-ten Horizontalroilu, voll $/$ und dop $\beta$-ten Vertikalrojhe aus $q$ stehen hat.

oder: Ilon rrhäl die e-te Horizontalreihe des Systems $\mu q$. iudrom man dire-tr Horizuntalreiluovon $p$ der Reihe nach mit den Vertikalroilon von q multipliziert.

Man iibryugt sirh lrioht. dab abso Drfinition mit der durch die cileichmen (8) gegubruen ïhereinstimmt.

Einier rinfarhe brispiele mögrn die lofinition "räutern. Weitere

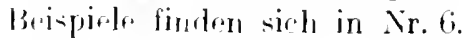

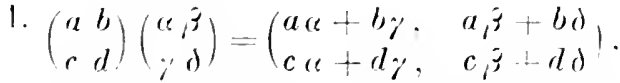

$$
\begin{aligned}
& \text { 2. }\left(\begin{array}{lr}
2-1 \\
3 & 4
\end{array}\right)\left(\begin{array}{lr}
1 & 1 \\
3 & -2
\end{array}\right)=\left(\begin{array}{ll}
2 \cdot 1-1 \cdot 3 . & 2 \cdot 1+1 \cdot 2 \\
3 \cdot 1+1 \cdot 3 . & 3 \cdot 1-4 \cdot 2
\end{array}\right)=\left(\begin{array}{rr}
-1 & 4 \\
15-5
\end{array}\right) \text {, } \\
& \therefore\left(\begin{array}{rr}
1 & 1 \\
; & -2
\end{array}\right)\left(\begin{array}{rr}
2 & -1 \\
3 & 4
\end{array}\right)=\left(\begin{array}{lr}
1 \cdot 2+1 \cdot 3, & -1 \cdot 1+1 \cdot 4 \\
3 \cdot 2-2 \cdot 3, & 3 \cdot-1-2 \cdot 4
\end{array}\right)=\left(\begin{array}{rr}
5 & 3 \\
0 & -11
\end{array}\right) \text {. }
\end{aligned}
$$




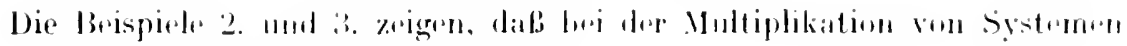

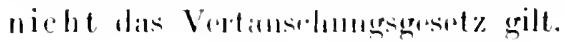

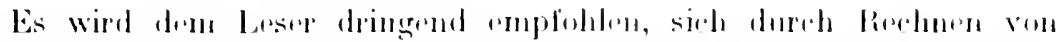
Zahlonbeispielen mit del Multiplikation von systemen ganz vertrant zu marleril.

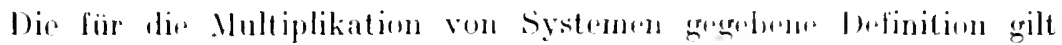
nicht nur für quadratisehe, sondern anch lür rechterkign Systrme, wenn

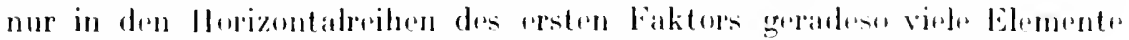
stehen wio in den Vortikalreilen des zwriten. Z. W.

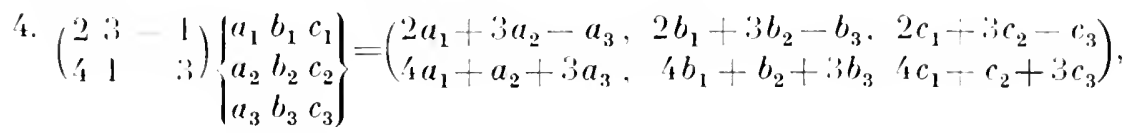

5. $\left(\begin{array}{lll}x & y & z\end{array}\right)\left|\begin{array}{ll}a & a \\ b & \beta \\ c & \gamma\end{array}\right|=(a r+b y+c z, \quad a x+\beta y+z z)$.

6. $\left\{\begin{array}{lll}a_{1} & b_{1} & c_{1} \\ a_{2} & b_{2} & c_{2} \\ a_{3} & b_{3} & c_{3}\end{array}|| \begin{array}{l}x \\ y \\ z\end{array}|=| \begin{array}{l}a_{1} x+b_{1} y+c_{1} z \\ a_{2} x+b_{2} y+c_{2} z \\ a_{3} x+b_{3} y+c_{3} z\end{array} \mid\right.$.

7. $\left(\Xi_{1}, \Xi_{2}, \Xi_{3}\right)\left(\begin{array}{lll}p_{11} & p_{21} & p_{31}\end{array} \mid=\left(p_{11} \Xi_{1}+p_{12} \Xi_{2}+p_{31} \Xi_{3}, \quad p_{21} \Xi_{1}+p_{22} \Xi_{2}+p_{23} \Xi_{3}\right.\right.$. $\left.\left|\begin{array}{lll}p_{12} & p_{22} & p_{32} \\ p_{13} & p_{23} & p_{33}\end{array}\right| \quad p_{31} \Xi_{1}+p_{32} \Xi_{2}+p_{33} \Xi_{3}\right)$

Aus dem letzen beispiel asehen wir: Bezeichnen wir die ans je aner Hori-

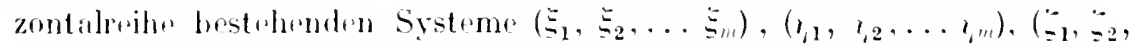

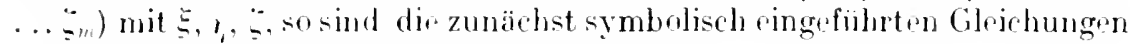
(3). (5), (7) Gluichumen zwischen Systemen.

Wie schon gaxigrt, gilt für die Multiplikation von Systemen nirht

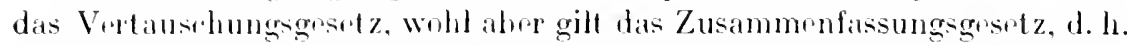
simbl $a$. $b$, e drei systeme, so ist es bei der berechnung von abe gleichgiiltig. oh man urst ab lustimmt mud das Ergobnis hinton mit c multiplizirt. oder ol, man erst be auserehnet und damn vorn mit a mult joliziert.

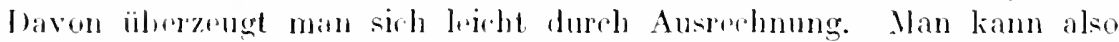

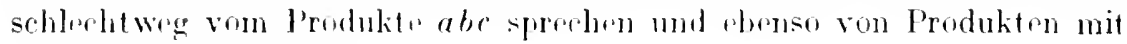
moler als leri liakloren.

Setzen wir das Multiplizieren von Systemen als bekannt voralls, so könnon wir, wir shun bemerkt, dir linearen Substitutionen (1), (4), (b) als Gleichungun zwiwhen Systemen in dor Form (3), (5), (7) schreiben, und esfolgt damm ams (:i) mod (5). und zwar nioht molnr nurformal. $5=\Xi p q$ und also dureh Verereigh mit (7) $r=p q$. 


\section{\$3. Quadratische Systeme.}

Wir betrachten in diesem $\S$, wo nichts anderes gesagt ist, quadratische Systeme von $m^{2}$ Elementen.

1. Die Determinante. Jeles quadratische System $p$ hat eine bestimmte Determinante, nämlich die aus den $m^{2}$ Elementen des Systems bestehende. Sie wird mit $p \mid$ bezeichnet. Wir betrachten im folgenden nur solche quadratische Systeme, deren Determinante nicht identisch Null ist. Vergleicht man den Multiplikationssatz der Determinanten mit der Definition des Produktes von Systemen, so ergibt sich

Satz I. Die Determinante eines Produktes von quadratischen Systemen ist gleich dem Produkt ihrer Determinanten.

2. Adjungierte Systeme. Vertauscht man in einem System $p$ die Horizontal- mit den Vertikalreihen, so heißt das neue System das adjungierte zu $p$ und wird mit $\bar{p}$ bezeichnet. Diese schon in $\S 3$ gegebene Definition ist hier der Vollständigkeit wegen wiederholt. Die Definition gilt nicht nur für quadratische Systeme. Ist z. B. ¿ wieder das nur aus der einen Horizontalreihe $\xi_{1}, \xi_{2}, \ldots \xi_{m}$ bestehende System, so hat das adjungierte System $\bar{\xi}$ nur die eine Vertikalreihe $\xi_{1}, \xi_{2}, \ldots \xi_{m}$. Da eine Determinante ihren Wert nicht ändert, wenn man Reihen mit Spalten vertauseht, so gilt:

Satz II. Ein quadratisches System und sein adjungiertes haben dieselbe Determinante.

Ferner gilt

Satz III. Das adjungierte des adjungierten Systems ist das ursprüngliche System.

Es seien $p$ und $q$ zwei Systeme und es sei

$$
p q=r, \quad \bar{q} \bar{p}=r^{\prime} .
$$

Wir erhalten das Element $r_{3 \alpha}$ von $r$, also das Element, das in der $\alpha$-ten Reihe und in der $\beta$-ten Spalte steht, indem wir die $\alpha$-te Horizontalreihe von $p$ mit der $\beta$-ten Vertikalreihe von $q$ multiplizieren. Wir erhalten ebenso das Element $r^{\prime}{ }_{\alpha 3}$ von $r^{\prime}$, indem wir die $\beta$-te Horizontalreihe von $\bar{q}$ mit der $\boldsymbol{t}_{\text {-ten }}$ Vertikalreihe von $\bar{p}$ multiplizieren. Da aber die Horizontalreihen von $\bar{q}$ die Vertikalreihen von $q$ sind und die Vertikalreihen von $\bar{p}$ die Horizontalreihen von $p$, so folgt $r_{\beta, t}=r^{\prime}{ }_{\alpha x}$. Das heißt aber, es ist $r^{\prime}=\bar{r}$. Aus $p q=r$ folgt also $\bar{q} \bar{p}=\bar{r}$ oder

$$
(p q)=\bar{q} \cdot \bar{p} .
$$

Sind $a, b, c$ drei Systeme, so ergibt sich durch wiederholte Anwendung des Gefundenen

$$
\overline{(a b c)}=\overline{(a b) c}=\bar{c} \cdot a b=\bar{c} \cdot \bar{b} \cdot \bar{a} .
$$

Daher haben wir: 
Satz IV. Das adjungierte System zu einem Produkte ist gleich dem I'rodukte der adjungierten, aber in umgekehrter Reihenfolge.

Ein System, das mit seinem adjungierten üloreinstimmt, heißt s y mmetriseh.

3. Das Einheitssystem. Bei der gewöhnlichen Multiplikation von Zahlen spiclt der Faktor 1 eine besondere Rolle, indom er zu einem Produkte beliehig hinzugrfügt werden kann, ohne den Wert des Produktes zu ändern. Es fragt sich, ob es nicht zwei Systeme $e$ und $e^{\prime}$ so giht, daß für jedes System $p$

$$
p e=p, \quad e^{\prime} p=p .
$$

Nehmen wir an, $e$ und $e^{\prime}$ existieren, dann folgt aus den Gleichungen (10), indem wir in der ersten $p=e^{\prime}$ und in der zweiten $p=e$ setzen,

$$
e^{\prime} e=e^{\prime}=e \text {. }
$$

Die $\alpha$-te Horizontalreihe von $e$ oder $e^{\prime}$ sei $e_{1}, e_{2}, \ldots e_{m}$. Wenn wir diese Reihe der Reihe nach mit den Vertikalreihen von $p$ multiplizieren, erhalten wir die $\alpha$-te Horizontalreihe von $e p$, was gleich $p$ sein soll, so daß sich die $a$-te Horizontalreihe von $p$ ergeben muß, gleichgültig wie $p$ beschaffen ist. Daraus folgt aber, $\mathrm{da} B e_{\iota}=1$ sein $\mathrm{mu} B$, während die anderen $e_{i}=0$ sind. In $e$ muB also in der $\alpha$-ten Horizontalroihe an $\alpha$-ter Stelle rine 1 stehen und an den anderen Stellen 0. Oder: Wenn ein System $e$ der verlangten Art existiert, so stehen in seiner Hauptdiagonale Einsen und an allen anderen Stellen Nullen. Wie man aber leicht sieht, hat dies System die gewünschte Eigensehaft. Es heißt das Einheitssystem und wird mit 1 bezeichnet. Es ist also für jedes System $p$

$$
p \cdot 1=1 \cdot p=p \text {. }
$$

Die Determinante des Einheitssystems ist offenbar gleich 1.

4. Reziproke Systeme. Wir fragen uns jetzt, ol es zu einem (quadratischen) System $p$ Systeme $p^{\prime}$ und $p^{\prime \prime}$ gibt, so daß

$$
p p^{\prime}=1, p^{\prime \prime} p=1 \text {. }
$$

Multiplizieren wir die zweite dieser Gleichungen hinten mit $p^{\prime}$, so erhalten wir unter Beachtung der ersten

$$
p^{\prime \prime} p p^{\prime}=p^{\prime \prime}\left(p p^{\prime}\right)=p^{\prime \prime}=p^{\prime} .
$$

Ist also $p p^{\prime}=1$, so ist auch $p^{\prime} p \doteq 1$. Weiter sehen wir, da $B$ es höchstens ein System $p^{\prime}$ der verlangten Art geben kann. Ist nämlich $p p_{1}^{\prime}=1$ und auch $p p_{2}^{\prime}=1$, also auch nach dem eben bewiesenen $p_{1}^{\prime} p=p_{:}^{\prime} p=1$, so folgt aus $p p_{1}^{\prime}=1$ durch vordere Multiplikation mit $p_{.2}^{\prime}$

$$
\left(p_{2}^{\prime} p\right) p_{1}^{\prime}=p_{2}^{\prime},
$$

also wegen $p_{2}^{\prime} p=1, p_{1}^{\prime}=p_{2}^{\prime}$. 
lats systrm p' heißt, wonn es existiert, das zu p reziproke System nud wired mit $p^{-1}$ bezelunet. Es ist

llieraus eremilht sich:

$$
p p^{1}=p^{1} p=1
$$

Satz V. las reziprolir systrm zu dem reziproken eines Sy-tems ist das urspoünglieho system.

Grhen wir in (1:2) zu den l)eterminanten über, so folgt

$$
p \quad p^{-1}=1 \text {. }
$$

also:

Satz VI. Din loterminanten reziproker Systeme sind proiproke Zahlen. l)araus sehließen wir, daß zu einem System $p$ das roziproke nur vortanten sein kann, wenn seine beterminante von Null verschieden ist. Wir setzen also voraus, daB

$$
l \neq 0 \text {. }
$$

lit aher diese Bedingung erfüllt, so existiert das reziproke System, und wir können es nach bekannten Determinantensätzen leicht angeben. Es $\therefore$ i $P_{1,1}$ die Unterdeterminante des Elementes $p_{h i}$ der Determinante $\mid p^{\prime}$. las quadratische System der $P_{l i}$ sei mit $P$ bezeichnet und es sei $p_{0}$ das Sistem von $m^{2}$ Elementen. in dessen Haupteliagonale überall die Determinante $y$ stoht, währund alle anderen Elemente Null sind. Dann können wir die Gleichungen, die zwischen den Elementen einer Determinante und ihren Unterdeterminanten bestehen, in der Form schreitien

l)araus folgt damn:

$$
\nu^{\prime} \bar{P}=p_{0}, \quad \bar{P} p=p_{0} .
$$

Satz VII. Jividiort mall in dem System $P$ der Unterdetorminatuten prster Ordung des Systems p alle Elemente durrh die leterminante $p \neq 0$ und vertauscht dann die Hurizontal-mit don Vertikalreihen, so ist das so orhaltene syotrin das roziperoke $p^{-1}$ ron $p$, d. h. es ist

$$
p p^{1}=p^{-1} p=1
$$

und dies Syotum ist das rinzige. das diese Eigensoluaft hat.

Sotzill wil $a d-b c=0$, so ist z. B.

$$
\left(\begin{array}{ll}
a & b \\
c & d
\end{array}\right)^{-1}=\left\{\begin{array}{rr}
d & b \\
d & \delta \\
c & a \\
-d & \delta
\end{array}\right\} .
$$

Sind $\%$, q. $r$ rlei Systeme mit von Null verschiedener Determinante, s" ist wegen des Znsammenfassungsgesetzos

$(p q r)\left(r^{-1} q^{-1} p^{-1}\right)=p q\left(r r^{-1}\right) q^{-1} p^{-1}=p\left(q q^{-1}\right) p^{-1}=p p^{-1}=1$. 
Es ist also $\quad(p q r)^{-1}=r^{-1} q^{-1} p^{-1}$

oder in Worten:

Satz VIII. llas rozproko Systrm rines looduktes ist gleieh dren lerodukt dor reziproken Systeme, abor in umgekehrter lirilienfolge.

5. Komplemontäre Systemo. Es sei wioder p rin System, dessen beterminante von Null versehieden ist, und es sei $p$ 'das adjumgierte seinus reziprokers, alsu

$$
p^{\prime}=\left(p^{-1}\right)
$$

Gehen wir in der Gleichnng $\rho p^{-1}=1$ links und rechts zu dru adjungierten Systrmon äber, so folgt, da das Einheitssystem dabni ungeändert lieilt, $p^{\prime} \bar{p}=1$, d. h. aber, es sind $p^{\prime}$ und $\bar{p}$ reziproke Systeme. Oder:

Satz IX. Das reziproke zum adjungierten eines Systems ist gleich dem adjungierten seines reziproken.

Dies System heißt das komplementäre System zu dem ursprünglichen. Da das adjungierte System $\bar{p}^{\prime}$ von $p^{\prime}$ gleich $p^{-1}$ ist und das reziprukt hiervon $p$, so folgt

Satz X. Ist ein System zu einem anderen komplementär, so ist auch das andere zum ersten komplementär.

Schließlich folgt noch aus den entsprechenden Sätzen für adjungirrt. und reziproke Systeme

Satz Xl. I)as komplementäre System zu rinem Produkt von Systemen ist gloich dem Produkt dor komplomentärrn System"in dorselben Rrihenfolge.

\section{$\$ 4$. Einige besondere Systeme.}

1. Multiplikationssysteme.

Es sei $M$ ein quadratisches Systיm, das auBerhalb der Hauptdiagonale nur Nullen hat. Die Elemente der Hauptdiagonale seien $M_{1}, M_{2}, \ldots M_{m}$. Ein solches System heißt Multiplikationssystem. Seine Determinante ist $M_{1} M_{2} \ldots M_{m}$.

Diese Bezeichnung wird sofort klar, wenn wir die Wirknng hetrachten, die die Multiplikation mit einem derartigen System hat. Es genügt, den Fall $m=-3$ zu nehmen. Es wird

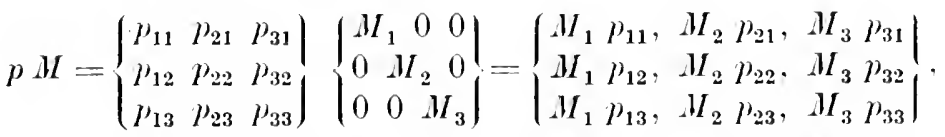

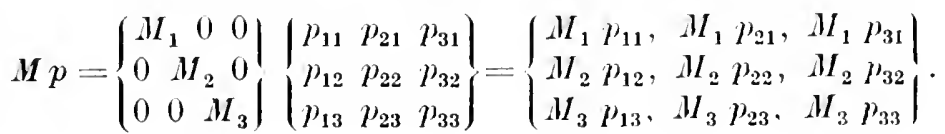


Also:

Satz Xll. Wird ein System mit einem Multiplikationssystem, dessen Diagonale $\boldsymbol{M}_{1}, \boldsymbol{M}_{2}, \ldots \boldsymbol{M}_{m}$ ist, hinten multipliziert, so werden seine Vertikalreihen, wird es vorne multipliziert, so werden seine Horizontalreihen der Reihe nach mit $M_{1}, M_{2}, \ldots M_{m}$ multipliziert.

has System, das multipliziert wird, braucht nicht quadratisch zu sein. Die Richtigkeit folgender beider Sätze erkennt man sofort:

Satz XII. Multiplikationssysteme sind vertauschbar.

Satz XIV. Das reziproke eines Multiplikationssystems ist das Multiplikationssystem, dessen Diagonalglieder die reziproken Werte derjenigen des ursprünglichen Systems sind.

2. Additionssysteme.

Es sei $A_{\alpha}$ das System, das aus dem Einheitssystem dadurch hervorgeht, daß die $\boldsymbol{c}$-te Vertikalreihe ersetzt wird durch

$$
i_{1}, i_{2}, \ldots \lambda_{u-1}, 1, \lambda_{\alpha+1}, \ldots \lambda_{m} \text {. }
$$

Ein solches System heißt Additionssystem. Seine Determinante ist 1. Es wird etwa für $m=4, \iota=3$

$$
\begin{aligned}
& p A=\left\{\begin{array}{llll}
p_{11} & p_{21} & p_{31} & p_{41} \\
p_{12} & p_{22} & p_{32} & p_{42} \\
p_{13} & p_{23} & p_{33} & p_{43} \\
p_{14} & p_{24} & p_{34} & p_{44}
\end{array}\right\}\left\{\begin{array}{llll}
1 & 0 & \lambda_{1} & 0 \\
0 & 1 & \lambda_{22} & 0 \\
0 & 0 & 1 & 0 \\
0 & 0 & \lambda_{4} & 1
\end{array}\right\}=
\end{aligned}
$$

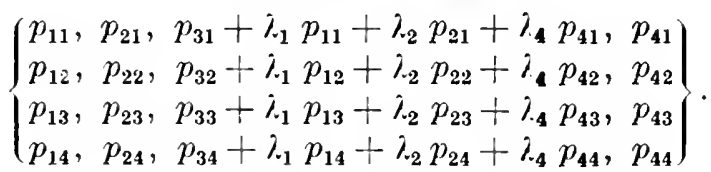

Also:

Satz XV. Wird ein System mit dem Additionssystem $\boldsymbol{A}_{\alpha}$ hinten multipliziert, so wird zu seiner a-ten Vertikalreihe das $\lambda_{i}$-fache der $i$-ten Vertikalreihe addiert $(i \neq \alpha)$.

bas inultiplizierte System braucht nicht quadratisch zu sein.

3. Vertauschungssysteme.

Es sei $V_{a,}$ das System, das aus dem Einheitssystem durch Vertauschen der $\alpha$-ten mit der $\beta$-ten Horizontalreihe hervorgeht oder, was auf dassell,e hirauskommt, durch Vertauschen der $\alpha$-ten und $\beta$-ten Vertikalreihe. Wir nennen ein solches System ein Vertauschungssystem. Seine Determinante ist -1 . 
$\S 5$. Quadratische Systeme, deren Elemente Funktionen einer Veränderlichen $x$ sind. 93

Wir nehmen als Beispiel $m=4, a=1, \beta=3$. Es wird

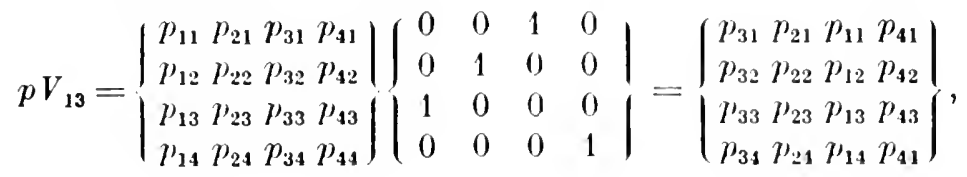

$$
\begin{aligned}
& V_{13} p=\left\{\begin{array}{llll}
0 & 0 & 1 & 0 \\
0 & 1 & 0 & 0 \\
1 & 0 & 0 & 0 \\
0 & 0 & 0 & 1
\end{array}\right\}\left\{\begin{array}{llll}
p_{11} & p_{21} & p_{31} & p_{41} \\
p_{12} & p_{22} & p_{32} & p_{42} \\
p_{13} & p_{23} & p_{33} & p_{43} \\
p_{14} & p_{24} & p_{34} & p_{44}
\end{array}\right\}=\left\{\begin{array}{llll}
p_{13} & p_{23} & p_{33} & p_{13} \\
p_{12} & p_{22} & p_{32} & p_{12} \\
p_{11} & p_{21} & p_{31} & p_{41} \\
p_{14} & p_{24} & p_{31} & p_{44}
\end{array}\right\} .
\end{aligned}
$$

Also :

Satz XVI. Durch hintere Multiplikation mit dem Vertauschungssystem $V_{\alpha \beta}$ werden die $\alpha$-te und $\beta$-te Vertikalreihe und durch vordere die $\alpha$-te und $\beta$-te Horizontalreihe vertauscht.

Auch hier braucht das multiplizierte System nicht quadratisch zu sein.

\section{$\$ 5$. Quadratische Systeme, deren Elemente Funktionen einer Veründerlichen $x$ sind.}

1. Definitionen.

Def. III. Sind die Elemente eines quadratischen Systems ganze rationale Funktionen von $x$ und ist seine Determinante von $x$ unabhängig und von Null verschieden, so nennen wir das System cine Einheit.

Def. IV. Sind die Elemente eines quadratischen Systems rationale Funktionen von $x$, die für $x=a$ nicht unendlich werden, oder gewöhliche Potenzreihen von $x-a$, und ist die Determinante des Systems eine Einheit für $x=a$, so nennen wir das System eine Einheit für die Stelle $x=a$.

2. Sätze.

Satz XVI. Produkte von Einheiten sind wieder Einheiten. Adjungierte, reziproke und komplementäro Systeme von Einheiten sind auch Einheiten. Das gilt auch von Einheiten für eine Stelle $x=a$.

Diese Sätze ergeben sich aus den für dic Einheiten gegebenen Definitionen in Verbindung mit den Regeln für die Multiplikation von Systemen.

Satz XVIH. Es bedeute $g$ das System, das aus der einen Horizontalreihe

$$
\left(g_{1}, g_{2}, \ldots g_{m}\right)
$$

besteht, wo die $g_{i}$ rationale Funktionen von $x$ sein sollen; 
es soi formor das System $p$ rime Einheit für $x=a$. Setzen wil

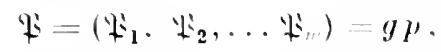

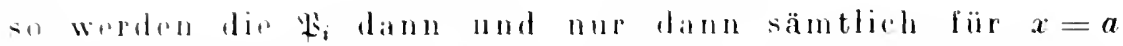

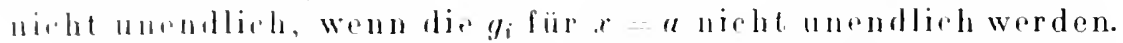

Zunärlsst orgibt sich sofort ans den Voraussotzungen, daß die $\mathfrak{F}_{i}$ simtlieh fïr $x=a$ nivht unendlich werden, wem die $g_{3}$ fïr die Umgebung vont $x=a$ ganz sind. Sind unigekwht allo 状 ganz fïr $x=a$, so folgern wir atus (15)

$$
y=\mathbb{\&} y^{-1} \text {. }
$$

1) ab aber $p^{-1}$ gledohzoitigr nit $p$ eine Einheit fïr $r=a$ ist, so folgt, dab die $g$ fïr $x=a$ nicht unendlich werden kömmen, wenn die i $_{i}$ es nicht werden.

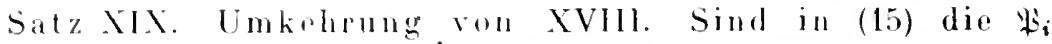
dann und nur dann ganz für $x=a$, wenn die $g_{i}$ rs sind, und ist $p$ nicht idnutish Null, so ist $p$ oine Einheit für $x=a$.

Wählen wie in (15) $g_{1}=1$ und diw anderen $g_{i}=0$, so wird

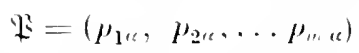

und daher minsen dir pir nach Voraussotzung ganz für $x=a$ sein. Also sind alle Elementr von $p$ nicht unendlich für $x=a$ und dasselbe gilt daher von der Determinante $|p|$. Bezeichnen wir das zu $p$ reziproke System mit $q$, so folgt aus (15)

$$
g=\mathfrak{P} q \text {. }
$$

Wählen wir hierin $\mathfrak{W}_{a}=1$ und allo anderen $\mathfrak{F}_{i}=0$, so wird

$$
g=\left(q_{1 *}, q_{2 \ll}, \ldots q_{m+\varepsilon}\right)
$$

und es sind daher narh Voraussetzung die $q_{2,}$ nicht unendlich für $x=a$. Es sind also auch die Elemente von $q$ ganz für $x=a$ und daher auch die Determinante $q$. Aber da $p$ und $q$ reziprok sind, so ist $p|\cdot| q \mid=1$ und es müssen daher $p$ und $q$ Einheiten für $x=a$ sein. Damit ist aber Satz XlX bewiesen.

Satz XX. Es surn in dem quadratischen system $p$ die Elrmente Potenzreilien von $x-a$, dio in ondlicher Zahl negative Potenzen enthaltull diirfon. Гjir Determinante $\mid p$ sei nicht iduntisch Null. Jann gibt $\ldots$ pin System $S$ folgender Art.

a) Die Elemente von $S$ sind rationale Funktionen von $x$.

b) Es ist $S$ eine Einheit für all endlichen Werte von $x$, hörhstens mit Ausnahmo von $x=a$.

r) Es ist das System

rine Einheit für $x=a$.

$$
q=p s
$$




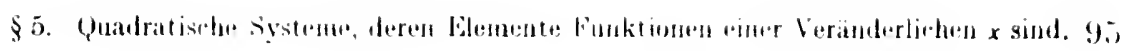

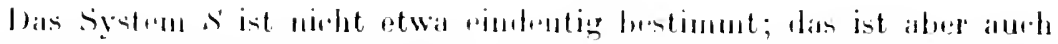

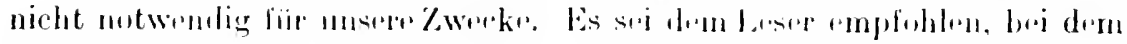

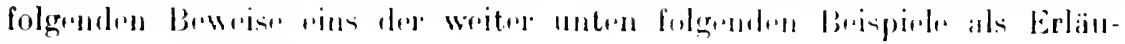

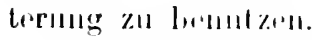

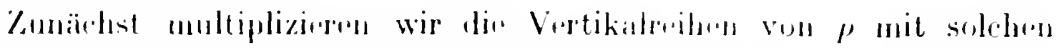

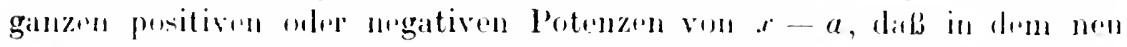

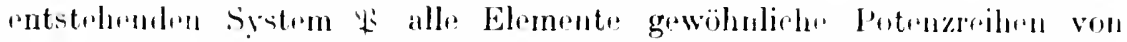
$x$ - a simd und laß in jeder Vertikalroihe mindestens ain Element strht, las für $r$ a ni.ht Null wird. Um das anszuführen, habun wir $p$ mit rinem Multiplikationssystem $S_{1}$ hinten zu nultiplizieren, in deson I)iagonale ganzaahlige Potenzen von $x-a$ stehen, so daß

$$
\mathbb{E}=\mu i_{1} \text {. }
$$

Dir beterminante von $S_{1}$ ist eine ganzzahlige Potenz von $a-a$ und a ist $s_{1}$ rin Einheit für alle endlichen Stellen $x$ mit höchstens der Ausnahmı $x=a$. Wom die Determinante $\$$ des Systems \& für $x=a$ von

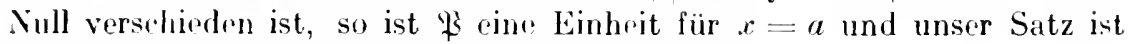
hewiesen. Es ist dann $S=S_{1}$ und $q=\$ 2$.

Es sei jetzl $\mathbb{b}=0$ für $x=a$, es sei also

$$
|\mathfrak{P}|=(x-a)^{\prime \prime} E^{\prime} \text {. }
$$

wo $">0$ und $E$ cine Einheit für $x=a$ ist. Wir bezcichnen den Wert. den das Element $\mathfrak{B}_{k l}$ von $\mathfrak{P}$ für $x=a$ annimmt, mit $r_{k l}$. Nach unserre Annahme ist die Determinante der $r_{k l}$ gleich Nill. Es gilst daher Konstanten, $\mu_{1}, \mu_{2}, \ldots \mu_{m}$, dir nicht sämtlich versohwinden, so daß die G](.ichungen bestohrin

$$
\mu_{1} r_{1 \ell}+\mu_{2} r_{2,}+\cdots+\mu_{m} r_{m u}=0, \quad(\alpha=1,2, \ldots m) \text {. }
$$

Ls soi z. B. " 1 von Null verschirden. Wir rlividieren rann mit " 1 und setzon $\mu_{i} \mu_{1}=\lambda_{i}$, so daB

$$
r_{1, t}+\lambda_{2} r_{2 u}+\cdots+\lambda_{m} r_{m u}=0 . \quad(\alpha=1,2, \ldots m) .
$$

Addieren wir daher in $\$$ das $\lambda_{2}$-fache der zweiten Vertikalreihe, das $i_{3}$-fach. der drittיn usw. zur ersten Vertikalreihe, so entsteht ein neurs System İ”. $^{\prime}$ in d'm dì Elemente der ersten Vertikalreihe sämtlich für $x=a$ vorschwinden, also mindestens durch die erste potenz von $x-a$ teilbar

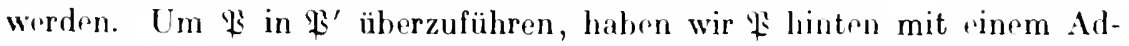
ditionssystem zu multiplizieren. Dies Systrm soi mit $S_{2}$ bezoichnet. Es geht aus dem Einheitssystem hervor, indem man dir Nullen von dessen erster Vertikalroihe durch $\lambda_{2}, \lambda_{3}, \ldots \lambda_{m}$ ersetzt. Es wird

$$
\mathfrak{P}^{\prime}=\mathfrak{P}^{\prime} S_{2} \text {. }
$$

Da $S_{2}=1$ und da die Elemente von $S_{2}$ konstant sind, so ist $S_{2}$ ein. Einheit. 
Wir dividieren die erste Vertikalreihe von l' mit $x-a$ oder, wenn möglich. durch eine höhere Potenz von $x-a$. Dazu müssen wir $\mathfrak{B}^{\prime}$ mit einem Multiplikationssystem $S_{3}$ hinten multiplizirren, das dadurch entsteht, daß in dem Einheitssystem die erste I durch eine passende negative ganzzahlige Potenz von $x-a$ ersetzt wird. Es ist $S_{3}$ eine Einheit für alle endlichen Stellen mit Ausnahme von $x=a$. Das neue System ist unter Benutzung von (16) und (18)

$$
\mathfrak{S}_{1}=\mathfrak{P}^{\prime} S_{3}=\mathfrak{L} S_{2} S_{3}=p S_{1} S_{2} S_{3} \text {. }
$$

Das System $F_{1}$ hat als Elemente geradeso wie \& gewöhnliche Potenzreihen von $x-a$. Seine Determinante ist nach (19)

$$
\left|\mathfrak{F}_{1}=\mathfrak{R}\right| S_{2}\left|S_{3}\right| \text {. }
$$

Da aber $S_{2} \mid=1$ und $S_{3}$ eine negative ganzzahlige Potenz von $x-a$ ist, so ergibt sich aus (20) und (17)

wo

$$
\mathfrak{F}_{1} \mid=(x-a)^{n_{1}} E \text {, }
$$

$$
0 \leq \varrho_{1}<\varrho .
$$

Ist $\varrho_{1}=0$, so ist $\mathfrak{F}_{1}$ eine Einheit für die Stelle $a$ und unser Satz ist bewiesen. Es wird $S=S_{1} S_{2} S_{3}$ und $q=\mathfrak{F}_{1}$.

Ist $\varrho_{1}$ noch größer als Null, so fahren wir in derselben Weise fort. Wie wir aus $\mathfrak{B}$ das System $\mathfrak{F}_{1}$ abgeleitet haben, können wir aus $\mathfrak{B}_{1}$ ein System $\mathfrak{F}_{2}$ herleiten, dessen Elemente auch gewöhnliche Potenzreihen von $x-a$ sind, dessen Determinante aber durch eine niedrigere Potenz von $x-a$ teilbar ist als $\mathfrak{F}_{1}$. So müssen wir schließlich zu einem System $b$ kommen, das eine Einheit für die Stelle $x=a$ ist, und das aus $p$ durch Multiplikation mit Systemen $S_{1}, S_{2}, S_{3}, \ldots S_{r}$ hervorgeht, deren Elemente konstant oder ganzzahlige Potenzen von $x-a$ sind und deren Determinanten gleich 1 oder gleich einer ganzzahligen Potenz von $x-a$ sind. Es wird

$$
q=p S_{1} S_{2} \ldots S_{r}=p S .
$$

Da aber die Systeme $S_{i}$ Einheiten für jede endliche Stelle mit Ausnahme von $x=a$ sind, so gilt dasselbe für ihr Produkt $S$. Damit ist unser Satz bewiesen.

Beispiel I. $a=0$.

$$
p=\left\{\begin{array}{c}
x^{2}, \quad x^{2}-\frac{1}{2} x^{4}+\cdots \\
x^{-1},-x^{-1}+\frac{1}{2} x+\frac{1}{8} x^{3}+\cdots
\end{array}\right\} .
$$

1. Multiplikation der beiden Vertikalreihen mit $x$. Substitution

$$
\left(\begin{array}{ll}
x & 0 \\
0 & x
\end{array}\right)=S_{1} \text {. }
$$


Das neur System ist

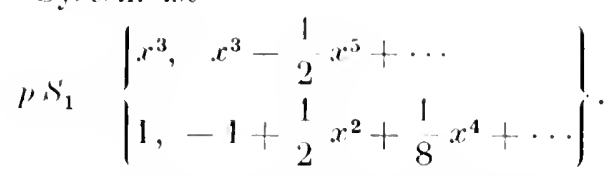

Dir lonterminante dieses Systems ist gleich Null fuir $x=a=0$.

2. Addieren der ersten Vertikalreihe zur zweiten. Substitution

$$
S_{2}=\left(\begin{array}{ll}
1 & 1 \\
0 & 1
\end{array}\right) \text {. }
$$

Das neue System ist

$$
p S_{1} S_{2}=\left\{\begin{array}{ll}
x^{3}, & 2 x^{3}-\frac{1}{2} x^{5}+\cdots \\
1, & 1 \\
1, & 2 \\
2 & 1 \\
8 & x^{4}+\cdots
\end{array}\right\} .
$$

Die im ganzen bisher ausgeïbte Sulstitution ist $S_{1} S_{2}$.

3. Multiplizieren der zweiten Vertikalreihe mit $x^{-2}$. Substitution

$$
s_{3}=\left\{\begin{array}{ll}
1 & 0 \\
0 & x^{-2}
\end{array}\right\}, \quad p S_{1} S_{2} S_{3}=\left\{\begin{array}{ll}
x^{3}, & 2 x-1 \\
1, & 1 \\
2 & +\frac{1}{8} x^{2}+\cdots
\end{array}\right\} .
$$

Die Determinante dieses Systems wird für $x=0$ noch zu Null. Die bisher im ganzen ausgeübte Substitution ist $S_{1} S_{2} S_{3}$.

4. Addieren des $\left\{\begin{array}{l}1 \\ 2\end{array}\right\}$-farhen der ersten Vertikalreihe zur zweiten. Substitution

$$
S_{\mathbf{4}}=\left\{\begin{array}{ll}
1- & 1 \\
1 & 2 \\
0 & 1
\end{array}\right\}, \quad p S_{1} S_{2} S_{3} S_{4}=\left\{\begin{array}{ll}
x^{3}, & 2 x-x^{3}+\cdots \\
1, & 1 \\
1, x^{2}+\cdots
\end{array} \mid .\right.
$$

5. Division der zweiten Vertikalreihe mit $x$.

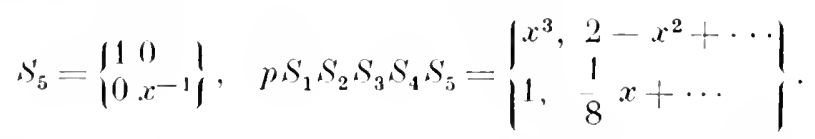

Die Determinante dieses Systems ist von Null versehieden für $x=0$, so da $B$ wir an Zinl sind. Es ist das System

$$
p S_{1} S_{2} S_{3} S_{4} S_{5}=q
$$

von der gewïnschten Beschaffenheit. Das gesuchte System ist

Es wird

$$
S=S_{1} S_{2} S_{3} S_{4} S_{5} .
$$

$$
S_{1} S_{2}=\left\{\begin{array}{ll}
x & x
\end{array} \mid, \quad S_{1} S_{2} S_{3}=\left\{\begin{array}{ll}
x & x^{-1} \\
0 & x
\end{array}\right\}, \quad x^{-1}\right\}, S_{2} S_{3} S_{4}=\left\{\begin{array}{l}
x, x^{-1}-1 \\
0, x^{-1}
\end{array}\right\},
$$


Beispiel 11. $a=1$.

$$
s=s_{1} s_{2} s_{3} s_{4} s_{5}=\left\{\begin{array}{l}
x, x^{-2}-1 \\
0, x^{2}
\end{array} \mid, \quad S=x^{-1} .\right.
$$

$$
p=\left\{\begin{array}{ll}
0 . & (x-1)-7 \\
1+(x-1) & 1 \\
2-2(x-1)^{2}+\cdots
\end{array}\right\} .
$$

1. Addieren des $\left\{\begin{array}{c}1 \\ 2\end{array}\right\}$-fachen der ersten Vertikalreihe zur zweiten.

$$
S_{1}=\left\{\begin{array}{ll}
1-\frac{1}{2} \\
0 & \frac{2}{1}
\end{array} \mid, p S_{1}=\left\{\begin{array}{ll}
0, & (x-1)-\frac{7}{4}(x-1)^{2}+\cdots \\
1+(x-1),-\frac{5}{2}(x-1)+\cdots
\end{array}\right\} .\right.
$$

2. Dividieren der zweiten Vertikalreihe mit $(x-1)$.

$$
\left.S_{2}=\begin{array}{ll}
10 & 0 \\
0(x-1)^{-1}
\end{array}\right\}, p S_{1} S_{2}=\left\{\begin{array}{ll}
0, & 1+\cdots \\
1+(x-1),-\frac{5}{2}+\cdots
\end{array}\right\} \text {. }
$$

Die Determinante dieses Systems ist für $x=1$ nicht Null. Es ist $S=S_{1} S_{2}$, also

$$
S=\left\{\begin{array}{ll}
1,-\frac{1}{2} & (x-1)^{-1} \\
0, & (x-1)^{-1}
\end{array}\right\}, \mid S=(x-1)^{-1} .
$$

Beispiel III. $a=0$. Wir setzen $\varepsilon=\frac{-1+i \mid 3}{2}$, so $\mathrm{da} \beta \varepsilon^{3}=1$, $\varepsilon^{2}+\varepsilon+1=0$.

$$
\begin{aligned}
& p=\left\{\begin{array}{l}
x^{-1}, x^{-1}-\frac{1}{3} x^{5}+\cdots, \quad x^{-1}+1+x+\cdots \\
1, \quad \varepsilon-\frac{\varepsilon}{3} x^{6}+\cdots, \quad \varepsilon^{2}+\varepsilon^{2} x+\varepsilon^{2} x^{2}+\cdots \\
x^{-2}, \varepsilon^{2} x^{-2}-\frac{\varepsilon^{2}}{3} x^{4}+\cdots, \varepsilon x^{2}+\varepsilon x x^{1}+\varepsilon+\cdots
\end{array}\right\} . \\
& S_{1}=\left\{\begin{array}{ccc}
x^{2} & 0 & 0 \\
0 & x^{2} & 0 \\
0 & 0 & x^{2}
\end{array} \mid, \quad p S_{1}=\left\{\begin{array}{lll}
x, & x+(7), & x+x^{2}+(3) \\
x^{2}, & \varepsilon x^{2}+(8), & \varepsilon^{2} x^{2}+(3) \\
1, & \varepsilon^{2}+(6), & \varepsilon+\varepsilon x+(2)
\end{array}\right\},\right.
\end{aligned}
$$

wo mit $(\alpha)$ eine Summe von Gliedern bezeichnet ist, die mindestens die a-te Potenz von $x$ enthalten.

$$
S_{2}=\left|\begin{array}{ccc}
1-\varepsilon^{2}-\varepsilon \\
0 & 1 & 0 \\
0 & 0 & 1
\end{array}\right|, p S_{1} S_{2}=\left\{\begin{array}{l}
x,\left(1-\varepsilon^{2}\right) x+(7),(1-\varepsilon) x+x^{2}+(3) \\
x^{2},\left(\varepsilon-\varepsilon^{2}\right) x^{2}+(8),\left(\varepsilon^{2}-\varepsilon\right) x^{2}+(3) \\
1,(6),
\end{array} \mid\right.
$$




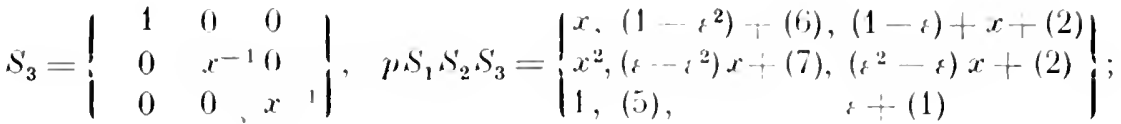

$$
\begin{aligned}
& S_{4}=\left|\begin{array}{lll}
1 & 0 & 8 \\
0 & 1 & 8 \\
0 & 0 & 1
\end{array}\right| \cdot p S_{1} S_{2} S_{3} S_{4}=\left|\begin{array}{l}
x,\left(1-8^{2}\right)+(6),(1-8) x+(2) \\
x^{2},\left(\varepsilon-8^{2}\right) x+(7), 3 \varepsilon^{2} x+(2) \\
1,(5),
\end{array}\right|: \\
& S_{5}=\left\{\begin{array}{lll}
1 & 0 & 0 \\
0 & 1 & 0 \\
0 & 0 & x^{-1}
\end{array}\left|, \mu_{1} S_{2} S_{3} S_{4} S_{5}=\right| \begin{array}{c}
(1),\left(1-\varepsilon^{2}\right)+(t),(1-\varepsilon)+(1) \\
(2),\left(\varepsilon-\varepsilon^{2}\right) x+(7), 3 \varepsilon^{2}+(1) \\
1,(5)
\end{array} \mid .\right.
\end{aligned}
$$

Für $x=0$ wird jedes $(x)=0$, wenn $x>0$. Also ist die Determinante des zuletzt gefundenen Systems

$$
\left|\begin{array}{lll}
0 & 1-\varepsilon^{2} & 1-\varepsilon \\
0 & 0 & 3 \varepsilon^{2} \\
1 & 0 & (0)
\end{array}\right|=3 \varepsilon^{2}\left(1-\varepsilon^{2}\right) \neq 0
$$

wo (0) irgendeine Konstante ist, auf deren Wert es nicht ankommt. Es ist

$$
\left.S=S_{1} S_{2} S_{3} S_{4} S_{5}=\mid \begin{array}{ccc}
x^{2}, & -\varepsilon^{2} x^{2}, \varepsilon^{2}-\varepsilon & x \\
0, & x, & ; \\
0, & 0, & 1
\end{array}\right\}, \quad S=x^{3}
$$

Beispiel IV. $a=0$.

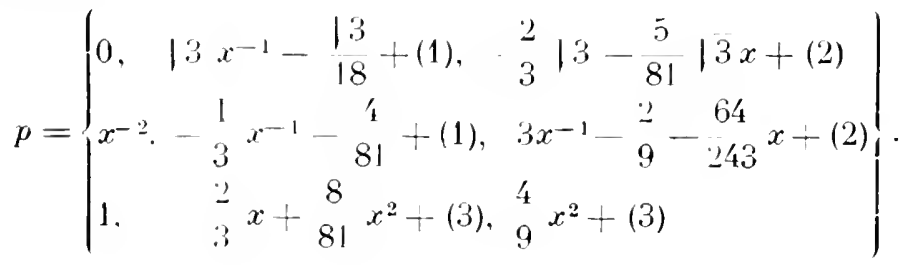

$S_{1}=\left\{\begin{array}{lll}x^{2} & 0 & 0 \\ 0 & x & 0 \\ 0 & 0 & x\end{array}\right\}, \quad S_{2}=\left\{\begin{array}{lll}1 & 0 & -3 \\ 0 & 1 & 0 \\ 0 & 0 & 1\end{array}\right\}, \quad S_{3}=\left\{\begin{array}{lll}1 & 0 & 0 \\ 0 & 1 & 0 \\ 0 & 0 & x^{-1}\end{array}\right\}, \quad S_{4}=\left|\begin{array}{lll}1 & \frac{2}{3} & 0 \\ 0 & 1 & 0 \\ 0 & 3 & 1 \\ 2 & 1\end{array}\right|$,

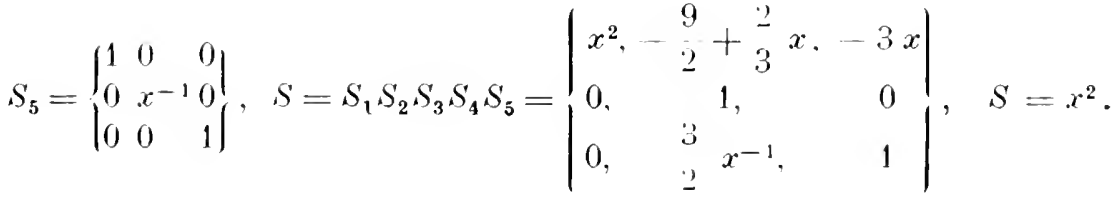




\section{Achtes Kapitel. \\ Die Vielfachen eines Divisors.}

\section{\$ 1. Die Iufuabe.}

In dirsm Kapitel werden wir uns mit der Aufgabe beschäftigen, Funktionen des Körpers mit gegebuen Null- und Unendlichkeitsstellen herznstıllen. Wir wählen eine Größe des Körper's, etwa $x$ als unabhängige Veränderliche und lassen zunächst die Stellen, für die $x$ unendlich groß ist, unberïcksichtigt. Wenn wir von einer funktion verlangen, sie soll an riner Stelle von der et-ten Ordnung Null werden, so soll das bedeuten, sie soll mindestens ron der Ordnung a Null werden. Wenn wir verlangen, ein" Funtion soll an einer Stelle von der Ordnung a unendlich werden, so sell das heißen, sje soll höchstens von der Ordnung \& unendlich werden.

Wir können der zu behandelnden Aufgabe auch eine andere Form geben. Wir bezeichnen die Funktionen, di nur für unendlich große Werte von $x$ unendlich werden. als ganze Funtionen, eine Bezcichnung, die solbstrerständlich wrsentlich von der Wahl der Größe $x$ abhängt, so daß wir, wrenn nötig. die Funktionen ganz in bezug auf $x$ nennen. Wir sagen ferner, ein Ilivisor a ist ganz in bezug auf $x$, wenn er im Nenner nur Primteiler enthält, die in $\pi_{x}$, dem Nenner von $x$, vorkommen. Er läßt sich dann. indem man mit einem passenden Divisor erweitert, darstellen in der Form

$$
\mathfrak{q}=\frac{\mathfrak{a}}{1 \mathrm{t}_{r}^{\prime}},
$$

wo n nin (absolut) ganzer Divisor ist. Fs ist klar, daß umgekehrt jeder Divisor von der Form (1), wo g] ein ganzer Divisor ist, ganz in bezug auf $x$ ist. Wir wollen forner einr Funktion $R$ des Körpers teilbar durch einen Divisor a oder "in Virlfaches des Inivisors a nennen, weun $\frac{R}{q}$ ein ganzer Divisor in bezug auf $x$ ist. Eigentlich müBten wir sagen: teilbar in bezug auf $x$, aber der Kürz wegn wollen wir winfach teillar sagen. Wir kömnen dir Aufgahe die uns in dirsem und den folgenden Paragraphen beschäftigen soll, so formulieren: Es sollen die Vielfachen eines gegoluenrn Divisors a bestimmt werden.

\section{s... Ein Beispiel.}

Vir betrachten zurrst ein cinfaches Beispiel. Der Körper sei definiert dureh die Gleichung

$$
y^{2}+x^{2}-1=0 .
$$


Die uber der $x$-Ebent ausgebreitete Kiemannsche Flache ist zweiblättrig. Sie hat Verewrigungspunkte erster (ordmung in $x=1$ und $x=-1$. Der zu dem ersten disser Punkte gehörendr l'rimtrilne sti mit b bezrichnet. Bei $x=0$ sind diw beiden Blätter gotronnt. Im einen ist $y=+1$, im anderen $y=-1$. Wie zu diesen Stellen gehörenden l'rimtriler bezoichnen wir mit $H_{1}$ und $w_{2}$. Wir wählon dann als Jivisor a den hivisor

$$
q=\frac{H_{2}}{4 H_{1}^{2}}
$$

und stellon uns die Aufgabe, die Virlfachen dieses Divisors zu bestımmen.

Wir können jede Funktion des Körpers nach Satz IX in Kap. IV, $\$ 7$ in der Form schreiben

$$
R=g_{1}+g_{2} y
$$

wo $g_{1}$ und $g_{2}$ rationale Funktionen von $x$ sind. Man nennt zwei solche Funktionen $z_{1}, z_{2}$ des Körpers von der Art, dab sich jede Funktion des Körpers in der Form

$$
g_{1} z_{1}+g_{2} z_{2}
$$

darstellen läßt, wo $g_{1}$ und $g_{2}$ rationale Funktionen von $x$ allein sind, ein Fundamentalsystem oder eine Basis. Es sind alsoz. B. die Funktionen 1 und $y$ ein solches Fundamentalsystem.

Wir haben $g_{1}$ und $g_{2}$ so zu bestimmen, daß die Funktion $R$ ein Vielfaches von q wird. Zunärhst sehen wir, daß $g_{1}$ und $g_{2}$ nur für $x=1$ und für $x=0$ unendlich werden. Es sei nämlich

$$
g_{1}+g_{2} y=\frac{g(x)+h(x) \underline{y}}{k(x)},
$$

wo $g, h, k$ ganze rationale Funktionen von $x$ ohne gemeinsamen Teiler sein sollen. Es enthalte $k(x)$ den Faktor $x-a$ und es sei $a$ weder 1 noch 0 . Da $R$ für $x=a$ in keinem der beiden Blätter der Riemannschen Fläche unendlieh werden darf, so dürfen die Funktionen

$$
\frac{g+h y}{x-a}, \quad \frac{g-h y}{x-a}
$$

für $x=a$ nicht unendlich werden, also auch nicht ihre Summe und Differenz, also nicht

$$
x-a, \quad \frac{2 h}{x-a} y .
$$

Daher müssen $g$ und $h$ durch $x-a$ teilbar sein und es würden gegen die Voraussetzung $g, h, k$ einen gemeinsamen Teiler haben. Es kann daher $k(x)$ in (5) nur die Faktoren $x$ und $x-1$ enthalten. Man sieht ferner 
leicht, daß $k$ den Faktor $x$ höchstens in der zweiten und $x-1$ höchstens in der ersten Potenz enthalten kann. Wir können daher setzen

Es wird

$$
k=x^{2}(x-1) \text {. }
$$

$$
R=\begin{gathered}
g+h y \\
x^{2}(x-1)
\end{gathered} .
$$

Wir betrachten $R$ zunächst in der Umgebung der Stelle $x=1$.

Es wird

$$
R=\frac{g(x)}{x^{2}(x-1)}+\frac{i h(x)}{x^{2} \mid x+1} \mid x-1 .
$$

Hieraus folgt, daß die Entwicklung von $R$ nach Potenzen von $\sqrt{x-1}$ mit $g(1)(\mid x-1)^{-2}$ beginnen würde, wenn nicht $g(1)=0$, d. h. wenn nicht $g(x)$ durch $x-1$ teilbar ist. Da aber $R$ für $x=1$ höchstens von der ersten Ordnung unendlich werden soll, also in der Entwicklung nur die $(-1)$ te Potenz von $\mid x-1$ vorkommen darf, so muß $g(x)$ durch $x-1$ teilbar sein. Wir setzen $g(x)=g_{1}(x)(x-1)$ und haben wegen (6)

$$
R=\frac{g_{1}(x)}{x^{2}}+\frac{h(x)}{x^{2}(x-1)} y
$$

und jede Funktion dieser Form, wo $g_{1}$ und $h$ ganze rationale Funktionen von $x$ sind, verhält sich an der Stelle $x=1$ so, wie wir wünschen.

Wir betrachten weiter die Stellen $x=0, y= \pm 1$. Es sei

$$
g_{1}(x)=a_{0}+a_{1} x+a_{2} x^{2}+\cdots, h(x)=b_{0}+b_{1} x+b_{2} x^{2}+\cdots
$$

Es ist

$$
\begin{aligned}
& y= \pm\left|1-\frac{1}{2} x^{2}-\frac{1}{8} x^{4}-\cdots\right|, \\
& y h= \pm\left[b_{0}+b_{1} x+\left(b_{2}-\frac{1}{2} b_{0}\right) x^{2}+\cdots\right],
\end{aligned}
$$

$=\operatorname{lic}_{x-1}^{x^{2}(x-1)}\left[\left(-a_{0} \pm b_{0}\right) x^{-2}+\left(a_{0}-a_{1} \pm b_{1}\right) x^{-1}+\left(a_{1}-a_{2} \pm b_{2} \mp \frac{1}{2} b_{0}\right)+\cdots\right]$,

wo das obere Zeichen für die Stelle $x=0, y=+1$ und das untere für die Stelle $x=0, y=-1$ gilt. Aus der Entwicklung geht hervor, da $B$ die Funktion an der Stelle $x=0, y=+1$ höchstens von der zweiten Ordnung unendlich wird, also dort so beschaffen ist, wie wir wünschen. Soll die Funktion an der Stelle $x=0, y=-1$ mindestens von der ersten Ordnung Null werden, so folgt aus (9)

$$
a_{0}+b_{0}=0, a_{0}-a_{1}-b_{1}=0, a_{1}-a_{2}-b_{2}+\frac{1}{2} b_{0}=0
$$


oder

$$
a_{0}=-b_{0}, a_{1}=-b_{0}-b_{1}, a_{2}=-\frac{1}{2} b_{0}-b_{1}-b_{2} .
$$

Es wird wegan (8)

$$
\begin{aligned}
& =-(x-1)\left|b_{0}+\left(b_{0}+b_{1}\right) x+\left(\frac{1}{2} b_{0}+b_{1}+b_{2}\right) x^{2}+x^{3} \gamma^{\prime}(x)\right| \\
& =b_{0}+b_{1} x+b_{2} x^{2}-\frac{1}{2} b_{0} x^{2}-\left(\frac{1}{2} b_{0}+b_{1}+b_{2}\right) x^{3}+x^{3} \gamma(x)(1-x),
\end{aligned}
$$

wo $y^{\prime}(x)$ eine ganze rationale Funktion von $x$ ist. Setzen wir noch

$$
h(x)=b_{0}+b_{1} x+b_{2} x^{2}+x^{3} \%(x),
$$

so nimmt wegen (7), (10) und (11) unsere Funktion $R$ die Form an

$$
\begin{aligned}
R & =\frac{1}{x^{2}(x-1)} \mid b_{0}\left(1-\frac{1}{2} x^{2}-\frac{1}{2} x^{3}+y\right)+b_{1}{ }^{\prime}\left(1-\ell^{2}+y\right) x \\
& +b_{2}(1-x+y) x^{2}+x^{3}(1-x) \gamma(x)+x^{3} \gamma(x) y \mid .
\end{aligned}
$$

Hier sind $b_{0}, b_{1}, b_{2}$ beliebige Konstante und $\gamma(x)$ und $\%(x)$ beliebige ganze rationale Funktionen von $x$. Alle Funktionen der Form (12) sind Vielfache des Divisors $q=t^{-1}+t^{-2} w_{2}$. Das Ergebnis läßt sich aber noch erheblich vereinfachen. Wir setzen zur Abkürzung

$$
f_{1}=1-\frac{1}{2} x^{2}-\frac{1}{2} x^{3}+y, f_{2}=\left(1-x^{2}+y\right) x, f_{3}=(1-x+y) x^{2}
$$

und brachten, daß folgende Gleichungen bestehen

$$
f_{1} x-f_{2}=\frac{1}{2} x^{3}(1-x) \cdot f_{1} x^{2}-f_{3}=x^{3}(1-x)\left(1+\frac{1}{2} x\right) \text {. }
$$

Es wird mit Hilfe dieser Gleichnngen

$$
R=\frac{1}{x^{2}(x-1)}\left[\left(b_{0}+b_{1} x+b_{2} x^{2}\right) f_{1}+x^{3}(1-x) \bar{\gamma}(x)+x^{3} \%(x) y\right] .
$$

wo $\bar{\gamma}(x)$ wieder eine beliebige ganze rationale Funktion von $x$ ist. Bedenken wir ferner, daß aus der ersten der Gleichungen (13) folgt

so haben wir

$$
y_{i}^{\prime}=f_{1}-(1-x)\left(1+x+\frac{1}{2} x^{2}\right),
$$

$$
\begin{aligned}
& R=\frac{1}{x^{2}(x-1)}\left\{\left(b_{0}+b_{1} x+b_{2} x^{2}+x^{3} \%(x)\right) f_{1}+x^{3}(1-x) \mid \bar{\gamma}(x)\right. \\
& \left.-\chi(x)\left(1+x+\frac{1}{2} x^{2}\right)\right]_{j}^{1} \text {. }
\end{aligned}
$$

Da aber $b_{0}, b_{1}, b_{2}$ beliebige Konstanten sind, so ist $b_{0}+b_{1} x+b_{2} x^{2}+x^{3} \%(x)$ eine beliebige ganzerationale Funktion von $x$, die mit $\lambda_{1}(x)$ bezeichnet 
sid. und denso ist $\bar{\gamma}(x)-\chi(x)\left(1+x+\frac{1}{2} x^{2}\right)$ eine beliebige ganze rationalir funktion von $x$, die mit $\lambda_{2}(x)$ bezelehnet sei. Setzen wir (1'i) $\because=\frac{1}{x^{2}(x-1)}=\frac{1}{x^{2}(\bar{x}-1)}\left|(1-x)\left(1+x+\frac{1}{2} x^{2}\right)+y\right|, \quad \zeta_{2}=x$, so wird schlieBlieh

$$
R=\lambda_{1}(x)=1+\lambda_{2}(x)=2,
$$

wo $\lambda_{1}$ und $\lambda_{2}$ beliebige ganze rationale Funktionen von $x$ sind.

Das Ergebnis ist sehr einfach und läßt sich in der Form aussprechen:

Es gibt zwei Funktionen $\zeta_{1}$ und $\zeta_{2}$ des Körpers von der Art, daß sich jedes Vielfache des gegebenen Divisors $q$ in der Form $\lambda_{1} \zeta_{1}+\lambda_{2} \zeta_{2}$ darstellen läßt, wo $\lambda_{1}$ und $\lambda_{2}$ ganze rationale Funktionen von $x$ sind, und da $B$ umgekehrt jede Funktion der Form $\lambda_{1} \zeta_{1}+\lambda_{2} \zeta_{2}$ ein Vielfaches von $a$ ist, wenn $\lambda_{1}$ und $\lambda_{2}$ ganze rationale Funktionen von $x$ sind.

Man nennt $\left(\zeta_{1}, \zeta_{2}\right)$ ein Fundamentalsystem oder eine Basis für die Vielfachen des Divisors a.

Wie wir gesehen haben, läßt sich jede Funktion $R$ des hier betrachteten Körpers durch die Basis $(1, y)$ in der Form darstellen

$$
R=h_{1} \cdot 1+h_{2} y,
$$

wo $h_{1}$ und $h_{2}$ rationale Funktionen von $x$ sind. Aus den Gleichungen (14) folgt

$$
1=\frac{1}{x} \cdot \zeta_{2}, y=x^{2}(x-1) \zeta_{1}-\frac{1-x}{x}\left(1+x+\frac{1}{2} x^{2}\right) \zeta_{2} .
$$

Führt man diese Werte von 1 und $y$ in (16) ein, so sieht man, daß man auch jede Funktion $R$ aus $(x y)$ darstellen kann in der Form

$$
R=\lambda_{1} \zeta_{1}+\lambda_{2} \zeta_{2},
$$

wo $\lambda_{1}$ und $\lambda_{2}$ rationale Funktionen von $x$ sind. Wir können das eben angegebene Ergebnis daher auch so aussprechen.

Es lassen sich zwei Funktionen $\zeta_{1}$ und $\zeta_{2}$ des Körpers finden von der Art, daß sich jede Funktion des Körpers in der Form $\lambda_{1} \zeta_{1}+\lambda_{2} \zeta_{2}$ darstollen läßt, wo $\lambda_{1}$ und $\lambda_{2}$ rationale Funktionen von $x$ sind. Die Funktion ist dann und nur dann ein Vielfaches von dem gegebenen Divisor $q$, wenn $i_{1}$ und $i_{2}$ gan $z$ sind.

Wir wollen zeigen, daß dies Ergebnis nicht nur für diesen besonderen Fall gilt, sondern da $B$ ein ganz entprechender Satz für jeden algebraischen Körper und für jeden Divisor a gilt.

\section{$\$ 3$. Basen des Körpers.}

Jer algebraische Körper $(x y)$, den wir untersuchen, war definiert durch die Gleichung 


$$
f(x, y)=0 \text {. }
$$

die in $y$ vom m-ton Grade sein solltu. Jerle Funktion des Körpers läßt sich, nuch satz IX, Kap. IV, \$ 7 darstedlen in dee form

$$
h_{1}+h_{2} y+h_{3} y^{2}+\cdots+h_{m} y^{\prime \prime \prime}{ }^{1} \text {. }
$$

wo die $h_{\text {a }}$ rationale Funktionen von $r$ sind, alsu als lineare lonogene Funktion der $m$ Funktionen $1, y, y^{2}, \cdots y^{-1}$.

Definition 1. Man nennt $m$ Größen des körpers $(x y)$ $\Xi_{1}, \Xi_{2}, \ldots \xi_{m}$ mit der Eigenschaft, daß sich jede Funktion $R$ dres Körpers in der Form darstellon läßt

$$
R=g_{1} \breve{s}_{1}+g_{2} \breve{s}_{2}+\cdots+g_{m} \xi_{m},
$$

wo die $g_{i}$ rationale Funtionen von $x$ sind, eine Basis des körpers.

Es bilden also z. B. nach Satz $\mathrm{IX}$ in Kap. IV, $\S 7$ die Funktionen

$$
\text { 1. } y, y^{2}, \ldots y^{m-1}
$$

eine Basis.

Es sei

ein System von $m$ Funktionen des Körpers $(x y)$. Es sei $\Xi_{u}=T_{u}(x, y)$, wo $T$ eine rationale Funktion von $x, y$ ist. Wir bezeichnen $y$ auch mit $y_{1}$ und wie früher die konjugierten Werte von $y$ mit $y_{1}, y_{2} \ldots y_{m}$. Wir bezeichnen ferner $\xi_{a}$ auch mit $\xi_{\text {u } 1}$ und setzen

$$
\xi_{\alpha 3}=T_{u}\left(x, y_{y}\right) \text {, }
$$

so daB $\xi_{a 1}$, $\xi_{u 2}, \ldots \xi_{u m}$ die $m$ kunjugierten Werte von $\xi_{a}=\xi_{u 1}$ sind. Wir rhalten so aus dem System (马), das nur aus einer IIorizontalreihe besteht, ein quadratisches System, dessen Elemente dio $\Xi_{\text {ar }}$ sind. Wir bezrichmen dies System mit $\left(\xi_{(1)}\right)$, setzen also

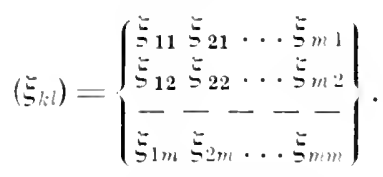

Satz I. Es gehortzu jedem System (G) ein quadratisches

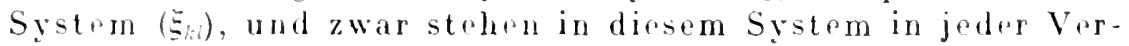
tikalroihe $m$ konjugierte Funktionen des Körpers (xy).

Wenn wir die Veränderliche $x$ in der einfachen $x$-Ebene einen geschlossenen Weg durchlaufen lassen, so tritt unter den $y_{1}$ höchstens eine Permutation ein. Es werden also in dem System $\left(\xi_{3 l}\right)$ dip Itorizontalreihen permutiert, so dab dir Determinante $\zeta_{43}$ hörhatens ihr Vorzcichen ändert. Daher bleibt das Quadrat der Determinante bei jedem solchen 
Umlauf von $x$ ungeändert, und da es eine algebraische funktion von $x$ ist, so muB es rational in $x$ sein. Also:

Definition II. Ist (5) ein System von $m$ Funktionen des Körpers und ist ( $\left.\Xi_{h}\right)$ das zugehörige quadratisclie System, so ist das Quadrat der Determinante $\left|5_{k}\right|$ eine rationale Funktion von $x$. Sie heißt die Diskriminante der $m$ Funktionen $\xi_{\alpha}$.

Zum Beispiel gehört zu der Basis (22) das quadratische System

$$
\left(y_{k}^{1-1}\right)=\left\{\begin{array}{lllll}
1 & y_{1} & y_{2}^{2} & \ldots & y_{1}^{m-1}-1 \\
1 & y_{2} & y_{2}^{2} & \ldots & y_{2}^{m-1}-1 \\
- & - & - & - & - \\
1 & y_{m} & y_{m}^{2} & \ldots & y_{m}^{m}-1
\end{array} \mid .\right.
$$

Die zugehörige Diskriminante ist nach (3), $\S 1$ und einem bekannten Satze der Algebra

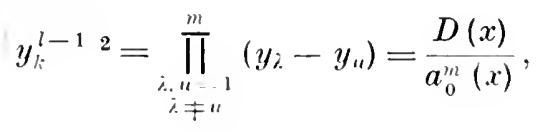

wo $D(x)$ die Diskriminante der Gleichung $(17)$ in bezug auf $y$ ist und $a_{0}(x)$ der Koeffizient von $y^{m}$ in $f(x . y)$. Es ist also bei unseren Voraussetzungen dip Diskriminante $\mathrm{d} r$ Basis (18) nicht identisch Null.

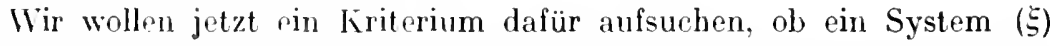
von $m$ Funktionen des Körpers eine Basis ist oder nicht. Es sei erstens $(\xi)$ eine Basis. Dann lassen sich alle Funktionen des Körpers $(x y)$ linear homogen durch die $\xi_{\text {k }}$ ausdrücken, also auch die Funktionen $1, y, \boldsymbol{y}^{2}$. $\ldots y^{m-1}$ der Basis (18). Es hestehen also Gleichungen der Form

$$
\begin{array}{r}
1=g_{11} \xi_{1}+g_{12} \xi_{2}+\cdots+g_{1 m} \xi_{m}, \\
y=g_{21} \xi_{1}+g_{22} \xi_{2}+\cdots+g_{2 m} \xi_{m}, \\
------------g_{m m} \xi_{m},
\end{array}
$$

wo die $g_{k l}$ rationale Funktionen von $x$ sind. Bezeichnen wir die Basis (18) kurz mit $(y)$ und mit $g$ das System

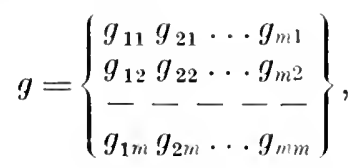

so können wir die Gleichungen (24) kürzer schreiben:

$$
(y)=(\xi) g \text {. }
$$

Es beschreibe $x$ einen geschlossenen Weg in der einfachen $x$-Ebene, so daß $y=y_{1}$ in $y_{k}$ übergeht. Dabei bleiben die identischen Gleichungen 


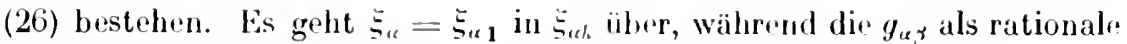
Funktionen von $x$ ungeändert bloiben. Walire folgt ans (26)

$$
\left(y_{k}^{l-1}\right)=\left(\Xi_{k l l}^{l}\right) g \text {. }
$$

Gehen wir hierhin zu den Determinanten über, so folgt

$$
y_{k}^{l-1}=\xi_{k l} \cdot g \text {. }
$$

Da aber die Diskriminante der Basis $(y)$ nicht identisch Null ist, so ergibt sich hieraus, daß die Determinanten $g$ und $\xi_{k l l} \mid$ auch nicht identisch Null sein können. Es ist also die Diskriminante einer Basis nicht identisch Null.

Es sei zweitens die Diskriminante des Systems ( $\xi$ ) nicht identisch Null. Da $(y)$ eine Basis ist, so bestehen Gleichungen der Form

$$
\text { (ら) }=(y) h \text {, }
$$

wo die Elemente des quadratisehen Systems $h$ rationale Funktionen von $x$ sind. Aus (29) folgt, geradeso wie aus (26) sich (27) ergab,

$$
\left(\xi_{k l}\right)=\left(y_{k}^{l-1}\right) h \text {. }
$$

Durch Übergang zu den Determinanten folgt aus (30)

$$
\left|\xi_{k l}\right|=y_{k}^{l-1} \cdot h
$$

und hieraus, weil nach Annahme $\xi_{k l} \neq 0$, daß $h \neq 0$. Daher existiert zu $h$ das reziproke System $h^{-1}$, ind aus (30) folgt

$$
\left(y_{k i}^{l-1}\right)=\left(\xi_{k l}\right) h^{-1}
$$

und also auch, indem wir uns links und rechts auf die erste Horizontalreihe beschränken,

$$
(y)=(\xi) h^{-1} \text {. }
$$

Ist jetzt $R$ irgendeine Funktion des Körpers $(x y)$, so können wir sie zunächst in der Form

$$
R=r_{1} 1+r_{2} y+\cdots+r_{m} y^{m-1}
$$

darstellen, wo die $r$ rationale Funktionen von $x$ sind. Setzen wir in (32) die Werte von $1, y, \ldots y^{m-1}$ ein, wie sie aus (31) folgen, so erhalten wir $R$ als lineare homogene Funktion der $\xi_{\text {_ }}$ mit rationalen Funktionen von $x$ als Koeffizienten. Damit ist aber bewiesen, daß (ङ) eine Basis ist.

Wir haben also:

Satz II. Ein System von $m$ Funktionen des Körpers $(x y)$

$$
\text { (ら) }=\left(\Xi_{1}, \Xi_{2}, \ldots \Xi_{m}\right)
$$

ist dann und nur dann eine Basis des Körpers, wenn die Diskriminante des Systems nieht identisch Null ist. 
Iliveras folgt weiter:

Satz 111. Zwischen den Funktionren piner Basis kann keine linrare homogene Gloirhung bestohem, deren koeffizienten rationale Funktionou vou $x$ sind.

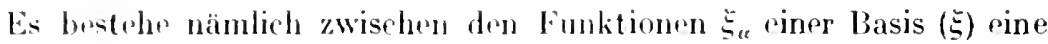
Gloichning

$$
g_{1} \Xi_{1}+g_{1} \Xi_{2}+\cdots+g_{m} \Xi_{m}=0,
$$

wo die $g$, rational. Funktionen von $x$ sind. Dirse Gleichung bleibt richtig bei analytischer Fortsetzmng. ladurch können wir $\xi_{a}=\Xi_{\nVdash 1}$ in jede konjugierte Funktion $\Xi_{a k}$ üborfuhron, während dif $g_{i}$ als rationale Funktionen ungeändert bleiben. Daher bestehen die $m$ linearen homogenen Gleichungen

$$
g_{1} \Xi_{1 k}+g_{2} \xi_{2 k}+\cdots+g_{m} \xi_{m k}=0,(k=1,2, \ldots m) \text {. }
$$

Daraus würde aber folgen, daB die Determinante $\left|\xi_{k l}\right|$ identisch Null wäre, was nicht sein kann. Eine Gleichung der Form (33) ist also, wie behauptet, nicht möglich.

Daraus sehließen wir

Satz IV. Eine Funktion $R$ des Körpers $(x y)$ läßt sich

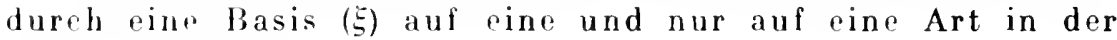
Form darstellen

$$
R=r_{1} \xi_{1}+r_{2} \Xi_{2}+\cdots+r_{m} \xi_{m},
$$

wo die $r_{1}$ rationale Funktionen von $x$ sind.

Gäbe es nämlich noch eine zweite Jarstellung

$$
R=s_{1} \xi_{1}+s_{2} \xi_{2}+\cdots+s_{m} \xi_{m},
$$

so wïrde durch Subtraktion folgen

$$
\left(r_{1}-s_{1}\right) \xi_{1}+\left(r_{2}-s_{2}\right) \xi_{2}+\cdots+\left(r_{m}-s_{m}\right) \xi_{m}=0 .
$$

Eine solche Gleichung kann aber naeh dem eben Bewiesenen nicht bestehen, wenn nicht alle Differenzen $r_{i}-s_{i}$ identisch Null sind.

Es seien jetzt ( () und $(r)$ zwei Basen des Körpers. Dann müssen sich die $\gamma_{i}$ linear durrh die $\xi_{i}$ darstellen lassen mit Koeffizienten, die rationale Funktionen von $x$ sind, d. h. es besteht eine Gleichung

$$
\left(u_{i}\right)=(\xi) g \text {, }
$$

wo $g$ rin quadratisches System ist, dessen Elemente rationale Funktionen von $x$ sind. Lassen wir $x$ einen gresrhlossenen Weg in der einfachen $x$-Ebene Jurchlaufen, so daß $y=y_{1}$ in $y_{k}$ übergeht, so geht $\xi_{\alpha}=\xi_{\alpha 1}$ in $\xi_{\alpha k}$ und $y_{a z}=r_{* 1}$ in $r_{i k k}$ über, während $g$ urgeändert bleibt. Da die Gleichungen (34) lri analytischer Fortsetzung bestehen bleiben, so folgt, daß auch

$$
\left(r_{i k}\right)=\left(\xi_{h}\right) g \text {. }
$$

Wurch C̈bergang zu den Drterminanten folgt

$$
\eta_{k \cdot l}=\left|\xi_{k i l}\right| \cdot g \text {. }
$$


Da $\eta_{k b_{1}} \neq 0$, weil $(1)$ eine Basis, so ist aur $h \neq 0$. Daraus aber folgt, dab das zu $g$ reziproke System $g^{-1}$ existiert, und ans (34) folgt daher

Wir haben also:

$$
(\xi)=(1) g^{-1} \text {. }
$$

Satz V. Je zwei Basen des Körpers $(x y)$ sind durch eine lineare homogene substitution miteinander verknipft, deren Elemente rationale Funktionen von $x$ sind und duren beterminante nicht identisch Null ist.

Ferner beweisen wir noch:

Satz VI. Sind zwei Systeme (5), (r) von je $m$ Funktionen des Körpers $(x y)$ durch eine lineare homogene Substitution miteinander verbunden, deren Determinante nicht identisch Null ist und deren Elemente rationale Funktionen von $x$ sind, so ist die eine dann und nur dann eine Basis, wenn die andere es ist.

Denn nach Voraussetzung besteht eine Gleichung der Form (34), aus der folgt, daß auch

$$
\left(v_{i k l}\right)=\left(\xi_{l i l}\right) g \text {. }
$$

Gehen wir zu den Determinanten über, so folgt

$$
r_{k l l}=\xi_{k l} \cdot g
$$

da aber nach Voraussetzung $g$ nicht identisch Null ist, so sund die Diskriminanten $\xi_{h l}{ }^{2}$ und ${\gamma_{i k l}}^{2}$ von $(\xi)$ und $(1$,$) gleichzeitig identisch Null oder$ nicht. Das ist aber nur ein anderer Ausdruck für die Behauptung.

\section{$\$$ 4. Basis für die Vielfachen eines Divisors.}

Es sei q ein Divisor, der keine Primteiler enthält, die zu Stellen gehören, wo $x$ unendlich groß ist. Wir nennen, wie wir schon in $\S 1$ definiert haben, eine Funktion $R$ (in Vielfaches von $q$, wenn der Divisor, der der Funktion $R$ entspricht, durch Division mit q übergeht in einen Divisor, der höchstens noch solche Primteiler im Nenner enthält, die zu Stellen gehören, wo $x$ unendlich wird. Die Definition ist also wesentlich abhängig von der Wahl der unabhängigen Veränderlichen $x$. Die Aufgabe, die uns jetzt beschäftigen wird, ist, alle Vielfachen eines gegebenen Divisors zu bestimmen. Wir lösen die Aufgabe so, daß wir eine Basis für die Vielfachen von q aufsuchen. Dabei verstehen wir unter einer Basis für die Vielfachen eines Divisors q folgendes:

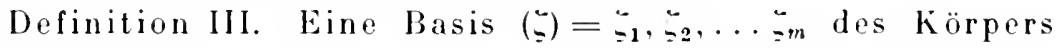
heißt eine Basis für die Vielfachen des Divisors $q$, wenn eine Funtion

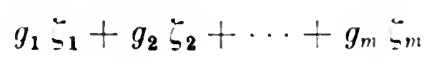


danu und nur dann pin Vielfarlas von o ist, wenn die rationalen Funktioneng ganz sind.

(relingt es uns, eine sulehe basis zu bestimmen, so haben wir eine Loisung der Aufgabe, die ganz so beshaffen ist wie die Lösung in dem in 5 - behandelton Beispiel.

Zunächst zeigen wir, daß und wie sich aus oiner Basis für die Vielfathen von q alle anderen herdeiton lassen, und dann zeigen wir, wie wir immer rine solehe Basis finden können.

Ls su ( $(5)$ eine Basis für die Vielfachen von qund ebenso $(\eta)$. Es ist jede Funktion der Form $g_{1} s_{1}+g_{2} 1_{2}+\cdots+g_{m} r_{m}$, wenn die $g$ ganze rationale Funktionen ron $x$ sind, ein Vielfaches ron a, also $z$. B. jede der Funktionen 1, sellst, so daß Gleichungen bestehen der Form

$$
(r)=(\xi) g \text {, }
$$

wo die $g_{4}$ gan ze rationale Funktionen von $x$ sind. Es sind aber auch die $\xi_{i}$ Vielfache von $q$ und sie müssen sich dureh die $y_{i}$ linear mit ganzen rationalen Funktionen von $x$ als Koeffizienten darstellen lassen. Es bestehen also auch Gleichungen der Form

$$
(\xi)=\left(y_{i}\right) h,
$$

wo auch die $h_{h l}$ gan ze rationale Funktionen von $x$ sind. Aus den Gleichungen (35) und (36) folgt, daß $g$ und $h$ zueinander reziprok sirid, so da

$$
g \cdot h=1 \text {. }
$$

Da die Elemente von $g$ und $h$ ganze rationale Funktionen von $x$ sind, so gilt dasselbe von ihren Determinanten, und da ihr Produkt gleich 1 ist, so müssen sie beide konstant sein. Es sind also nach der Definition III, Kap. VII, $\S 5$ die Systeme $g$ und $h$ Einheiten.

Wir beweisen hiervon auch die Umkehrung. Es sei $(\xi)$ eine Basis für die Virlfachen von qund es sei $g$ ein System von $m^{2}$ rationalen Funktionen von $x$, das eine Einheit ist. Das System ( $\left.b_{i}\right)$ sei durch (35) definiert. Um zu zeigen, daß (1) auch eine Basis für die Vielfachen von $q$ ist, haben wir zweierlei zu beweisen:

1. daß jedes viflfache $R$ von $q$ sich in der Form darstellen läßt

$$
R-r_{1} l_{11}+r_{2} r_{2}+\cdots+r_{m} \jmath_{m},
$$

wo die $r_{i}$ ganze rationale Funktionen von $x$ sind;

2. daß jede Funktion der Form (37) ein Vielfaches von $q$ ist.

1. Ist $R$ ein Vielfaches von $q$, so haben wir für $R$ eine Darstellung

$$
R=s_{1} \xi_{1}+s_{2} \xi_{2}+\cdots+s_{m} \xi_{m} \text {, }
$$

wo die $s_{1}$ ganze rationale Funktionen von $x$ sind. Aus (35) folgt aber (36), wo $h=g^{-1}$ ist. Da $g$ eine Einheit ist, so gilt nach Satz XVII, Kap. VII, $\S 5$ dasselb von $h$. Ersetzen wir also in (38) die $\xi_{i}$ durch die $\eta_{i}$, so er- 


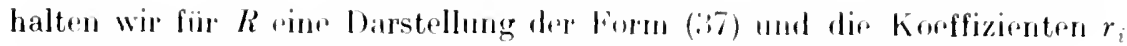
werden ganze rationale Funktionen von $x$.

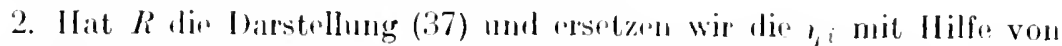
(35) durch die $\Xi_{i}$, so erhalten wir rine Cildichung der form (38), wo die $s$ ganze rationale funktionen von $x$ sind, da dasselter von den $r$, und von den Elementen von $y$ gilt.

Wir halwn also:

Satz VII. Zwisehen je zwoi bason für dir. Virllachen eines Divisors q besteht eine lineare homogeno Substitution, deren Elemente rationale Funktionen von $x$ sind und die eine Einheit ist, und wenn zwisehen zwei Systemen von je $m$ Größen des Körpers eine solche Substitution besteht, so ist die eine gleichzeitig mit der anderen eine Basis für die Vielfachen von q.

Zwischen zwei Basen $(\xi)$ und $(\eta)$ für die Vielfachen eines Divisors $q$ besteht eine Gleichung der Form (35), wo $g$ eine Einheit ist. Aus (35) folgt

und

$$
(\eta ! l)=(\xi, l) g
$$

$$
y_{k l}=\xi_{k l} \cdot g \text {. }
$$

Da $g$ konstant ist, so haben wir:

Satz VIII. Die Diskriminanten aller Basen für die Vielfachen eines Divisors q unterscheiden sich nur durch einen konstanten von Null verschiedenen Faktor.

\section{$\$ 5$. Normalbasen.}

Es sei $x=a$ irgendein endlicher Wert von $x$. Die Primteiler, die zu denjenigen Stellen der Riemannsehen Fläche gehören, wo $x=a$ ist, also die im Zähler $x-a$ enthaltenen Primteiler seien $p_{1}, t_{2}, \ldots p_{n}$. Es sei $p_{k}$ in $q$ genau in der Potenz $\tau_{k}$ enthalten. Wir definieren dann:

Definition IV. Eine Basis ( 5 ) heißt normal an der Stelle $x=a$ in bezug auf $q$, wenn eine Funktion

$$
g_{1} \xi_{1}+g_{2} \xi_{2}+\cdots+g_{m} \xi_{m}
$$

dann und nur dann den Divisor

$$
H_{1}^{r_{1}}{p_{2}^{\tau_{2}}}_{\tau_{2}} \ldots P_{n}^{{ }^{T_{n}}}
$$

enthält, wenn die rationalen Funktionen $g_{i}$ für $x=a$ nicht unendlich werden.

Eine Funktion enthält einen Divisor $\mathfrak{p}_{1}^{r_{1}} \mathfrak{p}_{2}^{r_{3}} \ldots \mathfrak{p}_{n}{ }^{{ }_{n}}$ soll hier und im folgenden bedeuten, sie enthält jeden Primteiler $w_{i}$ mindestens in del Potenz $\tau_{i}$. 
Es gelten die Sätze

Satz lX. Es sej die Basis (s) normal für $x=a$ in bezug auf don Divisor a, und es sei die Basis (1) definiert dureh

$$
(1)=(\Xi) g,
$$

wu gein quadratisches System ist, dessen Elemente rationale Funktionen von $x$ sind; so ist $(1$,$) dann und nur dann nor-$ mal für $x=a$ in bezug auf

Satz X. Sind (马) und (a) zwei Basun, die normal sind für $x=a$ in bezug auf den loivisor q, so unterseheiden sich ihre l)iskriminanten nur um rinen Faktor, der eine Einheit für $x=a \mathrm{ist}$.

Diese Sätze entsprechen denen des vorigen $\S$ und werden ganz ähnlich bewisen. Nämlich: Da (2) auch eine Basis sein soll, so muß in (41) $g$ eine I reterminante haben, die nicht identisch Null ist. Es existiert also das zu $g$ reziproke System $g^{-1}$, das mit $h$ bezeichnet sei. Aus (41) folgt dann

Aus (41) folgt ferner

$$
(\xi)=(\eta) h .
$$

$$
\left(y_{k l}\right)=\left(\xi_{k l}\right) g, r_{1 k l}=\xi_{k l} \cdot g \text {. }
$$

Nun seierstens ( $(3)$ auch normal für $x=a$. Dann sind alle Funktionen der Form (39), also im besonderen auch die $\xi$, durch den Divisor (40) teilbar und ebenso die $y_{i}$. Daher sind nach der Definition des Begriffes normal die Elemente in $g$ und $h$ ganz für $x=a$, also auch die Determinanten $g$ und $h$. Da aber $g$ und $h$ reziprok sind, so ist $g \cdot h=1$. Also müssen $g$ und $h$ Einheiten für $x=a$ sein. Hieraus folgt wegen (43) auch der zweite der angegebenen Sätze.

Zweitens sei $g$ und also auch $h$ eine Einheit für $x=a$. Um zu zeigen, laß clann $(1$,$) gleirhzeitig mit (\xi)$ normal für $x=a$ ist, haben wir wieder zweirelei zu zeigen:

1. daß jede Funktion $R$ des Körpers $(x y)$, die den Divisor $(40)$ enthält, in dre Form darstollbar ist:

$$
R=r_{1} !_{1}+r_{2} \imath_{2}+\cdots+r_{m} !_{i m},
$$

wo din $r$, rationale Funktionen von $x$ sind, die für $x=a$ nicht unendlich werden ;

2. daß rine Funktion der Form (41) immer den Divisor (40) enthält.

1. Zunärhst läßt sirh $R$ in der Form darstellen

$$
R=s_{1} \xi_{1}+s_{2} \xi_{2}+\cdots+s_{m} \xi_{m},
$$

wo dis $s$, für $x=a$ nicht unendlich werden. Führen wir hierin statt der $\xi$, die $r_{1}$, ein mit Hilfe der Gleichung (42), so erhalten wir, da die Elemente 
von $h$ für $x=a$ nicht unterdlinh werdell. fiir $R$ eine Darstralung der Forin (4í).

2. Aus (44) folgt mit Milfe von (4I) dis l)arstallnug (45), wo die $s$,

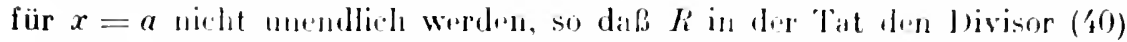
entbält. l)mu (汭) ist normal für $x=a$ in lorzug anf q.

Ist eine Basis (气) für alle rndlichen Stellen $x$ in lorzug auf a mormal, so ist eine Funktion $R=g_{1} \Xi_{1}+g_{2} \Xi_{2}+\cdots+g_{m} \Xi_{m}$ daun und nur dann durch o trilbar, wenn die $g$ für keinen endlichen Wert von $x$ unendlich werden, wemn es also ganze rationale Funktionen von $x$ siurl, d. h. abrer, (ङ) ist eine basis für die Vielfachen von q. Um zu einfr solchen liasis zu gelangen, werden wir so verfahron, dab wir von irgendeiner Basis ausgehen und sie Schritt für Schritt verwandeln in eine Basis, die an den Stellen. an denen die ursprüngliche nicht normal ist, auch normal ist. Dabei ist der erste der eben bewiesenen Sätze für uns sehr wichtig, wril er uns grstattet, dafür zu sorgen, daß nicht etwa, wenn wir cine Basis ( $引)$ in eine neue ( $\eta$ ) verwandeln, die an der Stelle $a$ normal ist, diese neue Basis $(r)$ an einer anderen Stelle $b$ nicht normal ist, an der ( $\Xi)$ normal war oder viplleicht mit vieler Wühe normal gemacht war.

\section{$\$$ 6. Herstellen einer Normalbasis. I.}

Es sei ( () ) irgendeine Basis des Körpers. Eine solche können wir immer finden. Wir können z. B. nehmen die Basis $1, y, y^{2}, \ldots y^{n-1}$. Wir brtrachten die Basis ( () ) an einer Stelle $x=a$ und zeigen, wie man entscheiden kann, ob sie normal ist für $x=a$ in bezug auf a und wie man, wenn das nicht der Fall ist, durch eine lineare Substitution aus ihr eine neue Basis herleiten kann, die an der Stelle $x=a$ normal ist, und die außerdem an allen Stellen normal ist, an denen ( $\Xi)$ normal ist. Wir unterscheiden zwoi Hauptfälle

I. Bei $x=a$ liegt kein Verzweigungspunkt.

II. Bei $x=a$ liegt mindestens ein Verzweigungspunkt.

I. Es sei $x=a$ eine im Endlichen liegende Stelle, und es liege in der Riemannschen Fläche bei $x=a$ kein Verzweigungspunkt.

Bei $x=a$ liegen dann genau $m$ Stellen der Riemannschen Fläche übereinander. Die zu ihnen gehörenden Primteiler seien $t_{1}, t_{2}, \ldots n_{n}$. Es sei $\%$ genau in der Potenz $\tau_{k}$ in q enthalten, wobei nicht ausgeschlossen ist, daB alle $\boldsymbol{r}_{k}$ gleich Null sind. Irgendeine Funktion $R$ des Körpers läßt sich darstellen in der Form

$$
R=g_{1} \xi_{1}+g_{2} \xi_{2}+\cdots+g_{m} \xi_{m} \text {, }
$$

wo die $g$ rationale Funktionen von $x$ sind. Die Werte von $R$ in der Umgebung der $m$ Stellen $\mathfrak{p}_{1}, \mathfrak{p}_{2}, \ldots \mathfrak{p}_{m}$ der Riemannschen Fläche sind

Jnng, Einfuhrung in dir Theorie. 


$$
\begin{aligned}
& R_{1}=g_{1} \check{亏}_{11}+g_{2} \check{亏}_{21}+\cdots+g_{m} \check{亏}_{m 1}, \\
& R_{2}=g_{1} 亏_{12}+g_{2} \Xi_{22}+\cdots+g_{m} 亏_{m 2}, \\
& \bar{R}_{m}=g_{1} \breve{s}_{1 m}+g_{2} \bar{\Xi}_{2 m}+\cdots+g_{m} \breve{s}_{m m} .
\end{aligned}
$$

Soll $R$ durch q teilhar sein, so muB w jodentalls den Divisor $\psi_{1}^{r_{1}} \psi_{2}^{r_{2}} \ldots \psi_{m}^{r}{ }_{m}$ enthalten. Wir nehmen die Bezeichnung so gewählt an, daß $R_{k}$ den Wert

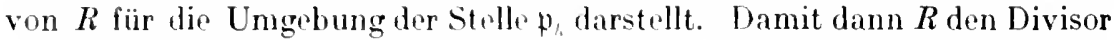
${ }_{1}^{r_{1}} w_{z}^{r_{2}} \ldots H_{m}^{m}$ enthält, muß die Entwicklung von $R_{k}$ nach steigenden ganzen Potenzen von $x-a$ mit riner Potenz von $x-a$ beginnen, deren Exponent mindestens gleich $\boldsymbol{I}_{k}$ ist. Setzen wir

$$
R_{k}=(x-a)^{\mathrm{r}} \text { : } \mathfrak{x}_{\mathrm{s}},
$$

so müssen die 愔 gwöhnliche Potenzreihen von $x-a$ sein. Setzen wir ferner

$$
\begin{aligned}
\Xi_{k l}=(x-a)^{r_{1}} p_{r_{1}}, \zeta_{k 2} & =(x-a)^{\tau_{2}} p_{k 2}, \ldots \xi_{k_{m}}=(x-a)^{r_{m}} p_{k m}, \\
(k & =1,2, \ldots m),
\end{aligned}
$$

so sind die $p_{a l}$ nach ganzen steigenden Potenzen von $x-a$ entwickelbar. Die Beziehung zwisehen den Systemen $\left(\xi_{k l}\right)$ und $\left(p_{k l}\right)$ ist die, daß das System $\left(\xi_{k l}\right)$ aue dem System $\left(p_{k l}\right)$ dadureh hervorgeht, daß man die Horizontalreihen von $\left(p_{1}\right)$ der Reihe nach multipliziert mit $(x-a)^{r_{1}},(x-a)^{\tau_{2}}$, ...(x-a ${ }^{\tau} m$. Bezeichnen wir das Multiplikationssystem, dessen Diagonalreihe $(x-a)^{\tau_{1}},(x-a)^{\tau_{2}}, \ldots(x-a)^{\tau_{m}}$ ist, mit $A$, so können wir die Gleichungen (48) in der Form schreiben

$$
\left(\xi_{k l}\right)=A\left(p_{k l}\right) \text {. }
$$

Da die Determinante von $A$ gleich $(x-a)^{r_{1}+r_{2}+\cdots+\tau_{m}}$ ist, so folgt hieraus

$$
\xi_{k l}=p_{k l}(x-a)^{\tau_{1}}+\tau_{2}+\cdots+r_{m} .
$$

Unter Benutzung der Gleichungen (47) und (48) gehen die Gleichungen (46) über in

$$
\begin{aligned}
& \mathfrak{Z}_{1}=g_{1} p_{11}+g_{2} p_{21}+\cdots+g_{m} p_{m 1}, \\
& \mathfrak{I}_{2}=g_{1} p_{12}+g_{2} p_{22}+\cdots+g_{m} p_{m 2}, \\
& ------------ \\
& \mathfrak{F}_{m}=g_{1} p_{1 m}+g_{2} p_{2 m}+\cdots+g_{m} p_{m m} .
\end{aligned}
$$

Es $n$ nthält $R$ den Divisor $\mathfrak{p}_{1}^{\tau_{2}} \mathfrak{p}_{2}^{\tau_{2}} \ldots \mathfrak{p}_{n}{ }^{{ }}{ }^{m}$, wenn die rationalen Funktionen $g_{i}$ so gewählt sind, daß die Funktionen $L_{i}$ für $x=a$ nicht unendlich werden.

Wir unterseheiden zwei Fälle.

Erster Fall. Das System $\left(p_{k l l}\right)$ ist eine Einheit für $x=a$.

Jann folgt aus XVIII, Kap. VII $\S 5$, daß die $\$_{i}$ dann und nur dann nicht unendlich werden für $x=a$, wenn die $g_{i}$ ganz für $x=a$ sind. D. h. aber, 
es ist danu das System (5) normal fïr $x=a$. Wunu aber umgekehrt dann und nur dann dir Li $_{\text {i für }} x=a$ ganz sind, wenn es die $g_{1}$ sind, wenn mit anderen Wurtun (ङ) normal ist für $x=a$, so folgt aus XIX, Kap. VII $\$ 5$, daß das System $(p, l)$ eine Einheit sein muß fïr $x=a$. Wir können jetzt folgenden Satz beweisen:

Satz XI. Wie Basis (5) ist dann und nur dann normal für $x=a$ in bezug auf den Divisor $q$, wenn die Funktionrn $\xi_{i}$ den Divisor

$$
H_{1}^{\tau_{1}} \psi_{2}^{r_{2}} \ldots \psi_{m}^{\tau_{m}}
$$

enthalten. und wenn die Diskriminante von ( $\xi$ ) genau durel die $2\left(\tau_{1}+t_{2}+\cdots+t_{m}\right)$-te Potenz on $x-a$ teilbar ist. Dabei ist angenommen, da $B$ bei $x=a$ kein Verzweigungspunkt liegt.

Es sei nämlich erstens (乡) normal für $x=a$. Dann ist nach dem eben Bewiesenen das System $\left(p_{k i}\right)$ eine Einheit für $x=a$. Seine Elemente $p_{n-l}$ sind also gewöhnliche Potenzreihen von $x-a$ und seine Determinante ist eine Einheit für $x=a$. Aus (48) folgt dann, daß die $\xi_{i}$ den Divisor (52) enthalten, und aus (50), daß die Diskriminante $\xi_{k l}{ }^{2}$ durch die angegebene Potenz von $x-a$ genau teilbar ist.

Enthalten zweitens die $\xi_{i}$ den Divisor (52), so sind wegen (48) die $p_{i l}$ gewöhnliche Potenzreihen von $x-a$ und dasselbe gilt dann von der Determinante $p_{l k}$. Aus (50) folgt weiter, da nach Voraussetzung $\Xi_{k l}$ genau durch die $\left(\tau_{1}+\tau_{2}+\cdots+\tau_{m}\right)$-te Potenz von $x-a$ teilbar ist, daß $p_{l i l}$ nicht Nul! wird für $x=a$. Also ist $\left(p_{k l l}\right)$ eine Einheit für $x=a$ und nach dem oben Bewiesenen ist ( $\xi$ ) normal für $x=a$ in bezug auf $q$.

Aus dem bewiesenen Satze können wir schließen, daß es nur eine endliche Zahl von Stellen $x$ gibt, für die eine Basis ( $)$ ) nicht normal ist in bezug auf einen gegebenen Divisor $q$. Denn die Bedingungen des eben angegebenen Satzes sind höchstens für folgende Stellen nicht erfüllt:

1. für die Stellen $x=a$, wo die Riemannsche Fläche Verzweigungspunkte hat;

2. für die Stellen $x=a$, an denen eine oder mehrere der Funktionen $\Xi$ oder ihrer konjugierten unendlich werden;

3. für die Stellen $x=a$, zu denen Primteiler gehören, die in q vorkommen ;

4. für die Stellen $x=a$, für die die Diskriminante von (ङ) verschwindet.

Die Zahl dieser Stellen ist aber endlich. Die Stellen lassen sich bestimmen, wenn $q$ gegeben und ( $\xi$ ) gewählt ist. Nur diese Stellen brauchen behandelt zu werden.

Zweiter Fall. Das System $\left(p_{\imath, l}\right)$ ist keine Einheit für $x=a$. In diesem Falle gibt es nach XX, Kap. VII, $\S 5$ cin System $S$ von $m^{2}$ 
rationalen Funktionen von $x$, das für alle andlichen Stellen eine Einheit ist aluber fuir $x=a$, so dials

$$
\left(p_{t: l}\right) s=(q: i)
$$

eine Einheit für $x=a$ ist. Setzen wir aber

so folgt aus (49), (53)

$$
(1,)=(5) S,(1,1)=(5) S,
$$

$$
\left(r_{i n}\right)=A\left(q_{i n}\right),
$$

wo $\left(q^{\prime}\right)$ eine Einheit ist. Waraus folgt aber nach dem ersten Fall, daß das System $(1$, normal ist in bezug auf $x=a$, und nach den Sätzen des vorigen $\$$ ist $\left(1_{1}\right)$ iuberall da normal, wo ( $)$ es war, da $S$ für alle endlichen Stellen mit alleiniger Ansnahme von $x=a$ eine Einhrit ist. Damit ist aber gezeigt, wie wir aus (引) eine Basis herleiten können, die an allen Stellen, wo kein Verzweigungspunkt liegt, normal ist.

\section{\$ 7. Herstellen einer Normalbasis. II.}

11. Es mögen an der Stelle $x=a$ ein oder mehrere Verzweigungspunkte liegen. Wir begnügen uns damit, zwei besondere Fälle zu betrachten, weil das genügt, um die Richtigkeit des Verfahrens auch im allgemeinsten Falle darzutun.

II a. Es sei erstens $m=5$ und es mögen an der Stelle $x=a$ in der Riemannschen Fläche zwei Verzweigungspunkte liegen, einer von der ersten und einer von der zweiten Ordnung.

Zum ersten gehöre der Primteiler $p_{1}$, zum zweiten $\downarrow_{2}$.

Es seien $\mathfrak{p}_{1}$ und $\mathfrak{p}_{2}$ in $q$ genau in den Potenzen $\tau_{1}, \tau_{2}$ enthalten. Eine Funktion $R$ enthält den Divisor $w_{1}^{\tau_{1}} k_{2}^{\tau_{2}}$, wenn in der Umgebung von $\mathfrak{p}_{\mathbf{1}}$ die Funktion $R(x-a)^{-{ }^{\gamma_{2}}}$ ? und in der Umgebung von $p_{2} R(x-a)^{-\frac{\tau_{2}}{3}}$ für $x=a$ nicht unendlich wird. In der Umgebung von $p_{1}$ läßt sich aber $R$ und also auch $R(x-a){ }^{2}$ nach ganzen steigenden Potenzen von $(x-a)^{\frac{1}{2}}$ entwickeln. Eine solche Entwicklung läßt sich, wie wir in $\S 6$, Kap. I gesehen haben, sehreiben

$$
R(x-a)^{\stackrel{\tau_{1}}{2}}=r_{1}+r_{2}(x-a)^{\frac{1}{2}},
$$

wo die $r$ nach ganzen Potenzen von $x-a$ fortschreitende Potenzreihen sind. Da in $r_{1}$ nur grade und in $r_{2}(x-a)^{2}$ nur ungrade Potenzen von $(x-a)^{2}$ vorkommen, so kann ein Glied in $r_{1}$ sich nicht fortheben gegen ein Glied in $r_{2}(x-a)^{\frac{1}{2}}$. Soll also $R(x-a)^{-\frac{\tau_{1}}{2}}$ für $x=a$ nicht unend- 
lich werden, so darf weder in $r_{1}$ noch in $r_{2}(x-a)^{2}$ eine negative Potenz von $x-a$ vorkonmen. Also müssin $r_{1}$ und $r_{2}$ für $x=a$ endlich srin.

In der Ungebung von $\psi_{2}$ wird $R$ und also aurh $R(x-a)^{-{ }^{r}: 3}$ aine Potenzreihe von $(x-a)^{\frac{1}{r}}$, die wir schreiben können

$$
R(x-a)^{-r_{3}^{3}}=s_{1}+s_{2}(x-a)^{3}+s_{3}(x-a)^{3} .
$$

wo die $s$ nach ganzen Potenzen von $x_{\star}-a$ fortschrriten. Ähnlich wie ehen schließen wir, daß $R(x-a)^{-}:$dann und nur dann für $x=a$ endlich bleibt, wenn die drei Reihen $s_{1} . s_{2}, s_{3}$ für $x=a$ endlich bleilın.

Wir haben also gefunden: Wir können eine Funktion $R$ in der Umgebung ron $\mathfrak{p}_{1}$ und $\mathfrak{p}_{2}$ in der Form schreiben

$$
\begin{aligned}
& R=(x-a)^{2} r_{1}+(x-a)^{\tau_{1}} r_{2}, \\
& R=(x-a)^{r^{3}} s_{1}-(x-a)^{\tau_{1}-1}{ }^{3} s_{2}+(x-a)^{\tau^{2}}{ }^{3} s_{3},
\end{aligned}
$$

wo die $r$ und $s$ nach ganzen Potenzen von $x$ - a fortschreitende Reihen sind. Die Funktion $R$ enthält dann und nur dann den Divisor $1_{1}^{T_{1}} \mathfrak{H}_{2}^{T_{2}}$, wenn die Reihen $r$ und $s$ für $x=a$ nicht unendlich werden. Es sei wieder (૬) irgendeine Basis. Wir setzen

$$
\Xi_{i_{1}}=(x-a)^{\circ} \mathfrak{1}_{1}-(x-a)^{\circ} p_{2} p_{1}, \quad(k=1,2 \ldots 5),
$$

w" $p_{1}$ und $p_{n 2}$ nach ganzen Potrnzen von $x$-a fortschreitende Potenzroihen sein sollen. Ersetzen wir hierin $(x-a)^{2}$ durch seinen anderen Wrert $-(x-a) \because$. so prhalten wir $\Xi: 2$, also

$$
\Xi_{2}=(-1)^{r}(x-a)-\left\{p_{1}-(x-a)^{2} p_{2}\right\}, \quad(k=1.2 \ldots 5) \text {. }
$$

Feruer setzen wir

(56) $\xi_{\llcorner 3}=(x-a)^{3}\left\{p_{13}+(x-a)^{3} p_{k 4}+(x-a)^{3} p_{3 i}, \quad(k=1,2, \ldots 5)\right.$, wo die $p_{i}$, nach ganzen Potenzen von $x$-- a fortschreitende Reihen sein sollen. Hivaus orhalten wir $\Xi_{4}$ und $\Xi_{5}$, indem wir $(x-a):$ durch seine beiden anderen Werte $\varepsilon(x-a)^{3}$ und $\varepsilon^{2}(x-a)^{\prime \prime}$ ersetzen, wo $\varepsilon$ cine primitive dritte Einheitswurzel ist. Also 


$$
\begin{aligned}
& \Sigma_{4}=\varepsilon^{r_{2}}(x-a)^{3}\left\{p_{k 3}+\varepsilon(x-a)^{3} p_{k 3}+\varepsilon^{2}(x-a)^{3} p_{k 5}\right\}, \\
& \Xi_{55}=\varepsilon^{2}(x-a)^{3}\left\{p_{k 3}+\varepsilon^{2}(x-a)^{3} p_{k 1}+\varepsilon(x-a)^{3} p_{k 5}\right\} .
\end{aligned}
$$

l) Be Beziehung zwischen dem System $\left(\xi_{k l}\right)$ und $\left(p_{k l}\right)$ können wir auch in folgender Weise sehreiben. Wir setzen

$$
\begin{aligned}
& r_{k_{1}}=p_{k 1}+(x-a)^{2} p_{k_{2}}, \\
& r_{k_{2}}=p_{k_{1}}-(x-a)^{2} p_{k \mathbf{2}}, \\
& r_{k 3}=p_{k 3}+(x-a)_{1}^{1} p_{i 4}+(x-a)_{2}^{3} p_{k 5}, \\
& r_{k 4}=p_{k 3}+\varepsilon(x-a)^{\frac{1}{3}} p_{k 4}+\varepsilon^{2}(x-a)^{3} p_{k 5} \text {, } \\
& r_{k 5}=p_{k 3}+\varepsilon^{2}(x-a)^{3} p_{k 4}+\varepsilon(x-a)^{3} p_{k 5} \text {. }
\end{aligned}
$$

Es wird dann

$$
\begin{aligned}
& \Xi_{k 1}=(x-a)^{2} r_{k 1}, \Xi_{k 2}^{r_{1}}=(-1)^{r_{1}}(x-a)^{2} r_{k 2}, \\
& \Xi_{k 3}=(x-a)^{\frac{r_{1}}{3}} r_{k 3}, \Xi_{k 4}=\varepsilon^{r_{2}}(x-a)^{3} r_{k 4}, \Xi_{k 5}=\varepsilon^{2 \tau_{2}}(x-a)^{\frac{\tau_{2}}{3}} r_{k 5} .
\end{aligned}
$$

Bezeichnen wir mit $A_{1}$ das Multiplikationssystem vòn $5^{2}$ Elementen, dessen Diagonale ist

$(x-a)^{2} . \quad(-1)^{\tau_{1}}(x-a)^{\gamma_{1}} . \quad(x-a)^{\tau_{3}}, \quad \varepsilon^{\tau_{2}}(x-a)^{\frac{\tau_{2}}{3}}, \quad \varepsilon^{2 \tau_{2}}(x-a)^{\frac{\tau_{2}}{3}}$, so ist

Setzen wir ferner

$$
\left(\xi_{k l l}\right)=A_{1}\left(r_{k l}\right)
$$

$$
\left\{\begin{array}{ccccc}
1 & (x-a)^{\frac{1}{2}} & 0 & 0 & 0 \\
1-(x-a)^{\frac{2}{2}} & 0 & 0 & 0 \\
0 & 0 & 1 & (x-a)^{\frac{1}{3}} & (x-a)^{\frac{2}{3}} \\
0 & 0 & 1 & \varepsilon(x-a)^{\frac{1}{3}} & \varepsilon^{2}(x-a)^{3} \\
0 & 0 & 1 & \varepsilon^{2}(x-a)^{\frac{2}{3}} & \varepsilon(x-a)^{\frac{2}{3}}
\end{array}\right\}=B
$$

$\rightarrow 0$ ist

und also

$$
\left(r_{k: l}\right)=B\left(p_{l: l}\right)
$$

$$
\left(\xi_{k l}\right)=A_{1} B\left(p_{h l}\right) \text {. }
$$

Das System $B$ ist aus zwei Systemen zusammengesetzt, nämlich aus einem System von $2^{2}$ und einem von $3^{2}$ Elementen, und zwar in der Weise, daß 
die Diagonalen der kleineren Systrin" sich zur Diagonale des Systems von $5^{2}$ Elmementen zasammensetzen und die fehlenden lilemente durch Nullen ersutzt sind. l)as System $B$ geht aus dem Systrin

$$
\left|\begin{array}{rrrrr}
1 & 1 & 0 & 0 & 0 \\
1 & -1 & 0 & 0 & 0 \\
0 & 0 & 1 & 1 & 1 \\
0 & 0 & 1 & \varepsilon & \varepsilon^{2} \\
0 & 0 & 1 & \varepsilon^{2} & 8
\end{array}\right|=A_{2}
$$

dadureh hervor, daß dessen Vertikalreihen der Keihe nach mit $1,(x-a)^{2}$, $1,(x-a)^{: 3},(x-a)^{3}$ multipliziert werden. Bezeichnen wir alsı das Multiplikationssystem, dessen Diagonalelemente $1,(x-a)^{2}, 1,(x-a)^{\frac{1}{3}}$, $(x-a)^{3}$ sind, mit $A_{3}$, so ist

und

$$
\begin{gathered}
B=A_{2} A_{3} \\
\left(\xi_{u}\right)=A_{1} A_{2} A_{3}\left(p_{! l}\right) .
\end{gathered}
$$

Das System $A_{2}$ ist geradeso wie $B$ aus zwei kleineren Systemen zusammengesetzt, nämlich aus den Systemen

$$
(a)=\left\{\begin{array}{cc}
1 & 1 \\
1 & -1
\end{array}\right\}, \quad(b)=\left\{\begin{array}{lll}
1 & 1 & 1 \\
1 & \varepsilon & \varepsilon \\
1 & \varepsilon^{2} & \varepsilon
\end{array}\right\} .
$$

Die Determinanten dieser beiden Systeme sind von Null verschieden, so da $B$ auch die Determinante von $A_{2}$, die nach rinem bekannten Determinantensatze gleich dem Produkt der Determinanten $a$ und $b$ ist, von Null verschirden ist. Wie die Systeme $(a)$ und $(b)$ gebildet sind, sieht man, wenn man sie folgendermaßen schreibt. Es seien $u_{1}=1$ und $u_{2}=-1$ die Wurzeln der Gleichung $z^{2}-1=0$ und $v_{1}=1, v_{2}=\varepsilon, v_{3}=\varepsilon^{2}$ die Wurzeln der Gleichung $z^{3}-1=0$. Dann ist

$$
(a)=\left\{\begin{array}{ll}
u_{1}^{0} & u_{1}^{1} \\
u_{2}^{\prime} & u !
\end{array} \mid, \quad(b)=\left\{\begin{array}{lll}
v_{1}^{0} & v_{1}^{1} & v_{1}^{2} \\
v_{2}^{0} & v_{2}^{1} & v_{3}^{*} \\
v_{0}^{0} & v_{3}^{1} & v_{3}^{\prime}
\end{array} \mid .\right.\right.
$$

Die Determinanten $a$ und $b$ sind also die Quadratwurzeln aus den Diskriminanten der Gleichungen $z^{2}-1=0$ und $z^{3}-1=0$.

Eine Funktion $R=g_{1} \Xi_{1}+g_{2} \Xi_{2}+\cdots+g_{5} \Xi_{5}$ hat in der Umgebung von $\psi_{1}$ die Darstellung

$$
\begin{array}{r}
R=(x-a)^{r_{1}}\left\{g_{1} p_{11}+g_{2} p_{21}+\cdots+g_{5} p_{51}\right\} \\
+(x-a)^{r_{1}+1}=\left\{g_{1} p_{12}+g_{2} p_{22}+\cdots+g_{5} p_{52}\right\}
\end{array}
$$

und in der Umgebung von $\$_{2}$ ist 


$$
\begin{array}{r}
R=(x-a)^{3} \quad\left\{g_{1} p_{13}+g_{2} p_{23}+\cdots+g_{5} p_{53}\right\} \\
+(x-a)^{\prime}:\left\{g_{1} p_{14}+g_{2} p_{24}+\cdots+g_{5} p_{54}\right\} \\
\left.+(x-a): \because g_{1} p_{15}+g_{2} p_{25}+\cdots+g_{5} p_{55}\right\} .
\end{array}
$$

lie Funktion $R$ enthält also dann und nur dann den Divisor $x_{1}^{r_{1}} w_{2}^{r}$, wenu die fünf Funktionen

$$
g_{1} p_{1:}+g_{2} p_{2 k}+\cdots+g_{5} p_{5 k},(k=1,2, \ldots 5)
$$

fiir $x=a$ nicht unendlich werden. Hieraus ergibt sich dann" ganz so wie im vorigen Paragraphen, daß die Basis ( $($ ) dann und nur dann normal für $x=a$ ist, wenn das System $\left(p_{h i}\right)$ eine Einheit für $x=a$ ist.

Aus (60) folgt

$$
\Xi_{u l}=A_{1} \cdot A_{2} \cdot \mid A_{3} \cdot p_{k l}=A_{2}(x-a)^{\tau_{1}+r_{2}} \cdot \frac{1}{2}: \because p_{k l},
$$

wo $A_{2}$ eine von Null versehiedene Konstante ist. Liegen an der Stelle $x=a$ allgemeiner drei Verzweigungspunkte von den Ordnungen $\alpha-1$, $\beta-1, \gamma-1$, so lautet der Exponent von $x-a$ in (62)

$$
\begin{aligned}
& t_{1}+r_{2}+r_{3}+\frac{1}{1}+\frac{2}{3}+\cdots+1-1+\frac{1}{3}+\frac{2}{\beta}+\cdots+\frac{\beta}{\beta}+\frac{1}{\gamma} \\
& +\frac{2}{y}+\cdots+y_{y}=r_{1}+r_{2}+r_{3}+\frac{1}{2}(1-1)+\frac{1}{2}(\beta-1)+\frac{1}{2}(\gamma-1)
\end{aligned}
$$

und es ist

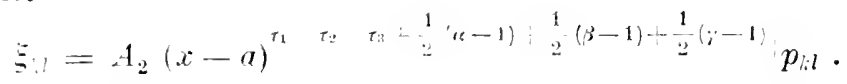

Ganz ähnlich wio in der vorigen Nummer haben wir

Satz XIl. An der Stelle $x=a$ mögen otwa drei Verzweigungspunkte der Ordnungen $x-1, \beta-1, y-1$ liegen. Die zu ihnen gelörenden Primteiler seien $p_{1}, f_{2}, f_{3}$ und es sei im livisor a gonau die $r_{i}$-te Potenz von pi enthalten. So ist die Basis (G) dann und nur dann normal für $x=a$ in bezug a uf 0 , wenn die $\xi_{i}$ den Divisor

$$
r_{1}^{r_{2}}+r_{2}^{T_{2}}+H_{3}^{r_{3}}
$$

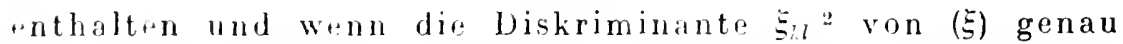
durch dic $\left[2\left(\tau_{1}+\tau_{2}+\tau_{3}\right)+(\alpha-1)+(\xi-1)+(\gamma-1)\right]$-te Potenz von $x-a$ trilbar ist.

Es sei nämlich erstens (乡) normal für $x=a$. So ist der Divisor (bif) in einer Funktion

$$
R=g_{1} \xi_{1}+g_{2} \xi_{2}+\cdots+g_{m} \xi_{m}
$$


dann und nur dann enthalten, wenn die $g$, lür $x=a$ endlich bleiben. Es sind also die Funktionen (61) dann und mur dann endlich für $x=a$, wenn dasselbe von den $g_{i}$ gilt. Daraus aber folgt nach XIX, Kap. VII 55 , daB $\left(p_{h l}\right)$ eine Einheit für $x=a$ ist. Aus (54) bis (57) vrgibt sich also, daß die $\xi_{i}$ den Divisor (64) enthalten, und aus (63), daß die Diskriminante von (5) $x-a$ genau in der angegehenen Potenz zum Faktor hat.

Ist zweitens der Vivisur $(64)$ in den $\check{\xi}_{i}$ enthalten und ist die Diskriminante genau durch die im Satze angegebene Potenz von $x-a$ teilbar, so folgt zunächst aus (54) bis (57), daß die $p_{k i}$ gewöhnliche Potenzreihen von $x-a$ sind. Dasselbe gilt dann von der Determinante $p_{k l}$ und aus 63) folgt, daß diese überdies eine Einheit für $x=a$ sein muB. D. h. aber, es ist $\left(p_{h l}\right)$ eine Einheit für $x=a$, womit bewiesen ist, daß (5) normal ist für $x=a$ in bezug auf $\mathfrak{q}$.

Ist $\left(p_{k l}\right)$ keine Einheit für $x=a$, so bestimmen wir wieder ein rationales System $S$, das für alle Stellen im Endlichen eine Einheit ist mit alleiniger Ausnahme von $x=a$, so daß

$$
\left(p_{k l}\right) S=\left(q_{i l}\right)
$$

aine Einheit für $x=a$ ist. Setzen wir dann

$$
(\eta)=(\xi) S,\left(\eta_{1}(l)=\left(\xi_{h l}\right) S,\right.
$$

so ergibt sich aus $(60),(65)$

$$
\left(v_{i l l}\right)=A_{1} A_{2} A_{3}\left(q_{i l}\right),
$$

und da $\left(q_{k l}\right)$ eine Einheit ist für $x=a$, so ist $\left(2_{1}\right)$ normal für $x=a$ und außerdem normal für alle Stellen, für dir (5) normal war, weil $S$ eine Einheit ist für alle Stellen, für die nicht $x=a$. Wir können also auch für solche Stellen $x=a$, wo Verzweigungspunkte liegen, aus einer Basis (5) eine neue bisis ( 1 ) herleiten, die normal ist, und zwar auch wieder, ohne an andern Stellen etwas zu verderben.

Damit ist aber gezeigt, wie wir aus irgendeiner Basis (G) eine Basis herleiten können, die an allen endlichen Stellen normal ist in bezug auf einen gegebenen Divisor, die also eine Basis für die Vielfachen von if ist.

Wir können jetzt noch folgenden Satz beweisen:

Satz XIll. Es gibt immer eine Funktion des Körpers $(x y)$, die an einer beliebigen Stelle genau von der ersten Ordnung Null wird.

Ist nämlich dir Basis (马) normal für $x=a$ in bezug auf q - und eine solche Basis gilut es immer, wie wir jetzt wissen - , so ist das System $\left(p_{? !}\right)$ in (54) bis (57) eine Einheit. Daraus folgt, da $B$ nicht alle $p_{\text {. }}$ und nicht alle $p_{k 3}$ gleich Null werden können für $x=a$, da sonst in $\mid p_{l l}$ eine Horizontalreihe durch $x-a$ teilbar sein würde und also auch $p_{k l}$ den Faktor 
$x$ - a hättp. Dann aher ist wegen (54) mindestens eine der Funktionen $\Xi_{11}$ genau durch $p_{1}^{r_{1}}$ und eine der Funktionen $\Xi_{k 3}$ wegen $(56)$ genau dureh $w^{r:}$ teilbar. Wählen wir $t_{1}=t_{2}=1$, so gibt es also eine Funktion des Körpers $(x y)$, die genan durch die erste Potenz von $p_{1}$, und eine, die genau durch die erste Potenz von $\mathrm{H}_{2}$ teilbar ist. Das gilt offenbar auch, wenn die Verhältnisse nicht so einfach liegen, wie in dem Fall, den wir zu unsern Beweisen benutzt haben.

Ferner folgt noch: Es können nicht alle Funktionen $\xi_{i}$ den Primteiler $\psi_{k}$ in einer höheren als der $t_{k}$-ten Potenz enthalten, wenn ( 5$)$ normal ist für $x=a$. Ist also ( $\zeta$ ) normal für alle endlichen Stellen von $x, \mathrm{~d}$. h. ist (引) eine Basis für die Vielfachen von q, so können nicht alle Funktionen $\xi_{\text {, }}$ einen Primteiler $w$ in höherer Potenz enthalten, als er in q enthalten ist. Das gilt auch für den Fall, wo der Primteiler in q nicht (oder in der nullten Potenz) enthalten ist. Denn der Fall, daß bei unsern Betrachtungen mehrere oder alle $\tau_{i}$ Null sind, ist ja nicht ausgeschlossen. Daher haben wir:

Satz XIV. Ist (今) eine Basis für die Vielfachen eines Divisors $q$, so ist q der größte gemeinsame Teiler der $\xi_{i}$, soweit es sich um Stellen handelt, wo $x$ endlich ist.

\section{\$ S. Herstellen einer Normalbasis. III.}

IIb. Wir betrachten zweitens noch den Fall, wo im Zähler von $x-a$ nur ein Primteiler enthalten ist, der mit $p$ bezeichnet sei. Bei $x=a$ liegt dann nur eine Stelle der Riemannschen Fläche, die also ein Verzweigungspunkt der Ordnung $m-1$ ist. Wir bezeichnen $m$ auch mit $\alpha$. Es sei $p$ in q genau in der Potenz $\tau$ enthalten. Wir schreiben dann in der Umgebung von $w$

$$
\xi_{k 1}=(x-a)^{\frac{\tau}{\alpha}}\left[p_{k 1}+(x-a)^{\frac{1}{\alpha}} p_{k 2}+\cdots+(x-a)^{\frac{a-1}{\alpha}} p_{k c}\right] \text {. }
$$

Aus dieser Gleichung ergibt sich für die konjungierten Funktionen die Darstellung

(67) $\xi_{k l}=\omega_{l}^{\tau}(x-a)^{a^{c}}\left[p_{k 1}+\omega_{l}^{1}(x-a)^{\frac{1}{\alpha}} p_{k 2}+\cdots+\omega_{l}^{a-1}(x-a)^{\frac{a-1}{\alpha}} p_{k \alpha}\right]$, wenn wir setzen

$$
\omega \omega_{l}=e^{\frac{2 \pi i l}{a}} \text {. }
$$

Die Gleichungen (66), (67) können wir in folgender Weise schreiben. Es seien bezeichnet mit $A_{1}$ und $A_{3}$ die Multiplikationssysteme mit den Diagonalen

$$
(x-a)^{\frac{\tau}{\omega}}, \omega_{1}^{\tau}(x-a)^{\frac{\tau}{\omega}}, \ldots \omega_{u-1}^{\tau}(x-a)^{\tau}
$$


Ferner sei

$$
\left.1,(x-a)^{1}, \ldots(x-a) "\right)^{\prime \prime} .
$$

Dann ist

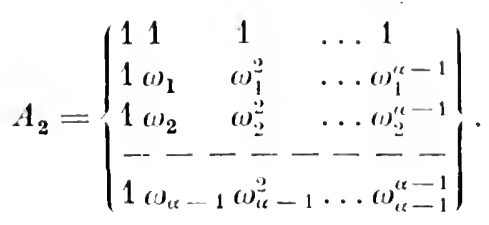

$$
\left(\xi_{k l}\right)=A_{1} A_{2} A_{3}\left(p_{k l}\right) \text {. }
$$

Eine Funktion $R=g_{1} \Xi_{1}+g_{2} \check{\Xi}_{2}+\cdots+g_{m} \Xi_{m}$ hat zufolge dieser Gleichungen in der Umgebung von $\mathfrak{p}$ die Darstellung

$$
\begin{aligned}
& R=(x-a)^{\frac{\tau}{\alpha}} \quad\left(g_{1} p_{\lambda \mathrm{\lambda}}+g_{2} p_{2 \mathrm{\alpha}}+\cdots+g_{\alpha} p_{\alpha_{1}}\right) \\
& +(x-a)^{\frac{\tau+1}{c}} \quad\left(g_{1} p_{12}+g_{2} p_{22}+\cdots+g_{c} p_{c 2}\right) \\
& +--\frac{-1}{\tau+a-1}--------
\end{aligned}
$$

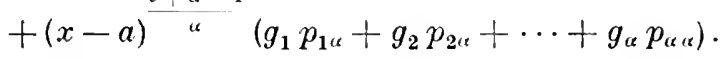

Die Funktion enthält also dann und nur dann den Divisor $\mathfrak{p}^{r}$, wenn die $\alpha$ Funktionen

$$
g_{1} p_{1 k}+g_{2} p_{2 k}+\cdots+g_{a k} p_{k a}, \quad(k=1,2, \ldots \alpha)
$$

für $x=a$ nicht unendlich werden. Ist das System $\left(p_{i r l}\right)$ eine Einheit für $x=a$, so ist das dann und nur dann der Fall, wie wir wissen, wenn die $g$ für $x=a$ nicht unendlich werden. Wenn aber $\left(p_{k l}\right)$ keine Einheit für $x=a$ ist, so können wir ein System $S$ so bestimmen, daß das System

$$
\left(q_{k l}\right)=\left(p_{k l}\right) S
$$

eine Einheit für $x=a$ ist. Dabei können wir $S$ so wählen, daß es für alle endlichen Stellen mit Ausnahme von $x=a$ eine Einheit ist. Definieren wir eine neue Basis durch die Gleichung

aus der folgt

$$
(\eta)=(\xi) S \text {, }
$$

$$
\left(\eta_{i l}\right)=\left(\xi_{k l}\right) S \text {, }
$$

so ergeben die Gleichungen (70), (71), (72)

$$
\left(\eta_{k l}\right)=A_{1} A_{2} A_{3}\left(q_{k l}\right) \text {. }
$$

Da aber $\left(q_{t: l}\right)$ eine Einheit für $x=a$ ist, so enthält eine Funktion $R=g_{1} \eta_{1}$ $+g_{2} \eta_{2}+\cdots+g_{m} \eta_{m}$ dann und nur dann $\mathcal{H}^{r}$, wenn die $g$ ganz sind; mit anderen Worten, die Basis $(\mathfrak{l})$ ist für $x=a$ normal in bezug auf $q$. Da die Substitution $S$, durch die $(\eta)$ aus $(\xi)$ hervorgeht, für alle endlichen 
vou a versehiedenen Stellen eine Einheit ist, so ist außerdem ( $y$ ) normal für alle Stellen, für die (引) es ist.

Aus der Gleichung (73) folgt durch C̈bergang zu den Determinanten

$$
y_{1}=\mid \begin{array}{ll:l|l}
A_{1} & A_{2} & A_{3} & q_{k l}
\end{array} .
$$

Da $A_{1}$ und $A_{3}$ Diagonalsysteme sind, so sind ihre Determinanten gleich dem Produkte ihrer Diagonalglieder, also nach (68) und (69) leicht zu berechnen. $A_{2}$ ist die Diskriminante der Gleichung $z^{t}-1=0$, also eine von Null verschiedene Konstante. Daher folgt aus (74)

$$
r_{k l}=\operatorname{konst}(x-a)^{r: "-1} \stackrel{2}{2} q_{k l} .
$$

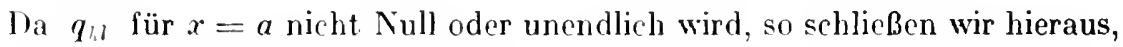
daß die Diskriminante $r_{k}^{2}$ genau durh dic $(2 t+a-1)$-te Potenz von $x-a$ teilbar ist.

\section{\$. D. Die Diskriminante einer Basis für die Vielfachen eines Dirisors $q$.}

1. Die Idealnorm. Definition V. Ist pein Primteiler und hat $x$ an der Stelle, zu der $\mathfrak{p}$ gehört, den endlichen Wert $a$, so heißt $x$-a die Idealnorm von $p$ in bezug auf $x$ oder auch kurz dir Idralnorm von $\%$. Wir schreiben

$$
N I(k)=x-a \text {. }
$$

Primteiler, dir zu Strllen gehören, wo $x$ unendlich wird, haben keine Idicalnorm.

l)iese Definition ïberträgt sich ohne welteres auf Divisoren. Ist

$$
\mathrm{r}=\mathrm{r}_{\mathrm{i}}^{\prime \prime}{ }_{i=1}^{\prime \prime 2} \ldots
$$

ain Divisur und nimmt $x$ an der Stelle des Primteilers $r_{i}$ den endlichen Wrert $a_{i}$ an, so ist die Idealnorm von ?

$$
N I(r)=\left(x-a_{1}\right)^{n_{1}}\left(x-a_{2}\right)^{j_{2}} \ldots\left(x-a_{i}\right)^{\prime \prime} \text {. }
$$

Satz XV. Die Idralnorm rines Divisors ist also immer eine rationalo Funktion von $r$, deren Ordnung - d. h. Differenz zwischen den Graden des Zälılers und Nenners - gleich der Ordnung des Divisors ist.

Es ist z. 13. die Idralnorm des Zählers von $x-a$ gleich $\left(x^{-}-a\right)^{m}$.

2. Die Diskriminante. Es sei q wieder rin Divisor, der nur Primtriler enthält, die zu endlichen Werten von $x$ gehören, und es sei $z^{\prime}$ der Teil des Verzweigungsdivisors von $x$, der zu den im Endlichen gelegenen Vrrzweigungsstellen gehört. Es sei $x=a$ irgendein endlicher Wert von $x$ und es mögen bei a die Primteiler $\mathfrak{p}_{1}, \mathfrak{k}_{2}, \ldots \mathfrak{p}_{r}$ liegen, und zwar sei $\mathfrak{p}_{i}$ 


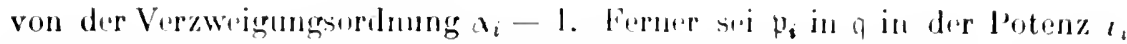

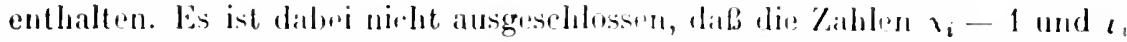
teilweise oder sämtlinh Null sind. lst (马) rine bissis für die Vielfachen

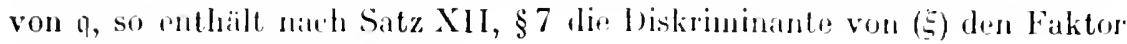
$(x-a)$ genilu in dor lotenz

$$
2 \pm \imath+\Sigma\left(x_{i}-I\right) \text {. }
$$

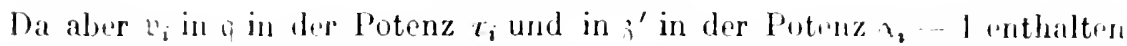
ist, so folgt, dab $(x-a)$ auch in der Idealnorm von $a^{2} z^{\prime}$ genau in der Potenz (76) molhalten ist. Da das für alle endlichen Stellen gilt, so folgt:

Satz XVI. Ist (引) eino Basis für die Vielfachen des Divisors $q$, so ist die Diskriminante

$$
\Xi_{: l}^{2}=\text { const. } N I\left(q^{2} z^{\prime}\right) \text {. }
$$

Vou diesem Satz gilt folgende Umkehrung:

Satz XVII. Ist ( $)$ eine basis und ist q der größte gemeinsame Teiler der $\xi_{i}$, soweit es sich um Stellen handelt, wo $x$ endich ist, und ist die Diskriminante

$$
\xi_{k l}^{2}=\text { const. } N I\left(q^{2} z^{\prime}\right),
$$

so ist ( $(\xi)$ eine Basis für die Vielfachen von q.

Denn wenn die Voraussetzungen dieses Satzes erfüllt sind, so sind für jede endliche Stelle $x=a$ die, Voraussetzungen des Satzes XII in $\S 7$ erfüllt, so daß ( 5 ) für jede endliche Stellen in bezug auf $q$ normal ist. Das ist aber nur ein anderer Ausdruck für die Behauptung.:

Nach dem Satz XV folgt aus (77) für den Fall, daß $z^{\prime}=-z$ ist:

Satz XVIII. Ist ( $\xi$ ) eine Basis für die Vielfachen eines Divisors $q$ von der Ordnung $q$ und liegt bei $x=\infty$ kein Verzweigungspunkt, so ist die Diskriminante von (ङ) von der Ordnung

$$
2 q+w
$$

wo $w$ die Verzweigungsordnung in bezug auf $x$ ist, oder, allders ausgedrückt, es wird die Diskriminante für unendliches $x$ von der Ordnung $2 q+w$ unendlich.

Ist ( $\xi$ ) irgendeine Basis, so gibt es nicht immer einen Divisor q von der Art, daB ( $\xi$ ) eine Basis für die Vielfachen von q wäre. Damit das der Fall ist, muß zunächst q nach Satz XIV in $\S 7$ der größte gemeinsame Teiler der $\xi_{i}$ sein, soweit die endlichen Werte von $x$ in Betracht kommen. Dann aber muB außerdem nach dem eben angegebenen Satze die Gleichung (77) bestehen. Das ist aber im allgemeinen keineswegs der Fall, wie folgendes einfache Beispiel lehrt. 
Es sei

$$
f(x, y) \quad y^{2}-x^{3}=0
$$

die den Körper $(x y)$ definierende Gleichung. Es ist

$$
\Xi_{1}=1, \Xi_{2}=y
$$

rine Basis. Ther größte gomeisame Triler von $\xi_{1}$ und $\xi_{2}$ ist $q=1$. Im Endlichen ist nur eine Verzweigungsstelle hei $x=0$, und zwar von der

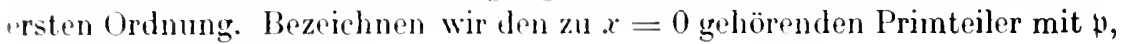
so ist also $i^{\prime}=p$ und

l)agegen ist

$$
N I\left(q^{2} i^{\prime}\right)=N I(\beta)=x .
$$

$$
5: l^{2}=\left|\begin{array}{l}
1 \\
1-y
\end{array}\right|^{2}=4 y^{2}=4 x^{3} \text {. }
$$

Es sei

\section{$\$$ 10. Beispiel 1 .}

$$
f(x, y)=x^{2}+y^{2}-1=0 .
$$

1)ie über der $x$-Ebene ausgebreitete Riemannsche Fläche ist zweiblättrig. Da $y=\mid 1-x^{2}$, so liegen bei $x=1$ und $x=-1$ je ein Verzweigungspunkt erster Ordnung. Wir bezeichnen die zugehörigen Primteiler mit $v_{1}, \mathrm{r}_{2}$. Es ist der Verzweigungsdivisor in bezug auf $x$

ınd die Idealnorm von $z$

$$
\hat{\jmath}=\mathfrak{v}_{1} \mathfrak{v}_{2}
$$

$$
N I(\mathfrak{z})=x^{2}-1 \text {. }
$$

An der Stelle $x=0$ liegen zwei Punkte der Riemannschen Fläche, $n_{1}$ und $H_{2}$. Die Entwicklungen von $y$ sind in ihrer Umgebung

$$
y=1-\frac{1}{2} x^{2}-\frac{1}{8} x^{4}+\cdots, \quad y=-1+\frac{1}{2} x^{2}+\frac{1}{8} x^{4}+\cdots .
$$

Es sei

$$
\mathfrak{q}=H_{1}^{-2}+n_{2}^{1} \mathfrak{v}_{1}^{-1} \text {. }
$$

Wir gehen aus von der Basis $(\xi)=(1, y)$. Das zugehörige System ist

$$
(\xi)=\left(\begin{array}{ll}
1, & y \\
1,-y
\end{array}\right)
$$

Jas System $\left(\xi_{01}\right)$ müssen wir betrachten in der Umgebung derjenigen Stellen $x$, an denm es nicht normal ist in bezug auf $q$. Das sind zunächst diejenigen Stellen, die einen Beitrag zu q liefern, also $x=0$ und $x=1$. Dat die Funktionen $\xi_{k l}$ für endliche Wrate von $x$ nicht unendlich werden, so kommen nur noch in Frage die Nullstellen der Jiskriminante $\mid \xi_{k l}{ }^{2}=4 y^{2}$ $=4\left(1-x^{2}\right)$, also außer der schon genannten Stelle $x=1$ noch die Stelle 
$x=-1$. Ls ist aber $5 / l^{2}$ genau dureh $N I\left(\mathrm{v}_{2}\right)$ teilbar, also normal in bezug auf $\mathrm{q}$ an der Stelle $x=-1$, da ja dir $5: l$ fiir $x=-1$ endlich sind. Das System ( ()$\left._{1}\right)$ ist also nur in der Umgebung von $x=0$ und $x=1 \mathrm{zu}$ betrachten. An allen anderen Stellen ist es normal in bezug auf $q$.

Wir betrachton $\left(\xi_{k_{l}}\right)$ zunächst in der Umgobung vou $x=0$ und setzen

$$
\begin{aligned}
& 1=x^{-2} \cdot x^{2}, \quad y=x^{-2}\left[x^{2}-\frac{1}{2} x^{4}+\cdots\right] . \\
& 1=x \cdot x^{-1}, \quad-y=x\left[-x^{-1}+\frac{1}{2} x+\frac{1}{8} x^{3}+\cdots\right] .
\end{aligned}
$$

Es ist also

$$
\left(p_{k l}\right)=\left\{\begin{array}{l}
x^{2}, x^{2}-\frac{1}{2} x^{4}+\cdots \\
x^{-1},-x^{-1}+\frac{1}{2} x+\frac{1}{8} x^{3}+\cdots
\end{array} \mid .\right.
$$

Wir haben eine Substitution $S$ zu suchen, die $\left(p_{l: l}\right)$ in eine Einheit für $x=0$ überführt. Diese entnehmen wir aus dem Beispiel I in $\S 6$ des Kap. VII. Janach können wir nehmen

Die Funktionen

$$
S=\left\{\begin{array}{l}
x, x^{-2}-\frac{1}{2} \\
0, x^{-2}
\end{array} \mid .\right.
$$

$$
\begin{aligned}
& \eta_{1}=x \xi_{1}+0 \cdot \xi_{2}=x, \\
& r_{2}=\left(x^{-2}-\frac{1}{2}\right) \xi_{1}+x^{-2} \xi_{2}=x^{-2}-\frac{1}{2}+x^{-2} y=\frac{1-\frac{1}{2} x^{2}+y}{x^{2}}
\end{aligned}
$$

bilden eine Basis für die Vielfachen von $\mathfrak{q}$, die normal ist für $x=0$. Es bleibt noch der Punkt $x=1$ zu betrachten. Wir haben in der Umgebung voln $x=1$

$$
\begin{aligned}
& y_{1}=1+(x-1), \\
& \eta_{2}=\frac{1}{2}-2(x-1)+\cdots+i \mid \overline{2}(x-1)^{\frac{1}{2}}\left[1-\frac{7}{4}(x-1)+\cdots\right] .
\end{aligned}
$$

Wir setzen

$$
\begin{aligned}
& y_{i 1}=0 \cdot(x-1)^{-\frac{1}{2}}+1+(x-1), \\
& r_{i 2}=i \mid 2\left[x-1-\frac{7}{4}(x-1)^{2}+\cdots \mid(x-1)^{-\frac{1}{2}}+\left[\frac{1}{2}-2(x-1)+\cdots\right] .\right.
\end{aligned}
$$

Es ist jetzt

$$
\left(p_{k l}\right)=\left\{\begin{array}{lc}
0 & i \mid 2\left[(x-1)-\frac{7}{4}(x-1)^{2}+\cdots\right] \\
1+(x-1) & \frac{1}{2}-2(x-1)+\cdots
\end{array}\right\} .
$$




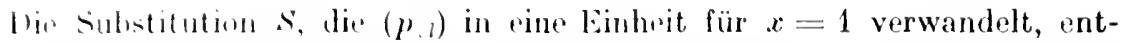
nehmon wir dem beispiel II in $\$ 6$ des Kap. VII. Der Falitor $i \sqrt{2}$ in der ersten $Z$ teile des hier auftretenden Systems $\left(p_{i l}\right)$ ist ohne EinfluB. Es ist

bin Funktione'n

$$
s=\left\{\begin{array}{ll}
1, & 1 \\
0, & (x-1)-1 \\
0, & (x-1)-1
\end{array} \mid .\right.
$$

$$
\ddot{g}_{1}=1 \cdot 1_{1}+0 \cdot 1_{2}, \quad \because=2=-\frac{1}{2}(x-1)^{-1} \cdot 1_{1}+(x-1)^{-1} \eta_{2}
$$

bilden eine Basis für die Vielfachen von 1 . Setzen wir die Werte von $\eta_{\mathbf{1}}$ und $r_{2}$ ein. so folgt

$$
\therefore=x, \begin{gathered}
=2 \\
=1=2
\end{gathered} \quad \begin{gathered}
1-\frac{1}{2} x^{2}-\frac{1}{2} x^{3}+y \\
x^{2}(x-1)
\end{gathered} .
$$

Las Ergebnis ist in voller Übereinstimmung mit dem früher in $\S 2$ gefundenen, wo wir dies Beispiel auf andere Art behandelt haben.

Wir berechnen norh die Diskriminante der Basis (־). Es ist

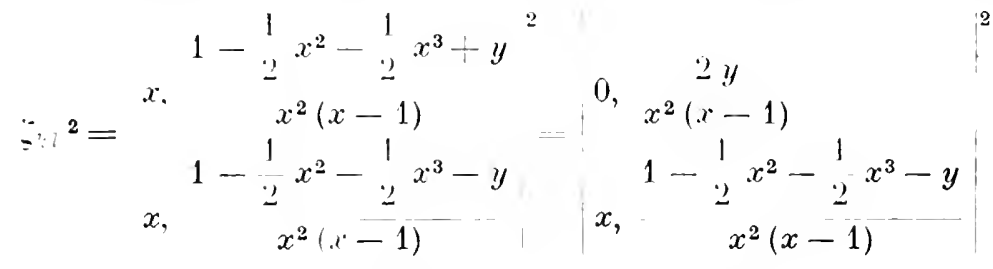

$$
\begin{aligned}
& =4 \begin{array}{c}
y^{2} \\
x^{2}(x-1)^{2}
\end{array}=-4 \frac{x+1}{x^{2}(x-1)} .
\end{aligned}
$$

Anderseits ist

und

$$
\begin{gathered}
N I\left(\mathfrak{p}_{1}\right)=x, \quad N I\left(\mathfrak{p}_{2}\right)=x, \quad N I\left(\mathfrak{b}_{1}\right)=x-1, \quad \text { also } \\
N I(\mathfrak{q})=\begin{array}{c}
1 \\
x(x-1)
\end{array}
\end{gathered}
$$

$$
[N I(q)]^{2} N I(z)=\frac{x+1}{x^{2}(x-1)},
$$

also, wio es sein muß, bis auf einen konstanten Faktor gleich der Diskriminante von $(\zeta)$.

Wir wollen auch noch eine Basis für die Vielfachen des Divisors

$$
x=4 n_{2}^{-1} n_{2}^{-1}
$$

bestimmen. Wir gehen wieder aus von der Basis $(1, y)$. Auch hier baben wir nur zwei Stellen, nämlich $x=0$ und $x=-1$, zu untersuchen. 
In der Umgebung von $x=0$ schereiben wir

$$
\begin{gathered}
1=x^{2} \cdot x^{2}, \quad y=x^{2}\left(x^{-2}-\frac{1}{2}-\frac{1}{8} x^{2}+\cdots\right), \\
1=x^{-1} \cdot x . \quad-y=x^{-1}\left(-x+\frac{1}{2} x^{3}+\frac{1}{8} x^{5}+\cdots\right),
\end{gathered}
$$

so daß

$$
\left(p_{i, 1}\right)=\left\{\begin{array}{l}
x^{-2} \quad x^{-2}-\frac{1}{2}-\frac{1}{8} x^{2}+\cdots \\
x-x+\frac{1}{2} x^{3}+\frac{1}{8} x^{5}+\cdots
\end{array}\right\} .
$$

Um $\left(p_{t: l}\right)$ in rine Einheit für $x=0$ zu verwandeln, wenden wir folgende Substitutionen an:

1. Wir multiplizieren $\left(p_{k l}\right)$ mit $x^{2}$.

$$
S_{1}=\left(\begin{array}{cc}
x^{2} & 0 \\
0 & x^{2}
\end{array}\right), \quad\left(p_{k l}\right) S_{1}=\left\{\begin{array}{c}
1, \quad 1-\frac{1}{2} x^{2}-\frac{1}{8} x^{4}+\cdots \\
x^{3},-x^{3}+\frac{1}{2} x^{5}+\cdots
\end{array}\right\} .
$$

2. Wir subtrahieren das $\left(1-\frac{1}{2} x^{2}\right)$-fache der ersten Vertikalreihe von der zweiten.

$$
S_{2}=\left|\begin{array}{l}
1,-1+\frac{1}{2} x^{2} \\
1
\end{array}\right|, \quad\left(p_{l, b}\right) S_{1} S_{2}=\left|\begin{array}{l}
1,-\frac{1}{8} x^{4}+\cdots \\
x^{3},-2 x^{3}+x^{5}+\ldots
\end{array}\right| .
$$

3. Wir dividieren die zweite Vertikalreihe mit $x^{3}$.

$$
S_{3}=\left(\begin{array}{ll}
1 & 0 \\
0 & x^{-3}
\end{array}\right) . \quad\left(p_{l: l}\right) S_{1} S_{2} S_{3}=\left\{\begin{array}{cc}
1, & -\frac{1}{8} x+\cdots \\
x^{3} & -2+\cdots
\end{array} \mid,\right.
$$

womit wir am Zirle sind, da die Determinante von $\left(p_{h l}\right) S_{1} S_{2} S_{3}$ für $x=0$ nicht mehr Null wird. Es wird

$$
S=S_{1} S_{2} S_{3}=\left\{\begin{array}{rr}
x^{2}, & -x^{-1}+\begin{array}{r}
1 \\
2 \\
0,
\end{array} \\
0, & x^{-1}
\end{array} \mid .\right.
$$

Die Basis $\left({ }_{i}{ }^{\prime}\right)=(1, y) S$, also

$$
\eta_{1}^{\prime}=x^{2}, \quad i_{2}^{\prime}=\left(-x^{-1}+\frac{1}{2} x\right)+x^{-1} y=\frac{-2+x^{2}+2 y}{2 x}
$$

ist daher normal für $x=0$ in bezug auf $\mathrm{r}$. 
In der Imgehung von $x=1$ set

$$
\begin{aligned}
& \therefore=(x+1)^{-2} \cdot 0+1 \cdot\left[1-2(x \quad 1)+(x+1)^{2}\right], \\
& \therefore=(x+1)=\left|-12(x+1) \quad 3 / 2(x+1)^{2}+\cdots\right|+ \\
& 1 \cdot\left|\begin{array}{l}
1 \\
2
\end{array}+\frac{3}{2}(x+1)+\cdots\right|
\end{aligned}
$$

so daB

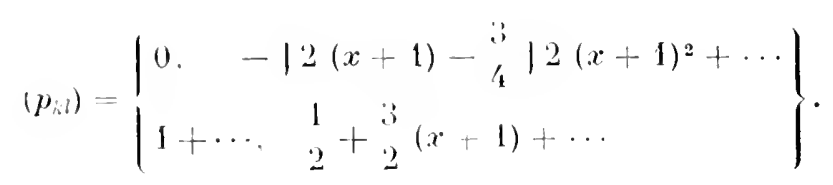

Dir anzuwendenden Substitutionen sind:

1. Subtraktion des $\frac{1}{2}$-fachen der ersten Vertikalreihe von der zweiten.

2. Division der zwriten Vertikalreihe durch $(x+1)$.

$$
S_{1}=\left\{\begin{array}{lr}
1 & -1 \\
0 & 2 \\
0 & 1
\end{array}\right\}, S_{2}=\left\{\begin{array}{lr}
1 . & 0 \\
0,(x+1)^{-1}
\end{array}\right\}, S=S_{1} S_{2}=\left\{\begin{array}{rr}
1, & -\frac{1}{2}(x+1)^{-1} \\
0, & (x+1)^{-1}
\end{array}\right\} .
$$

Es ist also $\left(\breve{\prime}^{\prime}\right)=\left(y^{\prime}\right) S$ oder

$$
\zeta_{1}^{\prime}=x^{2}, \quad \zeta_{2}^{\prime}=-\frac{1}{2}(x+1)^{-1} \eta_{1}^{\prime}+(x+1)^{-1} \eta_{2}^{\prime}=\frac{-2+x^{2}-x^{3}+2 y}{2 x(1+x)}
$$

eine Basis für die Vielfachen von $r$.

\section{Es sei}

\section{\$11. Beispiel 2.}

$$
f(x, y)=x^{6}+y^{3}-1=0 .
$$

Die über der $x$-Eben ausgebreitete Fläche ist dreiblättrig. Aus $y=\sqrt[3]{1-x^{6}}$ ersehen wir, daß wir sechs Verzweigungspunkte zweiter Ordnung haben, die Nullstellen von $x^{6}-1$. Wir bezeirhnen diese Stellen und die zugehörigen Primteiler mit $\mathfrak{v}_{1}, \mathfrak{v}_{2}, \ldots \mathfrak{v}_{6}$. Im besonderen gehöre $\mathfrak{v}_{1}$ zu der Stelle $x=1$.

Es wird

$$
;=v_{1}^{2} \mathfrak{v}_{2}^{2} \cdots v_{6}^{2}, \quad N I(3)=\left(x^{6}-1\right)^{2} .
$$

An der Stelle $x=0$ haben wir drei Punkte der Riemannschen Fläche übereinander. Die zugehörigen Primteiler seien $\mathfrak{p}_{1}, \mathfrak{p}_{2}, \mathfrak{p}_{3}$. Die Entwicklungen von $y$ sind in ihrer Umgebung 
$y=1-\frac{1}{3} x^{a}+\cdots, \quad y=8-\varepsilon_{3}^{t} x^{6} \cdots, \quad y \quad \varepsilon^{2} \quad \varepsilon^{2} x^{6}+\cdots$,

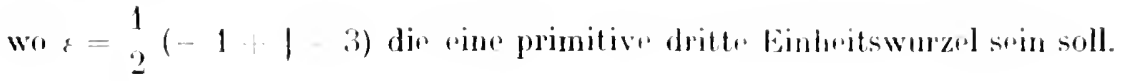

Es soi

$$
q=v_{1}^{-1} p_{1} p_{3}^{2} \text {. }
$$

Wir gehen aus von der Basis $(\xi)=\left(1, y, y^{2}\right)$, so daB

$$
\left(\Xi_{k l}\right)=\left\{\begin{array}{ccc}
1 & y & y^{2} \\
1 & \varepsilon y & \varepsilon^{2} y^{2} \\
1 & \varepsilon^{2} y & \varepsilon y^{2}
\end{array}\right\} .
$$

Es wird $\left|\xi_{h l}\right|^{2}=\operatorname{konst} y^{6}=\operatorname{kmnst}\left(1-x^{6}\right)^{2}$. Da die $\xi_{k l}$ für endliches $x$ nirgends unendlich werden, so ist das System $\left(\xi_{k l l}\right)$ nur $z, 2$ untersuchen in der Ungebung der Stellen $x=0, x=1$, die einen Beitrag zu q liefern, der Nullstellen von $\left|\xi_{h l}\right|^{2}$ und der Verzweigungsstellen. In unserm Falle wird $\left|\Sigma_{n l}\right|^{2}$ nur an den Verzweigungstellen Null, und an diesen genau so wie $N I(\xi)$. Hieraus schließen wir, da die $\xi_{k l}$ an diesen Stellen endlich bleiben, daß die Basis ( $\xi)$ an diesen Stellen normal ist in bezug auf $q$, soweit sie nicht einen Beitrag zu q liefern. Die einzigen Stellen, an denen

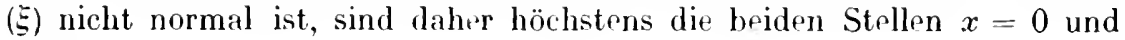
$x=1$.

In der Umgrbung von $x=1$ haben wir zu setzen

$$
\begin{aligned}
& \xi_{1}=1=(x-1)^{-\frac{1}{3}} \cdot 0+1 \cdot 1+(x-1)^{3} \cdot 0, \\
& \xi_{2}=y=(x-1)^{\frac{1}{3}} \cdot 0+1 \cdot 0+(x-1)^{\frac{1}{3}} \cdot|-| \frac{3}{6}+\cdots \mid, \\
& \xi_{3}=y^{2}=(x-1)^{-\frac{1}{3}}|3| 36(x-1)+\cdots \mid+1 \cdot 0+(x-1)^{\frac{1}{3}} \cdot 0 .
\end{aligned}
$$

Es ist also

$$
\left(p_{l . l}\right)=\left\{\begin{array}{ccc}
0 & 0 & 336(x-1)+\cdots \\
1 & 0 & 0 \\
0 & -16+\cdots & 0
\end{array}\right\} .
$$

Wir brauchen nur die letzte Vertikalreihe mit $x-1$ zu dividieren, um durch rine erlaubte Transformation das System $\left(p_{k l}\right)$ in eine Einheit für $x=1$ zu verwandeln. Das System $S$ ist ein Diagonalsystem mit der Diagonale $1,1,(x-1)^{-1}$. Die neue Basis $(\eta)$ ist

$$
\eta_{1}=\Xi_{1}=1, \quad \eta_{2}=\Xi_{2}=y, \quad \eta_{3}=\Xi_{3}(1-x)^{-1} \quad \frac{y^{2}}{1-x} .
$$

In der Umgehung von $x=0$ haben wir zu setzen 
$\left.\left.1_{11}=x \cdot x^{-1}, \quad\right\}_{21}=x\left(x-1-\frac{1}{3} x^{5}+\cdots\right), \quad\right\}_{31}=x\left(x^{-1}+1+x+\cdots\right)$, $1,13=1 \cdot 1 . \quad 1,22=1 \cdot\left(\varepsilon-\frac{3}{3} x^{6}+\cdots\right) . \quad 1,32=\varepsilon^{2}\left(1+x+x^{2}+\cdots\right)$, $1_{13}=x^{2} \cdot x^{-2}, \quad 1_{123}=x^{2}\left(\varepsilon^{2} x-r_{3}^{2} x^{4}+\cdots\right), 1_{33}=\varepsilon x^{2}\left(x^{-2}+x^{-1}+\cdots\right)$.

Es ist also jetzt $\left(p_{. l}\right)$ das System, das in Beispiel 111 in Kap. VII, $§ 6$ behandelt ist. Wir entnehmen von dort die erlanbte Substitution $S$, die $\left(p_{\wedge}\right)$ in , ine Einheit für $x=0$ verwandelt. Aus ihr folgt, da $B$ die Funktionen

$$
\Sigma_{1}=x^{2} \imath_{11}, \quad \zeta_{2}=-\varepsilon^{2} x^{2} \imath_{1}+x \imath_{12}, \quad \zeta_{3}=\left(\varepsilon^{2}-\varepsilon x\right) \eta_{1}+\varepsilon \eta_{2}+\eta_{3}
$$

eine Basis für die Vielfachen von a bilden. Es wird

$$
\check{S}_{1}=x^{2} . \quad \check{\zeta}_{2}=-\varepsilon^{2} x^{2}+x y, \quad \check{\zeta}_{3}=\left(\varepsilon^{2}-\varepsilon x\right)+\varepsilon y+\frac{y^{2}}{1-x} .
$$

Es muBsin

$$
5_{n i l}^{2}=\operatorname{konst}[N I(a)]^{2} N I(\xi)=\text { konst } x^{6} \frac{\left(1-x^{6}\right)^{2}}{(1-x)^{2}},
$$

was man leicht bestätigt.

\section{\$12. Beispiel 3 .}

Es sei

$$
f(x . y)=y^{3}-3 x y+2 x^{2}=0 .
$$

Lie über der $x$-Ebene ausgebreitete Riemannsche Fläche ist dreiblättrig. Die Diskriminante der Gleichung $f(x, y)=0$ in bezug auf $y$ ist $x^{3}(x-1)$. Verzwigungspunkte können daher nur sein bei $x=0, x=1, x=\infty$. Bei $x=0$ haben wir die Entwicklungen

$$
\begin{aligned}
& y_{1}=13 x^{\frac{1}{2}}-\frac{1}{3} x-\frac{15}{18} x^{2}-{ }^{4} x^{2}-\frac{35 \cdot 13}{8 \cdot 243} x^{\frac{5}{2}}+\cdots, \\
& y_{2}=-13 x^{2}-\frac{1}{3} x+\frac{13}{18} x^{2}-4 x^{4}-\frac{35 \cdot 13}{8 \cdot 243} x^{\frac{5}{2}}+\cdots \\
& y_{3} \quad \frac{2}{3} x+\frac{8}{81} x^{2}+\frac{32}{729} x^{3}-\cdots .
\end{aligned}
$$

Wir habm also bei $x=0$ einen Verzweigungspunkt erster Ordnung, den wir mit $v_{1}$. und einen gewöhnlichen Punkt, den wir mit $p$ bezeichnen. Bui $x=1$ haben wir ebenfalls einen Verzweigungspunkt erster Ordnung, der mit $\mathrm{r}_{2}$ bezeichnet sei, und einen gewönnlichen Punkt. Schließlich liegt bei $x=x$ ein Verzweigungspunkt zweiter Ordnung, den wir $\mathfrak{v}_{3}$ nennen. Es wird

$$
z=\mathfrak{v}_{1} \mathfrak{z}_{2}, \quad i^{\prime}=b_{1} \mathfrak{v}_{2}, \quad N I\left(z^{\prime}\right)=x(x-1) \text {. }
$$


Iss sil

$9 v_{1}^{3}$.

Wir gohen aun vou der Basis $(\xi)=\left(1, y, y^{2}\right)$. Wir Funktion y und damit

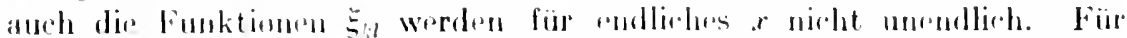
$x=1$ ist $\left(1, y, y^{2}\right)$ mormal. Es hloiht mur dir Stolle $x \quad 0$ zu hetrachten.

llier hisken wis \%us soten

$$
\begin{aligned}
& \Xi_{11}=1=x \quad: \quad 20+x^{2} \cdot x^{2}, \\
& \Xi_{21}=y=x^{-3}\left|13 x^{-1}-\frac{13}{18}+(1)\right|+x^{2}\left|\begin{array}{c}
1 \\
3
\end{array} x^{-1}-4^{4}+(1)\right| \text {, } \\
& \Xi_{31}=y^{2}=x^{\frac{3}{3}}\left|-\frac{2}{3}\right| 3-\frac{5}{81}|3 x+(2)|+x^{2}\left[3 x^{-1}-\frac{2}{9}-\frac{16}{243} x+(2)\right] \text {, } \\
& \xi_{13}=1, \quad \xi_{23}=\frac{2}{3} x+\frac{8}{81} x^{2}+(3), \quad \xi_{33}={ }_{9}^{4} x^{2}+(3) \text {. }
\end{aligned}
$$

Es ist daher das System $\left(p_{i l}\right)$ in diesem Falle im wesentlichen gleich dem im Beispiel IV in Kap. VII, $\$ 6$ behandelten. Wir entuelimen dort das System $S$. Es bilden dir Funktionen

$$
\xi_{1}=x^{2} \xi_{1}, \quad \check{s}_{2}=\left(-\frac{9}{2}+\frac{2}{3} x\right) \xi_{1}+\xi_{2}+\frac{3}{2} x^{-1} \xi_{3}, \quad \check{s}_{3}=-3 x \xi_{1}+\xi_{3}
$$

eine Basis für die Vielfachen von q. Es wird

$$
\check{s}_{1}=x^{2}, \quad \check{s}_{2}=-\frac{9}{2}+\frac{2}{3} x+y+\frac{3}{2} y^{2}, \quad \dot{s}_{3}=-3 x y+y^{2} .
$$

Ferner mub sein

$$
\left.\ddot{s}_{k l l}\right|^{2}=\operatorname{konst}[N I(q)]^{2} N I\left(i^{\prime}\right)=\text { konst } \cdot x^{2}(1-x) \text {, }
$$

was man loicht bestätigt.

\section{$\$ 13$. Komplementäre Basen.}

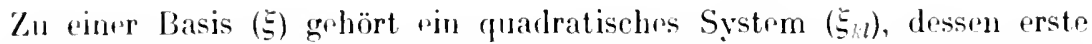
Zeile die Funtionen $\xi_{i}=\xi_{i}$ enthält, während die andern Reihen die zu den $\Xi_{i,}$ konjugierten Funktionen enthalten. Es liegt nahe, zu $\left(\Xi_{. l}\right)$ das $\mathbf{r}^{2-}$ ziproke System zu bilden und zu sehen, ob das nicht auch zu einer Basis gehört. Wir nohmen zunächst ein einfaches Brispiel. Es sei der Körpir $(x y)$ definiert durch

$$
f(x, y) \quad y^{2}-x=0
$$

und es sei $\xi_{1}=1, \xi_{2}=y$, also

$$
\left(\xi_{k i l}\right)=\left(\begin{array}{rr}
1, & y \\
1, & -y
\end{array}\right)
$$


1) a $\Xi_{k 1}=-2$ ! nicht identisch Null ist. so ist $(\xi)=(1, y)$ eine Basis. Das reziproke System zu $\left(\Xi_{k l}\right)$ sei mit $\left(\eta_{k: l}\right)$ bezeichnet. Es wird

$$
\left(\boldsymbol{y}_{k l}\right)=\left\{\begin{array}{cc}
1 & 1 \\
2 & 2 \\
1 & 1 \\
2 y, & 2 y
\end{array}\right\} .
$$

Di. Weterminante von $\left(\eta_{k l}\right)$ ist natürlich nicht identisch Null, da sie zu der von $\left(\Xi_{h l}\right)$ reziprok ist. Es gehört aber $(\eta, i l)$ doch nicht zu einer Basis, da nicht in jeder Vertikalreihe zueinander konjugierte Funktionen stehen. Wenn wir aber in $\left(\eta_{k i l}\right)$ die Zeilen mit den Spalten vertauschen, also das adjungierte System zu $\left(\eta_{k l l}\right)$ und das komplementäre zu $\left(\xi_{k l}\right)$ bilden, so erhalten wir ein System, das zu einer Basis gehört. Wir bezeichnen es mit $\left(\Sigma_{l !}^{\prime}\right)$. Es ist

$$
\left(\xi_{1}^{\prime}\right)=\left|\begin{array}{cc}
1 & 1 \\
2 & 2 y \\
1 & 1 \\
2 & -2 y
\end{array}\right|
$$

nudl dies gehört offonbar zu der Basis

$$
\left(\Xi^{\prime}\right)=\left(\begin{array}{cc}
1 & 1 \\
2 & 2 y
\end{array}\right) .
$$

Das ist num nicht nur in dem Beispiel so. Es gilt nämlich der Satz: Satz XIX. Es sei ( $)$ eineBasis undes sei $\left(\xi_{l l}^{\prime}\right)$ das zu $\left(\xi_{h l}\right)$ komplementäre System. Dann bilden die Funktionen

$$
\xi_{1}^{\prime}=\xi_{11}^{\prime}, \quad \xi_{2}^{\prime}=\xi_{21}^{\prime}, \ldots \quad \xi_{m}^{\prime}=\xi_{m 1}^{\prime}
$$

pine Basis (况) undes ist $\left(\xi_{n}^{\prime}\right)$ das zu dieser Basis gohörende quadratische System. Die beiden Systeme (ङ) und (ङ) heißen zurinander komplementär.

Div Dotrminante von $\left(\xi_{k l}^{\prime}\right)$ ist reziprok $z u$ der von $\left(\xi_{i l l}\right)$, also nicht identisch Null. Es bleibt noch zu zeigen, daß die $m$ Funktionen ( $\left.\xi_{k}^{\prime}\right)$ Funktionen des Körpers $(x y)$ sind und da $B$ in $\left(\xi_{k l}^{\prime}\right)$ in jeder Spalte $m$ konjugierte Funktionen stehen. Dazu beweisen wir zunächst folgenden

Hilfssatz. Es seien $y_{1}, y_{2}, \ldots y_{m}$ die $m$ konjugierten Werte von $y_{1}$. Jann ist eine rationale Funktion von $x, y_{1}, y_{2}, \ldots y_{m}$, die in den $m-1$ Funktionen $y_{2}, y_{3}, \ldots$ symmetrisch ist, darstellbar als rationale Funktion von $x$ und $y_{1}$. 
Die Größen $y_{1}, y_{2}, \ldots y_{m}$ genügenn der Gilrichung

$$
\begin{array}{ll}
f(x, y) \quad a_{0}(x) y^{m}+a_{1}(x) y^{m-1}+\cdots+a_{m}(x) \\
& a_{0}\left(y-y_{1}\right)\left(y-y_{2}\right) \cdots\left(y-y_{m}\right)=0 ;
\end{array}
$$

also genügen $y_{2}, y_{3}, \ldots y_{m}$ der Gleichung

$$
g(y) \quad \begin{aligned}
& f(x, y) \\
& y-y_{1}
\end{aligned} \quad a_{0}\left(y-y_{2}\right)\left(y-y_{3}\right) \cdots\left(y-y_{m}\right)=0 .
$$

Diese Gleichung können wir uns folgnndermaßen berstellen. Da $f\left(x, y_{1}\right) \quad 0$, so ist

$$
\begin{aligned}
& g(y) \quad \begin{array}{ccc}
f(x, y)-f\left(x, y_{1}\right) \\
y-y_{1}
\end{array} \quad \begin{array}{c}
y-y_{1} \\
y-y_{1}
\end{array} \quad \begin{array}{c}
y^{2}-y_{1}^{2} \\
y-y_{1}
\end{array}+\cdots+a_{0} y^{\prime \prime \prime}-y_{1}^{m} \\
& a_{m-1}+a_{m-2}\left(y+y_{1}\right)+\cdots+a_{0}\left(y^{m-1}+y^{m-2} y_{1}+\cdots+y_{1}^{m-1}\right) .
\end{aligned}
$$

Hieraus folgt, daß die Koeffizienten der Gleichung $g(y)=0$ rationale Funktionen von $x$ und $y_{1}$ sind. Nach dem Fundamentalsatze über rationale symmetrische Funktionen läßt sich aber jede symmetrische rationale Funktion der Wurzeln riner algebraischen Gleichung rational durch die Korfizienten ausdrücken. Daher läßt sich eine symmetrische rationale Funktion von $y_{2}, y_{3}, \ldots y_{m}$ rational dureh $x$ und $y_{1}$ darstellen. Jamit ist aber der Hilfssatz bwiesen.

Es sei

$$
\Xi_{1 k} \quad R_{1}\left(x, y_{k}\right), \quad \xi_{2 k}=R_{2}\left(x, y_{k}\right), \ldots \quad \xi_{m k}=R_{m}\left(x, y_{k}\right),
$$

und es sei $z_{: l}$ dir Unterdeterminante ron $\xi_{n l}$ in der Determinante $\xi_{k l}$. Dann hestehen für die Elemente der ersten Zeile von (E⿱:口) die Gleichungen (vol. Kap. VII, §3)

$$
\xi_{11}^{\prime}=\begin{aligned}
& z_{11} \\
& \xi_{k l}
\end{aligned}, \quad \xi_{\xi_{1}}^{\prime}=\begin{aligned}
& z_{21} \\
& \mid \xi_{k l}
\end{aligned}, \ldots \xi_{m 1}^{\prime}=\begin{aligned}
& z_{m 1} \\
& \xi_{k l}
\end{aligned} .
$$

Dirse Gröbrn sind also rationale Funktionen von $x, y_{1}, y_{2}, \ldots y_{n}$. Die Zählı sind dir Unterdeterminanten der Elemente drr ersten Zoile von $\xi_{k l}$, aliso lis aufs Vuzeichen rlie Jeterminanten derjenigen quadratischen Systeme, dir aus dem Systrom

$$
\left|\begin{array}{lll}
R_{1}\left(x, y_{2}\right), \quad R_{2}\left(x, y_{2}\right), \ldots & R_{m}\left(x, y_{2}\right) \\
R_{1}\left(x, y_{3}\right), \quad R_{2}\left(x, y_{3}\right), & R_{m}\left(x, y_{3}\right) \\
-\bar{R}-\overline{R_{1}\left(x, y_{m}\right),} \quad-R_{2}\left(x, y_{m}\right), & -\overline{R_{m}}\left(x, y_{m}\right)
\end{array}\right|
$$

hervorgehen, indem nan der Reihe nach eine Spalte fortstreicht. Permutieren wir also, die Größnn $y_{2}, y_{3}, \ldots y_{m}$ irgendwie, so ändern die $z_{i 1}$ höchstens ihr Zeichen, da in ihnen die Horizontalrwihen permutiert werden. Genau dieselbe Permutation tritt aber unter den $m-1$ letzten Zeilen von 
E: rin. Daher bleiben die Funktionen (79) ungeändert; d. h. aber sie sind symmotrisch in $y_{2}, y_{3}, \ldots y_{m}$. Nach unserm Hilfssatz lassen sie sich also als rationale Funktionen von $x$ und $y_{1}$ darstellen, etwa

$$
\xi_{11}^{\prime}=S_{1}\left(x, y_{1}\right), \quad \xi_{21}^{\prime}=S_{2}\left(x, y_{1}\right), \ldots \quad \xi_{m 1}^{\prime}=S_{m}\left(x, y_{1}\right) \text {. }
$$

Vertanshe man in der ganzen angestellten Betrachtung und in der Rechnung, die zu den Gleichungen (80) führt, $y_{1}$ mit $y_{\star}$, so geht $\xi_{k 1}^{\prime}$ in $F_{k u}^{\prime}$ über und es ist also allgemeiner

$$
\Xi_{1 \alpha}^{\prime}=S_{1}\left(x, y_{u}\right), \quad \Xi_{2_{u}}^{\prime}=S_{2}\left(x, y_{u}\right) \ldots \quad \Xi_{m \alpha}^{\prime}=S_{m}\left(x, y_{\alpha}\right) .
$$

Damit ist aber gezeigt, daß die Größen (79) Funktionen des Körpers sind und daß im System ( $\left.\xi_{1}^{\prime}\right)$ in jeder Spalte $m$ konjugierte Funktionen stehen, und unser Satz ist bewiesen.

Es spi jetzt (5) eine Basis für die Vielfachen eines Divisors q. Wir wollen sehen, ob die zu (૬) komplementäre Basis ( $\left.\xi^{\prime}\right)$ nicht auch eine Basis für die Vielfachen eines Divisors ist. Nach dem Satz XVII in $\S 9$ haben wir zunächst den größten gemeinsamen Teiler q̄ der Funktionen $\xi_{i}^{\prime}$ zu bestimmen, soweit es sich um Primtriler handelt, die zu endlichen Stellen von $x$ gehören. Es sei $x=a$ ein endlicher Wert von $x$. Wir unterscheiden wieder die Fälle wie in den $\S \S 6,7,8$ und benutzen die dortigen Bezeichnungen.

I. Es liege bei $x=a$ knin Verzweigungspunkt.

Nach (49) ist

$$
\left(\xi_{k l}\right)=A\left(p_{l l}\right) \text {. }
$$

Dabei ist $A$ das Multiplikationssystem mit der Diagonale

$$
(x-a)^{\tau_{1}}, \quad(x-a)^{\tau_{2}}, \ldots \quad(x-a)^{\tau_{m}}
$$

und $(p \cdot l)$ ist rine Einheit für $x=a$, da $(\xi)$ eine Basis für die Vielfachen vou $q$ ist. Bezeichnen wir die komplementären Systeme von $A$ und $\left(p_{k l}\right)$ mit $A^{\prime}$ und $\left(p_{, . l}^{\prime}\right)$, so folgt nach Satz XI in Kap. VII, §3 aus (82)

$$
\left(\xi_{l, l}^{\prime}\right)=A^{\prime}\left(p_{l, l}^{\prime}\right)
$$

und hier ist $\left(p_{i l}^{\prime}\right)$ auch eine Einhrit für $x=a$. Da $A$ als Multiplikationssystem symmetrisch ist, so ist $A^{\prime}$ gleich dem reziproken von $A$, also nach SatzXIV in Kap. VII, $\$ 4$ gleich dem Multiplikationssystem mit der Diagonale

$$
(x-a)^{-\tau_{1}}, \quad(x-a)^{-r_{1}} \ldots \quad(x-a)^{-\tau_{m}} .
$$

Aus (83) ergibt sich also z. B.

$$
\xi_{11}^{\prime}=(x-a)^{-r_{1}} p_{11}^{\prime}, \quad \xi_{21}^{\prime}=(x-a)^{-\tau_{1}} p_{2_{1}}^{\prime} \ldots \quad \xi_{m_{1}}^{\prime}=(x-a)^{-\tau_{1}} p_{m_{1}}^{\prime} \text {. }
$$

Hieraus folgt, daß die Funktionen $\xi_{i}^{\prime}$ den Primteiler $\mathfrak{p}_{1}$ alle mindestens 


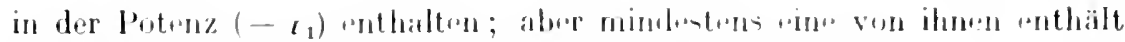

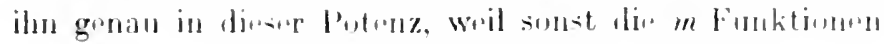

$$
p_{11}^{\prime}, \quad p_{21}^{\prime}, \ldots \quad p_{i=1}^{\prime}
$$

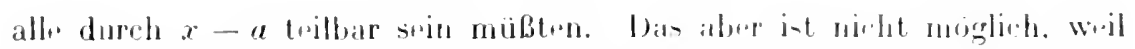

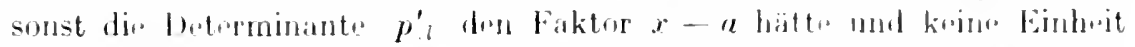

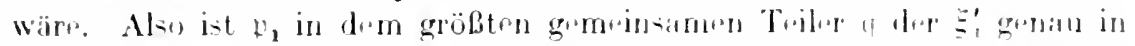
der Potenz $\left(-r_{1}\right)$ enthalten. Ebenso folgt, daballgomeiner p, in a genan

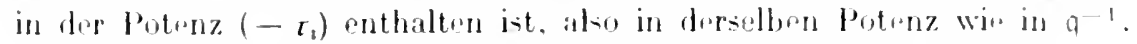

11. Es liege bei $r=a$ mindestrus ain Verzweigungipunkt.

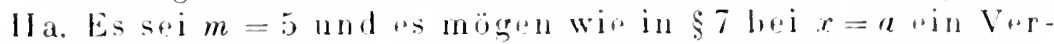
zwoigungspunkt erstrer und rinme zweiter Ordunng lingare

Niach (6) ist

$$
\left(\Xi_{i l}\right)=A_{1} A_{2} A_{3}\left(p_{: l}\right)
$$

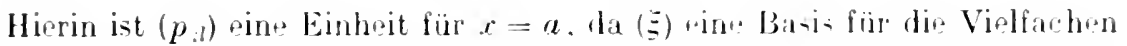
von $q$ ist. Dasselbe gilt daher von dom komplementäron System ( $\left.p^{\prime}\right)$. $A_{1}$ und $A_{3}$ sind Multiplikationssyteme mit den Jiagonalen

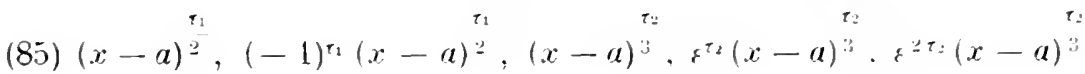

und

$$
1,(x-a)^{2}, \quad 1,(x-a)^{1},(x-a)^{3} \text {. }
$$

Die hierzu komplemrntären oder, was hier dassulbe ist, reziproken Systrme $A_{1}^{\prime}$ und $A_{3}^{\prime}$ sind Multiplikationsiystem mit den Diagonalen

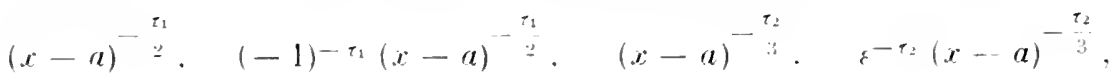

$$
\begin{aligned}
& f^{2}-212(x-a)^{-\frac{12}{3}}
\end{aligned}
$$

IIn)

$$
1,(x-a)^{-\frac{1}{2}}, \quad 1,(x-a)^{-\frac{1}{3}},(x-a)^{-\frac{2}{3}},
$$

deren Elemente reziprok zu denen der biagunalen (85) und (86) von $A_{1}$ und $A_{3}$ sind. Jas Sỵstom $A_{2}$ war

$$
A_{2}=\left|\begin{array}{rrrrr}
1 & 1 & 0 & 0 & 0 \\
1 & -1 & 0 & 0 & 0 \\
0 & 0 & 1 & 1 & 1 \\
0 & 0 & 1 & \varepsilon & \varepsilon^{2} \\
0 & 0 & 1 & \varepsilon^{2} & \varepsilon
\end{array}\right|
$$

Es sei $A_{2}^{\prime}$ das Sỵstem, das aus $A_{2}$ entsteht, wenn man di von Null verschiedenen Element" durch ihro reziproken Werte ersetzt. In unserm 
ninfichen Falle mitsteht $A_{2}^{\prime}$ aus $A_{2}$ durch Vertauschen von $\varepsilon$ und $\varepsilon^{2}$, da $\varepsilon^{3} \quad$ 1. Bedenkt man, daß $1+\varepsilon+\varepsilon^{2}=0$, so ergibt sich für das Produkt A. to das Multiplikationssystem mit der Diagonale

$$
2,2,3,3,3 \text {. }
$$

Dips sei mit $\ell$ be'zeichnet. Es ist damn

$$
A_{2} A_{2}^{\prime}=C, \quad A_{2}^{\prime}=A_{2}^{-1} C, \quad A_{2}^{-1}=A_{2}^{\prime} C^{-1},
$$

wo $C^{-1}$ das Multiplikationssystem mit der Diagonale

$\begin{array}{lllll}1 & 1 & 1 & 1 & 1 \\ 2 & 2 & 3 & 3\end{array}, \frac{3}{3}$.

गa $A_{2}$ symmetrisch ist, so ist das komplementäre System von $A_{2}$ gleich dem reziproken $A_{2}^{-1}$ und aus (84) folgt durch Übergang zu den komplementären Systrmen

$$
\left(\xi_{k l}^{\prime}\right)=A_{1}^{\prime} A_{2}^{\prime} C^{-1} A_{3}^{\prime}\left(p_{k l}^{\prime}\right) \text {. }
$$

Da aber Multiplikationssysteme vertauschbar sind, so baben wir weiter

$$
\left(\Xi_{1 .}^{\prime}\right)=A_{1}^{\prime} A_{2}^{\prime} A_{3}^{\prime}\left(q_{h l l}\right) \text {, }
$$

wenn wir $C^{-1}\left(p_{t . l}^{\prime}\right)=\left(q_{. l}\right)$ setzen. Es ist $\left(q_{l . l}\right)$ eine Einheit für $x=a$, da dasselbe von $C^{-1}$ und $\left(p_{., l}^{\prime}\right)$ gilt. Aus (87) folgt durch Ausmultiplizieren

$$
\begin{aligned}
& \xi_{k: 1}^{\prime}=(x-a)^{-\frac{\tau_{1}+1}{2}}\left\{q_{k: 1}(x-a)^{\frac{1}{2}}+q_{k \cdot 2}\right\} \text {. }
\end{aligned}
$$

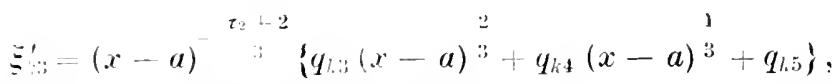

während $\xi_{! 2}$ die konjugierte Entwicklung zu $\xi_{1}^{\prime}$ ist und $\xi_{k 4}^{\prime}, \quad \xi_{l: 5}^{\prime}$ dir krnjugierten zu $\xi_{1.3}^{\prime}$ sind. Aus (88) rrgibt sich, daß die $\xi_{i}^{\prime}$ den Primteiler t) mindestens in der Potenz $-\left(r_{1}+1\right)$ enthalten. In einer höheren würden sie ihn sämtlich dann und nur dann enthalten, wenn alle Funktionen $q_{2}(k=1,2, \ldots 5)$ durch $x-a$ teilbar wären. Das geht aber nicht, da $\left(q_{k l l}\right)$ eine Einheit für $x=a$ ist und also dir Elemente der zweiten Zeile der Deterninante $i q_{.}$nicht sämtlich für $x=a$ verschwinden können. Waher ist $\mathfrak{p}_{1}$ in dem größten gemeinsamen Teiler $\bar{q}$ der $\xi_{i}^{\prime}$ genau in der Potenz $-\left(\tau_{1}-1\right)$ enthalten. Ebenso schließen wir aus (89), daß $\mathfrak{p}_{2}$ in $\bar{q}$ genau in der Potenz $-\left(\tau_{2}+2\right)$ enthalten ist. Da $\mathfrak{p}_{1}$ und $\mathfrak{p}_{2}$ Verzweigungsprimteiler erster und zweiter Orfnung sind und also im Verzweigung-divisor $z$ in der ersten und zweiten Potenz enthalten sind, so sind $p_{1}$ und $\mathfrak{p}_{2}$ in $\bar{a}$ in derselben Potenz enthalten wie in $q^{-1} z^{-1}$. Dies stimmt rnit dem in I gefundenen Ergebris überein, da dort die Primteiler $\mathfrak{p}_{1}$ in 3 richt vorkommen. 
IIb. Es liegobei $x$ a nureinestelle, diedannein Verzweigungspunkt dor Ordunug $m-1$ ist. Wir sclurrilen wieder für $m$ auch $\alpha$.

Es ist nach (70)

wo $\left(p_{k l}\right)$ eine Einheit für $x=a$ ist, da (引) eine Basis für die Vielfachen von $q$ ist. Nach $\S 8$ sind $A_{1}$ und $A_{3}$ die Multiplikationssysteme mit den Diagonalen

$$
(x-a)^{i}, \quad \omega_{1}^{r}(x-a)^{\imath}, \ldots \quad \omega_{a-1}^{\tau}(x-a)^{i}
$$

und

$$
1, \quad(x-a)^{1}, \ldots \quad(x-a)^{\frac{1 t-1}{\alpha}} .
$$

Daher sind die zu $A_{1}$ und $A_{3}$ komplementären Systeme $A_{1}^{\prime}, A_{3}^{\prime}$ die Multiplikationssysteme mit den Diagonalen

$$
\begin{aligned}
& (x-a)^{-r}, \quad \omega_{1}^{-r}(x-a)^{-\frac{r}{t}}, \ldots \quad \omega_{a-1}^{-\tau}(x-a)^{-\frac{r}{u}} ; \\
& \text { 1. }(x-a)^{-\frac{1}{\alpha}}, \ldots(x-a)^{-\frac{u-1}{"}} .
\end{aligned}
$$

Dabei ist gesetzt

$$
\omega_{l}=e^{\frac{2 \pi l l}{u}} \text {. }
$$

Ferner ist

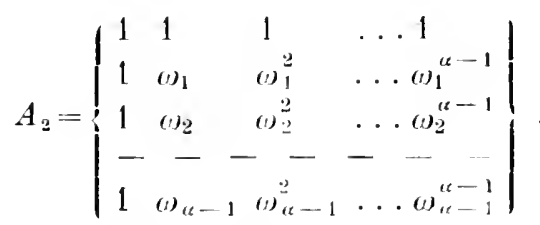

Wir bezrichnen das System, das aus $A_{2}$ dadurch hervorgeht, daß man seine Elemente durch ihre reziproken Werte ersetzt, mit $A_{2}^{\prime}$. Das System $A_{2}$ ist symmetrisch. Setzt man nämlich

so ist

$$
\omega=e^{2 \pi},
$$

$$
\omega_{l}^{k}=\omega^{k l} \text {. }
$$

Das reziproke System $A_{2}^{-1}$ von $A_{2}$ ist also auch komplementär zu $A_{2}$. Wir berchnen wie in IIa das Produkt $A_{2} A_{2}^{\prime}$. Die $(l+1)$-Zeile in $A_{2}$ ist wegen (91)

$$
1, \quad \omega^{l}, \quad \omega^{2 l}, \ldots \quad \omega^{(\alpha-1) l}
$$

und die $(k+1)$-te Spalte von $A_{2}^{\prime}$ ist

$$
1, \omega^{-k}, \omega^{-2 k}, \ldots \omega^{-k-1) k} .
$$


Setzen wir also

$$
\omega^{\prime-1}=\omega^{\prime}
$$

se ist das Produkt der beiden Reihen (92), (93)

$$
1+\omega^{\prime}+\omega^{\prime 2}+\cdots+()^{\prime} c-1 .
$$

Ist $l=k$, so ist $\omega^{\prime}=1$ und die Summe $\left(9^{\prime}\right)$ hat den Wert $\alpha$. Ist $l \neq k$ und also auch nicht $l=k(\bmod \alpha)$, diı $l$ und $k$ beide zwischen 0 und $\alpha-1$, die Grenzen eingeschlossen, liegen, so ist $\omega^{\prime}$ eine von 1 verschiedene Lösung der Gleichung $z^{t}-1=0$, also eine Lösung der Gleichung

$$
\frac{z^{u}-1}{z-1}=1+z+z^{2}+\cdots+z^{u-1}=0
$$

und es wird die Summe (94) zu Null. Daher wird $A_{2} A_{2}^{\prime}$ gleich dem Multiplikationssystem, in dessen Diagonale überall $\alpha$ steht. Bezeichnen wir dirs System mit $C$, so wird genau wie in IIa

und

$$
\begin{gathered}
A_{2}^{-1}=A_{2}^{\prime} C^{-1} \\
\left(\xi_{k l l}^{\prime}\right)=A_{1}^{\prime} A_{2}^{\prime} A_{3}^{\prime}\left(q_{k l}\right),
\end{gathered}
$$

wo $\left(q_{i l}\right)$ eine Einheit für $x=a$ ist. Hieraus folgt

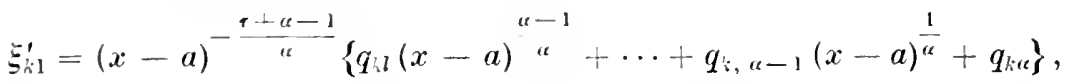

während $\xi_{k 2}^{\prime}, \xi_{k 3}^{\prime}, \ldots \xi_{k a}$ die hierzu konjugierten Entwicklungen sind. Da wieder $q_{1 / s}, q_{2 \alpha}, \ldots q_{u \alpha}$ nicht sämtlich für $x=a$ Null werden können, so ist der zu der betrachteten Stelle gehörende Primteiler $\mathfrak{p}$ in dem größten gemeinsamen Teiler $\bar{q}$ der $\xi_{i}^{\prime}$ genau in der Potenz $-(\tau+\alpha-1)$ enthalten, also in derselben Potenz wie in $q^{-1} z^{-1}$.

Fassen wir die Ergebnisse zusammen, so haben wir:

SatzXX. Ist ( (今) eine Basis für die Vielfachen des Divisors $a$, so haben die Funktionen $\xi_{i}^{\prime}$ der komplementären Basis $\left(\xi^{\prime}\right)$ den größten gemeinsamen Teiler

$$
\bar{q}=q^{-1} z^{\prime-1},
$$

wo $\dot{\lambda}^{\prime}$ der Teil des Verzweigungsdivisors 3 ist, der die zu endlichen Stellen von $x$ gehörenden Primteiler enthält.

Um nun zu sehen, ob ( $\left.\xi^{\prime}\right)$ eine Basis für die Vielfachen von $\bar{q}$ ist, haben wir die Diskriminante von ( $\left.\xi^{\prime}\right)$ zu berechnen. Da $\left(\xi_{k l l}\right)$ und $\left(\xi_{k l}^{\prime}\right)$ komplementär sind, so sind die Determinanten dieser Systeme reziprok zueinander. Also ist nach (77)

oder wegen (95)

$$
\left|\xi_{k l l}^{\prime}\right|^{2}=\left|\xi_{k l}\right|^{-2}=\text { konst } N I\left(\frac{1}{q^{2} z^{\prime}}\right)
$$

$$
\left|\xi_{k: l}^{\prime}\right|^{2}=\text { konst } N I\left(\bar{q}^{2} z^{\prime}\right) \text {. }
$$


Daher sind die Voraussetzungen des Satzes XVII in $\$ 9$ urfült, und wir haben

Satz XXI. Ist (5) eine Basis für dio Violfachen rinns Divisurs q, so ist dir komplementäro basis (朽) rino Basis für die Vielfachrol des loivisors

$$
\mathfrak{q}=\mathfrak{q}^{-1} i^{1-1},
$$

wo ó der Toil des Verzweigungsdivisors o ist, der dir zu endlirhen Strllun von $x$ gehörenden Primteiler enthält.

Wir fermulieren den Satz noch besonders für den Fall, daß bri $x=\infty$ krin Vorzwrigungspunkt liegt, und definieren zunäehst:

Definition VI. Ist a der Verzweigungsdivisor, so heiben zwei Jivisoren qund $q^{\prime}$ zueinander komplementär, wenn

Dann haben wir:

$$
q q^{\prime}=i^{-1} \text {. }
$$

Satz XXII. Liegt bei $x=\infty$ kein Verzweigungspunkt und ist $(5)$ eine Basis für die Vielfachen eines Divisors, so ist die komplementäre Basis eine Basis fürdie Vielfachen des komplementären Divisors.

Beispiel I.

\section{$\$ 14$. Beispiele zu $\$ 13$.}

Der Körper sei definiert dureh

$$
f(x, y) \equiv y^{3}-3 x^{2} y+2 x=0 .
$$

Ferner sei $q=1$. Zunächst bestimmen wir eine Basis für die Vielfachen von $\mathfrak{q}=1$, also für die in bezug auf $x$ ganzen Funktionen. Die Diskriminante von $f(y)=0$ ist

$$
\left|\begin{array}{lll}
1 & y_{1} & y_{1}^{2} \\
1 & y_{2} & y_{2}^{2} \\
1 & y_{3} & y_{3}^{2}
\end{array}\right|^{2}=D(x)=4 \cdot 27 x^{2}\left(x^{4}-1\right) .
$$

Verzweigungspunkte liegen daher höchstens bei $x=0, x= \pm 1, x= \pm i$, $x=\infty$. Jurch das in Kap. II auseinandergesetzte Verfahren findet man, daß bei $x=0$ ein dreifacher und bei den vier Punkten $x= \pm 1, x= \pm i$ je ein zweifacher Verzweigungspunkt liegt, während bei $x=\infty$ drei gewöhnliche Punkte vorhanden sind. Die zu den Verzweigungsstellen gehörenden Primteiler seien mit $v_{0}, v_{1}, \ldots v_{4}$ bezeichnet. Es ist

$$
\begin{aligned}
3 & =\mathfrak{v}_{0}^{2} \mathfrak{v}_{1} \mathfrak{v}_{2} \mathfrak{v}_{3} \mathfrak{v}_{4}, \quad w=6 . \\
N I(3) & =x^{2}\left(x^{4}-1\right) .
\end{aligned}
$$

Die Funktionen $\left(1, y, y^{2}\right)$ bilden eine Basis. Die Funktionen der Basis 
werden für endliehe Werte von $x$ uicht unendlich, und da sie nirgends gleichzeitig Null werden, so ist ihr größter gemeinsamer Teiler gleich 1, gheich q. Ferner ist de Diskriminante der Basis, nämlich $D(x)$, bis auf cinen konstanten Faktor gleich $N I(i)-N I\left(q^{2}\right.$ i). Nach Satz XVII, § 9 ist daher die Basis $\left(1, y, y^{2}\right)$ schon cinn lasis für die Vielfachen von 1 , so daß wir setzen tömnen $(\Xi)=\left(\Xi_{1}, \Xi_{2}, \Xi_{3}\right)=\left(1, y, y^{2}\right)$.

Wir gehen dazu über, die komplementäre Basis ( $\eta$ zu bestimmen. Es ist

$$
g(y)=\left(y-y_{2}\right)\left(y-y_{3}\right)=\begin{gathered}
f(x, y)-f\left(x, y_{1}\right) \\
y-y_{1}
\end{gathered}=y^{2}+y y_{1}+y_{1}^{2}-3 x^{2},
$$

woraus wir schlieBen

$$
y_{2}+y_{3}=-y_{1}, \quad y_{2} y_{3}=y_{1}^{2}-3 x^{2} .
$$

Frrmer ist in der Bezeichnung des vorigen Paragraphen

$z=\xi_{11}-\left(y_{1}-y_{2}\right)\left(y_{1}-y_{3}\right)\left(y_{3}-y_{2}\right)=g\left(y_{1}\right)\left(y_{3}-y_{2}\right)=-3\left(y_{1}^{2}-x^{2}\right)\left(y_{2}-y_{3}\right)$, $z_{11}=-y_{2} y_{3}\left(y_{2}-y_{3}\right), \quad z_{21}=y_{2}^{2}-y_{3}^{2}, \quad z_{31}=-\left(y_{2}-y_{3}\right)$,

also

$$
\begin{aligned}
D(x) \eta_{11} & =z^{2} \imath_{11}=z \cdot z_{11}=3\left(y_{1}^{2}-x^{2}\right) y_{2} y_{3}\left(y_{2}-y_{3}\right)^{2} \\
& =3\left(y_{1}^{2}-x^{2}\right)\left(y_{1}^{2}-3 x^{2}\right)\left[\left(y_{2}+y_{3}\right)^{2}-4 y_{2} y_{3}\right]= \\
& =-9\left(y_{1}^{2}-x^{2}\right)\left(y_{1}^{2}-3 x^{2}\right)\left(y_{1}^{2}-4 x^{2}\right) \\
& =-9\left(y_{1}^{6}-8 x^{2} y_{1}^{1}+19 x^{4} y_{1}^{2}-12 x^{6}\right) .
\end{aligned}
$$

Aus der Gleichung

folgt aber

$$
f\left(x, y_{1}\right)=y_{1}^{3}-3 x^{2} y_{1}+2 x \equiv 0
$$

so $\mathrm{da} B$ wird

$$
y_{1}^{3}=3 x^{2} y_{1}-2 x,
$$

$$
\begin{aligned}
D(x) \eta_{11} & =-9\left[\left(3 x^{2} y_{1}-2 x\right)^{2}-8 x^{2} y_{1}\left(3 x^{2} y_{1}-2 x\right)+19 x^{4} y_{1}^{2}-12 x^{6}\right] \\
& =-36 x^{2}\left(x^{2} y_{1}^{2}+x y_{1}-3 x^{4}+1\right) .
\end{aligned}
$$

Jaher wird, wenn wir den Wert von $D(x)$ einsetzen

Ebenso findet man

$$
r_{1}=\begin{gathered}
1-3 x^{4}+x y+x^{2} y^{2} \\
3\left(1-x^{4}\right)
\end{gathered}
$$

$$
y_{2}=\frac{-2 x^{2}-\left(2 x-3 x^{2}\right) y+y^{2}}{6 x\left(1-x^{4}\right)}, \quad y_{3}=\frac{-2 x+y+x y^{2}}{6 x\left(1-x^{4}\right)} .
$$

Die so gefundene Basis $(\eta)$ ist eine Basis für die Vielfachen des Divisors $r=q^{-1} j^{-1}=z^{-1}=\mathfrak{v}_{0}^{-2}\left(\mathfrak{v}_{1} b_{2} v_{3} b_{4}\right)^{-1}$. Es wird dem Leser empfohlen, nach den in diesem Kapitel auseinandergesetzten Methoden un- 
mittelhar pine Basis ( $\left.y^{\prime}\right)$ für die Virlfarfun von; 7 zu bestimmen und das Ergebnis mit dem hire gofundonmen zorgleinlun. Es braucht nicht

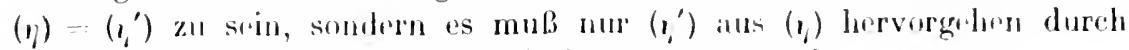
rum Substution, din ane Einbrit in broug anf $x$ ist.

Brispiel Il.

Wir betrachtendas Beispiel 1 in $\$ 10$. Dort hatten wir als basis für die Virlfachon von $1=p_{1}^{-2} p_{2}^{1} b_{1}^{-1}$ gefunden:

$$
\therefore=x, \quad s_{2}=\begin{gathered}
2-x^{2}-x^{3}+2 y \\
2 x^{2}(x-1)
\end{gathered},
$$

so $\mathrm{daB}$

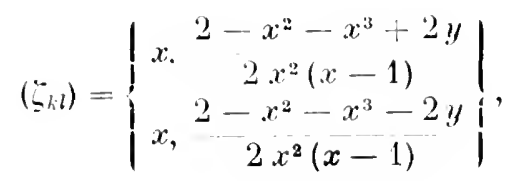

woraus sofort folgt, da

$$
\begin{aligned}
& \left|\zeta_{h l}\right|=\frac{-2 y}{x(x-1)}=\frac{-2 y^{2}}{x(x-1) y}=\begin{array}{c}
2(x+1) \\
x y
\end{array} \\
& \left(\eta_{k l}\right)=\left(\zeta_{k: l}\right)^{-1}=\left\{\begin{array}{ccc}
2-x^{2}-x^{3}-2 y & x y & x y \\
2 x^{2}(x-1) & 2(x+1) & -x \cdot 2(x+1) \\
2-x^{2}-x^{3}+2 y & x y & x y \\
2 x^{2}(x-1) & 2(x+1) & x \cdot 2(x+1)
\end{array} \mid,\right.
\end{aligned}
$$

also nach gehöriger Umformung unter Benutzung der Gleichung $f(x, y)$ $-y^{2}+x^{2}-1=0$

$$
\eta_{1}=\frac{2(1+x)-\left(2+2 x+x^{2}\right) y}{4 x(1+x)}, \quad y_{2}=-\frac{x^{2} y}{2(1+x)} .
$$

Es ist (y) eine Basis für die Vielfachen des Divisors

$$
r=q^{-1} i^{-1}=p_{1}^{2} p_{2}^{-1} v_{1} \cdot v_{1}^{-1} \mathfrak{v}_{2}^{-1}=p_{1}^{2} p_{2}^{-1} \mathfrak{v}_{2}^{-1}
$$

Für die Vielfachen dieses Divisors haben wir aber in $\$ 10$ auch eine Basis ( $\left(^{\prime \prime}\right)$ bestimmt. Es müssen also $(\eta)$ und $\left(\zeta^{\prime}\right)$ auseinander durch eine Substitution hervorgehen, div eine Einheit inbezug auf $x$ ist. Wir wollen das bestätigen. Setzen wir

$$
b=\left\{\begin{array}{cc}
1 & 0 \\
2 x & 0 \\
2+2 x+x^{2} & x^{2} \\
-4 x(1+x) & 2(1+x)
\end{array}\right\}, \quad c=\left\{\begin{array}{cc}
x^{2}, & -2-2 x+x^{2} \\
0 & 1 \\
0 & x(1+x)
\end{array}\right\},
$$

so ist

$$
(\eta)=(1, y) b
$$

$\left(\breve{c}^{\prime}\right)=(1, y) c$,

also

$$
(1, y)=\left(\zeta^{\prime}\right) c^{-1}, \quad(\eta)=\left(\zeta^{\prime}\right) c^{-1} b \text {. }
$$




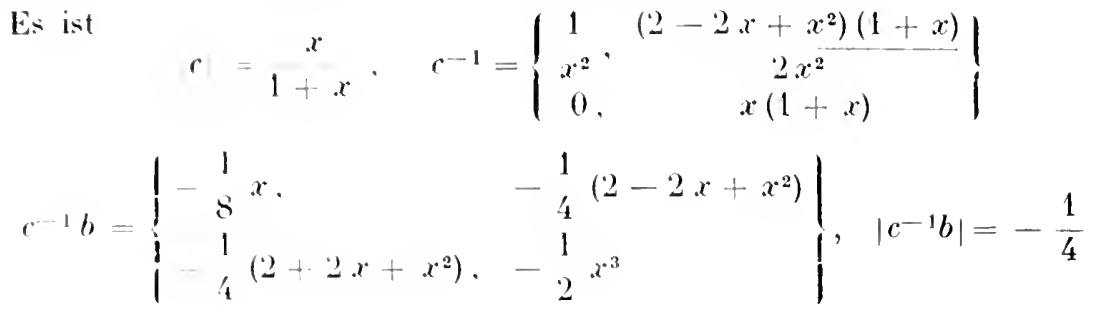

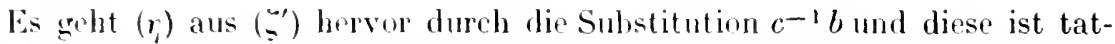
särhlich eine Einlseit in bezug auf $x$, da ihre Elemente ganze rationale Funktionen von $x$ sind und ihre Determinante eine von Null verschiedene Konstante ist.

Es wird dem Leser empfohlen, die Beispiele in $§ 11$ und $\S I 2$ ebenso zu behandeln.

Neuntes Kapitel.

\section{Ber Riemamn-Rochsche Satz.}

\section{$\$ 1$. Die ganzen Dirisoren einer Kílasse.}

Es sei ( $t$ ) irgendeine Klasse. Wir behandeln die Aufgabe, alle ganzen Divisoren der Klasse zu bestimmen, und im besonderen die Zahl der linear unabhängigen ganzen Divisoren der Klasse, also die Dimension der Klasse festzustellen.

Wir wählen aus der Klasse (t) irgendeinen Divisor $t$ aus. Dann nehmen wir statt $x$, wenn notwendig, eine neue unabhängige Veränderliche $x^{\prime}$. die wir durch die Gleichung

$$
\frac{1}{x-x_{0}}=x^{\prime}
$$

dיfinieren. Dabei soll $x_{0}$ so gewählt sein, daß erstens bei $x=x_{0}$ kein Verzwrigungspunkt liegt und daß zweitens keiner der in Zähler von $x-x_{0}$ vorkommenden Primteiler in $t$ enthalten ist. Wir bezeichnen die neue unabhängige Veränderliche wieder mit $x$. Wir können also annehmen, daß erstens bei $x=\infty$ kein Vrrzwigungspunkt liegt und daß zweitens keiner der im Nenner von $x$ enthaltenen Primteiler in t vorkommt.

Es sui g rin ganzer loivisor der Klasse (1). Dann ist $t^{-1}$ g eine Funktion d's Körpers und diese Funktion ist, da $t$ keinen Divisor enthält, der zu "in r im Unendlirhen lifgenden Stelle grhört, ein Vielfaches von $t^{-1}$ nach d.r Jefinition des vorigen Kapitels, und zwar eins, das für unendlirhe $x$ nicht unendlich wird. Ist umgekehrt $R$ ein Vielfaches von $\mathrm{t}^{-1}$, das für unendlich große Werte von $x$ nicht unendlich wird, und schreiben 


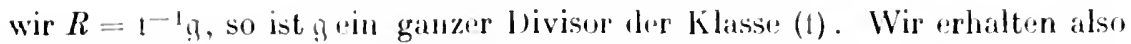
alle ganzen Divisoren der Klasse (1), wenn wir aus den Virlfachen von $\mathrm{t}^{-1}$ diejenigen aussuchen, die für unendlich großes $x$ nicht unendlich werden.

Es sei (a) rime Basis für die Vielfachen von $t^{-1}$, dann läßt sieh jedes Vielfache von t.1 $^{-1}$ in der Form schereihen

$$
R=g_{1} \ddot{亏}_{1}+g_{2} \check{\zeta}_{2}+\cdots+g_{m} \ddot{亏}_{m},
$$

wo die $g_{i}$ ganz' rationale Funktionen von $x$ sind, und umgrekehrt ist jede Funktion direr Form ein Vielfaches von $\mathrm{t}^{-1}$, wenn die $g_{2}$ ganz sind. WVie müssen die $g_{t}$ gewälılt werden, damit die Funktion $R$ für uncodliches $x$ nicht unendlich wird? Wir wollen diese Frage gleich in rtwas allgemeinerer Form stellen. Zunäehst definieren wir:

Defintion I. Wenn die Funktion $R$ des Körpers in keinem der m Punkte der Riemannschen Fläehe, in denen $x$ unendlich ist, von höherer als der r-ten Ordnung unendlich wird, aber wenigstens in einem dieser Punkte genau von der Ordnung $r$, so wollen wir $r$ die Ordnung der Funktion nennen.

Es ist dann $R x^{-r}$ im Unendlichen endlich und wenigstens in einem der $m$ im Unendlichen liegenden Punkte von Null verschieden. Wir wollen die Frage beantworten: Wie müssen die $g_{i}$ in (1) gewählt werden, damit $R$ höchstens von der gegebenen Ordnung $r$ ist? Für $r=0$ haben wir die spezielle oben gestellte Frage.

Die gesuchten Funktionen der Ordnung $r$ lassen sich, wenn wir den Nenner von $x$ mit 1 bezeichnen, in der Form schreiben

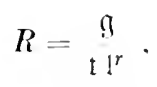

wo ạ ein ganzer Divisor ist. Die Zahl der linear unabhängigen Funktionen dieser Art ist also gleich der Zahl der linear unabhängigen ganzen Divisoren der Klasse $\left(t^{r}\right)$, d. h. gleich der Dimension $\left\{\mathrm{t}^{r}\right\}$ dieser Klasse. Nach unseren Voraussetzungen liegt bei $x=\infty$ kein Verzweigungspunkt. Die Funktionen $\zeta$ lassen sich also in allen $m$ Blättern der Riemannschen Fläche nach steigenden Potenzen von $x^{-1}$ entwickeln. Es seien die ganzen Zahlen $r_{1}, r_{2}, \ldots r_{m}$ so gewählt, daß die Funktionen $\zeta_{1} x^{-r_{1}}, \zeta_{2} x^{-r_{2}}, \ldots \zeta_{m} x^{-r_{m}}$ in keinem der $m$ Blätter für $x=\infty$ unendlich werden, daß aber jede von ihnen in wenigstens einem Blatte für $x=\infty$ nieht Null wird. Es sei also $r_{k}$ die Ordnung von $\zeta_{k}$. Wir setzen

$$
\zeta_{k l}=x_{k}^{r_{k}} \mathscr{H}_{k l}\left(\begin{array}{l}
1 \\
x
\end{array}\right)=x^{r} k\left(a_{k l}+b_{l l} \frac{1}{x}+c_{k: l} \frac{1}{x^{2}}+\cdots\right) \text {. }
$$

Es wird dann, wenn wir die $m$ konjugierten Werte von $R$ mit $R_{1}, R_{2}, \ldots$ $R_{m}$ bezeichnen,

Jung. Einfübrung in die Theorie. 


$$
\begin{aligned}
& R_{1}=g_{1} \cdot x^{r_{1}} \mathbb{L}_{11}+g_{2} \cdot x^{r_{2}} \mathbb{L}_{21}+\cdots+g_{m} \cdot x^{r_{m}} \mathscr{H}_{m 1}, \\
& k_{2}=g_{1} x^{r_{1}} \psi_{12}+g_{2} x^{r_{2}} \psi_{22}+\cdots+g_{m} x_{m} \psi_{m 2} \text {, } \\
& h_{m}=g_{1} x^{r_{1}} \psi_{1 m}+g_{2} x^{r_{2}} \psi_{2_{m}}+\cdots+g_{m} x^{r_{m}} \psi_{m m} .
\end{aligned}
$$

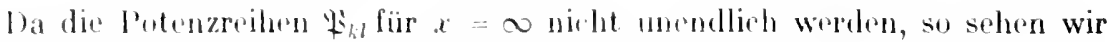
aus diesen Gileichungen sofort, dab kiner der konjugierten Werte $R_{1}$, $R_{2} \ldots R_{m}$ für $x=\infty$ von höheser als der $r$-twn ()rdnung unendlich wird, wenn wir den Crad von $g_{i}$ höchstens ghich $r-r$. wählen, wenn $r-r_{k} \geq 0$, und wenn wir $g_{i}$. 0 wählen, wrun $r-r, 0$. Es fragt sich, ob auch das Ungekehrte gilt oder ob auch, wenu die $g$. alle oder zum Teil von höherem Grade sind, die Funktion $R$ die Ordnung $r$ haben kann.

Es sei das Glied der höchsten Ordnung vong gleich $\lambda_{k} x^{\sharp-r}$, wo $s>r$; dabei schließnn wir nicht aus, daß die $\lambda_{k}$ zım Teil gleich Null sind. Nur dürfen sie nieht alle Null sein. Die Anfangsglieder der Entwicklungen der Funktionen $R_{k}$ sind

$$
\begin{aligned}
& x^{s}\left(\lambda_{11} a_{11}+\lambda_{2} a_{21}+\cdots+\lambda_{m} a_{m 1}\right), \\
& x^{s}\left(\lambda_{1} a_{12}+\lambda_{2} a_{22}+\cdots+\lambda_{m} a_{m 2}\right), \\
& \left.------------\lambda_{m} a_{m m}\right) .
\end{aligned}
$$

Soll $R$ in keinem Blatte für $x=\infty$ von höherer als der $r$-ten Ordnung unendlich werden, so müssen diese Anfangsglieder sämtlich verschwinden. Jas ist dann, aber auch nur dann möglich, wenn die Determinante $\left|a_{k l l}\right|$ gleich Null ist.

Wir unterscheiden daher die beiden Fälle, wo $\left|a_{l l}\right| \neq 0$ und wo $a_{k l} \mid=0$. Im ersten ist die Determinante $\$_{i . l}$ nicht Null für $x=\infty$; es ist also das System $\left(\mathfrak{F}_{1, i}\right)$ eine Einheit für $x=\infty$. Wir nennen in diesem Falle die Basis (c) normal für $x=\infty$. Aus den Gleichungen (3) folgt

$$
\left|S_{z: l}\right|=x^{r_{1}+r_{2}+\cdots+r_{m}}\left|\mathfrak{R}_{k i l}\right| \text {. }
$$

Setzen wir also

$$
\left|\zeta_{k i l}\right|=x^{0} E\left(\frac{1}{x}\right)
$$

wo $E\left(\frac{1}{x}\right)$ eine Einheit für $x=\infty$ sein soll, so ist im ersten Falle

$$
\varrho=r_{1}+r_{2}+\cdots+r_{m}
$$

und im zweiten

$$
\varrho<r_{1}+r_{2}+\cdots+r_{m} .
$$

Wir können daher definieren:

Jefinition II. Eine Basis heißt normal für $x=\infty$, wenn ihre Diskriminante für unendliches $x$ von einer Ordnung un- 


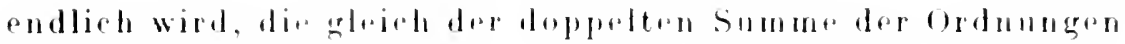
ihrer Funktionrn ist.

Wenn dir liasis (o) für $x=\infty$ nibht mormal ist, künmon wir inner

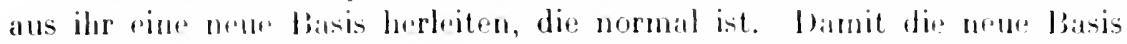
auch einr. basis liir dir. Vielfarlen von $t^{-1}$ ist, muB narh Satz VII, Kap. VIII,

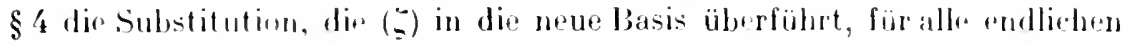
Stellen vou $x$ rim Einheit sein, d. h. sie muß ans ganzen rationalen liunktionen ron $x$ bestelen und ihre Jeterminante mub anc von Null verschiedene kumstante sein. Wï dïrfen laher z. B. die $\vdots$ brelielig vertauschen und wir dïrfen zu einer dor Funktionen die andern addieren, nachdem wir diese mit irgendwelchen ganzen rationalen Funktionen von $x$ multiplizirt haben. D. h. anders ausgedrückt, wir dürfrn $(\vdots)$ hinten multiplizieren mit Vertauschungssystemen und mit Additionssystemen, deren von 1 verschiedene Elemente ganze rationale Funktionen von $x$ sind.

Es sej jetzt $a_{h l}=0$, also $(\zeta)$ nicht normal für $x=\infty$. Wir denken uns die $\zeta_{i}$ so geordnet, dab

$$
r_{1}=r_{2} \leq r_{3} \ldots \ldots r_{m}
$$

Da $a_{k l}=0$, so können wir Konstante $\lambda_{1}, i_{2}, \ldots i_{m}$, die nicht alle Null sind, so bestimmen, daß die $m$ Gleichungen bestehen

$$
\lambda_{1} a_{1 k}+\lambda_{2} a_{2 k}+\cdots+\lambda_{m} a_{n k}=0 . \quad(k=1,2, \ldots m) .
$$

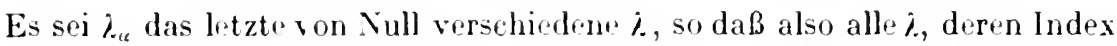
größer ist als $\alpha$, gleich Null sind, während $\lambda_{\text {q }}$ nieht Null ist. Wir können $\lambda_{\star s}=1$ annehmen, so dab rlie Gleichungen (6) lauten

$$
a_{u k}+\lambda_{1} a_{1 k}+i_{2} a_{2 k}+\cdots+\lambda_{u-1} a_{c-1 k}=0 . \quad(k=1,2, \ldots m) .
$$

Wir führen dann statt $=$ eine neue Funktion ein, während wir die andern Funktionen $\subsetneq$ : unverändert lassen. Wir nehmen nämlich statt $\varsigma_{u}$ die Funktion

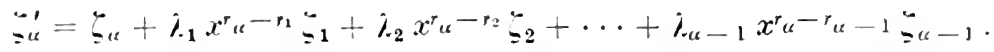

Mit Benutzung der Gleichungen (3) wird

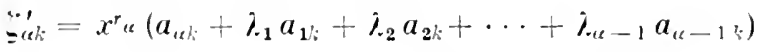

$$
\begin{aligned}
& +x^{r} u^{-1}\left(b_{u k}+\lambda_{1} b_{1 i}+\lambda_{2} b_{2 k}+\cdots+i_{u-1} b_{u-1 k}\right)+\cdots \text {. }
\end{aligned}
$$

Zufolge der Gleichungen (7) ist also die Ordnungszahl von $=$ : kleiner als $r_{u}$.

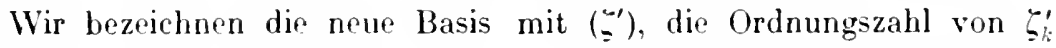
mit $r_{k}^{\prime}$. Es ist im allgemeinen $\zeta_{k}^{\prime}=\zeta_{n}^{\prime}, r_{k}^{\prime}=r_{k} ;$ nur $\zeta_{a}^{\prime}$ ist nicht gleich 
5 , sondern dureh die Gleichung (8) gegreben, und es ist $r_{u}^{\prime}<\boldsymbol{r}_{u}$, also

$$
r_{1}^{\prime}+r_{2}^{\prime}+\cdots+r_{m}^{\prime}-r_{1}+r_{2}+\cdots+r_{m} .
$$

Es ist nach (4)

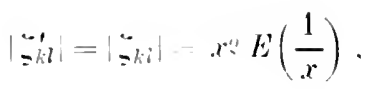

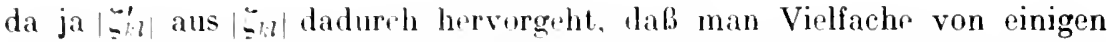
Vertikalreihen zu einer anteren aldiert. Da die Vertikalreihen von $\left|\zeta_{k}^{\prime}\right|$ der Reihe nach dureh $x_{1}^{r_{1}^{\prime}}, x_{2}^{r}, \ldots x_{m}^{r}$ trilbar sind, so folgt aus (10)

$$
r_{1}^{\prime}+r_{2}^{\prime}+\cdots+r_{n}^{\prime} \quad 0 \text {. }
$$

Wenn hier das Gleichheitszeichen richtig ist, so ist die Basis $\left(\zeta^{\prime}\right)$ normal für $x=\infty$. Ist das Gleichheitszeichen falsch, so können wir auf dem ben benutzten Wege eine neue Basis $\left(\zeta^{\prime \prime}\right)$ ableiten mit den Ordnungszahlen $r_{k}^{\prime \prime}$. so daß wieder entsprechend (9) und (11)

$$
\begin{gathered}
r_{1}^{\prime \prime}+r_{2}^{\prime \prime}+\cdots+r_{m}^{\prime \prime}<r_{1}^{\prime}+r_{2}^{\prime}+\cdots+r_{m}^{\prime}, \\
r_{1}^{\prime \prime}+r_{2}^{\prime \prime}+\cdots+r_{m}^{\prime \prime} \geq \varrho .
\end{gathered}
$$

Ist hier das Gleichheitszeichen richtig, so ist die Basis ( $\left.\zeta^{\prime \prime}\right)$ normal für $x=\infty$. Im anderen Falle leiten wir aus $\left(\zeta^{\prime \prime}\right)$ in der angegebenen Weise wieder eine neue Basis her. Da bei jedem Schritte die Summe der Ordnungszahlen der Elemente der Basis kleiner wird, diese Summe aber andererseits mindestens gleich der unveränderlichen Zahl $\varrho$ ist, so muß es nach einer endlichen Zahl von Schritten eintreten, daß wir eine Basis erhalten, bei der die Summe der Ordnungszahlen genau gleich $\varrho$ ist, die also für $x=\infty$ normal ist.

Nachdem wir gesehen haben, wie wir immer eine Basis finden können, die für $x=\infty$ normal ist, nehmen wir an, die Basis $(\zeta)$ sei normal für $x=\infty$. Dann ist wegen (5) und nach Satz XVIII, Kap. VIII, § 9

$$
r_{1}+r_{2} \cdots+r_{m}=-t+\frac{w}{2},
$$

wo $w$ die Verzweigungszahl in bezug auf $x$ ist und $t$ die Ordnung des Divisors t oder der Klasse (t). Eine Funktion

$$
R=g_{1} \zeta_{1}+g_{2} \check{\zeta}_{2}+\cdots+g_{m} \zeta_{m}
$$

ist, wie wir schon oben sahen, dann und nur dann ein Vielfaches von $\mathfrak{t}^{-1}$, das für $x=\infty$ höchstens von der Ordnung $r$ ist, wenn die $g_{i}$ ganze rationale Funktionen von $x$ sind und wenn der Grad von $g_{k}$ höchstens gleich $r-r_{k}$ ist für $r-r_{k} \geqq 0$ und wenn $g_{k}$ identisch Null ist für $r-r_{k}<0$. 


\section{\$. D. Der Riemannsche Satz.} setzen

Nach dem Ergubnis des vorigen Paragraphen können wir für $r-r_{k}-0$

$$
\text { g: } g_{k_{0}}+g_{k_{1}} x+\cdots+g_{k, r-r_{k}} x^{r-r_{k}} \text {, }
$$

wo die $g_{k i}$ bliphigh lionstante sind. Es enthält also für $r-r_{k} \geq 0$ die Funktion $g_{k}$ genau $r-r_{k}+1$ linear unabhängige kinstanten. Die $r-r_{k}+1$ Funktionen

$$
\check{\zeta}_{k}, \quad x \zeta_{k}, \quad x^{2} \check{\zeta}_{k}, \ldots x^{r-r_{k}} \zeta_{k}
$$

sind linear unabhängig und sind Vielfache von $t^{-1}$, die für $x=\infty$ höchstens die Ordnung $r$ haben. Solcher Funktionen gibt es also genau

$$
\underline{r}_{i}^{\prime}\left(r-r_{l_{i}}+1\right)
$$

linear unabhängige, wo die Summe über alle $k$ zu erstrecken ist, für die $r$ größer oder gleich $r_{k}$ ist, woran der Akzent am Summenzeichen erinnern soll. Wir können aber die Summe auch erstrecken über alle $k$, für die $r \geqq r_{k}-1$, da jeder Summand, in dem $r=r_{k}-1$ ist, den Wert Null hat. Nach dem Anfang dos vorigen Paragraphen ist aber die Zahl der linear unabhängigen Vielfachen von $\mathrm{t}^{-1}$, die für $x=\infty$ höchstens die Ordnung $r$ haben, gleich der Dimension der Klasse (t $i^{r}$ ). Wir haben also:

SatzI. lst $(\because)$ ) eine Basis für die Vielfachen des Divisors $t^{-1}$, die für $x=\infty$ normal ist, und ist $r_{t}$ die Ordnung von $\check{z}_{i}$, so ist dire Jimension der Klasse (tr). Wo t der Nenner von $x$ ist.

$$
\{1 r\}=\Sigma^{\prime}\left(r-r_{k}+1\right) \text {, }
$$

wo die Summe überalle $k z u$ erstrecken ist, fürdir $r-r_{k}+1 \geq 0$.

Wir betrachten zwei besondere Fälle. Es sei erstens $r$ so groß, da $B$ $r-r_{k}+1=0$ für alle $k$. Lann folgt aus (13)

oder wegen (12)

$$
\left\{\mathrm{t}^{r}\right\}=\sum_{k}^{m}\left(r-r_{1}+1\right)=m(r+1)-\sum_{k=1}^{m} r_{k}
$$

$$
\{\mathrm{t} 1 \mathrm{r}\}=m(r+1)+t-\frac{w}{2} .
$$

Nach (6), Kap. VI, §6 gilt aber für das Geschlecht $p$ des Körpers die Gleichung

$$
p={ }_{2}^{w}-m+1
$$

so daß aus (14) folgt: 
satzll. Ist i pin Divisor der Ordnung $t$ und I der Nenner von $x$. so ist bei genügend grobem $r$

$$
\{1 r\}=t-m r-p+1 \text {. }
$$

wo p las Geschlecht des körpers ist.

Wir wählen zweitens $r=0$. Nann folgt aus (13)

$$
\{\}=\mathbf{\Sigma}^{\prime}\left(1-r_{k}\right) \text {, }
$$

wo die Summe über alle $r_{\text {i }}$ zu erstrecken ist, die kleiner als 2 sind. Wir haken hiermit die Möglichkeit, die Dimensionen irgendeiner Klasse zu berechnen. Eine einfache Formel erhalten wir aber nicht. Wenn wir in (Ii) die Summe über alle $r_{k}$ erstrecken, so wird die rechte Seite höchstens zu klein, da nur negative Summanden hinzukommen können. Wir können daher schreiben

$$
\{t\}=\sum_{k=1}^{m}\left(1-r_{k}\right)-i=m-\underline{N} r_{k}-i,
$$

wo $i$ - 0 ist. Wegen (15) und (IS) hahen wir also

Satz III. Ist (:) eine Klasse der Ordnung $t$ und $p$ das Geschlecht des Körpers. so gilt für die Dimension der Klasse die Gleichung

wi) $i \cdots 0$.

$$
\{:\}=t-p-1-i
$$

Definition Ill. Wenn $i=0$. heißt die Klasse (t) regulär ader nichtspeziell. im andern Falle nicht reguläroder speziell. Di. Zall $i$ heibt der Index der speziollität.

Lieser Satz ist der Riemannsche Satz. Die Bedeutung von $i$ hat Rorh rkanut. Wir leiten sie im nächsten Paragraphen her.

\section{\$3. Der Riemamn-Rochsche Satz.}

Zunächst definieren wir:

Definition IV. Ist t ein Divisor der kanonischen Klasse, so heißon zwei Divisoren : und : Ergänzungsdivisoren. wenn (20)

$$
i t^{\prime}=t
$$

und es hoißen dir Klassen (i) und (:') Ergänzungsklassen, wenn

$$
\text { (i) }\left(i^{\prime}\right)=(\text { ( ) } \text {. }
$$

Für $t$ können wir wählen $(\S 6$, Kap. VI)

$$
\notin=3 !-2 \text {. }
$$


wo a wieder der Verzwigungsolivisor in berug anf $r$ ist mud 1 der Nenner von $x$. Es sind also die Klassin

$$
\text { (1) und }\left(1^{-1} ; 1^{-2}\right)
$$

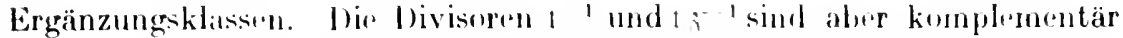
(Def. VI, Kap. VHII, \$ 13). lst daher $(G)$ wiedter wine Basis für dir Viel-

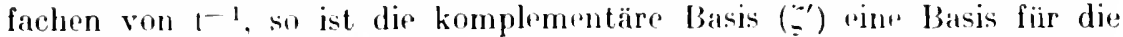
Vielfachen von $t ;-1$. Es sei $(\zeta)$ wieder normal für $x=\propto$ und die Ordmung von $\varsigma_{i}$ sei $r_{1}$. Nach $\$ 1$ halwn wir dann

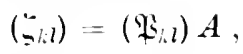

wo $A$ das Multiplikationssystem mit der Diagonalo

$$
x^{r_{1}}, x^{r_{2}}, \ldots x^{r_{m}}
$$

ist, während $\left(\mathfrak{B}_{h l}\right)$ eine Einheit für $x=\infty$ ist. Das komplementäre System $A^{\prime}$ zu $A$ ist das Multiplikationssystem mit der Diagonale

$$
x^{-r_{2}}, x^{-r_{2}}, \ldots x^{-r_{m}} .
$$

Das zu $\left(\mathfrak{P}_{. l}\right)$ komplementäre System $\left(\mathfrak{F}_{h l}^{\prime}\right)$ ist ebenso wie $\left(\mathfrak{B}_{k l}\right)$ eine Einheit für $x=\infty$. Aus (22) folgt

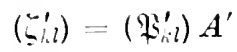

und daraus, daß auch $(\stackrel{-\prime}{)})$ normal für $x=\infty$ ist und daß die Ordnungszahl von $\stackrel{\because}{=}$ gleich $-r_{i}$ ist.

Dann aber folgt aus Satz 1 . $\$ 2$, indem wir crsetzen

durch

$$
\text { t. } r, \quad r_{i}
$$

$$
\left\{\mathrm{t}^{-1} \cdot \mathrm{j}^{-2}\right\}=\mathbf{N}^{\prime \prime}\left(-2+r_{k}+1\right)=\mathbf{N}^{\prime \prime}\left(r_{k}-1\right) \text {, }
$$

wo die Summe über alte $k$ zu erstrecken ist, für die $r_{k}>1$. Aus (17) und (23) folgt $\{1\}-\left\{t^{-1} ; 1^{-2}\right\}=\Sigma^{\prime \prime}\left(1-r_{k}\right)+\Sigma^{\prime \prime}\left(1-r_{k}\right)$

oder, wenn wir die Ergänzungsklasse von (i) mit ( $\left.t^{\prime}\right)$ bezeichnen

$$
\left\{1 ;-\left\{t^{\prime}\right\}=\Sigma^{\prime \prime}\left(1-r_{k}\right)+\Sigma^{\prime \prime}\left(1-r_{k}\right)\right. \text {. }
$$

Dir urste Summe ist über alle $r_{k} \leq 1$ und lie zwoite iuber alle $r_{k}>1 \mathrm{zu}$ erstrecken. Daher ist auch

$$
\left\{i-\left\{i^{\prime}\right\}=\mathbf{I}\left(1-r_{k}\right)=m-\mathbf{\Sigma} r_{k}\right.
$$

oder wegen (12) und (15)

$$
\{i-\{1\}=t-p+1 \text {. }
$$

Durch Vergleich mit (19) halen wir also den Satz von Roch: 
Satz IV. Mer Index der Speziellität piner Klasse ist gleich der Dimension ihrer Ergänzungsklasse.

Bezeichnen wir die Ordnung von ( $\left.t^{\prime}\right)$ mit $t^{\prime}$, so folgt aus (21), da die kanoniselue Klasse nach Definition die Ordnung $2 p-2$ hat,

$$
t^{\prime}=2 p-2-t .
$$

Daher können wir (24) auch in der symmetrischen Form schreiben

$$
\{1\}-\frac{t}{2}=\left\{t^{\prime} ;-\frac{t^{\prime}}{2}\right. \text {. }
$$

Fassen wir die Ergebnisse zusammen, so haben wir den RiemannRochschen Satz:

SatzV. Zwischen den Dimensionen zweier Ergänzungsklassen (t) und ( $\left.t^{\prime}\right)$ der Ordnungen $t$ und $t^{\prime}$ besteht die Gleichung

$$
\left\{t=t-p+1+\left\{t^{\prime}\right\}\right. \text {. }
$$

wo $p$ das Geschlecht des Körpers ist. Die Gleichung läßt sich auch in der Form schreiben

$$
\{\}-\frac{t}{2}=\left\{\imath^{\prime} ;-\frac{t^{\prime}}{2}\right. \text {. }
$$

Wir können hiernach die Dimension einer Klasse sehr einfach bestimmen, wenn wir die Dimension ihrer Ergänzungsklasse kennen. Auf einen Fall sei besonders hingewiesen. Wenn die Ordnung der Klasse $\left(t^{\prime}\right)$ negativ ist, wenn also die Ordnung von ( $t$ ) größer als $2 p-2$ ist, so kann $\left(t^{\prime}\right)$ keinen ganzen Divisor enthalten, so daß dann $\left\{t^{\prime}\right\}=0$. Also haben wir:

Satz VI. Wenn die Ordnung $t$ einer Klasse (t) größer ist a]s $2 p-2$, so ist ihre Dimension

$$
\{i=t-p+1
$$

wo p das Geschlecht des Körpers ist.

\section{\$ 4. Zahl der linear unabhängigen Differentiale erster Gattung.}

Wir wenden den Riemann-Rochschen Satz auf die kanonische Klasse ( $)$ an. Die Ergänzungsklasse ist die Hauptklasse (1). Deren Dimension ist aber 1 , da sie den einen und nur den einen ganzen Divisor 1 enthält. Da die Ordnung von (I) nach Definition (vgl. Kap. VI, §6) gleich $2 p-2$ ist, so folgt aus (26)

oder:

$$
\{\mathfrak{t} ;=2 p-2-p+1+1=p
$$

SatzVIl. Die Dimension der Differential-oder kanonischen Klasse ist gleich dem Gesehlechte des Körpers. 
Diesen Satz könmm wir in grewissem Simn nukrheren. Zunärhst besteht (vgl. den Schluß ron $\$ 5$, Kap. VI) dre

Satz VlII. Ist die Ordnungrincr Klasso o, soist ihrel)imension i. a. gleich 0 und nur dann gleieh 1, wonn die Klasso die If a uptklasse ist.

Hat aber eine Klasse die Ordnung $2 p-2$, so hat wegen (25) ihre Ergänzungsklasse die Ordnung 0. Unter Benutzung des wen angegebenen Satzes folgt aus (26):

Satz IX. Die Dimension einer Klasse der Ordnung $2 p-2$ ist i. a. gleich $p-1$ und nur dann gleich $p$, wenn die Klasse die Differentialklasse ist.

Dem Satze VII dieses Paragraphen können wir noch eine andere wichtige Form geben. Ist $\mathfrak{r}$ ein Divisor der kanonischen Klasse ( $\mathrm{t}$ ), so ist (vgl. Kap. VI, §6)

$$
r_{3}^{\mathfrak{l}^{2}} d x=R d x
$$

ein Differential des Körpers $(x y)$. Da $d x$ genau so Null und unendlich wird wie $y^{1-2}$, so wird $R d x$ genan so Null und unendlich wie $x$. Es wird also $R d x$ dann und nur dann nirgends unendlich, wenn $r$ ein ganzer Divisor der Differentialklasse ist. Wrinn wir definieren:

Definition V. Ein Differential des Körpers, das nirgends unendlich wird, heißt ein Differential erster Gattung, so haben wir wegen Satz. VII den

SatzX. Die Zahl der linear unabhängigen Differentiale erster Gattung ist gleich dem Gesehlechte $p$ des Körpers.

Dies ist historisch die ursprünglichr Definition des Geschlechtes.

Es ergibt sich nebenbei, daß $p$ immer eine ganze Zahl ist, die nicht negativ sein kann. Es ist also, wie schon in Kap. VI, $\$ 6$ angegeben, $2 p-2$ immer eine grade Zahl und aus der Gleichung (6) an derselben Stelle folgt dann, daB auch die Verzweigungszahl $w_{\text {s }}$ in bezug auf irgendrine veränderliche Größe des Körpers immer grade ist.

\section{$\$$ 5. Funktionen mit gegebenen Unendlichkeitsstellen.}

Es seien $a_{1}, a_{2}, \ldots a_{p} p$ linear unahhängige ganze Divisoren der Differentialklasse (f). Dann läßt sich jeder andere ganze Divisor a dieser Klasse in der Form darstellen

$$
\mathfrak{a}=a_{1} \mathfrak{a}_{1}+a_{2} \mathfrak{n}_{2}+\cdots+a_{p} \mathfrak{a}_{p},
$$

wo die $a_{i}$ konstant sind. Diese Gleichung besagt nach unsern Festsetzun- 
gn in Kap. VI, \$5 folgendes. Es sei y irgendein Divisor der Klasse (t). Dann soll zwischen den Funktionen

$$
A=\mathfrak{a q}^{-1}, \quad A_{1}=a_{1} q^{-1}, \ldots A_{n}=a_{n} q^{-1}
$$

des Körpers die Gleichung bestehen

$$
A=a_{1} A_{1}+a_{2} A_{2}+\cdots+a_{p} A_{n} .
$$

Vabei muB man, um die Funktionen $A_{i}$, die ja durch (29) nur bis auf einen konstanten Faktor bestimmt sind, vollkommen zu bestimmen, etwa vorschreiben, daß sie den Wert 1 an einer fest gewählten Stelle annehmen sollen, die natürlich von den Null- und Unendlichkeitsstellen der $A_{i}$ verschieden sein muß. Bei der Funktion $A$ kommt es auf einen konstanten Faktor nicht an.

Es sei

ein ganzer Divisor der Ordnung $g \leqq p$. Es seien also die $\mathfrak{p}_{i}$ Primteiler. Wir fragen uns, ob wir einen ganzen Divisor a der kanonischen Klasse ( $\mathrm{t})$ so bestimmen können, daß er durch $!$ teilbar wird. Es muß dann die Funktion $A$ in (29) an den $g$ Stellen $\mathfrak{p}_{1}, \mathfrak{p}_{2}, \ldots \mathfrak{p}_{g}$ Null werden. Bezeichnen wir $A_{i}$ als Funktion einer Stelle $p$ der Riemannschen Fläche mit $A_{i}(\mathfrak{p})$, so ergeben sich die $g$ Gleichungen

$$
\begin{aligned}
& a_{1} A_{1}\left(\mathfrak{p}_{1}\right)+a_{2} A_{2}\left(\mathfrak{p}_{1}\right)+\cdots+a_{p} A_{p}\left(\mathfrak{p}_{1}\right)=0, \\
& a_{1} A_{1}\left(\mathfrak{p}_{2}\right)+a_{2} A_{2}\left(\mathfrak{p}_{2}\right)+\cdots+a_{p} A_{p}\left(\mathfrak{p}_{2}\right)=0, \\
& --------------- \\
& a_{1} A_{1}\left(\mathfrak{p}_{g}\right)+a_{2} A_{2}\left(\mathfrak{p}_{g}\right)+\cdots+a_{p} A_{p}\left(\mathfrak{p}_{l}\right)=0,
\end{aligned}
$$

wobei wir annehmen, daß die $g$ Stellen $\mathfrak{p}_{i}$ voneinander verschieden sind. Diese $g$ Gleichungen sind, wenn nicht die $g$ Stellen $\mathfrak{p}_{i}$ eine besondere Lage haben, voneinander linear unabhängig, wie wir auf folgende Art einsehen.

Es seien die Gleichungen (32) bei beliebiger Wahl der $g$ Stellen $\mathfrak{p}_{i}$ linear abhängig. Dann ist der Rang der Matrix

$$
\left.\mid \begin{array}{llll}
A_{1}\left(\mathfrak{p}_{1}\right), & A_{2}\left(\mathfrak{p}_{1}\right), & \ldots & A_{p}\left(\mathfrak{p}_{1}\right) \\
A_{1}\left(\mathfrak{p}_{2}\right), & A_{2}\left(\mathfrak{p}_{2}\right), & \ldots & A_{p^{\prime}}\left(\mathfrak{p}_{2}\right) \\
-\bar{A}\left(\mathfrak{p}_{g}\right), & A_{2}\left(\mathfrak{p}_{g}\right), & \ldots & A_{p^{\prime}}\left(\mathfrak{p}_{g}\right)
\end{array}\right\}
$$

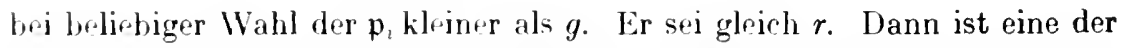
Determinanten $r$-ter Ordnung der Matrix von Null verschieden. Die Bezeichnung sei so gewählt, daß

$$
\left|\begin{array}{lll}
A_{1}\left(\mathfrak{p}_{2}\right) . & A_{2}\left(\mathfrak{p}_{2}\right), \quad \ldots A_{r}\left(\mathfrak{p}_{2}\right) \\
A_{1}\left(\mathfrak{p}_{3}\right), & A_{2}\left(\mathfrak{p}_{3}\right), \quad \ldots & A_{r}\left(\mathfrak{p}_{3}\right) \\
-- & ---\frac{-}{-}-
\end{array}\right| \neq 0
$$


Wir denken uns dir Stellen $p_{2}, p_{3}, \ldots p_{r}$, fest gewählt, so dab die. Ungleichheit (34) besteht. All, Determinanten $(r+1)$-ter Grinung der Matrix (33) sind identisch Null, also ist z. B. die Det reminante, die aus den ersten $r+1$ Zeilen und Spalten besteht, identisch Null. Entwirkehn wir diese nach Unterdeterminanten der ersten Zeile umel schreiben $p$ für $p_{1}$, so erhalten wir eine Gleichung der Form

$$
\lambda_{1} A_{1}(p)+\lambda_{2} A_{2}(p)+\cdots+\lambda_{r} \cdot A_{r} A_{r}(p)=0
$$

und hierin sind die $\lambda_{i}$ Konstante, da die Stellen $p_{2}, \mathfrak{p}_{3}, \ldots \mathfrak{p}_{r}$, fest gewählt sind, und es ist wegen (34) $\lambda_{1} \neq 0$. Daraus würde aber folgen, daß die $p$ Funktionen $A_{i}$ und also auch die $p$ ganzen Divisoren $a_{i}$ nicht linear unabhängig sind. Da das nicht geht, so ist unsere Amahme falsch und es sind die $g$ Gleichungen (32) bei nicht besonderer Wahl der g Strllen $\mathfrak{p}_{i}$ linear unabhängig.

Es gibt also bei nicht besonderer Wahl der $g$ Stellen $\mathfrak{p}_{i}$ genau $p-g$ linear unablängige ganze Divisoren der Klasse ( $\mathfrak{t}$ ), die durch a teilbar sind. Dies gilt für $g \leq p$. Ist $g>p$, so ist die Zahl der Gleichungen (32) größer als die Zahl der Unbekannten $a_{i}$, und da dann die Matrix der Koeffizienten den Rang $p$ hat, so haben die Gleichungen keine Lösung. Es ist also für $g>p$ die Zahl der linear unabhängigen ganzen Divisoren aus $(\mathfrak{f})$, die durch $\mathrm{g}$ teilbar sind, gleich Null. Wenn $g>2 p-2$, so ist die Zahl natürlich immer Null, da die Klasse (f) ja dir Ordnung $2 p-2$ hat.

Die Zahl, die wir bestimmt haben, hat eine einfache Bedeutung. Ist nämlich a durch $g$ teilbar, also $\mathfrak{a}=\mathfrak{n} \mathfrak{a}^{\prime}$, so ist $a^{\prime}$ ein ganzer Divisor der Klasse $\left(a \mathfrak{q}^{-1}\right)=\left(\mathfrak{t} \mathfrak{g}^{-1}\right)$ und umgekehrt, wenn $a^{\prime}$ ein ganzer Divisor dieser Klasse ist, so ist a' g rin ganzer durch o teilbarer Divisor der Klasse (i). 1) bestimmte Zahl ist also dir Dimmsion der Klasse ( $\left(\mathrm{g}^{-1}\right)$ und wir haben:

SatzXI. Ist a ein ganzer Divisor der Ordnung $g$, so ist

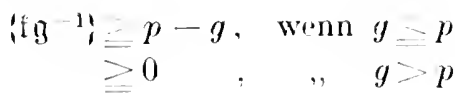

$$
\begin{aligned}
& =0 \quad, \quad, \quad g>2 p-2
\end{aligned}
$$

und :s gilt in (35) das Gleichhoitszeichen, wenn ạ nicht besonders gewïllt ist.

Da $\left(\mathfrak{f} \mathrm{q}^{-1}\right)$ die Ergänzungsklasse von (g) ist, so haben wir nach der Definition einer speziellen Klasse:

Satz XII. Ist geinganzer Divisor der Ordnung $g$, so ist die Klasse (n) für $g<p$ immer speziell, für $g>2 p-2$ immer regulär und für $p=g-2 p-2$ bei nicht besonderer Wahl von? regulär. 
Wir fragen uns jetzt, ob es Funktionen des Kö̈rpers gibt, die den Xenner n haben, oder genauer höchstens den Nenner an, die also die Form haben

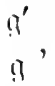

wo $q^{\prime}$ auch ein ganzer Divisor ist. Es muß $q^{\prime}$ - 9 sein, aber von $!$ versehieden, wenn wir von dem trivialen Fall absehen, wo die gesuchte Funktion konstant ist. Es muß also die Klasse (y) mindestens zwei linear unahhängige ganze Divisoren enthalten, d. h. es muß sein $\{9 ; \geq 2$. Aus dem liemann-Rochschen Satze folgt wegen Satz XI

Daraus folgt:

$$
\begin{aligned}
\{: & \geqq 1, \quad \text { wenn } g \\
& \geqq 2, \quad, \quad g, \quad g>p .
\end{aligned}
$$

Satz Xill. Ist g ein ganzer Divisor der Ordnung $g$, so gibt es für $g=p$ im allgemeinen keine Funktion des Körpers, die höehstens den Nenner n hat, abgesehen von der Konstanten. Ist $g>p$, so gibt es immer Funktionen der gew unschten Art.

Es sei jetzt $g>p$ und $\rho$ sei $q$ nicht besonders gewählt; dann wird nach dem Riemann-Rochschen Satz

$$
i g=g-p+1 \text {. }
$$

Es seien $q, a_{1}, \ldots \bigcap_{g-p} g-p+1$ linear unabhängige ganze Divisoren der Klasse (@). Wir setzen

$$
\frac{a}{!}=1 . \quad \frac{a_{1}}{!}=G_{1}, \ldots \quad \frac{a_{n-n}}{!}=G_{g-r} .
$$

Dann hat jedr Funktion

$$
G^{\prime}=c_{0}+c_{1} G_{1}+c_{2} G_{2}+\cdots+c_{y-l} G_{g-p},
$$

wo die $c_{2}$ konstant sind, höchstens den Nenner $g$ und andere Funktionen dieser Art giltt es nicht. Da wir $g-p+1$ linear homogen vorkommende Konstanten $c_{i}$ zur Verfügung haben, können wir noch $g-p$ Nullstellen willkürlich vorschreiben. Außer diesen Nullstellen hat $G$ noch $g-(g-p)$ $=p$ Nullstellen, da die Zahl der Nullstellen gleich der der Unendlichkeitsstellen sein muß. Wir haben also

Satz XIV. In einem algebraischen Körpervom Geschlechte $p$ kann man für $g>p$ immer eine Funktion finden, die $g$ gegebene Unendlichkeitsstellen und $g-p$ gegebene Nullstellen hat. Die Funktion ist beinicht besonderer Wahl der Null-und Unendlichkeitsstellen bis auf einen konstanten Faktor bestimmt. Die übrigen $p$ Nullstellen sind also durch die Angabe der $g$ Unendlichkeits- und $g-p$ Nullstellen i. a. eindeutig mitbestimmt. 
Im Falle $p=0$ kann man also dir Vull- und Unendlichkeitsstellen sämtlich willkiirlinh vorsetursibrn.

\section{$\$$ 6. Körper rom (ieschlechte $p=0$. Der Liirothsche Satz.}

IVir wir in Kap. IV, $\$ 6$ gesehen haben, nimmt jodk Funktion des Körpers $(x y)$ jedrul Wert gleich oft an, und zwar so oft, wis ihre Orduung angibt. Ei suig rine Funktion der (Ordnung $n>0$. Dann können wir 5 als unabhängig, Voränderliche wählen. Jedr Funktion $\eta$ des Körpers genügt dann einer unzerlegbaren Gleichung, deren Granl entwerler $n$ ist oder ein Teiler von $n$. Ist der Grad $n$, so lassen sich alle Funktionen des Körpers als rationale Funktionen von $\xi$, $\eta$ darstellen. (Satz II, §5, Kap V.) Wählen wir $\xi$ von möglichst niedrigner Ordnung, so erhalten wir zur Dיfinition des Körpers statt der Gleichung $f(x, y)=0$ eine Gleichung $\varphi\left(\xi, \eta_{i}\right)=0$, die in $\eta$ von möglichst niedrigem Grade ist.

Ist das Gesehlecht $p=0$, so gibt es nach dem vorigen Paragraphen immer eine Funktion $\xi$ erster Ordnung. Wir können sogar Null- und Unendlichkeitsstelle beliebig wählen. Nehmen wir diese Funktion 5 als unabhängige Veränderliche, so genügt jede andere Funktion einer Gleiehung ersten Grades, deren Koeffizienten rational in $\xi$ sind. D. h. aber, es wird jede Funktion des Körpers eine rationale Funktion von $\xi$.

Lassen sich umgekehrt alle Funktionen des Körpers als rationale Funktionen einer Größe $\lambda$ darstellen, so ist der Körper vom Geschlechte Null. Dabei braucht $\lambda$ dem Körper keineswegs anzugehören. Es gibt nämlich in einem solehen Körper kein Differential erster Gattung. Irgendein Differential läßt sich immer auf dir Form bringen $r\left(\lambda_{)}\right) d \lambda_{\text {, wo }} r\left(\lambda_{\text {. }}\right)$ eine rationale Funktion von $\lambda$ ist. Soll dies Differential nirgends unendlich worden, so muß $r\left(\lambda_{2}\right)$ überall endlich, also konstant sein. Dieser konstante Wert muß aber Null sein, da das Jifferential sonst für unendliches $\lambda$ doeh unendlich würde. Es ist also, wie behauptet, $p=0$. Also:

SatzXV. In einem Körper vom Gesehlechte $p=0$ gibt es immer Funktionen erster Ordnung. Ist $\xi$ eine solehe, so sind alle andern Funktionen des Körpers rationale Funktionen von 5 . Im besondern sind alle Funktionen erster Ordnung lineare (ganze oder gebrochene) Funktionen von $\xi$.

Satz XVI. Sind die Funktionen eines körpers alle als rationale Funktionen eines Parameters $\lambda$. darstollbar, so ist sein Gesehleeht Null.

Man nennt die Körper vom Gesehlechte Null daher auch rational. Körper. Wir können jetzt den Lürothsehen Satz*) heweisen:

*) Lüroth. Math. Ann. 9, S. 163. 
Satz XVll. Sind $x$, y rationale funktionen von $\lambda$, so ist

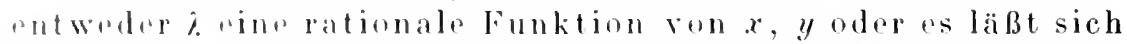
"ine rationale funktion uvon $x$, y finden, so da $B x$, y aneh rationate funktionen von "werden.

Zum beweise betrachten wir die Gesamtheit aller rationalen Funktionen von $x$. y, die einen algebraischen Körper bildet. Jie Funktionen dins's köpers sind aber noch Vuratusetzung rational durch $\lambda$ darstellbarr. so daf3 das Geschlecht des Körpers Null ist. Wir brauchen für $\mu$ nur irgendeine funktion ersten Grades dieses Körpers zu wählen. Dann werdon alle some Funktionon, also auch $x, y$, rational durch "darstellbar. Andererseits ist "l als Funktion des Körpers rational in $x, y$.

Buispinl 1 .

\section{s. . Beispiel 1 und 2.}

$$
f(x, y)=y^{3}-3 x y+2 x^{2}=0 .
$$

Wegen der Bezeichnungen vergleiche man das Beispiel III in $\$ 12$, Kap. VIII. Es ist der Verzweigungsdivisor $\mathfrak{h}=\mathfrak{v}_{1} \mathfrak{b}_{2} \mathfrak{v}_{3}^{2}$, seine Ordnung $w=4$. Da $m=3$, so ist $p=\frac{w}{2}-m+1=0$. Es gibt also im Körper $(x y)$ Funktionen erster Ordnung, und wenn $\xi$ eine solche ist, so werden $x$ und $y$ rationale Funktionen von $\xi$. Wir können die Nullstelle und Unendlichkeitsstelle von $\xi$ willkürlich vorschreiben. Bei $x=0$ liegt ein gewöhnlicher Punkt $\mathfrak{p}$ und ein Verzweigungspunkt $\mathfrak{v}_{1}$. Wir wollen $\xi=\mathfrak{v}_{1} \mathfrak{p}^{-1}$

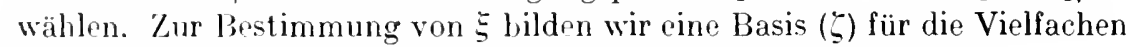
des Ijivisors $v_{1} p^{-1}$. Nach der Methode des Kap. VIII finden wir

$$
\check{s}_{1}=x, \quad \varsigma_{2}=y, \quad \varsigma_{3}=\frac{4}{9}-\frac{3}{x}+\frac{2}{3} \frac{y}{x}+\frac{y^{2}}{x^{2}} .
$$

Bei $x=\infty$ liegt nur der eine Punkt $\mathfrak{v}_{3}$, der ein Verzweigungspunkt zweiter ()rdnung ist. Die dort für $y$ geltende Entwicklung können wir aus Beispiel IV in $\$ 3$, Kap. II ntnthmen. Ersetzen wir dort nämlich $x$ durch $\frac{4}{27} x$ und $y$ durch $-\frac{2}{9} y$, so rrhalten wir dir hier betrachtete Gleichung zwischen $x$ und $y$. Wir finden

$$
y=-12 x^{3}-\frac{1}{2}\left|\frac{3}{4} x^{\frac{1}{3}}+1 \frac{3}{12}\right| 2 x^{-\frac{1}{3}}+\cdots
$$

Daher sind die Ordnungen der $\subsetneq$ für $x=\infty$

$$
r_{1}=1, \quad r_{2}=\frac{2}{3}, \quad r_{3}=0 .
$$


Die Ordnungazahlen sind also nirht gant, win wir os bei unstren allgemeinen Betrachtungen der Einfachleit hallue angenemunen haben. Wir

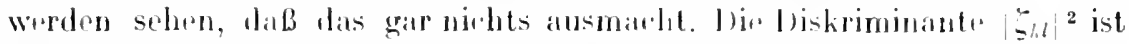

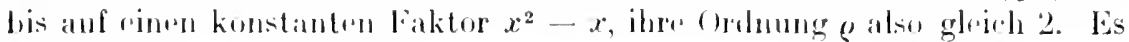
ist daher

$$
2 \underset{1}{\stackrel{3}{*}} r_{k}=\stackrel{10}{3}>0
$$

so daß die Basis (马) für $x=\infty$ nicht normal ist. Wenn as anch zur bestimmnng von $\Xi$ nicht notwendig ist, $(\zeta)$ in eine normale Basis für $x=\infty$ zu verwandeln, so wollen wir es doch der Übung halber tun. Es bedeute eine primitive dritte Einheitswurzel und es sei mit $(\alpha)$ eine Summe von Gliedern bezeichnet, die nur solche Potenzen von $x^{-1}$ enthalten, deren Exponenten mindestens gleich $\alpha$ sind. Dann ist in der Umgebung von $x=\infty$

$$
\left(\zeta_{k l}\right)=\left\{\begin{array}{llr}
x & -\sqrt[3]{2} x^{\frac{2}{3}}+\left(-\frac{1}{3}\right) & \frac{4}{9}-\sqrt[3]{2} x^{-\frac{1}{3}}+\left(\frac{2}{3}\right) \\
x & -\varepsilon^{2} \mid 2 x^{\frac{2}{3}}+\left(-\frac{1}{3}\right) & \frac{4}{9}-\varepsilon^{2} \mid 2 x^{-\frac{1}{3}}+\left(\frac{2}{3}\right) \\
x & -\varepsilon \mid 2 x^{\frac{2}{3}}+\left(-\frac{1}{3}\right) & \frac{4}{9}-\varepsilon \mid 2 x^{-\frac{1}{3}}+\left(\frac{2}{3}\right)
\end{array}\right\} .
$$

Hieraus ersehen wir, daß die Ordnungszahl von

$$
\because=\check{s}_{1}-\frac{9}{4} \zeta_{3} x
$$

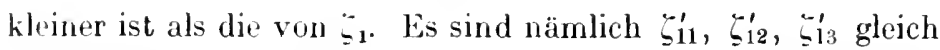

$$
9 \sqrt[3]{2} x^{3}+\left(-\frac{1}{3}\right), \quad 9 \varepsilon^{2} \sqrt[3]{2} x^{2}+\left(-\frac{1}{3}\right), \quad \frac{9}{4} \varepsilon \sqrt[3]{2} x^{\frac{2}{3}}+\left(-\frac{1}{3}\right),
$$

so daß die Ordnungszahl von $\zeta_{1}^{\prime}$ gleich $\frac{2}{3}$ ist. Wir sehen weiter, daß die Ordnungszahl von

$$
\zeta_{2}^{\prime}=\zeta_{2}+\frac{4}{9} \zeta_{1}^{\prime}=\zeta_{2}+\frac{4}{9} \zeta_{1}-\zeta_{3} x
$$

kleiner ist als die von $\zeta_{2}$. Es beginnen nämlich die Entwicklungen von $\zeta_{21}^{\prime}, \zeta_{22}^{\prime}, \zeta_{23}^{\prime}$ mit $x^{\frac{1}{3}}$, sc daß die Ordnungszahl von $\zeta_{2}^{\prime}$ gleich $\frac{1}{3}$ ist. Die Funktionen

$$
\zeta_{1}^{\prime}=\zeta_{1}-\frac{9}{4} x \zeta_{3}, \quad \zeta_{2}^{\prime}=\frac{4_{4}}{9} \zeta_{1}+\zeta_{2}-x \zeta_{3}, \quad \zeta_{3}^{\prime}=\zeta_{3}
$$


bilden anch eine Basis für die Vielfachen von $u_{1} p{ }^{1}$; ihre Ordnungszahlen sind

$$
r_{1}^{\prime}=\frac{2}{3}, \quad r_{2}^{\prime}=\frac{1}{3}, \quad r_{3}^{\prime}=0
$$

Ls ist. $2 \geq r^{\prime}=2=\varrho$, so daß die Basis $\left(\varrho^{\prime}\right)$ normal für $x=\infty$ ist. Eine Funktion

$$
R=g_{1}=\ddot{1}+g_{2}=2+g_{3} \dddot{=}
$$

ist also damn und nur dann rin Vielfaches von $v_{1} p^{-1}$ und hat höchstens die Ordnungazahl $r$, wenn die $g$ ganze rationale Funktionen von $x$ sind

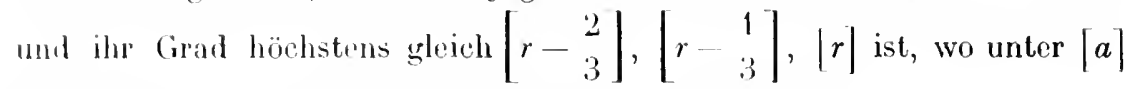
dir größte in a enthaltene ganze Zahl zu verstohen ist.

Die grsuchte Funktion $\Xi$ soll nur an der Stelle p unendlich werden, in Unendlichen also die Ordnungszahl Null haben. Es muß daher bis auf einen konstanten Faktor, der naturgemäß willkürlich bleibt, $\xi=\zeta_{3}^{\prime}$ suin. Diese Funktion ist, wie man leicht sieht, nicht konstant. Wir wollen norh zrigen, daß sich $x$ und $y$ wirklich als rationale Funktionen von $\xi$ darstellen lassen. Wir eliminieren zu diesem Zwocke aus den beiden Gleichungen

$$
\Xi=\frac{4}{9}-\frac{3}{x}+\frac{2}{3} \frac{y}{x}+\left(\begin{array}{l}
y \\
x
\end{array}\right)^{2}, \quad f(x, y) \quad \ldots y^{3}-3 x y+2 x^{2}=0
$$

erst $x$ und dann $y$. Bezeichnen wir $\frac{y}{x}$ mit $z$, so lassen sich die Gleichungen (37) schreiben

$$
\frac{3}{x}=\frac{4}{9}+\frac{2}{3} z+z^{2}-\xi, \quad x z^{3}=3 z-2,
$$

woraus durch Multiplikation folgt

order

$$
3 z^{3}=(3 z-2)\left(\frac{4}{9}+\frac{2}{3} z+z^{2}-\xi\right)
$$

$$
z=\frac{2}{27} \frac{9 \xi-4}{\xi} \text {. }
$$

Setzen wir diesen Wert von $z$ in die zweite der Gleichungen (38) ein, so erhalten wir $x$ und dann $y$ ans $y=z x$. Es ergibt sich

$$
x=\frac{3 \cdot 27^{2} \xi^{2}}{(4-9 \xi)^{3}}, \quad y=-\frac{6 \cdot 27 \xi}{(4-9 \xi)^{2}} .
$$

Beispiel II. In der lntegralrechnung werden gewisse Integrale, die aub $\cdot \mathrm{r}$ der Veränderlichen $x$ noch rine Quadratwurzel aus einer rationalen Funktion von $x$ enthalten, dadureh berechnet, daß man $x$ sowohl 


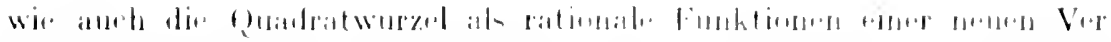

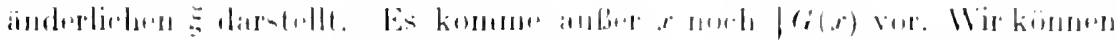

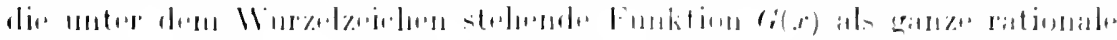

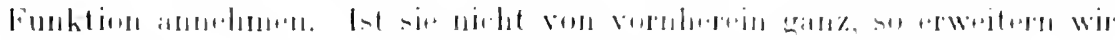

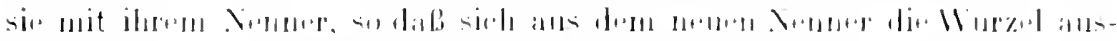

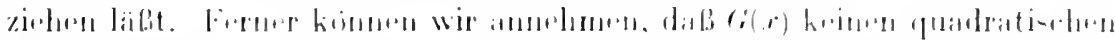

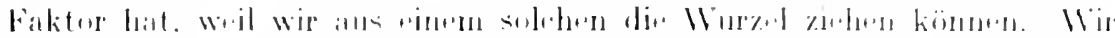

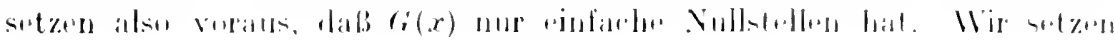

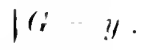

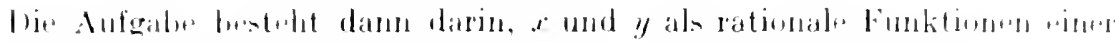

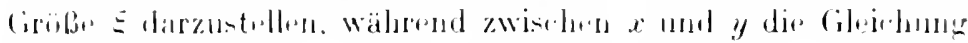

$$
f(x, y) \quad y^{2}-(r(x)-(1
$$

besteht. Wir wissen, dab dan damn mud nur lanm möglinh ist, wenn dup

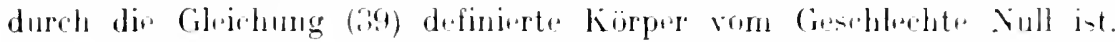

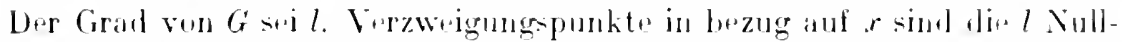
stellen von $G$ und, Wren $l$ ungrade ist, dir urendlich forme Strlle. Es ist daher das Gisclilerht

$$
\begin{aligned}
& p=\frac{1}{2}(l-2), \text { wenn l gradt. } \\
& p=\frac{1}{2}(l-1) \text {, wenn l ungradr, }
\end{aligned}
$$

also nur im Fallo $l=1$ oder $l=2$ glrich Null, so dab as unr in diesen boi den Fällen möglieh ist, $x$ und $y$ als rationale Funktionen einer neuen Gröb. Edarzustellen. Eime passende Größ. E findet man, indem man im Kör per $(x y)$ wine Funktion lwstiment, die nur rine Null- und piue Comdlichkeitsotelle lat. Dats kann so gechehen, wie in Beispiel l auseinandergesetzt. Es sti dem Leser "mpfohlen, die in der Integralrectinung gebräuhbichen Subutitutionen nit der allgemeinen Theorie zu vergheichen.

\section{\$ s. Körper rom Geschlechte 1 und 2 .}

II,nn das Grschirelit $p>0$, so kann as im Körper krine Funktion erster Ordnung geben, wie aus $\$ 6$ folgt. Wonn $p=1$ odrr 2 , sil giht es im Korper immor Funktionen zweiter Ordnung. Für $p=1$ folgt dios

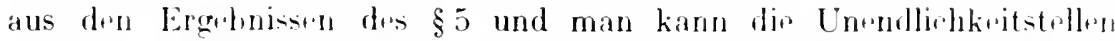

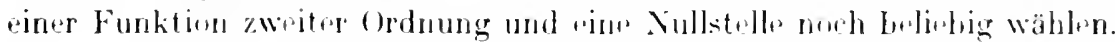
Ist $p=2$, su hat dir Differentialklasse zwoj linear unablıängige ganze Divisoren a. $q^{\prime}$ von der Ordnung $2 p-2=2$. Es ist also q $q^{\prime} 1$ rine Funktion zweiter Ordnung. Ist $p>2$, so können zwar Funktion»ll zwrite Ordnung vorhanden silu, sind es aber im allgemeinen nicht. 
Es soi E cine Funktion zweiter Ordnung und $y_{\text {p }}$ pine Funktion, die einer unzerlegharen Gleichung zwoiten Grades

$$
a y^{2}+2 b y+c=0
$$

genijgt. wo $a . b$ und c ganze ratimalr Punktionen von $\xi$ sind. Dann ist der Körper $(x y)$ gloich dem Körper $(\vdots, 1)$. Die Diskriminante von (41) ist

$$
D(\xi)=b^{2}-a c \text {. }
$$

Wir können sie in der Form schroihen

$$
D(\xi)=H^{2} G,
$$

wo $H$ und $(i$ ganzer rationale Funktionen von $\xi$ sein sollen, und zwar soll $G$ nur einfarhe Nullstellen haben. Wir setzen

Es folgt dann aus (41)

$$
\Sigma=\mid G \text {. }
$$

$$
y_{1}=-\frac{1}{a}\{b+H \zeta\} \text {. }
$$

Daher wird y eine rationale Funktion von $\xi, 5$ und umgekehrt $\zeta$ eine ebensolche Funktion von $\xi, 1$. Der Körper $(x y)$ ist daher auch identisch mit dem Körper $(\xi)$, der definiert ist durch die reine Gleichung

$$
\zeta^{2}-G(\xi)=0 \text {. }
$$

Ist der Grad von $G$ in $\Xi$ gleich $l$, so ist das Geschlecht des Körpers $(\xi)$, wie wir sehon in $\S \tau$, Beispiel 1 I bestimmt haben,

$$
\begin{aligned}
& p=\frac{1}{2}(l-2), \text { wenn } l \text { grade, } \\
& p=\frac{1}{2}(l-1), \text { wenn } l \text { ungrade. }
\end{aligned}
$$

Daher ist G für $p=1$ rom dritten oder vierten und für $p=2$ vom fünften oder serhsten Grade.

liörp.r vom Greshrehte 1 beißen auch plliptisch. Körper vom Gwollerlte $p=2$. die rine Funktion zwitrr Ordnung enthalten, sich aloo immor durch wine Gleirbung d.r Form (42) definieren lassen, heißen hyprolliptisch. Es sind also Körper rom Grschlechte 2 immer hyperalliptisch.

Wir bewisen noch:

Satz XVill. Sind 5 und $\xi_{1}$ zwi Funktionen zweiter Ordnung eines körpers rom Geschlechte 2, so ist die eine eine lineare Funtion der andern.

Wir beweisen den Satz auf zwei Arten. 
Erste Art. Da 5 von zwriter Ordume ist, so grnügt ह, nach Satz III, Kap. IV, \$ 2 rimer Giluchung zweiten Cirades, deren Koeffizienten rationale Funktionen von $\Xi$ sind.

$$
a \xi_{1}^{2}+2 b \xi_{1}+c=0 .
$$

Ebenso grnïgt aber auch $气$ einer Gleichung rersten oder zwriten Grades, deren Korffizienten rationale Funktionen von $\xi_{1}$ sind. laher sind in (43) dir Koeffizinten $a, b, c$ ganze rationale Funktionen von ersten oder zwiten Grade. Ist a nicht identisch Null, so ist nach Satz 11, §5, Kap. V dor Körper identisch mit dem Körper $\left(\xi \xi_{1}\right)$, also mit drm Körper ( $\zeta)$, wo $\zeta=b^{2}-a c$. Aler der Cirad vor $b^{2}-a c$ ist höchstens gleich vier, so daß $p<1$ sein wïrde. Waher ist $a=0$ und ebenso folgt, daß die Gleichung (43) auch in $\xi$ linear sein muB. Damit ist aber Satz XVIII bewiesen.

Zwrite Art. Es seien

$$
\xi=\frac{\Omega^{\prime}}{g}, \quad \xi_{1}=\frac{\Omega_{1}^{\prime}}{g_{1}}
$$

zwei Funktionen zweiter Ordnung, wo $\varrho, \Omega^{\prime}, \varrho_{1}$, $\eta_{1}^{\prime}$ ganze Divisoren zweiter Ordnung sein sollen. Dann ist die Dimension der Klasse (c) mindestens gleich zwei, da sie ja die beiden linear unabhängigen ganzen Divisoren $\mathfrak{g}$ und $\mathfrak{g}^{\prime}$ enthält. Wir finden also mit Hilfe des Riemann-Rochschen Satzes, da $p=2$, oder

$$
\begin{gathered}
\{g\}=1+\left\{f(\}^{-1}\right\} \geq 2, \\
\left.\{f\}^{-1}\right\} \geqq 1 .
\end{gathered}
$$

Die Ordnung der Klasse $\left(\mathrm{f}^{-1}\right)$ ist aber $(2 p-2)-2=0$. Nach Satz VIII, $\$ 4$ folgt also wegen (45), daß sie die Hauptklasse sein muß, daß also $₫$ und $q^{\prime}$ der Differentialklasse (.) angehören. Ceradeso ergibt sich, daß auch $?_{1}$ und ni der Klasse (.) angehören. Da aber () nur zwei linear unabhängige ganze Livisoren enthält, so bestehen Gleichungen der Form

$$
r_{1}=\alpha \beta+\beta q^{\prime}, \quad r_{i}^{\prime}=\gamma^{\prime} \beta+\delta a^{\prime} \text {. }
$$

wo $\varepsilon, \beta, \gamma, \delta$ konstant sind. Wrgen (44) folgt daraus

also die Behauptung.

$$
\xi_{1}=\frac{a \xi+\beta}{\gamma \xi+d}
$$

\section{\$. 9. Brispiel 3 und 4.}

Beispiol HJ. $f(x, y)=y^{4}+2\left(1-x-x^{3}\right) y^{2}+\left(1+x-x^{3}\right)^{2}=0$. Die Diskriminante dirser Gleichung virten Grades in $y$ rgibt sich nach der allgemeinen Formel für die Diskriminante einer Gleichung vierten Grades zu

$$
D(x)=64^{2} x^{2}\left(1-x^{3}\right)^{2}\left(1+x-x^{3}\right)^{2} .
$$


Es kömen daher im andlichen Verzwrigmugsumbte nur liegen bei $x=0$,

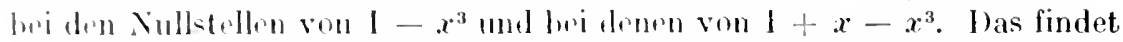
man hestatigt, wrun man die Cleichung $f$ - 0 narh $y$ auflöst. Das ergibt

$$
y=1 x+1 x^{3}-1 \text {. }
$$

llinraus sthen wil, dab bei $x=0, x \sim$ und bei den Nullstellen von $1-x^{3}$ je zwei Verzweigungepunkte rster Urdnung liegen. Daher ist die Verzweigungszahl in bezug auf $x$ gluich $w=10$ und das Geschlecht des kioppers $(x y)$

$$
\text { p } \quad 2 x-m-1+5-1+1=2 .
$$

Wir steflen uns die Aufgabe, rine Funktion 5 zweiter Ordnung zu bestimmen und die Funktionen des Körpers rational durch 5 und durch die Quadratwurzel aus einer rationalen Fuuktion von $\Xi$ darzustellen. Zu diesem Zwecke müssen wir zwei linear unabhängigr ganze Divisoren der Diffrentialklasse bestimmen. Dazu haben wir rine Basis für die Vielfarhen desjenigen Teiles $z^{\prime}$ des Verzweigungslivisor's is aufzusuchen, der sich auf die in Endlichen liegenden Stellen bezicht. Eine solche Basis finden wir bequemer, wenn wir erst eine Basis für die ganzen Funktionen, also für die Vielfachen des Divisors 1, aufsuchen und dazu die komplementäre Basis bestimmen. Wir wollen in dieser Weise verfahren.

Wir gehen aus von der Basis

$$
\left(\xi_{k}\right)=\left(1, y, y^{2}, y^{3}\right) \text {. }
$$

i) Diskriminante dieser Basis ist

$$
\left|\Xi_{k l}\right|^{2}=D(x)=64^{2} x^{2}\left(1-x^{3}\right)^{2}\left(1+x-x^{3}\right)^{2} .
$$

$$
N I\left(z^{\prime}\right)=x^{2}\left(1-x^{3}\right)^{2},
$$

und die $\xi_{i l}$ für endliches $x$ endlich sind, so folgt durch Vergleich von (47), mit (48), daß die Basis ( $)$ nur für die Nullstellen von $1+x-x^{3}$ nicht nicht normal ist. Wir bezeichnen die drei Nullstellen mit $\alpha, \beta, \gamma$ und setzen

$$
g(x)=1+x-x^{3}=-(x-\alpha)(x-\beta)(x-\gamma) .
$$

Wir betrachten die Basis (乡) zunächst in der Umgebung von $x=\alpha$. Bei $x=\alpha$ liegen vier gewöhnliche Punkte der Riemannschen Fläche ïbereinander. Wir erhalten alsn vier nach ganzen Potenzen von $x-\alpha$ fortschreitende Potenzreihen für $y$, nümlich

$$
\begin{aligned}
& y_{1}=2 \mid \bar{\alpha}+\frac{1+3 \alpha^{2}}{2 \downarrow \alpha}(x-\alpha)+\cdots \\
& y_{2}=\frac{1-3 \alpha^{2}}{2 \downarrow \alpha}(x-\alpha)+\cdots, \quad y_{3}=-y_{1}, \quad y_{4}=-y_{2},
\end{aligned}
$$


wie man an oinfarhsten aus (46) herloitre, wobei abre zu berïksichtigen

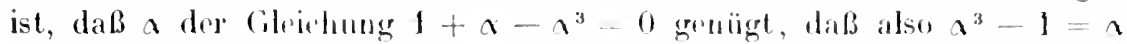
ist. Brzejehnen wir mit $(s)$ eine Summe vun Ciliedern, die mindestens durch dio $s$-tw Potrinz vou $x$ - a teilbar sind, so ist hirrnach in dor Umgebung voll ir $\quad$ r

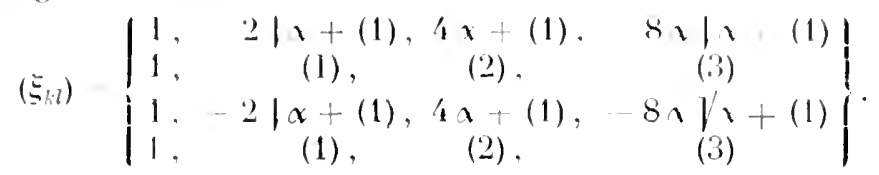

Djes System haben wir durch rine passende Substitution $a^{\prime}$ in rine Einheit für $x=x$ zu verwandeln. Wir subtrahieren zunächst das \& a-fache der zweiten Vertikalreihe von der vierten und dividieren dann die

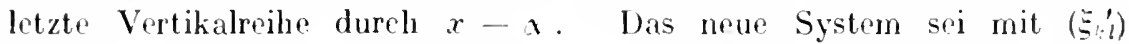
und die neue Basis mit (乡') bezeichnet. Wie wir schon olon festgestullt haben (vgl. 47), ist $\left|\xi_{k l}\right|$ genau durch die erste Potenz von $x-$ a teilhar. Daher ist die Determinante des neuen Systems für $x=\alpha$ endlich und von Null versehieden. Da ferner die $\xi_{l l}^{\prime}$ fïr $x=\alpha$ endlich sind, so ist $\left(\xi_{l l}^{\prime}\right)$ eine Einheit für $x=\alpha$. Die Substitution, durch die die Basis ( $\left.\Xi_{n}^{\prime}\right)$ aus der Basis $\left(\xi_{k}\right)$ hervorgeht, ist

$$
a^{\prime}=\left\{\begin{array}{cccc}
1 & 0 & 0 & 0 \\
0 & 1 & 0 & -4 x \\
0 & 0 & 1 & 0 \\
0 & 0 & 0 & 1
\end{array}|| \begin{array}{cccc}
1 & 0 & 0 & 0 \\
0 & 1 & 0 & 0 \\
0 & 1 & 0 & 0 \\
0 & 0 & 0 & (x-x)^{-1}
\end{array}\right\}=\left\{\begin{array}{cccc}
1 & 0 & 0 & 0 \\
0 & 1 & 0 & -4 \alpha(x-x)^{-1} \\
0 & 0 & 1 & 0 \\
0 & 0 & 0 & (x-x)^{-1}
\end{array} \mid .\right.
$$

biese Substitution ist nicht dir rinzige, die aus der basis (ङ) bine neue lervorgehen läßt, die für $x=\alpha$ in bezug auf den Divisor 1 normal ist, und die anch überall da normal ist, wo es $\left(\xi_{n}\right)$ ist. Wir können z. B. die lionstante $-4 x$ durch irgendeine ganze rationale Funktion von $x$ ersotzen, die für $x=x$ den Wert - 4 a annimmt. Wir nelimen die Funktion - 4x. Wir dürfen forner $x$ - a ersetzen durch cine rationale Funktion $h(x)$, die den Faktor $x-\alpha$ in der ersten Potenz entlält, wenn wir nur dafür sorgen, daB die Substitution eine Einheit bleibt für alle Stellen, für die ( $\xi$ ) normal ist. Daher darf die Funtion $h(x)$, die wir an Stelle von $x$ - a nehmen wollen, nur Null oder unendlich werden an den Nullstellen von $g(x)$, da (E) nur für diese Stollen nicht normal ist. Wir wollen $x$ - a durch $g(x)$ ersetzen. Die Substitution, dureh die wir uns aus der Basis $\left(\xi_{k}\right)$ eine noue Basis $(\leftrightarrows)$ luorleiten wollen, ist daher

$$
a=\left|\begin{array}{cccc}
1 & 0 & 0 & 0 \\
0 & 1 & 0 & -4 x g^{-1} \\
0 & 0 & 1 & 0 \\
0 & 0 & 0 & g^{-1}
\end{array}\right|
$$


Dureh diese Inderung erreichen wir einmal, daß wir die Wurzel $\alpha$ der Gilichung $g(x)=0$ nicht zu berechuen brauchen, dann aber wegen der Symmetrie von ( $(\xi)$ und $\alpha$ in bezug auf die drei Wurzeln $\alpha, \beta, \gamma$ von $g(x)=0$, daß die neue Basis (ڤ) nicht nur für $x=\alpha$, sondern auch für $x=\beta$ und $x=;$ normal ist in bezug auf den Divisor 1. Man kann in dieser Weise bei der Bestimmung einer Basis für die ganzen Funktionen immer mit dè Zahlen des Zahlkörpers auskommen, zu dem die Koeffizienten der Glichung $f=0$ gehören. Darauf soll aber hier nicht näher eingegangen werden. Die Basis ( $(\vdots)$ ist für alle Stellen im Endlichen normal in bezug auf den Divisor 1; sie ist also eine Basis für die ganzen Funktionen. Ihre Diskriminante ist

$$
\left.\zeta_{3, b}\right|^{2}=\left|\xi_{3 .,}\right|^{2}\left|a_{i, b}\right|^{2}=D(x) g^{-2}=64^{2} x^{2}\left(1-x^{3}\right)^{2} .
$$

Die komplementären Basen von $(\xi)$ und $(\xi)$ seien $\left(\eta^{\prime}\right)$ und $\left(\eta^{\prime \prime}\right)$. Es ist $\left(\zeta_{1}\right)=\left(\xi_{1}\right) a$. Bezeichnen wir mit $b$ das komplementäre System von $a$, so ist also

$$
\left(y^{\prime}\right)=\left(y^{\prime \prime}\right) b .
$$

Da in der Gleichung $f=0$ nur grade Potenzen von $y$ vorkommen, so ist gleichzeitig mit $y$ auch $-y$ eine Wurzel von $f=0$. Wir wählen, wie schon oben, die Bezeichnung so, daß $y_{3}=-y_{1}, y_{4}=-y_{2}$. Dann ist

$$
\xi_{h l}|=z=| \begin{array}{rrrr}
1 & y_{1} & y_{1}^{2} & y_{1}^{3} \\
1 & y_{2} & y_{2}^{2} & y_{2}^{3} \\
1 & -y_{1} & y_{1}^{2} & -y_{1}^{3} \\
1 & -y_{2} & y_{2}^{2} & -y_{2}^{3}
\end{array} \mid=-4 y_{1} y_{2}\left(y_{1}^{2}-y_{2}^{2}\right)^{2} .
$$

Ferner sind die Unterdeterminanten der ersten Zeile von $z$

so daß

$$
\begin{array}{ll}
z_{11}=2 y_{1} y_{2}^{3}\left(y_{1}^{2}-y_{2}^{2}\right), & z_{21}=2 y_{2}^{3}\left(y_{1}^{2}-y_{2}^{2}\right), \\
z_{31}=-2 y_{1} y_{2}\left(y_{1}^{2}-y_{2}^{2}\right), & z_{41}=-2 y_{2}\left(y_{1}^{2}-y_{2}^{2}\right),
\end{array}
$$

$$
\begin{aligned}
& r_{11}^{\prime \prime}=-\frac{y_{1} y_{2}^{2}}{2 y_{1}\left(y_{1}^{\prime}-y_{2}^{\prime 2}\right)}, \quad r_{21}^{\prime \prime}=-\frac{y_{2}^{2}}{2 y_{1}\left(y_{1}^{\prime 2}-\frac{\left.y_{2}^{2}\right)}{2}\right)} \\
& r_{31}^{\prime \prime}=\frac{y_{1}}{2 y_{1}\left(y_{1}^{2}-y_{2}^{2}\right)}, \quad r_{11}^{\prime \prime}=\frac{1}{2 y_{1}\left(y_{1}^{2}-y_{2}^{2}\right)} .
\end{aligned}
$$

Fabt man di: Gloichung $f=0$ auf als quadratische Gleichung für $y^{2}$, so ercilut sich aus ihr $y_{1}^{2}+y_{2}^{2}=-2\left(1-x-x^{3}\right)$.

Wa ferner

$$
\begin{aligned}
& \hat{c} \dot{\partial} y\left(x, y_{1}\right)=\left(y_{1}-y_{2}\right)\left(y_{1}-y_{3}\right)\left(y_{1}-y_{4}\right)=\left(y_{1}-y_{2}\right)\left(y_{1}+y_{1}\right)\left(y_{1}+y_{2}\right) \\
& =2 y_{1}\left(y_{1}^{2}-y_{2}^{2}\right),
\end{aligned}
$$

so folgt, wenn wir zum Schlub $y_{1}$ durch $y$ ersetzen, 
(51)

$$
\begin{array}{ll}
\partial f y_{1}^{\prime \prime}=y\left|y^{2}+2\left(1-x-x^{3}\right)\right| . & \partial f y^{\prime \prime}=y^{2}+2\left(1-x-x^{3}\right), \\
\partial f y^{\prime \prime}=y, & \partial f y=1 . \\
\partial y^{\prime 3}=y, & \partial y^{\prime \prime \prime}=1 .
\end{array}
$$

Als komplementäres System zu a findet man

$$
b=\left\{\begin{array}{cccc}
1 & 0 & 0 & 0 \\
0 & 1 & 0 & 0 \\
0 & 0 & 1 & 0 \\
0 & 4 x & 0 & g
\end{array}\right\}
$$

so da $B$ aus (50) und (51) folgt

$$
\begin{aligned}
& \partial f y_{i}^{\prime}=y\left[y^{2}+2\left(1-x-x^{3}\right)\right], \quad \frac{\partial f}{\partial y^{\prime}} \eta_{2}^{\prime}=y^{2}+2\left(1+x-x^{3}\right), \\
& \frac{\partial f}{\partial y} r_{3}^{\prime}=y \\
& \frac{\partial f}{\partial y} \eta_{i}^{\prime}=1+x-x^{3} \text {. }
\end{aligned}
$$

Es ist $\left(\eta^{\prime}\right)$ eine Basis für die Vielfachen von $z^{\prime}$.

Wir sehen zu, ob $\left(y^{\prime}\right)$ für $x=\infty$ normal ist. Es ist in der Umgebung von $x=\infty$, wie am einfachsten aus (46) folgt, $y_{1}=x^{\frac{3}{2}}+x^{\frac{1}{2}}+\cdots, \quad y_{2}=x^{\frac{3}{2}}-x^{\frac{1}{2}}+\cdots, \quad y_{3}=-y_{1}, \quad y_{4}=-y_{2}$. Hiermit findet man zunächst als Ordnungszahl von $\frac{\partial f}{\partial y}$ den Wert $\frac{7}{2}$ und dann als Ordnungszahlen der $r^{\prime}$

$$
r_{1}^{\prime}=\frac{2}{2}, \quad r_{2}^{\prime}=-\frac{1}{2}, \quad r_{3}^{\prime}=-\frac{4}{2}, \quad r_{4}^{\prime}=-\frac{1}{2} .
$$

Da das System $\left(g_{i}^{\prime} l\right)$ komplementär ist zu $\left(G^{\prime} l\right)$, so ist die Diskriminante von (a') reziprok zu der von (c), also nach (49)

Ihr Frad $\varrho$ ist -8 und daher

$$
\left|y_{1}^{\prime}\right|^{2}=61^{-2} x^{-2}\left(1-x^{3}\right)^{-2} \text {. }
$$

$$
2 \pm r_{k}^{\prime}=-4>\varrho,
$$

sodaß dir Basis $\left(y^{\prime}\right)$ für $x=\infty$ nicht normal ist. Wir wollen sie in eine normale verwandeln. Es ist in der Ungebung von $x=\infty$, wip aus (46) folgt,

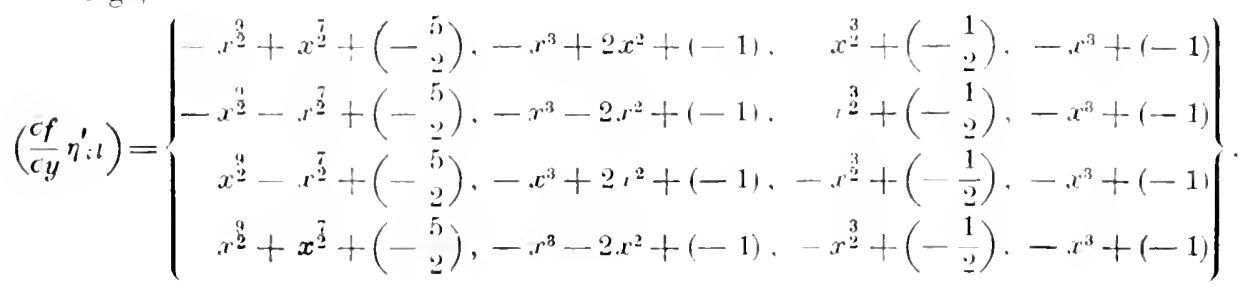


wo winder mit $(s)$ Glieder bezeichnet sind, die mindestens die $s$-te Potenz ron $x^{-1}$ enthatten. Hipraus arsehen wir, da $B$ die Funktionen

$$
r_{1}=y_{1}^{\prime}+x^{3} 1_{13}^{\prime}, \quad 1_{2}=y_{2}^{\prime}-y_{1}^{\prime}
$$

lithinere Ordnungszahlen haben als $y_{1}^{\prime}$ und $y_{2}^{\prime}$, und zwar 0 und $-\frac{3}{2}$. Die funktionen

$$
\eta_{1}=\eta_{1}^{\prime}+x^{3} \eta_{3}^{\prime}, \quad \eta_{2}=\eta_{2}^{\prime}-\eta_{4}^{\prime}, \quad \eta_{3}=\eta_{3}^{\prime}, \quad \eta_{4}=\eta_{4}^{\prime}
$$

bilden auch eine Basis für die Vielfachen von $z^{\prime}$; ihre Ordnungszahlen sind

$$
r_{1}=0, \quad r_{2}=-\frac{3}{2}, \quad r_{3}=-2, \quad r_{4}=-\frac{1}{2},
$$

so daß

$$
2 \Sigma r_{k}=-8=\varrho \text {. }
$$

Die Basis $\left(1_{1}\right)$ ist daher normal für $x=\infty$. Demnach ist rine Funktion des Körpers

$$
R=g_{1} \eta_{1}+g_{2} \eta_{2}+g_{3} \eta_{3}+g_{4} \eta_{4}
$$

dann und nur dann ein Vielfaches von $z^{\prime}$ und hat höchstens die Ordnungszahl $r$, wenn die $g$ ganze rationale Funktionen von $x$ sind, deren Grade höchstens gleich

$$
|r|, \quad\left[r+\frac{3}{2}\right], \quad|r+2| \cdot\left[r+\frac{1}{2}\right]
$$

sind, wo wieder $\lceil a\rfloor$ die größte in $a$ enthaltene ganze Zahl bedeuten soll. Soll das Differential $P d x$ auch für unendliches $x$ nicht unendlich werden, so muß die Ordnungszahl von $P$ kleiner als -1 sein. Es müssen also $g_{1}$ und $g_{4}$ identisch Null sein, während $g_{2}$ und $g_{3}$ konstant sein müssen. Daher erhalten wir, wie es sein muß, zwei linear unabhängige Differentiale erster Gattung

$$
R_{1} d x=\left(y^{2}+1+x-x^{3}\right) \frac{d x}{\frac{\partial f}{\partial y}}, \quad R_{2} d x=y \frac{d x}{\frac{\partial f}{\partial y}} .
$$

Ihr Quotient liefert uns die gesuchte Funktion 5 zweiter Ordnung

$$
\xi=\frac{y^{2}+1+x-x^{3}}{y} .
$$

Die Funktion $\xi$ hat eine sehr einfache Bedeutung. Aus der Gleichung $f=0$ folgt nämlich

und daraus

$$
\left(y^{2}+1+x-x^{3}\right)^{2}=4 x y^{2}
$$

$$
\xi=2 \sqrt{x} \text {. }
$$


Es ist also, in Primteiler zorlegt,

$$
\xi=\frac{v_{1} v_{2}}{v_{3} v_{1}}
$$

wenn mit $\mathrm{v}_{1}, \mathrm{v}_{2}$ dir bei $x=0$ liegenden und mit $\mathrm{v}_{3}, \mathrm{v}_{4}$ die bei $x=\infty$ lisgenden Verzweigungspunkte und Printeiler bezeichnet werden. Aus (46) und (56) folgt

$$
x=\frac{1}{4} \xi^{2}, \quad y=\frac{1}{2} \xi+\frac{1}{8} / \xi^{6}-\overline{64},
$$

so daB sich $x$ und $y$ und damit alle Funktionen des Körpers $(x y)$ rational durch $\xi$ und die Quadratwurzel aus $\xi^{6}-64$ ausdrücken lassen. Damit ist die Aufgabe, die wir uns gestellt hatten, gelöst.

Beispiel IV. Besonders einfach ist die Bestimmung einer Basis für die ganzen Funktionen und für die Vielfachen von $z$ oder $z^{\prime}$ in einem hyperelliptischen Körper. Nehmen wir die den Körper definierende Gleichung in der Form an

$$
y^{2}=G(x)
$$

wo $G(x)$ eine ganze rationale Funktion von $x$ ist mit nur einfachen Nullstellen, so ist schon $(1, y)$ eine Basis für die in bezug auf $x$ ganzen Funktionen. Denn die Funktionen der Basis werden für endliches $x$ nicht unendlich und die Diskriminante der Basis, nämlich $4 y^{2}$ ist im wesentlichen gleich der Idealnorm $G(x)$ von $z$ oder $z^{\prime}$.

Der Grad von $G(x)$ sei $l=2 p+2$ oder $2 p+1$, je naclidem $l$ grade oder ungrade ist. Als komplementär Basis zu $(1, y)$ ergiht sich, wenn wir den Faktor $\frac{1}{2}$ fortlassen.

$$
\left(\begin{array}{ll}
1 & , 1 \\
y & 1
\end{array}\right.
$$

Diese Basis ist normal für $x=\infty$. Die Ordnungszahlen ihrer Funktionen sind $r_{1}=-\frac{1}{2} l, r_{2}=0$, so daB alle und nur die Differentiale des Körpers, die im Endlichen nicht unendlich werden und für unendliches $x$ höchstens von der Ordnung $r+2$, sich darstellen lassen in der Form

$$
\left(\frac{g_{1}}{y}+g_{2}\right) d x
$$

wo $g_{2}$ und $g_{2}$ ganze rationale Funktionen von $x$ sind, und zwar höchstens vom Grade $r+\frac{1}{2} l$ und $r$. Wollen wir im besonderen die Differentiale erster Gattung haben, so muB $r \leq-2$ sein, also $g_{2}$ identisch Null und $g_{1}$ 
höchstens vom Grade $\frac{1}{2} l-2$ sein; wir erhalten so folgende $p$ linear unabhängigen Differentiale erster Gattung

$$
\frac{d x}{y}, \quad x d x, \quad \frac{x^{2} d x}{y}, \ldots \frac{x^{n-1} d x}{y} .
$$

\section{$\$ 10$. Weierstrabpunkte.}

Es sei $\mathfrak{p}$ eine Stelle des Körpers. Den zugehörigen Primteiler bezeichnen wir auch mit $p$. Ferner sei $g$ eine positive ganze Zahl. Wir fragen, ob es im Körper Funktionen gibt, die an der Stelle $p$ von der $g$-ten Ordnung unendlich werden und sonst nirgends, die also die Form haben

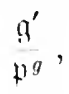

wo $q^{\prime}$ ein ganzer Divisor ist. Es ist dies dieselbe Frage wie in $\S 5$, nur daß die vorgesehriebenen Unendlichkeitsstellen hier alle zusammenfallen sollen. Es ist der dort mit n bezeichnete Divisor gleich $\mathfrak{p}^{7}$. Wenn $g \geqq p+1$, so gibt es immer eine Funktion der gewünschten Art. Denn nach dem Riemann-Rochschen Satz ist wegen $g \geqq p+1$

$$
\left\{p^{\prime}\right\} \geqq g-p+1 \geqq 2,
$$

so daß es einen ganzen zu $\mathfrak{p}^{g}$ äquivalenten Divisor $\mathfrak{g}^{\prime}$ gibt, der von $\mathfrak{p}^{q}$ linear unalhängig ist. Dagegen wird es für $g \leq p$ im allgemeinen eine solche Funktion nicht geben. Wir wollen sogar beweisen, daß die Zahl der Stellen $p$, für die eine Funktion der Art (59) mit $g \leq p$ existiert, endlich ist. Wir definieren:

Definition VI. Gibt es eine Funktion des Körpers, die nur an der Stelle punendlich wird und von einer Ordnung, die gröBer als Nullundkleinerals $p+1$ ist, so heißt die Stelle ein Weierstraßpunkt.

Jer Satz, den wir bewrisen wollen, lautet dann:

Satz XIX. Die Anzahl der Weierstraßpunkte ist endlich.

Es sei $\mathfrak{p}$ ein Weierstrabpunkt; rs sei (59) eine Funktion des Körpers und es sei $g \leqq p$. Dann hat lie Klasse $\left(p^{r}\right)$ zwei linear unabhängige ganze Divisoren, nämlich $\mathfrak{p}^{\prime}$ und $\mathfrak{p}^{p-g} \mathfrak{g}^{\prime}$. Hat umgekehrt die Klasse $\left(\mathfrak{p}^{\prime \prime}\right)$ zwei linear unabhängige ganze Divisoren $\eta^{\prime}$ und $p^{\prime \prime}$, so ist die Stelle $p$ ein Weierstrafpunkt, weil $g^{\prime} p^{-r}$ eine Funktion des Körpers ist, die nur an der Stelle $\mathfrak{p}$ unendlich wird und dort von geringerer als $(p+1)$-ter Ordnung.

Nach $\S 5$ ist aber $\left\{\mathfrak{p}^{\prime \prime} \geqq 2\right.$ dann und nur dann, wenn es in der kanonischen Klasse einen ganzen Divisor gibt, der dureh $p^{p}$ teilbar ist. Es habe $x$ an der Stelle $p$ den Wert $\xi$ und cs sei $p$ kein Verzweigungspunkt 
Zehntes Kapitel. Die Kurve $f(x, y)=0 . \$ 1$. Die mehrfachen Punkte. 171

und keine der Un'ndlichkeitsstellen der Fumktionen $A_{i}$ in (29), 5 . Dann lassen sich die fiunktionen $A_{2}$ und $A$ in (29), 55 nach ganzen positiven Potenzen von $x-\xi$ entwickeln, und wenn der $A$ entsprechende ganze Divisor a der kanonischen Klasse durch p"trilbar sein soll, so muß diese Entwicklung mit der $p$-ten Potenz von $x-\xi$ beginnen. Daraus ergeben sich nach dem Taylorschen Satze die Gleichungen

$$
\begin{aligned}
& a_{1} A_{1}(\xi)+a_{2} A_{2}(\xi)+\cdots+a_{p} A_{p}(\xi)=0, \\
& a_{1} A_{1}^{\prime}(\xi)+a_{2} A_{2}^{\prime}(\xi)+\cdots+a_{p} A_{p}^{\prime}(\xi)=0, \\
& -------------------- \\
& a_{1} A_{1}^{\prime p-1)}(\xi)+a_{2} A_{2}^{p-1)}(\xi)+\cdots+a_{p} A_{p}^{(p-1)}(\xi)=0 .
\end{aligned}
$$

Daraus folgt, da $\beta$ die Determinante der lineffizienten der $a_{i}$ gleich Null sein muß. Diese Determinante ist aber die Wronskische Determinante der $p$ Funktionen $A_{i}(\xi)$. Wäre sie identisch Null, so würden die $A_{i}$ linear abhängig sein, was nicht der Fall ist. Dann aber kann die Determinante nur für eine endliche Zahl von Werten $\xi$ Null werden, da sie eine algebraische Funktion von $\xi$ ist. Damit ist aber unser Satz bewiesen, da die beim Beweise ausgeschlossenen Stellen nur in endlicher Zahl vorhanden sind.

Wenn das Geschlecht $p=0$, so hat es keinen Sinn, von Weierstraßpunkten zu sprechen. Wenn $p=1$, so gibt es keine Weierstraßpunkte, da aus deren Existenz das Vorhandensein von Funktionen erster Ordnung folgen würde. Wenn $p>1$, gibt es immer Weierstraßpunkte. Wir gehen darauf aber nicht ein*).

Man vergleiche zu diesem Paragraphen Kap. XIII, §8.

Zehntes Kapitel.

\section{Die Kurve $f(x, y)=0$.}

\section{$\$ 1$. Die mehrfachen Punkte.}

Wir können die den Körper definierende Gleichung $f(x, y)=0$ als Gleichung einer kurve deuten, indem wir $x$ und $y$ als kartesische Koordinaten in riner Ebene betrachten. Dir so definierte kurve heißt eine algobraische kurve. Wir bezochnen sie mit $f$. Der Grad $(l, m)$ von $/(x, y)$ heißt dir Orduung derkurve. Es sei $P \quad(a, b)$ cin Punkt von $f$. Wenn $a$ oder $b$ unendlich ist, so hat man die Gleirhung $f(x, y)=0$ durch die Substitution $x=\frac{1}{x^{\prime}}$, oder $y=\frac{1}{y^{\prime}}$, umzuformen und dann die neue Glei-

*) Man sehe etwa H.-L. 28. Vorlesung, §2 und §3. 
chung in der l'mgedung von $x^{\prime}-0$ ader $y^{\prime} \quad 0$ zu betrachton. Wir können also im folgenden a und $b$ endlich ammhmen, da wir die Fälle, wo dies nicht dor Fall auf diesen Fall in der angegebenen Weise zurüiekführen. Wolln wir die knre $f$ in der Nähe von $P$ betrachten, so werden wir $f(x, y)$ nach Potenzen von $x-a, y-b$ antwikeln. Diese Entwicklung sei, nich homogenen Funktionen von $x$ a. $y-b$ geordunt,

$$
f(x, y)=u_{0}+u_{1+1}+\cdots .
$$

wi) $u_{k}$, ine homogene Funktion rom Grade $k$ in $x-a, y-b$ bedeuten silt. Wir nehmen an, daß die Glieder niedrigster Dimensionen, die in der Entwickhng vorkommen. die Dimension $v$ haben. Da der Punkt $(a, b)$ anf $f$ liegt, so ist $r=1$. Wer Punkt $P$ heißt ein $y$-lacher Punkt der Kurve. lst $y>1$, so heißt er ein mehrfacher Punkt, für $y=2$ auch im besonderen .in Doppelpunkt. Nach dem Taylorschen Satze ist

$$
u_{1}=\frac{\partial f}{\partial x}(a, b)(x-a)+\frac{\partial f}{\partial y}(a, b)(y-b) .
$$

Soll $v>1$ sein, so muß $u_{1}$ identisch verschwinden, "s müssen also die koordinaten $a, b$ den drei Gleichungen genügen

$$
f=0, \quad \frac{\partial f}{\partial x}=0, \quad \frac{\partial f}{\partial y}=0 .
$$

Ijiese Bedingungen sind aber aueh hinreichend. Wir haben also:

Ein Punkt $P$ ist dann und nur dann ein mehrfacher Punkt dur Kurvf. Wenn seine koordinaten den Gleiehungen genügen

$$
f=0 . \quad \frac{\partial f}{\partial x}=0 . \quad \frac{\partial f}{\partial y}=0 .
$$

lit $f$ keine mohrfachen Faktoren enthält, so können die drei Funktionen $f . \frac{\partial f}{\partial y}, \quad \partial y$ krinen gemeinsamen Faktor haben, und die Gleichungen (1) halen höchstens eine endliche Zahl von gemeinsamen Lösungen. Also:

Eine algebraische kurve hat höchstens eine endliehe Zahl von mehrfachen Punkten.

Es sei wir früher

$$
f=a_{0}(x) y^{m}+a_{1}(x) y^{m-1}+\cdots+a_{m}(x),
$$

wo die $a(x)$ ganze rationale Funktionen vom Grade $l$ in $x$ sind. Es ist

$$
\frac{\partial f}{\partial y}=m a_{0} y^{m-1}+(m-1) a_{1} y^{m-2}+\cdots+a_{m-1} .
$$


Es wird also $\begin{aligned} & \partial f \\ & \partial y\end{aligned}$

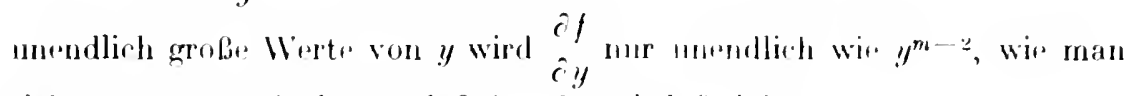

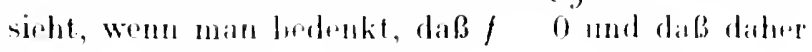

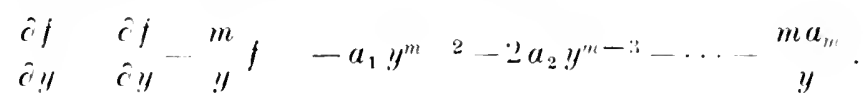

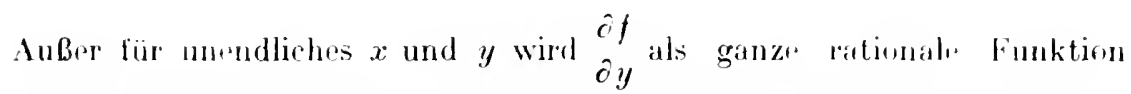
von $x, y$ nirht unendlich. Bezoiehnen wir den Nenner von $x$ mit $\mathfrak{l}$ und den von $y$ mit $m$, so können wir also setzen, wenn wir noch bedenken, daß ganz ähnliche Überlegungen für $\frac{\partial f}{\partial x}$ grelten,

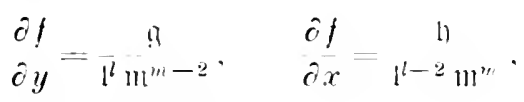

wo $g$ und $h$ ganze Divisoren sind.

Da ein Punkt von $f$ dann und nur dann ein mehrfacher Punkt ist, wenn in ihm $\frac{\partial f}{\partial x}$ und $\frac{\partial f}{\partial y}$ verschwinden, so können wir sagen: wenn p ein in 9 und in $h$ enthaltener Primteiler ist und wenn $x$ und $y$ an der Stelle $\mathrm{p}$ die Werte $a$ und $b$ annehmen, so ist $(a, b)$ ein mehrfacher Punkt von $f$ und umgekehrt muB einem mehrfachen Punkt von $f$ mindestens ein Primteiler $p$ entsprechen, der sowohl in ?q wie in h) aufgeht. Der größte gemeinsame Teiler von ! und b liefert uns also die mehrfachen Punkte von $f$.

Bezeichnen wir den Verzweigungsdivisor in bezug auf $x$ mit ${ }^{\prime}$ und den in bezug auf $y$ mit sy, so besteht nach (7) Kap. VI, $\S 6$ die Gleichung

$$
\frac{d y}{d x}=\frac{i_{i} I^{2}}{3 x 1^{2}} \text {. }
$$

Andererseits ist nach (3)

$$
\frac{d y}{d x}=-\frac{\partial x}{\frac{\partial f}{\partial y}}=\frac{g^{2}}{! m^{2}} .
$$

Es sind also die Divisoren $9 \lambda_{y}^{-1}$ und $h_{h x}{ }^{-1}$ einander gleich. Wir bezeichnen diesen Divisor mit $\mathrm{o}$. Wir haben dann die Darstellungen

$$
\frac{\partial f}{\partial x}=\frac{\partial z_{y}}{1^{-2} m^{\prime \prime}}, \quad \frac{\partial f}{\partial y}=\frac{\delta i_{x}}{l^{l} n^{n-2}} .
$$


Wir werden weiter unten sehen, daß der Divisor o immer ganz ist. llir lezirhnen den größten gemeinsamen Teiler von $j . x$ und sy mit $r$. lann ist der größte grmeinsame Teiler von a und h gleich o $x$. Der Divisor D. linfert uns also die mehrfachen Punkte von $f$. Wir werden aber sehen, daßB o immer durch $x$, sogar durch $x^{2}$ teilhar ist, so daß uns schon der Divisur o alle mohrfaten Punkte von $f$ liefort. Er heißt daher der Divis or der mehrfachen lenkte oder auch wohl der Doppelpunkt-Divisor inn $t$.

\section{\$2. Die Kinrrenzweige.}

U'm dir Besehaffenheit von $f$ in der Ungebung von $P$ kennenzulermen, benutzen wir die Reihenentwicklungen, die für $y$ in der Umgebung von $x=a$ gilten. Da der Punkt $(a, b)$ auf $f$ lingt, so muß unter den Reihen mindestens eine vorkommen, die für $y$ den Vert $b$ liefert, wenn man $x=a$ s.tzt. Eine solche Entwicklung sei

$$
y=y_{1}=b+b_{0}(x-a)^{\frac{u}{\lambda}}+b_{1}(x-a)^{\frac{u+1}{2}}+\cdots,\left(b_{0} \neq 0\right) .
$$

Sie sehreite also nach ganzen Potenzen von $(x-a)^{\frac{1}{k}}$ fort, und die Entwicklung von $y-b$ beginne mit der $\iota^{u-t e n}$ Potenz von $(x-a)^{\frac{1}{\lambda}}$. Es ist " $\geq 1$. Der zu der Entwicklung (6) gehörende Primteiler sei p. Er ist ein Verzweigungsprimteiler der Ordnung $\lambda-1$ in bezug auf $x$ und, da $y-b$ durch $p^{\prime \prime}$ teilbar ist, cin Verzweigungsprimteiler der Ordnung $\mu-1$ in bezug auf $y$. Es ist also $p$ in $\beta_{x}$ und $z$ genau in den Potenzen $\lambda-1$ und $u-1$ enthalten. Bezeichnen wir mit $\alpha$ die kleinere der beiden Zahlen i. - 1,,-1 oder, wenn sie gleich sind, die Zahl $\lambda-1=\mu-1$, so ist $p$ in dem größt en gemeinsamen Teiler $x$ von $z_{x}$ und $z_{y}$ genau in der Potenz $\alpha$ inthalten.

Aus der Funktion $y_{1}$, grhen, wenn $\lambda>1$, noch andere hervor, indem $x$ don Punkt a rinmal indre mehrer Male umkreist. Wir wollen sie bezhithren mit $y_{2}, y_{3}, \ldots y_{j}$. Die Bezeichnung soll so gewählt werden, daß $y_{1}$ ïlergellat in $y_{2}, y_{2}$ in $y_{3}, \ldots y_{2}$ in $y_{1}$, wenn $x$ don Punkt $a$ einmal in puitivem Sinne nmkrist. Lassen wir $x$ alle Werte in der Umgebung ron a durchlanfen, wobri rin rinmaliges oder mohrmaliges Umkreisen von a grotattet srin soll, so durehläuft der Punkt $(x, y)$ ein Stück der Kurw $f$. Wir nennen ein so definiertes Stüek einen durch $P$ gehenden Kurvonzwrig und nennen $P$ den Mittrlpunkt des Zwriges. Wir wollen den durrh $y=y_{1}, y_{2}, \ldots y_{2}$ definirerten Zwoig mit $p$ bezeichnen. Es können durrh $P$ noch andere Zwerge grhen. Srill ausdrücklich hervorgehoben wroten, daß $P$ als auf einem bestimmten $\mathrm{Zweige} \mathrm{liegend} \mathrm{betrachtet} \mathrm{werden}$ soll, so sprechen wir nicht vom Punkte $P$, sondern von der Stelle $P$. Ein 
Punkt $P$ von f zerfält also gewissermaben in so vid Stellen, wie Zweige durch ihn gehen.

Das rerodukt

$$
f_{1}(x, y) \quad\left(y-y_{1}\right)\left(y-y_{2}\right) \ldots\left(y-y_{i}\right)
$$

ist rine ganz" gationale Funlition vom Grate $\lambda$ in $y$. Wit lineffizinton sind symmetrishe aranze rationale Funktion von $y_{1}, y_{2}, \ldots y$, und daher grwöhnliche lentenzreihen von $x$-a. Die Funktion $f_{1}(x, y)$ wird in der Umgebung von $P$ Null nur für die Punkte des Zweiges p. Wï nennen $f_{1}$ dirzu $p$ grhörige Funktion. Es lommt bei der Funktion $f_{1}$ nicht an auf cinen Fatitor, der cine Einheit für die Stelle $(a, b)$ ist. Wir nennen daher auch jode Funktion, die sich von $f_{1}$ nur um einen solchen Falitor unterscheidet, zu $p$ grhörig. Das Wesentliche für eine solche Funtion ist, daß sir jn der Umgebung von $(a, b)$ nur für die Punkte von $p$ Null wird. $E s$ ist also auch nicht notwendig, daß sie eine rationale Funtion von $y$ ist.

\section{$\$ 3$. Gestalt reeller Kurvenzweige.}

Ehe wir weiter gehen, wollen wir uns von der Gestalt des recllen Teiles eines Kurvenzweiges eine Vorstellung machen. Ist $t$ eine Funktion, die an der Stelle p von der ersten Ordnung Null wird, so können wir den Zweig $p$ auch statt durch die Entwicklung (6) durch die Gleichungen

$$
x-a=A_{0} t^{\prime}+A_{1} t^{i+1}+\cdots, \quad y-b=B_{0} t^{u}+B_{1} t^{\prime \prime+1}+\cdots
$$

definieren, wo $A_{0}$ und $B_{0}$ von Null verschieden sind. Für das Folgende ist es zweckmäßig, diese Entwicklungen etwas anders zu schreiben. Es sei $\alpha$ die kleinere der beiden Zahlon $\lambda, "$, wenn $\lambda \neq \mu$ und $\alpha=\lambda=\mu$, wenn $\lambda=\mu$. Dann können wir schreiben

$$
x-a=c_{0} t^{\prime 2}+c_{1} t^{\prime \prime}+1+\cdots, \quad y-b=e_{0} t^{\mu}+e_{1} t^{\prime 2}+1+\cdots,
$$

wo jetzt $c_{0}$ und $e_{0}$ nirht beide Null sind. Setzen wir diese Entwirklungen in eine ganze rationale Funktion $g(x, y)$ von $x$ und $y$ oin, so wird sie eine grwöhnliche lotenzreihe von $t$ und wir liönnen setzen

$$
g(x, y)=t i E(t)
$$

wo $E(t)$ eine Einheit für $t=0 \mathrm{ist}$. Es ist dann nach früheren Festsetzungen $g$ durch pi teilbar. Deuten wir $g=0$ als kurve, so wird diese dureh $P$ dann und rut damn gohen, wenn $\gamma>0$, und je größor $\gamma$ ist, desto stärker werden die kurve $g=0$ und der Zwrig $p$ sich beribhen. Wir wollen sagen, die Kurve $g=0$ und der Zweig $p$ haben $\gamma$ Schnittunlite an der Stclle $P$. Wir werden witer unten auf diese Definition zurückiommen und ihre volle Berechtigung nachweisen. 
Wir betranten die geraden Linien, die durch den Punkt $p$ gehen.

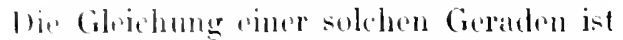

$$
g(x, y)=A(x-a)+B(y-b)=0 .
$$

w" A nnd $B$ kemstant sind. Sotzen wir die Entwieklungen (7) in die linke Siritr dieser Gleichung ein, so thatton wir

$$
y(x, y)=\left(A e_{0}+B e_{0}\right) t^{\prime \prime}+\left(.1 c_{1}+B e_{1}\right) t^{\prime \prime+1}+\cdots .
$$

Ha $e_{0}$ und $e_{0}$ nicht beide Null sind, so hat rine beliebige gerade Linie durch $P$ mit $p$ in $P$ a Schnittpunkte. Da in allgrmeinen $\lambda$ und $\mu$ und also auch $\checkmark$ gleich 1 sind, so ist in allgemeinen die Zahl der Schnittpunkte gleich 1 . Es giht nur rine endliche Zahl von Zweigen der Kurve $f$, für die $\alpha>1$. Wollen wir eine Gerade durel $P$ haben, dir melsr als a Schnittpunkte mit $p$ in $P$ hat, so mussen wir $A$ und $B$ so bestimmen, daß

$$
A c_{0}+B e_{0}=0 \text {. }
$$

Hierdurch ist das Verhältnis von $A$ und $B$ eindeutig bestimmt. Es gibt also immer eine und nur eine gerade Linie, die $p$ in $P$ in mehr Punkten schneidet als eine beliebige durch $P$ gehende Gerade. Diese Gerade wird sich dem Zweige $p$ von allen durch $P$ gehenden Geraden am stärksten anschmiegen. Sie ist die Tangente des $Z$ weiges im Punkte $P$. Jeder Kurvenzweig hat also in seinem Mittelpunkte eine gan z bestimmte Tangente. Iie Zahl der Sclnittpunkte der Tangente mit $p$ in $P$ wird im allgemeinen $\alpha-1$ sein. Sie ist nur größer, etwa gleich $\alpha+\beta$, wenn gleichzeitig mit (8) auch noch die Gleichungen bestchen

$$
A c_{1}+B e_{1}=0, \ldots A c_{\beta-1}+B e_{\beta-1}=0,
$$

während $A c_{\varsigma}+B e_{\varsigma} \neq 0$. Die Gestalt des Zweiges hängt, wie wir jetzt schen werden, wesentlich von den Zahlen $\alpha$ und $\beta$ ab.

Wir setzen $a$ und $b$ und die Koeffizienten $c$ und $e$ als reell voraus und ebenso $t$. Jann sind auch $x$ und $y$ reell. Wir machen durch eine Koordinatentransformation den Punkt $P$ zum Arfangspunkt, die Tangente zur 5 - und die Normale zur $r$-Achse eines neuen Koordinaten-Systems. Wir haben dann die Entwicklungen

$$
\Xi=g_{0} t^{\alpha}+g_{1} t^{\alpha+1}+\cdots, \quad y=h_{0} t^{\alpha+\beta}+h_{1} t^{\alpha+\beta+1}+\cdots .
$$

Um einen bestimmten Fall zu haben, nehmen wir $g_{0}$ und $h_{0}$ positiv an. Wir betrachten zunächst die einfachsten Fälle, nämlich folgende vier:

$$
\begin{aligned}
& \begin{array}{lll}
\text { 1. } \alpha=1 . & \beta=1 ; & \text { II. } \alpha=2, \quad \beta=1 ; \quad \text { III. } \alpha=1, \quad \beta=2 ;
\end{array} \\
& \text { IV. } \alpha=2, \beta=2 \text {. } \\
& \text { I. } \alpha=1, \quad \xi=1 . \quad \xi=g_{0} t+\cdots, \quad \eta=h_{0} t^{2}+\cdots \text {. }
\end{aligned}
$$


Für hinlänglich kloime Werte von $t$ kämonen wir uns and die Anfangs-

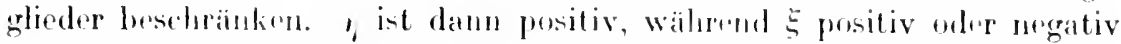

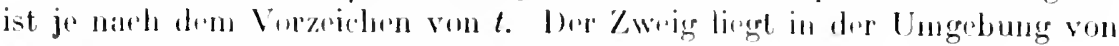
$P$ ginz auf rime Seite der Tangente $y=0$ ( $/ b, 1 \mathrm{r} .30)$.

$$
\text { II. } 12, \beta=1 . \quad \xi=g_{0} t^{2}+\cdots, \quad 1=h_{0} t^{3}+\cdots .
$$

Für kleine Werte von $t$ ist 5 positiv,, pesitiv oder negativ je nach dem Vorzeichen von $t$. Der Zweig liegt anl beiden Seiten der Tangente $y=0$ (Ab). 31). Der Zwrig hat in $P$, wie man sagt, eine Spitze. Fassen wir $t$ als Zeit auf und lassen wir $t$ die Werte eines kleinen den Nullpunkt enthaltenden Intervalles durchlaufen, so durchläuft der Punkt $(\xi, y)$ den Zweig. Die Riclitung der Bewegung ändert sich in $P$ um $180^{\circ}$. Der Punkt $(\xi, \eta)$ kehrt in $P$ um. Der Punkt heißt daher auch $R$ ückkehrpunkt. Die Geschwindigkeit des Punktes $(\xi, \eta)$ muß in $P$ gleich Null sein, da die Bewrgungstetigerfolgt und daher $\left(\xi, y_{i}\right)$ die entgegengesetzte Richtung erst einsehlagen kann, wenn srine Geschwindigkeit

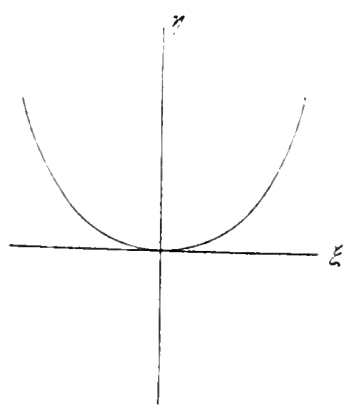

Abb. 30 .

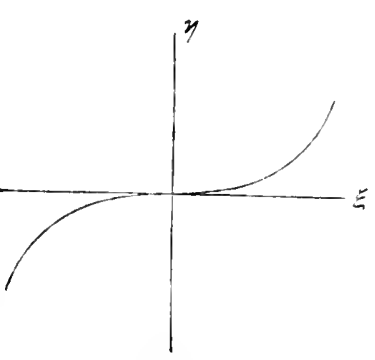

Abb. 32 .

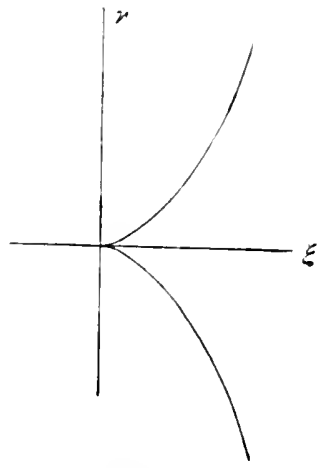

Ibb. 31 .

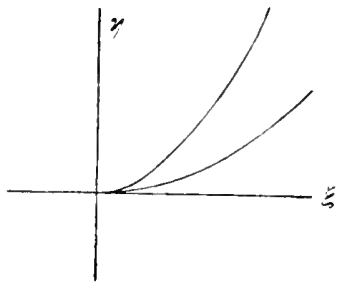

Abb. 33.

Null geworden ist. Der Punkt $P$ heißt daher auch stationärer Punkt. Die Tangente im Punkte $(\xi, \eta)$ dreht sich immer in demselben Sinne, wenn $\left(\xi, y_{1}\right)$ den Zweig durchläuft.

$$
\text { 111. } \alpha=1, \quad \beta=2 . \quad \xi=g_{0} t+\cdots, \quad \eta=h_{0} t^{3}+\cdots .
$$

Für klrine Werte von $t$ sind $\xi$ und $\eta$ positiv und negativ, je nachdem $t$ positiv oder negativ ist. Der Zweig liegt auf beiden Seiten der Tangente und auf beiden Seiten der Normale (Abb. 32). Fassen wir wieder $t$ als Zeit auf, so durchläuft der Punkt $(\xi, \eta)$ bei wachsender Zeit den Zweig ohne in $P$ anzuhalten, aber die Tangente in $(\xi, \eta)$ ändert beirn Durchgange des 
Punktes $(\xi, 1)$ dureh $P$ ihren Drehungrsinn. Sie hält daher in $P$ einen Angenblick an. Die Tangente in $P$ heißt taher stationäre Tangente oder auch wegen der Änderung drs Drehungssinnes Wondotangente und $P$ (in Wendepunkt.

IV. $\alpha=2, \quad \beta=2 . \quad \xi=g_{0} t^{2}+g_{1} t^{3}+\cdots, \quad \eta=h_{0} t^{4}+h_{1} t^{5}+\cdots$.

Wir können uns hier nicht auf die Anfangsglieder beschränken. Es würden dann die beiden Stücke des Zweiges, die positiven und negativen Werten von $t$ entsprechen, zusammenfallen. Für kleine Werte von $t$ überwiegen aber die Anfangsglieder, so daß $\zeta$ und, beide positiv sind für positive und negative kleine Werte von $t$. Der Zweig liegt ganz im ersten Quadranten. Die auf die Anfangsglieder folgomdon Glipder bewirken ein Abwoichen des Zweiges von der durch $\xi=g_{0} t^{2}, l_{i}=h_{0} t^{4}$ für reelles $t$ dargestellt'n Kurve (einer halben Parabel), und zwar ist das Abweichen verschieden, je nachdem $t$ positiv oder negativ ist (Abb. 33). Man nennt den Punkt $P$ eine Schnabelspitze. Lassen wir den Punkt $(\xi, \eta)$ den Zweig durchlaufen, so ändert er in $P$ seine Bewegungsrichtung um $180^{\circ}$, seine Geschwindigkeit ist also in $P$ Null. Ebenso ist die 1)rehgeschwindigkeit der Tangente des Punktes $(5, \eta)$ in $P$ Null, da die Tangente dort den Drehungssinn ändert. Der Punkt $P$ ist also stationär und die Tangente in $P$ auch.

Bei beliebigem $\alpha$ und $\beta$ hängt die Gestalt des Zweiges davon $a b$, ob $x$ und $\beta$ grade oder ungrade sind. Es sind so folgende vier Fälle zu unterscheiden.

$$
\begin{gathered}
\text { I. } x=1, \beta=1 ; \quad \text { II. } \alpha=2, \quad \beta=1 ; \quad \text { III. } \alpha=1, \beta=2 ; \\
\text { IV. } \alpha=2, \beta=2 ;(\bmod 2) .
\end{gathered}
$$

In roher Annäherung ist das Aussehen des Zweiges in jedem dieser vier Fälle so wie in dem eben betrachteten Falle mit derselben Nummer. Es wird dem Leser empfohlen, für verschiedene Werte von $\alpha$ und $\beta$ die $Z$ weige genau zu zeichnen. Man sieht dadurch am besten, wie die Gestalt von den Zahlen $\alpha$ und $\beta$ abhängt.

Wenn $\alpha>1$, so heißt $P$ stationär von der Ordnung $\alpha-1$, und wenn $\beta>1$, so heißt die Tangente in $P$ stationär von der Ordnung $\beta-1$. Wir bilden zwei Divisoren $r$ und $i$. In $r$ nelhmen wir jeden Primteiler in der Potenz auf, die angibt, von welcher (Ordnung die zugehörige Stelle stationär ist, und in $i$ in der Potenz, die angibt, von welcher Ordnung die Tangente an der zugehörigen Stelle stationär ist. Ier zur Stelle $P$ gehörende Primteiler ist also in $r$ in der Potenz $\alpha-1$ und in $i$ in der Potenz $\beta-1$ entbalten. Der Divisor $x$ ist identisch mit dem gemeinsamen Divisor von $z_{x}$ und $z_{y}$, in dem ja auch $p$ in der Potenz $\alpha-1$ enthalten ist. Die Ordnungen von $r$ und $i$ bezeichnen wir mit $r$ und $i$. Wenn nur Rückkehrpunkte und 
Wendetangenten erster Ordnung vorkommen, so sinel $r$ und $i$ de Anzahl dieser Punhte und Tangenten. Im allgemeinen Fallr künnen wir ihnen dieselbe Bedentung beilegen, wenn wir ïbremkonnen, jeden Rüekkehrpunkt und jede. Wendetangente se oft zu zahlen, wie ilere Ordnung angiltt.

\section{\$4. Zahl der Schnittpunkte zweier Kurvenzweige.}

Wir hetrachten außer $p$ noch einen zweiten durch $P$ gehenden Kurvenzweig $q$. Es braurht $q$ nicht derselben Kurve anzuguhören wie $p$. Die Zweige $p$ und $q$ seien in der Umgebung von $P$ definiort durch fie Entwicklungen

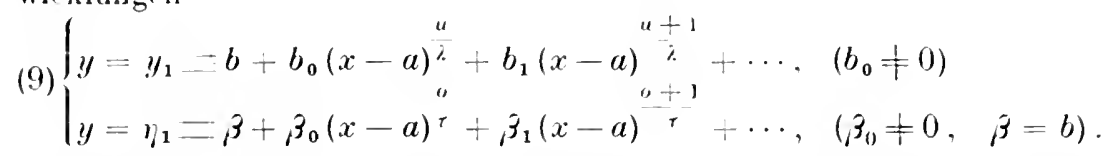

Sind $\lambda$ und $t$ größer als 1 , so bezeichnen wir dio adjungierten Entwicklungen mit $y_{2}, y_{3}, \ldots y_{i}$ und $\eta_{2}, \eta_{3}, \ldots, r_{1}$. Die zu $p$ und $q$ gehörenden Funktionen sind

$$
\begin{aligned}
& g(x, y)=\left(y-y_{1}\right)\left(y-y_{2}\right) \ldots\left(y-y_{j}\right), \\
& h(x, y)=\left(y-y_{1}\right)\left(y-r_{2}\right) \ldots\left(y-y_{r}\right) .
\end{aligned}
$$

Diese Funktionen lassen sich darstellen als gewöhnliche Potenzreihen von $x-a, y-b$. Setzen wir in $g(x, y)$ für $y$ die Entwicklung $\eta_{1}$ und entwickeln nach Potenzen von $(x-a)^{\tau}$, so mögen wir erhalten

$$
g\left(x, 3_{11}\right)=\left(3_{11}-y_{1}\right)\left(\eta_{1}-y_{2}\right) \ldots\left(\eta_{1}-y_{i}\right)=(x-a)^{\frac{n}{\tau}} E_{1},
$$

wo $E_{1}$ rine Einheit für $x=a$ sei. Wir wollen dann sagen, die Zweige $p$ und $q$ haben $\rho$ Schnittpunkte miteinander in $P$ gemeinsam. Wir bezeichnen die Zahl dieser Schnittpunkte mit $(p, q)$ oder $(q, p)$. Um die Berechtigung dieser Definition nachzuweisen, haben wir zu zeigen, daß sie symmetriseh von $p$ und $q$ abhängt. Lassen wir $x$ den Punkt $a$ in positivem Sinne umkreisen, so gehen aus der Gleichung (11) folgende Gleichungen hervor: $g\left(x, y_{k}\right)=\left(y_{i k}-y_{1}\right)\left(l_{i k}-y_{2}\right) \ldots\left(r_{k}-y_{k}\right)=(x-a)^{\frac{g}{r}} E_{k}, \quad(k=1,2, \ldots r)$, wo dir $E_{k}$ sämtlich Einheiten für $x=a$ sind. Durch Multiplikation dieser Gleichungen folgt

$$
\prod_{l, 1}^{+} g\left(x, \eta_{k}\right)=\prod_{k, l}\left(y_{i k}-y_{l}\right)=(x-a)^{\prime \prime} E .
$$

wo $E=E_{1} E_{2} \ldots E_{\text {q }}$ eine Einheit für $x=a$ ist. Wir können daher $(p, q)$ 
$=-(q . \mu)$ definieren als den Exponenten der l'otenz von $x-a$, durch die das l'rodukt $\Pi\left(y_{1}-y\right)$ trilhar ist. Es ist aber, wie aus

$$
\prod_{k, l}\left(b_{i}-y_{i}\right) \quad \prod_{l} g\left(x, l_{l}\right) \quad(-1)^{i r} \prod_{l} h\left(x, y_{l}\right)
$$

folgt. $\Pi(1,-y)$ im wosentlichen symmetrisch abhängig von $p$ und $q$. Iliraus folgt auch, daß $h\left(x, y_{i}\right)$ genau durch $(x-a)^{2}$ teilbar sein muB.

Es ist für das Folgende zweckmäßig. auch noch das Symbol $(p, p)$ in folgender Wrise zu definieren. Es sei

$$
\hat{\partial g}\left(x, y_{1}\right) \quad\left(y_{1}-y_{2}\right)\left(y_{1}-y_{3}\right) \ldots\left(y_{1}-y_{\lambda}\right)=(x-a)^{\frac{\omega}{2}} E_{1},
$$

wo $E_{1}$ eine Einheit für $x=a$ sei. Es ist für $k=2,3, \ldots \lambda$ nach (9)

$$
y_{1}-y_{i}=b_{0}(x-a)^{u}\left(1-e^{\frac{2 \pi i}{i} u k}\right)+\cdots,
$$

so daß $y_{1}-y_{:}$mindestens teilbar ist durch $(x-a)^{\frac{u}{\partial}}$. Wenn aber $\mu k$ durch $i$ teilbar ist, also z. B. dann, wenn $" \iota=\lambda$ ist, ist $y_{1}-y_{k}$ sogar mindestens teilbar durch $(x-a)^{\frac{\alpha+1}{i}}$. Es ist daher

(1) $\geq \mu(\lambda-1)$, wenn $\lambda \mp \mu ; \omega \geqq(\mu+1)(\lambda-1)=\mu^{2}-1$, wenn $\lambda==\mu$. Es ist jedenfalls $\omega \geq \lambda-1$. Wir definieren $(p, p)$ durch die Gleichung (1) $=(p, p)+\lambda-1$, so daB

$(p, p) \quad(\mu-1)(\lambda-1)$, wenn $\lambda \neq \mu ;(p, p) \geq \mu(\mu-1)$, wenn $\lambda=\mu$.

Wenn $\mu:=1$, so ist in der Gleichung (13) kein $\mu k$ durch $\lambda$ teilbar, also genau (1) $=\lambda-1$, und $(p, p)=0$. Ist fernor $\lambda=1$, so ist die Funktion (12) ielontisch gleich 1 und daher $\omega$ und damit $(p, p)$ gleich Null. Es ist also $(p, p)$ nur dann von Null verschieden, und dann immer positiv, wenn $i$ und $"$ bejde größer als 1 sind.

Wir wollen die Definition für $(p, p)$ noch umformen. Dabei werden wir auch sehen, daß die Jefinition gleichmäßig von $x$ und $y$ abhängt. E - ist für $y=y_{1}$ identisch $g(x, y)=0$, also $\frac{\partial g}{\partial x} d x+\frac{\partial g}{\partial y} d y=0$ oder mit Benutzung von (6) und (12)

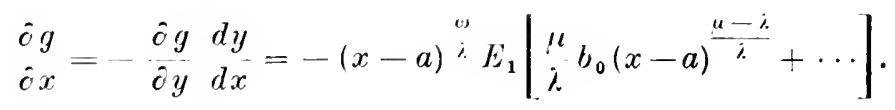

Setzen wir also

$$
\frac{\partial g}{\partial x}\left(x, y_{1}\right)=(x-a)^{\star *} E^{\prime}
$$


wo $E^{\prime}$ cine Einheit für $x=a$ ist, so ist $\varepsilon=(1)+\mu-\lambda=(p, p)+\mu-1$, während $\omega=(p, p)+\lambda-1$ ist. Wir orselen hirraus, daß die Zahl $(p, p)$ symmetrisch von $x$ und $y$ abhängt.

Die Zahl $(p, p)$ ist, wie wir jetzt zeigen, immer grate. Aus der Gleichung (12) gehen noch andere hervor, dadurch dab wir $x$ den Punkt $a$ umkreisen lassen. Wir erhalten so die Gleiehungen

$$
\begin{aligned}
& \frac{\partial g}{\partial y}\left(x, y_{k}\right) \quad\left(y_{k}-y_{\omega}\right) \ldots\left(y_{k}-y_{k-1}\right)\left(y_{k}-y_{k+1}\right) \ldots\left(y_{k}-y_{i}\right) \\
& =(x-a)^{i} E_{l:},
\end{aligned}
$$

wo $E_{k}$ wieder eine Einheit für $x=a$ ist. Durch Multiplikation folgt

$$
\prod_{k, l}^{\lambda}\left(y_{k}-y_{l}\right)=(x-a)^{a} E=(x-a)^{(p, p)+\lambda-1} E,
$$

wo $E=E_{1} E_{2} \ldots E_{k}$ eine Einheit für $x=a$ und außerdem eine gewöhnliche Potenzreihe von $x-a$ ist. Durch Ausziehen der Quadratwurzel folgt

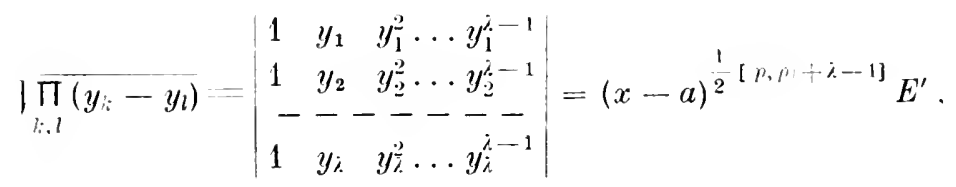

wo $E^{\prime}=\sqrt{E}$ eine Einheit für $x=a$ ist und nach ganzen Potenzen von $x-a$ fortschreitet. Lassen wir $x$ den Punkt $a$ einmal in positivem Sinne umlaufen, so geht $y_{1}$ in $y_{2}, y_{2}$ in $y_{3}, \ldots y_{i}$ in $y_{1}$ über. Es ändert daher in der Gleichung (14) die Determinante hierbei ihr Zeichen oder sie bleibt ungeändert, je nachdem $\lambda$ grade oder ungrade ist. Dasselbe muß von $(x-a)^{\left.\frac{1}{2}[m, p)+2-1\right]}$ gelten, da $E^{\prime}$ als gewöhnlich Potenzreihe von $x-a$ ungeändert bleibt. Also folgt

$$
\begin{aligned}
&(p, p)+\lambda-1- 0, \quad(\bmod 2) \\
& 1, \quad \text { wenn } \lambda=1 \\
& \text { wenn } \lambda=0
\end{aligned} \quad(\bmod 2)
$$

oder is ist immer, wir behauptet, $(p, p)-0(\bmod 2)$.

Wir fassen die Ergebnisse zusammen.

Es sei $p$ ein Kurvenzweig oiner algobraischen Kurve. Der zugehörige Primteiler sei p. Er sei cin Verzweigungsprimteiler der Ordnung $\lambda-1$ in bezugauf $x$ und der Ordnung $u-1$ in bezug auf $y$. Es sei ferner $g(x, y)$ die zu $p$ gehörige Funktion und es seien $\frac{\partial g}{\partial x}$ und $\frac{\partial g}{\partial y}$ genau teilbar durch $p^{\left(i_{1}\right.}$ und $\mathfrak{p}^{(w)}$. Dann sind die Zahlen $\omega_{1}-(\mu-1)$ und $\omega_{2}-(\lambda-1)$ einauder gleich und sollen mit $(p, p)$ bezeichnet werden. Für $(p, p)$ gelten folgende Sätze. 
Es ist $(p, p)=0$, wenn auch nur pinc der Zahlen $\lambda, \mu$ gleich I ist, wenn alsopuicht in $r$ enthalten ist. Im übrigen ist $(p, p)$ $>0$ und genaluer

$$
\begin{aligned}
(\mu, p) & \geq(\lambda-1)(\mu-1), \text { wenn } \lambda \neq \mu, \\
& \geq \lambda(\lambda-1)=\mu(\mu-1), \text { wenn } \lambda=\mu .
\end{aligned}
$$

sehließlich ist noch $(p, p)$ immer grade.

\section{\$ 5. Der Divisor der mehrfachen Punkte.}

Wir gehen dazu über, den Divisor o der mehrfachen Punkte genauer zu untersuchen. Er ist definiert durch die Gleichungen (5), von denen jede zu seiner Definition ausreicht. Wir benutzen die Gleichung

$$
\frac{\partial f}{\partial y}=\frac{D j x}{I^{1} m^{m-2}} .
$$

Es sei $P-(a, b)$ ein Punkt der Kurve $f$. Durch ihn mögen die Zweige $p_{1}, p_{2}, \ldots p_{r}$ gehen. Wir nennen den Punkt dann $r$-zweigig. Die zu $p_{k}$ gehörende Funktion sei $f_{k}(x, y)$. Jede der Funktionen $f_{k}$ besteht aus Faktoren der Form $y-y_{\text {c }}$, wo $y_{\text {c }}$ eine der Wurzeln der Gleichung $f=0$ ist. Es ist daher $f$ durch das Produkt der $f_{k}$ teilbar:

$$
t=f_{1} f_{2} \ldots f_{r} E,
$$

wo $E$ rine ganze rationale Funktion von $y$ ist, deren Koeffizienten gewöhnliche Potenzreihen von $x-a$ sind. Da aber $f$ in der Umgebung von $P$ nur längs der Zweige $p_{k}$ Null wird, so muß $E$ eine Einheit für $P \equiv(a, b)$ sein. Es sei $p_{1}$ definiert durch die Entwicklung

$$
y=y_{1}=b+b_{0}(x-a)^{\frac{u}{z}}+b_{1}(x-a)^{u+1}{ }^{\prime \prime}+\cdots
$$

und es sei $p_{1}$ der zugehörige Primteiler. Er ist ein Verzweigungsprimteiler der Ordnung $\lambda-1$ in bezug auf $x$, also genau in der Potenz $\lambda-1$ in $z_{x}$ enthalten. Es ist ferner

$$
\frac{\partial f}{\partial y}\left(x, y_{1}\right)=\frac{\partial f_{1}}{\partial y}\left(x, y_{1}\right) f_{2}\left(x, y_{1}\right) \ldots f_{r}\left(x, y_{1}\right) E\left(x, y_{1}\right) .
$$

Die rechte Seite dieser Gleichung ist genau teilbar durch die

$$
\left[\lambda-1+\left(p_{1}, p_{1}\right)+\left(p_{1}, p_{2}\right)+\cdots+\left(p_{1}, p_{r}\right)\right] \text {-te }
$$

Potenz von $(x-a)^{\frac{1}{2}}$. Durch dieselbe Potenz von $p_{1}$ ist also $\frac{\partial f}{\partial y}$ teilbar. Da ${ }_{3 x}$ genau durch $p_{i}^{-1}$ teilbar ist, so ist wegen (5) D genau teilbar durch die

$$
\left[\left(p_{1}, p_{1}\right)+\left(p_{1}, p_{2}\right)+\cdots+\left(p_{1}, p_{7}\right)\right] \text {-te }
$$


Potenz von p. Z̈hnliches gilt fïr dire zu den anderen Zweigen gehörenden Primteiler. Also:

Gehen dureh oinen Punkt $P$ rince algebraischen kurver Zweige $p_{1}, p_{2}, \ldots p_{r}$ und sind $p_{1}, p_{2}, \ldots p_{r}$ die zu diesen Zweigen gehörenden Primteiler, und bezeichnon wirdon linitrag, den $P$

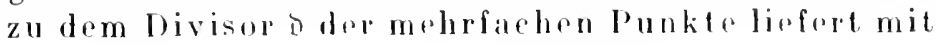

$\mathrm{so}$ is $\mathrm{t}$

$$
\mathfrak{p}_{1}^{d_{1}} \mathfrak{p}_{2}^{d_{2}} \cdots \mathfrak{p}_{r}^{d_{r}}
$$

$$
\delta_{k}=\left(p_{k}, p_{1}\right)+\left(p_{k}, p_{2}\right)+\cdots+\left(p_{k}, p_{r}\right)
$$

und der Beitrag, den $P$ zu der Ordnung $2 d$ von b liefert, ist

$$
\underset{l}{\mathbf{\Sigma}} \delta_{k}=\sum_{k, l}^{k}\left(p_{k}, p_{\mathrm{l}}\right)=\frac{\mathbf{N}}{k}\left(p_{k}, p_{k}\right)+2 \underset{k, l}{\mathbf{\Sigma}}\left(p_{k}, p_{l}\right) \text {, }
$$

also immer eine grade Zahl

Das letzte folgt daraus, daß $\left(p_{i}, p_{k}\right)$ immer gade ist.

Wir zeigen noch, daß o durch $x^{2}$ teilbar ist. Ist wieder $\alpha$ die kleinere der Zahlen $\lambda, \mu$, wenn $\lambda \neq \mu$ und $\alpha=\lambda=\mu$, wenn $\lambda=\mu$, so ist $p_{1}$, wie wir wissen, in $r$ genau in der Potenz $\alpha-1$ enthalten. Es ist aber $p_{1}$ in 5 enthalten in der Potenz $\delta_{1}$ und es ist, da die $\left(p_{t}, p_{t}\right) \geq 0$, und nach (15. 18)

$$
\begin{aligned}
\delta_{1}>\left(p_{1}, p_{1}\right) \geq & (\lambda-1)(\mu-1), \text { wenn } \lambda=\mu \\
& \lambda(\lambda-1), \text { wenn } \lambda=\mu .
\end{aligned}
$$

Wenu $\lambda \neq \|$ und $\alpha>1$, so ist die kleinere der Zahlen $\lambda$, $"$ glejeh $\alpha$ und die größere mindestens gleieh 3. Also $(\lambda-1)(u-1) \quad 2(\alpha-1)$. Dies ist aber für $\alpha=1$ auch richtig. da $\lambda$ und $\mu$ mindestens gleich 1 sind. Wenn $\lambda=\mu=\alpha$, so ist für $\alpha>1 \lambda(\lambda-1)=\alpha(\alpha-1)>2(\alpha-1)$ und dies ist anch richtig fïr $a=1$. Also ist immer

$$
\delta_{1}-2(a-1) \text {. }
$$

Während also $p_{1}$ in $r$ genau in der Potenz $\alpha-1$ enthalten ist, ist $p_{1}$ in b mindestens in der Potenz $2(\alpha-1)$ enthalten. Da das aber fïr jeden der Primteiler $\mathfrak{p}_{i:}$ gilt, und anch für alle anderen Primteiler, die in $r$ enthalten sind, so folgt:

Der Divisor o der melufachen Punkteist durch das Quadrat des Divisors r der liückehrpunkte teilbar.

Wir setzen

$$
D=D_{0} \mathfrak{r}^{2}
$$

und nennen Doden aigentliehen Divisordermehrfachen Punkte. Die Ordnungen von $D_{0}$ bezeiehnen wir mit $2 d_{0}$, so da

$$
d=d_{0}+r \text {. }
$$




\section{S 6. Beispiele.}

1. $y^{2}=x^{2}(x+a)$. Die Kurve lat in Punkte $x=y=0$ einen Doppelpunkt mit reellen verschiedenen Tangenten für $a>0$, mit imaginärem für $a<0$ (isolierter Punkt), und einen Doppelpunkt mit zusammenfallenden Tangenten für $a=0$.

2. $y^{2}=\frac{\left(x^{2}-a^{2}\right)^{2}}{1-x^{2}}, a^{2}<1$. Die Kurve hat für $a \neq 0$ in den Punkten $x=a, y=0$ und $x=-a, y=0$ Doppelpunkte mit getrennten Tangenten. Dir Geraden $x=1$ und $x=-1$ sind Asymptoten. Die Doppelpunkte fallen zusammen für $a=0$ und gehen über in einen Selbstberührungspunkt.

3. $y^{2}=\frac{x^{2}\left(x^{2}-a^{2}\right)^{2}}{1-x^{2}}, a^{2}<1$. Die Kurve hat die Geraden $x=1$ und $x=-1$ zu Asymptoten und liegt ganz zwischen diesen. Die Punkte $x=0, y=0 ; x=a, y=0 ; x=-a, x=0$ sind Doppelpunkte mit getrennten Tangenten. Für $a=0$ fallen sie zusammen in einen Selbstberührungspunkt zweiter Ordnung. Es wird dem Leser empfohlen, die Kurven der Beispiele 1 bis 3 für verschiedene Werte von a zu zeichnen.

4. Es habe $f=0$ im Nullpunkt einen $v$-fachen Punkt. Es sei also, nach homogenen Funktionen von $x, y$ geordnet,

$$
f=u_{v}+u_{v+1}+\cdots .
$$

Durch Drehung des Koordinatensystems um den Nullpunkt können wir erreichen, daß in $u_{v}$ das Glied mit $y^{r}$ vorkommt, wenn es nicht von vornherrin vorhanden sein sollte. Für kleine Werte von $x, y$ ist für die Punkte der Kurve annähernd

$$
u_{r}(x, y)=x^{v} u_{\imath}\left(1, \frac{y}{x}\right)=0 .
$$

Wir beschränken uns auf den Fall, daß die Gleichung $\nu$-ten Grades

$$
u_{v}(1, z)=0
$$

lanter verschiedene Wurzeln hat. Sie seien $m_{1}, m_{2}, \ldots m_{1}$. Dann ist

$$
u_{\imath}(x, y)=\text { konst } \cdot\left(y-m_{1} x\right)\left(y-m_{2} x\right) \ldots\left(y-m_{1} x\right) .
$$

Es ist also in der Umgelung des Nullpunktes die Kurve $f=0$ annähernd gleich den $v$ durch ihn gehenden Geraden $y=m_{l} x$. Wir schließen daraus, daß durch den Nullpunkt $v$ Zweige grhen, $p_{1}, p_{2}, \ldots p_{v}$, deren Tangenten die Geraden $y=m_{k} x$ sind. Die Entwicklung, die $p_{k}$ definiert, ist bei passender Wahl der Bezeichnung

$$
y=y_{k}=m_{k} x+\cdots .
$$


Es wird $\left(p_{k}, p_{k}\right)=0$ und $\left(p_{k}, p_{l}\right)=1$, wenn $k \neq l$. Ist $p_{k}$ der zu $p_{k}$ gehörende Primteiler, so ist der Beitrag, den $P$ z.11 o liefert,

$$
\mathfrak{p}_{1}^{y-1} \mathfrak{p}_{2}^{1}{ }^{-1} \ldots \mathfrak{p}_{1}^{1}{ }^{1}
$$

und der Beitrag, den $P$ zu $2 d$ liefert, $v(v-1)$. Man nennt $P$ einen $v$-fachen Punkt mit getrennten Tangenten. Er zählt, da er zudden Beitrag ${ }_{2}^{1} v(v-1)$ liefert, für $\frac{1}{2} v(v-1)$ Doppelpunkte. Er kann auch entstehen dadurch, daß $\frac{1}{2} v(v-1)$ Doppelpunkte zusammenfallen. Am einfachsten sieht man das, wenn man die zerfallende Kurve betrachtet, die aus $v$ geraden Linien besteht. Liegen die Geraden allgemein, so haben sie ${ }_{2}^{1} v(v-1)$ Schnittpunkte und jeder von ihnen ist ein Doppelpunkt der Kurve. Fallen die Schnittpunkte alle zusammen, gehen also die Geraden alle durch einen Punkt, bleiben aber voneinander verschieden, so hat die Kurve einen $\nu$-fachen Punkt mit getrennten Tangenten.

5. $f=y^{3}-x y^{2}-x^{3} y+1+x^{4}=0$. Welchen Beitrag liefert der Punkt $P=(x=\infty, y=\infty)$ zu o und zu $2 d$ ? Wir haben zu setzen $x=x^{\prime-1}$, $y=y^{\prime-1}$. Wir erhalten dann statt $f=0$ die Gleichung

$$
f^{\prime} \quad x^{4}-x^{\prime 3} y^{\prime}-x^{\prime} y^{\prime 2}+\left(1+x^{4}\right) y^{\prime 3}=0
$$

und wir habeu bei dieser Gleichung den Punkt $x^{\prime}=0, y^{\prime}=0$ zu betrachten. Wir finden nach den Methoden des Kap. II die Entwicklungen

$$
y^{\prime}=y_{1}-x^{\prime 3}+\frac{1}{2} x^{\prime 6}+\cdots, \quad y^{\prime}=y_{1}=x^{\prime}-x^{\prime 5}+\cdots .
$$

Der P'unkt $P$ ist also zweizweigig. Die zu den beiden Zweigen $p_{1}$ und $p_{2}$ gehörenden Funktionen sind

$$
\begin{aligned}
& f_{1}\left(x^{\prime}, y^{\prime}\right)\left(y^{\prime}-y_{1}\right)\left(y^{\prime}-y_{2}\right)=\left(y^{\prime}-x^{\prime 2}-\frac{1}{2} x^{6}-\cdots\right)\left(y^{\prime}+x^{2}-\frac{1}{2} x^{6}-\cdots\right) \\
&=y^{\prime 2}-\left(x^{6}+\cdots\right) y^{\prime}-\left(x^{3}+\cdots\right) \\
& f_{2}\left(x^{\prime}, y^{\prime}\right)-y^{\prime}-y_{1}=y^{\prime}-x^{\prime}+x^{\prime 5}-\cdots .
\end{aligned}
$$

Es wird

$$
\left(p_{1}, p_{1}\right)=2, \quad\left(p_{1}, p_{2}\right)=2, \quad\left(p_{2}, p_{2}\right)=0 .
$$

Sind $\mathfrak{p}_{1}, \mathfrak{p}_{2}$ die zu den Zweigen $p_{1}, p_{2}$ gehörenden Primteiler, so liefert $P$ zu o den Beitrag $p_{1}^{4} p_{2}^{\prime 2}$, also zu $d$ den Beitrag 3 . 


\section{\$ 7. Zahl der Schnittpunle zweier Kurven.}

Zunachst definieren wir die Zahl der Schnittpunkte, die zwei algehraishle Kurven $(i=0$ and $I J=0$ in cinem Punkt $P=(a, b)$ haben. Dureh $P$ mögen die Zweige $p_{1}, p_{2}, \ldots p_{r}$ von $G$ und die Zweige $q_{1}, q_{2}, \ldots q_{s}$ von $I I$ gehen. Dir z.11 $p_{k}, q_{k}$ gehörenden Funktionen seien $g_{k}(x, y)$ und $h_{s}(x, y)$. Lis ist dann in der Umgrebung von $P$

$$
G=g_{1} g_{2} \ldots g_{\mathrm{r}} E_{1} . \quad H=h_{1} h_{2} \ldots h_{s} E_{2},
$$

wo $E_{1}$ und $E_{2}$ Einheiten für die Stelle $(x:=0, y=0)$ sind. Wir setzen die Zahl der Schnittpunkte von $G=0$ und $I I=0$ in $P$ gleich der Summe der Zahl der Schnittpunkte, die die Zweige $p_{k}$ mit den Zweigen $q_{k}$ haben, also gleich

$$
\frac{\mathbf{N}}{l_{i, l}}\left(p_{l i}, q_{l}\right) \text {. }
$$

liese Zahl ist, da $\left(p_{k}, q_{l}\right)=\left(q_{l}, p_{k}\right)$ ist, symmetrisch von $G$ und $H$ abhängig. Als Beispiel nehmen wir an, $G$ habe in $P$ einen $r$-fachen Punkt mit getrennten Tangenten und $H$ rinen $\mu$-fachen, auch mit getrennten Tangenten. Außerdem sollen die Tangenten von $G$ in $P$ verschieden sein ron den Tangenten von $H$ in $P$. $G$ hat dann $v$ Zweige $p_{1}, p_{2}, \ldots p_{v}$ in $P$ und $I /$ hat $" Z$ weige $q_{1}, q_{2}, \ldots q_{n}$. Jeder Zweig von $G$ schneidet jeden Zweig ron $H$ in einem Punkt. $\Lambda$ lso ist $\left(p_{h}, q_{i}\right)=1$. Die Zahl der Schnittpunkte von $G$ und $H$ in $P$ ist also $w u$.

Es seien $G(x, y)$ und $H(x, y)$ vom Grade $(l, m)$ und $\left(l^{\prime}, m^{\prime}\right)$. In dem durch $G=0$ definierten algebraischen Körper seien $I$ und 11 dir Nenner won $x$ und $y$. Dann könmen wir $H$ in der form schreiben

$$
H=\frac{l)}{l^{\prime \prime} m l^{\prime \prime \prime}},
$$

wo h) ein ganzer Divisor ist. Wir betrachten $H$ in der Umgebung von $P$. Dir zu den Zweigen $p_{1}, p_{2}, \ldots p_{r}$ gehörenden Primteiler des Körpers $G=0$ seien $p_{1}, p_{2}, \ldots p_{r}$. Die $p_{k}$ definierende Entwicklung $y=y_{1}$ schreite nach ganzen Potenzen ron $(x-a)^{*}$ fort. Dann ist nach der Definition von $\left(p_{1}, q_{l}\right)$

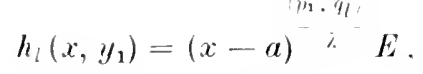

wo $E$ eine Einheit lür $x=a$ ist. Es wird also

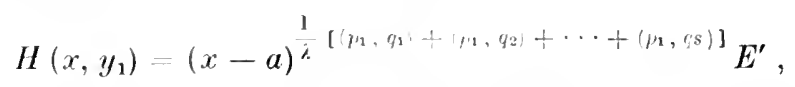

wo $E^{\prime}$ eine Einheit für $x=a$ ist. Das heißt aber, es enthält hy den Primteiler $\mathfrak{p}_{1}$ genau in der Potenz

$$
\left(p_{1}, q_{1}\right)+\left(p_{1}, q_{2}\right)+\cdots+\left(p_{1}, q_{s}\right) .
$$


Von den anderen Primteilern $p_{2}, p_{3}, \ldots p_{r}$ gilt Entsprechendes. Der Punkt $P$ liefert also ail h den Beitrag $p_{1}^{\alpha_{1}} p_{2}^{\varepsilon_{2}} \ldots p_{r}^{\alpha_{r}}$, wonn

$$
\alpha_{k}=\left(p_{k}, q_{1}\right)+\left(p_{k}, q_{2}\right)+\cdots+\left(p_{k}, q_{s}\right) .
$$

Zu der Ordnung von b liefert also $P$ den Beitrag $\boldsymbol{N} \alpha_{k}=\boldsymbol{N}\left(p_{k}, q\right)$, also einen Beitrag, der gleich der Zahl der Schnittpunkte von $G$ und $I$ in $P$ ist. Daher ist die Ordnung von h gleich der Gesamtzahl der Schnittpunkte von $G$ und $H$. Es ist aber zufolge (22)

$$
\text { h) } \sim \mathfrak{l}^{\prime \prime} \mathrm{m}^{n^{\prime}} \text {. }
$$

Da die Ordnung von $\{$ gleich $m$ und die von $m$ gleich $l$, so ist die Ordnung von h) gleich $l m^{\prime}+l^{\prime} m$. Also:

Zwei algebraische Kurven der Ordnung $(l, m)$ und $\left(l^{\prime}, m^{\prime}\right)$ haben $l m^{\prime}+l^{\prime} m$ Sehnittpunkte.

Beispiele.

1. $G \equiv 2 x-3 y=0, H=x+y=0 . \quad(l, m)=(1,1),\left(l^{\prime}, m^{\prime}\right)=(1,1)$.

Die Zahl der Schnittpunkte der Geraden $2 x-3 y=0, x+y=0$ ist also $l m^{\prime}+l^{\prime} m=2$. Wir können das leicht bestätigen. Die Geraden haben einen Schnittpunkt im Punkt $x=y=0$ und einen im Punkt $x=y=\infty$. Setzen wir nämlich $x=x^{\prime-1} \cdot y=y^{\prime-1}$, so erhalten wir die Gleichungen

$$
2 y^{\prime}-3 x^{\prime}=0, \quad y^{\prime}+x^{\prime}=0
$$

und die hierdurch dargestellten Kurven schneiden sich im Punkte $x^{\prime}$ $=y^{\prime}=0 ;$ also schneiden sich nach unserer Definition $G=0$ und $H=0$ im Punkte $x=y=\infty$.

2. $G \quad x-3=0, H \quad x+4=0 . \quad(l, m)=\left(l^{\prime}, m^{\prime}\right)=(1,0)$.

Die Zahl der Schnittpunkte ist $l m^{\prime}+l^{\prime} m=0$.

3. $G=x-4=0, H=y+3=0 . \quad(l, m)=(1,0),\left(l^{\prime}, m^{\prime}\right)=(0,1)$.

Zahl der Schnittpunkte ist 1.

Die Beispiele zeigen, daß die hier besprochene Art, die Schnittpunkte zweier Kurven zu bestimmen, mit der in der analytischen Geometrie üblichen nicht übereinstimmt. Es liegt das daran, daß wir die unendlich fernen Punkte anders definiert haben. Wir haben nämlich als unendlich ferne Punkte

1. die Punkte, wo $x=\infty, y$ beliebig,

2. die Punkte, wo $y=\infty, x$ beliebig.

Wir haben also in der $x y$-Ebene zwei unendlich ferne Gerade, die erste parallel zur $y$-Achse, die zweite zur $x$-Achse. Sie schneiden sich in dem 
$1 S S$ Elftes Kapitel. Ebene algebraische Kurven in homogenen Koordinaten.

Punkte $x=y=\infty$. Es gibt also nur einen Punkt, wo $x$ und $y$ gleichzeitig unendlich werden.

In der analytischen Geometric dag'gen pflegt man festzusetzen, daß alle unendlich fernen Punkte der Ebene auf einer Geraden, der sogenannten unendlich fernen Geraden liegen. Wie sich die Verhältnisse bei dieser Definition der unendlich fernen Punkte gestalten, darüber sehe man das nächste Kapitel.

\section{\$ S. Eine Formel für das Geschlecht des Körpers.}

Ist $\mathrm{t}$ ein Divisor der kanonischen Klasse, so ist nach Kap. VI, §6

$$
\mathfrak{E} \sim \frac{\dot{\partial}_{x}}{l^{2}}-\frac{z_{y}}{m^{2}} .
$$

Aus den Gleichungen (5) folgt aber, daß auch

$$
f \sim l^{l-2} m^{m-2} b^{-1} \text {. }
$$

Da die Ordnung eines jeden Divisors der kanonischen Klasse gleich $2 p-2$ ist, so folgt

oder der Satz:

$$
2 p-2=(l-2) m+(m-2) l-2 d
$$

Ist die Ordnung des Divisors der mehrfachen Punkte einer Kurve der Ordnung $(l, m)$ gleich $2 d$, so ist ihr Geschlecht

$$
p=(l-1)(m-1)-d .
$$

Elftes Kapitel.

\section{Ebene algebraische Kurven in homogenen Koordinaten*).}

Es seien

\section{$\$ 1$. Definition der Kurre.}

$$
a_{1}, a_{2}, a_{3} .
$$

drei äquivalente teilerfremde ganze Divisoren. Ihre Ordnung sei $n$. Ferner sei $a_{0}$ ein von ihnen linear alhängiger ganzer Divisor, also

$$
a_{0}=\lambda_{1} a_{1}+\lambda_{2} a_{2}+\lambda_{3} a_{3},
$$

wo die $i_{i}$ konstant sind. Wir setzen

$$
x_{1}=\frac{\mathfrak{a}_{1}}{\mathfrak{a}_{0}}, \quad x_{2}=\frac{\mathfrak{a}_{2}}{\mathfrak{a}_{0}}, \quad x_{3}=\frac{\mathfrak{a}_{3}}{a_{0}} .
$$

*) H.-L. 26 . Vorlesung S. 439 f. 
Fassen wir die $x$, die Funktionen drs Kërpers $(x y)$ sind, als hemogene Koordinaten rines Punktes $(x)$ riner Eherne auf, so hildet die Gresamtheit dieser Punkte $(x)$ eine algebraische Kurw $F$, deren Gleichung sei

$$
F(x) \quad F\left(x_{1}, x_{2}, x_{3}\right)=0,
$$

wo $F(x)$ ein" homugene ganze rationale Funktion dor $x_{i}$ ist. Der Grad von $F(x)$ ist, wie wir sehrn worden, gleich $n$ und heibt dir Ordnung der Kurve $F$.

Setzen wir

$$
\frac{x_{1}}{x_{3}}=\xi, \quad \frac{x_{2}}{x_{3}}=\eta,
$$

so besteht zwischen $\xi, \eta$ die Gleichung

$$
F(\xi, \eta, 1)=0
$$

und der hierdurch definierte algebraische Körper $(\xi \eta)$ soll mit dem Körper $(x y)$ identisch sein. Wäre er das nicht, so würden wir anstatt vom Körper $(x y)$ vom Körper $(\xi y)$ ausgehen.

Ist umgekehrt durch die Gleichung (4) eine algebraische ebene Kurve $F$ in homogenen Punktkoordinaten definiert, so wird gleichzeitig unter Benutzung von (5) ein algebraischer Körper $(\xi \eta)$ definiert. Zerlegen wir in diesem $\xi, \eta$ in Primteiler, so erhalten wir

$$
\xi=\frac{\mathfrak{a}_{1}}{\mathfrak{a}_{3}}, \quad \eta=\frac{\mathfrak{a}_{2}}{\mathfrak{a}_{3}},
$$

wo $a_{1}, \mathfrak{a}_{2}, \mathfrak{a}_{3}$ als ganze Divisoren ohne gemeinsamen Teiler angenommen werden können. Wir erhalten also wegen (5)

$$
x_{1}: x_{2}: x_{3}=a_{1}: a_{2}: a_{3},
$$

und kommen auf unsere erste Definition der Kurve $F$ zurück.

Es sei $p$ eine Stelle des Körpers $(x y)$ und $t$ eine Funktion, die dort von erster Ordnung Null wird. Ist für die Umgebung von $p$

$$
x_{i}=t^{-\lambda} \mathfrak{x}_{i}(t),
$$

wo dip $\mathfrak{P}_{\text {i }}$ gewöhnliche Potenzreihen von $t$ sein sollen, die nicht alle für $t=0$ versehwinden, so setzen wir*)

$$
a_{0}=t^{i}, \quad a_{1}=\mathfrak{F}_{1}(t), \quad a_{2}=\mathfrak{F}_{2}(t), \quad a_{3}=\mathfrak{P}_{3}(t) .
$$

Es sollen also an jeder Stelle die $a_{i}$ gewöhnliche Potenzreihen einer Größe $t$ des Körpers sein, die an der Stelle von erster Ordnung Null wird. Diese

*) Hierdurch werden die Betrachtungen gegenüber denen bei H.- L. 26. Vor lesung S. 439 f. formal etwas vereinfacht. 
Potunzrihion $n_{1}(t)$ sollen nur bestimmt sein, bis anf einen Faktor, der rime Eewöhnliche l'otenzreihe von $t$ ist. die für $t=0$ nicht versehwindet. biene l'nhestimmtheit müssen wir deshall zulassen, weil die Größe $t$ sulbst diese linhestimmtleit enthält.

Wir kimnen dam an joder stolle preradezu setzen

$$
x_{1}=a_{1}(t), \quad x_{2}=a_{2}(t), \quad x_{3}=a_{3}(t),
$$

da us bu den homogenen koordinaten $x_{i}$ auf einen gemeinsamen Faktor nicht ankommt. Wir könnes anch die Differentiale $d a_{i}, d^{2} a_{i}$ usw. der Divisoren $a_{i}$ hilden. Ist

$$
l a_{i}=t^{a_{i}} e(t) d t
$$

wo e ene Einheit für $t=0$ ist, so sagen wir $d a_{i}$ ist durch $p^{\prime i}$ teilbar. Wir können also für jeden Primteiler bestimmen, wie oft or in $d \mathfrak{a}_{i}$ enthalten ist und könmen daher von dem Divisor $d a_{i} s$ spexhen. Aher diese Divisoren $d a_{1}$ und inteprechend $d^{2} n_{i}, d^{3} a_{i}$ haben keinen bestimmten Sinn, weil $\mathfrak{a}_{i}$ für jede Stelle nur bis auf eine Einheit als Faktor bestimmt ist. Ist z. B. an dor stelle $\mathfrak{p}$

$$
a_{1}=1+t
$$

so ist $d n_{1}=d t$ und $d n_{1}$ enthält $\mathfrak{p}$ nicht. Wählen wir aber, was ja erlaubt ist,

$$
a_{1}=(1+t)(1-t)=1-t^{2},
$$

so wird $d a_{1}=-2 t d t$ und $d a_{1}$ enthält $\mathfrak{p}$ in der ersten Potenz. Wählen wir

$$
a_{1}=(1+t)(1+t)^{-1}=1 \text {, }
$$

so wird $d \mathfrak{a}_{1}=0 \cdot d t$ und es enthält $d \mathfrak{a}_{1} \mathfrak{p}$ sogar in beliebig hoher Potenz.

Daraus sehen wir, daß wir nur solche Differentialausdrücke benutzen dürfen, die auch nur eine Einheit als Faktor aufnehmen, wenn wir die (:) mit einer Einheit multiplizieren. Derartige Differentialausdrücke kommen aber inı folgenden auch nur vor und es können auch nur solche eine geometrische Bedeutung haben.

\section{$\$ 2 . \quad$ Die Reziprokalkurve.}

Es sei wieder $p$ eine Stelle des Körpers $(x y)$ und $t$ eine dort von erster Ordnung Null werdende Funktion. In der Umgebung von $p$ entspricht dann jerlem Werte von $t$ eine Stelle der Kurve $F$ und umgekehrt, so daß wir kurz von der Stelle $t$ sprechen können. Die Tangente von $F$ an der Stelle $t$ geht durch die Punkte mit den Koordinaten $a_{i}$ und $a_{i}+d a_{\hat{i}}$. Ihre Gleichung ist also

$$
\mid \begin{array}{lll}
y_{1} & y_{2} & y_{3} \\
a_{1} & a_{2} & a_{3} \\
d a_{1} & d a_{2} & d a_{3}
\end{array}=0 .
$$




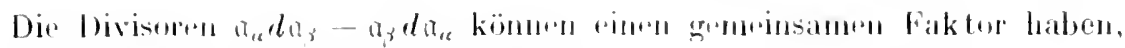
den wir mit $x$ begendelent. Wir settren

$$
a_{1} d a_{s}-a_{z} d a_{u}-r a_{;}^{\prime},
$$

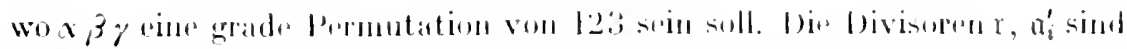
eindentig bestimnt. Ersetzen wir uämlich die $a_{i}$ dureh $e a_{i}$, wo o aine Ein-

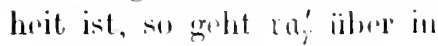

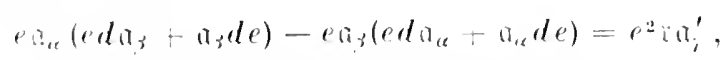

wird also nur mit der Einheit $e^{2}$ multipliziort.

Mit Benntzung von (9) geht dir Gleichung (8) der Tangente über in

$$
y_{1} a_{1}^{\prime}+y_{2} a_{2}^{\prime}+y_{3} a_{3}^{\prime}=0 \text {. }
$$

Es sind daher die a proportional zu den Linienkoordinaten der Tangente. Bezeichnen wir diese mit $x_{1}^{\prime}, x_{2}^{\prime}, x_{2}^{\prime}$, so können wir setzen

$$
x_{1}^{\prime}=\mathrm{a}_{1}^{\prime}, \quad x_{2}^{\prime}=\mathrm{a}_{2}^{\prime}, \quad x_{3}^{\prime}=\mathrm{a}_{3}^{\prime} .
$$

Die Divisoren $a_{i}^{\prime}$ sind zueinander äquivalent. Setzen wir nämlich

$$
\xi=\stackrel{a_{1}}{a_{3}}, \quad,=\frac{a_{2}}{a_{3}},
$$

so wird also

$$
\mathfrak{a}_{3}^{2} d \xi=a_{3} d \mathfrak{a}_{1}-a_{1} d \mathfrak{a}_{3}=\mathfrak{r} \mathfrak{a}_{2}^{\prime}, \quad a_{3}^{\prime 2} d_{\mathfrak{l}}=a_{3} d a_{2}-a_{2} d \mathfrak{a}_{3}=-r a_{1}^{\prime},
$$

$$
a_{1}^{\prime}=-\frac{d y}{d \xi}
$$

so daß $a_{1}^{\prime} / a_{2}^{\prime}$ eine Funktion des Körpers ist, da dasselbe von $\xi$ und $\eta$ gilt. Daher ist $a_{1}^{\prime} \sim a_{2}^{\prime}$ und cbenso folgt $a_{1}^{\prime} \sim a_{3}^{\prime}$. Setzen wir also

$$
\xi^{\prime}=\begin{aligned}
& a_{1}^{\prime} \\
& \mathfrak{a}_{3}^{\prime}
\end{aligned}, \quad y^{\prime}=\frac{\mathfrak{a}_{2}^{\prime}}{\mathfrak{a}_{3}^{\prime},}
$$

so besteht zwischen $\zeta^{\prime}$ und $\eta^{\prime}$ eine algebraische Gleichung, also zwischen $x_{1}^{\prime}, x_{2}^{\prime}, x_{3}^{\prime}$ eine homogene Gleichung

$$
F^{\prime}\left(x^{\prime}\right)-F^{\prime}\left(x_{1}^{\prime}, x_{2}^{\prime}, x_{3}^{\prime}\right)=0 .
$$

Dies ist die Gleichung von $F$ in Ebenenkoordinaten. Dentet man die $x_{i}^{\prime}$ als Punktkoordinaten, so nent man die dureh (1/4) definierte Kurve dir Reziprokalkurve von $F$.

Die Divisoren $a_{i}^{\prime}$ sind linear unabhängig. Bestände zwischen ihnen eine Gleichung

$$
c_{1} \mathfrak{u}_{1}^{\prime}+c_{2} \mathfrak{u}_{2}^{\prime}+c_{3} \mathfrak{u}_{3}^{\prime}=0,
$$


so wïrdr das aussagen, daß alle Tangenton von $F$ durch den Punkt $\left(c_{1}\right.$, $\left.c_{2} . c_{3}\right)$ gahen. Wenn wir von den trivialen Fällen absehen, wo $k$ rine Gerade ulde ein Punkt ist, ist das nicht mörlich. Wir können zur Definition dre Kurve $F$ aucli von iliren Tangenten statt von iliren Punkten ausgehen, also vou den Gleichungen (11) und (14) statt (3) und (4). Dann werden di. Ieterminanten $a_{u}^{\prime} d a_{3}^{\prime}-a_{x}^{\prime} d a_{c}^{\prime}$ proportional zu den Punktkoordinaten $a_{i}$ vun $F$. Bezeichnen wir also ihren größten gemeinsamen Teiler mit $\mathfrak{r}^{\prime}$, su habm wir dual zu (9)

$$
\mathfrak{a}_{u}^{\prime} d a_{i}^{\prime}-a_{j}^{\prime} d a_{t c}^{\prime}=r^{\prime} a_{i j} .
$$

Wir bestimmen noch die Tangenten der Kurve, die durch drei aufpinander folgende Stellen gehen. Sollen die drei aufeinander folgenden Stellen mit den Koordinaten $\left(a_{i}\right),\left(a_{i}+d a_{i}\right),\left(a_{i}+d a_{i}+\frac{1}{2} d^{2} a_{i}\right)$ auf einer Geraden liegen, so muß die aus diesen Koordinaten gebildete Determinante dritter Ordnung Null sein. Setzen wir also

$$
\begin{array}{lll}
a_{1} & a_{2} & a_{3} \\
d a_{1} & d a_{2} & d a_{3} \\
d: a_{1} & d^{2} a_{2} & d^{2} a_{3}
\end{array} \mid=\mathfrak{v},
$$

soliefern zu dem Divisor v die und nur die Stelleneinen Beitrag, deren Tangenten dureh drei aufeinander folgende Punkte gehen.

Dual ergibt sich: Setzen wir

$$
\begin{aligned}
& a_{1}^{\prime} \quad a_{2}^{\prime} \quad a_{3}^{\prime} \\
& d a_{1}^{\prime} \quad d a_{2}^{\prime} \quad d a_{3}^{\prime}=\mathfrak{v}^{\prime}, \\
& d^{2} \mathfrak{a}_{1}^{\prime} \quad d^{2} \mathfrak{a}_{2}^{\prime} \quad d^{2} \mathfrak{a}_{3}^{\prime}
\end{aligned}
$$

so liefern zu dem Divisor $v^{\prime}$ die und nur die Stellen einen Beitrag, die Berührungspunkte zu drei aufeinander folgenden Tangenten sind.

Die Livisoren $\mathfrak{v}$ und $\mathfrak{b}^{\prime}$ sind eindeutig bestimmt, da z. B. $\mathfrak{v}$ den Faktor $e^{3}$ annimmt, wenn wir die $a_{i}$ durch $a_{i} e$ ersetzen.

Sind $c_{1}^{\prime}, c_{2}^{\prime}, c_{3}^{\prime}$ Konstante, so wird die Funktion

$$
c_{1}^{\prime} a_{1}+c_{2}^{\prime} a_{2}+c_{3}^{\prime} a_{3}
$$

an den Stellen von $F$ Null, wo die Gerade $\left(c_{i}^{\prime}\right)$ mit der Gleichung

$$
c_{1}^{\prime} y_{1}+c_{2}^{\prime} y_{2}+c_{3}^{\prime} y_{3}=0
$$

die Kurve $F$ trifft. Der (18) entsprechende Divisor ist aber von der Ordnung $n$, enthält daher $n$ Primteiler und wird an den Stellen Null, zu denen diese Primteiler gehören. Eine Gerade schneidet also die Kurve $F$ in $n$ 
Punkten, wronn $n$ die Ordnung der l)ivisorem a, ist. Jarans folgt aber auch, dab die Funktion $F(x)$ vom $n$-ten Grarle in den $x_{2}$ ist, da sich aus dren Gleichungen

$$
F(x)=0, c_{1}^{\prime} x_{1}+c_{2}^{\prime} x_{2}+c_{3}^{\prime} x_{3}=0
$$

eine Gleichung $n$-ten Girades zur Bestimmung der Vurhältuisse der $x_{i}$ urgeben muß.

Ebenso folgt, daB die Gleichung $F^{\prime}\left(x^{\prime}\right) \quad 0$ dre Karre $F$ in Linienkoordinaten rom Grade $n^{\prime}$ ist, wenn $n^{\prime}$ die Ordnung der loivisoren $a_{i}^{\prime}$ ist. Und es ist $n^{\prime}$ dual zu der Bedentung von $n$ gleich der Zahl der Tangenten von $F$, dir durch rinen Punkt gehen. Es ist also $n^{\prime}$ dir Klasso von $F$.

\section{$\$$ 3. Die Divisoren $\mathfrak{r}$ und $\mathfrak{r}^{\prime}$.}

Wenn wir statt der $x_{i}$ durch eine Koordinatentransformation neur homogene Koordinaten $\xi_{i}$ einführen, so werden die Determinanten

$$
x_{a} d x_{3}-x_{3} d x_{a}=a_{a} d a_{3}-a_{3} d a_{a}=r a_{i}^{\prime}
$$

auch nur linear homogen transformiert. Ihr größter gemeinsamer Teiler bleibt also unverändert. Dasselbe ergibt sich für $\mathfrak{r}^{\prime}$. Um also die Bedeutung von $\mathfrak{r}$ und $\mathfrak{r}^{\prime}$ zu erkennen, können wir von einer passenden Koordinatentransformation Gebrauch machen.

Es sei p eine Stelle von $F$. Das Koordinatensystem sei so gewählt, daß an der Stelle $\mathfrak{p} x_{1}=x_{2}=0$ ist. Es ist dann $x_{3}=0_{3}$ an der Stelle $\mathfrak{p}$ eine Einheit. Wir dürfen also die $\mathfrak{a}_{i}$ mit $\mathfrak{a}_{3}$ dividieren, d. h. wir können $a_{3}=1$ annehmen. Ist wieder $t$ eine Größe des Körpers, die an der Stelle $p$ von der ersten Ordnung Null wird, so werden $\mathfrak{a}_{1}$ und $\mathfrak{a}_{2}$ beide durch $t$ teilbar. Sie mögen beide den Faktor $t^{\prime \prime}$ haben, aber nicht beide eine höhere Potenz von $t$ enthalten. Wir können damn annehmen $a_{2}=t^{\alpha} e(t)$, wo $e$ eine Einheit für $t=0$ ist. Wir setzen

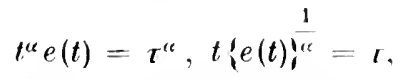

woraus $t$ als gewöhnliche Potenzreilue von $\tau$ folgt, die mit der ersten Potenz von $t$ beginnt. Es ist dann $a_{\mathfrak{a}}=\tau^{\prime \prime}$, und es sei

$$
\mathrm{a}_{3}=c_{1} \tau^{\prime \ell}+c_{2} \tau^{\alpha+1}+\cdots \text {. }
$$

Indem wir pin passendes Vielfaches von $a_{2}$ von $a_{3}$ subtrahiereu, also durch Koordinatentransformation, erreichen wir, daß die Entwicklung von $a_{3}$ mindestens mit der $(\alpha+1)$-ten Potenz von $r$ beginnt. Wir haben alsu für die Umgehung von $p$

$$
\begin{gathered}
a_{1}=1 . \quad a_{2}=t^{\prime c}, \quad a_{3}=a_{0} t^{t+\beta}+a_{1} t^{t+}+\beta+1+\cdots, \\
(\alpha>0, \quad \beta>0) .
\end{gathered}
$$


Hiordurh wird ein dureh p gehender Kurvenzweig $p$ von $F$ definiert. Ist

$$
c_{2} y_{2}+c_{3} y_{3} \ldots 0
$$

irgendine durch p gotende Gerade, so wird

$$
c_{2} a_{2}+c_{3} a_{3}=l^{\prime \prime}\left(c_{2}+c_{3} a_{0} r^{3}+\cdots\right)
$$

durch p" toilhar. Die Geradr hat also a zusammenfallende Schnittpunkte mit $f$ in p. Es ist also nach dor l, finition in Kap. X, $\$ 3 p$ cin Rückkehrpunkt der Ordnung a - 1. Eime und nur eim Grade hat mehr als $\alpha$ Schnittfunktr in $p$ mit $\mu$. nëmliel dir Geride $y_{3} \quad 0$. Sie hat $\alpha+\beta$, so da $p$ in Windepunkt der Ordumg $\beta-1$ ist. AuBerdem ist die Gerade $y_{3}=0$ natch früherer l),finition (vgl. Kap. X, \$3) und narh $\$ 2$ Tangente des Zweiges $p$ in $p$.

Es wird wegen (19)

$$
\begin{aligned}
& a_{2} d a_{3}-a_{3} d n_{2}=\quad ; \beta a_{0} t^{2 a+\gamma-1}+\cdots ; d \tau=r n_{j}^{\prime}, \\
& a_{3} d a_{1}-a_{1} d a_{3}=-(x+\beta) a_{0} t^{(t+1-1}+\cdots ; d r=\mathfrak{r} \mathfrak{a}_{2}^{\prime}, \\
& a_{1} d a_{2}-a_{2} d a_{1}=\quad \alpha \tau^{a-1} \quad d \tau=r a_{3}^{\prime} .
\end{aligned}
$$

Daraus ergibt sich, daß $\mathrm{r}$ genau durch $\mathrm{p}^{\prime-1}$ teilbar ist. Es ist also $x$ der Divisor der Rückkehrpunkte.

Ferner ergilht sifh

$$
a_{1}^{\prime}={ }_{\alpha}^{\beta} a_{0} r^{a+\gamma}+\cdots \quad a_{2}^{\prime}=-\frac{\alpha+\beta}{\alpha} a_{0} \tau^{\gamma}+\cdots \quad a_{3}^{\prime}=1
$$

and daraus wegen (15), daß der größte gemeinsame Teiler $\mathfrak{x}^{\prime}$ der Divisoren

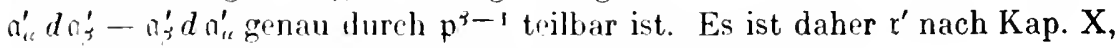
$\$ 3$ der Divisor der Wondrpunkte. Wir haben also:

Die sich dual entsprechenden Divisoren $r$ und $r^{\prime}$ sind die Divisoren der stationäron Punkt: und stationären Tangenten.

Die Ordnungen von $r$ und $\mathrm{r}^{\prime}$ bezeichnen wir mit $r$ und $r^{\prime}$. Im einfachsten Falle ist $r$ dir Zahl der Rürkkehrpunkte und $r^{\prime}$ die Zahl der Wendetangenten von $F$.

$$
\begin{aligned}
& \text { Aus (16) folght wegen (19) } \\
& 1, I^{a} \text {, } \\
& v=10, x \tau^{\alpha}:, \quad(x+\beta) a_{0} \tau^{\alpha \cdot y-1}+\cdots \\
& a_{0} \tau^{\alpha+3}+\cdots \\
& \text { 0. } \alpha(\alpha-1)^{\alpha-2},(\alpha+\beta)(\alpha+\beta-1) a_{0} \tau^{\alpha-\alpha} \alpha^{2}+\cdots \\
& \left\{\alpha, \beta(\alpha+\beta) a_{0} \tau^{2 / \alpha} 1+\beta-1+\cdots\right\} d \tau^{3}, \\
& d \tau^{3}
\end{aligned}
$$

so dab v den Primteiler $p$ genau in derscilben Potenz enthält wie $r^{2} r^{\prime}$. Da das für alle Stellen gilt, so folgt, $\mathrm{da} b v=\mathfrak{r}^{2} \mathfrak{r}^{\prime}$. Nehmen wir das dual entsprechende Ergebris hinzu, so haben wir

$$
\mathrm{v}=\mathrm{r}^{2} \mathrm{r}^{\prime}, \quad \mathrm{v}^{\prime}=\mathrm{r} \mathrm{r}^{\prime 2} \text {. }
$$




\section{$\$ 4$. Der Divisor der mehrfachen Punkte und der der mehr. fachen 'Tangenten.}

Aus der in $t$ identischen Gloichung

$$
F(a) \quad F\left(a_{1}, a_{2}, a_{3}\right)=0
$$

folgt wegen des Eulersehen Satzes ïber homngene Funktionm und durch Differentiation, whn wir Alleitungen nach a, dur h don fudex $i$ bezeichnen,

$$
\begin{aligned}
& F_{1} a_{1}+F_{2} a_{2}+F_{3} a_{3} n F=0 \\
& F_{1} d a_{1}+F_{2} d a_{2}+F_{3} d a_{3}=0 .
\end{aligned}
$$

Lis sind dabrer dir. $F_{i}$ proportional zu den beterminantru $a_{,} d a_{3}-a_{3} d a_{a}$. so dab wir haben

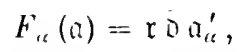

wo der I)ivisor ô durch diese Gleirhungen definiert ist.

Ebenso findet man dual

$$
F_{a c}^{\prime}\left(\mathfrak{a}^{\prime}\right)=\mathfrak{r}^{\prime} \bar{d}^{\prime} \mathfrak{a}_{\alpha} .
$$

Um die Bedeutung von $\bar{\delta}$ und $\bar{\delta}^{\prime}$ kennenzulernen, wollen wir unhomogene Koordinaten einführen. Wir wählen diesen Weg, um zugleich den Zusammenhang von $\bar{\delta}$ mit dem in (5) Kap. X, $\S 1$ definierten Divisor o kennenzulernen. Wir setzen

$$
x=\frac{\mathfrak{a}_{1}}{\mathfrak{a}_{3}}=\frac{\mathrm{l}_{0}}{1}, \quad y=\frac{\mathfrak{a}_{2}}{\mathfrak{a}_{3}}=\frac{\mathrm{m}_{0}}{\mathrm{~m}} .
$$

Dabei sollen $l_{0}$ und $\mathfrak{l}$ ganze Divisnren ohne gemeinsamen Teiler sein und ebenso $m_{0}$ und $m$. Setzen wir

$$
F\left(\begin{array}{ll}
x_{1} \\
x_{3}
\end{array}, \begin{array}{c}
x_{2} \\
x_{3}
\end{array}, 1\right)=F(x, y, 1)=f(x, y) .
$$

so ist $f=0$ die neue Gleichung der Kurve $F$ oder $f$. Aus (26) folgt

und daraus

$$
F(\mathfrak{a})=\mathfrak{a}_{3}^{n} f(x, y)
$$

$$
F_{1}(\mathfrak{a})=\mathfrak{a}_{3}^{n} \frac{\partial f}{\partial x} \cdot \frac{\partial x}{\partial \mathfrak{a}_{1}}=\mathfrak{a}_{3}^{n-1} \frac{\partial f}{\partial x} .
$$

Wegen (23) und (5) Kap. X, §1 ergibt sich

$$
r \bar{b} a_{1}^{\prime}=a_{3}^{n-1} \frac{\mathfrak{D} \hat{A}_{y}}{\mathfrak{d}^{b-2} \mathrm{ll}^{m}} \text {. }
$$

Aus (9) und (25) folgt aber

$$
\mathrm{r} \mathrm{a}_{1}^{\prime}=\mathfrak{a}_{2} d a_{3}-a_{3} d \mathfrak{a}_{1}=\mathfrak{a}_{3}^{2} d y=\mathfrak{a}_{3}^{2}{ }_{m^{2}}^{\lambda^{2}},
$$

so da $B$ wir aus (29) schließen

$$
\overline{\mathfrak{D}}=\frac{\mathfrak{a}_{3}^{n-3} \mathfrak{d}}{\mathfrak{l}^{l-2} \mathfrak{m}^{m-2}} .
$$


196 Elftes Kapitel. Ebene algebraische Liurven in homogenen Koordinaten.

Wir arsohen hieraus, daß $\bar{\delta}$ der Divisor der melerfachen Punkte von $F$

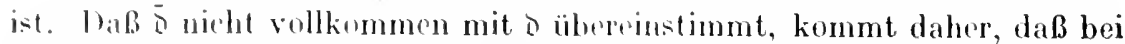
der friilwern und der jetzigen betrachtung die unendlich fornen Punkte rerschieden definiert sind. (Vgl. Beispiel I und 2 in $\$ 10$.)

Mir nehmen noch zwei hesondere Fälle.

1. Es seicn $a_{1}$ und $\mathfrak{a}_{3}$ teilerfremel und olonso $\mathfrak{a}_{2}$ und $a_{3}$. 1)ann ist nath (25) $a_{3}=1=11$ und $n=l-m$, also

$$
\text { (1) } a_{3}^{n-1} \text {. }
$$

2. Es seien 1 und $m$ trilerfremd. Dann wird $a_{3}$ als Hauptnenner der Venner I und un gleich I m, also $n=l+m$ und

$$
\bar{\delta}=1^{m-1} m^{l}+i \text {. }
$$

Dual ergiht sich, daß $\bar{\delta}$ der bivisor der mehrfachen Tangenten ist. Die halben Urdnungen $\bar{d}$ und $\bar{d}^{\prime}$ von $\bar{\delta}$ und $\bar{\delta}^{\prime}$ sind im einfachsten Falle die Zahl der Doppolpunkte und die Zahl der Doppeltangenten von $F$.

Geradeso, wie in Kap. X, $\$ 5$ folgt, daß $\bar{\delta}$ durch $\mathfrak{r}^{2}$ und $\bar{\delta}$ durch $\mathfrak{r}^{\prime 2}$ teilbar ist. Wir setzen

$$
\bar{D}=r^{2} \bar{\zeta}_{0}, \quad \bar{D}^{\prime}=r^{\prime 2} \nabla_{0}^{\prime} .
$$

Bezeichnen wir die Ordnungen von $\bar{\delta}_{0}$ und $\bar{\delta}_{0}^{\prime}$ mit $2 \bar{d}_{0}$ und $2 \bar{d}_{0}^{\prime}$, so ist

$$
\bar{d}=\bar{d}_{0}+r, \quad \bar{d}^{\prime}=\bar{d}_{0}^{\prime}+r^{\prime} .
$$

\section{$\$$ i. Die Hessesche Kurve.}

Wir setzen

$$
H(x)=\left|F_{h l}(x)\right|, \quad H^{\prime}\left(x^{\prime}\right)=\left|F_{k l}^{\prime}\left(x^{\prime}\right)\right|,
$$

wo dir Indizes die Ableitungen nach $x_{k}, x_{l}$ and $x_{k}^{\prime}, x_{l}^{\prime}$ bedeuten sollen. Di" Kurve $H(x)=0$ hoißt die Hessesch. Kurve von $F$ und $H^{\prime}\left(x^{\prime}\right)=0$ ist die $z u H(x)=0$ duale Kurve.

Um zu bestimmen, wo $H(x)=0$ dic Kurve $F$ trifft, haben wir in $H(x)$ cinzusetzen $x_{i}=a_{i}(t)$ und zu sehen, für welche Werte von $t H(a)$ verschwindet. Mit andren VWrten, wir habrn den Divisor $H(a)$ zu bestimmrn.

Wir setzen, unter $\xi_{1}, \xi_{2}, \xi_{3}$ unbestimmte Größen verstehend,

$$
F_{u 1} \xi_{1}+F_{u 2} \xi_{2}+F_{u 3} \xi_{3}=\xi_{u}^{\prime} \text {. }
$$

Aus (2.3) folgt wegen der Eulerschen Satzes über homogene Funktionen und durch Differentiation

$$
\begin{aligned}
& F_{a 1} a_{1}+F_{u 2} a_{2}+F_{u 3} a_{3}=(n-1) \mathfrak{r} \delta a_{u}^{\prime},
\end{aligned}
$$

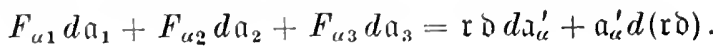


Forner ergibt sich aus (9)

$$
\begin{aligned}
& \Xi_{1} \quad \Xi_{2} \quad \Xi_{3} \\
& a_{1} \quad a_{2} \quad a_{3}=\imath \geq \xi_{i} a_{1}^{\prime} . \\
& \begin{array}{llll}
d u_{1} & d u_{2} & d u_{3}
\end{array}
\end{aligned}
$$

Hieraus und aus (36), (37), (38) folgt nach dem Multiplikationssatz von Determinanten

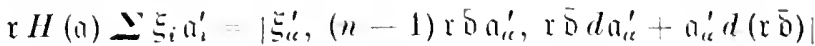

$$
\begin{aligned}
& =(n-1) r^{2} \delta^{2}\left|\xi_{a}^{\prime}, a_{a}^{\prime}, d a_{a}^{\prime}\right|=(n-1) r^{2} r^{\prime} D^{2} \geq \xi_{\imath}^{\prime} a_{i} \text {. }
\end{aligned}
$$

Aus (37) rrgibt sich abor in Verbindung mit (38) wegen $F_{a \beta}=F_{\zeta u}$

Hiermit folgt aus (39)

$$
\sum \xi_{i}^{\prime} a_{i}=(n-1) r \overline{0} \geq \xi_{i} a_{i}^{\prime} \text {. }
$$

$$
H(\mathfrak{a})=(n-1)^{2} \mathfrak{x}^{2} \mathfrak{x}^{\prime} \delta^{2} .
$$

Dual hierzu folgt

$$
H^{\prime}\left(\mathfrak{a}^{\prime}\right)=\left(n^{\prime}-1\right)^{2} \times \mathfrak{r}^{\prime 2} \overline{\mathfrak{D}}^{\prime 3} \text {. }
$$

Aus (40) und (41) folgt:

Die Hessesche Kurve von $F$ geht nur durch die Rückkehrpunkte, die Wendepunktr und die mehrfachen Punkte von $F$.

Die zur Hesseschen Kurve duale Kurve hat die Tangenten in den Rückkehrpunkten von $F$, die Wende- und melirfachen Tangenten von $F$ zu Tangrnten und sonst keine Tangenten mit $F$ gemeinsam.

Weiter wollen wir hirraul nicht eingehen.

\section{\$ (6. Plïckersche Formeln.}

Lst $\Xi$ eine Größe des Körpers $(x y)$, so ist der $d \xi$ entsprechende Divisor ein Divisor der Differentialklasse und daher von der Ordnung $2 p-2$, wenn $p$ das Geschlecht des Körpers ist. Ist, irgendeine andere Funktion des Körpers, so ist auch $\frac{d \xi}{d_{y_{j}}}$ und also auch $\frac{d}{d \eta}\left(\begin{array}{l}d \xi \\ d_{\eta}\end{array}\right)=\frac{d^{2} \xi}{d_{y^{2}}{ }^{2}}$ eine Funktion des Körpers, der ihr entsprechende Divisor also von der Ordnung Null. Daher hat $d^{2} \xi$ dieselbe Ordnung wie $\left(d_{1}\right)^{2}$, also $4 p-4$.

Nun ist

$$
a_{1} d a_{2}-a_{2} d a_{1}=a_{1}^{2} d\left(\begin{array}{c}
a_{2} \\
a_{1}
\end{array}\right) \text {. }
$$

wo $\mathfrak{a}_{2} / \mathfrak{a}_{1}$ eine Funktion des Körpers ist. Daher ist dic Ordnung der Divisoren $a_{a} d a_{3}-a_{3} d a_{c}$ gleich $2 n+2 p-2$ und die der Divisoren $a_{t c}^{\prime} d \mathfrak{a}_{3}^{\prime}$ $-a_{3}^{\prime} d a_{4}^{\prime}$ gleich $2 n^{\prime}+2 p-2$.

Ferner ist

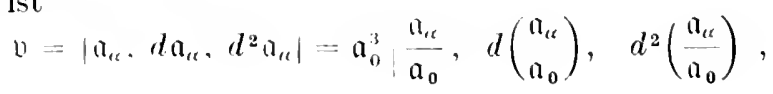


Ly EJfes Kapitel. Ebene algebraische Kurven in homogenen Koordinaten.

alin, da dir a $a_{\text {a }}$ Funktionen des lïrpers siud, von der Ordnung $3 n$ - (ip - 6. Entsprechend ist $u^{\prime}$ von der Ordumng $3 n^{\prime}+6 p-6$.

Vergloichen wir jetzt in den Glevhumgen (9), (15), (20), (23), (24) die Grdunngen der links und rechts stehenden bivisoren, so erhalten wir folgende Gleichungen, die die Pliickreschen genannt werden:

$$
\left\{\begin{array}{c}
2 p-2=r+n^{\prime}-2 n-3 n+n-2 n^{\prime}, \\
6 p-6=2 r+r^{\prime}-3 n-2 r^{\prime}+r-3 n^{\prime}, \\
n(n-1)=r+2 \bar{d}+n^{\prime}=3 r+2 d_{0}+n^{\prime}, \\
n^{\prime}\left(n^{\prime}-1\right)=r^{\prime}+2 d^{\prime}+n=3 r^{\prime}+2 \bar{d}_{0}^{\prime}+n^{\prime} .
\end{array}\right.
$$

Von den beiden ersten Gleichungspaaren sind nur drei Gleichungetn voneinander unabhängig. Aus (4-) folgen noch die Gleichungen

$$
p=\stackrel{1}{2}_{2}^{1}(n-1)(n-2)-\bar{d}=\frac{1}{2}\left(n^{\prime}-1\right)\left(n^{\prime}-2\right)-\bar{d}^{\prime} .
$$

\section{$\$$ 7. Zahl der Schnittpunkte zweier Kurven.}

\section{Es sei durch $\quad G(x) . G\left(x_{1}, x_{2}, x_{3}\right)=0$}

"ine zweite algebraische Kurvo definiert. Sie sei von der $m$-ten Ordnung. In der Umgebung einer Stelle $p$ wird $G(a)$ eine gewöhnliche Potenzreihe von $t$, wenn wieder $t$ eine in $p$ von erster Ordnung versehwindende Funktion ist. Es spi

$$
G(a)=t^{\prime} e(t)
$$

wo $e$ eine Einheit für $t==0$ sein soll. Es ist dann der Divisor $G(\mathrm{a})$, d. h.

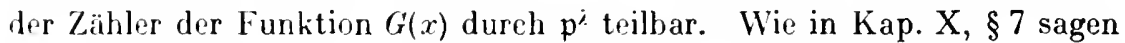
wir, die kurven $F$ und $G$ haben $\lambda$ gemeinsame Schnittpunkte in $p$. Daß lies. Definition symmetrisch von $F$ und $G$ abhängt, ist geradeso wie a. a. O. zu zeigen. Daraus folgt dann, daß die Gesamtzahl der Schnittpunkte gleich der Ordnung des livisors $G(a)$ ist. Da $G(x)$ vom Grade $m$ und lie $a_{i}$ von der Ordnung $n$, so ist $G(a)$ von der Ordnung $m \boldsymbol{n}$. Also haben wir den Bezoutschen Satz:

Zwei algebraisehe Kurven der Ordnungen $m$ und $n$ haben $m n$ Silhnittpunktr.

\section{\$ 8. Unendlich ferne Punkte.}

Wir wollen zu nicht homogenen Koordinaten übergehen und setzen

$$
\begin{gathered}
x=\frac{a_{1}}{a_{3}}, \quad y=\frac{a_{2}}{a_{3}}, \\
F(x, y, 1) \quad g(x, y),
\end{gathered}
$$

$$
g(x, y)=0
$$




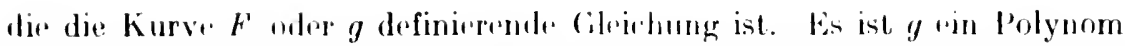
$n$-ten Grades und g aine Kurve n-ker ()rdunng. Wrun $a_{1}$ und $a_{3}$ keinen gemoinsamen Trilde haben, so ist $x$ vent der $n$-ten (Ordmung und die Gleichung $g=0$ ist nach Kap. IV, $\$ 2$ in $y$ vem $n$-tun Cirate, d. h. es kommt damn in $g(x, y)$ das cilied mit $y^{n}$ vor. Wir kïmm"n immer arreichen, da $\beta$ das der Fall ist, und zwar dureh einfache lorehung des $x$, y Koordinatensystems. Wa einer solehen Transformation anch nur rine KoordinatenIransformation der ursprünglichen homogenen Koordinaten entspricht, so bleibt dabei der llivisor $x \bar{D}$ als größter gemeinsamer Teiler der $F_{k}(\mathfrak{a})$ ungeändert. Da aber $\mathrm{r}$ nach $\$ 3$ sich nicht ändert, so bleibt auch $\bar{\delta}$ ungeändert.

Die Art, die Kurve $g$ zu definieren, unterscheidet sich von der früheren Definition der Kurve $f$ in Kap. $X, \S 1$ durch eine andere Definition der unendlich fernen Stellen. Hier gelten als unendlich fern die Stellen, die einen Beitrag zu dem Hauptnenner $\mathfrak{a}_{3}$ von $x$ und $y$ liefern, also die Punkte der Geraden $y_{3}=0$ bei Verwendung der homogenen Koordinaten. Um also $g$ in der Umgebung eines mendlich fernen Punktes zu untersuchen, haben wir hier folgendermaßen zu verfahren. Der Einfachhoit wegen nehmen wir an, da $B$ der Punkt $y_{1}=y_{3}=0$ nicht auf der Kurve liegt, was ja durch eine Koordinatentransformation immer zu erreichen ist.

Ist daun $P$ ein auf der Geraden $y_{3}=0$ liegender Punkt von $F$, so hat in $P y_{2} / y_{1}$ einen endlichen Wert $a$. Wir führen dann statt $y_{2}$ durch die Transformation

$$
\bar{y}_{2}=y_{2}-a y_{1}
$$

eine neue Koordinate ein. Es ist dann $P$ die Ecke $\bar{y}_{2}=0, y_{3}=0$ des neuen Koordinatendreiecks. Um die Gestalt von $F$ oder $g$ in der Umgebung dieses Punktes zu bestimmen, setzen wir dann

$$
\begin{array}{ll}
\bar{y}_{2} \\
y_{1}=\xi, & y_{3}= \\
y_{1}
\end{array}
$$

und trhalten eine Gileichung zwischen $\Xi$ und, , die in der Umgebung von $\xi=y_{i}=0$ zu untersuchen ist. Ghen wir zu nicht homogenen Koordinaten über, so hahen wir folgendes:

Um die Kurve $g$ in der Umgebung eines ihrer unendlich fernen Punkte zu untersuehen, setze man zunächst

$$
x=\frac{y_{1}}{y_{3}}, \quad y=\frac{y_{2}}{y_{3}},
$$

wodurch $g=0$ übergehen möge in $F(y)=0$. und bestimme die Werte, die sich aus $F(y)=0, y_{3}=0$ für

$$
\frac{y_{2}}{y_{1}}=\frac{y}{x}
$$


ergoben. l)ureh koordinatentransformation kann man erroichen, da B diesesämtlich endlich sind. Ist a einer der Werte, sil sintzr man

$$
x=\frac{1}{1} \quad y-\frac{a+\xi}{1}
$$

und untersuchedieaus $g=0$ hervorgehende Gleichung zwischen End, in der Umgebung von $\Xi=y_{i}=0$.

Will man in Falle, wo sich für $\frac{y}{x}$ aus $F(y)=0, y_{3}=0$ der Wert $\infty$ rrgilut, die Koordinatentransformation vermeiden, so benutzt man an Stelle von $\frac{y}{x}$ umgekehrt ${ }_{y}^{x}$, was dann den Wert 0 annimmt. Man hat dann entsprechend (47) unter Vertauschen von $x$ und $y$ zu setzen (da jetzt $a=0$ )

$$
x=\frac{\xi}{3} . \quad y=\frac{1}{\eta} .
$$

Es ist klar, daß das Verhalten einer Kurve $f$ oder $g$ im Unendlichen ganz verschieden ist, je nach der Definition der unendlich fernen Punkte. llan vergleiche die Beispiele in $\$ 10$.

\section{\$ 9. Der Noethersche Satz. Adjungierte Funktionen.}

Wir nennen jetzt eine Funktion des Körpers ganz, wenn sie nur unendlich wird in den unendlich fernen Punkten von $g$, wenn sie also in der Form dargestellt wrorden kann

$$
\frac{i}{a_{3}^{v}}
$$

wo c ein ganzer Divisor ist. Diese Definition hängt also ab von der Wahl des Divisors $a_{3}$.

Unter den ganzen Funktionen sind alle Funktionen enthalten, die sich als ganze rationale Funktionen von $x, y$ darstellen lassen. Aber es gilt keineswegs das Umgekehrte, wie schon folgendes einfache Beispiel zeigt. Es sei

$$
g(x, y)-y^{3}-x^{2}=0
$$

die $g$ definicrende Glrichung. Da $y=x^{3}$, so haben wir sowohl bei $x=0$ wie bei $x=\infty$ einen Verzweigungspunkt zweiter Ordnung. Die zugehörigen Primteiler seien $p_{0}$ und $p$. Dann wird

$$
x=\frac{\mathfrak{p}_{0}^{3}}{\mathfrak{p}^{3}}, \quad y=\frac{\mathfrak{p}_{0}^{2}}{\mathfrak{p}^{2}}=\frac{\mathfrak{p}_{0}^{2} \mathfrak{p}}{\mathfrak{p}^{3}} .
$$

alses

$$
a_{1}=p_{0}^{3}, \quad a_{2}=p_{i j}^{2} p, \quad a_{3}=p^{3} .
$$


Es wird

$$
\begin{aligned}
& x \\
& y
\end{aligned}=\frac{\mathfrak{p}_{0} \mathfrak{p}^{2}}{\mathfrak{p}^{3}}=\frac{\mathfrak{p}_{0} \mathfrak{p}^{2}}{\mathfrak{a}_{3}},
$$

so daß nach unserer lofinition $x / y$ ganz ist. Es läßt sich aber $x / y$ nicht als ganze rationale Funktion von $x, y$ darstellen, auch nicht unter Benutzung der Gleichung $g=0$.

Ein cinfaches Kriterium dafür, wann eine ganze Funktion sich als ganze rationale Funktion von $x, y$ darstellen läßt, gibt es nicht. Wohl aber gibt es eine hinreichende Bedingung, die besonders wirhtig ist. Es gilt nämlich der Satz:

Ist $\bar{D}$ der Divisor der mehrfachen Punkte einer Kurveg und ist $a_{3}$ der Hauptnennervon $x$ und $y$ in dem dureh $g=0$ definierten algebraischen Körper, so läßt sich jede Funktion der Form

$$
R=\frac{\mathfrak{r} \overline{\mathfrak{d}}}{\mathfrak{a}_{3}^{\mathfrak{r}}}
$$

wo $x$ ein ganzer Divisor ist, als ganze rationale Funktion $y$-ten Grades von $x, y$ darstellen.

Eine ganze Funktion läßt sich also dann sicher als ganze rationale Funktion von $x, y$ darstellen, wenn ihr Zähler durch $\bar{b}$ teilbar ist.

Ehe wir zum Beweise schreiten, sind einige Vorbetrachtungen nötig.

Aus (44),. (45) folgt

$$
a_{3}^{n} g(x, y)=F\left(a_{1}, a_{2}, a_{3}\right)=F(a)
$$

und daraus durch Differentiation nach $a_{2}$ und wegen (23)

$$
a_{3}^{n-1} \frac{\partial g}{\partial y}=F_{2}(a)=r \bar{b} a_{2}^{\prime} \text {. }
$$

Es war aber

$$
\mathfrak{a} \mathrm{a}_{2}^{\prime}=\mathrm{a}_{3} d \mathrm{a}_{1}-\mathrm{a}_{1} d \mathrm{a}_{3}=\mathrm{a}_{3}^{2} d_{\mathrm{a}_{3}}^{\mathrm{a}_{1}}=\mathrm{a}_{3}^{2} d x .
$$

Der $d x$ entsprechende Divisor ist nach Kap. VI, $\S 6_{j x^{2}} \mathfrak{l}^{-2}$, wenn ix der Verzweigungsdivisor in bezug auf $x$ ist und $I$ der Nenner von $x$. Wir wollen aunehmen, daß in $g(x, y)$ das Glied mit $y^{n}$ vorkommt, daß also $a_{1}$ und $a_{3}$ keinen gemeinsamen Teiler haben. Dann ist $\mathfrak{l}=\mathfrak{a}_{3}$ und der $d x$ entsprechende. Divisor $z_{x} a_{3}^{-2}$, so daß nach (52) $\mathfrak{r a}_{2}^{\prime}=3_{x}$. Aus (51) folgt also

$$
\partial g=\frac{\bar{b} \dot{s}_{x}}{\partial y} \cdot \mathfrak{a}_{3}^{n-1}
$$

Die Voraussetzung, daß $y^{n}$ in $g(x, y)$ vorkommen soll, ändert an dem zu beweisenden Satze nichts, da sie durch Koordinatentransformation zu erreichen ist, und da durch eine solche $\bar{\delta}$ nicht geändert wird und eine 
202 Elftes Kapitel. Ebene algebraisehe Kurven in homogenen Koordinaten.

ganze rationale Funktion wieder in eine solche Funktion desselben Grades iibergiht.

Der Beweis unseres Satzes stützt sich auf folgenden Hilfssatz:

Hat die Funktion of des körpers die Form

$$
\mathscr{\gamma}={ }_{i_{x}}^{\mathfrak{q}} \mathfrak{a}_{3}^{\prime \prime},
$$

wo q ein ganzer Divisor ist, und sind $y_{1}, y_{2}, \ldots y_{n}$ die konjugierten Funktionen von $y$, so ist die Spur von $\mathscr{f}$, nämlich

$$
S(\varphi)=\varphi\left(x, y_{1}\right)+\varphi\left(x, y_{2}\right)+\cdots+\varphi\left(x, y_{n}\right)
$$

eine ganze rationale Funktion von $x$ höchstens vom Grade $\mu$.

Zunächst ist $S(\gamma)$ als symmetrische Funktion der $y_{i}$ rational in $\boldsymbol{x}$. Dann kann $S(r f)$ wegen (54) unendlich werden:

erstens für unendliches $x$, und zwar höchstens von $\mu$-ter Ordnung;

zweitens für solche Werte von $x$, wo ein Verzweigungspunkt liegt. Ist $x=a$ ein derartiger Wert, so kann dort $\varphi$ und also auch $S(\varphi)$ höchstens unendlich werden wie eine echt gebrochene negative Potenz von $x-\boldsymbol{a}$. Aber $S(\mathscr{f})$ kann als rationale Funktion von $x$ nur unendlich werden wie eine ganze negative Potenz von $x-a$.

Daher wird $S(r)$ nur für unendliches $x$ unendlich und höchstens von der $\mu$-ten Ordnung, ist also, wie behauptet, eine ganze rationale Funktion von $x$ höchstens rom Grade $\mu$.

Es habe jetzt $R$ die Form (49). Zunächst können wir $R$ in der Gestalt schreiben

$$
R=r_{i}+r_{1} y+\cdots+r_{n-1} y^{n-1} .
$$

wo die $r_{i}$ rationale Funktionen von $x$ sind. Wir haben zu zeigen, daß es ganze Funktionen sind und da $r_{h}$ höchstens vom Grade $v-h$ ist.

Nach der Lagrangeschen Interpolationsformel ist, wenn wieder $y_{1}$, $y_{\mathbf{2}}, \ldots y_{n}$ die konjugierten Werte von $y$ sind,

Setzen wir also

$$
R(x, y)=g(x, y)\left\{\begin{array}{c}
R\left(x, y_{1}\right) \\
\left(y-y_{1}\right) \frac{\partial g}{\partial y}\left(x, y_{1}\right)
\end{array}+\cdots+\frac{R\left(x, y_{n}\right)}{\frac{\partial g}{\partial y}\left(x, y_{n}\right)}\right\}
$$

so wird

$$
\begin{gathered}
r(u)=r_{0}+r_{1} u+\cdots+r_{n-1} u^{n-1} \\
\varphi(x, y)=\frac{g(x, u)}{u-y} \frac{R(x, y)}{\frac{\partial g}{\partial y}(x, y)}
\end{gathered}
$$

$$
r(u)=S(\varphi) .
$$


Als Funktion des Körpers $(x y)$ wird. da $g(x, y)$ identisch Null ist,

oder, wenn wir setzen

$$
\begin{aligned}
& g(r, u) \quad g(r, u) \cdots g(r, y) \\
& u-y \quad u-y
\end{aligned}
$$

(60)

$$
\begin{aligned}
& g-g_{11}+g_{1} !+\cdots+g_{n} y^{\prime \prime}, \\
& \left\{\begin{aligned}
g(x, u)=g_{1}+g_{2}(y+u) & +g_{3}\left(y^{2}+y u+u^{2}\right)+\cdots \\
u-y & +g_{n}\left(y^{\prime \prime-1}+y^{n-2} u+\cdots+u^{n} \quad\right. \\
= & \left(g_{1}+g_{2} y+\cdots+g_{n} y^{n-1}\right)+\left(g_{2}+g_{3} y+\cdots\right. \\
& \left.+g_{n} y^{n-2}\right) u+\cdots \\
= & h_{0}+h_{1} u+\cdots+h_{n-1} u^{n-1},
\end{aligned}\right.
\end{aligned}
$$

wo $h_{i}$ eine ganze rationale Funktion von $x, y$ höchstens vom Grade $n-i-1$ ist. Es hat daher $h_{i}$ die Form

wo $\mathrm{h}_{i}$ ein ganzer Divisor ist.

$$
h_{i}=\frac{h_{1}}{n_{i}^{n-i-1}} .
$$

Setzen wir

so wird nach (57) bis (60)

$$
\begin{gathered}
R(x, y) \\
h_{k} \frac{\partial g}{\partial y}(x, y)
\end{gathered}=\varphi_{! k},
$$

$$
r_{k}=S\left(f_{k}\right) .
$$

Wegen (49), (53), (61) und (62) ist aber

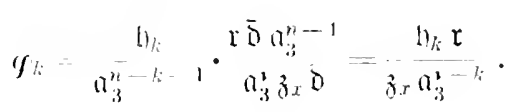

Nach dem Hilfssatz ist daher $r_{k}$ cine ganze rationale Funktion von $x$ höchstens vom Grade $y-k$. Damit ist aber unser Satz bewiesen.

Dieser Satz kann an Stelle des Noetherschen Fundamentalsatzes die Grundlage der Noethersshen 'Theorie der algebraischen Funktionen bilden*). Der Northersche Satz selbst gibt für drei ganze rationale Funktionen $A, B, C$ von $x, y$ die notwendigen und hinreichenden Bedingungen dafür. daß sich rine der Funktionen, etwa $C$, in der Form darstellen läßt

$$
C=g A+h B
$$

wo $g$ und $h$ auch ganze rationale Funktionen von $x$ sint. Der hier bewiesene Satz gilst hierfür eine hinreichende Bedingung. Die gewünschte Darstellung ist nämlich dann sicher möglich, wenn die Funktion $C / B$

*) Man vergleiche hierzu II.- I. 25. Vorlesung, $\$ 4$. S. $434 \mathrm{f}$. 
dureh den Divisor der mehrfachen Pmokte der kurve $A=0$ teilbar ist. 1):unn läbt sich nämlich $C / B$ unter der Voraussetzung $A=0$ als ganze rationale Funktion $h$ von $x$ und y darslollon, d. h. e- besteht eine ldentität der firm

$$
\text { C } 1 \text { l } \quad l+A \text {. }
$$

wo $r$ rine rationale Funktion von $x, y$ ist, deren Nenner sicher $n A$ teilerfremul ist. Sutzt man $r B=g$, so folgt die zu beweisende Gleichung. Denn ans dir-er Glebhong, die identish in $x, y$ hesteht, folgt, daB $g A$ ganz ist und also, da der Kinner von $g$ sieher teilerfermel zu $A$ ist, da $B$ anch $g$ ganz ist.

Man nennt ganze Funktionen, de den Faktor $\overline{0}$ haben, adjungierte Fanktionnn. Sir lasson sich also immer als ganze rationale Funktionen von $x, y$ darstellen. Sot man wim solde Funktion gleich Null, so nennt man die dadureh definierte Kurve pine adjungierte Kurve von $g$.

bir adjungierten Funktionen werden benutzt zur Darstellung der lifferentialo des Körpers, die für endliche Werte von $x, y$ nirgends unendjich werden. Ist $R$ d $x$ ein bifferential dieser Art, das für unendliches $x, y$

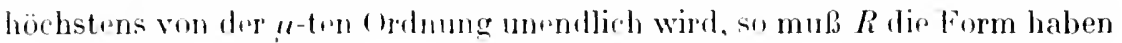

$$
R=\frac{\mathrm{r}}{i_{x} \mathrm{a}_{3}^{\prime \prime \prime}-2},
$$

wo $z$, nin ganzer Divisor ist, dat $d r$ Null and unendlich wird wie $z_{x} a_{3}^{-2}$. D)araus folgt wegen (53)

$$
R \frac{\partial g}{\partial y}=\frac{r \bar{b}}{a_{3}^{n}+u-3} .
$$

a daß $R_{\partial y}^{\partial g}$ eine adjungierte Funktion $\varphi(x, y)$ der Ordnung $n+\mu-3$ wird. Wir haben also im besonderen für $u=0$ :

Ein Jifferential erster Gattung läßt sich in der Form darstollon

$$
\begin{gathered}
\varphi(x, y) d x \\
\partial g \\
\hat{o} y
\end{gathered}
$$

wo g eine adjungierto Funktion $(n-3)$-ten Grades ist. Und umgekehrtist jedressolclo Jifferentialvonderersten Gattung.

\section{$\$ 10$. Beispiele.}

1. Wir betrachten zunächst zwri Beispiele für das Verhalten von Kurven im Unendliclern. Unter a) Irenutzen wir die in Kap. X, §1 gegebone Definition rler mondlich fromen Stellen und unter b) die in $\$ 8$ gegeterere. 
Es sei die kure/ definiert dureh

$$
f(x, y) \quad y^{3}-x^{2} y+x^{2}-1 \quad 0 .
$$

a) Zur Linteramehung der unendlieh formen stellen haben wir folgende drei Sulstitutinnen aus\%uführen:
I. $x \quad \frac{1}{x^{\prime}}, y-y$.
II. $x \cdot x, y=\begin{aligned} & 1 \\ & y^{\prime}\end{aligned}$.

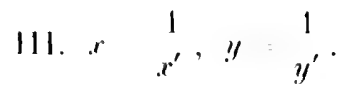

Wir rehalten die Gilichungen

$$
\begin{aligned}
& \text { I: } \quad(y-1)-x^{2}-x^{\prime 2} y^{3}=0, \\
& \text { II: } \quad 1+y^{3}-x^{2} y^{\prime 2}+x^{2} y^{3}=0, \\
& \text { HI }: x^{\prime 2}-y^{\prime 2}+y^{\prime 3}+x^{\prime 2} y^{\prime 3}=0 .
\end{aligned}
$$

lis geht also $f$ durch die unendlich fornen Punkte

$$
\text { 1. } x=\infty, y=1 ; 2 . x=\infty, y=\infty
$$

und hat in dem zweiten einen Doppelpunkt mit getrennten Tangenten.

b) Da $y^{3}$ in der Gleichung $f=0$ vorkommt, luaben wir nur zu setzen (vgl. §8)

und erhalten

$$
x=\frac{1}{?}, \quad y=\frac{\xi}{3}
$$

$$
1-\xi+\xi^{3}+1^{3}=0 \text {. }
$$

Hieraus folgt für $\eta=0: \xi=0,1,-1$. Es geht also $f$ bei dieser Betrachtungsart dureh dir unendlich fernen Punkte, dio definiert sind durch
1. $\lim \frac{!}{x}=0$.
2. $\lim \frac{y}{x}=1$,
3. $\lim _{r}^{y}=-1$.

Durch jeden dieser Punkte geht $f$ nur einfach.

2. Es sei die Gileirhung von $g$.

$$
g-y^{3}-x y^{2}-x^{3} y+1+x^{4}=0
$$

a) In der Umgebung des Punktes $x=\infty, y=\infty$ haben wir dies" Kurve srhon in Kap. X, $\$ 6$ Beispiel 5 untersucht. Durch andere unendlich ferme Punkte geht die Kurve nicht. Der Beitrag des Punktes $x=y=\infty$ $z u$ o ist gefunden zu $p_{1}^{4} p_{2}^{2}$. Ferner werden $x$ und $y$ nur an diesen Stellen unendlich. Aus den Entwicklungen für $y^{\prime}$ ergibt sich für dir Nenner ! und $m$ ron $x$ und $y$

$$
1=\mathfrak{p}_{1}^{3} \mathfrak{p}_{2}, \quad \amalg=\mathfrak{p}_{1}^{3} \mathfrak{p}_{2} .
$$

Das kleinste gemeinschaftliche Vielfache $a_{3}$ von $I$ und $m$ ist also

$$
\mathfrak{a}_{3}=m=\mathfrak{p}_{1}^{3} \mathfrak{p}_{2} .
$$


¿0) Elftes Kapitel. Ebene algebraische Kurven in homogenen Koordinaten.

b) lia $g$ vom virrten Grade ist mud das Glied mit $x^{4}$ in $g$ vorkommt, -1) beuntzen wir dir Substitution (48)

nud erhalten

$$
x=5, \quad y=\begin{aligned}
& 1 \\
& y
\end{aligned}
$$

Fiir ,

$$
\bar{g}(\Xi, y) \quad \text { y- }
$$

$$
\Xi=0 \text { und } \Xi=1 \text {. }
$$

1. $\Xi=0, y=0$. Dies ist vin cinfacher l'unkt der Kurve. Man findet

$$
1=\Xi^{3}+\cdots .
$$

so daB der l'unkt ein Wendepunkt ist.

2. $\Xi-1, y=0$. Setzen wir $\Xi-1=\Xi$, so erbalten wir

$$
\Xi^{\prime}+3 \Xi^{\prime 2}+3 \Xi^{\prime}+\Xi^{\prime}-\eta \Xi^{\prime}+y^{4}=0 \text {. }
$$

Es ist also anch dieser Punkt ein einfarher Punkt. Es ergibt sich

$$
\xi^{\prime}=-\eta^{4}+\cdots .
$$

Jer Punkt ist also ein Wendepunkt zweiter Ordnung.

Die unendlich fernen Punkte liefern zu D also keimen Beitrag. Da endlich Stellen zu o und $\delta$ denselben Beitrag liefern, so ist

Andererseits ist nach (31)

$$
\overline{\mathfrak{D}}=\mathfrak{D} \mathfrak{p}_{1}^{-4} \mathfrak{p}_{2}^{-2}
$$

$$
\overline{\mathrm{j}}=\mathrm{a}_{3}^{n-3} \mathrm{~b} .
$$

Setzen wir hierin die Werte für $a_{3}, \mathfrak{l}$, m aus (64) und (65) ein, so erhalten wir wegen $l=4, m=3, n=4$ tatsächlich (66).

3. Es sei

$$
F(x) \quad 4\left(x_{1}^{2}+x_{2}^{2}\right)^{3}-4\left(x_{1}^{2}+x_{2}^{2}\right)^{2} x_{3}^{2}+x_{2}^{2} x_{3}^{4}=0 .
$$

Wählen wir $x_{3}=0$ als unendlich ferne Gerade und $x_{1}, x_{2}$ als rechtwinklige kartesische Koordinaten, so ist $F$ die zweiblättrige Rosenkurve. (Vgl. G. Loria, Spezielle algebraische und transzendente ebene Kurven [B. G. Trubner 1910] Bd. I, Tafel Xl, Fig. 81.)

Wir bestimmen zunächst die mehrfachen Punkte. In diesen werden $F_{1}(x), F_{2}(x), F_{3}(x)$ gleichzeitig Null. Setzen wir zur Abkürzung

si erhalten wir die Gleichungen

$$
x_{1}^{2}+x_{2}^{2}=z
$$

$$
\left\{\begin{array}{l}
F_{1}-12 z^{2} \cdot 2 x_{1}-8 z \cdot 2 x_{1} x_{3}^{2}=8 z x_{1}\left(3 z-2 x_{3}^{2}\right)=0 \\
F_{2}-12 z^{2} \cdot 2 x_{2}-8 z \cdot 2 x_{2} x_{3}^{2}+2 x_{2} x_{3}^{4} \equiv 2 x_{2}\left\{4 z\left(3 z-2 x_{3}^{2}\right)+x_{3}^{4}\right\}=0 \\
F_{3}-8 z^{2} x_{3}+4 x_{2}^{2} x_{3}^{3}=-4 x_{3}\left(2 z^{2}-x_{2}^{2} x_{3}^{2}\right)=0
\end{array}\right.
$$




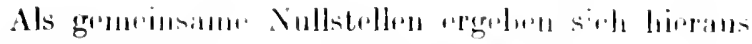

$\left.1: x_{1} \quad x_{2} \quad 1\right) ; 11: x_{1}=0, x_{2}: x_{3}=1: 12: 111: x_{1}=0, x_{2}: x_{3}=-1: 12 ;$ $\mathrm{W}: x_{1}: x_{2} \quad i: 1 . x_{3} \quad 0 ; \quad \mathrm{V}: x_{1}: x_{2}-\cdots: 1, x_{3}=0$.

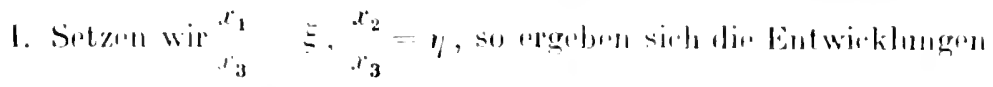

$$
1,25^{2}+\cdots \text { und } y=-25^{2}+\cdots \text {. }
$$

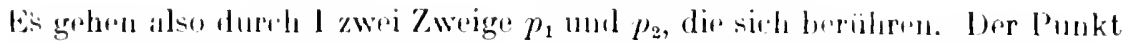
$r_{1}=x_{2}=0$ ist ein Solbstbrö̈hrungapunkt. Wie Tangente in ihn ist $y_{2}=0$. liu zugehörigen Primtriler seien $p_{1}$ und $p_{2}$. Nan findet nach Kap. X, \$4 und $\$ 5$

$$
\left(p_{1}, p_{1}\right)=\left(p_{2}, p_{2}\right)-0,\left(p_{1}, p_{2}\right)=2 .
$$

l)er Beitrag von I zu d ist $\mathrm{p}_{1}^{2} \mathrm{p}_{2}^{2}$. lungen

II. Hier setzen wir. wie in I $\frac{x_{1}}{r_{3}}=\Xi, x_{2}^{x_{2}}=\eta$ und finden die Entwiek-

$$
r=\frac{1}{2} \mid 2+\frac{5}{2}+\cdots \text { und } \quad r_{1}=\frac{1}{2} \mid \overline{2}-\frac{5}{2}+\cdots
$$

Es gehen also aueh durch II zwei Zweige $p_{3}, p_{4}$, die sich schneiden. Die zugehörigen Primteiler seien $p_{3}, p_{4}$. Es wird

$$
\left(p_{3}, p_{3}\right)=\left(p_{4}, p_{4}\right)=0, \quad\left(p_{3}, p_{4}\right)=1,
$$

$\therefore$ daB der Britrag von $I 1$ zu $\overline{\mathrm{D}}$ gleich $\mathrm{p}_{3} \mathrm{p}_{4}$ ist.

III. Die Ergebnisse für diesen Punkt erhalten wir aus denen für II, da statt $\mid 2$ nur - $\mid 2$ zu schreiben ist. Es gehon also dureh III zwoi Zwrige $p_{5}, p_{8}$. Sind $p_{5}, p_{6}$ die zugehörigen Primteiler, so ist der Beitrag von 111 zu $\overline{\mathrm{D}}$ gleich $\mathfrak{p}_{5} \mathfrak{p}_{6}$.

IV. Wir sotzen $\frac{x_{3}}{x_{1}}=5, \frac{x_{2}}{x_{1}}=\eta$ und erhalten

$$
1=-i-\left.\int_{4}^{1}\right|^{3} i \xi^{4}+\cdots
$$

Jurch IV geht ein Zweig $p_{7}$ von $F$. Der zugehörige Primteiler soi $p_{7}$. Man findet $\left(p_{7}, p_{7}\right)=6$, so $\mathrm{da} B p_{7}$ in $\bar{\delta}$ in der sechsten Potenz enthalten ist.

$\mathrm{V}$. In den Betrachtungen IV ist $i$ durch $-i$ zu ersetzen. Dureh $\mathrm{V}$ geht ein Zwrig $p_{8}$ von $F$ und der zugehörige Primteiler $p_{8}$ liefert zu $\bar{\delta}$ den Beitrag $\mathfrak{p}_{,}^{6}$.

Es wird also

$$
D=p_{1}^{2} p_{2}^{2} p_{3} p_{\mathbf{s}} p_{\mathbf{s}} p_{\mathbf{6}} p_{7}^{6} p_{5}^{6}, \quad 2 \bar{d}=20
$$


Nach (43) wird also das Geschlecht $p$ von $F$

$$
p=\frac{1}{2}(n-1)(n-2)-10=0 .
$$

Bedentest trine Größe, tie an der betroffenden Stelle von der ersten Grduung Null wird, so haben wir folgende Darstellungen an den einzelnen Stellen

(71)

$$
\left\{\begin{array}{llll}
p_{1}: & a_{1}=t, & a_{2}=2 t^{2}+\cdots, & a_{3}=1 ; \\
p_{2}: & a_{1}=t . & a_{2}=-2 t^{2} \cdots, & a_{3}=1 ; \\
p_{3}: & a_{1}=t, & a_{2}=\frac{1}{2} \mid 2+\frac{1}{2} t+\cdots, & a_{3}=1 ; \\
p_{4}: & a_{1}=t, & a_{2}=\frac{1}{2} \mid 2-\frac{1}{2} t+\cdots, & a_{3}=1 ; \\
p_{5}: & a_{1}=t . & a_{2}=-\frac{1}{2} \mid 2+\frac{1}{2} t+\cdots, & a_{3}=1 ; \\
p_{8}: & a_{1}=t . & a_{2}=-\frac{1}{2} \mid 2-\frac{1}{2} t+\cdots, & a_{3}=1 ; \\
p_{7}: & a_{1}=1, & a_{2}=-i-\frac{1}{4} \mid \frac{3}{2} i t^{4}+\cdots, & a_{3}=t^{3} ; \\
\mathfrak{p}_{8}: & a_{1}=1, & a_{2}=i+\frac{1}{4} \mid \frac{3}{2} i t^{4}+\cdots, & a_{3}=t^{3} .
\end{array}\right.
$$

Da der Livisur der Rückkehrpunkte $r$ auch gemeinsamer Teiler von $F_{1}(a), F_{2}(a), F_{3}(0)$ ist, so können in $r$ nur die Primteiler $\mathfrak{p}_{1}$ bis $\mathfrak{p}_{8}$ enthalten sein. Um $r$ zu bestimmen, brauchen wir den größten gemeinsamen Teiler der Divisoren $\mathfrak{a}_{a} d \mathfrak{a}_{s}-\mathfrak{a}_{j} d \mathfrak{a}_{a}$ also nur für die acht Stellen $\mathfrak{p}_{\mathbf{1}}$ bis $\mathfrak{p}_{\mathbf{z}}$ zu berechnen. Wir finden so

$$
\mathrm{r}=\mathfrak{p}_{7}^{2} \mathrm{p}_{s}^{2}, \quad r=4 .
$$

Aus den Plückerschen Gleichungen (42) ergibt sich wegen $p=0$, $n=6 \cdot r=4 . d=10$

$$
r^{\prime}=4, d^{\prime}=10, d_{0}^{\prime}=d^{\prime}-r^{\prime}=6, n^{\prime}=6 .
$$

Die Kurve hat also vier Wendepunkte und sechs Doppeltangenten. Aus der Form der Kurve sieht man, daß die Wendepunkte imaginär sind, während dir Doppeltangenten alle reell sind. Dabei muß man bedenken, daß di Tangente in dem Selbstberührungspunkt doppelt zählt unter den Joppoltangenten. Denkt man sich nämlich die beiden Kurvenzweige, die sich im Selbstberührungspunkt berühren, etwas voneinander entfernt, so hat dis Kurve zwej Doppeltangenten, die so liegen wie die inneren Tangenten zweier Kreise. Läßt man dann die Kurvenzweige sich nähern, bis sie einander herühren, so fallen diese beiden Joppeltangenten zusammen. 
Da $p=0$ ist, so können wir dir $x_{i}$ als rationale Funktionen eines Parameters darstellen. Z. B. auf folgente Art. Setzen wir

$$
r_{1}^{\ddot{2}}+x_{2}^{\ddot{2}}=z x_{3}^{2}, \quad x_{2} \quad x_{3} y, \quad x_{1}=x x_{3} .
$$

also $x^{2}+y^{2} z$, so lautet die Gileichung (67) von $F$

$$
4 z^{3}-4 z^{2}+y^{2}=0 .
$$

Hierin setzen wir $y=2 z u$ und finden zunäclst $z \quad 1-u^{2}$. Dann folgt aus $y=2 z u$, da $3 y=2 u\left(1-u^{2}\right)$ und unter Verwendung der für $y$ und $z$ gefundenen Werte ergibt $\operatorname{sich} x$ aus $x^{2}+y^{2}=z$. Wir haben also

$$
x=\left(1-2 u^{2}\right) / 1-u^{2}, \quad y=2 u\left(1-u^{2}\right), \quad z=1-u^{2} .
$$

Um die in $x$ noch vorkommende Wurzel fortzuschaffen, setzen wir

Wir erhalten dann

$$
\mid 1-u^{2}=1-u v \text {. }
$$

$$
x=\frac{x_{1}}{x_{3}}=\frac{\left(1-v^{2}\right)\left(1-6 v^{2}+v^{4}\right)}{\left(1+v^{2}\right)^{3}} . \quad y=\frac{x_{2}}{x_{3}}=\frac{4 v\left(1-v^{2}\right)^{2}}{\left(1+v^{2}\right)^{3}},
$$

so daß wir setzen können

$$
x_{1}=\left(1-v^{2}\right)\left(1-6 v^{2}+v^{4}\right), \quad x_{2}=4 v\left(1-v^{2}\right)^{2}, \quad x_{3}=\left(1+v^{2}\right)^{3} .
$$

Der Parameter $v$ ist eine Funktion des Körpers. Das folgt schon daraus, daß die $x_{i}$ rationale Funktionen seehster Ordnung mit demselben Nenner - nämlich 1 - sind und die Gleichung zwisehen den $x_{i}$ auch vom sechsten Grade ist. Wir können aber auch leicht unmittelbar zeigen, daß $v$ dem Körper angehört. Aus (75) folgt

$$
u=\stackrel{y}{2 z}, \quad \mid 1-u^{2}=\begin{gathered}
x \\
2 z-1
\end{gathered},
$$

so da $B$ wegn $(76) v$ eine rationale Funktion von $x, y, z$ wird. Es sind aber $x, y, z$ nach (7/) Funktionen des Körpers.

Es entspricht also jeder Stello des Körpers ein Wert von $v$ und umgekehrt. Die Werte, die $v$ an den Stellen $\mathfrak{p}_{i}$ hat, sind folgende:

$$
\begin{gathered}
\mathfrak{p}_{1}: 1, \mathfrak{p}_{2}:-1, \mathfrak{p}_{3}:-1+12, \mathfrak{p}_{4}: 1+1 \overline{2}, \mathfrak{p}_{5}:-1-12, \\
\mathfrak{p}_{8}: 1-1 \overline{2}, \quad p_{7}:-i, \quad \mathfrak{p}_{8}: i
\end{gathered}
$$

Es ist also z. B. $p_{1}$ der Zähler von $v-1, \mathfrak{r}$ gleich dem Zähler von $(v+i)$ : $(v-i)^{2}=\left(1+v^{2}\right)^{2}$. Wenn wir $v$ also auf endliche Werte beschränken. können wir geradezu setzen

(79)

$$
\mathrm{r}=\left(1+v^{2}\right)^{2}
$$

und wegen (69) 
210 Elftes Kapitel. Ebene algebraische Kurven in homogenen Koordinaten.

$8(1)$

$$
\left\{\begin{array}{lll} 
& b & \left(1-v^{2}\right)^{2}\left(1-b v^{2}+v^{4}\right)\left(1+v^{2}\right)^{6}, \\
b^{2} & b_{0} & \left(1-v^{2}\right)^{2}\left(1-b v^{2}+v^{4}\right)\left(1+v^{2}\right)^{2} .
\end{array}\right.
$$

Fur endliches $v$ wird wegen $(77)$

(b) $a_{1}-\left(1-r^{2}\right)\left(1-6 r^{2}+r^{4}\right) . \quad a_{2} \quad a\left(1-v^{2}\right)^{2} . \quad a_{3}=\left(1+v^{2}\right)^{3}$.

lst $r_{0}$ rin endlicher Wert von e und will man an der entsprechenden Stelle

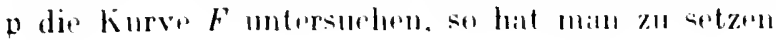

$$
r r_{0}+1
$$

und es ist dann $t$ aine Grobe, die in p von erster Urdnung Null wird. Aus (\$') folgt $d v=d t$, so daB an allen mollithen Stellen $d v$ nicht Null oder unendlich wird. Fïr mondliches $v$ hat man zu setzen

$$
v=\frac{1}{t}
$$

Für die Umgelunng are diesem Werte entsprechenden stelle hat man - nach Nultiplikation mit $t^{6}-$

(83) $\mathrm{a}_{1}=-\left(1-t^{2}\right)\left(1-6 t^{2}+t^{4}\right), \quad \mathrm{a}_{2}=4 t\left(1-t^{2}\right)^{2}, \quad \mathrm{a}_{3}=\left(1+t^{2}\right)^{8}$

Diese Jarstellung ist nur zu bemutzen für kleine Werte von $t$. Da für $t=0, \mathrm{a}_{1}=-1, \mathrm{a}_{2}=0, \mathrm{a}_{3}=1$, so hat der $v=\infty$ entsprechende Punkt die Koordinaten $(-1,0,1)$. Es ist ein regulärer Punkt und auch die Tangente in ihm hat keine Besonderheiten. Wir werden uns daher im folgenden auf endliche. Werte von $v$ beschränken.

Wir bestimmen zunächst die Jivisoren $a_{i}^{\prime}$. Zur bequemeren Rechnung fïhren wir folgende Abkürzungen ein

so $\mathrm{daB}$

$$
1+v^{2}=i, \quad 1-v^{2}=\mu,
$$

Von diesen Gleichungen werden wir öfter Gebrauch machen. Es wird

$$
u_{1}=\mu\left(2 \mu^{2}-\lambda^{2}\right), \quad a_{2}=4 v \mu^{2} . \quad a_{3}=\lambda^{3} .
$$

Daraus folgt

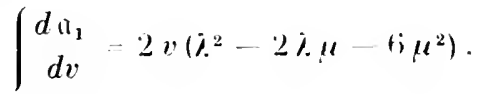

$$
\begin{aligned}
& \mid \begin{array}{l}
d a_{2} \\
d v
\end{array}=4 u(3 u-2 i), \quad d a_{3}=6 \lambda^{2} v .
\end{aligned}
$$

Aus (86), (87) regiht sich wegen (9). §2 unter Fortlassung des Faktors 2 also

$$
\text { r } a_{i}^{\prime}=2 \lambda^{2} \mu\left(2 \lambda^{2}-3 \mu^{2}\right), \quad r a_{2}^{\prime}=2 v \lambda^{2}\left(\lambda^{2}-6 \mu^{2}\right), \quad \mathfrak{i} a_{3}^{\prime}=2 \lambda^{3} \mu^{2},
$$

(88) $\quad \imath=2 \lambda^{2}, \quad a_{1}^{\prime}=\mu\left(2 \lambda^{2}-3 \mu^{2}\right) . \quad a_{2}^{\prime}=v\left(\lambda^{2}-6 \mu^{2}\right), \quad a_{3}^{\prime}=\lambda \mu^{2}$. 
Der gefundene. Wort fiur 2 stimmt mit drm in (79) gegrbenen überein, da "s hei loivisuren auf rinen konstanten Faktor ja nirht ankommt. Fiihren wir statt $\lambda, !$ wieder $v$ rin, so wirl

(89) $\quad a_{1}^{\prime} \quad\left(1 \quad r^{2}\right)\left(1 \quad 10 v^{2}+v^{4}\right) . \quad a_{2}^{\prime} \quad v\left(5-14 v^{2}+5 v^{4}\right)$,

$$
13-\left(1+v^{2}\right)\left(1-v^{2}\right)^{2} \text {. }
$$

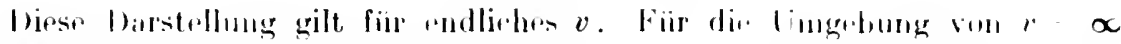
hätren wir statt " rinzulühron $t^{-1}$.

Un dir dileithung von $F$ in Linienkoordinaten hrorzuleiten, ortzen wir

$$
\Xi=\left(\begin{array}{l}
a_{1}^{\prime} \\
a_{3}^{\prime}
\end{array}\right)^{2}-\left(\begin{array}{l}
x_{1}^{\prime} \\
x_{3}^{\prime}
\end{array}\right)^{2}, \quad,=4\left(\begin{array}{c}
a_{2}^{\prime} \\
a_{3}^{\prime}
\end{array}\right)^{2}=4\left(\begin{array}{c}
x_{2}^{\prime} \\
x_{3}^{\prime}
\end{array}\right)^{2} \text {. }
$$

Hirratu folgt wogen (85) und (88)

oder, w'nn wir

$$
\xi=\frac{\left(2 \lambda^{2}-3 \mu^{2}\right)^{2}}{\lambda^{2} \iota^{2}}, \quad,=\frac{\left(\eta^{2}-\mu^{2}\right)\left(\lambda^{2}-6 \mu^{2}\right)^{2}}{\lambda^{2} \mu^{4}}
$$

$$
\lambda^{2}=\rho u^{2}
$$

setzen

$$
\xi=\frac{(2 \varrho-3)^{2}}{\varrho}, \quad r=\frac{(\rho-1)(\varrho-6)^{2}}{\rho} .
$$

Aus diesen beiden Gleichungen ist $\varrho$ zu eliminieren. Die Gleichung für $\xi \eta$, die sich ergibt, sei $h\left(\xi, y_{j}=0\right.$. Da $\xi$ und $\eta$ Funktionen dritter Ordnung mit demselben Nenner sind, so ist $h$ vom dritten Grade in $\xi, \eta$. Da $\Xi$ nur von zweiter Ordnung ist, so ist $h$ in , nur vom zweiten Grade. Da aber y nur unendlich wird, wenn $\xi$ es wird, nämlich für $\varrho=0$ und $\varrho=\infty$, so ist der Faktor von $y^{2}$ konstant, d. h. es fehlt das Glied $\xi \eta^{2}$. Daher hat $h$ dic Form

$$
h(\xi, \eta)=a \xi^{3}+b \xi^{2} y+c \xi^{2}+\partial \xi_{1}+e \eta^{2}+f \xi+g \eta+k .
$$

Für $\varrho=0$ werden $\xi, \eta$ beide unendlich und wegen $(92) \lim \underset{\eta}{\xi}=-4$. Setzru wir also dif Glieder höchster Ordnung von $h$ gleich Nisll.

$$
a \xi^{3}+b \xi^{2} \eta=0 \text {. }
$$

so muB hioraus unter andrem $\eta=-4 \xi$ folgen. Es ist daher

$$
a=4 b \text {. }
$$

Es wird $y_{y}=0$ für $\varrho=1$ und zweimal für $\varrho=6$. Die zugehörigrn Werte von $\xi \operatorname{sind} \xi=1$ und $\xi=\frac{27}{2}$. Diese müssen sich fiir,$=0$ aus $h(\xi, \eta)=0$ rergeben. Daher wird 


$$
h(\xi 0) \quad a \xi^{3}+c \xi^{2}+f \xi+k \quad a(\xi-1)\left(\xi-\frac{27}{2}\right)^{2} .
$$

Es wird $\Xi=0$ zwimal für $\varrho=\frac{3}{2}$. Der zugehörige Wert von $y$ ist $\frac{27}{4}$. Daller wird

$$
h\left(0, y_{i}\right) \quad c z^{2}+g_{1}+k \quad e\left(y_{1}-\frac{27}{4}\right)^{2} .
$$

Ans (95) und (96) folgt unter anderem

$$
k=-a_{4}^{27^{2}}=e^{27^{2}} 16 .
$$

Wählen wir $a=4$, so haben wir wegen (94) und (97)

$$
a=4, \quad b=1, \quad e=-16, k=-27^{2}
$$

und es wird wegen (95) und (96)

$$
\begin{aligned}
h(\xi, \eta) & =h(\xi .0)+h(0, \eta)-k+b \xi^{2} \eta+\partial \xi \eta \\
& =4(\xi-1)\left(\xi-\frac{27}{2}\right)^{2}-16\left(\eta-\frac{27}{4}\right)^{2}+27^{2}+\xi^{2} \eta+\partial \xi \eta
\end{aligned}
$$

oder

(98) $h(\xi, 1)=(\xi-1)(2 \xi-27)^{2}-8 \eta(2 \eta-27)+\xi^{2} \eta+\partial \xi \eta=0$.

Um endlich $\partial \mathrm{zu}$ bestimmen, betrachten wir die Umgebung von $\varrho=6$. Setzen wir $\varrho=6+6 t$, so wird

$$
(2 \varrho-3)^{2}=(9+12 t)^{2}=81+216 t+\cdots \cdot \frac{1}{\varrho}=\frac{1}{6}(1-t+\cdots)
$$

und wegen (92)

$$
2 亏-27=45 t+\cdots, \quad y=30 t^{2}+\cdots .
$$

Jaher ist für $g \rightarrow 6$

$$
(2 \xi-27)^{2}=\frac{45^{2}}{30}=\begin{gathered}
5 \cdot 27 \\
2
\end{gathered}, \quad \xi=\frac{27}{2}, \quad y=0 .
$$

Dividieren wir (98) durch , und bedenken, daß die entstehende Gleichung identisch besteht, so folgt wegen (99) für $\varrho \rightarrow 6$

$$
\frac{25}{2} \cdot \frac{5 \cdot 27}{2}+8 \cdot 27+\frac{27^{2}}{4}+\partial \cdot \frac{27}{2}=0 \text {. }
$$

also

$$
\partial=-92 \text {. }
$$

Es ist daher andgültig

$$
\text { (100) } h\left(\xi, r_{1}\right) \quad(\xi-1)(2 \xi-27)^{2}-8 y_{i}(2 \eta-27)+\xi^{2} \eta-92 \xi \eta=0
$$

die Gleichung zwischen $\xi$ und $r_{i}$. 
Wegen (90) folgt

$$
F^{\prime \prime}\left(x^{\prime}\right) \quad F^{\prime}\left(x_{1}^{\prime}, x_{2}^{\prime}, x_{3}^{\prime}\right) \quad x_{3}^{\prime 6} h\left(\begin{array}{ll}
x_{1}^{\prime 2} \\
x_{3}^{\prime 2}, & 4 \\
x_{2}^{\prime 2} \\
x_{3}^{\prime 2}
\end{array}\right)
$$

order

$$
\begin{aligned}
& F^{\prime}\left(x^{\prime}\right)=\left(x_{1}^{\prime 2}-x_{3}^{\prime 2}\right)\left(2 x_{1}^{\prime 2}-27 x_{3}^{\prime 2}\right)^{2}-3 x_{3}^{\prime 2} x_{3}^{\prime 2}\left(8 x_{2}^{\prime 2}-27 x_{3}^{\prime 2}\right) \\
&+4 x_{1}^{\prime} x_{2}^{\prime 2}-368 x_{1}^{\prime 2} x_{2}^{\prime 2} x_{3}^{\prime 2} .
\end{aligned}
$$

Um die Wende- and Doppeltangenten zu bestimmen, körnten wir so verlahren, wir wir es bei den Rückkehr- und boppelpunkten getan haben. Wir wollen aber einen anderen Weg einschlagert.

Aus (88) folgt

$$
\frac{d a_{i}^{\prime}}{d v}=-2 v\left(2 \lambda^{2}-4 \lambda \mu-9 \mu^{2}\right), \quad \frac{d a_{3}^{\prime}}{d v}=2 v \mu(\mu-2 \lambda) .
$$

Daher wird nach (15), §2

$$
\mathrm{r}^{\prime} \mathrm{a}_{2}=4 v \mu^{2}\left(2 \lambda^{2}+3 \mu^{2}\right),
$$

wobei von der Gleichung $\lambda+\imath=2$ Gebrauch gemacht ist. Da $a_{2}=4 v \iota^{2}$, so folgt

$$
\mathfrak{l}^{\prime}=2 \lambda^{2}+3 \mu^{2}=\mu^{2}(2 \varrho+3)=5-2 v^{2}+5 v^{4} .
$$

Durch Auflösen der Gleichung vierten Grades $\mathfrak{r}^{\prime}=0$ erhalten wir die Stellen, wo unsere Kurve $F$ Wendepunkte hat. Es ergeben sieh die Werte

$$
\sqrt{3}+i|\overline{2}, \quad-13+i| 2, \quad|\overline{3}-i| \sqrt{2}, \quad-|\sqrt{3}-i| \overline{2} \text {. }
$$

Sie sind also voneinander verschieden. Da wir wissen, daß nur vier Wendepunkte vorhanden sind $\left(r^{\prime}=4\right)$, so sind sie von der ersten Ordnung.

Aus (101) folgt

$$
\begin{aligned}
F_{2}^{\prime}\left(a^{\prime}\right)= & 8 a_{3}^{\prime} a_{3}^{\prime} \frac{\partial h}{\partial \eta}=8 a_{2}^{\prime} a_{3}^{\prime}:-32 \eta+216+\xi^{2}-92 \xi \\
= & -\frac{8 a_{2}^{\prime} a_{3}^{4}}{\varrho^{2}}: 16 \varrho^{4}+48 \varrho^{3}-108 \varrho-81 ; .
\end{aligned}
$$

Ls muß aber $F_{2}^{\prime}\left(a^{\prime}\right)$ durch $\mathrm{r}^{\prime 3}$, also wegen $(103)$ durch $(2 \varrho+3)^{3}$ teilbar sein. Das ist tatsächlich der Fall. Es wird nämlich

$$
F_{2}^{\prime}\left(n^{\prime}\right)=-\frac{8 a_{2}^{\prime} r_{3}^{\prime}{ }^{4}}{\varrho^{2}}(2 \varrho+3)^{3}(2 \varrho-3)=-80_{2}^{\prime} a_{3}^{\prime 4}\left(2 \lambda^{2}+3 u^{2}\right)^{3}\left(2 \lambda^{2}-3 u^{2}\right) \text {. }
$$

Wrgen (24), $\$ 4$ und (88) folgt also

$$
F_{2}^{\prime}\left(u^{\prime}\right)=\imath^{\prime} \iota^{\prime} a_{2}=r^{\prime 3} \bar{b}_{0}^{\prime} u_{2}=-8 r^{\prime 3} v u^{4}\left(\lambda^{2}-6 u^{2}\right)\left(2 \lambda^{2}-3 u^{2}\right) \text {. }
$$

also wegen (86) und (8'i)

$$
\begin{aligned}
\mathrm{B}_{0}^{\prime} & -u^{2}\left(\bar{\lambda}^{2}-6 u^{2}\right)\left(2 \lambda^{2}-3 u^{2}\right) \\
& =\left(1-v^{2}\right)^{2}\left(5-14 v^{2}+5 v^{1}\right)\left(1-10 v^{2}+v^{4}\right) .
\end{aligned}
$$

Die Gleichung $\widehat{\delta}_{0}^{\prime}=0$ liefert uns die Berührungspunkte der Doppeltangenten ron $F$. 
Zwölftes Kapitel.

\section{Die Art \\ des Zusammenhangs einer Riemannschen Fläche.}

\section{\$1. Definitionen.}

Wir betrachten im folgenden solche Eigenschaften von Flächen, die mngeändert bleiben, wenn man die Flächen irgendwie stetig verändert, whe sip zu zerriben oder neue Verbindungen herzustellen. Man nennt diese Betrachtungsart $A_{n}$ alysis situs. Die Flächen müssen, soll unsere Betrachtungsart anwendhar sein, mindestens eine Begrenzungslinic haben, sie müssen mit anderen Worten berandet sein. Hat eine Fläche keine Begrenzungslinie, wie z. B. eine Kugelfläche oder eine Riemannsehe Fläche, s) muß man ihr eine geben. Dies geschieht dadurch, daß man die Fläche, mit einem kleinen Loch versieht, daß man die Fläche, wie man sagt, punktiert.

Es soll nicht ausgeschlossen sein, daß eine Fläche aus mehreren getrennten Stücken besteht.

Zunächst geben wir einige Definitionen.

Definition 1. Ein Schnitt, der von einem Punkte der Begrenzung nach einem anderen Punkte der Begrenzung führt, soll Querschnitt heißen. Der schon ausgeführte Teil des Schnittes soll schon zur Grenze gehören, so daß ein Quersehnitt in sich selbst zurücklaufen kann.

Definition II. Lin Schnitt, der von einem Punkte im Innern nach einem Punkte des Randes führt, soll Riß heißen. Auch der schon ausgeführt: Teil eines Risses soll zur Grenze gehören. Es kann daher ein Riß in sich zurücklaufen; im besonderen kann er im Anfangspunkte enden, so dab eine geschlossene Linie entsteht.

Definition III. Ein Schnitt, der von einem im Innern liegenden Pınkte nach rin'm andereu Punkte im Innern führt, soll ein Loch heißen. Dadureh, daß man die länder eines Loches anseinanderbiegt, kann man ihm z. B. die Grestalt eines Kreises geben. Wie sehon oben gesagt, verwandeln wir dureh Anbringung eines Loches, das beliebig klein gewählt werden kann, eine unberandete. Fläche in cine berandete.

Definition IV. Eine aus einem Stüek bestehende Fläche, die durch jeden Querschnitt in zwei getrennte Teile zerlegt wird, heißt einfach 7.nsammenhängend.

Definition V. Eine Fläche, die erst durch eine endliche Zahl von Querschnitten in eine oder mehrere einfach zusammenhängende Flächen zrrlegt werden kann, heißt mehrfach zusammenhängend. 
Beispiele tür einfach zusannmenhängronde Flärhen sind the Fläche

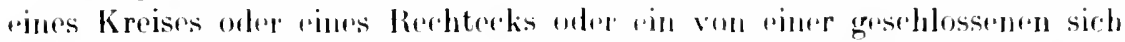

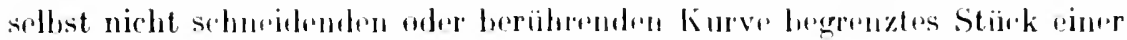

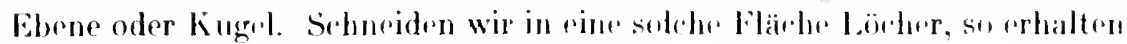
wir mehrfach zositumenhängende fläthen, Bwhahtrn wir z. l?. die Fläche, dir aus demjenigen Teil riner Ebene besteht, der innerbalb eines Kroises $K_{0}$ und anBerhalb zwoier im hnern von $K_{0}$ liegender sich nicht schneidender onder berïhrender Kreise $K_{1}$ und $K_{2}$ liegt. Ziehen wir rinen Querschnitt von $K_{0}$ nach $K_{1}$, so zerfällt die Fläche nicht in zwei getrennte Trile; sie ist daher nieht einfach zusammenhängend. Zirhen wir rinen zweiten Qurrsehnitt von $K_{0}$ nach $K_{2}$, so rrhalten wir rine Flärhe, die durch joden weiteren Querschnitt in zwei getrennte Teile zerlegt wird, die also einfurh zusammenhängend ist. Diese Fläche hat nur eine Begrenzungslinie und kann durch stetige Deformation z. B. in ein Rechterk verwandelt werden.

\section{\$2. Einfach zusammenhängende Flächen.}

Wir beweisen eine Reihe von Sätzen.

Satz 1. Die Teile, in die eine einfach zusammenhängende Fläehe dureh einen Querschnitt zerfällt, sind rinfach zusammenhängend.

Es zerfalle die einfach zusammenhängende Fläehe ' $T$ ' dureh den Quersehnitt $Q=A B$ in die beiden Trifflächen $T_{1}$ und $T_{2}$. Von diesen sei etwa $T_{1}$ nieht einfach zusammrnhängend. Dann können wir in $T_{1}$ einen Querschnitt $S$ ausführen, der $T_{1}$ nicht zerstüekt, so daß es in $T_{1}$ riurn Whig $w$ gibt, der von der einen Seite von $S$ auf die andere führt. Dieser Weg liegt auch inmerhatb $T$. Liegen Anfang und Ende von $S$ beide nieht auf $Q$, so ist $S$ auch rin Quersehnitt von $T$, muß also $T$ in zwei getrennte Teile zerlegen. Andererseits führt der Weg $w$ innerhalb, $T$ von der einen Seite von $S$ anf die andere, was cinen Widersprueh rrgibt. liegt ein Endpunkt $C$ von $S$ auf $Q$ und der andere auf der (irrnze von ' $T$ ', so bildet das Stück $A C$ von $Q$ zusammen mit $S$ eineu Qurersehnitt von $T$ und wieder führt der Weg $w$ von der einen Seit. dieses Querschnittes innerhalb $T$ auf die andere. Liegen endlich beide Endpunkte $C, D$ von $S$ auf $Q$, so sei dir Bezeichnung so gewählt, daß man erst naeh $C$ kommt, wenn man $Q$ von $A$ nach $B$ durchläuft. Es bildet dann der Tril $A C$ von $Q$ zusammen mit $S$ und dem Teil $D B$ von $Q$ einen Querschnitt von $T$ und es ist $w$ wieder ein Weg, der innerhalb $T$ von der einen Seite dirses Quersehnittes zur anderen führt. Wir haben also in allen Fällen denselben Widerspruch, so daß unsere Annahme, $T_{1}$ sei nicht einfach zusammenhängend, falseh sein muß. 
216 Zwöltes Kapitel. Die Art des Zusammenhangs einer Riemannschen Flächo.

Satz II. Eineberandeteeinfach zusammenhängende Fläche lat nur rine Begrenzungslinie.

Die Fläche habe die Begrenzungslinien $a$ und $b$ und vielleicht noch irgendwelehe andere. Wir zerschneiden die Fläche längs eines Querschnittes $Q$, der von einem Punkte von $a$ nach einem von $b$ führt. Durch $Q$ muB die Fläche in zwei getrennte Teile $T_{1}$ und $T_{2}$ zerfallen. Der eine Rand des Querschnittes $Q$ muB zum liande von $T_{1}$, der andere zu dem von $T_{2}$ gohören. Andererseits bilden aber die Begrenzungslinie $a$, der eine Rand von $Q$, die Begrenzungslinie $b$ und der andere Rand von $Q$ in dieser Reihenfolge eine einzige geschlossene Kurve. Der Widerspruch, auf den wir so gefïhrt werden, zeigt uns, daß eine berandete einfach zusammenhängende Fläche nicht mehr als eine Begrenzungslinie haben kann.

\section{\$ 3. Die Ordnung des Zusammenhangs einer Flïche.}

Satz III. Wirdeine Flächedaseine Maldureh q Querschnitte in $n$ einfach zusammenhängende Flächen zerlegt und ein andermal dureh $q^{\prime}$ Querschnitte in $n^{\prime}$ einfach zusammenhängende Flächen, so ist

$$
q-n=q^{\prime}-n^{\prime}
$$

Die Fläche heiße $T$. Dic das erstemal ausgeführten Querschnitte seien $Q_{1}, Q_{2}, \ldots Q_{1}$ und die das zweitemal benutzten $Q_{1}^{\prime}, Q_{2}^{\prime}, \ldots Q_{q}^{\prime}$. Man vergleiche Abb.34, wo die Querschnitte $Q$ ausgezogen und die $Q^{\prime}$ punktiert

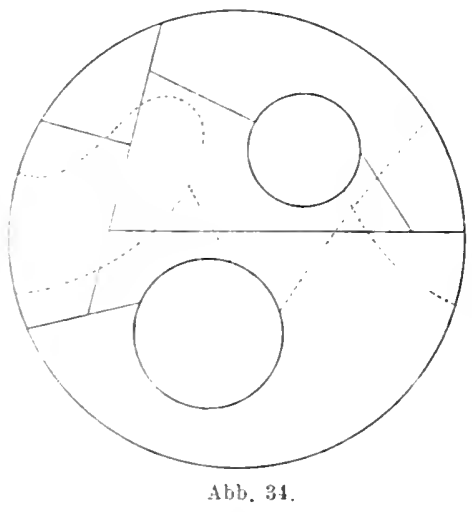
sind. Es ist in der Abb. $q=6, n=5, q^{\prime}=$ 5, $n^{\prime}=4$, also $q-n=q^{\prime}-n^{\prime}=1$. Wir berechnen auf zwei Arten, in wie viele einfach zusammenhängende Flächen $T$ zerfällt, wenn wir die Querschnitte $Q$ und $Q^{\prime}$ zusammen ausführen. Wirnehmen an, daß keiner der Querschnitte $Q$ mit einem der $Q^{\prime}$ ganz oder teilweise zusammenfällt. Sollte ein derartiges Zusammenfallen stattfinden, so verschieben wir die Querschnitte stetig so, daB unsere Annahme erfüllt ist, daß also die Querschnitte nur einzelne Punkte gemeinsam haben. Durch das stetige Verschieben der Querschnitte können die Zahlen $n$ und $n^{\prime}$ nicht geändert werden, wenn nur darauf gearhtret wird, daß die Querschnitte Querschnitte bleiben.

Zunächst werde $T$ durch die $q$ Querschnitte $Q$ in $n$ einfach zusammenhängende Teile $T_{1}, T_{2}, \ldots T_{n}$ zerlegt. Wir führen dann den Querschnitt $Q_{1}^{\prime}$ aus. Er brginne bei $A$ und ende bei $B ;$ s sind $A$ und $B$ Punkte auf dem 


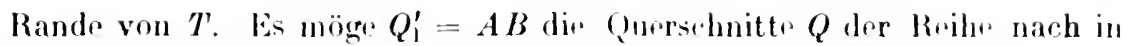
den Punkton $A_{1}, A_{2}, \ldots A_{h}$ schneiden. Jodles der stürke $A A_{1}, A_{1} A_{2}, \ldots$

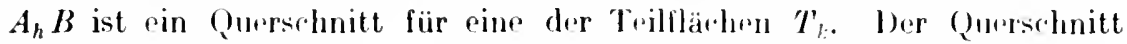
$A A_{1}$ zerlegt die Teilfäche, durch die or gelıt, in zwoi cinfach zusammenhängende Flächen, so daß die Zahl der einfarh zusammonhängenden Tuilflächen durch das Zichen von $A A_{1}$ um 1 auf $n+1$ erhöht wird. Ebenso wird durch das Ziehen von $A_{1} A_{2}$ die Zahl der nummolir vorhandenen Teilflächen um 1 auf $n+2$ vermehrt. Schließen wir so woiter, so sehen wir, daB durch den Querschnitt $Q_{1}^{\prime}=A B$ die Zahl der pinfach zusammenhängenden Teilflächen um $h+1$ auf $n+h+1$ erhöht wird; dabei bodeutet $h$ die Zalıl der Schnittpunkte von $Q_{1}^{\prime}$ mit den Querschnitten $Q_{\mathbf{1}}$, $Q_{2}, \ldots Q_{7}$.

Die Fläche, die aus $T$ durch Ziehen von $Q_{1}^{\prime}$ und der $q$ Querschnitte $Q$ entsteht, sei mit $T^{\prime}$ bezeichnet. $T^{\prime}$ besteht aus $n+h+1$ einfach zusammenhängenden Stücken. Wir schließen genau so wie eben, daß durch Ziehen von $Q_{2}^{\prime}$ die Zahl der $n+h+1$ Teilflächen um $k+1$ auf $n+h+k+2$ erhöht wird, wenn $k$ die Zahl der Schnittpunkte von $Q_{2}^{\prime}$ mit den $q$ Querschnitten $Q$ ist. Dabei ist zu beachten, daß $Q_{1}^{\prime}$ und $Q_{2}^{\prime}$ einander ihrer Definition nach zwar treffen, aber nicht schneiden können. Schließen wir so weiter, so finden wir, daß durch die Querschnitte $Q$ und $Q^{\prime}$ die Fläch $T$ in

$$
n+s+q^{\prime}
$$

einfach zusammenhängende Flächen zerlegt wird, wenn $s$ die Zahl der Schnittpunkte der Querschnitte $Q$ mit den Querschnitten $Q^{\prime}$ ist.

Führen wir erst die Querschnitte $Q^{\prime}$ und dann die Querschnitte $Q$ aus, so finden wir durch dieselben Überlegungen, daß $T$ durch die zusammen ausgeführten Querschnitte $Q$ und $Q^{\prime}$ in

$$
n^{\prime}+s+q
$$

cinfach zusammenhängende Flächen zerfällt, wo $s$ dieselbe Bedeutung hat wie oben. Dic beiden Zahlen (1) und (2) müssen gleich sein und daraus folgt die Behauptung $q-n=q^{\prime}-n^{\prime}$.

Die Zahl $q-n$ ist also unabhängig davon, wie die Fläche $T$ durrh Querschnitte zerlegt wird, wenn nur die Teilflächen einfach zusammenhängend sind. Wir wrorden weiter unten sehen, daß jede aus einem St ürk bestrhende mehrfach zusammenhängende Fläche immer durrh passende Querschnitte in eine einfach zusammenhängende Fläche zerlegt werten kann. Wie das aurh geschehen mag, de Zahl der dazu notwendigen Qurrschnitte ist nach dom eben hewiesenen Satze immer dieselhe. Sie sei mit $r$ bezeichnet; $\rho$ ist dann $r-1=q-n$. Jie Zahl $r$ und damit auch $q-n$ gibt uns ein Maß dafür, wievielfach die Fläche $T$ zusamnrmhängt; denn je mehr Querschnitte notwendig sind, um $T$ in eine einfach zusammen- 
214 Zwolfes lapitel. Die Art des Zusammenhangs einer Riemannschen Fläche.

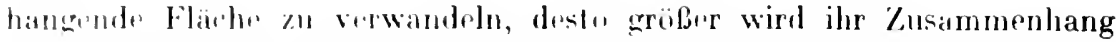
-rin. lamit sieh fïr rime einfach zusammenhängende Flärhe dir Zahl 1 "reght. mhmen wir als Mab für die Stäli" des Zusammenhangs die Zahl " $n$. 2 und nenmon sie die Ordmung des Zusammenhangs der Fläche, und жwar allh lann. wenn dir Flärlw ans mehreren Stïcken besteht. Be-

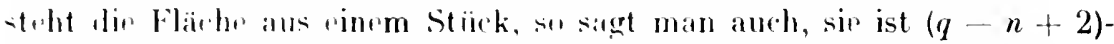
fach zusammenhängend. Da dureh einen (Gerschnitt dio Zahl der vorhambenen nieht miteinandor zusanımenhängonden Teilflächen höchstens um 1 vermehrt werden kamn. so ist für riun aus rinem Stück bestehende mehrfarh zusammenhängende Fläche immer $n-q+1$, also $q-n+2$

1. Alles dirs geilt zunärhst mur für hrandetr Flärhen, da wir auf einer unherandeten Fläıh" kinen Querschnitt ziehen können. Wir müssen eine unherandete Flähe erst punktieren und künen dann mosere Definition inwenden. Wir definieren also:

l)efinition VI. Wird rine mehrfach zusammenhängende berandete Fähly Hurch q Querschnitte in $n$ einfach zusammenhängende Flächen zrrolegt, so heiBt $q-n+2$ die Ordnung des Zusammenhangs der Fäche. Bestrht die Fuärhr aus rinem Stück, so sagt man auch, sie ist $(q-n+2)$-fach zusammenhängend. J)j Zahl $q-n+2$ ist nach Satz II Imabhängig von dor Wahl der Quersohnitte. Sie ist für eine aus einem stück bestehende Fläche immer positiv. Die Ordnung des Zusammenhangs riner unberandeten Fläche soll glrich der (ordnung dor aus ihr durch pentitieren hervorgehendon Fläche sein.

\section{\$4. Beispiele.}

1. Eine lireisfläch" mit zwi löchern. Wie aus Abb. 35 hervorgeht, ist hier $q-n-2=3$, so daß die Fläche droifach zusammonhängend ist.

2. Ior Mantral rines Kroiszylinders. Jie Fläche hat zwei Ränder. dir brjdrn Grundkrejse. Z rochneiden wir den Mantel längs einer Seitenlinie, di" zwoi l'unkto dor Begrenzung verbindot und daher ein Querschnitt ist, so erhalten wir eirn Flärhe, die wir in ein Rechteck auseinanderbiegen können und dir daher rinfarh zusammanhängend ist. Es ist $q=1, n=1$, $\eta-n+2$ 2. dir Flärh» also zwrifarh zusammenhängend.

3. Eine Kingflärhr, wir sie z. B. "ntsteht, wenn ein hreis um eine ihn nirht schureidende Arhso rotirert. Hirso. Fläche hat krinen Rand, so da $B$ wir sie urst punktieren müssen. Jas geschehe durch Anbringung eines kejuen loches. Wir zirhen dann rintn Querschnitt von dem Rande des L.relım lis wirefer dahin zuräck längs rines Meridianes und denken uns dir Flärho läng: des Quersehnittres zerschnitten. Wir können den Ring dann alusfinandrorbirgen, so daß or dir Gestalt eines Zylindermantels annimmt. Jjesen kr̈nnon wir. wir wir in dom vorigen Beispiel gesehen haben, 


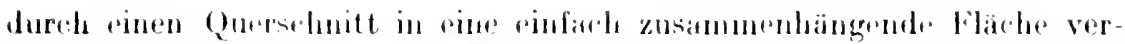
wandoln. Es ist hirr $q-2, n-1, q-n+2 \quad 3$. Fiir dir Analysis situs

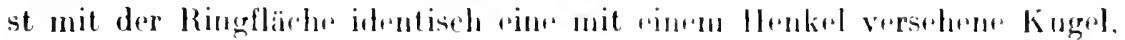

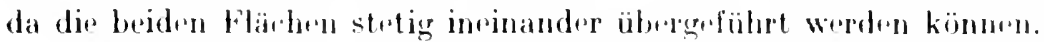

Andere Beispiele sind rine Kugelfäche mit $r$ llonkelu orler dir. Fläche, die mntstohl, wenn man in rine Kugel $r$ löroher hohrt, die sich gegenseitig nitht treffen.

4. Wir brtrachten noch ein Beispiel, das wir spater benutzen. Hor. Fläche bestehr aus $m$ untereinander nicht zusammenhängenden voneinander getrennten cinfach zusammenhängenden Flächen. Es ist $n:=m$, $q=0$, die Ordnung des Zusammenhangs also $q-m+2=-m+2$. Demnach kann die Ordnung des Zusammenhangs anch negativ werden.

\section{\$. T. Zerlegung einer Fläche in eine einfach zusammenhängende.}

Satz IV. Durch einen Querschnitt wird die Ordnung des Zusammenhangs um 1 vermindert.

Die Fläche heiße $T$. Die Fläche, die durch das Ziehen eines Querschnittes $Q$ entsteht, heiße $T^{\prime}$. Die Ordnung des Zusammenhangs der Flächen sei mit $O$ und $O^{\prime}$ bezeichnet. Es werde $T$ durch $q$ Querschnitte $Q_{1}, Q_{2}, \ldots Q_{q}$ in $n$ einfach zusammenhängende Flächen zerlegt. so daß

$$
O=q-n+2 \text {. }
$$

Zeichnen wir noch den Querschnitt $Q$, so wird $T$, wie wir beim Beweise von Satz III gesetien haben, in $n+h+1$ rinfach zusammenhängende Flächen zerlegt, wenn $h$ die Zahl der Schnittpunkte von $Q$ mit den $q$ Querschnitten $Q_{k}$ bedeutet. Es wird also auch die Flächr $T^{\prime}$ durch die $q$ Linien $Q_{k}$ in $n+h+1$ einfach zusammenhängende Flächen zerlegt. Aber die Linien $Q_{k}$, die für $T$ Querschnitte sind, brauchen es nicht zu sein für $T^{\prime}$, da zur Begrenzung von $T^{\prime}$ auch die beiden Ränder vou $Q$ gehören. Sehneidet $Q_{k}$ den Querschnitt $Q$ in $h_{k}$ Punkten, so zerfällt $Q_{k}$ in $h_{k}+1$ Stücke und jodes dieser Stücke ist ein Querschnitt von $T^{\prime}$. Die Linien $Q_{1}, Q_{2}, \ldots Q_{q}$ bilden daher $\left(h_{1}+1\right)+\left(h_{2}+1\right)+\cdots+\left(h_{q}+1\right)=h+q$ Querschnitte von $T^{\prime}$. Es zerfällt also $T$ durch $h+q$ Querschnitte in $n+h+1$ einfach zusammenhängende Flächen, so daB $O^{\prime}=(h+q)-(n+h+1)+2$ $=q-n+1$. Vergleichen wir dies mit (3), so folgt die Bohauptung $O^{\prime}=O-1$.

Satz V. Dureh einen RiB wird die Ordnung des Zusammenhangs nicht geändert.

Die Fläche heiße wieder $T$ und die aus ihr durch den RiB $R$ entstehend Fläche $T^{\prime}$. Dir Ordnung des Zusammenhangs der beiden Flächen sei bezeichnet mit $O$ und $O^{\prime}$. Wir fïhren cinen Schnitt $Q$ von dem im Innern 
ron $T$ liegenden Ausgangspunkt von $R$ nach rinem Punkt der Grenze von $T$, der woder $R$ noch die Grenze von $T$ schneidet. Die dadurch aus $T^{\prime}$ entstchende Fläche sei mit $T^{\prime \prime}$ bezrichnet. Die Ordnung des Zusammenhangs ven $T^{\prime \prime}$ sui $O^{\prime \prime}$. Es ist $Q$ ein Quersehnitt von $T^{\prime}$, so daß nach Satz IV

$$
O^{\prime \prime} O^{\prime}-1 \text {. }
$$

Ferner ist der aus $R$ und $Q$ bestehende Schnitt ein Querschnitt von $T$, so da $B$ nach Satz IV $O^{\prime \prime}=O-1$. Durch Vergleichen mit (4) folgt $O^{\prime}=O$, also die Behauptung.

Satz VI. Durch ein Loch wird die Ordnung des Zusammenhangs einer berandeten Fläche um 1 erhöht.

Die Fläche heiße wieder $T$ und die aus ihr durch Anbringung eines Loches $L$ entstchende Fläche $T^{\prime}$, die Ordnungen des Zusammenhangs der Flächen $O$ und $O^{\prime}$. Wir verbinden den einen Endpunkt von $L$ mit einem Punkte des Randes von $T$ durch einen Schnitt $Q$, der weder $L$ noch den Rand von $T$ schneidet. Die dadurch aus $T^{\prime}$ entstehende Fläche heiße $T^{\prime \prime}$, die Ordnung ihres Zusammenhanges $O^{\prime \prime}$. Es ist $Q$ ein Querschnitt von $T^{\prime}$, so daß nach IV.

$$
O^{\prime \prime}=O^{\prime}-1 .
$$

Ferner ist der aus $L$ und $Q$ bestehende Schnitt ein $\operatorname{RiB}$ von $T$, so da $B$ nach V. $O^{\prime \prime}=O$. Durch Vergleichen mit (5) finden wir die Behauptung $O^{\prime}=O+1$.

SatzVII. Jede einfach zusammenhängende Fläche wird durch eine geschlossene sich selbst und die Grenze nicht treffende Linie in zwei nichtmiteinanderzusammenhängende Teile zerlegt, vondenender eine einfach, der andere zweifach zusammenhängend ist. Wir nennenden einfach zusammenhängenden Teil das Innere, den anderen das ÄuBere der geschlossenen Linie.

Die Fläche heibe wieder $T$, die aus ihr durch Zerschneiden längs der geschlossenen Jinie $R$ hervorgehende Fläche $T^{\prime}$; die Ordnungen ihrer Zusammenhänge seien $O$ und $O^{\prime}$. Nach Voraussetzung ist $O=1$. Der Schnitt $R$ ist ein Riß von $T$, so da $B$ nach $\mathrm{V} . O^{\prime}=O=1$ ist. Wir führen den Beweis indirekt, nehmen also an, es zerfalle $T$ nicht in zwei getrennte Stürkr. Dann können wir von der einen Seite von $R$ nach der anderen gelangen, ohne $T^{\prime}$ zu verlassen odir die Grenze von $T$ zu überschreiten. Es sei $Q$ ein Weg, der von rinem Punkte $P$ von $R$ wieder nach $P$ führt, aber auf die andere Seite von $R$, und zwar ohne $R$ oder die Grenze von $T$ zu treffen. $Q$ zerlegt $T$ nicht in zwei getrennte Teile, da wir von der einen Srite von $Q$ auf die andere kommen können, ohne $Q$ oder die Grenze von $T$ zu überschreiten; wir brauchen zu diesem Zwecke nur der Linie $R$ entlang zı wandern. Die aus $T^{\prime \prime}$ durch Zerschneiden längs $Q$ entstehende 


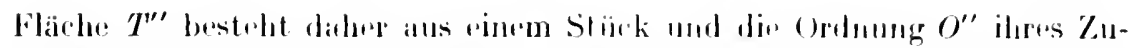
sammenhanges ist gröber als Null. Es ist aber Q $Q$ in Quershente von $T^{\prime \prime}$ und daher nach $1 \mathrm{~V}$. $O^{\prime \prime} O^{\prime}-1=0$, wats d.m rben gefundenen Ergebnis $O^{\prime \prime}>0$ widersprieht. Es $m u B$ daher $T$ durh $R$ in zwoi getrennte Teile zerfallen.

Die beiden Trile mögren $T_{1}$ und $T_{2}$ heißen, die Crdnungen ihrer Zu-

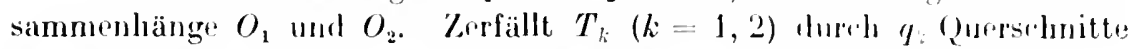
in $n_{:}$einfach zusammenhängende Flächen, so zerfällt $T^{\prime \prime}$ durch $q_{1}+q_{2}$ Querschnitte in $n_{1}+n_{2}$ einfach zusammenhängende Flächn'n, sn diab nach Definition

$O_{1}=q_{1}-n_{1}+2, \quad O_{2}=q_{2}-n_{2}+2, \quad O^{\prime}=\left(q_{1}+q_{2}\right)-\left(n_{1}+n_{2}\right)+2$, woraus folgt

$$
O^{\prime}=O_{1}+O_{2}-2 .
$$

Andererseits ist aber, wie wir schon bemerkt haben, $O^{\prime}=O=1$. Wir schließen daher aus (6)

$$
O_{1}+O_{2}=3 \text {. }
$$

Da die Flächen $T_{1}$ und $T_{2}$ jede aus einem Stück bestehen, so sind $O_{1}$ und $\mathrm{O}_{2}$ beide größer als Null, so daß wir aus (7) folgern, daß, wie behauptet, die eine der beiden Zahlen $O_{1}, O_{2}$ gleich 1 und die andere gleich 2 ist.

Satz VIII. Fine aus einem Stück bestehende mehrfach zusammenhängende Fläche läßt sich durch eine endliche Zahl von Querschnitten in eine einfach zusammenhängende Fläche zerlegen.

Da die Fläche nicht einfach zusammenhängend ist, so können wir in ihr einen Querschnitt ziehen, der sie nicht zerlegt. Dadurch wird nach IV. die Ordnung ihres Zusammenhanges um 1 erniedrigt. Ist die so erhiltene Fläche noch nicht einfach zusammenhängend, so können wir auch in ihr einen Querschnitt zichen, der sie nicht zerlegt, wodurch wieder die Ordnung des Zusammenhangs um 1 verringert wird. Ist die so entstehende Fläche noch nicht einfach zusammenhängend, so können wir in derselben Weise fortfahren. Da die Ordnung des Zusammenhangs immer weiter, und zwar jedesmal um 1 erniedrigt wird, und die Ordnung eine positive ganze Zahl ist, so muß sehließlich nach einer endlichen Zahl von Querschnitten eine einfach zusammenhängende Fläche entstehen.

\section{\$ 6. Ordnung des Zusammenhangs einer Riemannschen Flïche.}

Satz IX. Die Ordnung des Zusammenhangs einer m-blättrigen Riemannschen Fläche ist

$$
w-2 m+3=2 p+1,
$$

wenn $w$ die Verzweigungszahl der Fläche und $p$ das feschlecht des zugehörigen algebraischen Körpers ist. 
Zum Bowoise zorlegen wir die Riemanusche Fläche, die mit $T$ bezeichunt si, in die $m$ einfach zusammenhängendon Ebenen $E_{1}, E_{2}, \ldots E_{m}$, aus druen wir sir zusammengesetzt halon. Die Ebenen $E_{k}$ sind einander kongruent und sind längs $h$ Linion $l_{1}, l_{2}, \ldots l_{h}$ zerschnitteu, die von einem

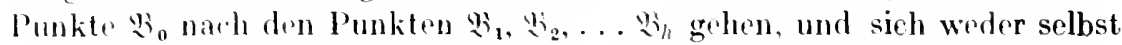
norh grensitig treffon. Die Fläche $T$ rntsteht aus diesen $m$ Ebenen, indem man dir händer der Sihnith l passend miteinander verbindet. bie llärhe $T$ hat keinen Rand. Zersehneiden wir sie längs der Linien $l$, $\therefore$ erhalten wir wieder die $m$ einfach zusammenhängenden Ebenen $\boldsymbol{E}$. Wir wothen den Punkten $\aleph_{k}$ und den Schnitten $l_{k}$ einen Index anfügen, der angeben soll, in weleher Ebene der Punkt oder der Schnitt liegt. Jeder dor Punkte $S_{k}$ und Schnitte $l_{k}$ kommt ja $m$-mal vor, einmal in jeder Ebene. Dir Pinkte $\&_{01}, \$_{02}, \ldots \$_{0 m}$ liegen in $T$ getrennt, während die anderen Punkte $\mathbb{S}_{\text {a }}$ sich zum Teil zu Verzweigungspunkten vereinigen. Von jedem

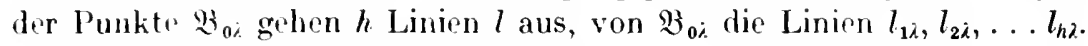

Wir bezeichen die gesuchte Ordnung des Zusammenhangs von $T$ mit $O$. Zunächst zerschneiden wir $T$ von den $m$ Punkten $\mathfrak{B}_{0 i}$ aus längs eines kleinen Stückes der Linien $l_{1 \lambda}$, so da $B$ in $T m$ Löcher entstehen. Die dadurch aus $T$ hervorgehende Fläehe heiße $T^{\prime}$, die Ordnung ihres Zusammenhangs $O^{\prime}$. Von den $m$ Löehern, die $T^{\prime}$ hat, dient eins dazu, $T$ in eine berandete Fläche zu verwandeln. Die durch dies Loch aus $T$ entstehende Fläche hat nach Definition dieselbe Ordnung des Zusammenhangs wie $T$. Die anderen $m-1$ Löcher sind aber in eine berandete Fläche geschnitten, so daß nach Satz VI durch jedes von ihnen die Ordnung um 1 erhöht wird. Daher ist $O^{\prime}=O+m-1$.

Es sei $p$ ein $\alpha$-facher Verzweigungspunkt von $T$. In ihm seien etwa die Punkte $\mathfrak{B}_{11}, \mathfrak{S}_{12}, \ldots \mathfrak{B}_{1 \alpha}$ vereinigt. Von $\mathfrak{p}$ aus gehen nach den Punkten $\mathfrak{B}_{01}, \mathfrak{B}_{02}, \ldots \mathfrak{B}_{0, \mathrm{~d}}$ dif Linien $l_{11}, l_{12}, \ldots l_{1 \alpha}$. Zerschneiden wir $T$ längs dieser Linien, so ist der erste Schnitt ein Riß, da er von dem im Innern von $T^{\prime}$ liegenden Punkt $p$ nach einem Punkte der Grenze von $T^{\prime}$ geht, nämlich nach einem der kleinen Löcher von $T^{\prime}$. Nach Satz $\mathrm{V}$ wird dadurch die Ordnung von $T^{\prime}$ nicht geändert. Der zuerst ausgeführte Schnitt ist ein Teil der Grenze der durch ihn aus $T^{\prime}$ entstehenden Fläche, so daß nunmehr $p$ ein Punkt der Grenze ist. Die anderen $\alpha-1$ Schnitte sind daher Quersehnitte, so daß nach Satz IV durch jeden von ihnen die Ordnung um 1 erniedrigt wird. Jaher wird durch das Zerschneiden von $T^{\prime}$ längs der von einem $\alpha$-fachen Verzweigungspunkt ausgehenden Linien $l$ die Ordnung des Zusammenhangs um $\alpha-1$ erniedrigt. Dies gilt auch, wenn $\alpha=1$, wenn also $p$ ein gewöhnlicher Punkt der Fläche ist. Da für die anderen Verzweigungspunkte dasselbe gilt, so wird durch Zerschneiden von $T^{\prime}$ längs aller Linien $l$ die Ordnung $O^{\prime}$ um $\Sigma(\alpha-1)=w$ erniedrigt. 


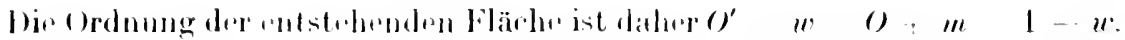

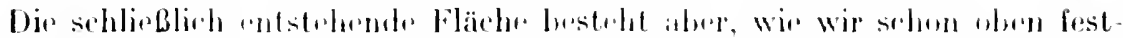
gestellt haben, ans den $m$ einfach zusammenhängenden Ehonen $E$, die

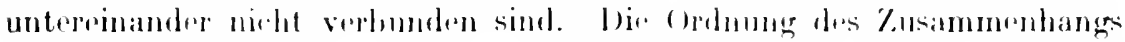

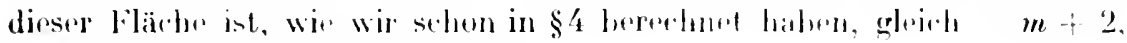

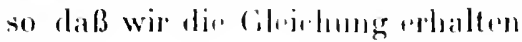

$$
0+m-1-m-m+2 .
$$

ans der sids dir liehauptung ergibt.

\section{$\$ 7$. Kanonische Zerschneidung der Riemannschen Fläche.}

Eine Rirmannschr Fläelıe ist also im Falle $p=0$ "infach zusammenhängend, in allen anderen Fällen aber melırfarh, und zwar ist dir Ordnung des Zusammenhangs immer ungrade. Zur Untersuchung der Int.grale von Differentialen des zu riner Rirmanusehen Fläche gehörenden algebraisehen Körper's ist es notwendig, die Flärhe durrh geeignete Quer-

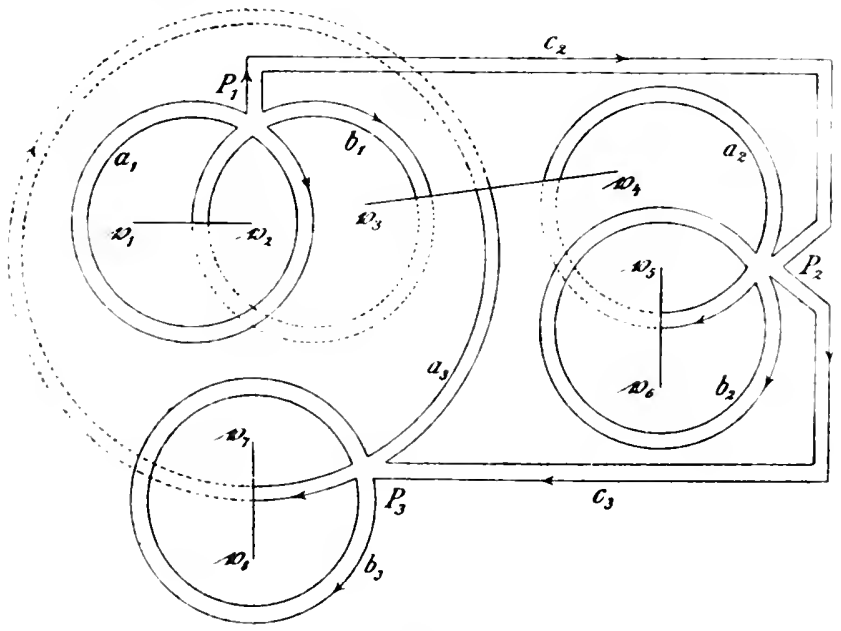

$A b \mid, 35$

schnitte in eine einfach zusammenhängende zu verwandeln. Die Zahl der dazu notwendigen Querschnitte ist $2 p$, also immer eine grade Zahl. Es ist am besten für unseren Zweek, die Fläche in folgender, dor sogenannten kanonisehen, Art zu zerschneiden. Nan vergleiche die $\mathrm{Abb} .35$, in der die beiden Ränder der Sclınitte der Deutlichkeit wegen getrennt gezeichnet sind.

Die Fläche heiße wieder $T$, die Ordnung ihres Zusammenhangs sei $O=2 p+1$, und es sei $p>0$. Wir punktieren $T$ zunächst, und zwar 
2.24 Zwolftes Kapitel. Die Art des Zusammenlangs einer Riemannschen Fläche.

beri tin'm gewönlichen l'unkte $P_{1}$ der Fläehe. Wir zersehneiden $T$ dann lange oins Gursehnittes $a_{1}$. der $T$ nicht in zwei getrennte Teile zerlegt. lin entsthende Fäehe heiße $T_{1}$. Wer Qursehnitt beginnt bei $P_{1}$ und wir kënn"n ihn auch in $P_{1}$ enden lassen. Wenn er nämlich nicht in $P_{1}$ antet, sundern in einem seiner Punkte, su liönnen wir den Endpunkt auf

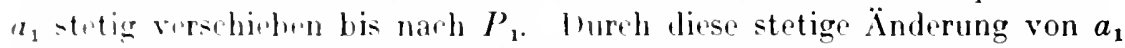
kinn $T_{1}$ nicht zerfallen. Der Querschnitt $a_{1}$ bildet zusammen mit dem bleinen bri $A_{1}$ angelirachten Loch eine geschlossene Kurve und wir wollen im folgenden unter $a_{1}$ lisse gesehlossene kurve verstehen. Da $T$ durch $a_{1}$ nielit in zwei Teile zerlegt wird, so gilht es einen Weg in $T$, der von irgendrinem Punkte zu irgendeinem anderen führt, ohne $a_{1}$ zu schneiden, also auch rinen Weg $b_{1}$, der von $P_{1}$ nach $P_{1}$ führt. und dabei von der einen Seite ron $a_{1}$ auf die andere. Wie zerschneiden $T_{1}$ längs $b_{1}$ und nennen die entstehende Fläche $T_{2}$. Der Querschnitt $b_{1}$ zerstückt $T_{1}$ nicht, da man von der inen Seite von $b_{1}$ auf die andere gelangen kann, ohne $a_{1}$ oder $b_{1}$ zu überschreiten. Man braucht zu diesem Zwecke nur den Querschnitt $a_{1}$ entlang zu gehen. Wir setzen für die Querschnitte $a_{1}$ und $b_{1}$ eine positive Richtung fest und unterscheiden zwischen ihrem rechten und linken Cfer. Wir bezeichnen die rechten Ufer mit $a_{10}, b_{10}$ und die linken mit $a_{1 ;} . b_{1}$. Die Ufer der Schnitte $a_{1}, b_{1}$ schließen sich zu einer einzigen geschlossenen kurve zusammen, und zwar, wenn man die Richtung von $a_{1}$ und $b_{1}$ so wählt wie in der Abb. 35, in der Reihenfolge $b_{1 \dot{1}}, a_{10}, b_{10}, a_{1 \dot{\alpha}}$. Es hat demnach $T_{2}$ eine einzige Begrenzungslinie. Der Sehnitt $a_{1}$ ist ein Querschnitt von $T$ und der Schnitt $b_{1}$ einer von $T_{1}$, so da $B$ die Ordnung des Zusammenhangs von $T_{2}$ um 2 kleiner ist als die von $T$, also gleich $2 p-1$. Ist daher $p=1$, so ist $T_{2}$ einfach zusammenhängend. Beispiele liof rn auB r Abb. 35 auch die Abbildungen_6 und 9 , die Riemannsche Flächen rom Geschlechte 1 darstellen, auf denen zwei geschlossene Kurven $a$ und b) arzichnot sind, die sich nur in einem Punkte $P$ selneiden.

Ist $p>1$, so ist $T_{2}$ noch mehrfach zusammenhängend. Es gibt also winen Qureshitt $Q$, der $T_{2}$ nicht in zwei getrennte Teile zerlegt. Da $T_{2}$ nur eine berenzung-linie hat und auf ihr der Anfangspunkt von $Q$ liegt, a) könn'n wir dureh tetign Änderung von $Q$ creichen, dab der Anfangspunkt mit $P_{1}$ zusammonfällt. Wenn der Endpunkt von $Q$ nicht schon auf $Q$ liegt, so können wir ihn durch stritige Verschiebung erst nach $P_{1}$ und dann narh einem Punktr von $Q$ bringen, so daß $Q$ ein Rüekkehrsehnitt wirl. Der Endpuntit won $Q$ hribr $P_{2}$. Wir bezeichnen das von $P_{1}$ bis $P_{2}$ reirhend" Stürk von $Q$ mit $c_{2}$ und das von $P_{2}$ bis wieder nach $P_{2}$ gehende Stürk mit $a_{2}$. I) Fir Färche, die aus $T_{2}$ durch Zerschneiden längs $c_{2}$ und $a_{2}$ entstrit, hriße $T_{3}$. Da $T_{3}$ aus einem Stucke besteht, so gibt es in $T_{3}$ immer eiren Wigg, der von einem Punkte in $T_{3}$ nach irgendeinem anderen Punkte von $T_{3}$ fürt, also auch einen Weg $b_{2}$, der von $P_{2}$ wieder nach $P_{2}$ geht, 


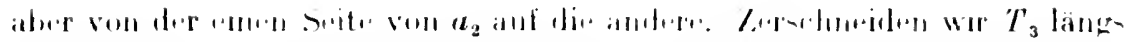

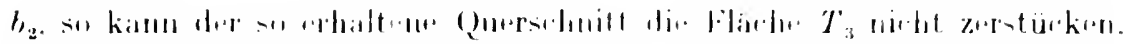

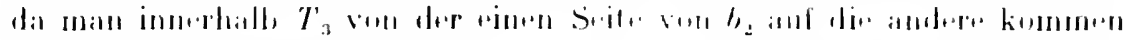

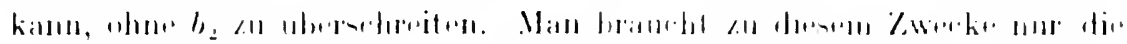

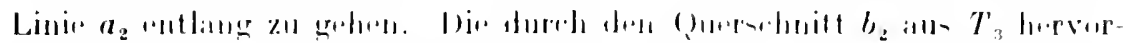

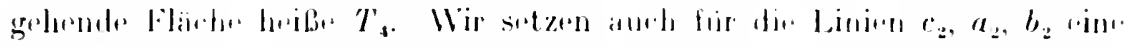

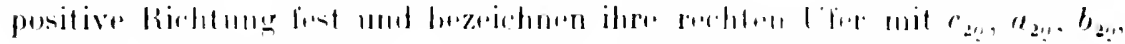

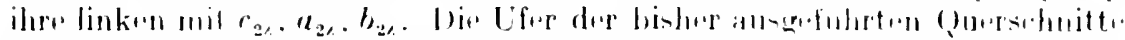

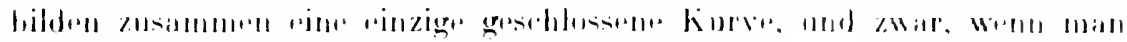

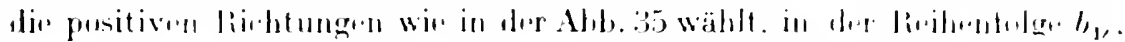

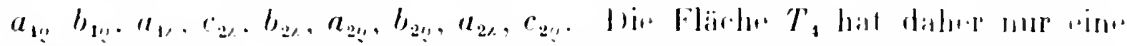

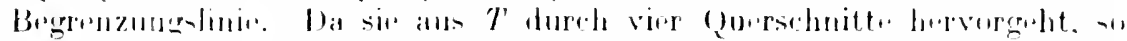

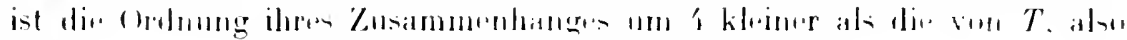

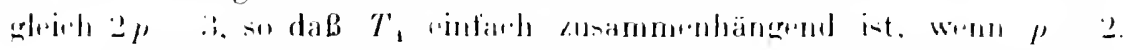

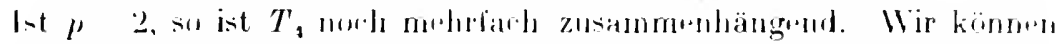

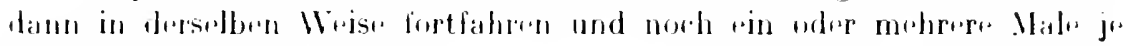

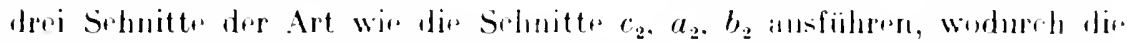
Grdnumg den Zusammenhangs jedemal um 2 erniedrigt wird, so dab wir

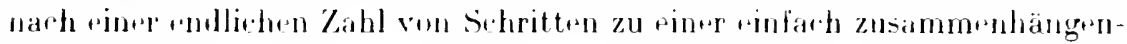

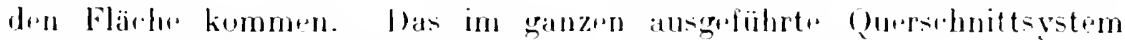
können wir folgendermaBen beschreiben. Auf der Riumannsehen Fläche $T$

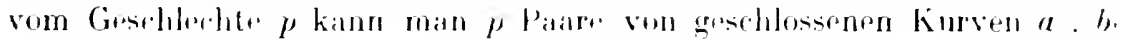

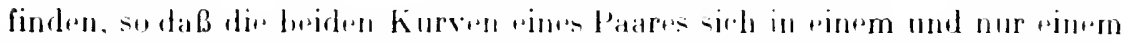

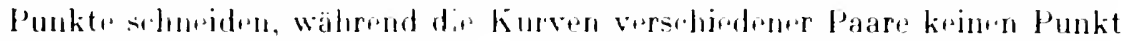

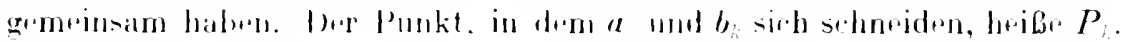

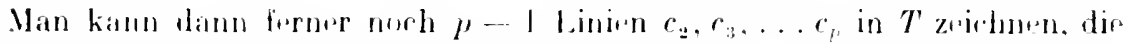

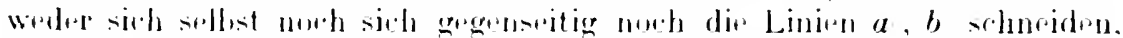

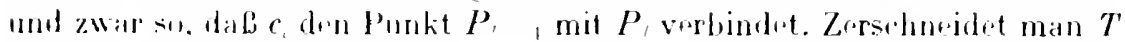

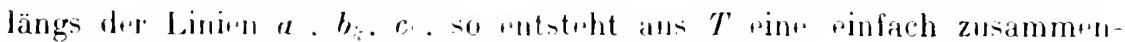
hängende Flärhe, dir mit $T^{\prime}$ brzeirhnet werden soll. Diese Fläche hat

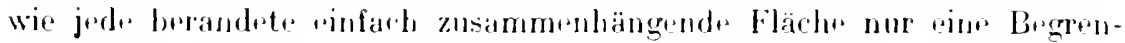
zung:linie. Wählen wir dị positiw Rirhtung der Srhnittr $a, b, r$ in

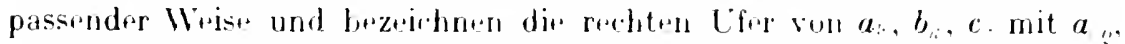

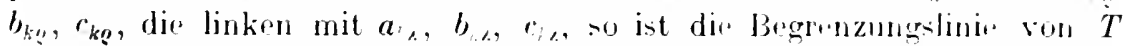
aus den Ufern der ausgnführten Sobnitte in folgender Reilunfolgezusammen-

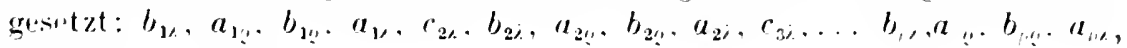
$r_{1, \ldots,}, r_{n}, 1, \ldots, r_{2 !:}$. 


$$
\text { l) roizeluter Kapitrl. }
$$

\section{Die Integrale.}

\section{$\$ 1$. Definition der Integrale.}

Jedes l)ifferential des Körpers läBt sich in der Form sehreiben $R d x$, wo $R$ eine rationale Funktion von $x$. $y$ ist. Mir wir in $\$ 6$, Kap. VI gesehen haben, gehört zu jedem Differential ein llivisor der Differentialklasse, der angiht, wie das Differential Null und mendlich wird. Dieser Divisor heißt der loifforentialtriler des Differntials. Umgrkehrt gehört zu jedem bivisor der Difforentialklasse ein bis auf rinrin konstanten Faktor be-timmtes Differential des Körpers.

Zur Definition und zur Untersuchung dor Eigenschaften der Integrale von bifferentialen des körpers benutzen wir die über der $x$-Ebene ausgehreitete Riemammsche Fläche, die wir mit $T$ bezeichnen wollen. Es seien $p_{0}$ und $p$ zwei Punkte von $T$ und $s$ ein Weg, der beide verbindet. Wir setzen voraus, daß weder $p_{0}$ und p noch rin Punkt von $s$ rin Verzweigungspunkt oder eine Unendlichkeitsstelle lus Differentials $R d x$ ist. Wir unterscheiden versehiedene Fälle.

1. Es liege $p$ und der ganze Weg $s$ in einer Umgebung $U$ von $\mathfrak{p}_{0}$, die keine Unendlichkeitsstelle von $R d x$ enthält. Hat $x$ in $\mathfrak{p}_{0}$ den Wert $a$. so ist innerhalb $U$

$$
R=\mathfrak{P}(x-a) \text {. }
$$

wo $\mathfrak{P}(x-a)$ cine in $U$ überall konvergirrende gewöhnliche Potenzreihe von $x-a$ ist. Wir können $\mathfrak{F} d x$ daher bestimmen, indem wir die Reihe gliedweise integrieren. Wir erhalten so eine gewöhnliche Potenzreihe von $x$-a, die auch in $U$ konvergiert; wir bezeichnen sie mit $P(x)$. Um sie vollkommen zu bestimmen, setzen wir fest, daß die Integrationskonstante so bestimmt werden soll, daß $P(x)$ in $p_{0}$ den Wert Null annimmt, daß alsor $P(a)=0$. Es ist hann

$$
\int_{i} \mathfrak{B}(x-a) d x \quad P(x) .
$$

Indem wir muter $x$ den Wert verstehen. Ien $x$ in $p$ hat, definieren wil

$$
\int_{\mathfrak{j}_{0}}^{\mathfrak{p}} R d x=\int_{h} \mathfrak{B}(x-a) d x=P(x)
$$

und momen das so definierte Introgral $j R d x$ das von $p_{0}$ nach $\mathfrak{p}$ über $s$ erstrefktי Intregral. Da bei der Definition von flem Wege $s$ kein Gebrauch gemacht ist, so ist der Wert des Integrals in dem betrachteten Falle 1. von ler Wahl des Weges Inabhängig. 


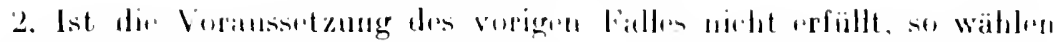
wir auf $s$ rill. liejhe vou $r$ l’unkten $p_{1}, p_{2}, \ldots p_{r}$, dab für jerles Stürk $p_{0} p_{1}, p_{1} p_{2}, \ldots p_{r} p$ des Integrationsweges $s$ dir Voranssetzungen des

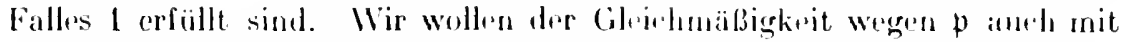

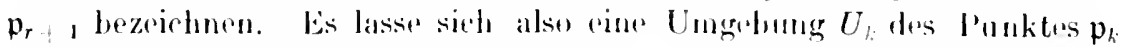
so angeben, dafs $\mathfrak{p}_{k}$, und das zwischen $\mathfrak{p}_{k}$ und $\mathfrak{p}$, , liegrande Stürk von $s$ ganz in $U_{k}$ lingt. Nimmt $x$ in $\boldsymbol{p}_{k}$ den Wert a itl, st ist in $U$.

$$
R-\mathfrak{H}_{k}\left(x-a_{k}\right) \text {. }
$$

wo $\mathscr{F}_{\%}\left(x-a_{h}\right)$ eine gewöhnliche Potenzreihe von $x-a$ ist. Das Integral fil $d x$ können wir durch gliedweise Integration berechnen. Die dadurch erhaltene Reihe sei mit $P_{k}(x)$ bezeichnet; sie sei vollkommen bestimmt durch die Bedingung, da $B$ sie für $k>0$ in $\mathfrak{p}_{k}$ denselben Wert haben soll wie $P_{k}{ }_{1}(x)$ und da $\beta$ im besonderen $P_{0}(x)$ in $p_{0}$ verschwinden soll. Es soll also sein

$$
P_{\mathbf{0}}\left(a_{0}\right)=0 . \quad P_{k}\left(a_{k}\right)=P_{h} \quad{ }_{1}\left(a_{k}\right) . \quad(k>0) .
$$

Wir definieren dann

$$
\begin{aligned}
& \int_{\mathfrak{b}_{0}}^{p} R d x=\int_{\mathfrak{b}_{0}}^{\mathfrak{p}_{1}} R d x+\int_{\mathfrak{p}_{1}}^{p_{2}} R d x+\cdots+\int_{\mathfrak{b}_{r}}^{\mathfrak{p}_{r}} R d x=\int_{a_{0}}^{a_{2}} \mathfrak{B}_{0} d x+\int_{a_{1}}^{a_{2}} \mathfrak{P}_{1} d x+\cdots+\int_{a_{r}}^{a_{r}} \mathfrak{P}_{r} d x \\
& =\left[P_{0}\left(a_{1}\right)-P_{0}\left(a_{0}\right)\right]+\left[P_{1}\left(a_{2}\right)-P_{1}\left(a_{1}\right)\right]+\cdots+\left[P_{r}\left(a_{r+1}\right)-P_{r}\left(a_{r}\right)\right] .
\end{aligned}
$$

Unter Benutzung der Gleichungen (1) wird, wenn wir wieder unter $x$ den Wert von $x$ in $\mathfrak{p}$ verstehen, wenn wir also $a_{r+1}=x$ setzen,

$$
\int_{\mathbb{p}_{n}}^{p} R d x=P_{r}(x) \text {. }
$$

In dem $U_{h}$ und $U_{k-1}$ gemeinsamen Getiet, das mit $G_{k}$ bezeichnet spi, ist

$$
\frac{d P_{i}}{d x} \quad-\mathfrak{H}_{k}=\mathfrak{q}_{-1}=\frac{d P_{k-1}}{d x} .
$$

da $\mathfrak{L}_{k}$ und $\mathbb{H}_{\%}$, beide in $G_{k}$ gleich $R$ sind und $R$ in der Riemannschen Fläche $T$ rindentig ist. Daher können sich $P_{k}(x)$ und $P_{k-1}(x)$ in $G$ nur dureh einen konstanten Summanden unterscheideu. Wa sie aber nach (1) in $\mathfrak{p}_{k}$ übereinstimmen, so sind sie in dem ihnen gemeinsamen Konvergenzgebiet $G_{k}$ miteinander identiseh. Wit anderen Worten, es ist $P_{k}(x)$ die analytische Fortsetzung von $P_{k},(x)$ und damit $P_{r}(x)$ die analytische Fortsetzung von $P_{0}(x)$ längs des Weges $s$. Daraus schließen wir, daß der Wert des von $\mathfrak{p}_{0}$ nach $\mathfrak{p}$ längs $s$ erstreckten Integrals von $R d x$ wohl von $s$ abhängen kann, aber nicht von der Wahl der IIilfspunkte $\mathfrak{p}_{1}, \mathfrak{p}_{2}, \ldots \mathfrak{p}_{\text {r }}$. 


\section{\$‥ Verhalten der Integrale in der Umgebung einer Stelle.}

Wäbrend wir dir untere Grenze po bisthalten wollen, nehmen wir die ubere p als beweglich an, betrahten also das Integral als liunktion der

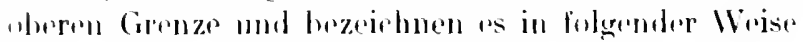

$$
\int_{i=0}^{\prime \prime} R d x \quad J(x, y) \quad J(p) \text {. }
$$

Wir wollen zunärhst shen, wir sirh t in der Ungebung irgendeines Punkles der Riomannsehen Fläh $T$ verhält. J)azu müssen wir die Definition von $I$, in wenig orwertern. Es sei q der Punkt von $T$, in desen Umgebung wir das Verhalten von J untersurhen wollen. Es sei $U$ erine solehe Umgehung von $q$, in der, abgesohen vielleicht von q selhst, kine Unendlichkeitsstelle ron $R d x$ liegt. Wir nehmen an, daß die obere Grenze von $J$, nämlich der Punkt p. so nabe bei q liegt, daß er sich innerhalb von $U$ befindet. Es sei ferner $p_{1}$ ein in $U$ liegender Punkt des Integrationswerges $s$. Wir lassen das Stiick $\mathfrak{p}_{0} \mathfrak{p}_{1}$ von $s$ unverändert, während wir $\mathfrak{p}$ und das Stück $\mathfrak{p}_{1} \mathfrak{p}$ von $s$ als veränderlich annehmen, aber so, daß es immer in $U$ bleibt. Ist $t$ eine Funktion des Körpers, dir in q von der ersten Ordnung Null wird, so ist in $I$

$$
R d x-\left[c_{j} t^{-1}+c_{j}, t^{-i} \quad 1+\cdots+c_{1} t^{1}+\mathscr{H}(t)\right] d t=Q(t) d t,
$$

wo 忟 $(t)$ eine grähnliche Potenzreihre von $t$ ist. Wir haben dabei angenommen. daß $R d x$ in a von der ()rdnung $\lambda$ unendlich wird. Wir setzen

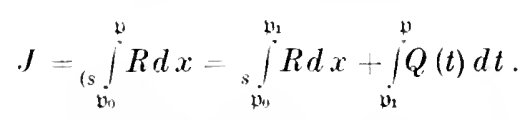

Das erste Intrgral rechts in dieser Gleichung ist eine Konstante, das zweite können wir durch gliedweise Integration berechnen. Wir erhalten dadureh

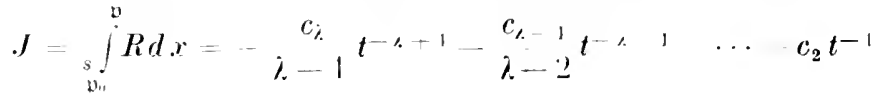

$$
\begin{aligned}
& +c_{1} \lg t+P(t) \text {. }
\end{aligned}
$$

wo $P(t)$ eine gewöhnliche Potenzreilw. von $t$ ist, dir in $U$ konvergiert. Wir rsehen daraus, daß sich $J$ in der Umgebung einer Stelle der Riemannshen Fläche ähnlich verhält wie die Funktionen des Körpers. Im allgemeinen läßt sich $J$ in der Umgebung einer Stelle $q$ in eine nach steigenden ganzen Poterizen von $t$ fortschreitende Potenzreihe entwickeln, wenn $t$ eine Funktion des Körpers ist, die in q von der ersten Ordnung Null wird. Nur wenn $\mathfrak{q}$ eine der in endlicher Zahl vorhandenen Unendlichkeitsstellen von $R d x$ ist, kann diese Entwicklung eine endliche Zahl von nega- 


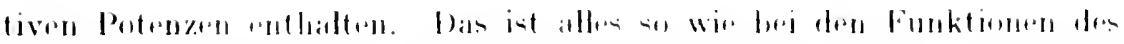

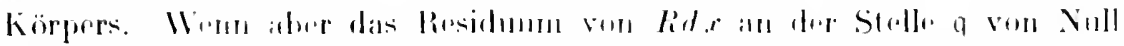

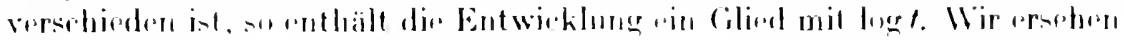

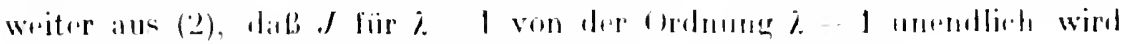

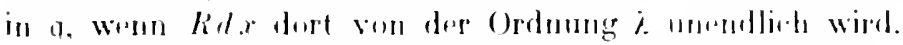

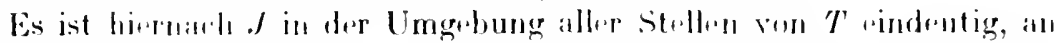

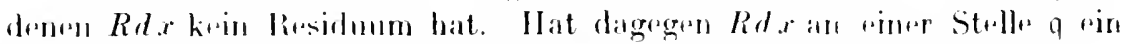

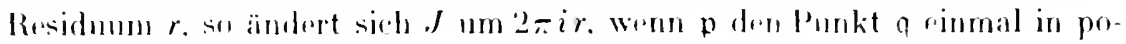
sitivem sinne umkroist.

Es halw Rdx in q koin Residnum. sodals $J(p)$ in $l$ rindentig ist. Es

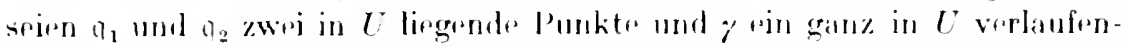

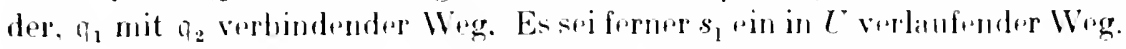
dor $p_{1}$ init $\theta_{1}$ verhindet. Dann ist narb der Dofinition von $I(p)$.

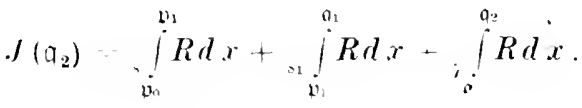

$$
\begin{aligned}
& f\left(a_{1}\right)=\int_{j=1}^{p_{1}} R d x+\sum_{j 1}^{a_{1}} R d x .
\end{aligned}
$$

so $\mathrm{daB}$

$$
\int_{\dot{o}_{2}}^{q_{2}} R d e=J\left(a_{2}\right)-J\left(q_{1}\right) .
$$

J) $J(p)$ eindeutig in $U$ ist, su folgt hieraus. wenn wir im besonderen $q_{1}=q_{2}$ annehmen. also miter $\gamma^{\prime}$ rinen in $C$ verlaufenden geschlossenen WVg verstehen.

$$
\text { i } R d x=-0
$$

\section{\$ 3. Verhalten der Integrale auf der Riemannschen Fläche.}

Wir brtrarhten jetzt of in der ganzen Riemanuschen Fläche $T$. Wir verwandeln zunärhst $T$ durrh kanonische Zerschneidung. wir es am Endr des vorigen Kapitels anseinandergesetzt ist, in eine rinfach zusammenhängende Fläch» $T^{\prime}$. Es mögo $R d x$ an den Strellen $r_{1}, r_{2}, \ldots r_{\mathfrak{b}}$ von Null verschiedene Residurn haben. Wir ziehen dann von diesen Punkten aus Linien $d_{1}, d_{2} \ldots d_{1}$ narh $P_{1}$, dir innerhalh $T^{\prime}$ verlaufen und sich weder solbst, noch untereinander schneiden. Wir zerschneiden $T^{\prime}$ längs dieser Linien und rernen dir su entstrhende Fläche $T$ ". Da die Sehnitte de nach der im vorigen Kapitel, \$ I angewandten bezeichnung lisse sind. so wird durch sio die (Ordnung des Zusammenhangs njcht geändert, so da $B$ aueh $T^{\prime \prime}$ "inlach zusammenhängt. Wir hetrarliten zunächst das Verhalten von $J$ in diłsèr rinfarch zusammenhäıgenden Flärhe $T$ ". Ẅ̈r erhalten dann, da die obere Grenze in der nuzerselenittenen Fläche $T$ nicht frei beweglich ist, nicht dir ganze Funktion $J(p)$, sondern nur einen Teil. den wir weiter unten mit $J^{*}(\mathfrak{p})$ bezeiblonen werden. Wir werden zeigen. 
deB $J$ in dieser Fläche eine eindeutige analytische Funktion ist, die sich in ihrem Innern überall wie eine Funktion des Körpers verhält.

Aus der Definition von $J$ ergeben sich folgende einfache Sätze. Eis ist

$$
\int_{p_{0}}^{p_{1}} R d x=\int_{p_{1}}^{p_{0}} R d x
$$

wenn dir lntegrale über denselhen Weg $s$ erstreckt werden, und es ist

$$
\int_{\mathfrak{n}_{1}}^{\mathfrak{p}} R d x=\int_{\mathfrak{b}_{1}}^{\mathfrak{v}_{1}} R d x+\int_{\mathfrak{b}_{1}}^{\mathfrak{p}_{2}} R d x+\cdots+\int_{\mathfrak{b}_{r}}^{\mathfrak{p}} R d \dot{x},
$$

wemn die Punkte $\mathfrak{p}_{1}, \mathfrak{p}_{2}, \cdots \mathfrak{p}_{r}$ auf dem W'ege $s$ liegen, auf dem das links stehende Integral erstreekt wird, während die rechts stehenden Integrale übrr die zwischen den Punkten $\mathfrak{p}_{k}$ liegenden Teile von $s$ zu erstrecken sind.

Wir beweisen den Satz von der Eindeutigkeit von $J(p)$ in $T^{\prime \prime}$ indirekt, nehmen also an, er sei falseh. Dann gibt es in $T^{\prime \prime}$ zwei Punkte $\mathfrak{p}_{1}$ und $\mathfrak{p}_{2}$ und zwei sie verbindende Wege $s_{1}$ und $s_{2}$, so daß die von $\mathfrak{p}_{1}$ nach $\mathfrak{p}_{2}$ über $s_{1}$ und $s_{2}$ erstreckten Integrale von $R d x$ einander nicht gleich sind. daß also

$$
\int_{s_{1}}^{p_{p_{1}}} R d x \int_{p_{1}}^{p_{2}} R d x \neq 0 \text {. }
$$

Bezeichnen wir den aus $s_{1}$ und $s_{2}$ bestehenden geschlossenen Weg mit $\gamma$, $\therefore$ folgt nach (4) und (5) hicraus

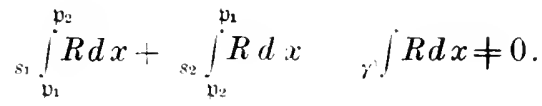

Ist daher der zu beweisende Satz falsch, so gibt es in $T^{\prime \prime}$ einen geschlossenen Wing $\gamma^{\prime}$, sodaß das über $\gamma^{\prime}$ erstreckte Integral von $R d x$ von Null verschieden ist. Jurch $\gamma$ wird $T^{\prime \prime}$ in zwei Teile zerlegt, von denen nach Satz VII in Kap. XII, § 2 der eine, den wir das Innere von $\gamma$ nennen, einfach zusammenhängend ist. Zirhen wir durch das Innere von $;$ einen Querschnitt $q$, so wird $\gamma$ in zwei einfach zusammenhängende Teile zerlegt, die mit $G_{1}$ und $G_{1}^{\prime}$ bezeichnet seien; ihre Grenzen seien mit $\gamma_{1}$ und $\gamma_{i}^{\prime}$ benannt. Es besteht $\gamma_{1}$ aus einem Teile $\gamma^{\prime}$ von $\gamma^{\prime}$ und aus $q$; ebenso besteht $\gamma_{1}^{\prime}$ aus einem Teile $\gamma^{\prime \prime}$ von $\gamma$ und aus $q$. Sind $\mathfrak{q}_{1}$ und $\mathfrak{q}_{2}$ die Endpunkte von $q$, so ist bei passender Wahl der Bezeichnung

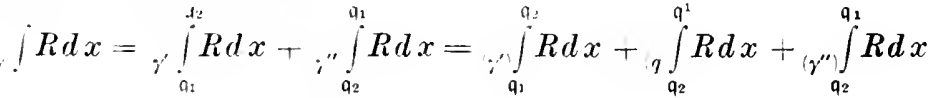

$$
\begin{aligned}
& { }_{(1,} \prod_{q_{2}}^{q_{2}} R d x={ }_{y_{1}} \int R d x+{ }_{y_{1}^{\prime}} \int R d x \text {. }
\end{aligned}
$$




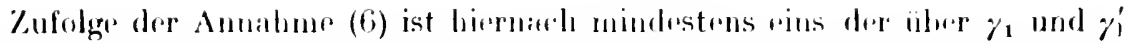

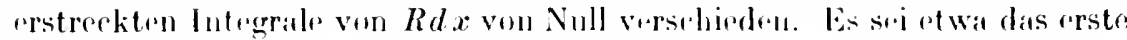
nirlit Null, su daßs

$$
{ }_{n} \int R d x \neq 0 .
$$

Dir Ungleirhheit (7) ist genau von derselben form wire dir Ungleichheit (6). Aber dir Kurve $y_{1}$ schlirßt nur einen Tril des Gebirtes rin, das y rinsehließt. Wir können in derselben WVise frortfahren und writere gesthlosseme Kurven $\gamma_{2}, \gamma_{3}^{\prime}, \ldots$ lereroiten, so daß jedr von ilonem rinen Tril des Gebirtes rinsthlie Bt, das dir vorhergehende hegrenzt, und daß das über irgendeine dieser liurven erstreckte Integral von $R d x$ von Null verschieden ist. Hierdurch rrhalten wir nach einer endlichen Zahl von Schnitten eine geschlossene Kurve $y_{\prime \prime}$, die mit ilırem Innern ganz in einer Umgebung $U$ rines Punktes q von $T^{\prime \prime}$ lirgt, in der, abgesehen vielleicht ven q selbst, keine Unendlichkeitsstelle von $R d x$ liegt. Aber wir haben oben (vgl. (3)) gesehen, daß in diesem Falle das über $\gamma_{n}$ erstreckto Integral von $k d x$ gleich Null sein muß. Es ergibt sich also ein Widerspruch, woraus wir schließen, daß unsere Annahme falsch ist, daß daher $J$ in $T^{\prime \prime}$ eine eindentig. Funktion der oberen Grenze ist. Wir nemnen diese Funktion den Hauptwert des Integrals

$$
J(\mathfrak{p})=\int_{\mathcal{V}_{1}}^{\mathfrak{p}} R d x
$$

und bezeichnen sir mit $J^{*}(p)$. Sie ist innerhalb $T^{\prime \prime}$ eine eindeutige analytische Funktion, dir im Inuern von $T^{\prime \prime}$ als singuläre Stellen höchstens rine rndliche Zahl von Polen hat.

Es seien $\mathfrak{p}_{1}$ und $\mathfrak{p}_{2}$ irgend zwei P'unkte in $T^{\prime \prime}$ und $s$ ein in ' $T^{\prime \prime}$ liegender Weg, der beidre verbindet. Ist $s^{\prime}$ irgendein Weg. der in $T^{\prime \prime}$ voll $\mathfrak{p}_{0}$ nach $\mathrm{p}_{1}$ führt. so ist

$$
\int_{\mathfrak{p}_{1}}^{\mathfrak{p}_{2}} R d x \quad \int_{\mathrm{s}^{\prime}}^{\mathfrak{p}_{1}} R d x \int_{\mathfrak{p}_{1}}^{\mathfrak{p}_{1}} R d x=J^{*}\left(\mathfrak{p}_{2}\right)-J *\left(\mathfrak{p}_{1}\right) .
$$

\section{\$4. Die Periodizitatsmoduln.}

1)ir Funktion $J^{*}$ ist in $T^{\prime \prime}$ überall stetig; sie ist "s also auch im allgemeinen in der unzerschuittenen Fläehe $T$. Nur an don Querschnitten $a_{k}, b_{k}, c_{k}, d_{i}$ kann $J^{*}$ zwerdentig und unstetig sein. Das wollen wir genauer untersuchen. Es sei a an Punlit von $a_{1}$. Wir bezidenen ilnn mit $a_{0}$ oder mit $a_{i}$, je nachdem wir ihn als auf dem rerhten oder dem linken Ufer von $a_{1}$ liegend letrachten. Wir sitzen

$$
J *(a ;)-J *(a, 0)=A_{1}
$$




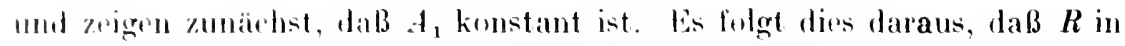

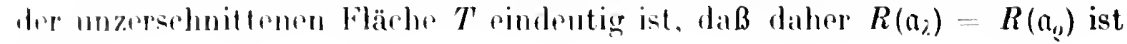
inil

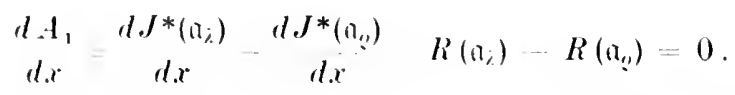

IIir linden dassolbe Ergebnis, wenn wir $A_{1}$ in folgender Weise berechnen. Sirch (S) ist $t_{1}$ gloich dem von $a_{\text {, }}$ marh $a$, erstreckten Integral von $R d x$, wron wir den im übrigen beliebigen lntegrationsweg in $T^{\prime \prime}$ wählen. Wir wählıu ihn in folgender Wrise. Wir gehen von a, in negativer Riehtung längs $a_{1}$ nach $P_{1}$, dann in negativer Richtung längs $b_{1}$ bis wieder nach $P_{1}$ and schlicßlich längs $a_{1}$ in positiver Richtung nath $a_{\%}$. Die Integrale über den ersten und letzen Teil dieses Weges heben einander auf, da sie sich nur in der lichtung des Integrationsweges motersehriden. Es ist daher

$$
A_{1}=\ldots, \int R d x
$$

wo durch das Mimnseichen angegeben werden soll, da $\beta$ über $b_{1}$ in negativer Kichtung zu integrieren ist. Ganz ebenso beweisen wir allgemeiner, wenn as rin Punkt von $a_{1}$ ist,

$$
J *\left(a_{k \lambda}\right)-J *\left(a_{k, a}\right)=A_{h}=\left(-b_{k}\right) / R d x .
$$

Es sei forner $b_{k}$ rin Punkt von $b_{k}$; wir muterselueiden wioder zwisehen bly, und $\mathfrak{b}_{k} ;$ Dif Differenz

$$
J *\left(\mathfrak{b}_{l ; i}\right)-. J *\left(\mathrm{~b}_{k !}\right)=B_{\text {/ }}
$$

ist nach (8) gleirh dem von $b_{k, 0}$ nach $b_{k j}$ erstreckten Integral, wenn wir len Integrationsweg in $T^{\prime \prime}$ wählen. Wir wählen ihn so: Wir gehen von $\mathfrak{b}_{l, \text { längs }} b_{k}$ in positiver Richtung bis $P_{l k}$, dann in positiver Richtung längs $a_{l}$ wieder nach $P_{k}$ und schließlich in negativer Richtung längs $b_{k}$ nach $b_{h}$. Die Integrale über den ersten und letzten Teil des Weges heben einander wieder auf, da sie sich wieder nur durch die Richtung des Integrationsweges unterseheiden. Es ist daher

$$
J *\left(\mathfrak{b}_{k i \lambda}\right)-J *\left(\mathrm{~b}_{k, k}\right)=B_{k}=\left(+\mu_{i,}\right) \int R d x .
$$

Es spi c, rin Punkt von $c_{k}$. Die Jifferenz

$$
J *\left(c_{h i}\right)-J *\left(c_{k ! n}\right)=C_{l}
$$

berechnen wir, indem wir von $c_{h o}$ nach $c_{k ;}$ auf folgendem Wege integrieren. Wir integrieren von $c_{k,}$ längs $c_{k}$ in negativem Sinne nach $P_{k-1}$, dann längs dor Ränder der Querschnitte $a_{l}, b_{l}, c_{l}$, deren Index $l$ kleiner ist als $k$, in der Reihenfolge $b_{k-1 \%}, a_{k-1 \varrho}, b_{k-1 \varrho}, a_{k-1 \%,} c_{k-1, \vdots,} b_{k-2, \lambda,} a_{k-2, \varrho}, b_{k-2, \varrho}$,

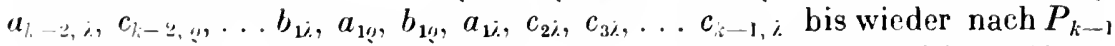
und integriren schließlich von $P_{k-1}$ nach $c_{k i}$ längs $c_{k}$ in positivem Sinne. 


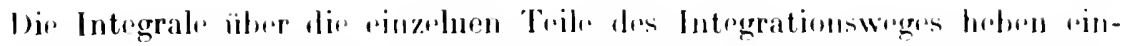

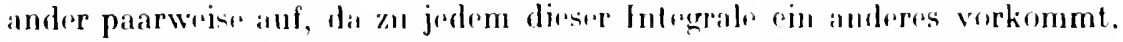
das sich von ihm nur durrh dir kirhtung des lntegrationswregre muter-

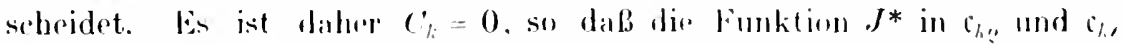
denselhen Wert hat. sibh also stetig ändert. Wrmu p den Quersinhitt $e_{l}$ iiberschreitet.

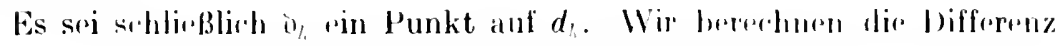

$$
J *\left(\delta_{k i \lambda}\right)-J *\left(D_{k: 0}\right)=D_{k} .
$$

indem wir von $\nabla_{l, 0}$ nach $D_{k: \text { in }}$ integrieren auf folgendem Wige. Wir inte.

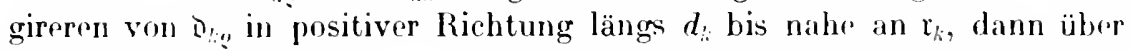
rinen kleinen Kreis um $r_{k}$ in positivem Sinne und schließlich längs $d_{k}$ in negativer lichtung bis $\lesssim_{k}$. Die Integrale über den ersten und latzten Teil des Weges heben einander wieder auf. Das Integral über den kleinen Kreis ist, wir wir schon oben gesehen haben, gleich $2 \pi i r_{l}$, wenn wir wieder mit $r_{\text {. }}$ das Residuum von $R d x$ an der Stelle $\mathfrak{r}_{k}$ bezeichnen. Also ist

$$
J *\left(D_{k i}\right)-J *\left(D_{k q}\right)=D_{k}=2 \pi i r_{k} .
$$

Wir wollen jetzt die obere Grenze $p$ des Integrals $J(p)$ einmal den Querschnitt $a_{1}$ überschreiten lassen, und zwar in positiver lichtung, d. h. vom linken Ufer nach d'm rechten. Dann aber soll $p$ in $T^{\prime \prime}$ bleiben. Wir bezeichnen die Funktion, die wir auf diese Weise erhalten und die wieder wegen der Beschränkung. die wir der oberen Grenze $p$ auferlegen, nur $\in$ in Teil der Funktion $I_{(p)}$ ist, mit $J_{1}(p)$. Diese Funktion steht in sehr einfacher lieziehung zu dom Hauptwerte des Integrals $J(\mathfrak{p})$. Es ist, wenn der Integrationsweg den Quursichnitt $a_{1}$ bei a überschreitet,

$$
J_{1}(p)=\dddot{a}_{\mathfrak{i},}^{a} k d x+\int_{\mathfrak{a}_{0}}^{p} R d x=J *\left(\mathfrak{a}_{2}\right)-J *\left(\mathfrak{p}_{0}\right)+J *(\mathfrak{p})-J *\left(\mathfrak{a}_{0}\right) .
$$

also, da $J^{*}\left(\mathfrak{p}_{0}\right)=0$ und wegen $(9)$

$$
J_{1}(\mathfrak{p})=J^{*}(\mathfrak{p})+A_{1} .
$$

Allgemeiner schließen wir hieraus, da für die anderen Querschnitte $a_{h}$ und für dir Querschnitte $b_{k}$ und $d_{k}$ Ähnliches gilt:

Überschreitet die obere Grenze des Integrals $J(p)$ den Querschnitt $a_{h} a_{k}$-mal in positiver und $\alpha_{k}^{\prime}$-mal in negativer Richtung, den Querschnitt $b_{k} \quad \beta_{k}$-mal in positiver und $\beta_{k}^{\prime}$-mal in nrgativer Richtung und schließlich den Querschnitt $d_{k} \delta_{i}$-mal in positiver und $\delta_{k}^{\prime}$-mal in negativer Richtung, so ist

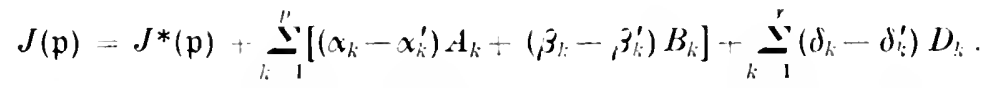

Es ist also $J(p)$ unendlinh virldeutig in $T$. Die verschiedenen Werte, die 
$J(p)$ au derselben Stelle p annehmen kann, unterseheiden sich voneinander durh ganzzahlige Vielfache der Konstanten $A_{k}, B_{k}, D_{k}$. Diese Konstanten heiben die Periodizitätsmoduln oder auch kürzer die Perioden des lntegrals $J(p)$; im besonderen heiBen die $A_{l}$. $B_{l}$ zyklische und die $D_{\text {in }}$ logarithmische Perioden.

Es sei noch bemerkt. da $B$ ein Integral $J(p)$, dessen Perioden alle gleich Null sind, eine Funtion des Körpers sein muB.

Zunächst kann dann nämlich $J(p)$ kein Residuum haben, da es sonst logarithmische Perioden hätte. Daher verhält sich $J$ in $T$ überall wie eine Funktion des Körpers und da $J$ außerdem wegen des Versehwindens aller Perioden in $T$ eindeutig ist, so ist es nach Satz VIII, $\$ 7$ Kap. IV eine Funktion des Körpers.

\section{$\$$ 5. Integrale erster Gattung.}

Man unterseheidet drei Arten von Integralen:

1. Intigrale, die an keiner Stelle unendlich werden. Sie heißen In tegrale prster Gattung.

2. Intrgrale, die nur unendlich werden wie die Funktionen des Körpers. Sie heißen Integrale zweiter Gattung.

3. Integrale, die sich nicht überall wie die Funktionen des Körpers verhalten, die also an mindestens einer Stelle eine Entwicklung haben, die einen Logarithmus enthält. Sie heißen Integrale dritter Gattung.

Wir wollen die Integrale dieser drei Gattungen etwas genauer betrachten. Dabei nehmen wir wieder die untere Grenze $\mathfrak{p}_{0}$ als fest und die obere $\mathfrak{p}$ als beweglich an.

Ein Integral wird dann und nur dann nirgends unendlich, wenn das integrierte Differential nirgends unendlich wird, wenn also das Differential von der ersten Gattung ist. Soleher Differentiale gibt es $p$ linear unabhängige. Es seien $d u_{1}, d u_{2}, \ldots d u_{t}, p$ linear unabhängige Differentiale erster Gattung; dann sind

$$
u_{1}, u_{2}, \ldots u_{p^{\prime}}
$$

$\mu$ Integrale erstır Gattung und sie sind in dem Sinne linear unabhängig, daß keine Gleichung zwischen ihnen bestehen kann von der Form

$$
c_{1} u_{1}+c_{2} u_{2}+\cdots+c_{p} u_{p}=c_{0} .
$$

wo die $c$ konstant sind. Ist $u$ irgendein Integral erster Gattung und also $d u$ ein Jifferential erster Gattung, so ist $d u$ in der Form darstellbar

$$
d u=i_{1} d u_{1}+i_{2} d u_{2}+\cdots+i_{p} d u_{p},
$$

wo die 7 konstant sind. Es it also $u$ darstellbar in der Form

$$
u=\lambda_{1} u_{1}+\lambda_{2} u_{2}+\cdots+\lambda_{p} u_{p^{\prime}}+\lambda_{0},
$$

wro auch $\lambda$, konstant ist. 


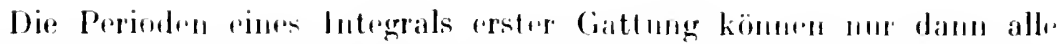
gleich Null sein, wenu es konstant ist, wenu also das zugrhörige Defferential identisch Null ist. Dem rin soleles Integral wörd" one Funktion des Körpers sein, dir nirgends unendlith wird.

\section{$\$$ (integrale zweiter Gattung.}

Wir zeigen zunächst, daß es immer ein lntegral $J$ zweiter Gattung gibt, daß an irgendeiner gegebenen Stelle q gena von gegehener Ordnung $\lambda$ unendlich wird. Soll $J$ in q von der Ordnung $\lambda$ unendlifh werden; so muB das Differential von der Ordnung $\lambda+1$ in $q$ unendlich werden, der zugehörige Differentialteiler also die Form haben

$$
\text { a) } q^{-k+1}
$$

wo !n ein ganzer Divisor ist. Da jeder Differentialteiler der kamonischen Klasse (i) angehört, so ist p ein ganzer Divisor der Klasse ( $\left.q^{j+1}\right)$. Die Zahl der linear unabhängigen Differentiale, die nur in y und dort höehstens von der Ordnung $\lambda+1$ unendlich werden, ist daher gleich der Zahl der linear unabhängigen ganzen Divisoren der Klasse $\left(\mathfrak{f} q^{j+1}\right)$, also gleich der Dimension dieser Klasse. Diese Dimension, die mit $n_{;}$bezeichnet sei, ist für $\lambda \geqq 0$ nach dem Riemann-Rochschen Satze

$$
n_{i}=\left\{\mathrm{fq}^{i}+1\right\}=2 p-2+\lambda+1-p+1=i,
$$

da die Ergänzungsklasse von negativer Ordnung und ihre Dimension daher Null ist.

Da für $\lambda \ldots 1$

$$
n_{i}-n_{i-1}=i-(i-1)=1>0,
$$

so schließen wir, daß es mindestens rin Differential geben muß, das nur in $q$ und dort genau von der Orduung $\lambda+1$ unendlich wird, und daraus folgt dann, daß es auch mindestens ein Integral gibt, das nur in q und dort genau von der Ordumg $i$. unendlich wird. Wir bezeichnen mit $z_{a}^{1}$ ein Integral, das nur in a und dort von der ersten Ordnung unendlich wird. Es habe $x$ in q den Wert $a$ und es sei q ein Verzweigungspunkt der Ordunng $\alpha-1$. Wir setzen zur Abkürzung $(x-a)^{\frac{1}{x}}=t$. Dureh Hinzufügrn eines konstanten Faktors können wir erreichen, daß die Entwieklung von $z_{q}^{(1}$ in der Umgebung von q folgende Gestalt hat

$$
\tilde{z}_{\mathfrak{a}}^{(1)}=t^{-1}+\psi_{1}(t) \text {, }
$$

wo $\mathfrak{H}_{1}(t)$ eine gr'wöhnliehe Potenzreihe von $t$ ist. Es sei ferner $z$ ein Integral, das nur in q und dort genau von der zweiten Ordnung unendlieh wird. Die Entwicklung von $z$ in der Umgebung von a sei

$$
z-a_{1} t^{2}+a_{2} t^{-1}+\mathfrak{B}(t) \text {. }
$$


wo Integral $a_{1}-1\left(z-a_{2} z_{a}^{(1)}\right)$ mit $z_{a}^{(2)}$. Die Entwicklung von $z_{a}^{(2)}$ in der Umgrobung von o ist

$$
z_{4}^{2}=t^{2}+\mathscr{L}_{2}(t) \text {. }
$$

wo $W_{2}(t)$ einn gewöhnliche Potenzreihe von tist. Fahren wir in dieser Weise fort, so erhalten wir für jedes $\lambda$. 0 e ein Integral $z_{0}^{(i)}$, das nur in $q$ und dort genau von der Ordnung $\lambda$. unendlieh wird und dessen Entwicklung in der I'mgehung von o sel aussieht:

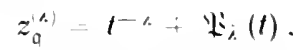

"ow: $(t)$ eine gewöhnliche Potenzreihe ven $t$ ist. Diese lntegrale sind nicht vollständig bestinmt. Ist $z^{\prime}$ ein Integral, das dieselben Eigensehaften hat wie $z_{0}^{(i)}$, so ist $z^{\prime}-z_{a}^{(i)}$ ein lntegral, das nirgends unendlich wird, also rin Integral erster Gattung. Es sind daher die Integrale $z_{\mathfrak{a}}^{(\lambda)}$ durch ihre Eigenschaften nur hestimmt bis auf Integrale erster Gattung, die man beliebig hinzufügen kann, ohne ihre wesentlichen Eigenschaften zu ändern.

\section{$\$$ 7. Elementarintegrale zweiter Gattung.}

Es seien $p_{1}, p_{2}, \ldots p_{p}$, irgend $p$ Stellen der Riemannschen Fläche $T$ dienicht sprziell liegen, $d$. h. nach $\$ 4$ und $\$ 5$, Kap. IX es soll keinenganzen Divisor der Differentialklasse geben, der durch den Divisor $\mathfrak{p}=\mathfrak{p}_{1} \mathfrak{p}_{2} \ldots \mathfrak{p}_{n}$ teilbar ist. Wïe wir in $\$ 5$, Kap. IX gesehen haben, folgt daraus, daß es keine Funktion des Körpers gibt, die nur an den Stellen $\mathfrak{p}_{1}, \mathfrak{p}_{2}, \ldots \mathfrak{p}_{p}$, und zwarhöchstens von der orsten Ordnung unendlich wird. Wir bezeichnen die $p$ Integrale $z_{\mathfrak{b}_{1}}^{1}, z_{p_{2}}^{\prime 1}, \ldots z_{\mathfrak{b},}^{1)}$ kürzer mit $v_{1}, v_{2}, \ldots v_{p}$. Solche Integrale, die also nur an einer Stelle und dort von der ersten Ordnung unendlich werden, heißen Elementarintegrale zweiter Gattung.

Nan nennt $s$ Integrale zweiter Gattung $J_{1}, J_{2}, \ldots J_{s}$ linear unabhängig, wenn zwischen ihnen keine Gleichung besteht von der Form

$$
c_{1} J_{1}+c_{2} J_{2}+\cdots+c_{s} J_{s}=S(x, y)+u,
$$

wo die $c$ kunstant sind und wo $S(x, y)$ eine Funktion des Körpers und $u$ nin Integral erster Gattung ist. Wir behaupten, die $p$ Elementarintegrale zweiter Gattung $v_{1}, v_{2}, \ldots v_{p}$ sind in diesem Sinne linear unabhängig. Es bestrhe nämlich zwischen ihnen eine Gleichung der Form

$$
c_{1} v_{1}+c_{2} v_{2}+\cdots+c_{p} v_{\prime \prime}=S(x, y)+u,
$$

wo dir "und $S$ und $u$ lieselbe Bedeutung haben wie eben, so würde $S(x, y)$, wie ans (13) folgt, eine Funktion des Körpers sein, die nur an den Stellen $\mathfrak{p}_{1}, \mathfrak{p}_{2}, \ldots \mathfrak{p}_{p}$, und zwar hïrhst was nicht möglich ist. 


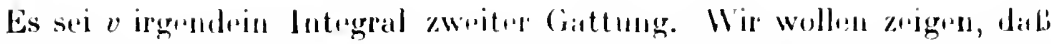

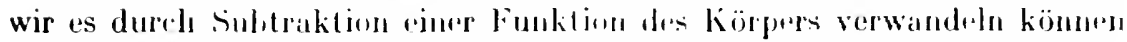
in ein Integral, das nur nerch an den Stollen $p_{1}, p_{2} \ldots p_{\mu}$, und zwar höchstens von der ersten Ordnung unendlich wird. Es sei a rine Unendlich-

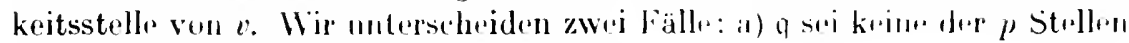
$\mathfrak{p}_{l}$; b) q sei ritur der Siellen $\mathfrak{p}_{k}$, rtwa $\mathfrak{p}_{1}$.

a) Wir zoigen zunäehst, daß as eine Funtion lwo Korjer's gibt, dlie

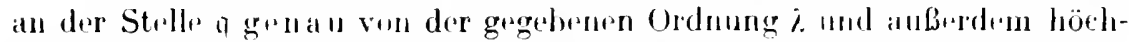

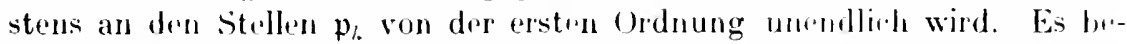

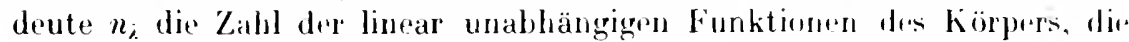

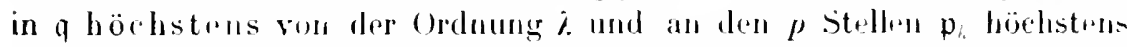
von der resten (Ordnung unendlich werden. Diese Funktionen sind ron der Form nq $q^{-i} p$ ', wo $q$ ein ganzer l)ivisor ist und wo zur Al,kurzung $p=$ $p_{1} p_{2} \ldots p_{n}$, gesetzt ist. I) Ze Zahl der linear unabhängigen dieser Funktion’u ist daher gleich der Dimension der Klasse (a’p), alsu narh dem RirmannRochschen Satze

$$
n_{i}=\left\{q^{*} p\right\}=i+p-p+1+\left\{\mathfrak{k} q^{-\alpha} p^{\prime}\right\} .
$$

Die Ergänzungsklasse kann keinen ganzen Divisor enthalten; wäre nämlich $g^{\prime}$ ein soleher, so wäre $\eta^{\prime} p g^{\prime}$ ein ganzer Divisor der Differentialklasse, der durch $p$ teilbar wäre. Wir haben aber ausdrücklich vorausgesetzt, dat? es cinen solchen nicht gibt. Daher ist die Dimension der Ergänzungsklass. Null und aus (14) folgt für $\lambda=0$

und hieraus fiir $\lambda .1$

$$
n_{i}=i+1
$$

$$
n_{,}-n_{,-1}-1>0 \text {, }
$$

woraus wir schlieben, daß es einr Funktion den Körpers geben muB, di. in $q$ g e $\mathrm{n}$ a $u$ von der $\hat{\lambda}_{\text {-ten }}$ und an den $p$ Stellen $\mathfrak{p}_{k}$ höchstens von der ersten Ordnung unendiich wird, während sie sonst übreall endlich ist.

Wird $v$ in q von der Ordnung $\mu$ unendlich, so bestimmen wir eine Funktion $R$ des körpers, die in q genau von der $\iota$-ten und außerdem höchstens an den Stellen $\mathfrak{p}_{k}$ von der ersten Ordnung unendlich wird. Es sei q eine Verzweigungsstelle der Ordnung $\alpha-1$ und es habe $x$ in a den Wert $a$. Sind die Anfangsglieder in den Entwicklungen von $v$ und $R$ in der Umgebung von a

$$
a(x-a) " \text { " und } \quad b(x-a) \text { " }
$$

so wird $v-\frac{a}{b} k$ ein Integral zweiter Gattung, das in q von geringerer Ordnung unendlich wird als $v$. Ist also $q$ nicht eine von den $p$ Stellen $\mathfrak{p}_{l}$, su können wir durch Subtraktion einer Funktion $R$ des Körpers $v$ in ein Inte- 
gral zweiter Gattung verwandeln, das in if von geringerer Ordnung unendlich wird als $r$ und das als neue Unendlichkeitsstellen höchstens solche r rster Ordnung in den $p$ Punkten $p$ hat. J)urch wiederholtes Anwenden dirses Verfahrens könurn wir $v$ in ein Integral zweiter Gattung verwandeln, das nur noch an den Stollen $\mathfrak{p}_{k}$ unendlich wird. Es sei $v^{\prime}$ dies Integral und $s_{1}(x, y)$ die im ganzen von $r$ subtralierte Funktion. Dann ist

$$
\text { r. } S_{1}(x, y)=r^{\prime} \text {. }
$$

b) Wir beweisen zunächst, daß $\rho$ für $i>1$ immer eine Funktion des Kërpers gibt, die in $p_{1}$ gen au von der i-ten und sonst nur an den übrigen Stellen $\mathfrak{p}_{i}$, und zwar höchstens von der ersten Ordnung unendlich wird. E- bedeute $m_{\text {; }}$ die Zahl der linear unabhängigen Funktionen des Körpers die in $\mathfrak{p}_{1}$ höchstens von der $\lambda_{\text {-ten }}$ und außerdem höchstens in den anderen Punkten $\mathrm{p}_{k}$ von der ersten Ordnung unendlich werden. Solch eine Funktion ist von der Form $n \mathfrak{p}_{1}^{-i-1} \mathfrak{p}^{-1}$, wo $g$ ein ganzer Divisor ist. Es ist daher $m_{\text {. }}$ gleieh der Dimension der Klasse $\left(\mathfrak{p}_{j}^{j-1} \mathfrak{p}\right)$, so daß nach dem RiemannRoehschen Satze für $i \geq 1$

$$
m_{i}=\left\{\mathfrak{p}_{i}^{i-1} \mathfrak{p}\right\}=\dot{j}-1+p-\varrho+1+\left\{\mathfrak{f} \mathfrak{p}_{1}^{1-i} \mathfrak{p}^{-1}\right\} .
$$

Die Ergänzungsklasse $\left(\mathfrak{f} \mathfrak{p}_{1}^{1-\prime} \mathfrak{p}^{-1}\right)$ kann wieder keinen ganzen Divisor $\mathfrak{g}^{\prime}$ enthalten, da sonst $\mathfrak{p}_{1}^{j-1} p q^{\prime}$ ein ganzer Divisor der Differentialklasse wäre, der durch $p$ teilbar ist. Daher ist die Dimension der Ergänzungsklasse Null und nach (15) für $i \geq 1$

und für $\dot{\lambda} \geq 2$

$$
m_{i}=i
$$

$$
m_{i}-m_{i-1}=\lambda-(\lambda-1)=1>0,
$$

worans wir sehließen. daß es für $i \geq 2$ eine Funktion mit den verlangten Eigenschaften gibt.

Es werde $v$ in $p_{1}$ von der Ordnung $" \geq 2$ unendlich. Wir bestimmen dann eine Funktion $Q$ des Körpers, die in $p_{1}$ genau von der $u$-ten Ordnung unendlich wird. Es sei $\mathfrak{p}_{\text {: }}$ ein Verzweigungspunkt der Ordnung $\alpha_{k}-1$ und $x$ habe in $\mathfrak{p}_{k}$ den Wert $a_{k}$. Zur Abkürzung sei gesetzt $\left(x-a_{i:}\right)^{\frac{1}{\alpha_{k}}}=t_{k}$. Sind die Anfangsglierdor in den Entwicklungen von $v^{\prime}$ und $Q$ in der Umgeloung von $\mathfrak{p}_{1}$

$$
a t_{1}^{-u} . b t_{1}{ }^{u} .
$$

so ist $v^{\prime}-{ }_{b}^{a} Q$ cin Integral zweiter Gattung, das in $p_{1}$ von geringerer Ordnung umendlich wird als $v$. Wir können also aus $v^{\prime}$ durch Subtraktion einer Funktion des Körpers ein Integral zweiter Gattung herleiten, das in $p_{1}$ von geringerer Ordnung unendlich wird, wenn $v^{\prime}$ dort von höherer als der ersten Ordnung unendlich wird. An neuen Unendlichkeitsstellen 
können höchstens solche erster Ordnung inl den Stellen $p_{2}, p_{3}, \ldots p_{p}$ hinzukommen. Durch wiederholtes Anwenden dieses Verfahrens rrhalten wir ein Integral zweiter Gattung $J$, das höchstrus an den $p$ Stell'n $p_{k}$, und zwar höchstens von der ersten Ordnung unendlich wird.

Die im ganzen von " subtrahierte Funktion si mit $S(x, y)$ bezeichnet, so da $B$

$$
v-S^{\prime}(x, y)=J .
$$

Es sei in der Entwicklung von $J$ in der Umgebung von $p_{k}$ der Koeffizient von $t_{\ell}{ }^{1}$ gleirh $c_{l}$. Dann wird das Integral

$$
u=J-c_{1} v_{1}-c_{2} v_{2}-\cdots-c_{\rho} v^{\prime \prime}
$$

nirgends mehr unendlich, ist also von der ersten Gattung. Aus (16) und (17) folgt, daß wir jedes Integral zweiter Gattung mit Hilfe der $p$ Elementarintegrale $v_{t}$ in der Form darstellen können

$$
v=S(x, y)+u+c_{1} v_{1}+c_{2} v_{2}+\cdots+c_{p} v_{p},
$$

wo die $c$ konstant sind und wo $S$ eine Funktion des Körpers und $u$ ein Integral erster Gattung bedeutet. Wir hatten oben gesehen, daß die $p$ Integrale $v_{k}$ linear unabhängig sind. Jetzt haben wir nachgewiesen, da $B$ alle anderen Integrale zweiter Gattung von den $p$ Integralen $v_{k}$ linear abhängig sind. Es gibt also genau p linear unabhängige Integrale zweiter Gattung.

\section{\$ 8. Der WeierstraBsche Lückensatz.}

Wir wollen zeigen, wie wir uns noch auf eine andere Art linear unabhängige Integrale zweiter Gattung herstellen können, von denen auch alle anderen Integrale zweiter Gattung linear abhängig sind. Ist $r$ die Zahl dieser Integrale, so muß nach dem eben Bewiesenen $r=p$ sein. Wir werden auf diese Art zu einem interessanten Satze kommen.

Wir betrachten die Funktionen des Körpers, die nur an einer Stelle a unendlich werden und sonst nirgends. Wird eine solche Funktion in $q$ von der Ordnung $\lambda$ unendlich, so ist $\lambda$ die Ordnung der Funktion. Es wird wirht zu jeder Zahl $\lambda$. eine Funktion des Körpers geben, die nur in $q$ und durt genau von der Ordnung $\lambda$ unendlich wird. So gibt es für $p>0$ uberhaupt keine Funktion erster Ordnung, also auch keine Funktion, die uur in $q$ und dort von der ersten Ordnung unendlich würde. Schreiben wir also die Ordnungszahlen aller derjenigen Funktionen des Körpers auf, die nur in q un ndlich werden, so werden in dieser Reihe für $p>0$ nicht alle positiven ganzen Zahlen vorkommen. es werden einige Ordnungszahlen fehlen. Wir zeigen zunächst, da $B$ die fehlenden Ordnungszalılen kleiner sind als $2 p$, woraus dann fọtgt, daß ihre Anzahl endlich ist. Es be- 
dentr. $n$, dir Zahl der linear unabbängigron Funktionen des Körpers, die nur in q. und zwar höhstens von der lordnung i. nnendlich werden. Es

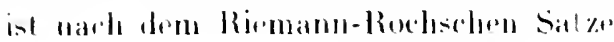

$$
n ;\left\{q^{\prime}\right\} ; p+1-\{t a\}
$$

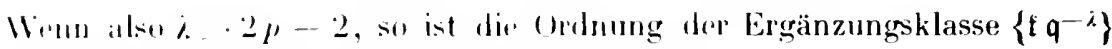
megativ und daher ilue Dimension Null, al daß

$$
n_{i}-i, \cdots+1 . \quad(\lambda, 2 p \quad 2) \text {. }
$$

Didher wird far $i \quad 2 p-1$

$$
n,-n ;-10 \text {. }
$$

Whatls wir sehlieben, dab es für $i .2 p-1$ immer eine funktion des kïrpers gilit. dir nur in 17 und dort genau vent der Ordnung $\lambda$ unendlich wird. E kann also, wie behauptet, keine Zahl, die größer ist als $2 p$ zu

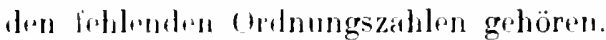

1) ferhlenders Ordnungsablen seien $\sigma_{1}, \sigma_{2}, \ldots \sigma_{r}$. Thre Anzahl sei alsu $r$. lanu ind die when definirrten Integrale

$$
z_{i}^{(n)}, \quad z_{i}^{\prime \prime 2} \ldots z_{a}^{(n r}
$$

linear unahhängig. Wärde nämlich rine Gloidhung der Form

$$
c_{1} z_{a}^{(n)}+c_{2} z_{a}^{(n z}+\cdots c_{r} z_{a}^{\prime \prime r^{\prime}}=S(x, y)+u
$$

bestehen, wo dir $c$ konstant sind und wo $S$ eine Funktion des Körpers und " "in Integral "rster fiattung ist, so würde $s$ einr Funktion sein, die nur in a unendlich wird und ihre Ordnungszahl würde gleich einer der fehlenden Ordnungszahlen swin.

Wir zeigen writer, daß jedes andere Integral zweiter Gattung linear von dיn $r$ Introgralon (19) abhängt. Es genügt, dies für die $p$ Integrale $v_{k}$ nachzuwisun, von den'n ja allo anderen linear abhängen. Wir beweisen "s tür $e_{1}$; für dir anderen $v_{l}$ : gilt dann dasselbe. Zunächst läßt sich immer eine Funktion $Q$ des Körpers finden, die in $p_{1}$ genau von der ersten Ordnung und aubrdem nur noch in qunendlich wird. Es bedeute nämlich $m_{\text {; }}$ dir. Zahl der linear unabhängigen Funktionen des Körpers, die in $\mathfrak{p}_{1}$ hörhstuns von der ersten und in a höchstens von der $\lambda$-ten Ordnung unesullich werden, und $n$; sei dir Zahl der linear unabhängigen Funktionen des Körpers, die nur in $a$, und zwar höchstens von der (Ordnung $\lambda$ unendlich werden. Wir nrhmen $i>2 p-2$ an, damit de Ergänzungsklassen von $\left(p q^{j}\right)$ und $\left(q^{\prime}\right)$ von negativer ()rdnung sind. Narh den Riemann-Rochschen Satze ist dann

$$
m_{i}=\left\{\mathrm{pq}^{\prime}\right\}=i-1-p+1=i-p+2 . \quad n_{i}=\left\{q^{i}\right\}=\lambda-p+1
$$

und itso,

$$
m_{i}-n_{i}=1=0
$$


woraus wir seluliebun, daß es cine Funktion $Q$ les liörpers gibt, die in $p_{1}$

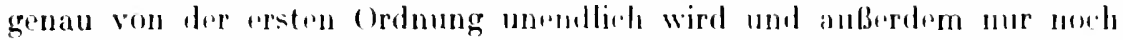
in $q$. Ist a dur liorflizint von $t^{-1}$ in der Entwicklung von $Q$ in der Ungehung von $p_{1}$, so ist

$$
r_{1}-{ }_{a}^{1} Q \ldots . J
$$

rin Integral zwriter Gattung, das nur noch in q Imendlinh wird.

Es sei qutwa rin Verzweigungspunkt der ()rdumes $x-1$ und $x$ hab" in $q$ den Wirt $a$. Zur Abkürzung sri grsetzt $(x-a)^{\prime \prime} t$. Es werde $J$ in o von dep ()rdnung " unendlich. Das Anfangsgliol in dur Entwicklung von $J$ in der Limgroung von q sei

$$
a t^{-u} \text {. }
$$

Wie untrrscheiden zwei Fälle: 1. "gehört nicht zu den fehlenden Ordnungszahlen; 2. " gehört dazu. Im ersten Falle gibt "s rine Funktion $Q_{1}$ des Körpers, die nur in q und dort gwnau von der Oranung " unendlich wird. Ist $b t^{-"}$ das Anfangsglied in der Entwicklung von $Q_{1}$ in der Umgebung vou $\mathfrak{q}$, so ist $J-\frac{a}{b} Q_{1}$ ein Integral zweiter Gattung, das auch nur in $\mathfrak{q}$, aber von geringerer Ordnung als $J$ unendlich wird. Ist zweitens $\mu$ eine der fehlenden Ordnungszahlen, so ist $J-a z_{0}^{\prime \prime \prime}$ ein Integral zweiter Gattung, das nur in q und von geringerer Ordnung unendlich wird als $J$. Wir können also immer aus cinenı Integral $J$, das nur in q unembllich wird, durch Subtraktion entweder riner Funktion des Körpers oder eines mit einer gerigneten konstantin multiplizierton Integrals (19) ein neues Intrgral zweiter Gattung horleiten, das auch nur in a unendlich wird, aber von geringerer Ordnung als $J$. Durch wied rholtes Awwenden dieses Verfahrens können wir $J$ verwandeln in ein Integral $u$, das überhaupt nicht mehr unendlich wird, also von der ersten fattung ist. Die im ganzen von $J$ subtrahierte Funktion sei $Q_{2}$ und die subtrahierte lineare Kombination der Integrale (19) sei $e_{1} z_{q}^{\left.o_{1}\right)}+e_{2} z_{q}^{\left(\omega_{2}\right)}+\cdots+e_{r} z_{q}^{\left(\sigma_{r}\right)}$, so daß

$$
J-Q_{2}-\left(e_{1} z_{\mathfrak{q}}^{\left(\omega_{1}\right)}+e_{2} z_{\mathfrak{q}}^{\left(\omega_{2}\right)}+\cdots+e_{r} z_{\mathfrak{q}}^{\left(\sigma_{r}\right.}\right)==\| \text {. }
$$

sitzen wir $\frac{1}{a} Q-Q_{2}=S$, so folgt aus (20) und (21), daß wir setzen können

$$
r_{1} S(x, y)+u+e_{1} z_{\mathrm{q}}^{\left(o_{1}\right)}+e_{2} z_{\mathrm{q}}^{\left(\sigma_{2}\right)}+\cdots+\rho_{r} z_{\mathrm{q}}^{\left(\sigma_{r}\right.} .
$$

Allgemeiner lrstehen Gleichungen ron der Form

$$
r_{l_{k}} \quad S_{k}(x, y)+u^{(k)}+e_{1 k} z_{\mathrm{q}}^{\left(\sigma^{1}\right)}+e_{2 k} z_{\mathrm{q}}^{\left(a_{2}\right)}+\cdots+e_{r k} z_{\mathrm{a}}^{a_{r}},
$$

wo die $e_{1 k}$ konstant sind und wo die $S_{k}$ Funktionen des liörpers und die $u^{(k)}$ Integralu erstre Gattung sind. Wir haben oben bewiesen, dab wir jedes Jung, Einfuhrung in die Theorim. 
Intrgtal zweiter Gattung in der Form (18) linear durch die $p$ Elementarintegrate $v_{k}$ darstellen kömnen. Durch Benutzung der Gleichungen (22) rhalten wir also fiir irgendein lntegral zweiter Gattung $v$ dic Darstellung

$$
l=P(x, y)+\vec{u}+c_{1} z_{\mathfrak{q}}^{\left(w_{1}\right)}+c_{\mathbf{2}} z_{q}^{\left(b_{2}\right)}+\cdots+\rho_{r} z_{\mathfrak{q}}^{\left(i_{r}\right)} \text {. }
$$

wo die $e$ konstant sind und wo $P$ eine Funktion des körpers und $\bar{u}$ ein Integral erster Gattung ist. Damit ist gezeigt, daß die $r$ Integrale (19) nicht nur linear unabhängig sind, wie wir ohen festgestellt haben, sondern daß auch jedes andere Integral zweiter Gattung linear von ihnen abhängt. Daher ist die Zahl der linear unabhängigen Intrgrale zweiter Gattung genau gleich $r$. Wir haben aber oben gofunden, daß diese Zahl gleich $p$ ist. Waher ist $r=p$ mol wir haben damit den Wrierstraßschen Lückensatz bewiesen:

In der Reihe der Ordnungszahlen derjenigen Funktionen des käpors. die nur an einer Stelle unendlich werden, fehlen immer $p$ Zahlen, wo $p$ das Geschlecht des Körpers ist.

Lje fehlenden Ordnungszahlen sind im allgemeinen die Zahlen $1,2, \ldots$ $p$; nur für eine endliche Zahl von Stellen sind dies nicht die fehlenden Ordnungszahlen. Diese Stellen heißen Weierstraßpunkte. (Vgl. Kap. IX. $\$ 10$.

\section{$\$ 9$. Integrale dritter Gattung.}

soll ein Integral an ciner Stelle logarithmisch unendlich werden, so muß das Differential an der Stelle von der ersten Ordnung unendlich werden, also dort ein Residuum haben. Da dir Summe der Residuen eines bifferentials gleich Null ist, so kann es kein Differential geben, das nur an einer Stelle und dort ron der frsten Ordnung unendlich wird, also auch kein Integral, das nur an einer Stelle und dort logarithmisch unendlich wird. Das infachste Integral dritter Gattung ist daher eins, das nur an zwei Stellen und dort logarithmisch unendlich wird. Wir wollen zeigen, daß cin solches immer vorhanden ist. Es seien $q_{1}$ und $\mathrm{a}_{2}$ irgend zwei Stcllen. Soll ein Differential nur an diesen beiden Stellen, und zwar von der ersten Ordnung verschwinden, so muß der zugehörige Differentialteiler von der Form $\mathrm{Sq}_{1}^{-1} \mathrm{q}_{2}^{-1}$ sein, wo g ein ganzer Divisor ist, und zwar ein Diviror lle Klasse $\left(\mathfrak{f} \mathfrak{q}_{1} \mathfrak{q}_{2}\right)$, wenn wieder mit $(\mathfrak{f})$ die kanonisch lilasse bezeichnet wird. Die Zahl $m$ der linear unabhängigen bifferentiale, deren Diffrentialteiler rlie Form $\bigcap_{1}^{-1} q_{2}^{-1}$ hat, ist daher gleich der Dirn'nsion der Klasse $\left(\mathfrak{f} \mathfrak{a}_{1} \mathfrak{q}_{2}\right)$, so daß

$$
m=\left\{q_{1} q_{2}\right\}=2 p-2+2-p+1=p+1 .
$$

Es fragt sich, w) unter den $p-1$ linear unabhängigen Differentialen, die nur in $q_{1}$ und $q_{2}$ unendlich wrrden, und zwar höchstens von der ersten 
Ordnung, atuh wolche volkonmen. dir wirklich sowohl in $q_{1}$ wie in $q_{2}$ unendlich werden. Nehmen wir an, das wibe nieht der Fall. Dann würden die $p+1$ linear mahhängrgen l)ifferentiale, da es Differentiale, die nur an einer Stollı von der ersten Ordnung unendlich werdin, nicht gibt, überhaupt nicht unendlich werden, würden also von der erstrn Gattung sein, und wir hätten $p+$ I linear unabhängige Diffrementiale erstrer Gattung. Es gibt daher immı ein Differential $d w_{a_{1} a_{2}}$, das nur an den loriden beliebig gegebenen Strllon $\mathfrak{q}_{1}$ und $\mathfrak{q}_{2}$, und zwar genau von der ersten Ordnung unendlich wird. Durel Multiplikation mit rinem konstanten Faktor können wir erreichen, daB das hesidum von $d w_{a+9}$ in $q_{1}$ gleich 1 ist. Da die Summe der Residuen gleich Null ist, so is das Residum von $d w_{a_{2}} a_{2}$ in $q_{2}$ gleich - 1. Bedeutet $t$ eine Funktion des Körpers, die an der Strlle q von der resten Ordnung Null wird, so ist in der Umgebung von $\mathfrak{\eta}_{k}$

$$
w_{\mathrm{q}_{1} \mathrm{a}_{2}}=(-1)^{i_{i}+1} \ln t+P(t)
$$

wo $P(t)$ eine gewöhnliche Potenzreihe von $t$ ist. Das Integral $w_{a_{1} q}$, heißt Elementarintegral dritter Gattung.

Es sei $w$ irgendein Integral des Körpers. Das Diflerential $d w$ habe Residuen an den Stellen $r_{1}, r_{2}, \ldots r_{1,}$, und es sei das Residumm an der Stelle $r_{k}$ gleich $r_{k}$. Es besteht die Gleichung

$$
r_{1}+r_{2}+\cdots+r_{0}=0
$$

la die Summe der Rersidurn cines jerlen Differentials des Körpers Null ist. Ist $r_{0}$ irgend eine von den Stellen $r_{1}, r_{2}, \ldots r_{0}$ verschiedene Stellr, so hat das Differential

$$
d v=d w-\cdots r_{1} d w_{\mathrm{r}_{1} \mathrm{r}_{0}}-r_{2} d w_{\mathrm{r}_{2} \mathrm{r}_{u}}-\cdots-r_{q} d w_{\mathrm{r}_{2} \mathrm{r}_{1}}
$$

an den Stellen $r_{1}, r_{2}, \cdots r_{0}$ lioin Residuum mehr, aber auch an der Stelle $r_{n}$ nicht. Denn dort wïrde das Residuum sein

$$
\left(r_{1}+r_{2}+\cdots+r_{n}\right)
$$

was nach (23) gleich Null ist. Daher ist $d v$ cin bifferential zweiter Gattung. ledes Integral $w$ des Körpers läBt sich also in der Form darstellen

$$
w=v-\mathrm{r}_{1} w_{\mathrm{r}_{1} \mathrm{r}_{1}}+\mathrm{r}_{2} w_{\mathrm{r}_{2} \mathrm{r}_{n}}+\cdots+\mathrm{r}_{n} w_{\mathrm{r}_{0} \mathrm{r}}
$$

wo $v$ ein Integral zweiter Gattung ist. Benulzen wir noch die Sätze über die Darstellbarkeit der Integrale erster und zweiter Gattung, so haben wir:

Sind $u_{1}, u_{2}, \ldots u_{p} p$ lincar unabhängige Integrale erster und $v_{1}, v_{2}, \ldots v_{p} p$ linoar unahhängige Intugralezweiter Gattung. 
so läbt sich jedes Integral $w$ des Körpers in der Form darstellen

$$
\begin{gathered}
u^{\prime}=S(x, y)+a_{1} u_{1}+a_{2} u_{2}+\cdots+a_{p} u_{p}+b_{1} v_{1}+b_{2} v_{2}+\cdots+b_{p} v_{p} \\
-r_{1} u_{\mathrm{r}_{1} \mathrm{r}_{0}}+r_{2} u_{\mathrm{r}_{2} \mathrm{r}_{n}}-\cdots+r_{v} u_{\mathrm{r}_{0} \mathrm{r}_{0}},
\end{gathered}
$$

wo suine Funktion des Körpers ist und wo dir $a, b, c$ Konstante sind. Dabei ist angenommen, daß das Differential $d w$ nur an den $\varrho$ Stellen $1_{1}, r_{2}, \ldots r_{0}$ Residuen hat und daß das Residuum in $r_{t}$ gleich $r_{r}$ ist.

Als Beispiele für die Betrachtungen dieses hapitrls seien dem Leser die Integrale eines elliptisehen oder hyperelliptischen hörpers vom Ges'hlechte 2 empfohlen. 


\section{Namen- und Sachregister.}

Abel 1.

Additionssysteme 92.

Adjungierte Entwicklungen 15.

Funktionen ?1)!

hilrven 201.

- Sirsteme 85, -

Algebraische Funktion 5 .

- Kurve 171, 189.

Agebraischer Kürper t?.

Analysis situs 214 .

Basis des liörpers 105.

- für die Vielfachen eines Divisor's 104. 119.

Bezoutscher Satz 198.

Jirationale Transformation 62 .

Brill 2, 4.

Cauchy 1.

Clebsch̆ 2.

Dedekind 3.

Deseartes 1.

Diagramm von Pujsellx 21 .

Differential erster Giattung 153, 20.4.

Differentialklasse 8i.

Differentialt iler 206 .

Dimension einer Klasse is.

Dirichlet?.

Diskriminante cincr algebraischen Gileichung 5.

- einer Basis 106.

bivisor der mehrfathen Punkte 1it, 183, 196.

ter mehrfachen Tangenten 196.

der stationären Punle 17s, 183, 194.

der stationären 'Tangenten 17s. 194.

1)ivisoren 72 .

livisorenklassen it.

loppelpunktdivisor $17 t$.

Einfache zusanmenhängende Flächen 214 .

Einheiten (Potenzreihen) 11.

- (Systeme) 93.

Einheitssytem $\rightarrow 9$.

Element einer algebrais hen Funktion $7,16$.

Elementarintegrale "2. 1iattung 236 .

- 3. Gattuner $24:$.

Elliptische Körper 115:.

Ergänzungsdivisoren 150 !.

Ergällzungsklassen $13^{\prime \prime}$.
Fundamentalsstem für die Vielfachen rines Divisors 104.

Ganze Divisoren i:.

Gauls. F. 1.

(ieschlecht eines algebraishen Kijpers oder piner algebraischen bunktion order kurse (4), $152,153,158$.

Hauptilasse it.

Hauptwert eines hutegrals 2:31.

Ilensel, K. 4, 84, 171, 189, 2019.

Hessesche Kurve 196.

Hyperelliptische Kiirper 162.

Idealnorm 124.

Index der spezicllität 150.

Integrale 226 .

- 1., 2., 3. Gattung 23t.

Jakobi 2 .

Jordan 2.

Kanonische Klar.ie oly.

Kanonisches Zerschneiden einer Riemannschen Fläche 223 .

Klasse einer Kurve 193.

Klassen (von Divisoren) $7 t$.

Komplementïre Basen 134.

- Divisoren 141

- Systeme 91.

Konjugierte Entwicklungen 1i.

- Funktionen 50 .

kronecker 84.

hurvenzweig 17 t.

Landsberg, 6. 4, 171, 189, 26:3.

Linear unabhängige Divisoren is.

- Integrale 1. Gattung 2031.

- - - 2. Gattung $239,2.2$.

Lineare Cnabhängigkeit ron Integtalen 1. Gatting 234 .

- - - 2. Gattung esiti.

Joch 214 .

logarithmische Perioden 234.

loria. G. 2uri.

lückensatz von Wuierstrab 24:-

Lïrothscher satz $15 \%$.

Mehrfach zusammenhängende Flichen 214.

Mehriache Punkte einer liurse 17:. 
Mehriache Tangenten einer liurve 196.

Iehrfacher l'unkt mit epetremnten Tamgenten 15.5 .

Multiplikation von systemen $86,87$.

Inttiplikattionssystemic 91.

Newton 1.

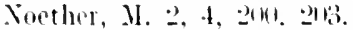

Noetherseher satz 203.

Norm $51 \%$.

Normalbasen fïr endliches $x 111$.

- - mnentliches $x$ 1ti.

Ordnumg des Zusammenhanges einer Fladede 21 s.

- Riemannschen Fläble 2.2.).

eines llivisurs $7: 2$.

einer Funktion 60, 145 .

lilasse 74

liurve 1ii, 193.

Perioden oder Periodizitätsmodun 234.

Plïcker, J. 197, 195.

Pliiekersche Formeln 19s.

Pole 55.

Primteiler i2.

Produkt von Systemen sti.

- zweier lilasien 75.

Puiseux 1. 21.

Punkt riner Rirmannschen fiärhe und seine limgelsung tij.

Querschnitt 21t.

Guotint zweier lilassen 75.

Rationale Kürper $15 \overline{\text { i }}$

Reguläre lilassen 15!l.

Residuen 55, 2.99.

Reziprokalkurve 191.

Reziproke Systeme s.t.

Pirmann, B. 1, 2. 32. 37, 15), 152. 2214.

Riemann-Rochschur Satz 152 .

Piemannsche Flï he 32, 37. ㄴ14.

Riemannscher Satz 151 .

RiB 214.
Roch 150, 15:.

Rosinkurve 206 .

Riickkehrpunkt $17 \%$.

Schnabelspitze 178.

sichnittpunkte zweier algebraischen liurven $187,198$.

Selbstberülıruugspunkt 184 .

sieveri. F. 4.

cinguläre Werte der unabhängigen Verinderlichen 6 .

sipezielle klassen 150 .

sipitze 17 .

sipur 511.

Stationire 'angente 178, 194.

Stationïrer l'mikt 177, 194.

stelle riner algehraischen Kurve 174.

systeme \&4.

Tangente 17i, 1!M!

Unendlich ferme Punkte einer algebraischen Kurve $171,15 \bar{i}, 19 \%$.

Vertausehungssysteme !).2.

Verzweigungsdivisoren it.

Verzweigungsordnung 48.

Verzweigungsprimteiler $\mathrm{it}$.

Verzweigungspunkt 48 .

Verzweigmoszahl einer Riemannschen Fläche +5 .

- in bezng auf eine Veränderliche 74 .

Vielfithe eines Divisors 100.

Weber, II. 3.

Weierstrab, k. 3, 4, 170, 242.

Weierstraßpunkte $170,242$.

Wende-Punkte und Tangenten 178.

Windungspunkt 48 .

Zugehörige Funktion zu einem 'sweig 175.

Zweiblättrige Riemannsche Flächen 45 .

Zweig einer algebraischen Funktion 7, 16.

_ _ - Kurve 174.

7rklische Perioden 234. 
$=6$

b.

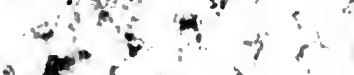

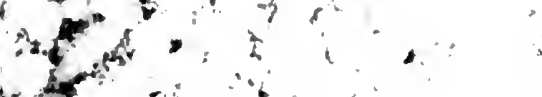

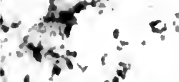

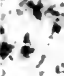

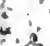

$\log ^{4}$

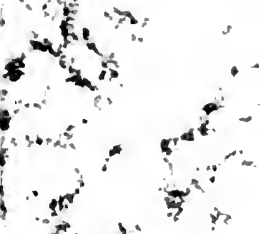

th +2

t?

(2)

and

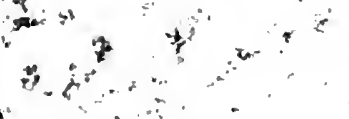

5

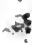

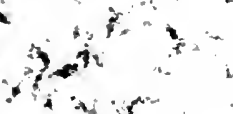

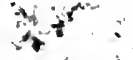

i.

$\because \cdots$

$\div$

7

i.
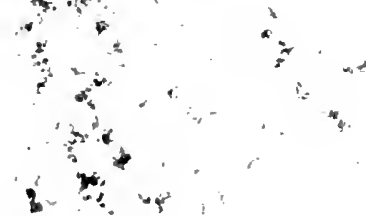

2. $\because 4$

$\because a^{\circ} \quad \&=$

,

s?

tis

$x=6 a^{\circ}$

is.

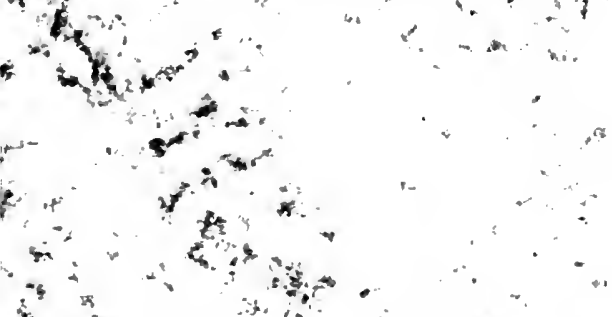


